\title{
AJOM new records and collections of fungi: 1-100
}

Hyde $\mathrm{KD}^{1,2,3}$, de Silva $\mathrm{NI}^{1,6,7^{*}}$, Jeewon $\mathrm{R}^{14}$, Bhat $\mathrm{DJ}^{26}$, Phookamsak $\mathrm{R}^{6,17,18}$, Doilom $\mathbf{M}^{6,17,18}$, Boonmee $S^{1,2}$, Jayawardena $\operatorname{RS}^{1,2,3}$, Maharachchikumbura $\mathrm{SSN}^{4}$, Senanayake IC ${ }^{19}$, Manawasinghe IS $^{1,2,3,11}$, Liu NG ${ }^{2,5,13}$, Abeywickrama PD ${ }^{1,2,11}$, Chaiwan $\mathrm{N}^{1,2,3}$, Karunarathna $\mathrm{A}^{6,8,12}$, Pem $\mathrm{D}^{1,2,3}$, Lin $\mathrm{CG}^{1,2,3,4}$, Sysouphanthong $P^{1,21}$, Luo $Z^{23}$, Wei $D^{1,2,3,6}$, Wanasinghe $D^{6}$, Norphanphoun $C^{1,2,3}$, Tennakoon $\mathrm{DS}^{1,2,3,12}$, Samarakoon $\mathrm{MC}^{4,7}$, Jayasiri $\mathrm{SC}^{1,2,3}$, Jiang $\mathrm{HB}^{1,2,3,6}$, Zeng $\mathrm{XY}^{1,22}$, Li $\mathrm{JF}^{1,2,3,6}$, Wijesinghe $\mathrm{SN}^{1,2,3,20}$, Devadatha $\mathrm{B}^{1,15}$, Goonasekara ID ${ }^{1,2,3,6}$, Brahmanage RS $^{1,2,3,11}$, Yang EF ${ }^{6}$, Aluthmuhandiram JVS ${ }^{1,2,11}$, Dayarathne MC $\mathbf{C}^{1,20}$, Marasinghe $\mathrm{DS}^{2,3,12}$, Li WJ ${ }^{1,2,3,6}$, Dissanayake $\mathbf{L S}^{22}$, Dong $\mathrm{W}^{1,3,8,24}$, Huanraluek $\mathrm{N}^{1,2}$, Lumyong $\mathrm{S}^{7,10}$, Liu $\mathrm{JK}^{4}$, Karunarathna $\mathrm{SC}^{6,17}$, Jones $\mathbf{E B G}^{25}$, Al-Sadi $\mathbf{A M}^{16}$, Xu JC ${ }^{6,17,18}$, Harishchandra $\mathrm{D}^{1,2,3,11}$, Sarma $\mathrm{VV}^{15}$ and Bulgakov $\mathrm{TS}^{27}$

${ }^{1}$ Center of Excellence in Fungal Research, Mae Fah Luang University, Chiang Rai 57100, Thailand

${ }^{2}$ School of Science, Mae Fah Luang University, Chiang Rai 57100, Thailand

${ }^{3}$ Mushroom Research Foundation, 128 M.3 Ban Pa Deng T. Pa Pae, A. Mae Taeng, Chiang Mai 50150, Thailand

${ }^{4}$ School of Life Science and Technology, University of Electronic Science and Technology of China, Chengdu 611731, People's Republic of China

${ }^{5}$ Guizhou Key Laboratory of Agricultural Biotechnology, Guizhou Academy of Agricultural Sciences, Guiyang 550006, People's Republic of China

${ }^{6}$ Key Laboratory for Plant Biodiversity and Biogeography of East Asia (KLPB), Kunming Institute of Botany, Chinese Academy of Science, Kunming 650201, Yunnan, People's Republic of China

${ }^{7}$ Department of Biology, Faculty of Science, Chiang Mai University, Chiang Mai, 50200, Thailand

${ }^{8}$ Department of Entomology and Plant Pathology, Faculty of Agriculture, Chiang Mai University, Chiang Mai 50200, Thailand

${ }^{9}$ Research Center of Excellence in Bioresources for Agriculture, Industry and Medicine, Faculty of Science, Chiang Mai University, Chiang Mai, 50200, Thailand

${ }^{10}$ Academy of Science, The Royal Society of Thailand, Bangkok 10300, Thailand

${ }^{11}$ Beijing Key Laboratory of Environment Friendly Management on Fruit Diseases and Pests in North China, Institute of Plant and Environment Protection, Beijing Academy of Agriculture and Forestry Sciences, Beijing, 100097, People's Republic of China

${ }^{12}$ Department of Plant Medicine, National Chiayi University, 300 Syuefu Road, Chiayi City 60004, Taiwan

${ }^{13}$ Faculty of Agriculture, Natural Resources and Environment, Naresuan University, Phitsanulok, 65000, Thailand

${ }^{14}$ Department of Health Sciences, Faculty of Science, University of Mauritius, Reduit,Mauritius

${ }^{15}$ Fungal Biotechnology Laboratory, Department of Biotechnology, Pondicherry University, Kalapet, Pondicherry605014, India

${ }^{16}$ Department of Crop Sciences, College of Agricultural and Marine Sciences, Sultan Qaboos University, P.O. Box 34, Alkhoud, 123 Muscat, Oman

${ }^{17}$ East and Central Asia Regional Office, World Agroforestry Centre (ICRAF), Kunming 650201, Yunnan, China

${ }^{18}$ Centre for Mountain Futures (CMF), Kunming Institute of Botany, Kunming 650201, Yunnan, People's Republic of China

${ }^{19}$ Shenzhen Key Laboratory of Microbial Genetic Engineering, College of Life Science and Oceanography, Shenzhen University, 3688,Nanhai Avenue, Nanshan, Shenzhen 518055, People's Republic of China

${ }^{20}$ Department of Plant Pathology, Agriculture College, Guizhou University, Guiyang, Guizhou Province, 550025, People's Republic of China

${ }^{21}$ Ecology Division, Biotechnology and Ecology Institute, Ministry of Science and Technology, P.O.Box: 2279, Vientiane Capital, Lao PDR

${ }^{22}$ Engineering Research Center of the Utilization for Characteristic Bio-Pharmaceutical Resources in Southwest, 
Ministry of Education, Guizhou University, Guiyang, Guizhou Province 550025, People's Republic of China

${ }^{23}$ College of Agriculture and Biological Sciences, Dali 671003, Yunnan Province, People's Republic of China

${ }^{24}$ Yunnan Institute of Food Safety, Kunming University of Science and Technology, Kunming 650500, People's Republic of China

${ }^{25}$ Department of Botany and Microbiology, College of Science, King Saud University, Riyadh, Kingdom of Saudi Arabia; dNantgaredig, Southsea, UK

${ }^{26}$ Formerly Department of Botany, Goa University, Goa, India; No. 128/1-J, Azad Housing Society, Curca, Goa Velha, India

${ }^{27}$ All-Russian Scientific Research Institute of Floriculture and Subtropical Crops, Sochi, Krasnodar region, Russia

Hyde KD, de Silva NI, Jeewon R, Bhat DJ, Phookamsak R, Doilom M, Boonmee S, Jayawardena RS, Maharachchikumbura SSN, Senanayake IC, Manawasinghe IS, Liu NG, Abeywickrama PD, Chaiwan N, Karunarathna A, Pem D, Lin CG, Sysouphanthong P, Luo ZL, Wei DP, Wanasinghe DN, Norphanphoun C, Tennakoon DS, Samarakoon MC, Jayasiri SC, Jiang HB, Zeng XY, Li JF, Wijesinghe SN, Devadatha B, Goonasekara ID, Brahmanage RS, Yang EF, Aluthmuhandiram JVS, Dayarathne MC, Marasinghe DS, Li WJ, Dissanayake LS, Dong W, Huanraluek N, Lumyong S, Liu JK, Karunarathna SC, Jones EBG, Al-Sadi AM, Xu JC, Harishchandra D, Sarma VV 2020 AJOM new records and collections of fungi: 1-100. Asian Journal of Mycology 3(1), 22-294, Doi 10.5943/ajom/3/1/3

\begin{abstract}
This paper is the first in the AJOM series in which we report 100 new collections of fungi which include new species, host and country records. In all, nine new species, 90 new records and one new combination are introduced. The purpose of this series is to provide an outlet for publishing collections with sequence data, so that these observations will not be wasted and mycologists can use the information to update fungal classification and better identification of species. Previously, numerous species were described from the first collection and no further data on the species were published as it was considered low impact. This series will, therefore, increase the knowledge on the host occurrence, biogeography and sequence variability in each taxon dealt with. The distribution and hosts for each listed species are added if backed up by sequence data.
\end{abstract}

Key words -9 new taxa - 90 new records - Ascomycota - Dothideomycetes - Leotiomycetes Molecular phylogeny - Sordariomycetes - Taxonomy

Table of contents

Phylum Ascomycota

Class Dothideomycetes

Subclass Dothideomycetidae P.M. Kirk et al.

Capnodiales Woron.

Phaeothecoidiellaceae K.D. Hyde \& Hongsanan

1. Chaetothyrina guttulata Hongsanan \& K.D. Hyde, Mycosphere 8: 142 (2016) (contributed by Zeng XY)

Subclass Pleosporomycetidae C.L. Schoch et al.

Hysteriales Lindau

Hysteriaceae Chevall.

2. Gloniopsis calami Konta \& K.D. Hyde, Fungal Diversity 80: 34 (2016) (contributed by Boonmee S)

3. Gloniopsis calami Konta \& K.D. Hyde, Fungal Diversity 80: 34 (2016) (contributed by Luo ZL)

4. Hysterium angustatum Alb. \& Schwein., Consp. fung. (Leipzig): 55 (1805) (contributed by Boonmee S) 
5. Rhytidhysteron thailandicum Thambug. \& K.D. Hyde, Cryptog. Mycol. 37(1): 110 (2016) (contributed by Doilom M)

6. Rhytidhysteron thailandicum Thambug. \& K.D. Hyde, Cryptog. Mycol. 37(1): 110 (2016) (contributed by Wanasinghe DN)

Pleosporales Luttrell ex M.E. Barr

Anteagloniaceae K.D. Hyde, J.K. Liu \& A. Mapook

7. Anteaglonium parvulum (Schwein.) Mugambi \& Huhndorf, Syst. Biodiv. 7(4): 460 (2009) (contributed by Boonmee $S$ )

Astrosphaeriellaceae Phookamsak \& K.D. Hyde

8. Astrosphaeriella neostellata D.Q. Dai, Phookamsak \& K.D. Hyde, Fungal Diversity 74: 172 (2015) (contributed by Jiang HB)

Camarosporidiellaceae Wanas., Wijayaw., Crous \& K.D. Hyde

9. Camarosporidiella celtidis (Shear) Thambug., Wanas. \& K.D. Hyde, Stud. Mycol. 87: 226 (2017) (contributed by Tennakoon DS)

10. Camarosporidiella laburni (Pers.) Wanas., Bulgakov, Camporesi \& K.D. Hyde. Stud. Mycol. 87: 233 (2017) (contributed by Tennakoon DS)

Corynesporaceae Locq.

11. Corynespora doipuiensis J.F. Li \& Phookamsak, sp. nov. (contributed by Li JF)

12. Corynespora submersa Z.L. Luo, H.Y. Su \& K.D. Hyde, sp. nov. (contributed by Luo ZL)

13. Corynespora lignicola Z.L. Luo, H.Y. Su \& K.D. Hyde, sp. nov. (contributed by Luo ZL)

Dictyosporiaceae Boonmee \& K.D. Hyde

14. Dendryphiella eucalyptorum Crous \& E. Rubio, Persoonia 32: 231 (2014) (contributed by Liu NG)

15. Neodendryphiella tarraconensis González, Gené \& Dania García, Mycokeys 37: 30 (2018) (contributed by Liu NG)

Didymellaceae Gruyter, Aveskamp \& Verkley

16. Ascochyta herbicola Qian Chen \& L. Cai, Stud. Mycol. 82: 187 (2015) (contributed by Abeywickrama PD)

17. Calophoma humulicola Chaiwan, T.C Bulgakov, Jayaward \& K.D. Hyde, sp. nov. (contributed by Chaiwan $\mathrm{N}$ )

18. Epicoccum latusicollum Qian Chen, Crous \& L. Cai, Stud. Mycol. 87: 144 (2017) (contributed by Manawasinghe IS)

19. Nothophoma pruni Chethana, J.Y. Yan, X.H. Li \& K.D. Hyde, Mycosphere 10(1): 520 (2019) (contributed by Manawasinghe IS)

20. Nothophoma quercina (Syd. \& P. Syd.) Qian Chen \& L. Cai, Stud. Mycol. 82: 213 (2015) (contributed by Manawasinghe IS)

21. Phomatodes nebulosa (Pers.) Qian Chen \& L. Cai, Stud. Mycol. 82: 191 (2015) (contributed by Manawasinghe IS)

Didymosphaeriaceae Munk = Montagnulaceae M.E. Barr, Mycotaxon 77: 194 (2001)

22. Neokalmusia didymospora D.Q. Dai \& K.D. Hyde (contributed by Karunarathna A)

23. Paracamarosporium fagi Crous \& R.K. Schumach., Sydowia 67: 109 (2015) (contributed by Pem D)

24. Paraconiothyrium cyclothyrioides Verkley, da Silva, Wicklow \& Crous, Stud. Mycol. 50(2): 330 (2004) (contributed by Devadatha B) 
Leptosphaeriaceae M.E. Barr

25. Plenodomus collinsoniae (Dearn. \& House) Gruyter, Aveskamp \& Verkley, Stud. Mycol. 75: 21 (2012) (contributed by Brahmanage RS)

\section{Lophiostomataceae Sacc.}

26. Vaginatispora nypae Jayasiri, E.B.G. Jones \& K.D. Hyde, Mycosphere 10(1): 84 (2019) (contributed by Boonmee $S$ )

Lophiotremataceae K. Hiray. \& Kaz.

27. Atrocalyx bambusae (Phookamsak, S.C. Karunarathana \& K.D. Hyde) N.I. de Silva \& K.D. Hyde, Phytotaxa 333: 204 (2018) (contributed by Jiang HB)

28. Lophiotrema neohysterioides M.E. Barr, Mycotaxon 45: 208, 1992 (contributed by Jayasiri $\mathrm{SC})$

Massariaceae Nitschke

29. Massaria anomia (Fr.) Petr., Annls Mycol. 21:114 (1923) (contributed by Pem D)

Massarinaceae Munk

30. Pseudosplanchnonema phorcioides (I. Miyake) Chethana, Camporesi \& K.D. Hyde, Phytotaxa 231(2): 139 (2015) (contributed by Pem D)

Melanommataceae G. Winter (= Pseudodidymellaceae A. Hashim. \& Kaz. Tanaka)

31. Byssosphaeria musae Phookamsak \& K.D. Hyde, Fungal Diversity 72: 119 (2015) (contributed by Yang EF/Phookamsak R)

Morosphaeriaceae Suetrong et al.

32. Helicascus elaterascus (Shearer) H. Zhang \& K.D. Hyde, Cryptog. Mycol. 33: 158 (2012) (contributed by Boonmee S)

Paradictyoarthriniaceae Doilom et al.

33. Paradictyoarthrinium diffractum Matsush., Matsush. Mycol. Mem. 9:18 (1996) (contributed by Doilom M)

34. Paradictyoarthrinium hydei N.G. Liu \& J.K. Liu, Phytotaxa 338(3): 290 (2018) (contributed by Dong W)

Phaeosphaeriaceae M.E. Barr

35. Leptospora rubella Rabenh., Klotzschii Herb. Viv. Mycol., Edn 2: no. 532 (1857) (contributed by Yang EF/Phookamsak R)

36. Pseudoophiobolus pseudoitalicus Senan., \& K.D. Hyde, sp. nov. (contributed by Senanayake IC)

Pleosporaceae Nitschke

37. Bipolaris yamadae (Y. Nisik.) Shoemaker, Can. J. Bot. 37(5): 884 (1959) (contributed by Karunarathna A)

38. Curvularia hawaiiensis (Bugnic. ex M.B. Ellis) Manamgoda, L. Cai \& K.D. Hyde, Fungal Diversity 56(1): 141 (2012) (contributed by Maharachchikumbura SSN)

39. Curvularia muehlenbeckiae Madrid, K.C. Cunha, Gené, Guarro \& Crous, Persoonia 33: 56 (2014) (contributed by Maharachchikumbura SSN)

40. Curvularia subpapendorfii (Mouch.) Manamgoda, Rossman \& K.D. Hyde, Stud. Mycol. 79: 282 (2014) (contributed by Maharachchikumbura SSN)

41. Curvularia verruculosa Tandon \& Bilgrami ex M.B. Ellis, Mycol. Pap. 106: 20 (1966) (contributed by Maharachchikumbura SSN) 
42. Pyrenophora trichostoma (Fr.) Fr., Jb. nassau. Ver. Naturk. 23-24: 215 (1870) [1869-70] (contributed by Goonasekara ID)

Roussoellaceae J.K. Liu et al.

43. Pseudoneoconiothyrium rosae (Phukhams., Camporesi \& K.D. Hyde) Phukhams., Camporesi \& K.D. Hyde, Index Fungorum 357: 1 (2018) (contributed by Wijesinghe SN)

44. Pseudoneoconiothyrium rosae (Phukhams., Camporesi \& K.D. Hyde) Phukhams., Camporesi \& K.D. Hyde, Index Fungorum 357: 1 (2018) (contributed by Wijesinghe SN)

45. Roussoella siamensis Phook., Jian K. Liu \& K.D. Hyde, Phytotaxa 181(1): 18 (2014) (contributed by Karunarathna A)

Sporormiaceae Munk

46. Forliomyces uniseptatus Phukhams., Camporesi \& K.D. Hyde, Cryptog. Mycol. 37(1): 84 (2016) (contributed by Abeywickrama PD)

Tetraplosphaeriaceae Kaz. Tanaka \& K. Hiray

47. Polyplosphaeria fusca Kaz. Tanaka \& K. Hiray., Studies in Mycology 64: 193 (2009) (contributed by Jiang HB)

48. Tetraploa sasicola (Kaz. Tanaka \& K. Hiray.) Kaz. Tanaka \& K. Hiray. Fungal Diversity 63: 253 (2013) (contributed by Karunarathna A)

Torulaceae Corda

49. Dendryphion nanum (Nees) S. Hughes, Canadian Journal of Botany 36 (6): 761 (1958) (contributed by Liu NG)

50. Torula chromolaenae J.F. Li, Phookamsak, A. Mapook \& K.D. Hyde, Mycol Progress 16:447461 (2017) (contributed by Phookamsak R)

Asterinales M.E. Barr ex D. Hawksw. \& O.E. Erikss. (=Asterotexales Firmino et al.)

Asterinaceae Hansf. (= Lembosiaceae Hosag.)

51. Asterostomella grewiae Petr., Sydowia 12: 485 (1959) [1958] (contributed by Zeng XY)

Botryosphaeriales C.L. Schoch et al.

Aplosporellaceae Slippers et al.

52. Aplosporella javeedii Jami, Gryzenh., Slippers \& M.J. Wingf., Fungal Biology 118(2): 174 (2013) (contributed by Phookamsak R)

53. Aplosporella prunicola Damm \& Crous, Fungal Diversity 27(1): 39 (2007) (contributed by Samarakoon MC)

Botryosphaeriaceae Theiss. \& H. Syd.

54. Barriopsis stevensiana A.J.L. Phillips \& Pennycook, Fungal Diversity: 10.1007/s13225017-0386-0, [56] (2017) (contributed by Doilom M)

55. Botryosphaeria dothidea (Moug.) Ces. \& De Not., Comm. Soc. crittog. Ital. 1(fasc. 4): 212 (1863) (contributed by Manawasinghe IS)

56. Diplodia galiicola Dissanayake, Camporesi \& K.D. Hyde, Fungal Diversity, 75: 54 (2015) (contributed by Aluthmuhandiram JVS)

57. Dothiorella alpina (Y. Zhang ter \& Ming Zhang) Phookamsak \& Hyde, comb. nov. (contributed by Phookamsak R)

58. Dothiorella sarmentorum (Fr.) A.J.L. Phillips, J. Luque \& A. Alves, Mycologia 97: 522 (2005) (contributed by Aluthmuhandiram JVS)

59. Dothiorella symphoricarposicola W.J. Li, Jian K. Liu \& K.D. Hyde, Cryptog. Mycol. 35(3): 265 (2014) (contributed by Abeywickrama PD)

60. Lasiodiplodia theobromae (Pat.) Griffon \& Maubl., Bull. Soc. Mycol. Fr. 25: 57 (1909) 
(contributed by Doilom M)

61. Neofusicoccum parvum (Pennycook \& Samuels) Crous, Slippers \& A.J.L. Phillips, Stud. Mycol. 55: 248 (2006) (contributed by Doilom M)

Venturiales Y. Zhang ter, C.L. Schoch \& K.D. Hyde

Sympoventuriaceae Y. Zhang ter, C.L. Schoch \& K.D. Hyde

62. Ochroconis musae (G.Y. Sun \& Lu Hao) Samerp. \& de Hoog, Mycological Progress 14 (2/6): 8 (2015) (contributed by Chaiwan N)

Class Eurotiomycetes Tehler ex O.E. Eriksson \& K. Winka

Chaetothyriales M.E. Barr

Trichomeriaceae Chomnunti \& K.D. Hyde

63. Trichomerium camporesii Marasinghe \& K.D. Hyde sp. nov. (contributed by Marasinghe DS)

Class Lecanoromycetes O.E. Erikss. \& Winka

Lecanoromycetidae families incertae sedis

Ostropales Nannf

Stictidaceae Fr.

64. Stictis urceolata (Ach.) Gilenstam (Ach.) Gilenstam, Lichenologist 37(1): 74 (2005) (contributed by Karunarathna A)

Class Leotiomycetes O.E. Erikss. \& Winka

Helotiales Nannf

Vibrisseaceae Korf

65. Phialocephala humicola S.C. Jong \& E.E. Davis, Mycologia 64 (6): 1352 (1972) (contributed by Liu NG)

Rhytismatales genera incertae sedis

66. Apiculospora spartii Wijayaw., W.J. Li, Camporesi, A.J.L. Phillips \& K.D. Hyde, Fungal Diversity:(2016) (contributed by Li WJ)

Class Sordariomycetes O.E. Erikss. \& Winka

Subclass Diaporthomycetidae Senan. et al.

Atractosporales H. Zhang et al.

Conlariaceae H. Zhang, K.D. Hyde \& Maharachch.

67. Conlarium aquaticum W. Dong., H. Zhang \& K.D. Hyde, Fungal Diversity 85: 75-110 (2017) (contributed by Phookamsak R / Yang EF)

Diaporthales Nannf.

Cryphonectriaceae Gryzenh. \& M.J. Wingf.

68. Aurifilum marmelostoma Begoude, Gryzenh. \& Jol. Roux, Antonie van Leeuwenhoek 98 (3): 273 (2010) (contributed by Samarakoon MC)

Cytosporaceae Fr.

69. Cytospora cedri Syd., P. Syd. \& E.J. Butler, Annls mycol. 14(3/4): 193 (1916) (contributed by Senanayake IC)

70. Cytospora fraxiicola Chaiwan, Bulgakov, T.C., Jayaward \& Hyde, sp. nov. (contributed by Chaiwan N)

71. Cytospora malicola Z. Urb., Česká Mykol. 10(4): 209 (1956) (contributed by Senanayake IC)

72. Cytospora melnikii Norphanphoun, Doilom, Daranagama, Phookamsak, Wen, Bulgakov \& 
Hyde, Mycosphere 8(1): 68 (2017) (contributed by Chaiwan N)

Diaporthaceae Höhn. ex Wehm.

73. Diaporthe ambigua Nitschke, Pyrenomyc. Germ. 2: 311 (1870) (Abeywickrama PD)

74. Diaporthe foeniculina Udayanga \& Castl. Persoonia 32: 95 (2014) (Abeywickrama PD)

75. Diaporthe rumicicola Manawasinghe, Camporesi \& K.D. Hyde Fungal Diversity [138] (2019) (contributed by Aluthmuhandiram JVS)

76. Diaporthe sojae Lehman, Ann. Mo. bot. Gdn 10: 128 (1923) (contributed by Manawasinghe IS)

Melanconidaceae G. Winter

77. Melanconis stilbostoma (Fr.) Tul. \& C. Tul., Select. fung. carpol. (Paris) 2: 115 (1863) (contributed by Pem D)

Schizoparmaceae Rossman

78. Coniella eucalyptorum (Crous \& M.J. Wingf.) L.V. Alvarez \& Crous, Stud. Mycol. 85: 15 (2016) (contributed by de Silva NI)

Subclass Hypocreomycetidae O.E. Erikss. \& Winka

Glomerellales Chadef.

Glomerellaceae Locq. ex Seifert \& W. Gams

79. Colletotrichum fructicola Prihast., L. Cai \& K.D. Hyde, Fungal Diversity 39: 96 (2009) (contributed by Jayawardena RS/Huanraluek N)

80. Colletotrichum siamense Prihast., L. Cai \& K.D. Hyde, Fungal Diversity 39: 98 (2009) (contributed by Jayawardena RS/Huanraluek N)

81. Colletotrichum viniferum Li J. Peng, L. Cai, K.D. Hyde \& Zi Y. Ying, Mycoscience 54(1): 36 (2013) (contributed by Jayawardena RS/Huanraluek N)

Hypocreales Lindau

Cordycipitaceae Kreisel ex G.H. Sung et al.,

82. Cordyceps pruinosa Petch, Trans. Br. mycol. Soc. 10(1-2): 38 (1924) (contributed by Wei DP)

83. Cordyceps rosea Kobayasi \& Shimizu, Bull. natn. Sci. Mus., Tokyo, B 8(4): 112 (1982) (contributed by Wei DP)

Microascales Luttr. ex Benny \& Kimbr

Microascaceae Luttr. ex Malloch

84. Parascedosporium putredinis (Corda) Lackner \& de Hoog, IMA Fungus 2(1): 44 (2011) (contributed by Dayarathne MC)

Subclass Xylariomycetidae O.E. Erikss \& Winka

Amphisphaeriales D. Hawksw. \& O.E. Erikss.

Apiosporaceae K.D. Hyde et al.

85. Nigrospora oryzae (Berk. \& Broome) Petch, J. Indian bot. Soc. 4: 24 (1924) (contributed by Li JF)

86. Nigrospora sphaerica (Sacc.) E.W. Mason, Trans. Brit. Mycol. Soc. 12: 158. (1927) (contributed by Jayawardena RS/Huanraluek N)

Beltraniaceae Nann.

87. Beltrania dushanensis C.G. Lin, Jian K. Liu \& K.D. Hyde, sp. nov. (contributed by Lin CG)

88. Beltraniella brevis C.G. Lin, Jian K. Liu \& K.D. Hyde, sp. nov. (contributed by Lin CG) 
Sporocadaceae Corda

89. Bartalinia robillardoides Tassi, Bulletin Labor. Orto Bot. de R. Univ. Siena 3: 145 (1900) (contributed by Li WJ)

90. Discosia pini Heald, Mycologia 1(5): 216 (1909) (contributed by Wei DP)

91. Pestalotiopsis diploclisiae Maharachch., K.D. Hyde \& Crous, Stud. Mycol. 79: 160 (2014) (contributed by Norphanphoun C)

92. Pestalotiopsis kenyana Maharachch., K.D. Hyde \& Crous, Stud. Mycol. 79: 166 (2014) (contributed by Norphanphoun C)

93. Pseudopestalotiopsis kubahensis Lateef, M. Sepiah \& Bolhassan, Current Research in Environmental \& Applied Mycology 5(4): 378 (2014) (contributed by Goonasekara ID)

Xylariales Nannf.

Lopadostomataceae Daranag. \& K.D. Hyde

94. Whalleya microplaca (Berk. \& M.A. Curtis) J.D. Rogers, Y.M. Ju \& F. San Martín, Mycotaxon 64: 48 (1997) (contributed by Abeywickrama PD)

Xylariaceae Tul. \& Tul. (as 'xylariei')

95. Rosellinia convexa Q.R. Li \& J.C. Kang, Mycoscience 57(3): 166 (2016) (contributed by Dissanayake LS)

Sordariomycetes orders incertae sedis

Catabotryales K.D. Hyde \& Senan.

Catabotrydaceae Petr. ex M.E. Barr

96. Catabotrys deciduum (Berk. \& Broome) Seaver \& Waterston, Mycologia 38(2): 184 (1946) (contributed by Devadatha B)

Phylum Basidiomycota

Class Agaricomycetes Doweld

Subclass Agaricomycetidae Locq.

Agaricales Underw.

Agaricaceae Chevall.

97. Lepiota angusticystidiata J.F. Liang \& Z.L. Yang, Mycologia 110(3): 496 (2018) (contributed by Sysouphanthong P)

98. Lepiota attenuata J.F. Liang \& Zhu L. Yang, Mycologia 103(4): 821 (2011) (contributed by Sysouphanthong P)

99. Lepiota magnispora Murrill, Mycologia 4: 237 (1912) (contributed by Sysouphanthong P)

100 Lepiota thrombophora (Berk. \& Broome) Sacc., Syll. fung. (Abellini) 5: 53 (1887) (contributed by Sysouphanthong P)

\section{Introduction}

Fungal diversity is the key to many biological processes on earth. Fungi act as major decomposers and help in the recycling of nutrients in the environment (e.g. Hyde et al. 1998, Tang et al. 2005, Jeewon et al. 2003). Many others live in symbiotic associations with plants and exhibit endophytic lifestyles (Promputtha et al. 2005, 2007, Doilom et al. 2017a, Tibpromma et al. 2018a). A number of fungi also cause damage to crops and are economically important plant pathogens (Jayawardena et al. 2019a, b), but their diversity, nomenclature and roles still warrant further investigation (Hyde \& Soytong 2008, Jeewon et al. 2017). Current fungal diversity estimates range from 2.2 to 3.8 million (Hawksworth \& Lucking 2017) and it has been suggested that a large proportion of new species still awaits discovery and they possibly lie in tropical regions such as Thailand (Hyde et al. 2018). Our understanding of the ecological and evolutionary context of many fungi relies on their discovery from their natural environment. 


\section{Why is there a need for publications of new records based on molecular data?}

Taxonomic evaluation is an ongoing process, but a common misconception among many mycologists is that new fungal records are obsolete. The goal in fungal taxonomy lies in reconstituting biological knowledge and providing all information necessary to unravel species relationships and properly describing species. In this context, publishing of new records (even from new habitats or from new hosts) plays a significant role in species documentation. Provision of such data in easily accessed journals is beneficial as this acts as a pool of taxonomic information that can be used and shared by many mycologists and further provides a platform allowing taxonomists to properly identify species and infer more accurate fungal diversity estimates. Publishing and redescriptions of extant taxa should also be encouraged as these 1) provide supplementary data and sometimes insights into better descriptions of morphological data; 2) result in additional information on phylogenetic relationships especially when data are made available from protein and rDNA sequence data; 3) assist in reevaluating, updating and validating current taxonomic classification schemes which are always in transition; 4) provide additional taxonomic documentation to help manage the increasing number of new species and new records; 5) and allow opportunities for mycologists to update nomenclature of taxa and check for potential synonyms and amend species concepts.

Another important question to ask with regards new fungal records/collections is: "how are these fungi emerging from new hosts?" With documentation from these new host records, it will be easier for mycologists to gain insights into the extent to which fungi are evolving, their host range, jumping to novel hosts, host shift speciation and how they adapt to different environments. Previous checklists of plant pathogens (Jayawardena et al. 2019a, b), freshwater fungi (Luo et al. 2018), terrestrial fungi (Wanasinghe et al. 2018b, Hyde et al. 2019, Phookamsak et al. 2019, Pem et al. 2019) and marine fungi (Jones et al. 2019, Dayarathne et al. 2020) were based mostly on morphological identifications supplemented with DNA based sequence data. The application of phylogenetics based on DNA sequence data in the last decade has largely improved species identification and understanding of taxonomic relationships (Jayasiri et al. 2019, Zeng et al. 2019, Zhang et al. 2019a). These approaches have often shown species to be cryptic, as in genera such as Colletotrichum (Jayawardena et al 2016, Veloso et al. 2018) and Diaporthe (Senanayake et al. 2017), but in other cases species classification remains unresolved (e.g. Jeewon et al. 2018, Shang et al. 2020). Fungal host association has often been used to either delineate species or establish new species, but this has been reported to be erroneous (Jeewon et al. 2004). However, it is important to document species and gather data that can provide further insights into taxonomic complexity and fungal plant interactions useful for optimization of conservation practices. In this paper, we provide new data on the distribution and host records of taxa mostly in the Ascomycota with emphasis on phylogenetic inferences derived from DNA sequences of commonly used genes in molecular systematics. The paper complements Fungal Diversity notes (Hyde et al. 2019, Phookamsak et al. 2019) and Mycosphere notes (Hyde et al. 2018), where new species have been introduced and new records have been described with detailed descriptions, illustrations and updated information. With additional data, we anticipate that this paper will provide a more comprehensive taxonomic understanding of species.

\section{Materials and methods}

Materials and methods follow the previous Fungal Diversity Notes (Hyde et al. 2019, Phookamsak et al. 2019). Fresh and dried specimens in this study were collected from China, India, Italy, Oman, Russia, Taiwan, Thailand and Ukraine (further details for each taxon studied are given in the taxonomy section). Phylogenetic analyses were performed based on Bayesian inference (BI), maximum likelihood (ML) and maximum parsimony (MP) with details as outlined by Tang et al. (2007), Zhang et al. (2008) and Phukhamsakda et al. (2019). Establishment of new species and species differences are based on the guidelines outlined by Jeewon \& Hyde (2016). Faces of Fungi numbers and Index Fungorum numbers were registered as described in Jayasiri et al. (2015) and 
Index Fungorum (2020), respectively. The numbers of taxa in this study are organized as in the "Outline of Ascomycetes" (Wijayawardene et al. 2018).

\section{Taxonomy}

\section{Phylum Ascomycota}

Class Dothideomycetes

Subclass Dothideomycetidae P.M. Kirk et al.

For recent treatments of Dothideomycetes, we follow Liu et al. (2017b) and Wijayawardene et al. (2018).

\section{Capnodiales Woron.}

Phaeothecoidiellaceae K.D. Hyde \& Hongsanan

Phaeothecoidiellaceae was introduced by Hongsanan et al. (2017a) to accommodate Chaetothyrina, Houjia and Phaeothecoidiella in Capnodiales.

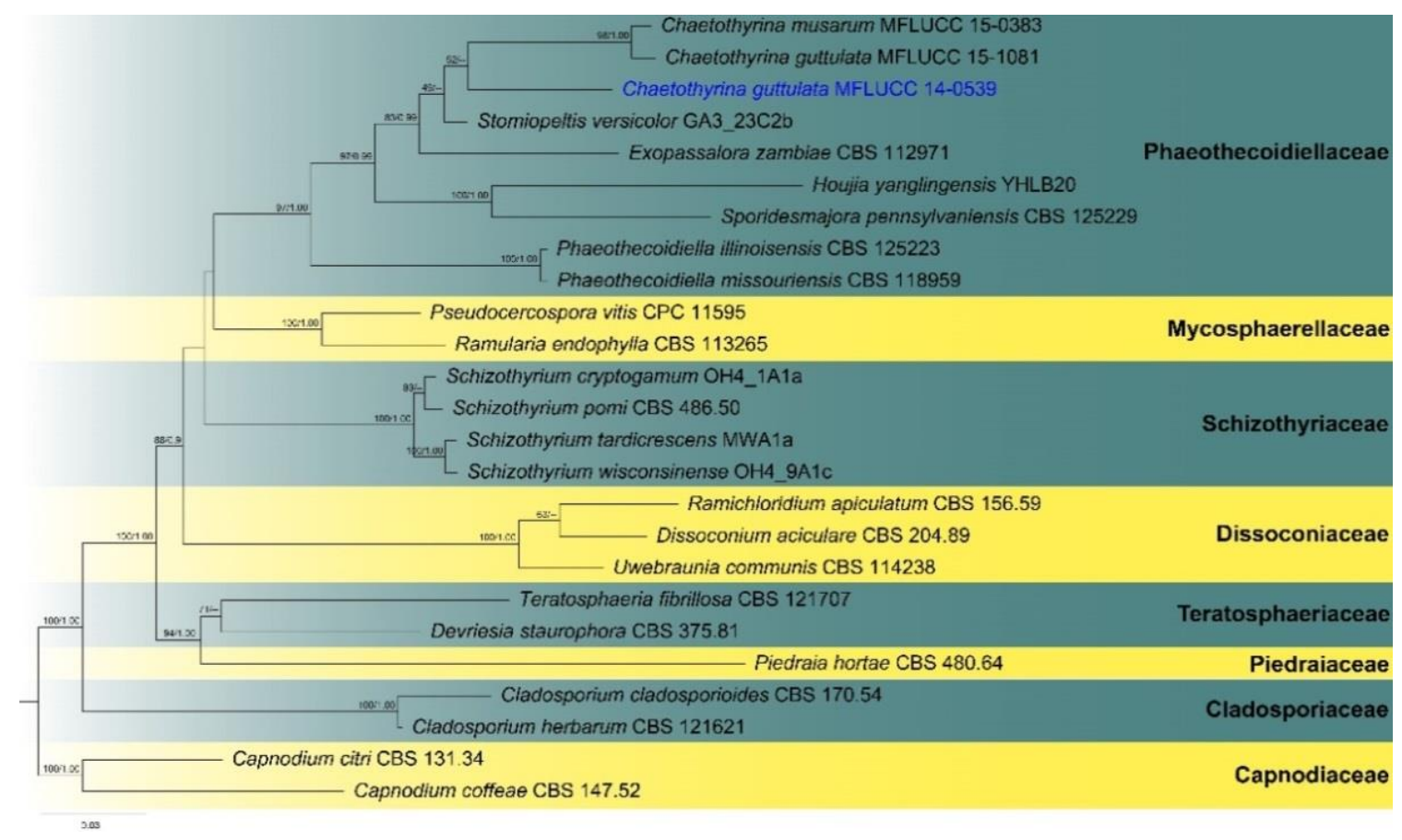

Fig. 1 - Phylogram generated from maximum likelihood analysis based on combined LSU, ITS and SSU sequence data. Twenty-five strains are included in the combined gene analyses comprising 2501 characters after alignment (850 characters for LSU, 634 characters for ITS, 1017 characters for LSU). Capnodium citri (CBS 131.34) and C. coffeae (CBS 147.52) are used as the outgroup taxa. The tree topology derived from the Bayesian analysis was similar to that derived from the maximum likelihood analysis. The best RaxML tree with a final likelihood value of -12303.332942 is presented. The matrix had 775 distinct alignment patterns, with $24.61 \%$ undetermined characters or gaps. Estimated base frequencies were as follows: $\mathrm{A}=0.244506, \mathrm{C}=0.238481, \mathrm{G}=0.282732, \mathrm{~T}$ $=0.234281$; substitution rates $\mathrm{AC}=1.302334, \mathrm{AG}=1.595992, \mathrm{AT}=1.294978, \mathrm{CG}=1.166465$, $\mathrm{CT}=5.090925, \mathrm{GT}=1.000000$; gamma distribution shape parameter $\alpha=0.428977$. Bootstrap values for maximum likelihood equal to or greater than 50 and Bayesian posterior probabilities equal or greater than 0.9 are placed above the branches. The newly generated sequence is indicated in blue.

\section{Chaetothyrina Theiss.}

Chaetothyrina was introduced as a member of Microthyriaceae. Singtripop et al. (2016) illustrated the type specimens of Chaetothyrina, along with a reference specimen $C$. musarum 
(MFLU 16-0884). Hongsanan et al. (2017a) transferred it to Phaeothecoidiellaceae based on phylogenetic analyses.

Chaetothyrina guttulata Hongsanan \& K.D. Hyde, Mycosphere 8: 142 (2016)

Fig. 2

Facesoffungi number: FoF01884

Epiphytes on surface of leaves and petioles of Mangifera indica. Sexual morph: Thyriothecia up to $170 \mu \mathrm{m}$ in diameter, superficial, solitary, subdense, dark brown, circular, flattened, with an irregular central ostiole. Upper wall comprising cells of textura angularis, seated on a thin, hyaline basal stroma. Pseudoparaphyses filiform, septate, hyaline. Asci $26-45 \times 11-17 \mu \mathrm{m}(\bar{x}=35 \times 14$ $\mu \mathrm{m}, \mathrm{n}=30$ ), bitunicate, 8-spored, fusoid, fusiform or clavate, with a short pedicel. Ascospores 11$15 \times 3-5 \mu \mathrm{m}(\bar{x}=13 \times 4 \mu \mathrm{m}, \mathrm{n}=30), 2-3$-seriate, hyaline, ellipsoidal to oblong, 1-septate, constricted at the septum, with 2 guttules in each cell,. Asexual morph: Undetermined.

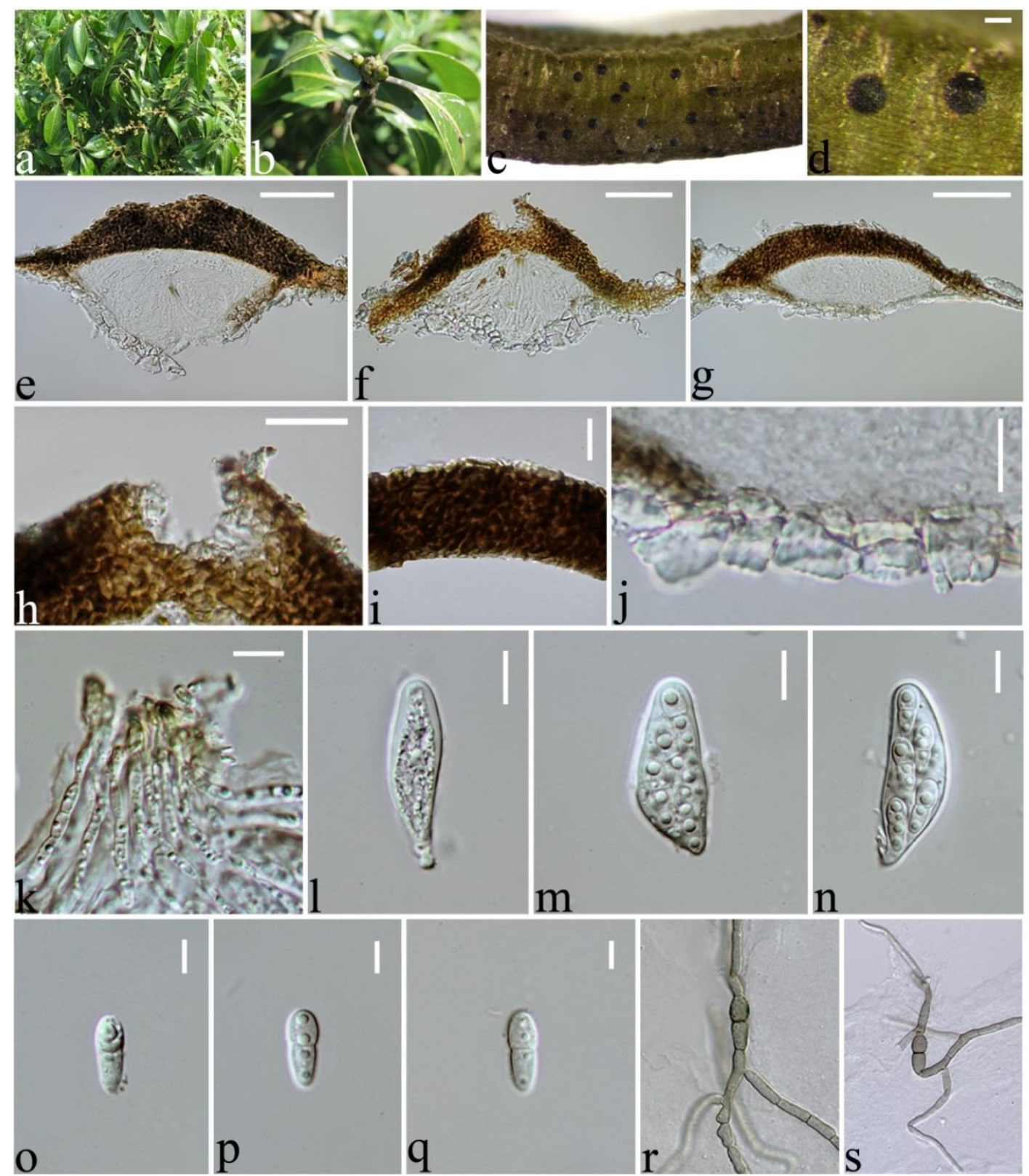

Fig. 2 - Chaetothyrina guttulata (MFLU 14-0006, new sequence data). a, b Host plant. c, d Ascomata. e-g Ascomata cross sections. h Ostiole. i Peridium. j Basal wall of ascoma. $\mathrm{k}$ Pseudoparaphyses. 1-n Asci from immature to mature stage. $\mathrm{o}-\mathrm{q}$ Ascospores from immature to mature stage, $r-s$ Germinating ascospores. Scale bars: $d=100 \mu \mathrm{m}, \mathrm{e}-\mathrm{g}=50 \mu \mathrm{m}, \mathrm{h}=20 \mu \mathrm{m}, \mathrm{i}-\mathrm{n}=$ $10 \mu \mathrm{m}, \mathrm{o}-\mathrm{q}=5 \mu \mathrm{m}$. 
Known distribution (based on molecular data) - Thailand (Hongsanan et al. 2017b, this study). study).

Known hosts (based on molecular data) - Mangifera indica (Hongsanan et al. 2017b, this

Material examined - Thailand, Chiang Rai, Tasud, Ban Doo, Pong Phra Bat, on living leaves of Mangifera indica (Anacardiaceae), 10 January 2014, X.Y. Zeng (MFLU 14-0006, new sequence data), living culture (MFLUCC 14-0539).

GenBank numbers - ITS-LSU: MN462949; SSU: MN364417; TUB: MN482717; GAPDH: MN482716.

Notes - This new collection was from the same host and locality as the type specimen, and has identical morphological features. However, there are quite a number of base-pair differences between the two collections (Fig. 1). This species is different from the generic type Chaetothyrina musarum as it lacks setae. Although the author described the characteristic of setae, the latter could not be observed in its photoplate (Hongsanan et al. 2017b). Examination of more samples is needed to understand this species.

Hysteriales Lindau

Hysteriaceae Chevall.

This family currently comprises 14 genera (Wijayawardene et al. 2018, Jayasiri et al. 2019). The latest treatment of Hysteriaceae is by Jayasiri et al. (2019) and a new record for Gloniopsis calami is reported here.

\section{Gloniopsis De Not.}

The genus comprises 68 epithets in Index Fungorum (2020), but only five species have sequence data to represent the generic placement. In addition, several species in this genus are polyphyletic (Boehm et al. 2009a, Jayasiri et al. 2019). In this study, we provide an updated phylogenetic tree based on available sequence data.

Gloniopsis calami Konta \& K.D. Hyde, Fungal Diversity 80: 34 (2016)

Figs 4, 5

Facesoffungi number: FoF02366

Saprobic on dead wood. Sexual morph: Ascomata 174-229 $\mu \mathrm{m}$ high $\times 136.5-209 \mu \mathrm{m}$ diameter $(\bar{x}=196.5 \times 162 \mu \mathrm{m})$, hysterothecial, erumpent to entirely superficial, solitary, or gregarious, oval or ellipsoid to greatly elongate, straight to curved, scattered, dark, carbonaceous, brittle, with a sunken longitudinal slit-like opening. Peridium $27-40.5 \mu \mathrm{m}$ wide, composing thick layers of dark brown cells of carbonaceous texture, inner layers composed of several layers of hyaline to brown cells of textura angularis. Hamathecium comprising $1.5-2 \mu \mathrm{m}$ thin, cylindrical, hyaline, branched, septate, anastomosing pseudoparaphyses. Asci 53-78 $\times 13-19 \mu \mathrm{m}(\bar{x}=64 \times 16$ $\mu \mathrm{m}, \mathrm{n}=10$ ), 8-spored, bitunicate, fissitunicate, saccate, broadly clavate to cylindrical, slightly curved, short pedicellate, with knob-like pedicel, apically rounded, with a well-developed ocular chamber. Ascospores 15-20 × 7-9 $\mu \mathrm{m}(\bar{x}=18 \times 8 \mu \mathrm{m}, \mathrm{n}=20), 2$-seriate, overlapping, reddishbrown to brown, dictyosporous, fusiform, oblong or ellipsoidal to cylindrical, 4-6-trans-septate and with 2-4 vertical septa, constricted at the septa, smooth-walled. Asexual morph: Undetermined.

Culture characteristics - Ascospores germinating on WA within $12 \mathrm{~h}$ and germ tubes produced from spore cells. Colonies on MEA $18 \mathrm{~mm}$ after 7 days at $28{ }^{\circ} \mathrm{C}$. Mycelium superficial, low convex, slightly effuse hairy, edge entire, dark brown. Aerial mycelium, radiating outwards, superficial, septate, hyaline to brown, smooth-walled.

Known distribution (based on molecular data) - Thailand (Phang-Nga Province) - Hyde et al. (2016), Chiang Rai Province (this study).

Known substrates (based on molecular data) - On dead Calamus sp. (Arecaceae) Hyde et al. (2016), on dead wood of unidentified host (this study).

Material examined - Thailand, Chiang Rai, Huai Kang Pla, on dead wood of unidentified host, 25 October 2010, S. Boonmee, HPK02 (MFLU 10-0973, new geographical record); living 
culture (MFLUCC 10-0927 = BCC 52145).

GenBank numbers - ITS: MN608546, LSU: MN577415, SSU: MN577426.

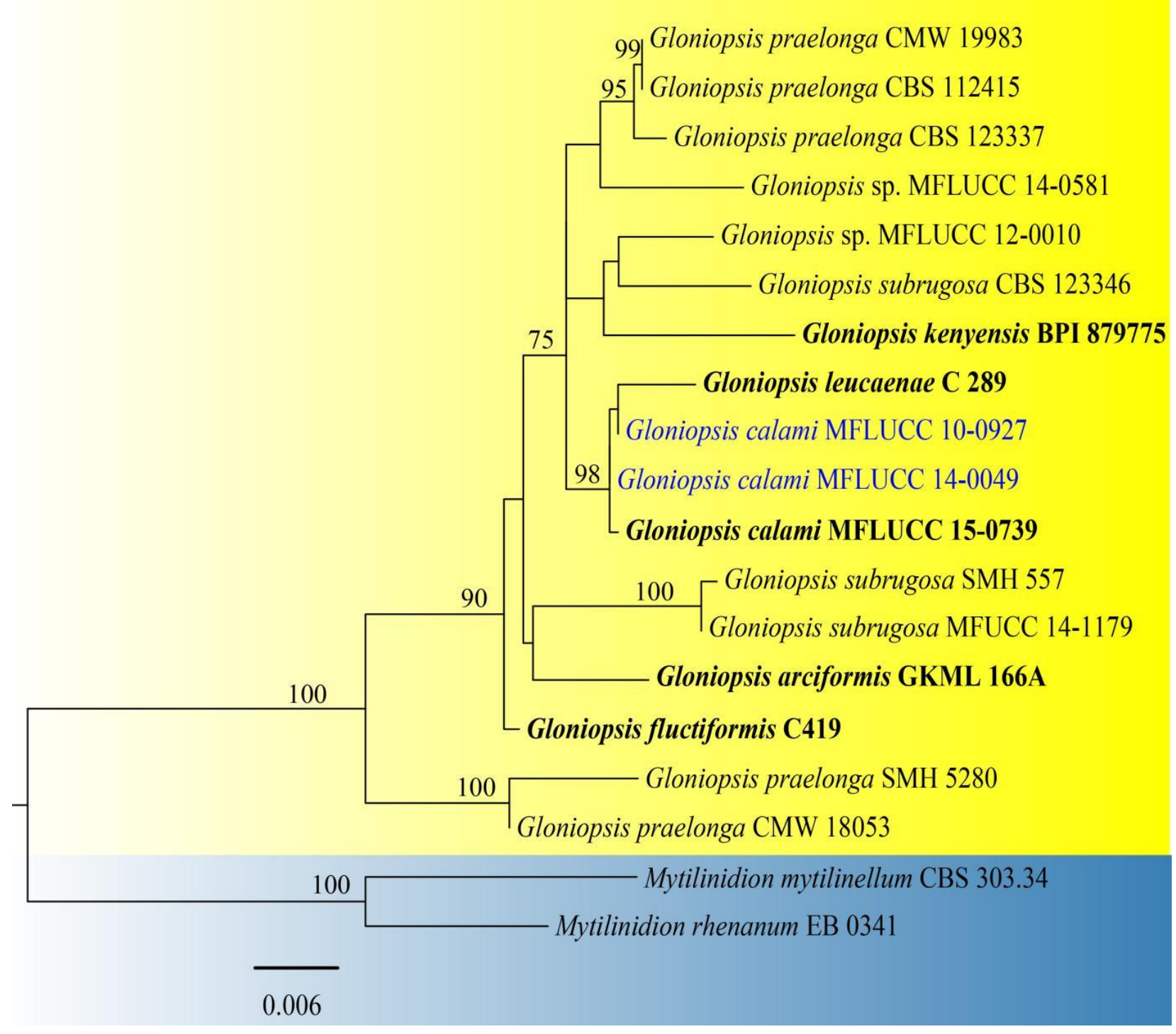

Fig. 3 - Phylogram generated from maximum likelihood analysis based on combined LSU and SSU sequence data. Nineteen taxa are included in the combined gene analyses comprising 1715 characters after alignment (821 characters for LSU, 894 characters for SSU). Mytilinidion mytilinellum (CBS 303.34) and M. rhenanum (EB 0341) are used as the outgroup taxa. The best RaxML tree with a final likelihood value of -3823.854846 is presented. Estimated base frequencies were as follows: $\mathrm{A}=0.251300, \mathrm{C}=0.211072, \mathrm{G}=0.294664, \mathrm{~T}=0.242964$; substitution rates $\mathrm{AC}$ $=0.566402, \mathrm{AG}=1.996467, \mathrm{AT}=0.358447, \mathrm{CG}=0.000000, \mathrm{CT}=6.395101, \mathrm{GT}=1.000000$; gamma distribution shape parameter $\alpha=0$. Bootstrap values for maximum likelihood equal to or greater than 75 are placed above or below the branches. Ex-type strains are in bold and black. The newly generated sequences are indicated in blue.

Notes - Gloniopsis calami was introduced by Hyde et al. (2016) based on morphology and phylogenetic analysis. This species was described from a palm from southern Thailand. Our strain shares similar characteristics such as ascomata, asci and ascospores with Gloniopsis calami (MFLUCC 15-0739). Further, phylogenetic analysis based on LSU and SSU data indicated that our strain clusters with an asexual species, Gloniopsis leucaenae (C289) with poor support (Fig. 3). Comparison of ITS regions of Gloniopsis leucaenae (C289) and G. calami MFLUCC 10-0927 reveals 6 base pair $(0.92 \%)$ differences. However, base pair comparison of ITS, LSU and SSU of 
Gloniopsis calami MFLUCC 15-0739 and G. calami MFLUCC 10-0927 are 100\% similar (data not shown here). Therefore, we identify our strain as Gloniopsis calami and this is the first report of G. calami from dead wood in northern Thailand.

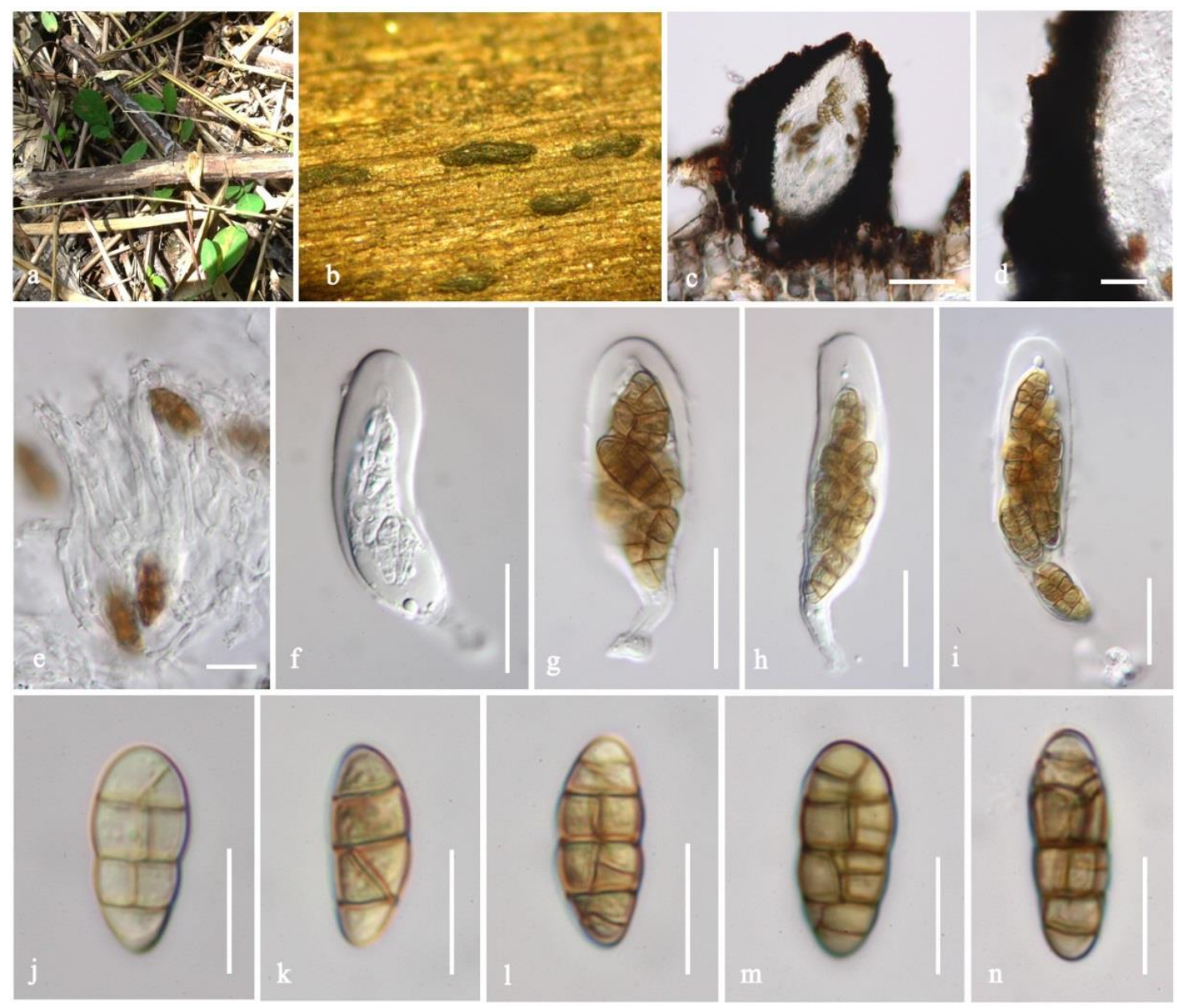

Fig. 4 - Gloniopsis calami (MFLU 10-0973, new geographical record). a, b Material and Appearance of ascomata on woody substrate. c Section of ascoma. $d$ Peridium. e Pseudoparaphyses. f-i Asci. j-n Ascospores. Scale bars: $c=50 \mu \mathrm{m}, \mathrm{d}, \mathrm{f}-\mathrm{i}=20 \mu \mathrm{m}, \mathrm{e}, \mathrm{j}-\mathrm{n}=10$ $\mu \mathrm{m}$.

Gloniopsis calami Konta. \& K.D. Hyde, Fungal Divers 81: 34 (2016)

Fig. 6

Facesoffungi number: FoF02366

Saprobic on decaying wood submerged in freshwater habitats. Sexual morph: Hysterothecia 130-200 $\mu \mathrm{m}$ high, 150-180 $\mu \mathrm{m}$ diameter, erumpent to superficial, solitary to gregarious, scattered, dark, straight to flexuous. Peridium 25-50 $\mu \mathrm{m}$ thick, carbonaceous, thick-walled, relatively smooth on the outer surface. Hamathecium comprising dense, branched, hyaline, septate pseudoparaphyses. Asci $107-127 \times 17-21 \mu \mathrm{m}(\bar{x}=117 \times 19 \mu \mathrm{m}, \mathrm{n}=10), 8$-spored, bitunicate, fissitunicate, cylindrical to cylindric-clavate, short pedicellate, apically rounded, with a well-developed ocular chamber. Ascospores 23-27 $\times 10-12 \mu \mathrm{m}(\bar{x}=25 \times 11 \mu \mathrm{m}, \mathrm{n}=20)$, overlapping 1-2-seriate, pale brown to dark brown, dictyosporous, fusiform, slightly curved to straight, 3-5-trans-septate and with 1-4 vertical septa, constricted at the septa, smooth-walled. Asexual morph: Undetermined.

Culture characteristics - Ascospores germinating on PDA within $14 \mathrm{~h}$ and germ tubes produced from spore cells. Colonies on MEA reached $20 \mathrm{~mm}$ after 10 days at $25{ }^{\circ} \mathrm{C}$. Mycelium superficial, low convex, slightly effuse hairy, edge entire, dark brown.

Known distribution (based on molecular data) - Thailand, Phang-Nga Province (Hyde et al. 2016), Chiang Rai Province (this study). 


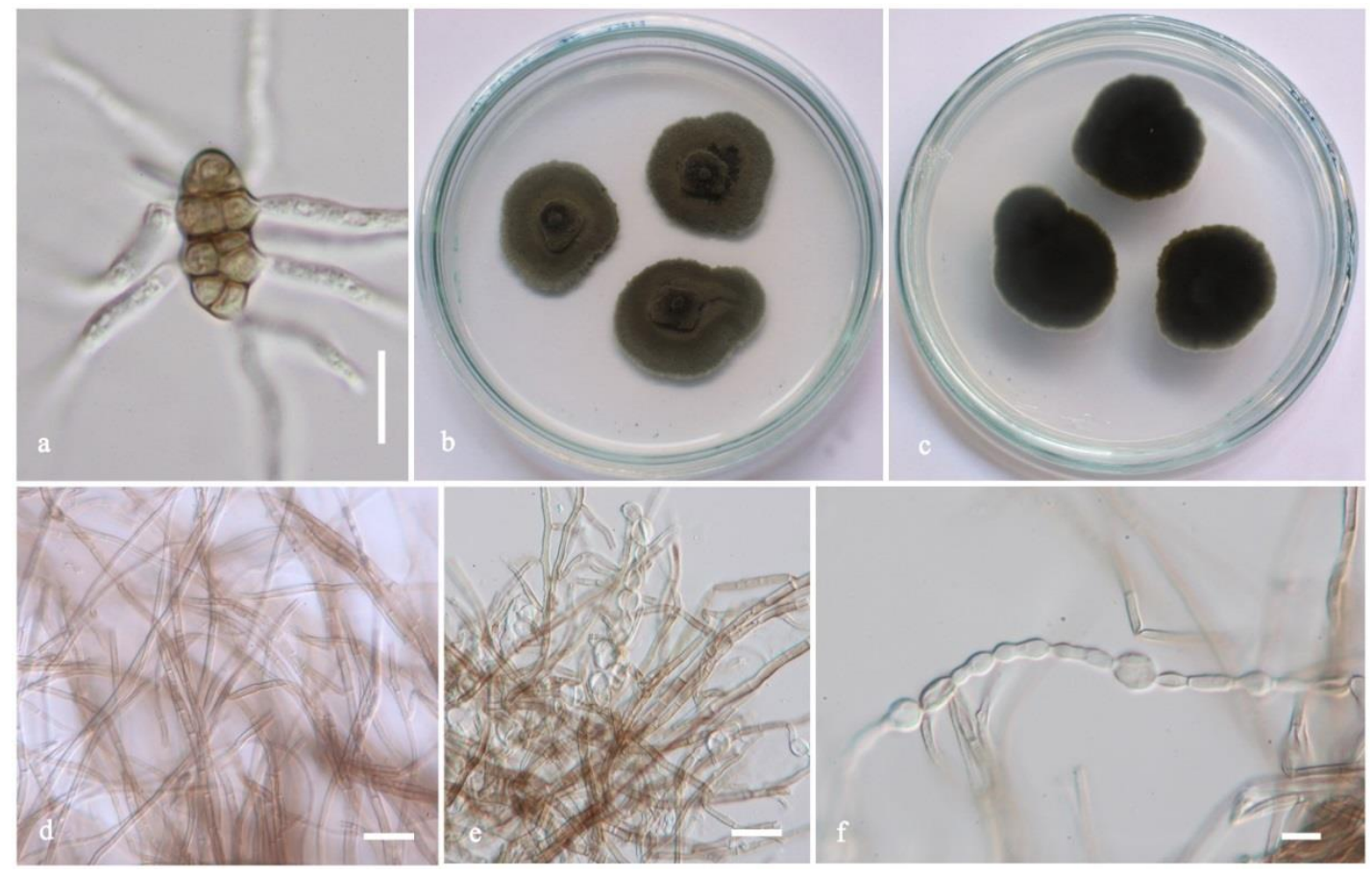

Fig. 5 - Gloniopsis calami (MFLUCC 10-0927, living culture). a Germinating spore. b, c Culture colonies on MEA from surface and reverse. $\mathrm{d}-\mathrm{f}$ Aerial hyphae in culture. Scale bars: $\mathrm{a}, \mathrm{d}-\mathrm{f}=10$ $\mu \mathrm{m}$.

Known hosts (based on molecular data) - On dead Calamus sp. (Arecaceae) (Hyde et al. 2016), on submerged dead wood of unidentified host (this study).

Material examined - Thailand, Chiang Mai Province, saprobic on decaying wood submerged in a freshwater pond, January 2014, Z.L. Luo, ZL-27 (MFLU 15-0074, new to freshwater habitats), living culture MFLUCC 14-0049.

GenBank numbers - ITS: MN 860550, LSU: MN 860555.

Notes - Our strain (MFLUCC 14-0049) shares similar characteristics such as ascomata, asci and ascospores with Gloniopsis calami (MFLUCC 15-0739). Further, phylogenetic analysis based on LSU and SSU data indicated that our strain clusters with Gloniopsis calami with high statistical support (98\% ML) (Fig. 3). This is the first report of G. calami from submerged dead wood in freshwater habitats.

\section{Hysterium Pers.}

Hysterium is one of the largest genera of Hysteriales with a cosmopolitan distribution and comprises 435 epithets (Index Fungorum 2020). The genus is characterized by hysterothecial ascomata, carbonaceous, with a longitudinal slit, bitunicate, fissitunicate, cylindrical clavate asci and fusiform, septate, pigmented ascospores (Boehm et al. 2009a). Members of this genus are saprobic on dead wood in terrestrial habitats. An updated phylogenetic tree is provided here.

Hysterium angustatum Alb. \& Schwein., Consp. fung. (Leipzig): 55 (1805)

Figs 8,9

Facesoffungi number: FoF 04579

Saprobic on dead wood. Sexual morph: Ascomata 207.5-294.5 $\mu \mathrm{m}$ high $\times 242.5-346 \mu \mathrm{m}$ diameter $(\bar{x}=238.5 \times 278 \mu \mathrm{m})$, hysterothecial, erumpent to entirely superficial, solitary, or gregarious, oval or ellipsoid to greatly elongate, straight to curved, scattered, dark, carbonaceous, brittle, with a sunken longitudinal slit-like opening. Peridium 42-57 $\mu \mathrm{m}$ wide, composing thick layers of dark brown cells of carbonaceous texture, inner layers composed of several layers of light brown to brown cells of textura angularis. Hamathecium comprising 1-2 $\mu \mathrm{m}$ thin, cylindrical, hyaline, branched, septate, anastomosing pseudoparaphyses. Asci 67.5-92 × 13-20.5 $\mu \mathrm{m}(\bar{x}=80 \times$ 
$17 \mu \mathrm{m}, \mathrm{n}=10$ ), 8-spored, bitunicate, fissitunicate, saccate, broadly clavate to cylindrical, slightly curved, short pedicellate, with knob-like pedicel, apically rounded, with a well-developed ocular chamber. Ascospores 20-31 $\times 7-10 \mu \mathrm{m}(\bar{x}=23.5 \times 8.5 \mu \mathrm{m}, \mathrm{n}=20)$, overlapping 2-seriate, brown to greenish brown, fusiform, oblong or ellipsoidal to cylindrical, 3-septate, constricted at the median septum, smooth-walled. Asexual morph: Undetermined.

Culture characteristics - Ascospores germinating on WA within $12 \mathrm{~h}$ and germ tubes produced from both cells. Colonies on MEA $0.2 \mathrm{~mm}$ after 7 days at $28{ }^{\circ} \mathrm{C}$. Mycelium superficial, low convex, slightly effuse hairy, edge entire, grayish-brown to brown. Aerial mycelium, radiating outwards, superficial, septate, hyaline to brown, smooth-walled.

Known distribution (based on molecular data) - Kenya, New Zealand, South Africa, United States (Boehm et al. 2009a), Italy (Jayasiri et al. 2018) and Thailand (this study).

Known hosts (based on molecular data) - On bark of living Pinus rigida (Boehm et al. 2009a), on dead aerial branch of Rubus sp. (Jayasiri et al. 2018), on dead wood of unidentified host (this study).

Material examined - Thailand, Chiang Mai, Chom Thong, on dead wood, 16 November 2010, R. Phookamsak, ITN06 (MFLU 11-0004, new geographical record), living culture (MFLUCC 11-0004 = BCC 52154).

GenBank numbers - ITS: MN608547, LSU: MN577416, SSU: MN577427.

Notes - The strain MLFUCC 11-0004 that was isolated and described in this study, is phylogenetically related to other Hysterium angustatum isolates with high statistical support (94\% ML/1.00 BYPP, Fig. 7). In addition, our strain shares similar morphologies in shape, size and pigment of ascomata, asci and ascospores with Hysterium angustatum (CBS 236.34, CBS 123334, CMW 20409, GKM 243a, GKM 5211, MFLUCC 16-0623, SMH 5216). Therefore, based on morphology and phylogenetic affinity, our strain MFLUCC 11-0004 is identified as Hysterium angustatum and it is reported here as a new geographical record from Thailand.

\section{Rhytidhysteron Speg.}

Rhytidhysteron was classified in Patellariaceae (Barr 1987, Lumbsch \& Huhndorf 2010) but recently accommodated in Hysteriaceae based on multi-gene phylogenetic analyses (Boehm et al. 2009a, b, de Almeida et al. 2014, Wijayawardene et al. 2014). Rhytidhysteron species are endophytes, saprobes and pathogens on plants and humans (Yacharoen et al. 2015, Chander et al. 2017). Several new species have been recently introduced based on morphology and phylogeny (Thambugala et al. 2016b, Doilom et al. 2017b, Kumar et al. 2019).

Rhytidhysteron thailandicum Thambug. \& K.D. Hyde, Cryptog. Mycol. 37(1): 110 (2016)

Facesoffungi number: FoF01841

Fig. 11

Saprobic on dead twigs of Afzelia xylocarpa. Sexual morph Ascomata 1300-1700 $\mu \mathrm{m}$ long $\times 380$ $790 \mu \mathrm{m}$ high $\times 1160-1815 \mu \mathrm{m}$ wide $(\bar{x}=1500 \times 585 \times 1505 \mu \mathrm{m}, \mathrm{n}=10)$, hysterothecial with a longitudinal slit, black, solitary to aggregated, semi-immersed to superficial, with irregular opening when wet, folded at the margin when dry, with striation, subiculum, coriaceous, yellow at the center, forming an elongate slit. Exciple 50-100 $\mu \mathrm{m}(\bar{x}=75 \mu \mathrm{m}, \mathrm{n}=15)$, composed of textura angularis, comprising two cell layers, outer layer comprising black, thick-walled cells, inner layer comprising dark reddish to hyaline, thin-walled cells. Hamathecium comprising 1.-2.3 $\mu \mathrm{m}$ wide, dense, septate, filiform, cellular pseudoparaphyses, forming epithecium above the asci and enclosed in a gelatinous matrix. Asci $140-157 \times 10-12 \mu \mathrm{m}(\bar{x}=150 \times 11 \mu \mathrm{m}, \mathrm{n}=15), 8$-spored, bitunicate, subcylindrical to cylindrical, with a short pedicel, apically rounded with an ocular chamber. Ascospores $21-25.5 \times 7.5-9.6 \mu \mathrm{m}(\bar{x}=23.5 \times 8.7 \mu \mathrm{m}, \mathrm{n}=20)$, uni-seriate, partially overlapping, hyaline to pale brown, becoming pale brown to dark brown, initially subglobose, 1-septate, slightly constricted at the central septum, becoming 3-septate, ellipsoidal to fusiform, slightly rounded or pointed at both ends, guttulate, without a mucilaginous sheath. Asexual morph Undetermined (see Thambugala et al. $2016 \mathrm{~b}$ for the type). 

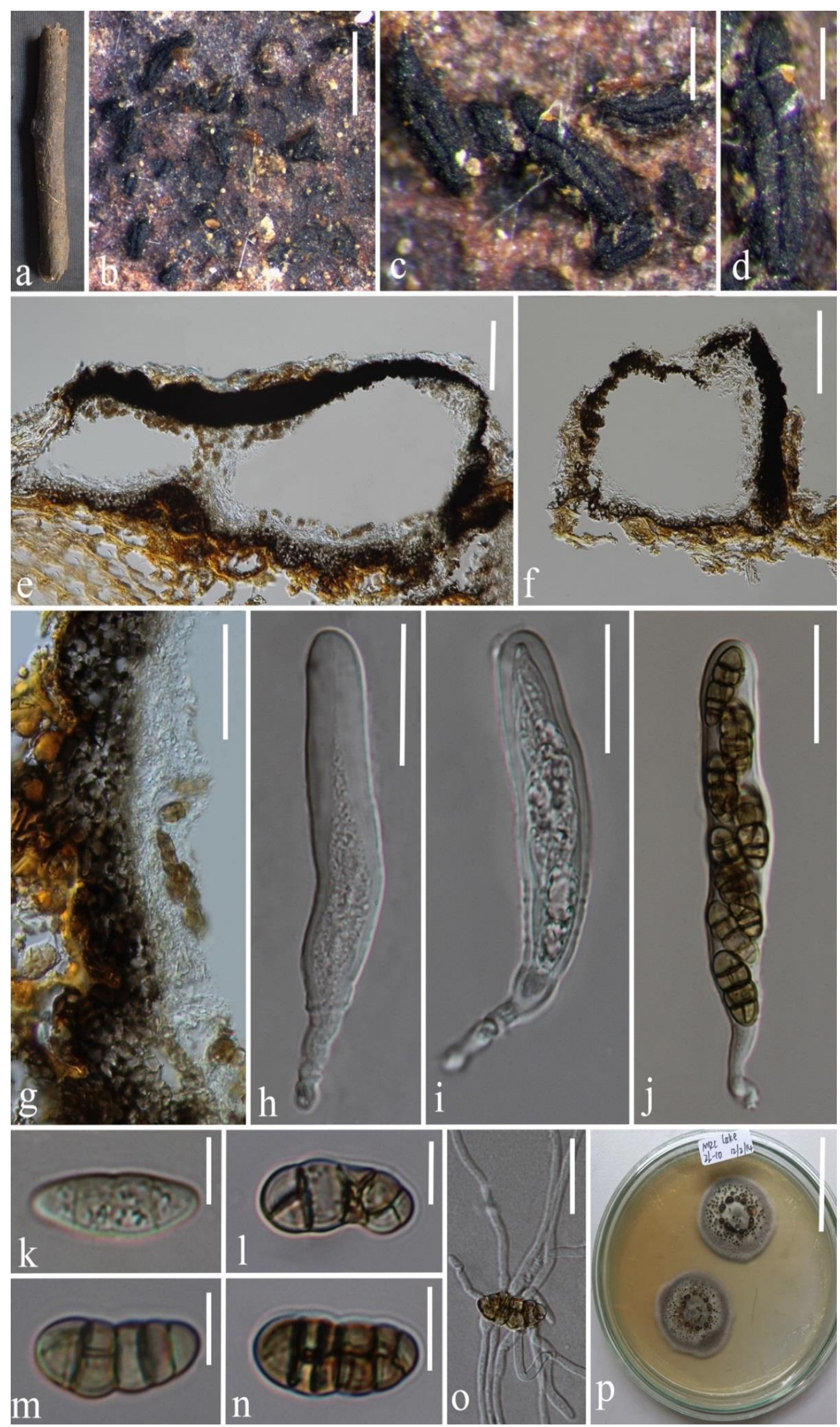

Fig. 6 - Gloniopsis calami (MFLU 15-0074, new to freshwater habitats). a Specimen. b-d Appearance of hysterothecia on host. e, f Section of hysteriothecium. g Structure of peridium. $\mathrm{h}-\mathrm{j}$ Asci. $\mathrm{k}-\mathrm{n}$ Ascospores. o Germinating ascospore. $\mathrm{p}$ Colony on MEA. Scale bars: $\mathrm{b}=1000 \mu \mathrm{m}$, $\mathrm{c}=200 \mu \mathrm{m}, \mathrm{d}=150 \mu \mathrm{m}, \mathrm{e}, \mathrm{f}=60 \mu \mathrm{m}, \mathrm{g}-\mathrm{j}=30 \mu \mathrm{m}, \mathrm{k}-\mathrm{n}=10 \mu \mathrm{m}, \mathrm{o}=20 \mu \mathrm{m}, \mathrm{p}=30 \mathrm{~mm}$. 


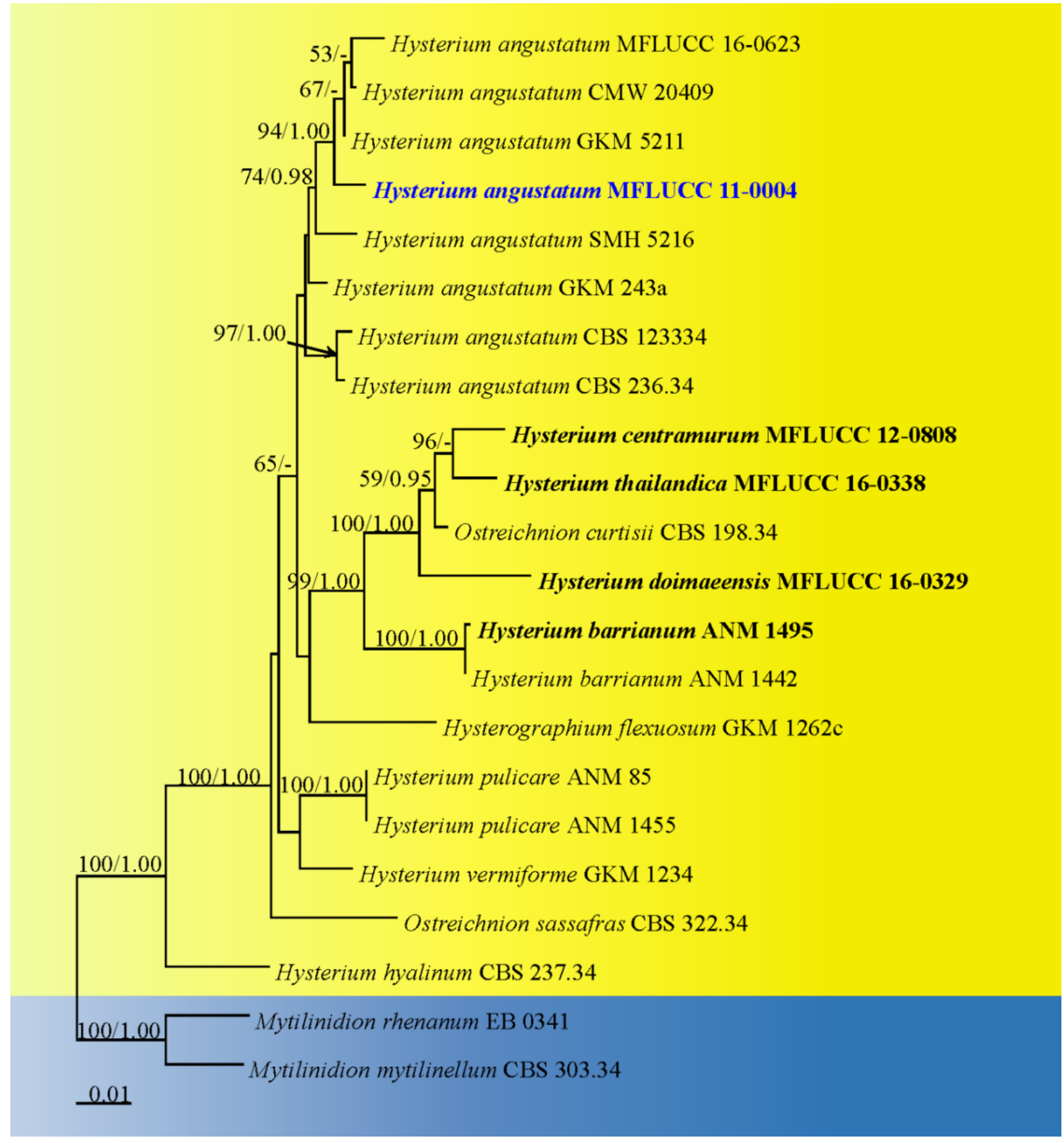

Fig. 7 - Phylogram generated from maximum likelihood analysis based on combined LSU and SSU sequence data. Twenty-one taxa are included in the combined gene analyses comprising 2065 characters after alignment (1043 characters for LSU and 1022 characters for SSU). Mytilinidion mytilinellum (CBS 303.34) and M. rhenanum (EB 0341) are used as the outgroup taxa. The best RaxML tree with a final likelihood value of -5388.433176 is presented. The matrix had 332 distinct alignment patterns, with $32.25 \%$ undetermined characters or gaps. Estimated base frequencies were as follows: $\mathrm{A}=0.254589, \mathrm{C}=0.217165, \mathrm{G}=0.291200, \mathrm{~T}=0.237046$; substitution rates $\mathrm{AC}=$ $0.327416, \mathrm{AG}=3.974093, \mathrm{AT}=1.042057, \mathrm{CG}=0.582695, \mathrm{CT}=12.493723, \mathrm{GT}=1.000000$; gamma distribution shape parameter $\alpha=0.075104$. Bootstrap values for maximum likelihood equal to or greater than 50 and Bayesian posterior probabilities equal or greater than 0.95 are placed above or below the branches. Ex-type strains are in bold and black. The newly generated sequence is indicated in blue. 


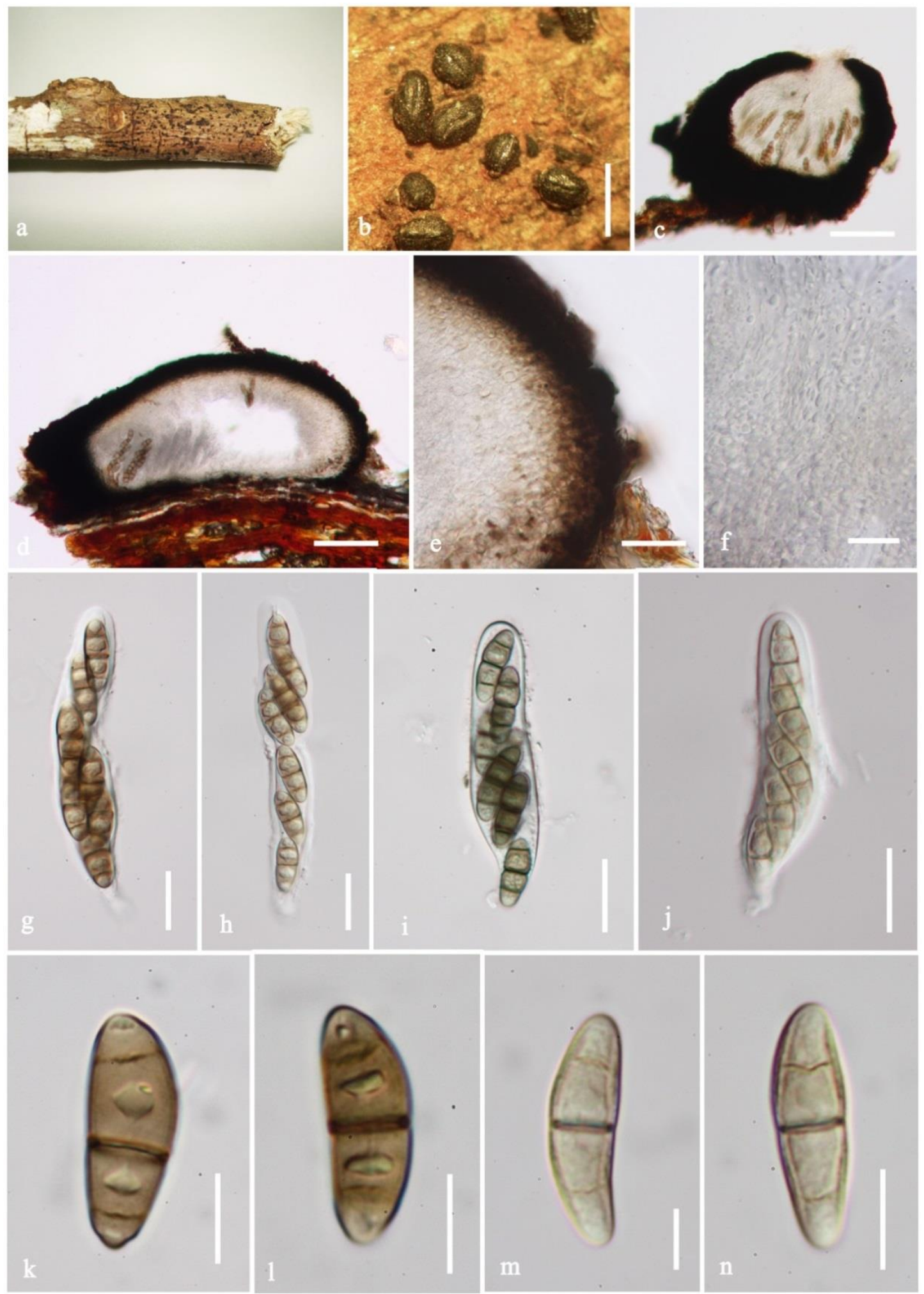

Fig. 8 - Hysterium angustatum (MFLU 11-0004, new geographical record), a Material. b Appearance of ascomata on woody substrate. c, d Sections of ascomata (Fig. c transverse section and Fig. d longitudinal section). e Peridium. f Pseudoparaphyses. g-j Asci. k-n Ascospores. Scale bars: $\mathrm{b}=500 \mu \mathrm{m}, \mathrm{c}, \mathrm{d}=100 \mu \mathrm{m}, \mathrm{e}=50 \mu \mathrm{m}, \mathrm{f}, \mathrm{k}-\mathrm{n}=10 \mu \mathrm{m}, \mathrm{g}-\mathrm{j}=20 \mu \mathrm{m}$.

Culture characteristics - Ascospores germinating on PDA within $24 \mathrm{~h}$ and germ tubes produced from one or both ends or second or third cells. Colonies on PDA filamentous to irregular in shape, undulate edge, flat, initially white, becoming grey. 


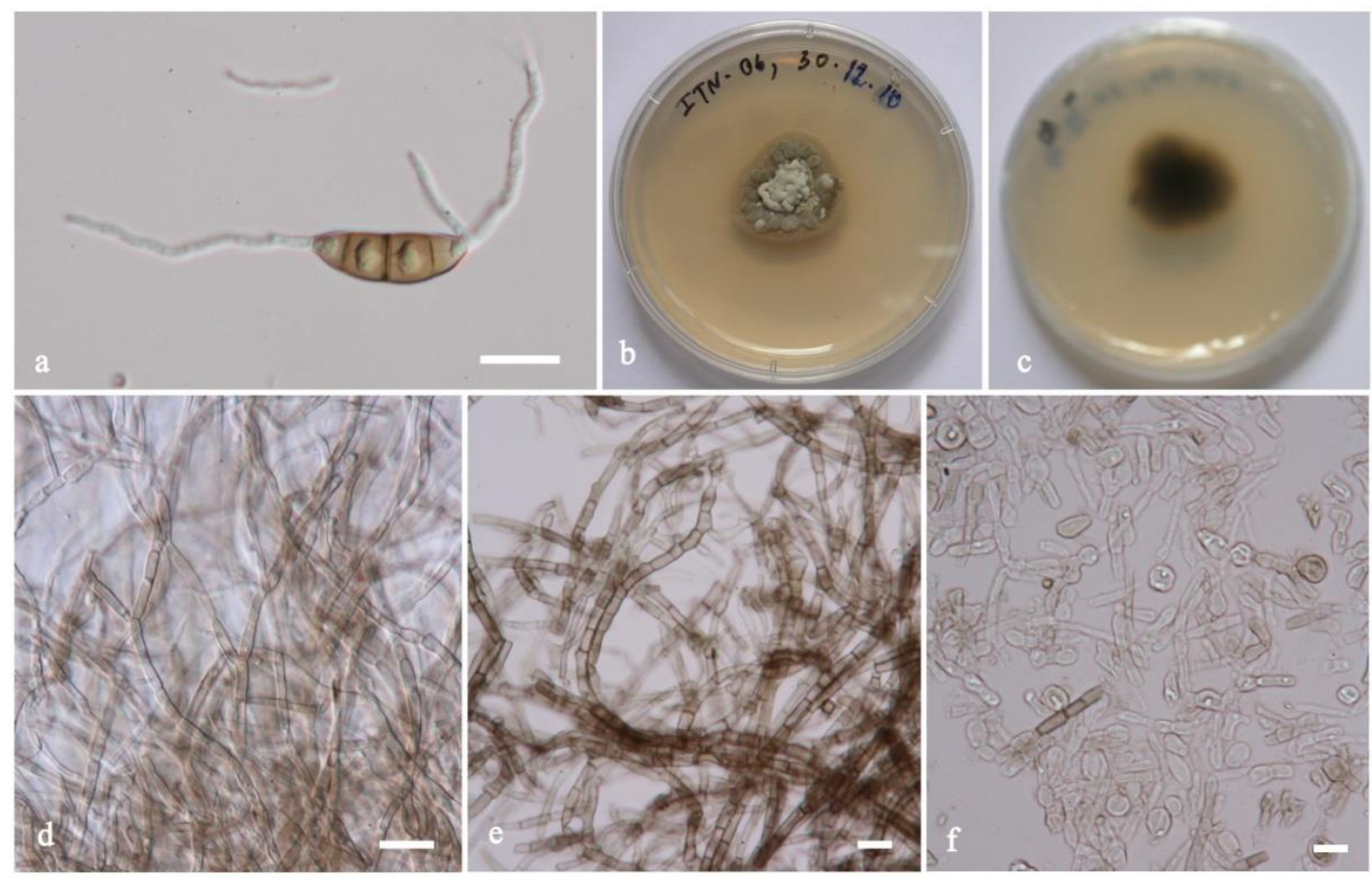

Fig. 9 - Hysterium angustatum (MFLUCC 11-0004, living culture). a Germinating spore. $\mathrm{b}, \mathrm{c}$ Culture colonies on MEA from surface and reverse at 30 days. $\mathrm{d}-\mathrm{f}$ Aerial hyphae in culture. Scale bars: $a, d-f=10 \mu \mathrm{m}$. study).

Known distribution (based on molecular data) - Thailand (Thambugala et al. 2016b, this

Known hosts (based on molecular data) - Afzelia xylocarpa (this study).

Material examined - Thailand, Chiang Rai Province, Mae Suai District, on dead twigs of Afzelia xylocarpa (Leguminosae), 20 November 2012, M. Doilom (MFLU 19-2701, new host record), living culture (MFLUCC 13-0051).

GenBank numbers - ITS: MN509433, LSU: MN509434, tef1: MN509435.

Notes - The holotype of Rhytidhysteron thailandicum has been reported from a dead twig in Thailand (Thambugala et al. 2016b). Our collection has similar morphologies to the holotype (Thambugala et al. 2016b) in its clavate to cylindrical asci, ellipsoidal to fusiform ascospores with 3 septa, but they are different with regards to the hysterothecia on host substrate along with their sizes. In combined analysis of sequence data of LSU, ITS and tef1 of our strain (MFLUCC 130051) clusters with $R$. thailandicum (MFLUCC 14-0503, ex-type) and (MFLUCC 12-0530) with high bootstrap and Bayesian probabilities (100\% ML/ 1.00 PP) (Fig. 10). Thus, we identify our collection as $R$. thailandicum. This is the first record of $R$. thailandicum on Afzelia xylocarpa.

Rhytidhysteron thailandicum Thambug. \& K.D. Hyde, Cryptog. Mycol. 37(1): 110 (2016)

Facesoffungi number: FoF 01841

Fig. 12

Saprobic on decaying wood. Sexual morph: Hysterothecia $1000-1800 \mu \mathrm{m}$ long $\times 300-500$ high $\times 500-1200 \mu \mathrm{m}$ diameter $(\bar{x}=1400 \times 420 \times 850 \mu \mathrm{m}, \mathrm{n}=10)$, arising singly or in small groups, sessile, slightly erumpent from the substrate. Receptacle cupulate, black, flat or slightly concave, yellowish brown when fresh, with slightly dentate margin. Excipulum 40-70 $\mu \mathrm{m}$ wide, ectal excipulum narrow layered, deep, thick-walled, with black cells of textura globulosa to textura angularis; medullary excipulum composed of narrow, long, thin-walled, hyaline to brown cells of textura angularis. Hamathecium comprising 2-5 $\mu \mathrm{m}$ wide, numerous, propoloid, apically swollen, branched and pigmented, branched paraphyses, exceeding asci in length, apices form a layer on the hymenium to develop the epithecium. Asci $120-150 \times 12-16 \mu \mathrm{m}(\bar{x}=130.1 \times 15.3 \mu \mathrm{m}, \mathrm{n}=30), 8$ - 
spored, long cylindrical, short pedicellate, rounded at the apex. Ascospores $20-32 \times 7.5-10.5 \mu \mathrm{m}(\bar{x}$ $=23.3 \times 8.6 \mu \mathrm{m}, \mathrm{n}=40$ ), uniseriate, dark brown, ellipsoid with conical ends, regularly 3 -septate, smooth-walled, guttulate. Asexual morph: coelomycetous. See Thambugala et al. (2016b, Pages 15, 16) for more details.

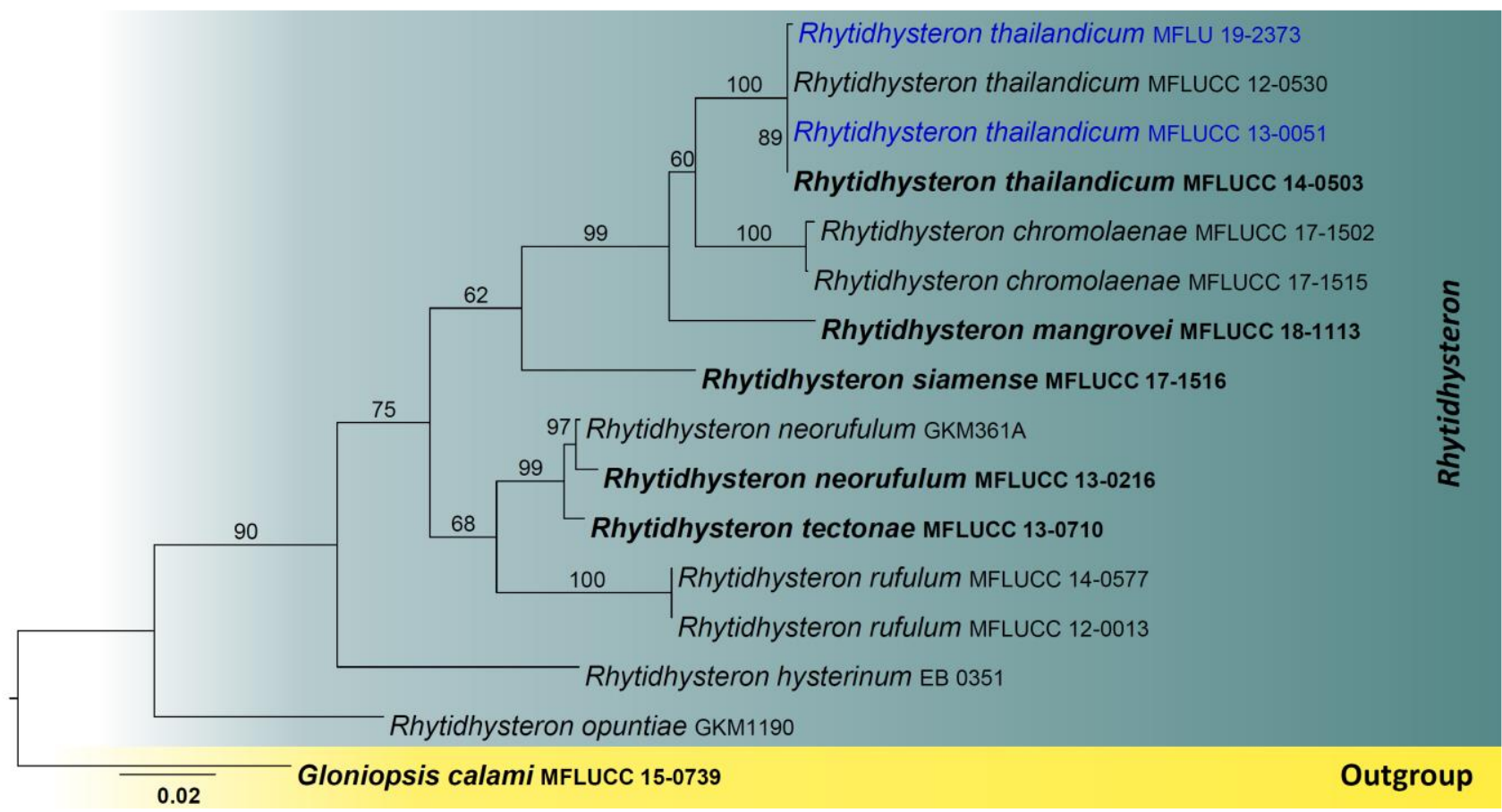

Fig. 10 - Phylogram generated from maximum likelihood analysis based on combined LSU, ITS and tefl sequence data. Sixteen strains are included in the combined gene analyses comprising 2358 characters after alignment (868 characters for LSU, 637 characters for ITS, 853 characters for tef1). Gloniopsis calami (MFLUCC 15-0739) is used as the outgroup taxon. The best RaxML tree with a final likelihood value of -6327.236134 is presented. The matrix had 393 distinct alignment patterns, with $15.91 \%$ undetermined characters or gaps. Estimated base frequencies were as follows: $\mathrm{A}=$ $0.229826, \mathrm{C}=0.263523, \mathrm{G}=0.280734, \mathrm{~T}=0.225917$; substitution rates $\mathrm{AC}=1.425723, \mathrm{AG}=$ 2.098406, $\mathrm{AT}=0.841566, \mathrm{CG}=0.571540, \mathrm{CT}=7.348273, \mathrm{GT}=1.000000$; gamma distribution shape parameter $\alpha=0.502627$. Bootstrap values for maximum likelihood equal to or greater than 60 and Bayesian posterior probabilities equal or greater than 0.95 are placed above or below the branches. Ex-type strains are in bold and black. The newly generated sequences are indicated in blue.

Known distribution (based on molecular data) - Thailand, Chiang Rai, Phitsanulok (Yacharoen et al. 2015, Thambugala et al. 2016b), China, Yunnan Province (this study).

Known hosts (based on molecular data) - deciduous plants

Material examined - China, Yunnan Province, Qujing, 24.68703 ${ }^{\circ} \mathrm{N}, 104.24653^{\circ} \mathrm{E}, 1618 \mathrm{~m}$, on dead twigs of an undetermined tree, 6 May, 2019, Dhanushka N. Wanasinghe (MFLU 19-2373).

GenBank numbers - ITS: MN989428, LSU: MN989429, SSU: MN989430, tef1: MN989431.

Notes - Rhytidhysteron thailandicum was introduced by Thambugala et al. (2016b), which was collected from Thailand (on a dead twig). During our investigation on diversity of microfungi in China, a specimen was recovered from a dead twig in Qujing, Yunnan Province. Morphological characters such as ascomata, asci and ascospores fit well within the sexual morph of Rhytidhysteron thailandicum. We did not obtain an isolate and therefore we isolated DNA directly from the fruiting bodies. Comparison of ITS and tefl sequence data reveals there is no significant difference $(<3 \mathrm{bp}$ differences) between our new collection and the type strain (MFLUCC 14-0503). Therefore, we introduce our new collection as a new geographical record herein. 


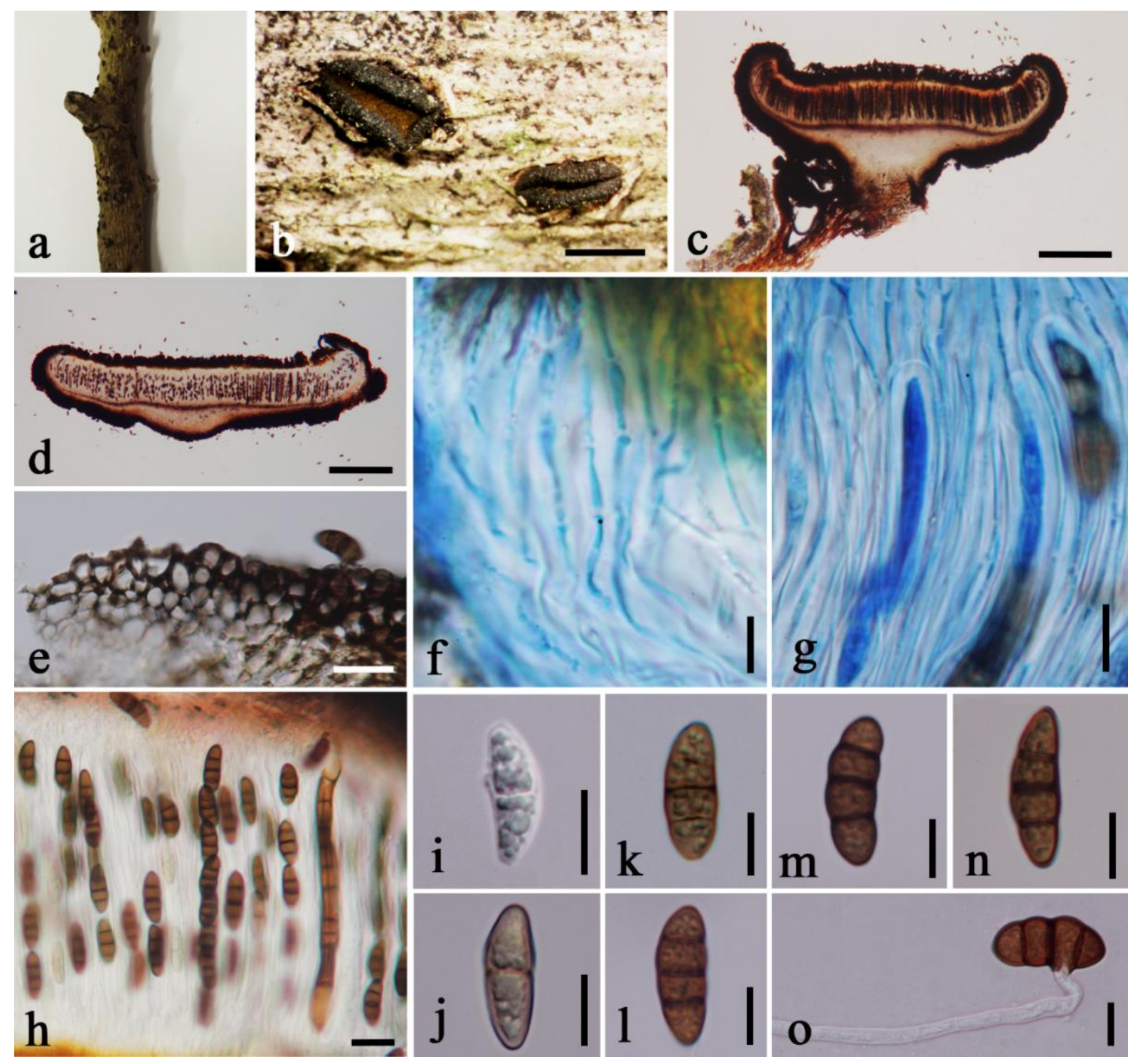

Fig. 11 - Rhytidhysteron thailandicum (MFLU 19-2701, new host record). a Host substrate. b Appearance of hysterothecia on host. c, d Vertical section through hysteriothecia. e Exciple. f Pseudoparaphyses. g Pseudoparaphyses and immature and mature asci. $\mathrm{h}$ Asci and ascospores enclosed in a gelatinous matrix. i-1 Ascospores. o Germinated ascospore. Scale bars: $\mathrm{b}=1000 \mu \mathrm{m}, \mathrm{c}, \mathrm{d}=300 \mu \mathrm{m}, \mathrm{e}, \mathrm{h}=20 \mu \mathrm{m}, \mathrm{g}, \mathrm{i}-\mathrm{O}=10 \mu \mathrm{m}, \mathrm{f}=5 \mu \mathrm{m}$.

\section{Pleosporales Luttrell ex M.E. Barr}

Anteagloniaceae K.D. Hyde, J.K. Liu \& A. Mapook

Anteagloniaceae comprises two genera i.e., Anteaglonium and Flammeascoma (Hyde et al. 2013, Jayasiri et al. 2016, Wijayawardene et al. 2018). Its members are characterized by hysterothecial to conical or lenticular ascomata, uni- to bi-loculate, with a longitudinal slit- to porelike opening, bitunicate, fissitunicate, cylindric-clavate asci and 1-septate, hyaline ascospores. The latest treatment of Anteagloniaceae is by Jayasiri et al. (2019) and is followed here.

Anteaglonium Mugambi \& Huhndorf

Anteaglonium is characterized by hysterothecial ascomata, carbonaceous navicular with a longitudinal slit, bitunicate, fissitunicate, cylindrical clavate asci and 1-septate, hyaline ascospores (Mugambi \& Huhndorf 2009). Members of this genus are saprobes and are commonly found on dead wood in terrestrial habitats and have a cosmopolitan distribution. The genus currently includes eight species (Index Fungorum 2020). An updated phylogenetic tree is provided here. 

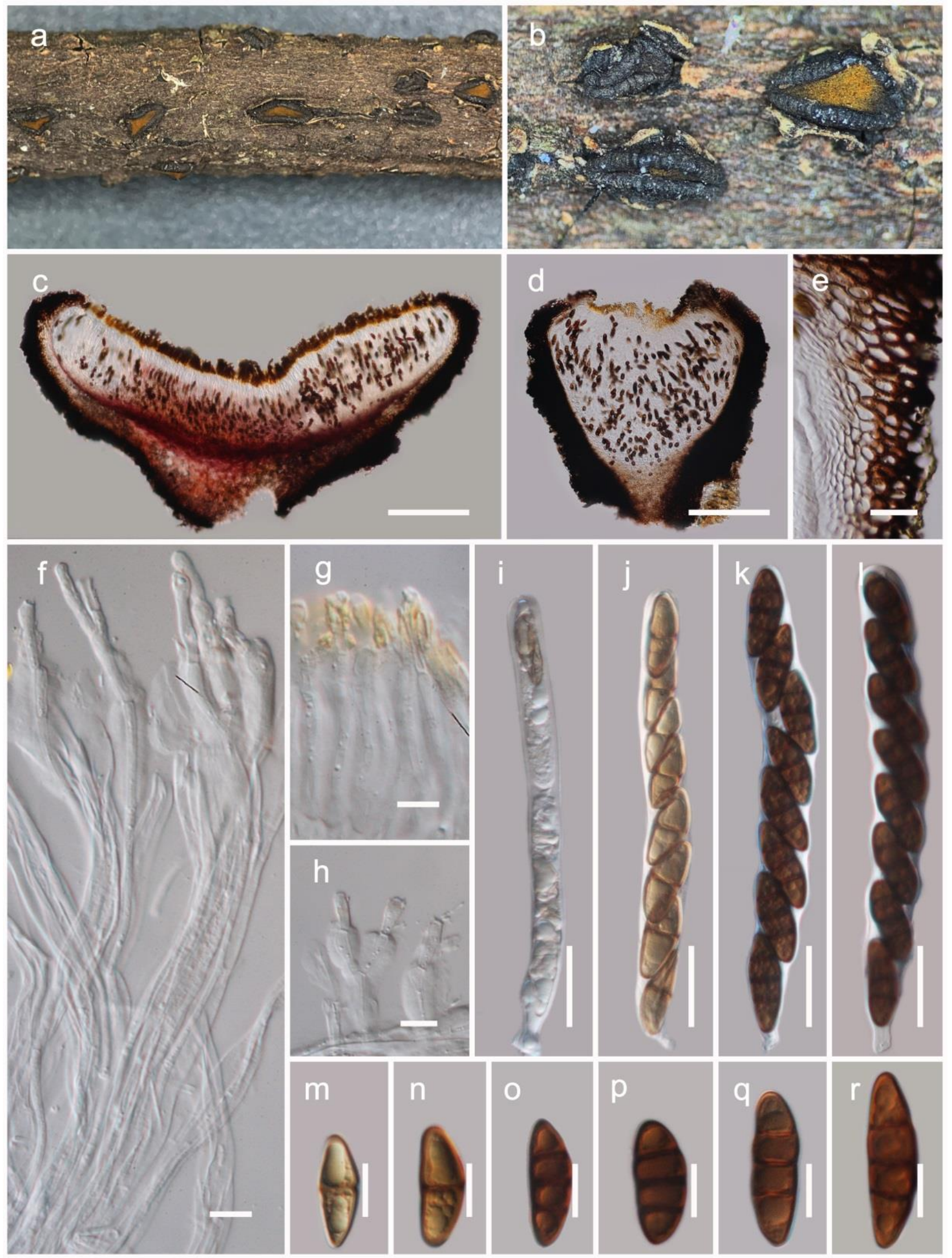

Fig. 12 - Rhytidhysteron thailandicum )MFLU 19-2373, new geographical record(. a, b Appearance of hysterothecia on host. c, d Vertical section through hysteriothecium. e Cells of peridium. $\mathrm{f}-\mathrm{h}$ Pseudoparaphyses. i-1 Asci. m-r Ascospores. Scale bars: $\mathrm{c}, \mathrm{d}=200 \mu \mathrm{m}, \mathrm{e}, \mathrm{i}-\mathrm{l}=20$ $\mu \mathrm{m}, \mathrm{f}-\mathrm{h}, \mathrm{m}-\mathrm{r}=10 \mu \mathrm{m}$. 


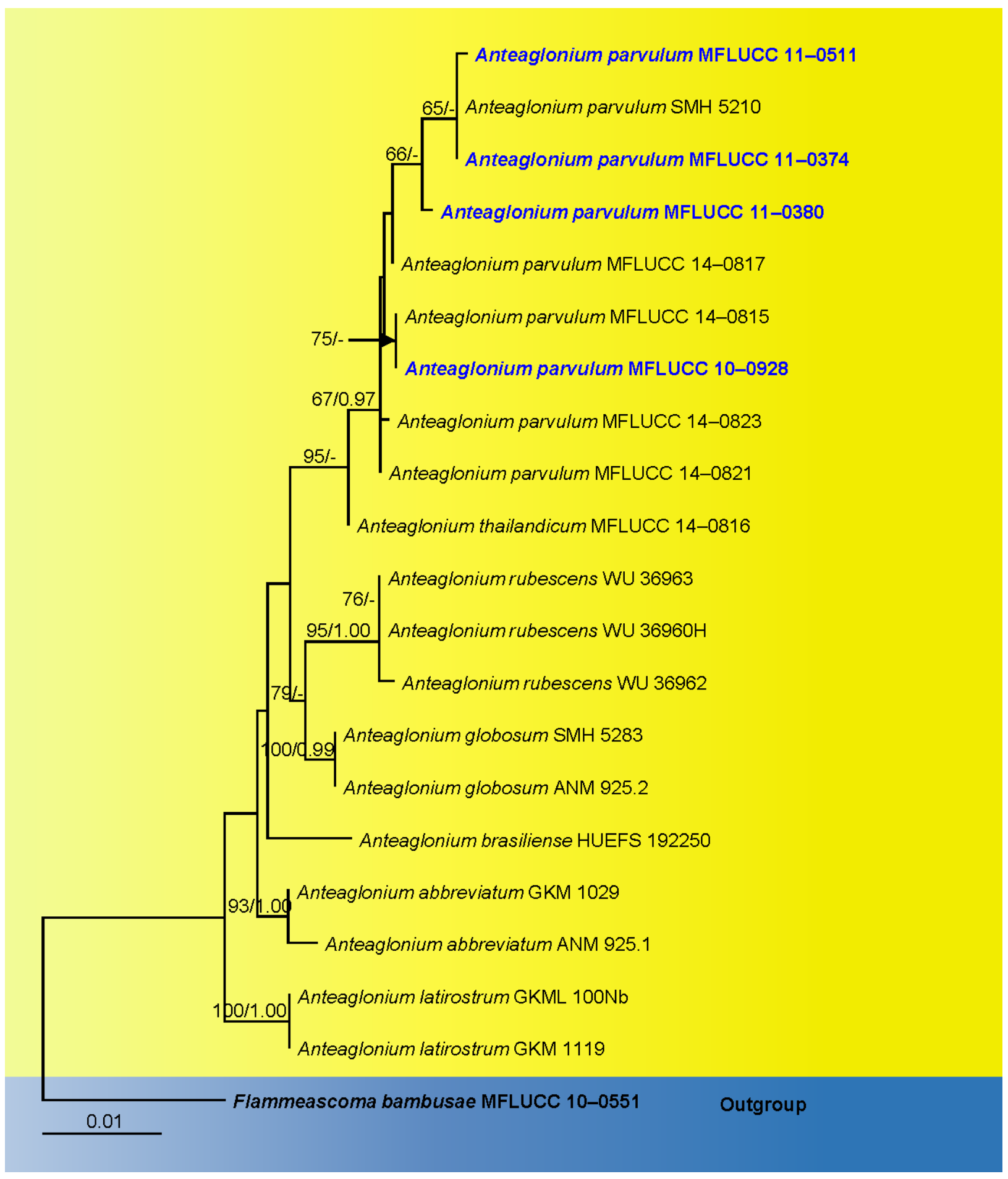

Fig. 13 - Phylogram generated from maximum likelihood analysis based on combined LSU and SSU sequence data. Twenty-one taxa are included in the combined gene analyses comprising 1769 characters after alignment (827 characters for LSU, 942 characters for SSU). Flammeascoma bambusae (MFLUCC 10-0551) is used as the outgroup taxon. The best RaxML tree with a final likelihood value of -3014.773692 is presented. The matrix had 139 distinct alignment patterns, with $36.86 \%$ undetermined characters or gaps. Estimated base frequencies were as follows: $\mathrm{A}=$ $0.247411, \mathrm{C}=0.226484, \mathrm{G}=0.303542, \mathrm{~T}=0.222563$; substitution rates $\mathrm{AC}=0.807595, \mathrm{AG}=$ 3.459156, $\mathrm{AT}=1.159395, \mathrm{CG}=0.412993, \mathrm{CT}=12.612093, \mathrm{GT}=1.000000$; gamma distribution shape parameter $\alpha=0.020000$. Bootstrap values for maximum likelihood equal to or greater than 50 and Bayesian posterior probabilities equal or greater than 0.95 are placed above or below the branches. Ex-type strains are in bold and black. The newly generated sequences are indicated in blue. 


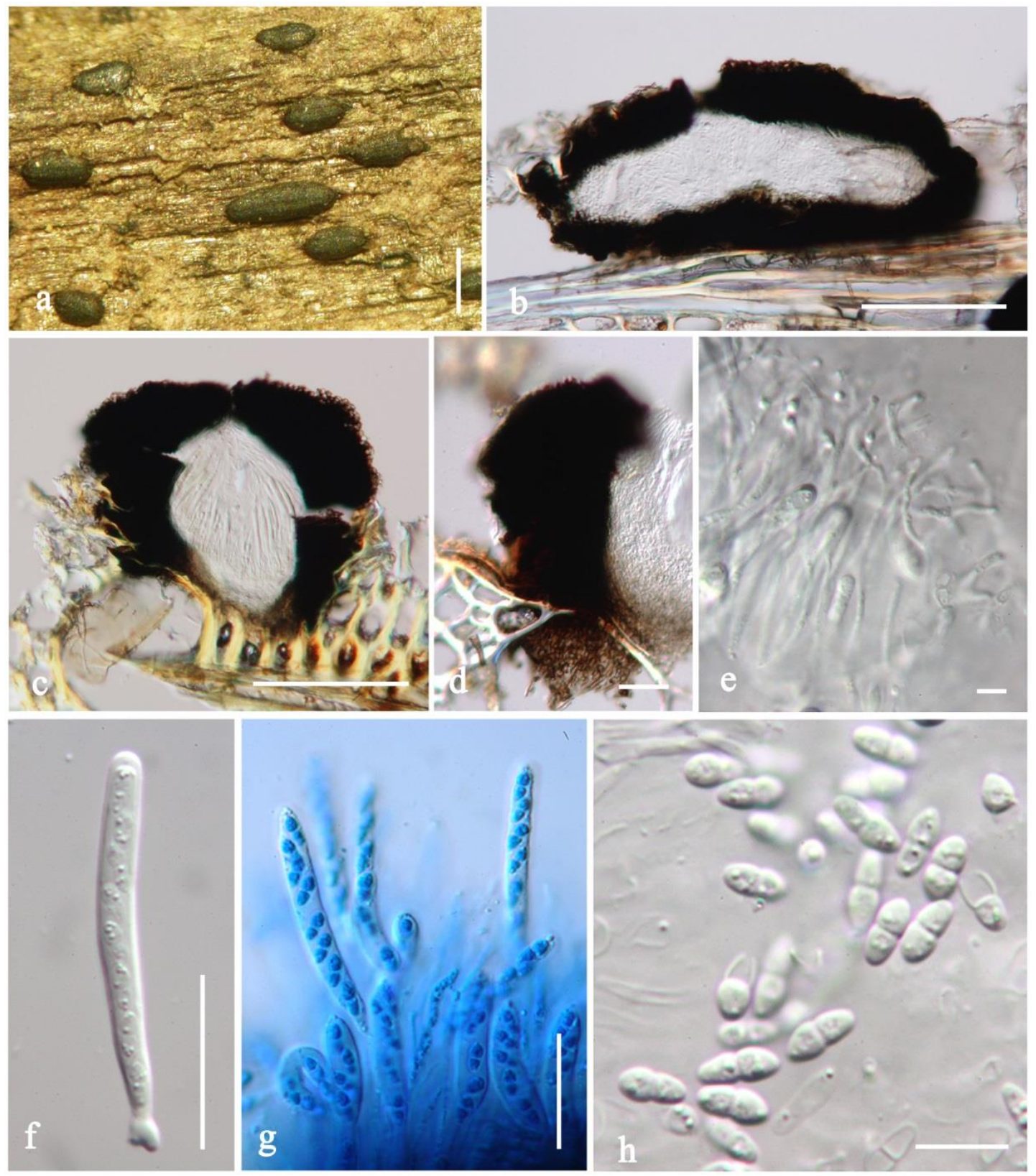

Fig. 14 - Anteaglonium parvulum (MFLU 10-0974, new geographical record). a Appearance of ascomata on woody substrate. b, c Longitudinal and vertical sections of ascomata with slightly hyphae penetrating into the host tissue. d Peridium. e Pseudoparaphyses. f, g Asci (Fig. g stained in cotton blue reagent). $\mathrm{h}$ Ascospores. Scale bars: $\mathrm{a}=500 \mu \mathrm{m}, \mathrm{b}, \mathrm{c}=100 \mu \mathrm{m}, \mathrm{d}, \mathrm{f}, \mathrm{g}=20 \mu \mathrm{m}, \mathrm{e}=5$ $\mu \mathrm{m}, \mathrm{h}=10 \mu \mathrm{m}$.

Anteaglonium parvulum (Schwein.) Mugambi \& Huhndorf, Syst. Biodiv. 7(4): 460 (2009)

Facesoffungi number: FoF01931

Figs 14, 15

Saprobic on dead wood. Sexual morph: Ascomata 128-192 $\mu \mathrm{m}$ high $\times 134-304 \mu \mathrm{m}$ diameter $(\bar{x}=223 \times 166.5 \mu \mathrm{m})$, hysterothecial, superficial, semi-immersed or sunken at the base, oval to elongate, or subglobose, black, carbonaceous, straight or curved, with slightly hyphae penetrating into the host tissue. Ostiole central, with longitudinal slit. Peridium 34-58 $\mu \mathrm{m}$ thick, relatively thick, strongly carbonaceous texture, composed of thick-walled dark angular or relatively compressed pseudoparenchymatous cells, inner layers composed of hyaline to brown cells of textura angularis. Hamathecium comprising 1-1.5 $\mu \mathrm{m}$ thin, cylindrical to filiform, hyaline, branched, septate, anastomosing pseudoparaphyses. Asci $34-53 \times 4-5.5 \mu \mathrm{m}(\bar{x}=44 \times 4.5 \mu \mathrm{m}, \mathrm{n}=$ 
20), 8-spored, bitunicate, fissitunicate, elongate cylindric-clavate, straight or slightly curved, sessile or with short pedicel, apically rounded, with a minute ocular chamber. Ascospores 5-8 $\times 2.5-3 \mu \mathrm{m}$ $(\bar{x}=6 \times 3 \mu \mathrm{m}, \mathrm{n}=20), 1$-seriate, hyaline, ellipsoidal, 1-septate, constricted at the septa, upper cell wider and tapering towards the narrow ends, guttulate, smooth-walled. Asexual morph: Coelomycetous. Conidiomata 42-85 $\mu \mathrm{m}$ high, 42-77 $\mu \mathrm{m}$ diameter, superficial, grouped to scattered, subiculum, uniloculate or multi-loculate, subglobose to globose, dark brown. Conidioma wall 42-77 $\mu \mathrm{m}$ wide, pseudoparenchymatous, composed of dark brown cells of textura angularis to subglobosa. Conidiophores 11-21 $\mu \mathrm{m}$ long, 2-2.5 $\mu \mathrm{m}$ wide, cylindrical, hyaline. Conidiogenous cells phialidic, hyaline, simple, smooth, with a conspicuous collarette at the apex. Conidia $3-4 \times$ 1.5-2 $\mu \mathrm{m}(\bar{x}=4 \times 2 \mu \mathrm{m}, \mathrm{n}=20)$, 1-celled, oblong to ellipsoidal or oval, rounded ends, slightly curved, aseptate, hyaline, smooth-walled.

Culture characteristics - Ascospores germinating on MEA with $12 \mathrm{~h}$ and germ tubes produced from both ends. Colonies on MEA reaching $9 \mathrm{~mm}$ after 7 days at $28{ }^{\circ} \mathrm{C}$, slightly effuse, radially with undulate edge, greyish to light brown, with reddish pink pigmented in media. Mycelium superficial, branched, septate, hyaline to light brown, smooth-walled, asexual state formed within 60 days.

Known distribution (based on molecular data) - USA (Louisiana and Michigan States) (Mugambi \& Huhndorf 2009), India (Hongsanan et al. 2020), Thailand (Chiang Rai Province) (Jayasiri et al. 2016, and this study).

Known substrates (based on molecular data) - On dead wood (this study).

Material examined - Thailand, Chiang Rai, Huai Kang Pla, on dead wood, 25 October 2010, S. Boonmee, HPK03 (MFLU 10-0974, new geographical record); living culture (MFLUCC 10$0928=$ BCC 52146).

GenBank numbers - ITS: MN608542, LSU: MN577411, SSU: MN577422.

Notes - The new collection is a typical Anteaglonium species, and it shares similar morphological characters such as hysterothecial ascomata, black, carbonaceous, cylindric-clavate, bitunicate, fissitunicate asci and uniseriate, ellipsoidal, hyaline, 1-septate, small ascospores (less than $10 \mu \mathrm{m}$ ). In addition, this species always developed a coelomycetous asexual morph in culture (Fig. 15). Multi-gene phylogenetic analysis placed these four strains (MFLUCC 10-0928, MFLUCC 11-0374, MFLUCC 11-0380, MFLUCC 11-0511) with other Anteaglonium parvulum isolates (SMH 5210, MFLUCC 14-0815, MFLUCC 14-0817, MFLUCC 14-0821, MFLUCC 140823) with moderate-support and type sequence of this species is unavailable (Fig. 13). These four strains share similar sexual morphological features, but they differ in culture and asexual characteristics.

Anteaglonium parvulum (Schwein.) Mugambi \& Huhndorf, Syst. Biodiv. 7(4): 460 (2009)

Facesoffungi number: FoF01931

Figs 16, 17

Saprobic on dead wood. Sexual morph: Ascomata 155-261 $\mu \mathrm{m}$ high $\times 180-284 \mu \mathrm{m}$ diameter $(\bar{x}=196 \times 240 \mu \mathrm{m})$, hysterothecial, superficial, semi-immersed or sunken at the base, oval to elongate, or subglobose, uniloculate or multi-loculate, carbonaceous, brittle, straight or curved, black. Ostiole central, with longitudinal slit. Peridium 32-48(-77.5) $\mu \mathrm{m}$ thick, relatively thick, strongly carbonaceous texture, composed of thick-walled dark angular or relatively compressed pseudoparenchymatous cells, inner layers composed of hyaline to brown cells of textura angularis. Hamathecium comprising 1-2.5 $\mu \mathrm{m}$ thin, cylindrical to filiform, hyaline, branched, septate, anastomosing pseudoparaphyses. Asci $28-41 \times 4-5.5 \mu \mathrm{m}(\bar{x}=35.5 \times 4.5 \mu \mathrm{m}, \mathrm{n}=20)$, 8-spored, bitunicate, fissitunicate, elongate cylindric-clavate, straight or slightly curved, sessile or with short pedicel, apically rounded, with a minute ocular chamber. Ascospores 5-8 $\times 2-3 \mu \mathrm{m}(\bar{x}=6.5 \times 2.5$ $\mu \mathrm{m}, \mathrm{n}=20$ ), 1-seriate, hyaline, ellipsoidal, 1-septate, constricted at the septa, upper cell wider and tapering towards the narrow ends, guttulate, smooth-walled. Asexual morph: Coelomycetous. Conidiomata grouped to scattered, superficial, semi-immersed at the base, subglobose to globose, dark brown. Conidioma wall pseudoparenchymatous, composed of dark brown cells of textura 
angularis. Conidiophores 9-21 $\mu \mathrm{m}$ long, 1.5-3 $\mu \mathrm{m}$ wide, cylindrical, hyaline. Conidiogenous cells holoblastic. Conidia 2.5-4 × 2-3 $\mu \mathrm{m}(\bar{x}=3 \times 2 \mu \mathrm{m}, \mathrm{n}=20)$, 1-celled, globose, subglobose to ellipsoidal, aseptate, hyaline, guttulate, smooth-walled.

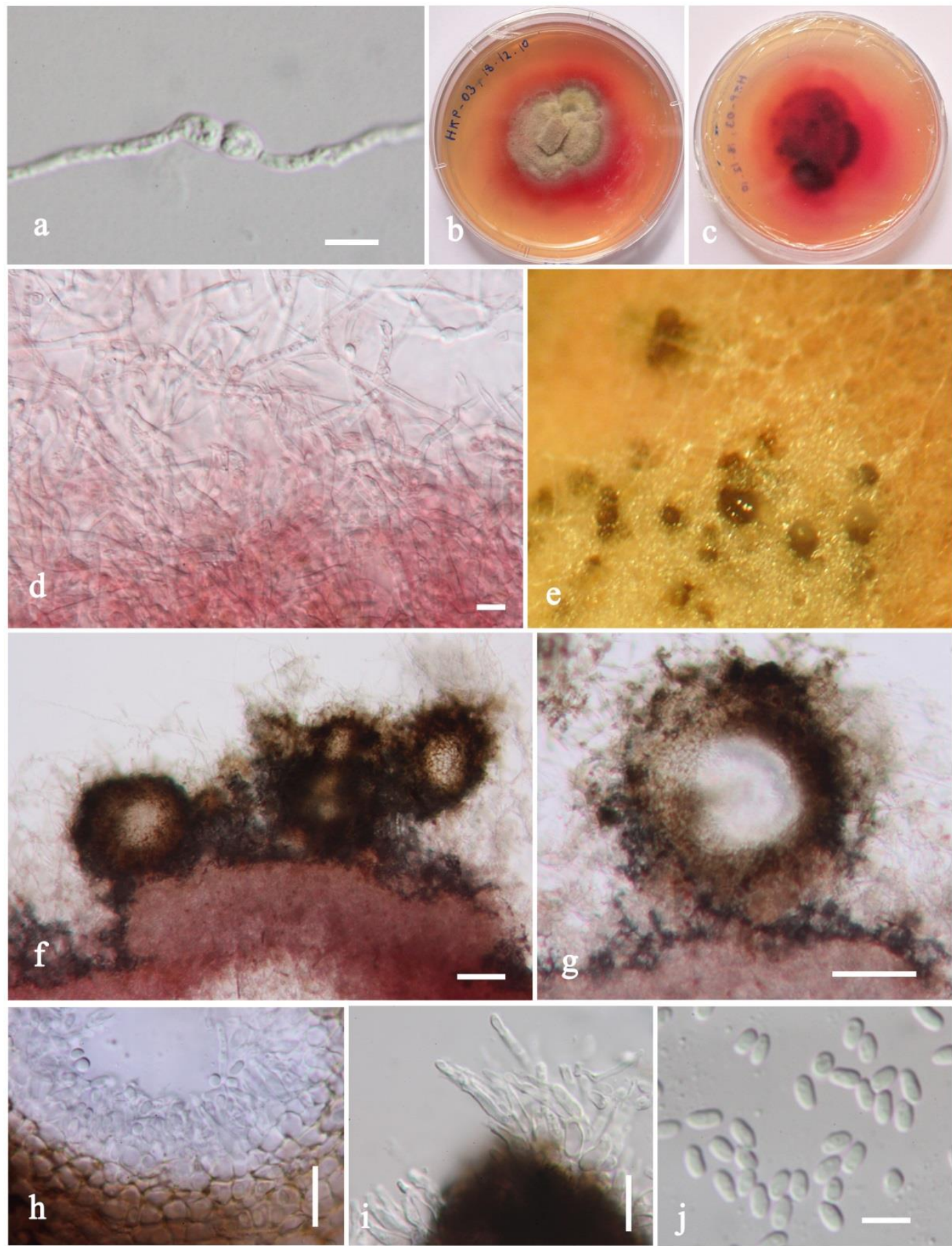

Fig. 15 - Anteaglonium parvulum (MFLUCC 10-0928, living culture). a Germinating spore. $\mathrm{b}, \mathrm{c}$ Culture colonies on MEA from surface and reverse. $d$ Aerial hyphae in culture. e Developing conidiomata in culture. $\mathrm{f}, \mathrm{g}$ Section of conidiomata. $\mathrm{h}$, i Peridium with developing of conidiophores and conidiogenous cells. $\mathrm{j}$ Conidia. Scale bars: $\mathrm{a}, \mathrm{d}, \mathrm{j}, \mathrm{i}=5 \mu \mathrm{m}, \mathrm{f}, \mathrm{g}=20 \mu \mathrm{m}, \mathrm{h}=10 \mu \mathrm{m}$.

Culture characteristics - Ascospores germinating on MEA with $12 \mathrm{~h}$ and germ tubes produced from both ends. Colonies on MEA reaching $8 \mathrm{~mm}$ after 7 days at $28^{\circ} \mathrm{C}$, slightly effuse, radially with undulate edge, greyish, light brown, reddish pink. Mycelium superficial, branched, 
septate, hyaline to light brown, with reddish pink pigmented, smooth-walled, asexual state formed within 60 days.

Known distribution (based on molecular data) - USA, Louisiana and Michigan States (Mugambi \& Huhndorf 2009), India (Hongsanan et al. 2020), Thailand, Chiang Rai Province (Jayasiri et al. 2016, this study).

Known substrates (based on molecular data) - On dead wood (this study).

Material examined - Thailand, Chiang Rai, Muang, Doi Pui, on dead wood, 10 May 2011, S. Boonmee, DP05 (MFLU 11-0389, new geographical record); living culture (MFLUCC 11-0380 = BCC 52031).

GenBank numbers - ITS: MN608544, LSU: MN577413, SSU: MN577424

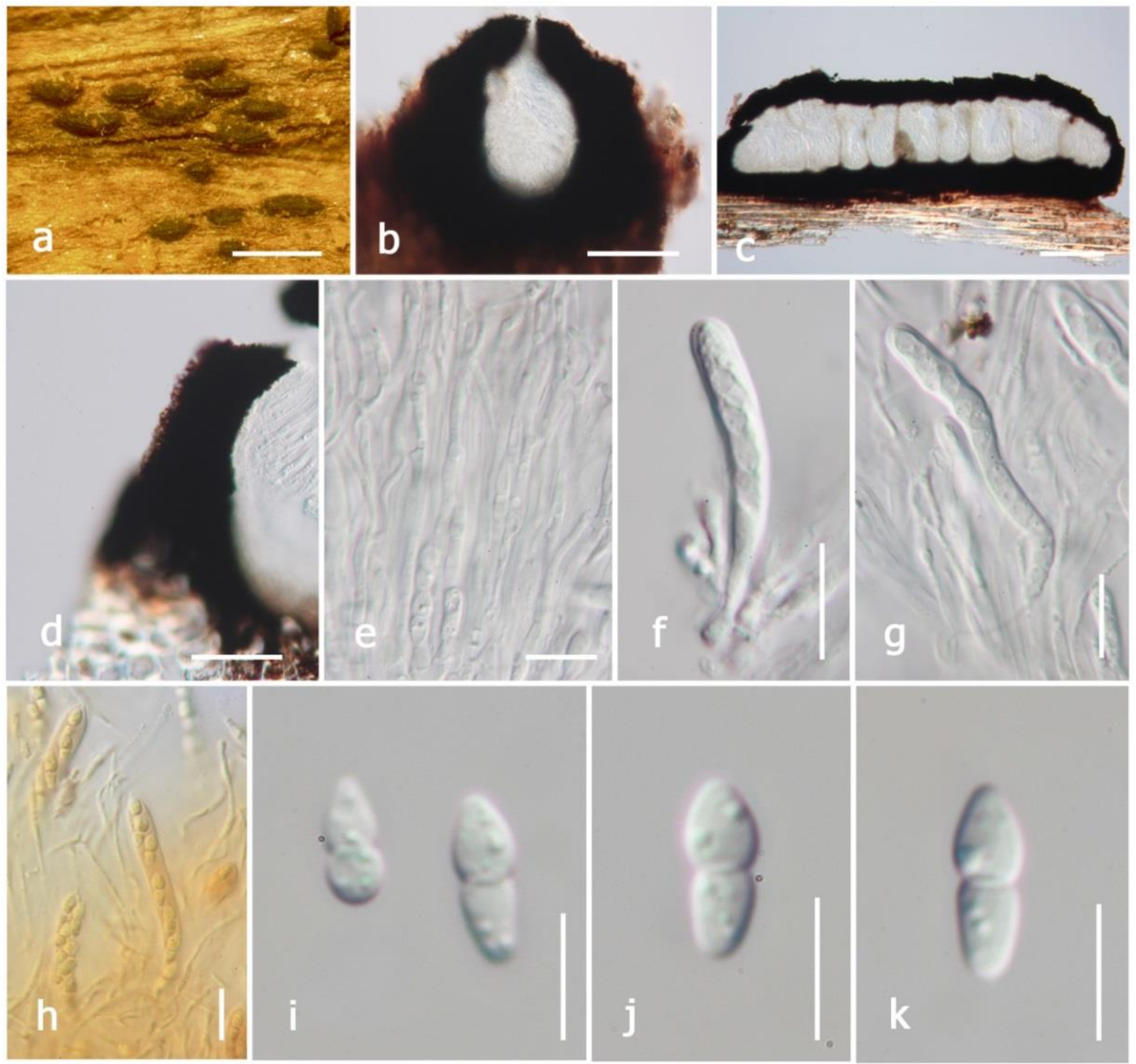

Fig. 16 - Anteaglonium parvulum (MFLU 11-0389, new geographical record). a Appearance of ascomata on woody substrate. b, c Vertical and longitudinal sections of multi-loculate stromata. d Peridium. e Pseudoparaphyses. $\mathrm{f}-\mathrm{h}$ Asci (Fig. $\mathrm{h}$ stained in Melzer's reagent). $\mathrm{i}-\mathrm{k}$ Ascospores. Scale bars: $\mathrm{a}=1000 \mu \mathrm{m}, \mathrm{b}, \mathrm{c}=100 \mu \mathrm{m}, \mathrm{d}=40 \mu \mathrm{m}, \mathrm{e}-\mathrm{h}=10 \mu \mathrm{m}, \mathrm{i}-\mathrm{k}=5 \mu \mathrm{m}$.

Anteaglonium parvulum (Schwein.) Mugambi \& Huhndorf, Syst. Biodiv. 7(4): 460 (2009)

Facesoffungi number: FoF01931

Figs 18, 19

Saprobic on dead wood. Sexual morph: Ascomata 230-259 $\mu \mathrm{m}$ high $\times 209-268 \mu \mathrm{m}$ diameter $(\bar{x}=$ $247.5 \times 239 \mu \mathrm{m})$, hysterothecial, superficial, semi-immersed or sunken at the base, oval to 
elongate, or subglobose, carbonaceous, brittle, straight or curved, black. Ostiole central, with longitudinal slit. Peridium 52-69 $\mu \mathrm{m}$ thick, relatively thick, strongly carbonaceous texture, composed of thick-walled dark angular or relatively compressed pseudoparenchymatous cells, inner layers composed of hyaline to brown cells of textura angularis. Hamathecium comprising 1-2 $\mu \mathrm{m}$ thin, cylindrical to filiform, hyaline, branched, septate, anastomosing pseudoparaphyses. Asci 38.5$59 \times 3.5-6 \mu \mathrm{m}(\bar{x}=49 \times 5 \mu \mathrm{m}, \mathrm{n}=20), 8$-spored, bitunicate, fissitunicate, elongate cylindricclavate, straight or slightly curved, sessile or with short pedicel, apically rounded, with a minute an ocular chamber. Ascospores 5-9 $\times 2-4 \mu \mathrm{m}(\bar{x}=6.5 \times 3 \mu \mathrm{m}, \mathrm{n}=20), 1$-seriate, hyaline, ellipsoidal, 1-septate, constricted at the septa, upper cell wider and tapering towards the narrow ends, smoothwalled. Asexual morph: Coelomycetous. Conidiomata grouped to scattered, superficial, subglobose to globose, dark brown, surrounded by aerial mycelia. Conidiophores and conidiogenous cells not observed. Conidia 2.5-5 × 3-5 $\mu \mathrm{m}(\bar{x}=3 \times 4 \mu \mathrm{m}, \mathrm{n}=20)$, 1-celled, globose to subglobose, aseptate, hyaline, guttulate, smooth-walled.

Culture characteristics - Ascospores germinating on MEA with $12 \mathrm{~h}$ and germ tubes produced from both ends. Colonies on MEA reaching $10 \mathrm{~mm}$ after 7 days at $28{ }^{\circ} \mathrm{C}$, effuse, velvety to hairy, radially with entire edge, dark brown and sparse mycelium in the outer ring on the surface with light brown margin. Mycelium superficial and partially immersed, branched, septate, light brown, smooth-walled, asexual state formed within 60 days.

Known distribution (based on molecular data) - USA, Louisiana and Michigan States (Mugambi \& Huhndorf 2009), India (Hongsanan et al. 2020), Thailand, Chiang Rai Province (Jayasiri et al. 2016), Thailand, Chiang Mai Province (this study).

Known substrates (based on molecular data) - On dead wood (this study).

Material examined - Thailand, Chiang Mai, Muang, on dead wood, 21 April 2011, S. Boonmee, DST02 (MFLU 11-0133, new geographical record); living culture (MFLUCC 11-0374 $=$ BCC 52032).

GenBank numbers - ITS: MN608543, LSU: MN577412, SSU: MN577423.

Anteaglonium parvulum (Schwein.) Mugambi \& Huhndorf, Syst. Biodiv. 7(4): 460 (2009)

Figs 20, 21

Facesoffungi number: FoF01931

Saprobic on dead wood. Sexual morph: Ascomata 155-242 $\mu \mathrm{m}$ high $\times 216-292 \mu \mathrm{m}$ diameter $(\bar{x}=206 \times 240 \mu \mathrm{m})$, hysterothecial, superficial, semi-immersed or sunken at the base, oval to elongate, or subglobose, black, carbonaceous, straight or curved. Ostiole central, with longitudinal slit. Peridium 34-83 $\mu \mathrm{m}$ thick, relatively thick, strongly carbonaceous texture, composed of thickwalled dark angular or relatively compressed pseudoparenchymatous cells, inner layers composed of hyaline to brown cells of textura angularis. Hamathecium comprising 1-2 $\mu \mathrm{m}$ thin, cylindrical to filiform, hyaline, branched, septate, anastomosing pseudoparaphyses. Asci 45-55 $\times 3-6 \mu \mathrm{m}(\bar{x}=50$ $\times 4 \mu \mathrm{m}, \mathrm{n}=20$ ), 8 -spored, bitunicate, fissitunicate, elongate cylindric-clavate, straight or slightly curved, sessile or with short pedicel, apically rounded, with a minute ocular chamber. Ascospores 6-8 $\times 3-4 \mu \mathrm{m}(\bar{x}=7 \times 3.5 \mu \mathrm{m}, \mathrm{n}=20), 1$-seriate, hyaline, ellipsoidal, 1-septate, constricted at the septa, upper cell wider and tapering towards the narrow ends, smooth-walled. Asexual morph: Coelomycetous. Conidiomata grouped to scattered, superficial, subglobose to globose, dark brown. Conidiophores holoblastic, cylindrical, hyaline. Conidiogenous cells not observed. Conidia $2-4 \times 2$ $\mu \mathrm{m}(\bar{x}=3 \times 2 \mu \mathrm{m}, \mathrm{n}=20)$, 1-celled, globose to subglobose, oblong to ellipsoidal or oval, aseptate, hyaline, smooth-walled.

Culture characteristics - Ascospores germinating on MEA with $12 \mathrm{~h}$ and germ tubes produced from both ends. Colonies on MEA reaching $8 \mathrm{~mm}$ after 7 days at $28^{\circ} \mathrm{C}$, effuse, radially with entire edge, initially white cream, eventually turning unevenly brown after 4 weeks. Mycelium superficial, branched, septate, brown, smooth-walled, asexual state formed within 60 days.

Known distribution (based on molecular data) - Thailand, USA, Louisiana and Michigan States (Mugambi \& Huhndorf 2009), India (Hongsanan et al. 2020), Thailand, Chiang Rai Province (Jayasiri et al. 2016), Thailand, Chiang Mai Province (this study). 
Known substrates (based on molecular data) - On dead wood (this study).

Material examined - Thailand, Chiang Mai, Mae Taeng, Mushroom Research Center, on dead wood, 23 June 2011, S. Boonmee, MRC04 (MFLU 11-1147, new geographical record); living culture (MFLUCC 11-0511= BCC 52384).

GenBank numbers - ITS: MN608545, LSU: MN577414, SSU: MN577425.

Astrosphaeriellaceae Phookamsak \& K.D. Hyde

Astrosphaeriellaceae was introduced by Phookamsak et al. (2015) to accommodate Astrosphaeriella and Pteridiospora. Another five genera viz. Astrosphaeriellopsis (Wanasinghe et al. 2018a), Javaria (Wijayawardene et al. 2018), Pithomyces (Wanasinghe et al. 2018a), Quercicola and Xenoastrosphaeriella (Jayasiri et al. 2019) were included in this family. The family is characterized by conical, carbonaceous ascostromata, bitunicate asci with trabeculate pseudoparaphyses, fusiform or obclavate ascospores with or without sheath and both coelomycetous and hyphomycetous asexual morphs (Phookamsak et al. 2015, Wanasinghe et al. 2018a).

Astrosphaeriella Syd. \& P. Syd.

Astrosphaeriella introduced by Sydow \& Sydow (1913) with A. fusispora as the type, is a common genus and can be found on bamboos, palms and stout grasses (Barr 1990a, Zhou et al. 2003, Tanaka \& Harada 2005, Hu 2010, Zhang et al. 2012, Phookamsak et al. 2015, this study). The genus is similar to Acrocordiopsis, Caryospora, Caryosporella, Mamillisphaeria and Trematosphaeria in having carbonaceous ascostromata, and trabeculate pseudoparaphyses (Hawksworth 1981, Hyde \& Fröhlich 1998, Hu 2010, Liu et al. 2011, Zhang et al. 2012), however, it can be distinguished based on asci and ascospores shape and hosts (Phookamsak et al. 2015). The genus has coelomycetous asexual morph as seen in the holomorph of Astrosphaeriella bambusae (Phookamsak et al. 2015).

Astrosphaeriella neostellata D.Q. Dai, Phookamsak \& K.D. Hyde, Fungal Diversity 74: 172 (2015)

Fig. 23

Facesoffungi number: FoF01226

Saprobic on dead bamboo culms. Sexual morph: Ascomata 300-700 $\mu \mathrm{m}$ diameter, 250-500 $\mu \mathrm{m}$ high, black, scattered, solitary, 2-3-gregarious, erumpent to superficial, breaking the host tissue, conical, uni-loculate, glabrous, carbonaceous, with centrally ostiole. Peridium 40-50 $\mu \mathrm{m}$ wide, of unequal thickness, poorly developed at the base, composed of dark brown to black pseudoparenchymatous cells arranged in textura angularis. Hamathecium comprising dense, 1-1.5 $\mu \mathrm{m}$ wide, branched, filamentous pseudoparaphyses, anastomosing at the top, embedded in gelatinous matrix. Asci 130-200 × 10-14 $\mu \mathrm{m}(\bar{x}=165 \times 12 \mu \mathrm{m}, \mathrm{n}=20), 8$-spored, bitunicate, fissitunicate, cylindrical to clavate, with a long furcate pedicel, apically rounded with an indistinct ocular chamber. Ascospores 40-50 × 4.5-5.5 $\mu \mathrm{m}(\bar{x}=45 \times 5 \mu \mathrm{m}, \mathrm{n}=20)$, overlapping 1-2-seriate, hyaline to subhyaline, fusiform, tapering at both ends, rarely aseptate, normally 1-septate, constricted at the septum, smooth-walled, guttulate, surrounded by a thin gelatinous sheath. Asexual morph: Undetermined.

Culture characteristics - Ascospores germinating on PDA within $48 \mathrm{~h}$, at $23-28^{\circ} \mathrm{C}$. Colonies growing on PDA, reaching $15 \mathrm{~mm}$ diameter in 2 weeks at $23-28^{\circ} \mathrm{C}$, cottony, circular, umbonate, pale brown at the middle, dark brown at the margin from above; dark brown from below. Mycelium superficial to immersed in media, with dark brown, branched, septate, smooth hyphae.

Known distribution (based on molecular data) - Thailand, Chiang Rai (Phookamsak et al. 2015), China, Yunnan (this study).

Known hosts (based on molecular data) - Dead bamboo culms (Phookamsak et al. 2015, this study).

Material examined - China, Yunnan Province, Chuxiong Yi Autonomous Prefecture, Chuxiong City, Biji Village, on dead bamboo culms, 5 February 2019, H.B. Jiang, CX003 (KUN- 
HKAS 101775, new geographic record), living culture (KUMCC 19-0218).

GenBank numbers - ITS: MN629351, LSU: MN629352, SSU: MN629353, tef1: MN635787.

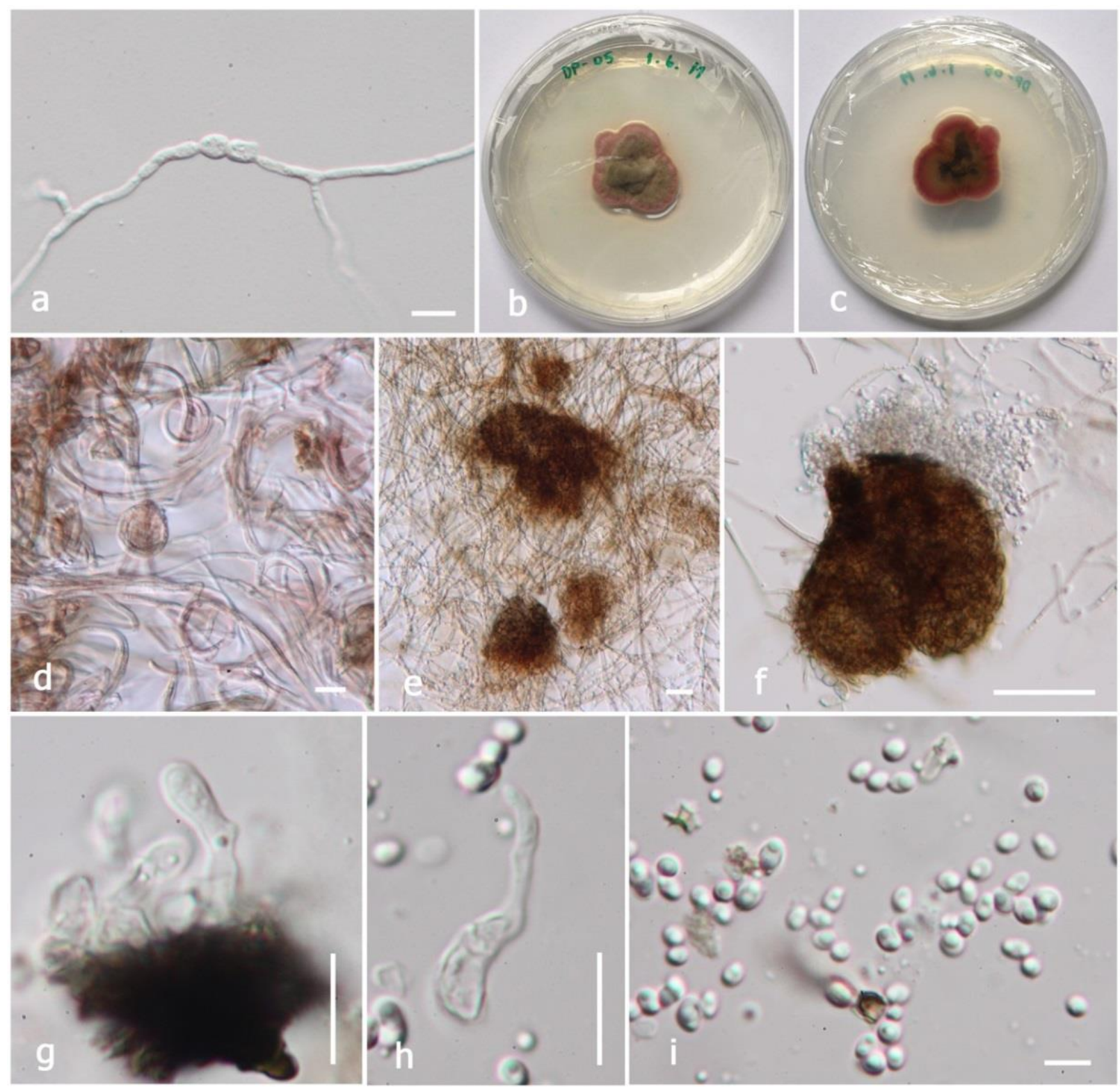

Fig. 17 - Anteaglonium parvulum (MFLUCC 11-0380, living culture). a Germinating spore. $\mathrm{b}$, c Culture colonies on MEA from surface and reverse. d, e Aerial hyphae in culture. e Compressed hyphae and developing conidiomata in culture. f Squash mount of conidioma. $\mathrm{g}$, $\mathrm{h}$ Developing of conidiophores and conidiogenous cells. i Conidia. Scale bars: $\mathrm{a}, \mathrm{d}, \mathrm{g}, \mathrm{h}=10 \mu \mathrm{m}$, $\mathrm{e}=20 \mu \mathrm{m}, \mathrm{f}=50 \mu \mathrm{m}, \mathrm{i}=5 \mu \mathrm{m}$.

Notes - The new strain forms a sister clade to Astrosphaeriella neostellata (MFLUCC 110625) with high statistical support (Fig. 22). It also shares similar morphological characteristics with the type specimen (MFLU 15-1195) of A. neostellata and has small differences in the size of ascomata. A comparison of the LSU, SSU and tef1 nucleotides of these two strains reveals less than $1.5 \%$ nucleotide differences, which demonstrates that our new collection is Astrosphaeriella neostellata (Jeewon \& Hyde 2016). Based on the known distribution of Astrosphaeriella neostellata (Phookamsak et al. 2015), the new collection is reported in China for the first time in this study. 

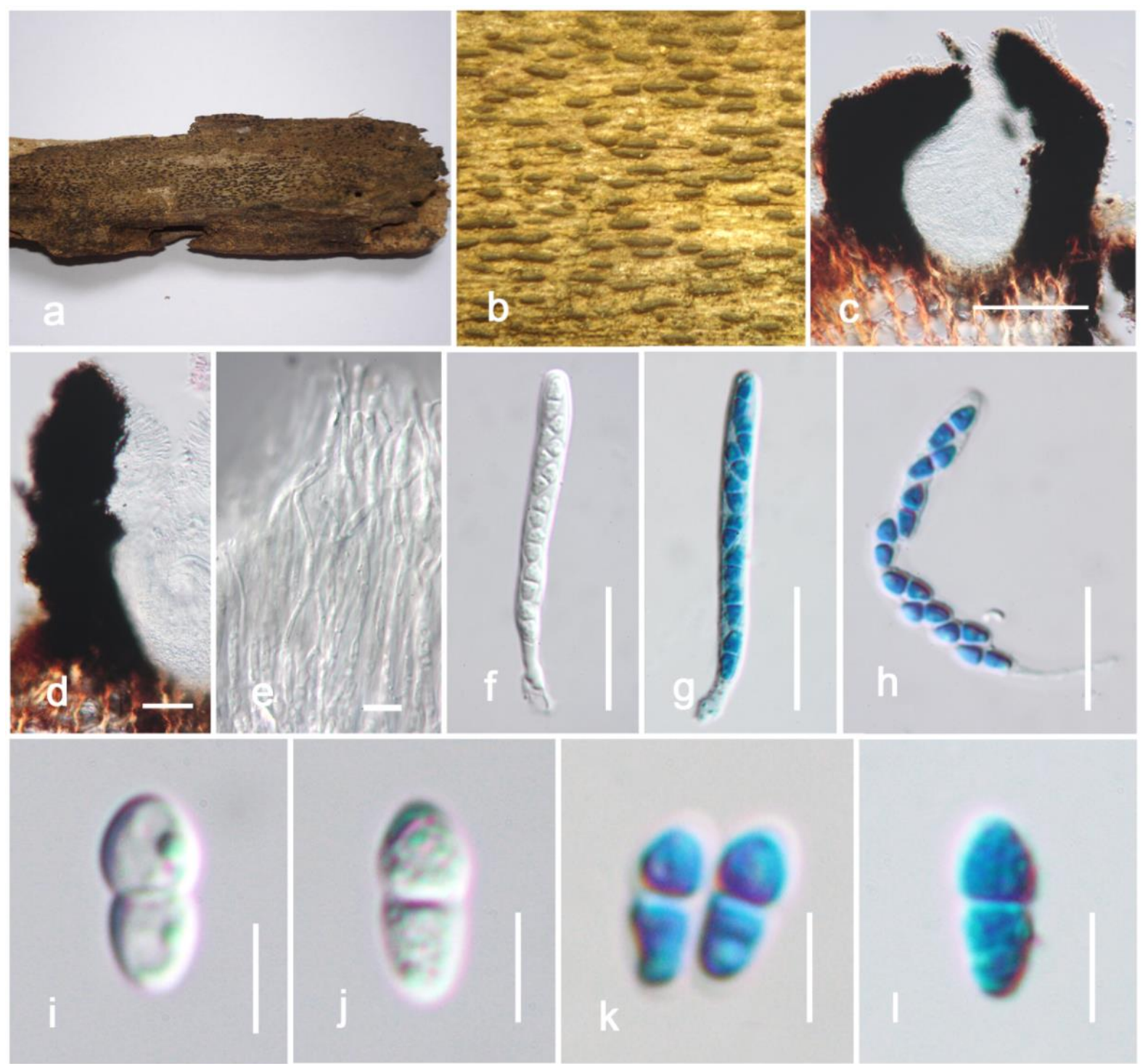

Fig. 18 - Anteaglonium parvulum (MFLU 11-0133, new geographical record). a, b Appearance of ascomata on woody substrate. b Vertical section of ascoma. d Peridium. e Pseudoparaphyses. $\mathrm{f}-\mathrm{h}$ Asci (Figs. g, h stained in cotton blue reagent). i-l Ascospores (Figs. g, h stained in cotton blue reagent). Scale bars: $c=100 \mu \mathrm{m}, \mathrm{d}=40 \mu \mathrm{m}, \mathrm{e}, \mathrm{i}-\mathrm{l}=5 \mu \mathrm{m}, \mathrm{f}-\mathrm{h}=20 \mu \mathrm{m}$.

Camarosporidiellaceae Wanas., Wijayaw., Crous \& K.D. Hyde

Based on broad morphology and phylogeny investigations, Wanasinghe et al. (2017) introduced Camarosporidiellaceae to accommodate species with conidial morphology resembling Camarosporium sensu stricto and other camarosporium-like genera. The family includes a single genus Camarosporidiella (Wanasinghe et al. 2017, Wijayawardene et al. 2018). The members of Camarosporidiellaceae have coelomycetous asexual morphs, comprising pycnidial conidiomata, with a single, papillate ostiole, enteroblastic, annellidic, integrated to discrete, doliiform, lageniform or cylindrical, hyaline conidiogenous cells and pale to dark brown conidia that are phragmosporous to muriform and mostly ellipsoidal. Sexual morphs are gregarious to solitary, globose to subglobose ascomata having a papillate, central ostiole, a peridium containing cell layers of textura angularis, cylindrical, (2-)4-8-spored asci and uniseriate, ellipsoidal, brown, muriform ascospores. Camarosporidiellaceae members are cosmopolitan in distribution and exist as saprobes, endophytes or pathogens of various host species (Wanasinghe et al. 2017, Hyde et al. 2018). An updated phylogenetic tree for the family is presented in Fig. 24 and we introduce a new host record of Camarosporidiella laburni from Colutea cilicica (Fabaceae). 


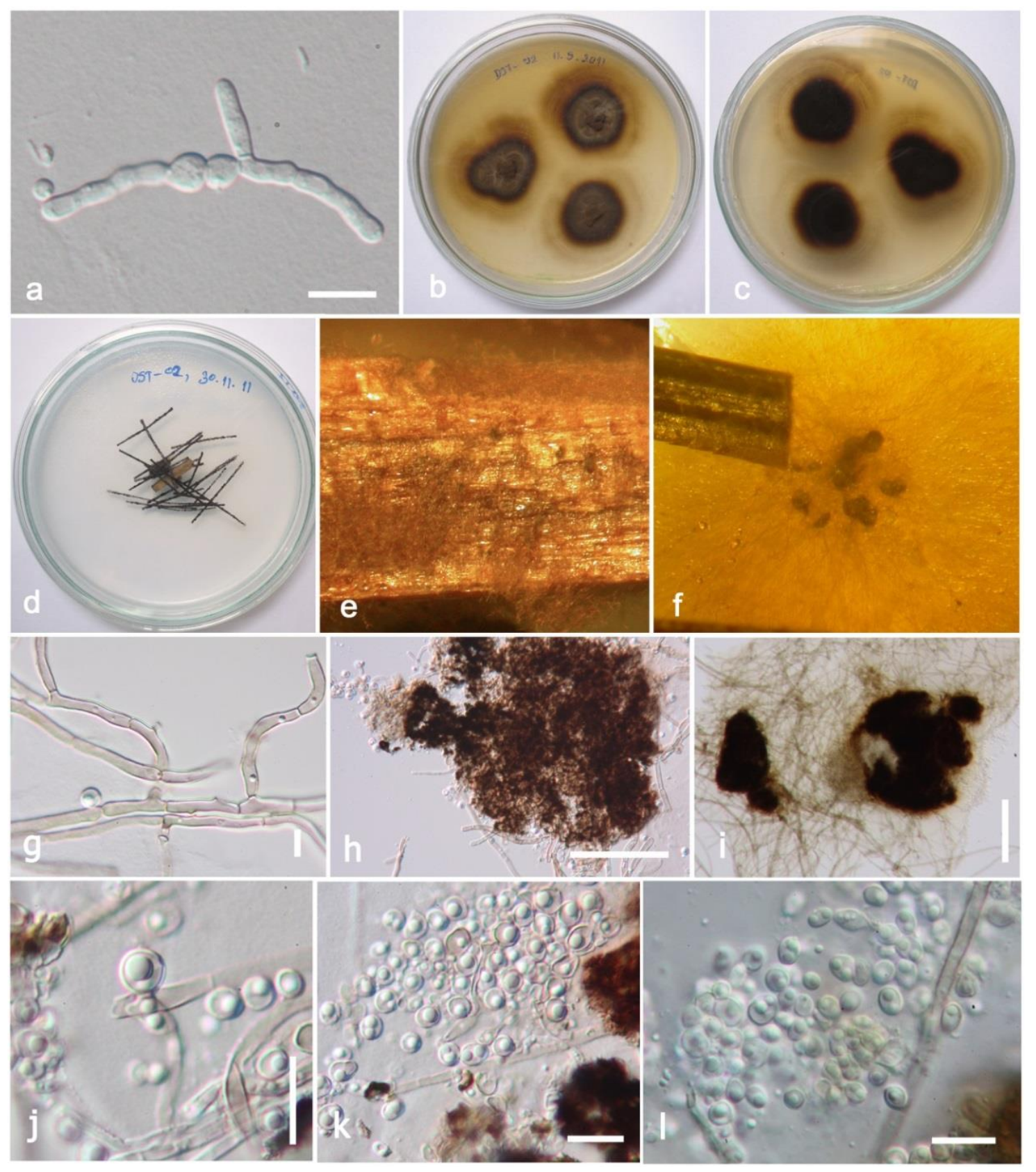

Fig. 19 - Anteaglonium parvulum (MFLU 11-0374, living culture). a Germinating spore. $\mathrm{b}$, c Culture colonies on MEA from surface and reverse. $\mathrm{d}-\mathrm{f}$ Growth of asexual state on plant tissues and developing conidiomata in water agar culture. g Aerial hyphae in culture. $h$, i Squash mount of conidiomata. $\mathrm{j}$ Close up of conidiophore and conidiogenous cell with developing of conidium at the apex. $\mathrm{k}, 1$ Conidia. Scale bars: $\mathrm{a}, \mathrm{j}-\mathrm{l}=10 \mu \mathrm{m}, \mathrm{g}=5 \mu \mathrm{m}, \mathrm{h}=50 \mu \mathrm{m}, \mathrm{i}=100 \mu \mathrm{m}$.

Camarosporidiella Wanas., Wijayaw. \& K.D. Hyde

Camarosporidiella was introduced by Wanasinghe et al. (2017) to accommodate $C$. caraganicola as the type species. Camarosporidiella is characterized in having camarosporium-like asexual morph and cucurbitaria-like sexual morph. Based on morphology and phylogeny support, Wanasinghe et al. (2017) placed 20 species in this genus and Hyde et al. (2018) introduced another new species, Camarosporidiella populina from Russia. Camarosporidiella species show a wide distribution as saprobes, endophytes or pathogens of various host species. In this study, we follow the latest treatment and updated account of Camarosporidiella in Wanasinghe et al. (2017) and Hyde et al. (2018). Camarosporidiella laburni is introduced as a new host record from Colutea cilicica (Fabaceae). 

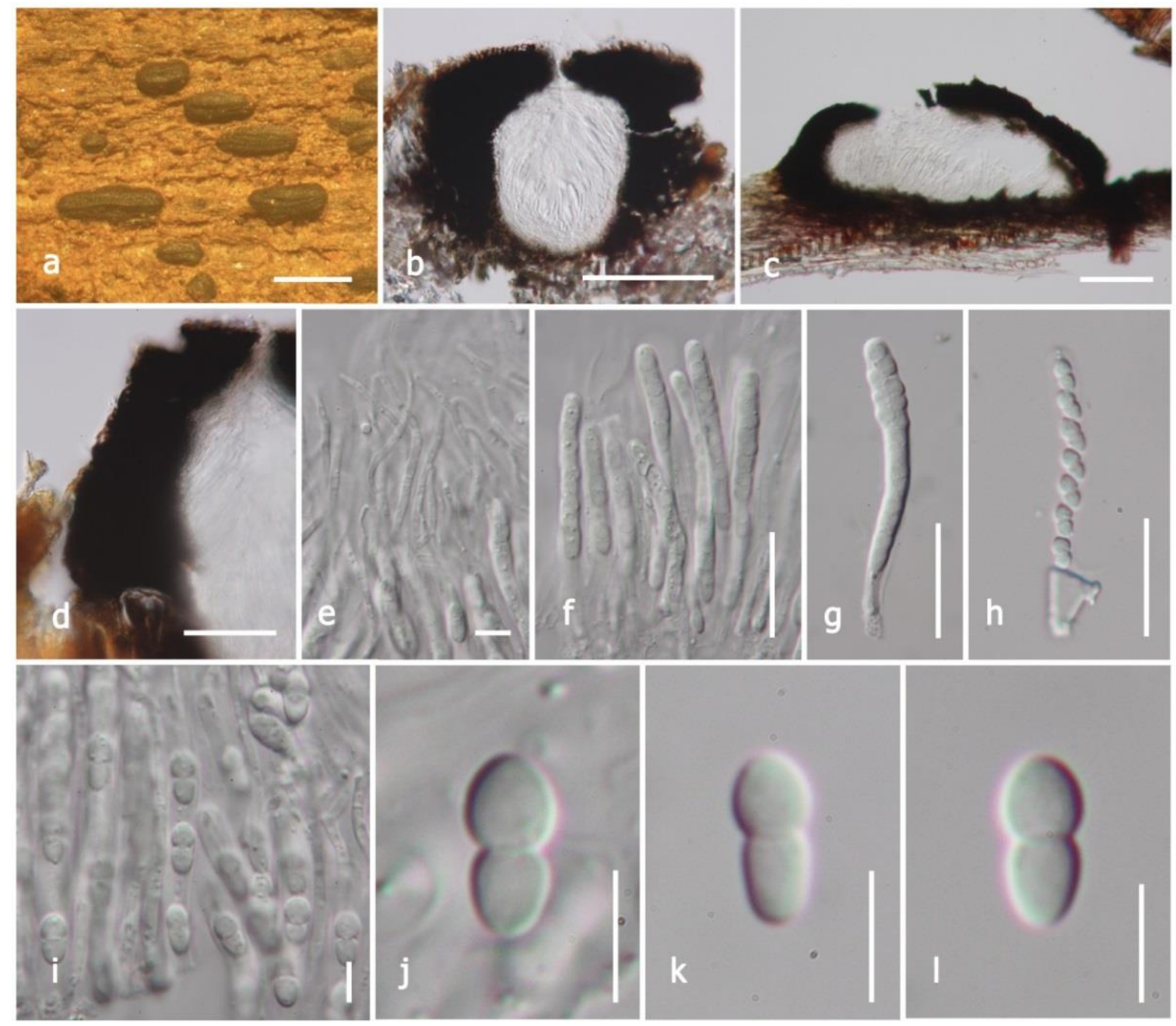

Fig. 20 - Anteaglonium parvulum (MFLU 11-1147, new geographical record). a Appearance of ascomata on woody substrate. b, c Vertical and longitudinal sections of ascomata. $d$ Peridium. e Pseudoparaphyses. $\mathrm{f}-\mathrm{h}$ Asci. i-1 Ascospores. Scale bars: $\mathrm{a}=500 \mu \mathrm{m}, \mathrm{b}, \mathrm{c}=100 \mu \mathrm{m}, \mathrm{d}=50 \mu \mathrm{m}$, $\mathrm{e}, \mathrm{i}-\mathrm{l}=5 \mu \mathrm{m}, \mathrm{f}-\mathrm{h}=20 \mu \mathrm{m}$.

Camarosporidiella celtidis (Shear) Thambug., Wanas. \& K.D. Hyde, Stud. Mycol. 87: 226 (2017)

Facesoffungi number: FoF 03533

Saprobic on dead twigs and thin branches. Asexual morph: Conidiomata pycnidial, 310-350 $\mu \mathrm{m}$ high, $330-400 \mu \mathrm{m}$ diameter $(\bar{x}=323.5 \times 358.8 \mu \mathrm{m}, \mathrm{n}=10)$, solitary or gregarious, black, immersed to semi-erumpent, unilocular. Pycnidial wall multi-layered, 22-26 $\mu \mathrm{m}$, thick, comprising 4-5 layers, light-brown cells of textura angularis, cells towards the inside lighter. Conidiophores reduced to conidiogenous cells. Conidiogenous cells enteroblastic, annellidic, doliiform, integrated, solitary, hyaline, smooth-walled, and formed from the inner layer of pycnidium wall. Conidia 11$14 \times 5-7 \mu \mathrm{m}(\bar{x}=12.8 \times 5.7 \mu \mathrm{m}, \mathrm{n}=30)$, oblong, straight, rounded at both ends, sometimes narrowly rounded ends, 3-transversely septate, without longitudinal septa, smooth-walled, initially hyaline, becoming brown to dark brown at maturity. Sexual morph: See Wanasinghe et al. (2017).

Culture characteristics - Colonies on PDA reaching $25 \mathrm{~mm}$ diameter after 2 weeks at 20 $25{ }^{\circ} \mathrm{C}$, colonies medium sparse, circular, flat, surface slightly rough with edge entire, margin well defined, cottony to fairly fluffy with sparse aspects, colony from above: light brown to yellowish the margin, light brown to grey at the centre; reverse, yellowish at the margin, dark brown to black at the centre; mycelium light brown to whitish grey with tufting; not producing pigments in PDA. 
Known distribution (based on molecular data) - USA (Shear 1902), Russia (Wanasinghe et al. 2017 and this study).

Known hosts (based on molecular data) - Ailanthus altissima, Betula pendula, Celtis occidentalis, Elymus repens, Gleditsia tracanthos, Maclura pomifera, Morus alba, Prunus padus, Spiraea sp. (Shear 1902, Wanasinghe et al. 2017), Prunus armeniaca (this study)

Material examined - Russia, Krasnodar region, Novorossiyssk City, trees near Sudzhuk lagoon $\left(44.6836366^{\circ} \mathrm{N} 37.7952325^{\circ} \mathrm{E}\right.$ ), on dead twigs of Prunus armeniaca (Rosaceae), 14 June 2016, Timur S. Bulgakov, NK071 (MFLU 17-0804, new host record), living culture, MFLUCC $17-1765$.

GenBank numbers - LSU: MN750588, SSU: MN750603, ITS: MN750609.

Notes - Camarosporidiella celtidis was introduced as Cucurbitaria celtidis by Shear (1902) from Celtis occidentalis. Thambugala et al. (2016a) placed this species in Camarosporium based on DNA sequence data from a fresh collection and introduced $\mathrm{Cm}$. uniseriatum. Based on morphology and phylogeny evidence, Wanasinghe et al. (2017) accommodated Cucurbitaria celtidis in Camarosporidiella and the asexual morph of the species is described and illustrated herein.

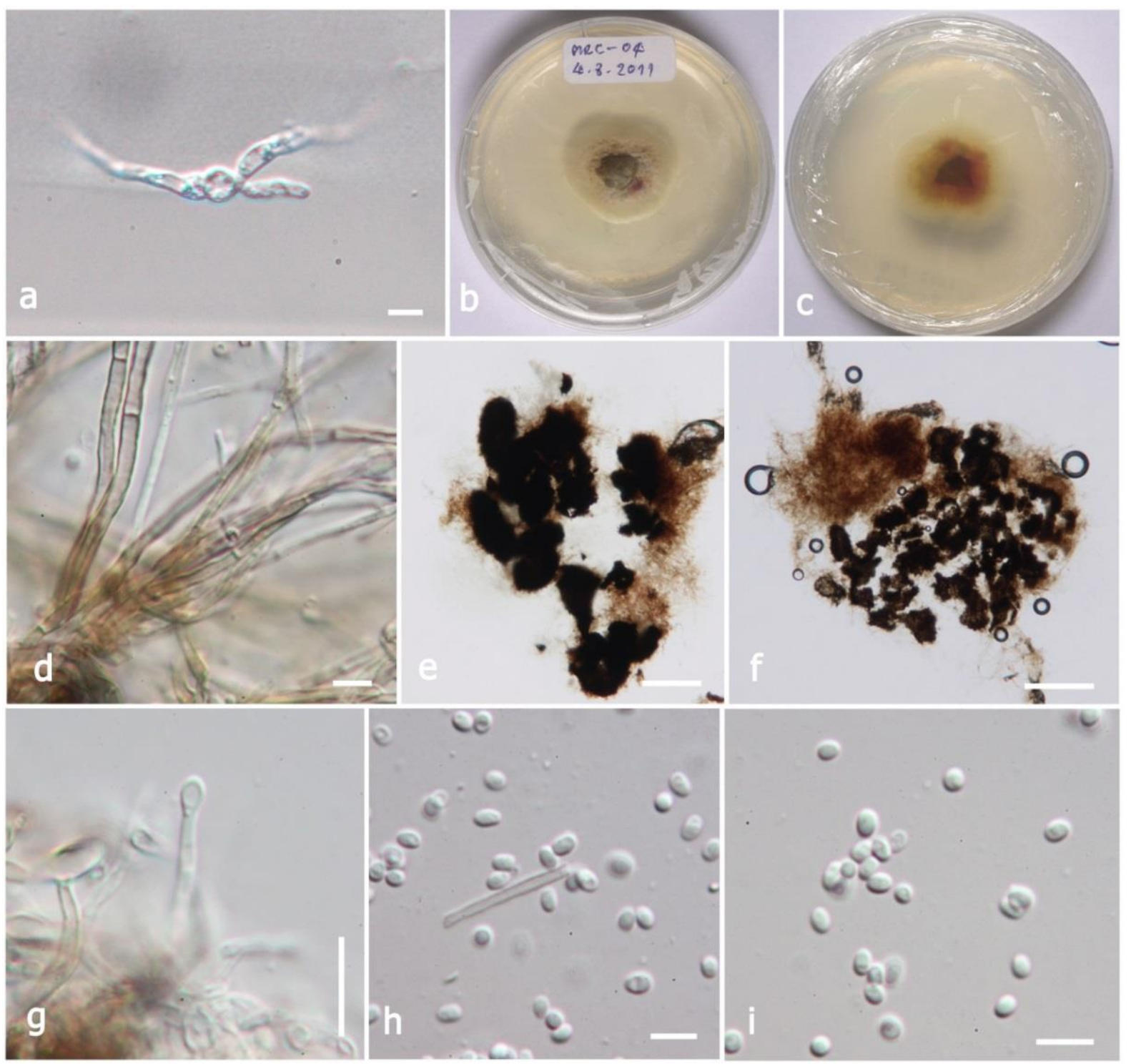

Fig. 21 - Anteaglonium parvulum (MFLUCC 11-0511, living culture). a Germinating spore. b, c Culture colonies on MEA from surface and reverse. d Aerial hyphae in culture. e, f Squash mount of conidiomata. g Close up of conidiophore and conidiogenous cell with developing of conidium at the apex. $\mathrm{h}$, i Conidia. Scale bars: $\mathrm{a}, \mathrm{h}, \mathrm{i}=5 \mu \mathrm{m}, \mathrm{e}, \mathrm{f}=200 \mu \mathrm{m}, \mathrm{g}=10 \mu \mathrm{m}$. 


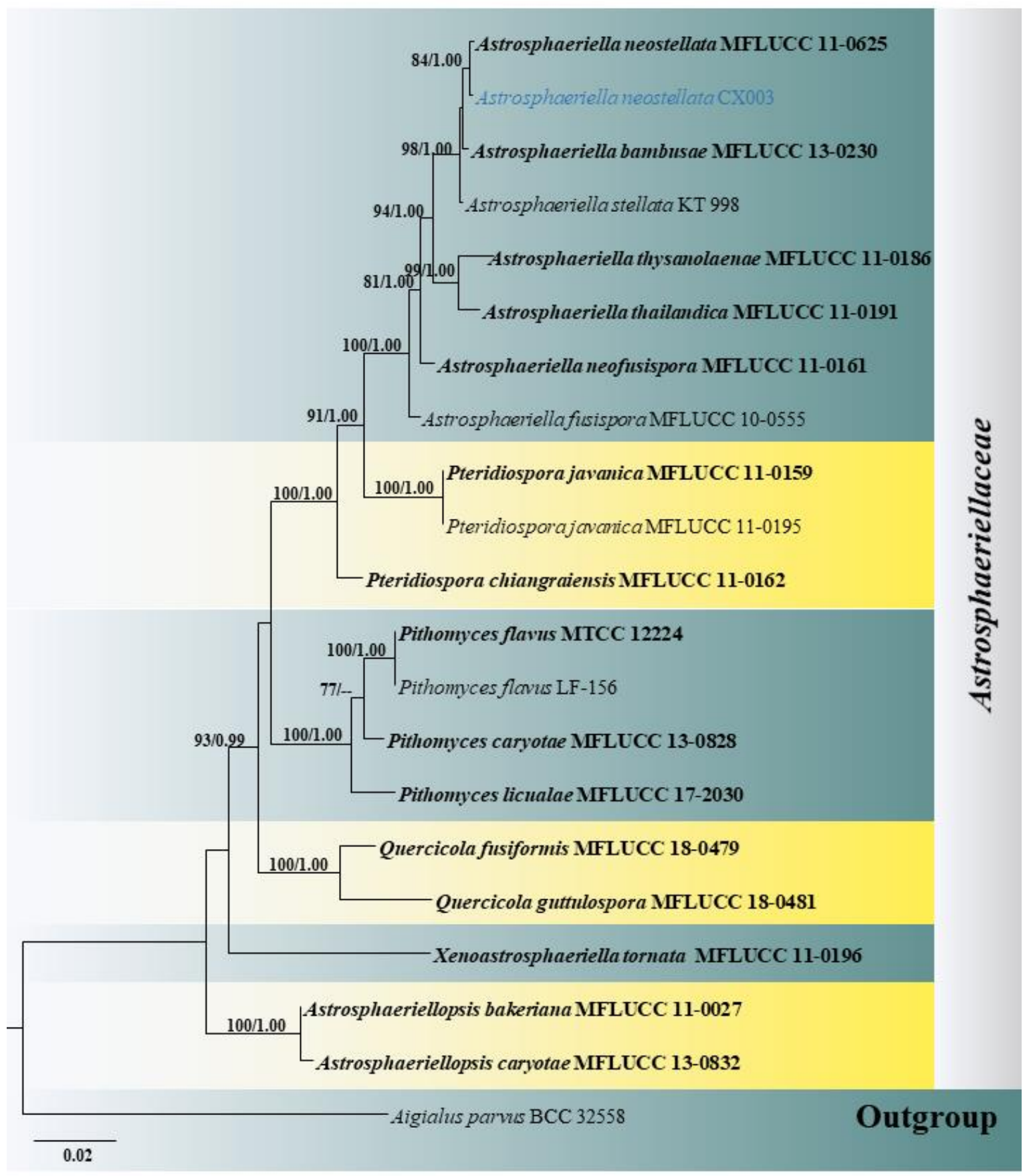

Fig. 22 - Phylogram generated from maximum likelihood analysis based on a combined LSU, SSU and tefl sequence dataset. Twenty-one strains are included in the combined gene analyses comprising 2,768 total characters including gaps (LSU: 1-855 bp, SSU: 856-1896 bp, tef1: 1897$2768 \mathrm{bp}$ ). The ML tree with the best scores is selected to represent the phylogenetic relationships of Astrosphaeriella species, with the final ML optimization likelihood: -7773.014991. The matrix had 482 distinct alignment patterns, with $19.13 \%$ undetermined characters or gaps. Estimated base frequencies were as follows: $\mathrm{A}=0.244553, \mathrm{C}=0.251159, \mathrm{G}=0.280413, \mathrm{~T}=0.223875$; substitution rates $\mathrm{AC}=1.475476, \mathrm{AG}=4.502665, \mathrm{AT}=0.996575, \mathrm{CG}=1.808357, \mathrm{CT}=$ 17.545595, GT $=1.000000$; Tree-Length $=0.380939$; gamma distribution shape parameter $\alpha=$ 0.724752; The proportion of invariable sites $\mathrm{I}=0.641084$. Bayesian posterior probabilities (BYPP) from MCMC were evaluated with a final average standard deviation of split frequencies of 0.009646. Bootstrap support for ML greater than $70 \%$ and Bayesian posterior probabilities greater than 0.95 are defined above the nodes as MLBP/BYPP. The tree is rooted to Aigialus parvus (BCC 32558). The type strains are indicated in bold and newly generated sequence is shown in blue. 
As morphological characters examined largely overlap with Camarosporidiella celtidis (MFLU 17-0466), we, therefore, report our collection (MFLU 17-0804) as a new host record of $C$. celtidis from dying twigs of Prunus armeniaca (Rosaceae). The multi-gene phylogeny herein also shows our collection clusters with other Camarosporidiella celtidis species with high bootstrap support (88\% ML, 1.00 BYPP) (Fig. 24).
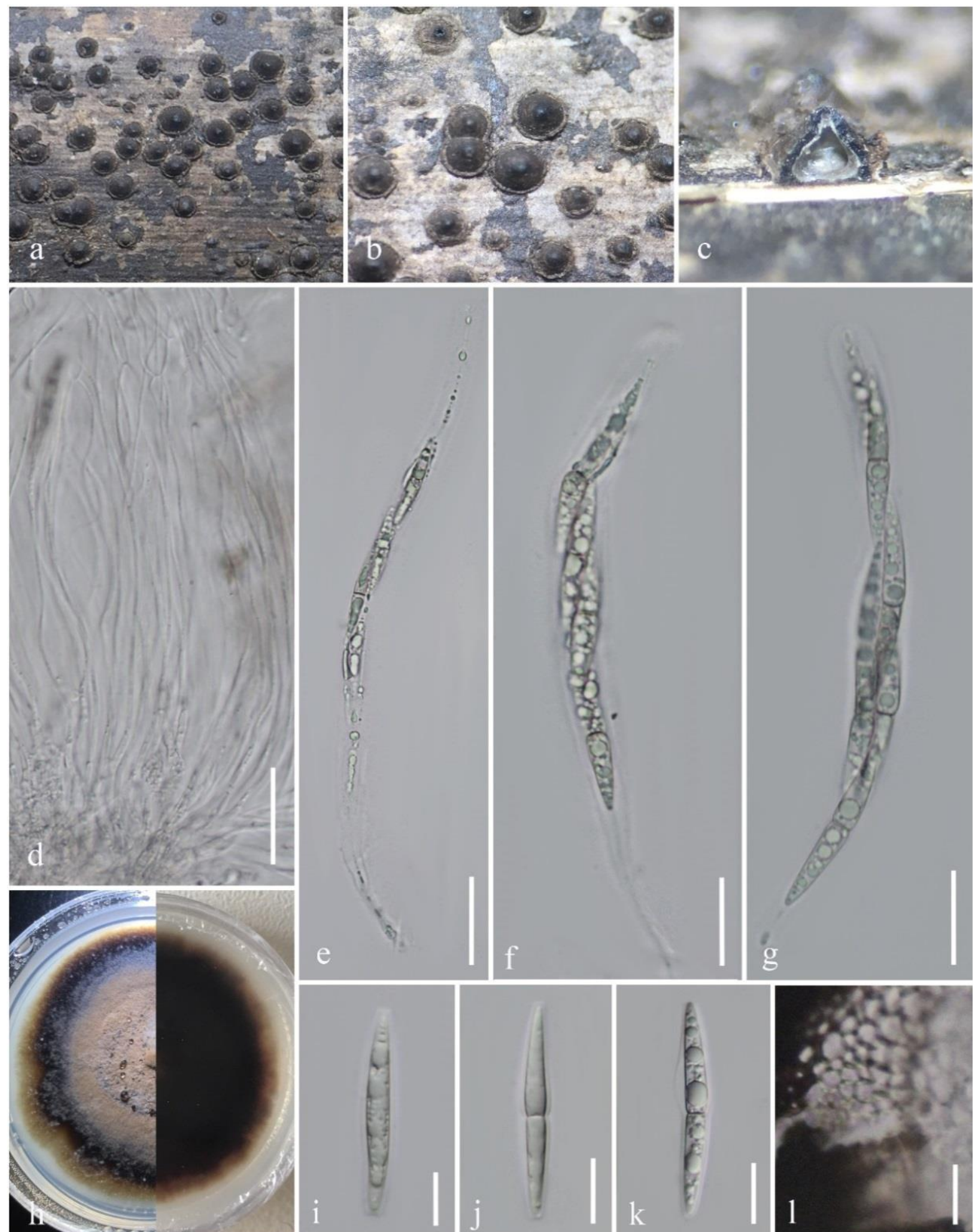

Fig. 23 - Astrosphaeriella neostellata (KUN-HKAS 101775, new geographic record). a, b Ascomata on host surface. c Vertical section through ascoma. d Pseudoparaphyses. e-g Asci. h Culture frontage and back. i-k Ascospores. 1 Peridium. Scale bars: $d, e=30 \mu \mathrm{m}, \mathrm{f}, \mathrm{g}=20 \mu \mathrm{m}$, $\mathrm{j}, \mathrm{k}=15 \mu \mathrm{m}, \mathrm{i}, \mathrm{l}=10 \mu \mathrm{m}$. 


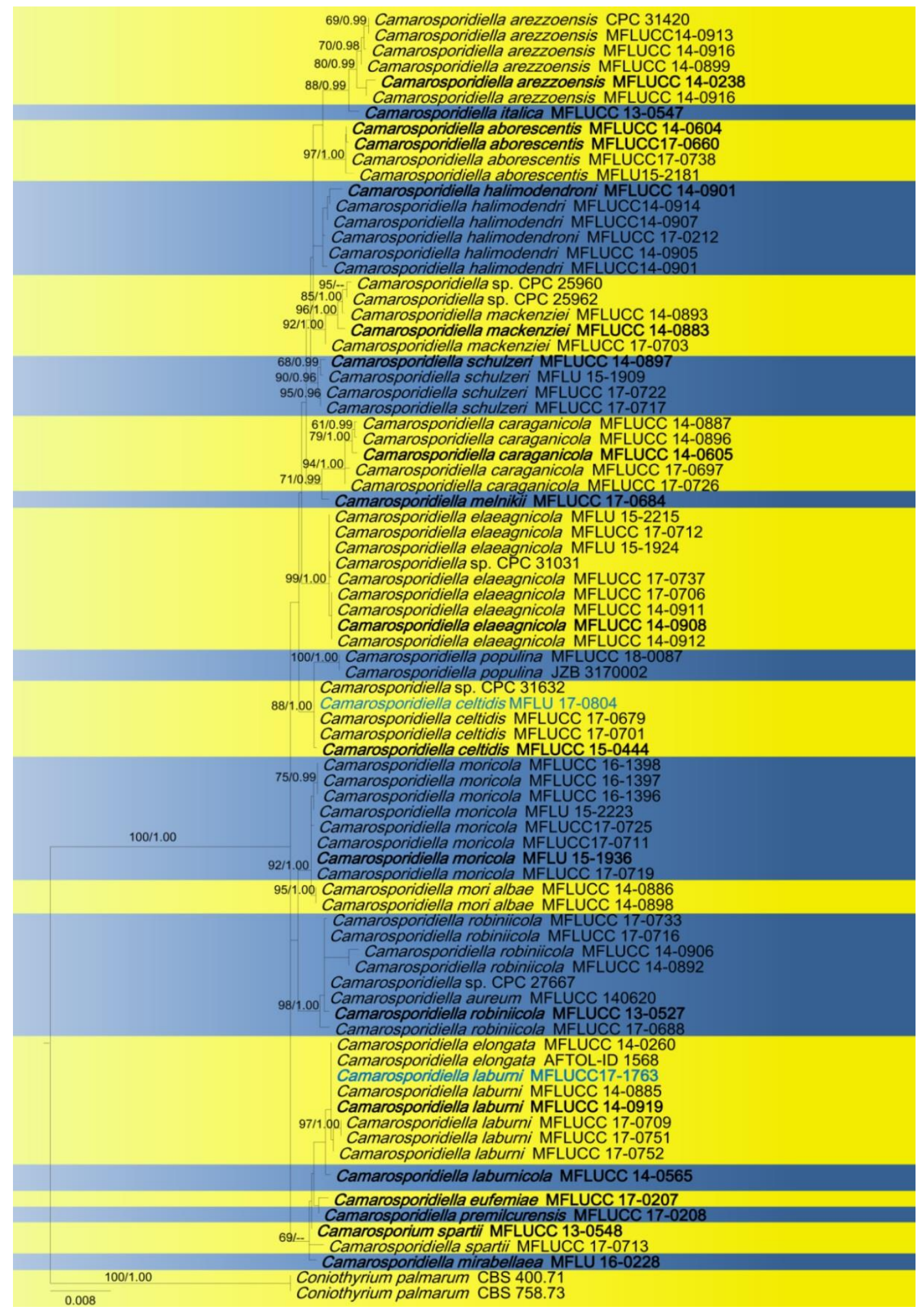

Fig. 24 - Phylogram generated from maximum likelihood analysis based on combined LSU, SSU, ITS, and tefl sequence data. Eighty three strains are included in the combined gene analyses comprising 3334 characters after alignment. Coniothyrium palmarum (strains CBS 400.71 and CBS 758.73) are used as the outgroup taxa. The tree topology derived from the Bayesian analysis was similar to that derived from the maximum likelihood analysis. The best RaxML tree with a final likelihood value of -6761.700472 is presented. The matrix had 323 distinct alignment patterns, with $12.37 \%$ undetermined characters or gaps. Estimated base frequencies were as follows: $\mathrm{A}=$ $0.242758, \mathrm{C}=0.240900, \mathrm{G}=0.267664, \mathrm{~T}=0.248678$; substitution rates $\mathrm{AC}=1.300383, \mathrm{AG}=$ 5.109489, $\mathrm{AT}=2.415853, \mathrm{CG}=0.406655, \mathrm{CT}=8.867918, \mathrm{GT}=1.000000$; gamma distribution 
shape parameter $\alpha=0.705373$. Bootstrap values for maximum likelihood equal to or greater than 50 and Bayesian posterior probabilities equal or greater than 0.95 are placed above or below the branches. Ex-type strains are in bold and black. The newly generated sequence is indicated in blue.

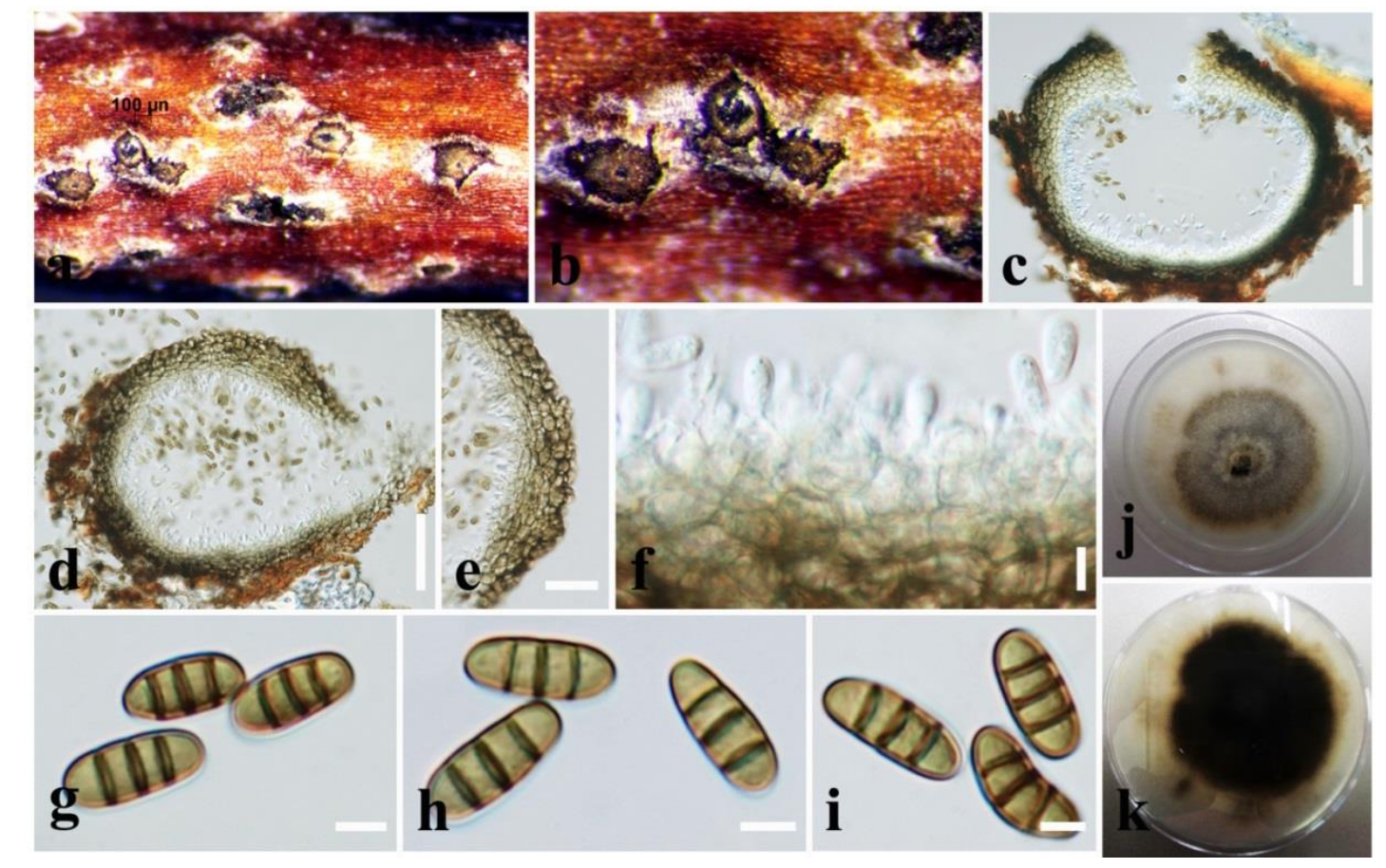

Fig. 25 - Camarosporidiella celtidis (MFLU 17-0804, new host record). a Conidiomata on host surface. b Close-up of conidiomata. c, d Vertical section through conidioma. e Conidioma wall. $\mathrm{f}$ Conidiogenous cells producing conidia. $\mathrm{g}-\mathrm{i}$ Conidia. $\mathrm{j}$ Colony from above. $\mathrm{k}$ Colony from below. Scale bars: $\mathrm{c}, \mathrm{d}=100 \mu \mathrm{m}, \mathrm{e}=20 \mu \mathrm{m}, \mathrm{C}=20 \mu \mathrm{m} ; \mathrm{f}-\mathrm{i}=10 \mu \mathrm{m}$.

Camarosporidiella laburni (Pers.) Wanas., Bulgakov, Camporesi \& K.D. Hyde. Stud. Mycol. 87: 233 (2017)

Fig. 26

Facesoffungi number: FoF 03540

Saprobic on woody branches. Sexual morph: Ascomata 350-500 $\mu \mathrm{m}$ high, 400-550 $\mu \mathrm{m}$ diameter $(\bar{x}=425.8 \times 452.8 \mu \mathrm{m}, \mathrm{n}=10)$, black, superficial to semi-immersed, clustered, fully or partly erumpent, globose, multi-loculate, with an ostiole. Peridium 44-60 $\mu \mathrm{m}$ wide, thick, comprising 7-9 layers, outermost layer heavily pigmented, thin-walled, comprising blackish to dark brown amorphous layer, middle layer thick-walled, light brown, loosely packed cells of textura angularis, inner layer composed of 3-4 layers, hyaline, flattened, thick-walled cells of textura angularis. Hamathecium comprising numerous, $2-3 \mu \mathrm{m}(\mathrm{n}=30)$ wide, filamentous, branched septate, pseudoparaphyses. Asci 155-180 × 11-14 $\mu \mathrm{m}(\bar{x}=169.4 \times 12.4 \mu \mathrm{m}, \mathrm{n}=40)$, 8-spored, bitunicate, fissitunicate, cylindrical, short-pedicellate, apex rounded, with a minute ocular chamber. Ascospores $25-30 \times 8-11 \mu \mathrm{m}(\bar{x}=27.5 \times 9.3 \mu \mathrm{m}, \mathrm{n}=30)$, overlapping uniseriate, initially hyaline, becoming pale brown at maturity, with slightly paler ends, muriform, mostly ellipsoidal, 5-7transversely septate, with 4-5 longitudinal septa, deeply constricted at the middle septum, slightly constricted at remaining septa asymmetrical, conical and narrowly rounded at the ends, not surrounded by a mucilaginous sheath. Asexual morph: See Wanasinghe et al. (2017).

Culture characteristics - Colonies on PDA reaching $25 \mathrm{~mm}$ diameter after 2 weeks at 20$25^{\circ} \mathrm{C}$, colonies medium sparse, circular, flat, surface slightly rough with entire edge, margin well defined, cottony to fairly fluffy with sparse aspects, colony from above and reverse: greenish grey; mycelium grey to whitish grey with tufting; producing pigments (pink) in PDA.

Known distribution (based on molecular data) - Italy, Russia (Wanasinghe et al. 2017 and this study). 
Known hosts (based on molecular data) - Laburnum anagyroides (Wanasinghe et al. 2017), Colutea cilicica (this study).

Material examined - Russia, Republic of Crimea, Bakhchysarai District, Sel-Bukhra Mountain, shrubs on the slopes $\left(44.736148^{\circ} \mathrm{N}, 33.989779^{\circ} \mathrm{E}\right)$, on dying twigs of Colutea cilicica Boiss. \& Balansa (Fabaceae), 4 July 2016, Timur S. Bulgakov, CR088 (MFLU 17-0795, new host record), living culture, MFLUCC17-1763.

GenBank numbers - LSU: MN750589, SSU: MN750604, ITS: MN750610.
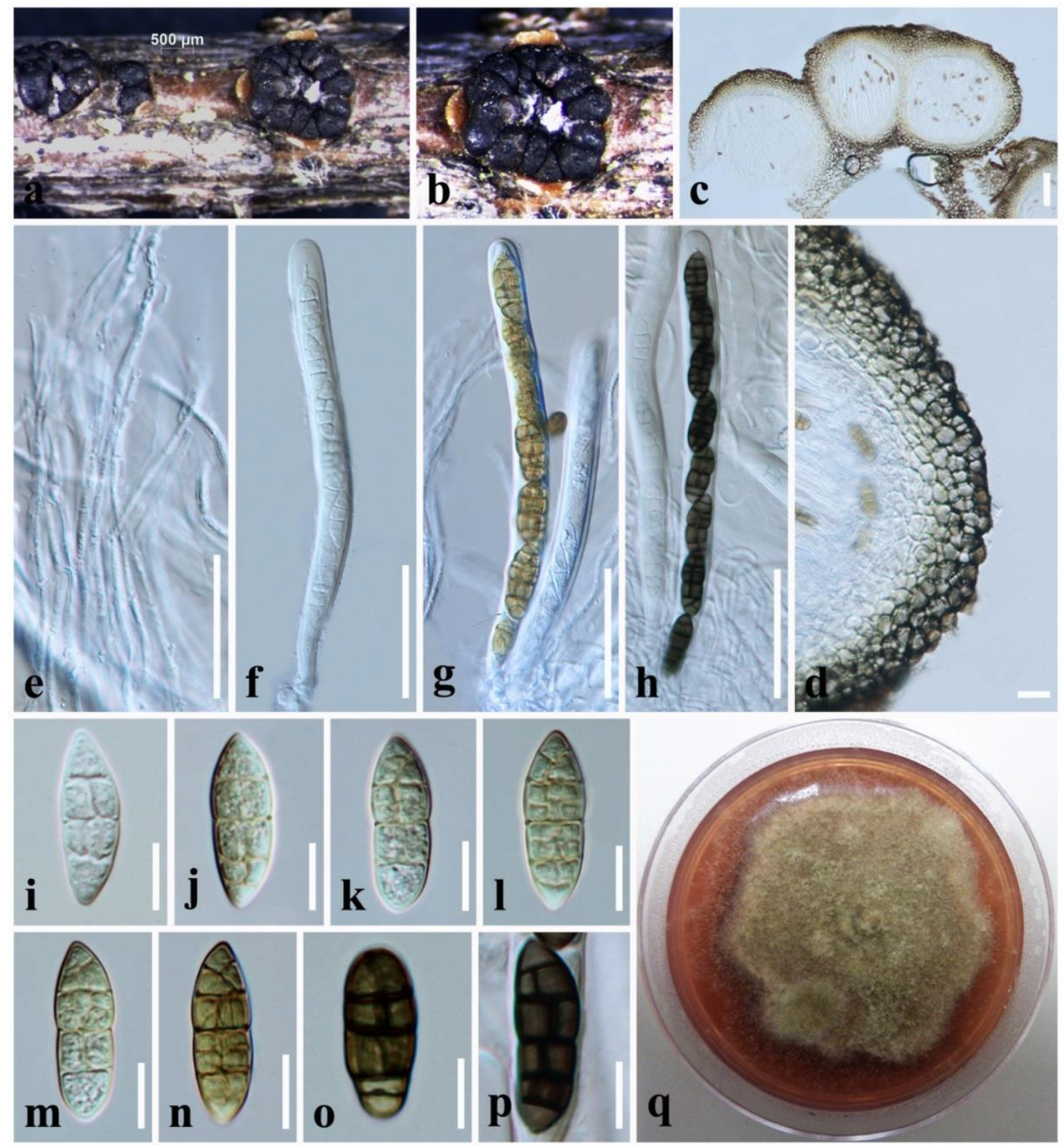

Fig. 26 - Camarosporidiella laburni (MFLU17-0795, new host record). a Appearance of ascomata on host substrate. b Close-up of ascomata. c Vertical section of ascomata. d Section through peridium. e Pseudoparaphyses. $\mathrm{f}-\mathrm{h}$ Asci. i-p Ascospores. q Colony from above. Scale bars: $\mathrm{c}=100$ $\mu \mathrm{m}, \mathrm{d}=20 \mu \mathrm{m}, \mathrm{e}-\mathrm{h}=50 \mu \mathrm{m}, \mathrm{i}-\mathrm{p}=10 \mu \mathrm{m}$.

Notes - As morphological characters examined largely overlap with Camarosporidiella laburni (MFLU 16-0094), we report our collection (MFLU 17-0795) as a new host record from dying twigs of Colutea cilicica (Fabaceae). Both species share a similar morphology, viz. black, superficial to semi-immersed, clustered ascomata, cylindrical, short-pedicellate asci and muriform, mostly ellipsoidal ascospores (Wanasinghe et al. 2017). The multi-gene (LSU, SSU, ITS and tef1) 
phylogeny herein, also shows that our collection clusters with other Camarosporidiella laburni species (Fig. 24). Camarosporidiella laburni species have been recorded from Italy and Russia (on dead aerial branches of Laburnum anagyroides) and this is the first record of Camarosporidiella laburni from Colutea cilicica (Fabaceae).

\section{Corynesporascaceae Sivan.}

Corynesporascaceae introduced by Sivanesan (1996) with Corynesporasca caryotae as the type species, was collected from a decaying leaf of Caryota urens collected in Sri Lanka and linked the sexual (Corynesporasca caryotae) and asexual (Corynespora) state were linked in culture. Rossman et al. (2015) recommended to use Corynespora rather than Corynesporasca in case of Corynespora has been widely used and includes approximately 200 species names. Corynespora is accepted in Corynesporaceae, but this family is invalidly published (Index Fungorum 2020). In the previous phylogenetic analysis, species of Corynespora form a distant clade together with the generic type, C. cassiicola, and is classified in Corynesporascaceae (Voglmayr \& Jaklitsch 2017).

\section{Corynespora Güssow}

Corynespora was described by Güssow (1906), and more than 200 epithets have been recorded (Index Fungorum 2020). However, there are only six species in this genus with DNA sequence data (Crous et al. 2019b). Corynespora has a widespread distribution (Kirk et al. 2008a) and are saprobes, pathogens, and endophytes on woody and herbaceous plants, other fungi, nematodes, and human skin (Dixon et al. 2009, Kumar et al. 2012, Singh et al. 2012). In this study, an additional three new species are described.

Corynespora doipuiensis J.F. Li \& Phookamsak, sp. nov.

Fig. 28

Index Fungorum number: IF557020; Facesoffungi number: FoF07056 Thailand.

Etymology - Name reflects the location from which it was collected, Doi Pui, Chiang Rai,

Holotype - MFLU 14-0388

Saprobic on branches of hanging dead branch of unidentified plant. Sexual morph: Undetermined. Asexual morph: Hyphomycetous, colony on natural substrate effuse, dark brown or black. Colonies effuse, thin, cottony, pale to dark grey. Mycelium partly superficial, consisting of branched, septate, smooth, thin-walled, pale hyphae. Conidiophores $212-426 \times 10-15 \mu \mathrm{m}(\bar{x}=$ $313.45 \times 13.89 \mu \mathrm{m}, \mathrm{n}=40$ ), macronematous, setiferous, erect, straight or flexuous, often arising in groups, septate, unbranched, thick-walled, dark brown, sometimes light brown at the tip, percurrently proliferating from cut ends. Conidiogenous cells $27-5 \times 10-16 \mu \mathrm{m}(\bar{x}=33.71 \times 13.34$ $\mu \mathrm{m}, \mathrm{n}=100)$ monotretic, integrated, terminal, light to moderately dark brown, often percurrently proliferating. Conidia 136-165 × 5-25.5 $\mu \mathrm{m}(\bar{x}=143.7 \times 21.31 \mu \mathrm{m}, \mathrm{n}=100)$, solitary, simple, obconical, curved, subhyaline to moderately brown, acrogenous, pseudoseptate, guttulate, thickwalled, smooth, with a dark basal scar, wide at lower part, narrow and elongate at the upper part.

Culture characteristics - Conidia germinating on PDA within 12 hours and germ tubes produced from both ends. Colonies growing on PDA, cottony, white to pale grey, reaching $5 \mathrm{~mm}$ in 7 days at $30{ }^{\circ} \mathrm{C}$, mycelium partly superficial, partly immersed, slightly effuse, radially striate, with irregular edge, white; Asexual and sexual spores are not formed within 60 days.

Material examined - Thailand, Chiang Rai Provice, Doi Pui, on dead herbaceous branch, 9 January 2014, J.F Li, H-15 (MFLU 14-0388, holotype), ex-type living culture at MFLUCC 140022.

GenBank numbers - ITS: MN648322, LSU: MN648326, SSU: MN648318.

Notes - In this study, our strain (MFLUCC 14-0022) shares a size range of the conidial characters with the type and other representative specimens (Voglmayr \& Jaklitsch 2017). However, Corynespora doipuiensis is unique in having lanky, curved, hyaline to light brown conidia with long and brown conidiophores. Furthermore, phylogenetic analysis shows that our strain (MFLUCC 14-0022) forms a well-supported subclade (0.99 BYPP) (Fig. 27) with Corynespora 
submersa (MFLUCC 16-1101) with support. Our specimen is identified as a novel species Corynespora doipuiensis collected from herbaceous aerial dead branches from Chiang Rai, Thailand.

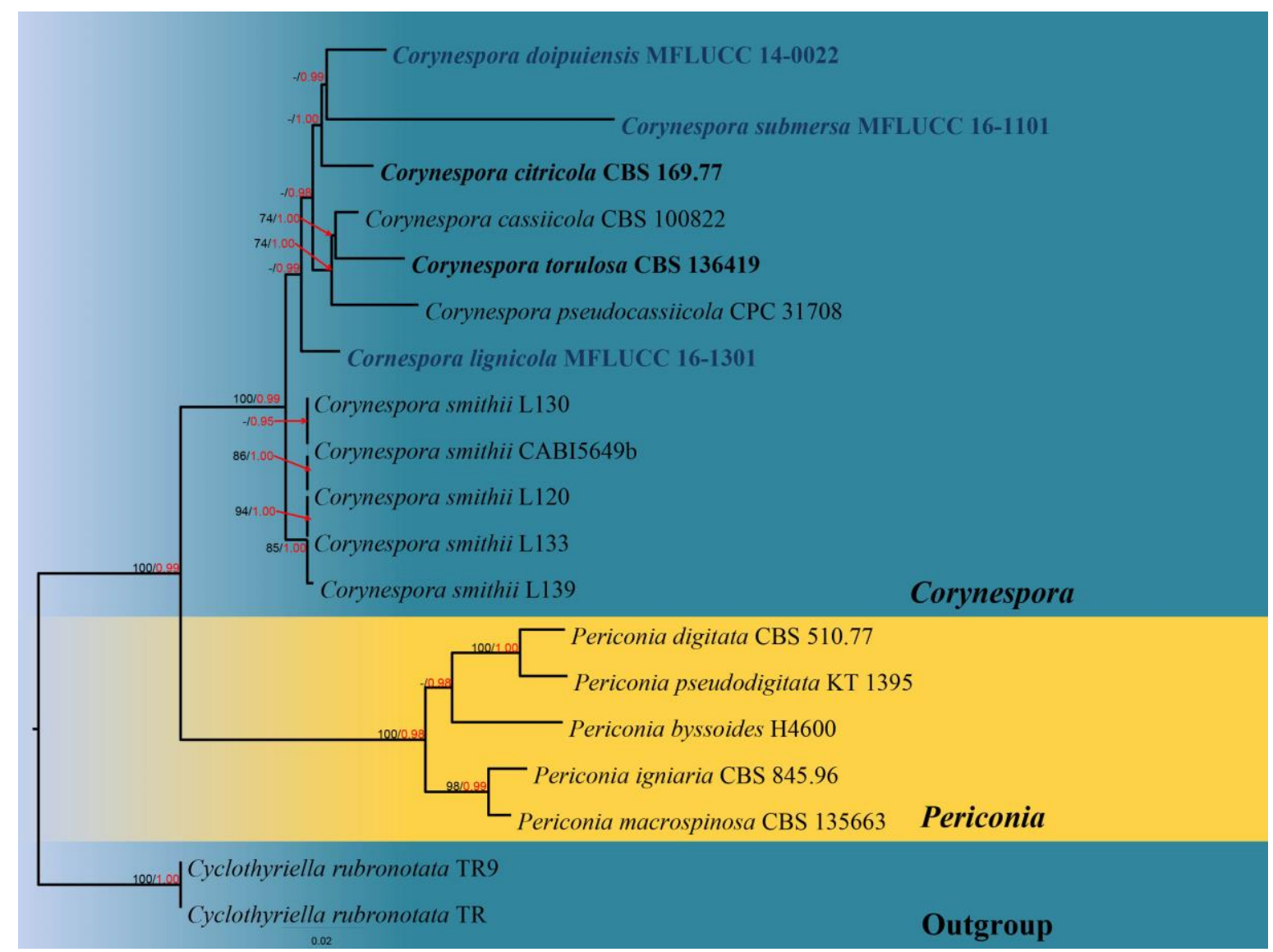

Fig. 27 - Phylogram generated from the best scoring of the RAxML tree based on combined ITS, LSU, SSU and tefl sequenced data of taxa in Corynespora and other related families (Periconiaceae). Cyclothyriella rubronotata (TR9) and C. rubronotata (TR) were selected as the outgroup taxa. The tree topology derived from the Bayesian analysis was similar to that derived from the maximum likelihood analysis. The best RAxML tree with a final likelihood value of 9787.150160 is presented. The value of Tree-Length is 0.422339 .The matrix had 590 distinct alignment patterns, with $26.28 \%$ undetermined characters or gaps. Estimated base frequencies were as follows: $\mathrm{A}=0.240881, \mathrm{C}=0.256132, \mathrm{G}=0.272893, \mathrm{~T}=0.230094$; substitution rates $\mathrm{AC}=$ 1.532399, $\mathrm{AG}=2.164946, \mathrm{AT}=1.539029, \mathrm{CG}=1.017301, \mathrm{CT}=7.622600, \mathrm{GT}=1.000000$; gamma distribution shape parameter $\alpha=0.169372$. Bayesian posterior probabilities (BYPP) from MCMC were evaluated with final average standard deviation of split frequencies $=0.008695$. Bootstrap support for maximum likelihood (ML, black) equal to or greater than 70\%. BYPP (red) equal to or greater than 0.95 are given above or below the nodes. Newly generated sequences are indicated in blue.

Corynespora submersa Z.L. Luo, H.Y. Su \& K.D. Hyde, sp. nov.

Fig. 29

Index Fungorum number: IF557058; Facesoffungi number: FoF07072

Etymology - Referring to the submerged habitat.

Holotype - HKAS 92703

Saprobic on decaying wood submerged in freshwater habitats. Sexual morph Undetermined. Asexual morph Colonies on natural substrate effuse, dark brown to black. Mycelium partly superficial, partly immersed in the substrate, composed of branched, septate, subhyaline to brown, smooth-walled hyphae. Conidiophores $150-370 \mu \mathrm{m}(\bar{x}=260 \mu \mathrm{m}, \mathrm{SD}=110, \mathrm{n}=10)$ long, 10-12 
$\mu \mathrm{m}(\bar{x}=11 \mu \mathrm{m}, \mathrm{SD}=1, \mathrm{n}=10)$ wide, macronematous, mononematous, erect or ascending, simple, straight or flexuous, pale brown to dark brown, septate, with up to four successive cylindrical proliferations. Conidiogenous cells monotretic, cylindrical, pale brown to brown, often with proliferation through the apical pore and formation of another conidium at the apex of the proliferation. Conidia 100-150 $\mu \mathrm{m}(\bar{x}=125 \mu \mathrm{m}, \mathrm{SD}=25, \mathrm{n}=20)$ long, 16-24 $\mu \mathrm{m}(\bar{x}=20 \mu \mathrm{m}, \mathrm{SD}=$ $4, \mathrm{n}=20$ ) wide, formed singly or in a short chains through a wide pore at the apex of the conidiophore, almost obclavate, sometimes rostrate, straight or slightly curved, smooth, subhyaline to golden brown, 9-13-distoseptate.

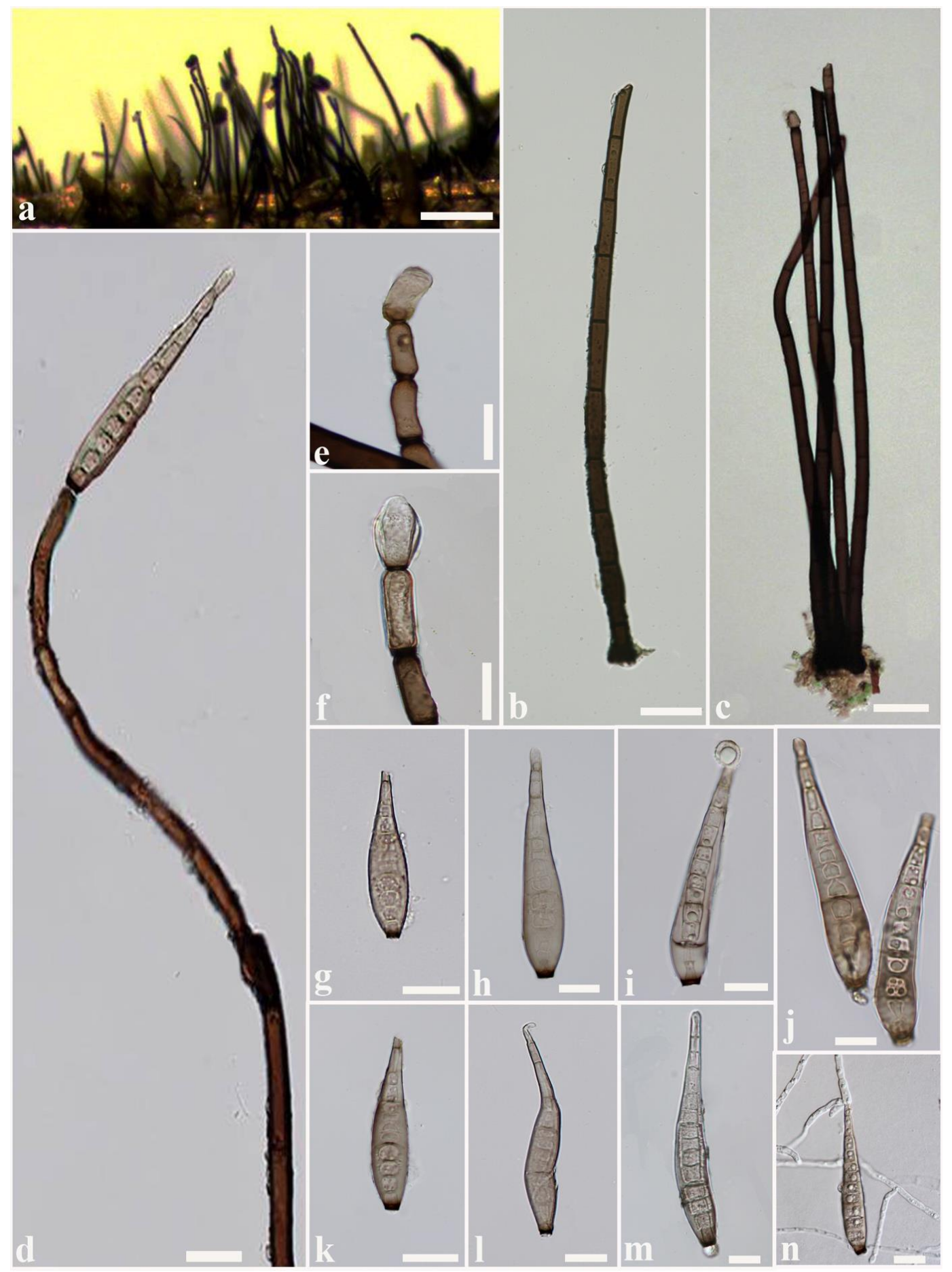

Fig. 28 - Corynespora doipuiensis (MFLU 14-0388, holotype). a Colonies on handing dead herbaceous branch. $\mathrm{b}-\mathrm{c}$ Conidiophores. $\mathrm{d}$ Conidiophores bearing conidia. e-d Conidiogenous cells. g-f Conidia. $\mathrm{n}$ Germinated conidia. Scale bars: $\mathrm{a}=200 \mu \mathrm{m}, \mathrm{b}-\mathrm{i}, \mathrm{k}, 1, \mathrm{n}=20 \mu \mathrm{m}, \mathrm{j}, \mathrm{m}=10 \mu \mathrm{m}$. 
Culture characteristics - Colonies on PDA attaining $20 \mathrm{~mm}$ diameter within 25 days at $25^{\circ} \mathrm{C}$ under natural light, velvety, centrally raised, pale brown or greyish olivaceous, reverse dull green or grey olivaceous.

Material examined - China, Yunnan Province, saprobic on decaying wood submerged in Dulong River, May 2015, Z.L. Luo, S-504 (HKAS 92703, holotype), ex-type living culture MFLUCC 16-1101.

GenBank numbers - ITS: MN860548, LSU: MN860553.

Notes - Corynespora submersa resembles $C$. titarpaniensis in having macronematous, erect, straight or flexuous, smooth conidiophores, straight or slightly curved, smooth, distoseptate conidia. However, Corynespora submersa differs from $C$. titarpaniensis in having subhyaline to golden brown, smaller conidia which are formed singly or in a short chain through a wide pore at the apex of the conidiophore (Kushwaha et al. 2017). Phylogenetic analysis also shows that Corynespora submersa is distinct from other species presently known from culture or DNA sequence.

Corynespora lignicola Z.L. Luo, H.Y. Su \& K.D. Hyde, sp. nov.

Fig. 30

Index Fungorum number: IF557059; Facesoffungi number: FoF07073

Etymology - Referring to this taxon dwelling on wood.

Holotype - HKAS 92792

Saprobic on decaying wood submerged in freshwater habitats. Sexual morph Undetermined. Asexual morph Colonies on natural substrate effuse, dark brown to black. Mycelium partly superficial, partly immersed in the substrate, composed of branched, septate, subhyaline to brown, smooth-walled hyphae. Conidiophores (350-)470-670(-700) $\mu \mathrm{m}(\bar{x}=570 \mu \mathrm{m}, \mathrm{SD}=100, \mathrm{n}=10)$ long, 9-13 $\mu \mathrm{m}(\bar{x}=11 \mu \mathrm{m}, \mathrm{SD}=2, \mathrm{n}=10)$ wide, macronematous, mononematous, erect or ascending, simple, straight or flexuous, septate, smooth. Conidiogenous cells monotretic, cylindrical, pale brown to brown, often with proliferation through the apical pore and formation of another conidium at the apex of the proliferation, branched. Conidia $110-156 \mu \mathrm{m}(\bar{x}=133 \mu \mathrm{m}, \mathrm{SD}$ $=23, \mathrm{n}=20)$ long, 7-9 $\mu \mathrm{m}(\bar{x}=8 \mu \mathrm{m}, \mathrm{SD}=1, \mathrm{n}=20)$ wide, formed singly or in a short chain through a wide pore at the apex of the conidiophore, cylindrical, straight or slightly curved, smooth, subhyaline to pale brown, distoseptate.

Culture characteristics - Colonies on MEA attaining $15 \mathrm{~mm}$ diameter within 20 days at $25^{\circ} \mathrm{C}$ under natural light, velvety, centrally raised, pale brown or greyish olivaceous, reverse grey olivaceous to dark brown.

Material examined - China, Yunnan Province, saprobic on decaying wood submerged in Nujiang River, May 2015, S.M. Tang, S-334 (HKAS 92792, holotype), ex-type living culture MFLUCC 16-1301.

GenBank numbers - ITS: MN 860549, LSU: MN860554.

Notes - Corynespora lignicola resembles C. encephalarti in having macronematous, erect, straight, smooth-walled conidiophores, monotretic, cylindrical conidiogenous cells and distoseptate conidia which are similar in size. However, Corynespora lignicola differs from C. encephalarti in having cylindrical, straight or slightly curved, subhyaline to pale brown conidia, while $C$. encephalarti has obclavate, medium olivaceous brown to dark brown conidia (Crous et al. 2019b), and Corynespora lignicola is phylogenetically distinct from all species that have DNA sequences.

\section{Dictyosporiaceae Boonmee \& K.D. Hyde}

Dictyosporiaceae was validly introduced by Boonmee et al. (2016) based on type genus, Dictyosporium Corda. Typical asexual morphs in Dictyosporiaceae are characterized by cheiroid conidia (Boonmee et al. 2016). Currently, 13 genera are accepted in Dictyosporiaceae (Boonmee et al. 2016, Liu et al. 2017b, Iturrieta-González et al. 2018, Yang et al. 2018). 

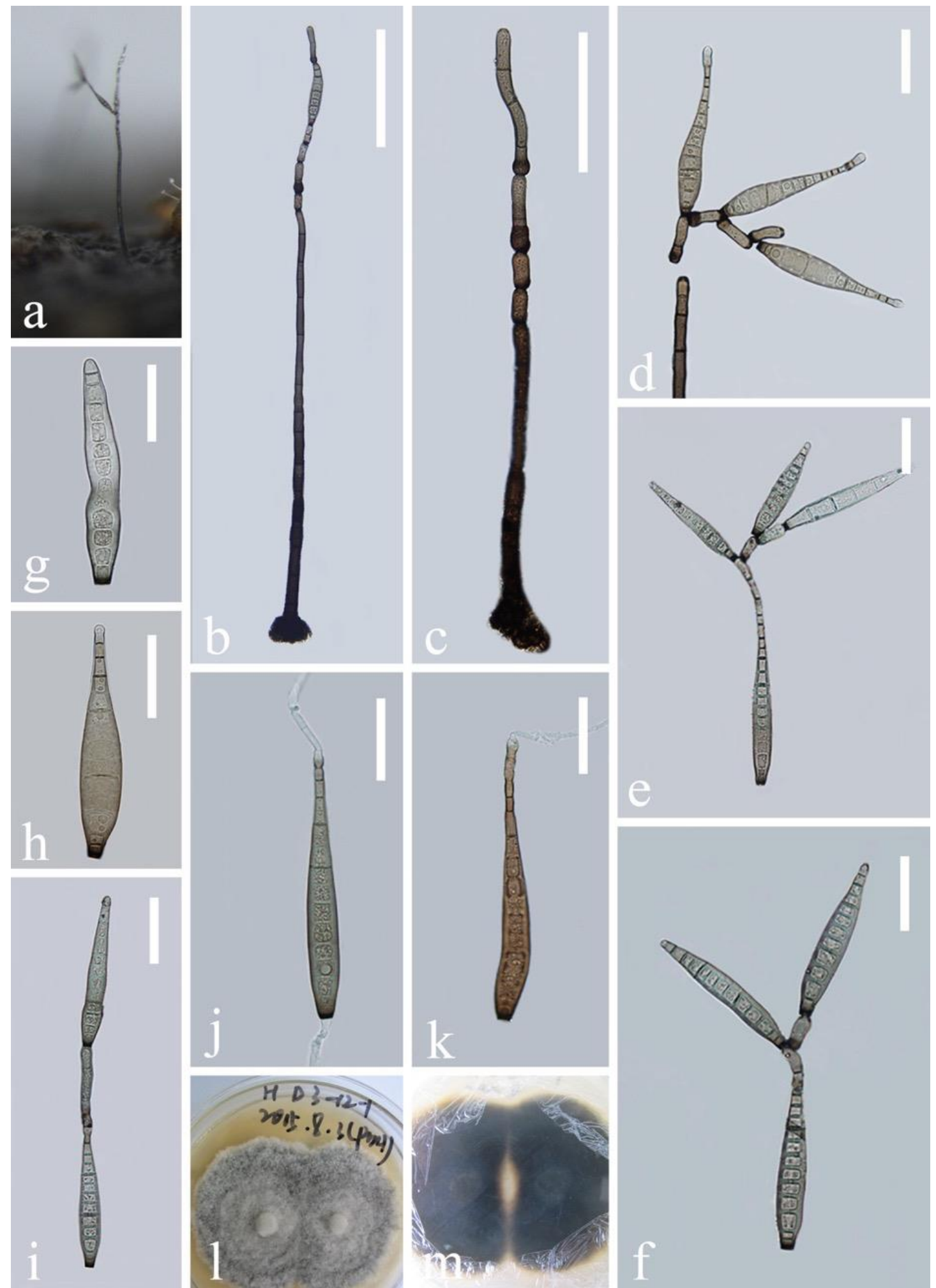

Fig. 29 - Corynespora submersa (HKAS 92703, holotype). a Conidiophore with conidia on natural substrate. $\mathrm{b}$ Conidiophore with conidia. c Conidiophore with conidiogenous cells. d Conidiogenous cells with conidia. e-i Conidia. j, k Germinating conidia. 1, m Colony on MEA. Scale bars: $\mathrm{b}, \mathrm{c}=100 \mu \mathrm{m}, \mathrm{d}-\mathrm{k}=50 \mu \mathrm{m}$.

Dendryphiella Bubák \& Ranoj.

Dendryphiella was established by Ranojevic (1914) with the type species D. interseminata (Berk. \& Ravenel) Bubák. Fifteen species are accepted in this genus (Crous et al. 2016a, Liu et al. 2017c, Hyde et al. 2018, Iturrieta-Gonzalez et al. 2018). 
Facesoffungi number: FoF06712

Saprobic on decaying wood in terrestrial habitats. Sexual morph: Undetermined. Asexual morph: Hyphomycetous. Colonies on natural substrate superficial, effuse, greyish brown. Mycelium partly immersed, composed of septate, branched, hyaline hyphae, 1-2 $\mu \mathrm{m}$ wide. Conidiophores up to $320 \mu \mathrm{m}$ long, macronematous, mononematous, solitary, erect, brown, thick-walled, straight or slightly flexuous, finely verruculose, septate, branched, wider at the subsection. Conidiogenous cells $22-41 \mu \mathrm{m}$ long $(\bar{x}=31 \mu \mathrm{m}, \mathrm{n}=15)$, polytretic, terminal, later becoming subterminal, proliferating asymmetrically, integrated, brown, finely verrucose, enlarged at vertex. Conidia 16-26 $\times 4-6 \mu \mathrm{m}(\bar{x}=21 \times 5 \mu \mathrm{m}, \mathrm{n}=30)$, catenate in acropetal chain, fusiform to ellipsoidal, rounded at apex, subtruncate at base, pale brown, aseptate when young, brown or dark brown, 3septate when mature, slightly constricted at the septa, thick-walled, verrucose.

Culture characteristics - Conidia germinating on water agar within $24 \mathrm{~h}$. Germ tubes produced from one or both ends. Mycelia superficial, circular, with entire edge, mycelia dense at center, sparse towards circumference, yellowish white from above, yellow at center, paler towards circumference from below.

Known distribution (based on molecular data) - Spain, Asturias (Crous et al. 2014b) and China (this study).

Known hosts (based on molecular data) - Eucalyptus globulus (Myrtaceae) (Crous et al. 2014b) and decaying wood (this study).

Material examined - China, Guizhou Province, Guiyang, Guiyang Botanical Garden, on decaying wood, 8 August 2017, Ningguo Liu, ZWY006 (GZAAS 20-0002, new geogrophical record), living culture, GZCC 20-0001.

GenBank numbers - ITS: MN999925; LSU: MN999929.

Notes - Dendryphiella eucalyptorum was introduced by Crous et al. (2014b) on small branches of Eucalyptus globulus (Myrtaceae) in Spain. Our collection differs from the holotype (CBS H-21699) (Fig. 31) in having shorter conidiophores (up to $320 \mu \mathrm{m}$ vs up to $500 \mu \mathrm{m}$ ), but there are no nucleotide differences in the ITS and LSU regions and therefore, we identify it as $D$. eucalyptorum. This is a new geographical record of $D$. eucalyptorum from China.

\section{Neodendryphiella Iturrieta-González}

Neodendryphiella was introduced by Iturrieta-González et al. (2018) based on the type species N. tarraconensis Iturrieta-González, Gené \& Dania García. Neodendryphiella species have been reported as coprophilous, saprobic and soil fungi. Only three species are accommodated to this genus (Iturrieta-González et al. 2018).

Neodendryphiella tarraconensis Iturrieta-González, Gené \& Dania García, MycoKeys 37: 30 (2018)

Fig. 33

Facesoffungi number: FoF06713

Saprobic on decaying wood in terrestrial habitats. Sexual morph: Undetermined. Asexual morph: Hyphomycetous. Colonies on natural substrate superficial, effuse, gelden brown, velvety. Mycelium mostly immersed, composed of septate, branched, median brown hyphae, 1-2 $\mu \mathrm{m}$ wide. Conidiophores up to $530 \mu \mathrm{m}$ long, 3-5 $\mu \mathrm{m}$ wide at tip, 8-10 $\mu \mathrm{m}$ at base, macronematous, mononematous, erect or slightly flexuous, branched or unbranched, septate, cylindrical, dark brown at base, slightly paler towards the apex, smooth to finely verrucose, thick-walled. Conidiogenous cells polyblastic, terminal and intercalary, mostly cylindrical. Ramoconidia 0 -1-septate, pale brown, smooth to verruculose, mostly cylindrical. Conidia $9-15.5 \times 3-5 \mu \mathrm{m}(\bar{x}=11 \times 4 \mu \mathrm{m}, \mathrm{n}=30)$, catenate, ellipsoidal or subcylindrical with more or less rounded ends, 0-2-septate, slightly constricted at the septum; when 2-septate, usually constricted at only one septum, pale brown, verruculose.

Culture characteristics - Conidia germinating on water agar within $24 \mathrm{~h}$. Germ tubes produced from one or both ends. Mycelia superficial, circular, slightly umbonate at center with 
entire edge, olivaceous brown from above, dark brown at center, paler towards circumference from below.

Known distribution (based on molecular data) - Spain (Tarragona) (Iturrieta-González et al. 2018) and China (this study).

Known hosts (based on molecular data) - From garden soil (Iturrieta-González et al. 2018) and decaying wood (this study).

Material examined - China, Guizhou Province, Zunyi, Wangcao $\left(28^{\circ} 12.30^{\prime} \mathrm{N}, 107^{\circ} 10.24^{\prime} \mathrm{E}\right)$, on decaying wood, 15 September 2018, N.G. Liu, KKS016 (GZAAS 20-0003, new geogrophical record), living culture, GZCC 20-0002.

GenBank numbers - ITS: MN999922, LSU: MN999927.
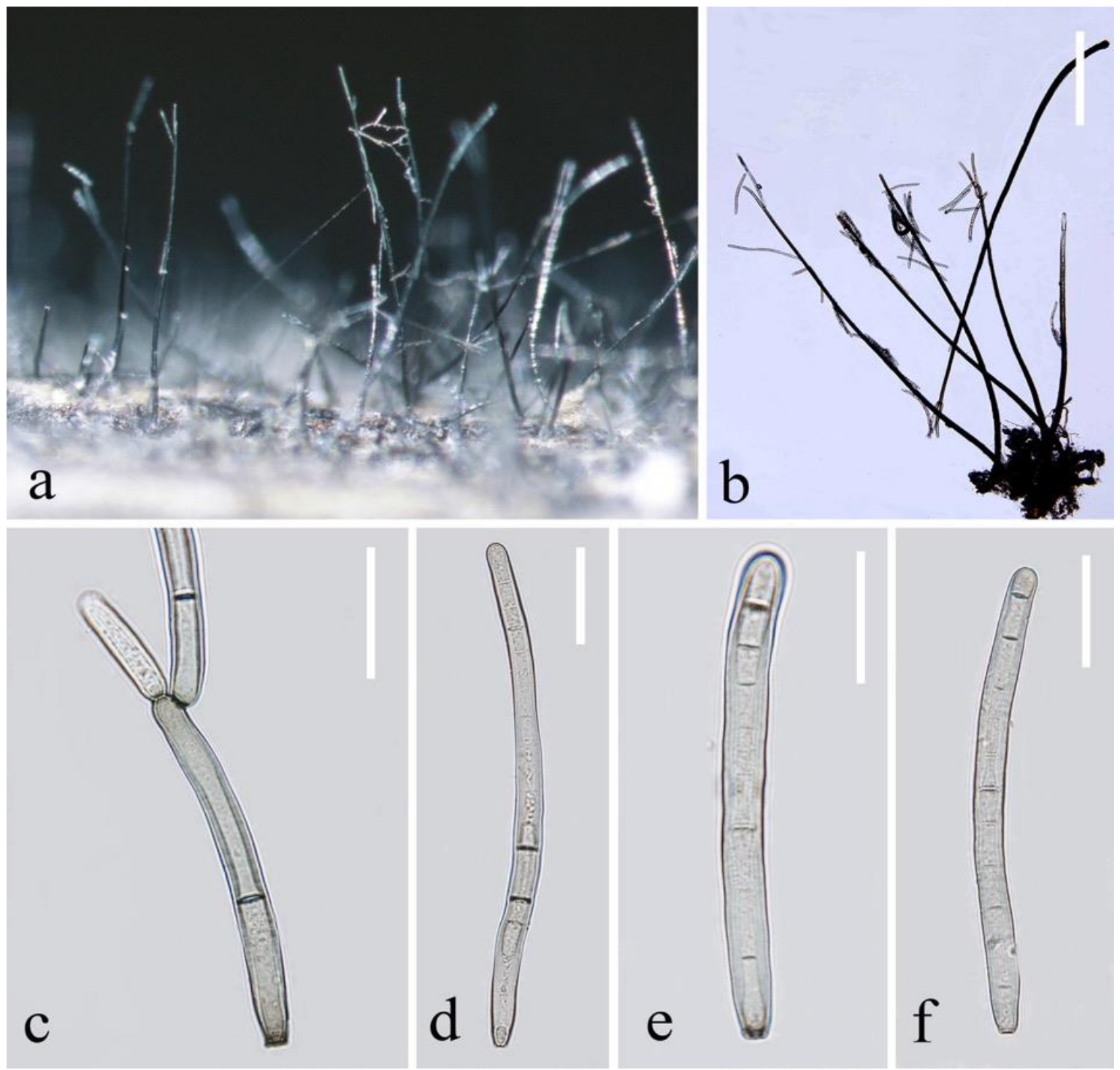

Fig. 30 - Corynespora lignicola (HKAS 92792, holotype). a Colony on natural substrate. b Conidiophore with conidia. $\mathrm{c}-\mathrm{f}$ Conidia. Scale bars: $\mathrm{b}=150 \mu \mathrm{m}, \mathrm{c}-\mathrm{f}=30 \mu \mathrm{m}$.

Notes - Neodendryphiella tarraconensis was introduced by Iturrieta-González et al. (2018) from soil in Spain. This species was previously identified as Dendryphiella sp. Our collection differs from the holotype (CBS H-23479) in having much longer conidiophores (up to $530 \mu \mathrm{m}$ vs 19-185 $\mu \mathrm{m}$ ). This may be because of the differences when grown in natural substrate (our collection) and in culture (holotype). ITS comparison between our strain and FMR 16234 showed that there are only 3 bp differences in a total of $452 \mathrm{bp}$. Thus, we identify it as Neodendryphiella tarraconensis and it is a new geographical record for China. 


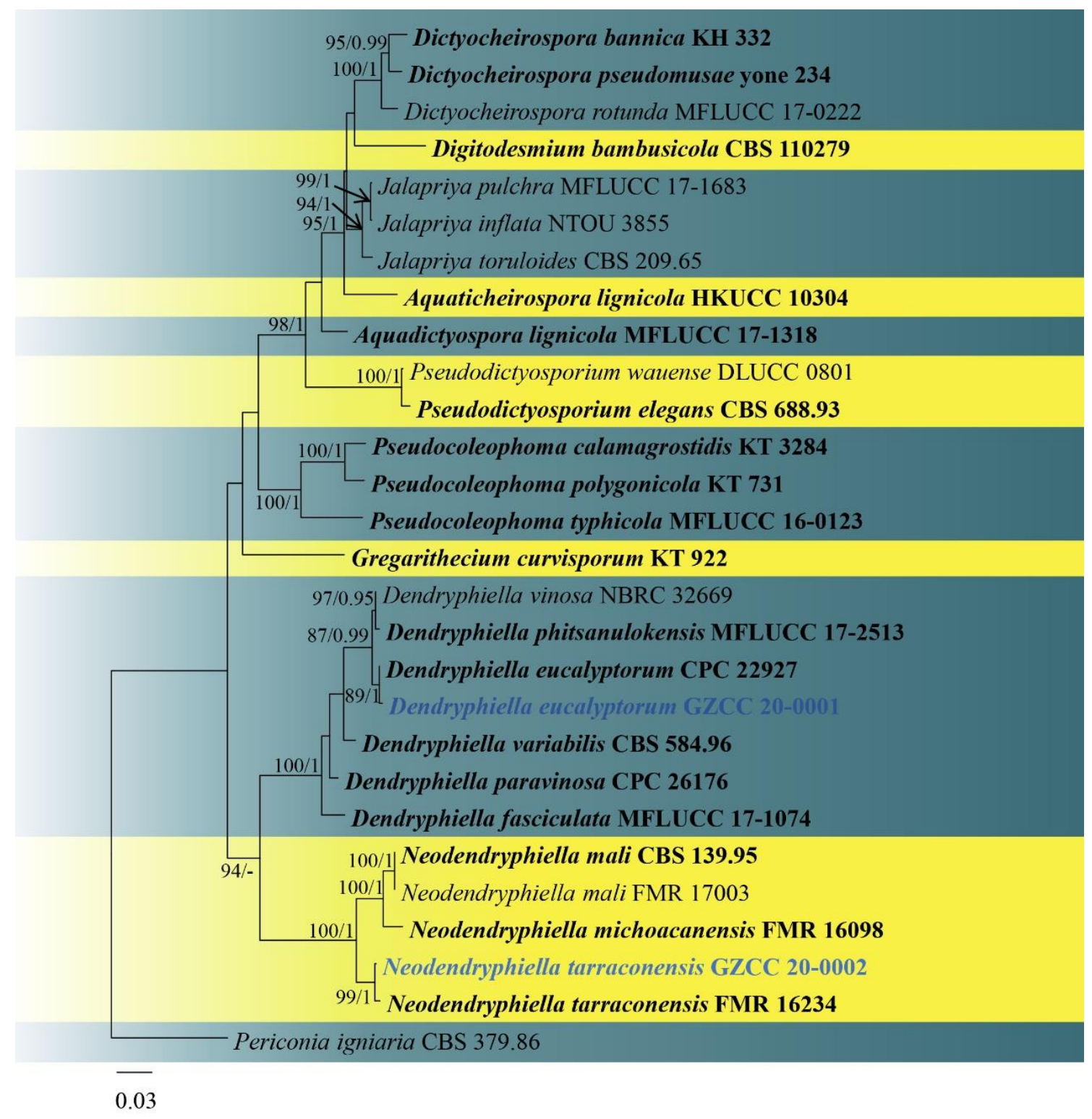

Fig. 31 - Phylogram generated from maximum likelihood analysis based on combined LSU and ITS sequence data. Twenty-eight strains are included in the combined gene analyses comprising 1404 characters after alignment (825 characters for LSU, 579 characters for ITS). Periconia igniaria (CBS 379.86) is used as the outgroup taxon. The tree topology derived from the Bayesian analysis was similar to that derived from the maximum likelihood analysis. The best RaxML tree with a final likelihood value of -7037.019449 is presented. The matrix had 473 distinct alignment patterns, with $12.81 \%$ undetermined characters or gaps. Estimated base frequencies were as follows: $\mathrm{A}=0.238381, \mathrm{C}=0.235435, \mathrm{G}=0.277387, \mathrm{~T}=0.248797$; substitution rates $\mathrm{AC}=$ 1.542553, $\mathrm{AG}=2.179153, \mathrm{AT}=2.337602, \mathrm{CG}=0.362520, \mathrm{CT}=6.291882, \mathrm{GT}=1.000000$; gamma distribution shape parameter $\alpha=0.834067$. Bootstrap values for maximum likelihood equal or greater than $75 \%$ and Bayesian posterior probabilities equal or greater than 0.95 are placed above or below the branches. Ex-type strains are in bold and black. The newly generated sequences are indicated in bold and blue.

Didymellaceae Gruyter, Aveskamp \& Verkley

The most recent taxonomic treatments of Didymeallaceae are from Valenzuela-López et al. (2018), Wanasinghe et al. (2018a), and Jayasiri et al. (2019). This family was established by de Gruyter et al. (2009) with species which are traditionally classified as Phoma and phoma-like. 

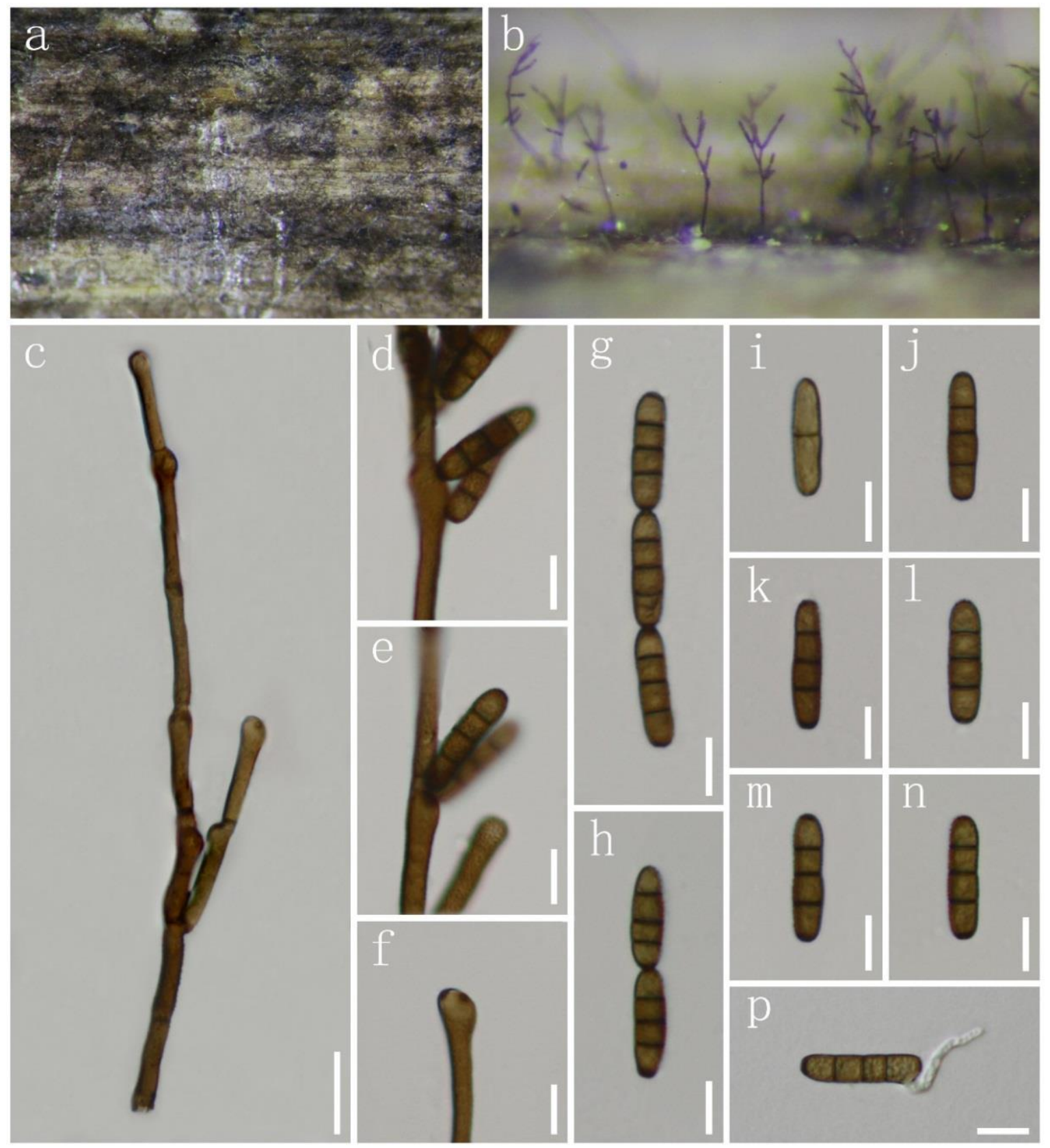

Fig. 32 - Dendryphiella eucalyptorum (GZAAS 20-0002, new geogrophical record). a, b Colonies on natural substrate. c Conidiophore. $\mathrm{d}-\mathrm{f}$ Conidiogenous cells and conidia. $\mathrm{g}-\mathrm{n}$ Conidia. p Germinated conidium. Scale bars: $c=20 \mu \mathrm{m}, \mathrm{d}-\mathrm{p}=10 \mu \mathrm{m}$.

\section{Ascochyta Lib.}

Ascochyta with Ascochyta pisi Lib. as its type species, contains numerous endophytic, pathogenic and saprobic species associated with a wide range of hosts worldwide (Wijayawardene et al. 2017).

Ascochyta herbicola Qian Chen \& L. Cai, Stud. Mycol. 82: 187 (2015)

Fig. 35

Facesoffungi number: FoF07122

Saprobic on dead aerial stem of Orobanche sp. Sexual morph: Undetermined. Asexual morph: Coelomycetous. Conidiomata visible as dark round dots on the host surface. Conidiomata 120-340 $\mu \mathrm{m}$ diameter, pycnidial, solitary, scattered or gregarious, globose to irregular, semi- immersed to immersed, sometimes erumpent, unilocular, black. Conidioma wall thick, 2-4 layered, hyaline to dark brown cells of textura angularis, Conidiogenous cells hyaline, phialidic, globose. Conidia 5-7 
$\times 2-3 \mu \mathrm{m}$ wide, cylindrical to subcylindrical, guttulate, aseptate.
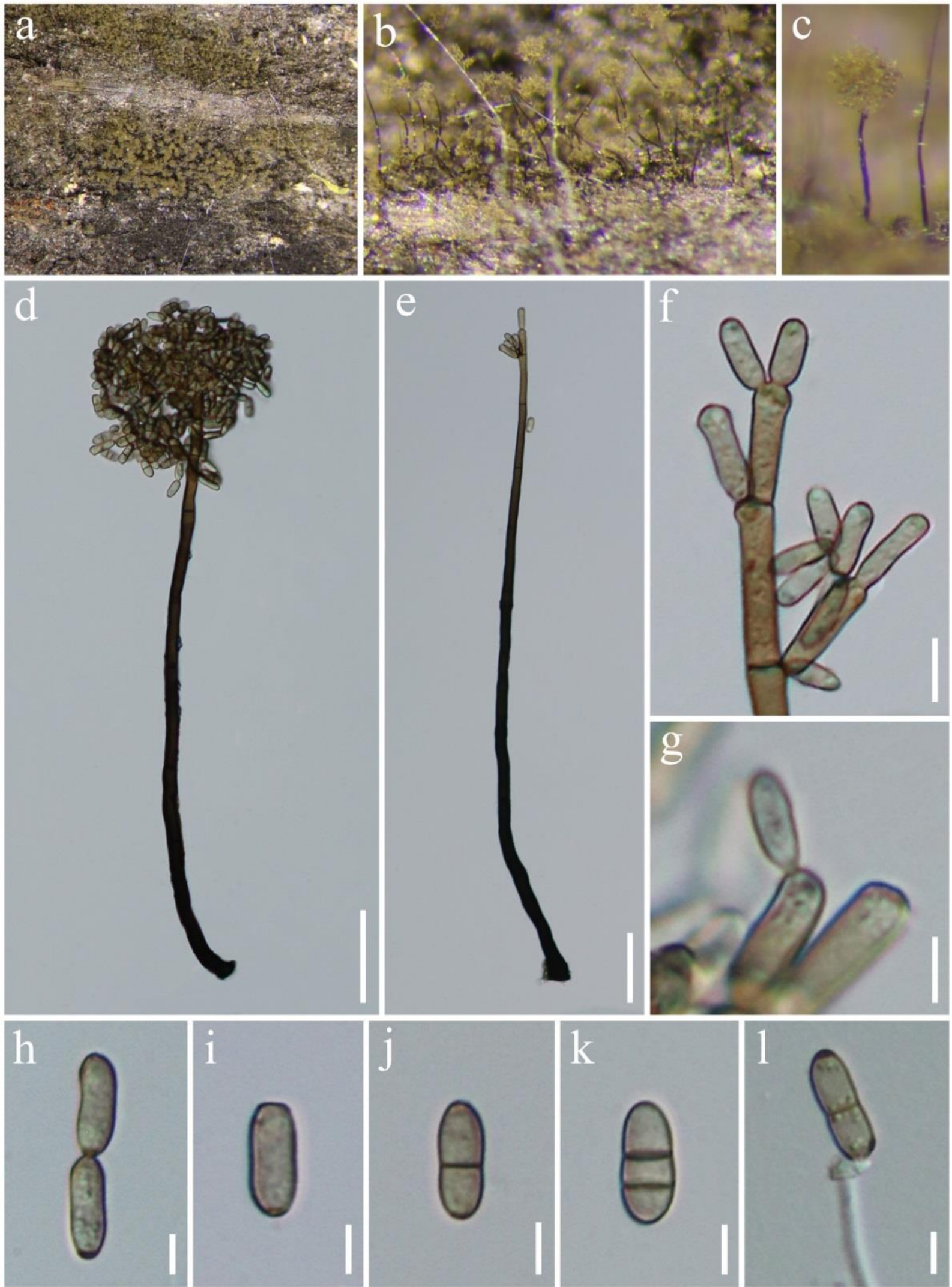

Fig. 33 - Neodendryphiella tarraconensis (GZAAS 20-0003, new geogrophical record). $\mathrm{a}-\mathrm{c}$ Colonies on natural substrate. $\mathrm{d}$, e Conidiophores and conidia. $\mathrm{f}, \mathrm{g}$ Conidiogenous cells and conidia. $\mathrm{h}-\mathrm{k}$ Conidia. 1 Germinated conidium. Scale bars: $\mathrm{d}$, $\mathrm{e}=50 \mu \mathrm{m}, \mathrm{f}, \mathrm{g}=10 \mu \mathrm{m}, \mathrm{h}-1=5 \mu \mathrm{m}$. 


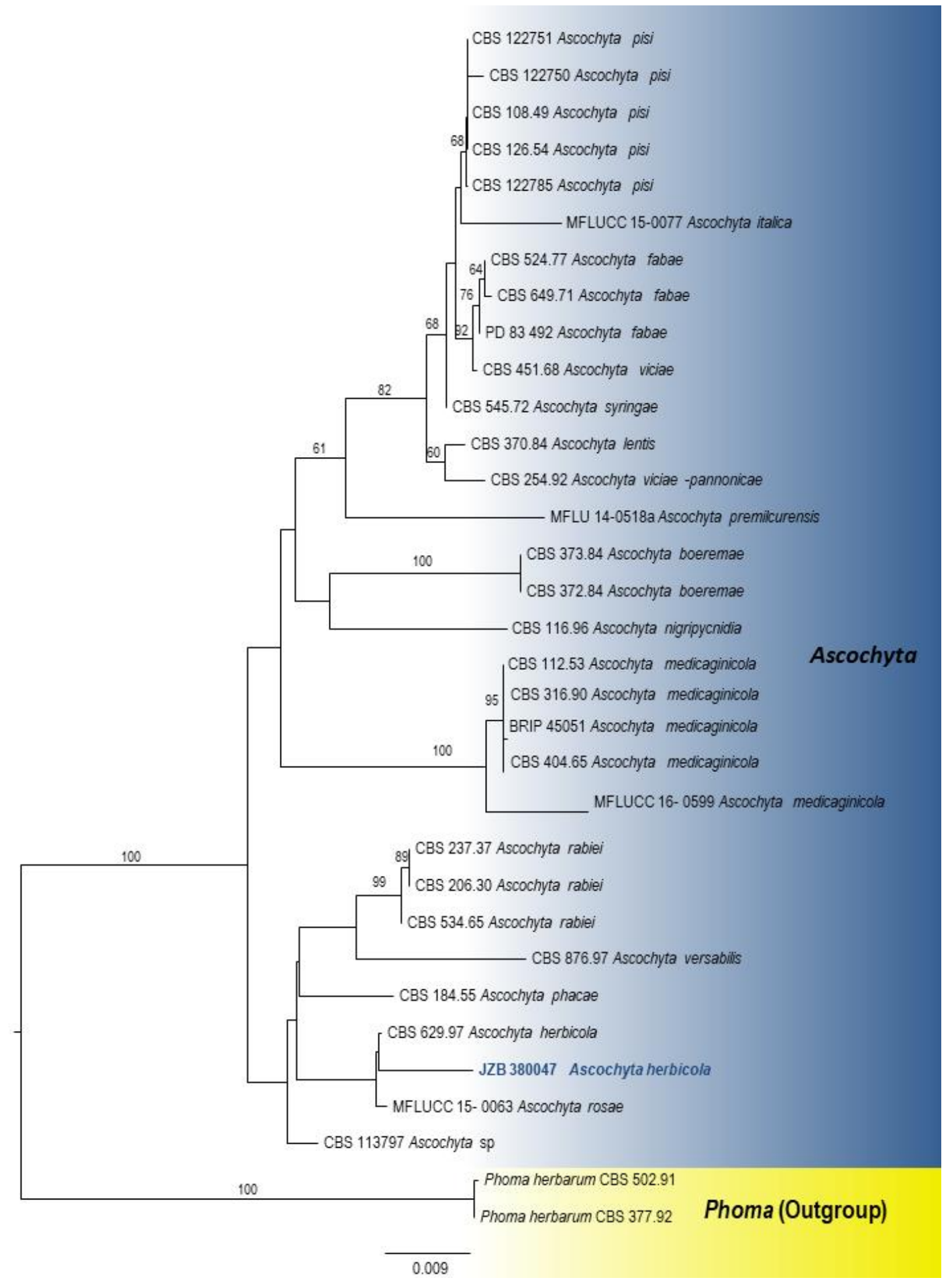

Fig. 34 - Phylogram generated from maximum likelihood analysis based on combined LSU, ITS, RPB2 and TUB sequence data. Thirty-three strains are included in the combined gene analyses and Phoma herbarum (CBS 502.91 and 377.92) strains are used as the out-group taxa. The tree topology derived from the Bayesian analysis was similar to that derived from the maximum likelihood analysis. The best RaxML tree with a final likelihood value of -5091.923675 is presented. The matrix had 214 distinct alignment patterns, with $22.94 \%$ undetermined characters or gaps. Estimated base frequencies were as follows: $\mathrm{A}=0.234220, \mathrm{C}=0.256120, \mathrm{G}=0.244025, \mathrm{~T}=$ 0.265634; substitution rates $\mathrm{AC}=0.657233, \mathrm{AG}=4.062723, \mathrm{AT}=1.701821, \mathrm{CG}=0.583489, \mathrm{CT}$ $=9.457386, \mathrm{GT}=1.000000$; gamma distribution shape parameter $\alpha=0.669698$. Bootstrap values for maximum likelihood equal to or greater than 60 and Bayesian posterior probabilities equal or greater than 0.90 are placed above or below the branches. The newly generated sequences are indicated in blue. 

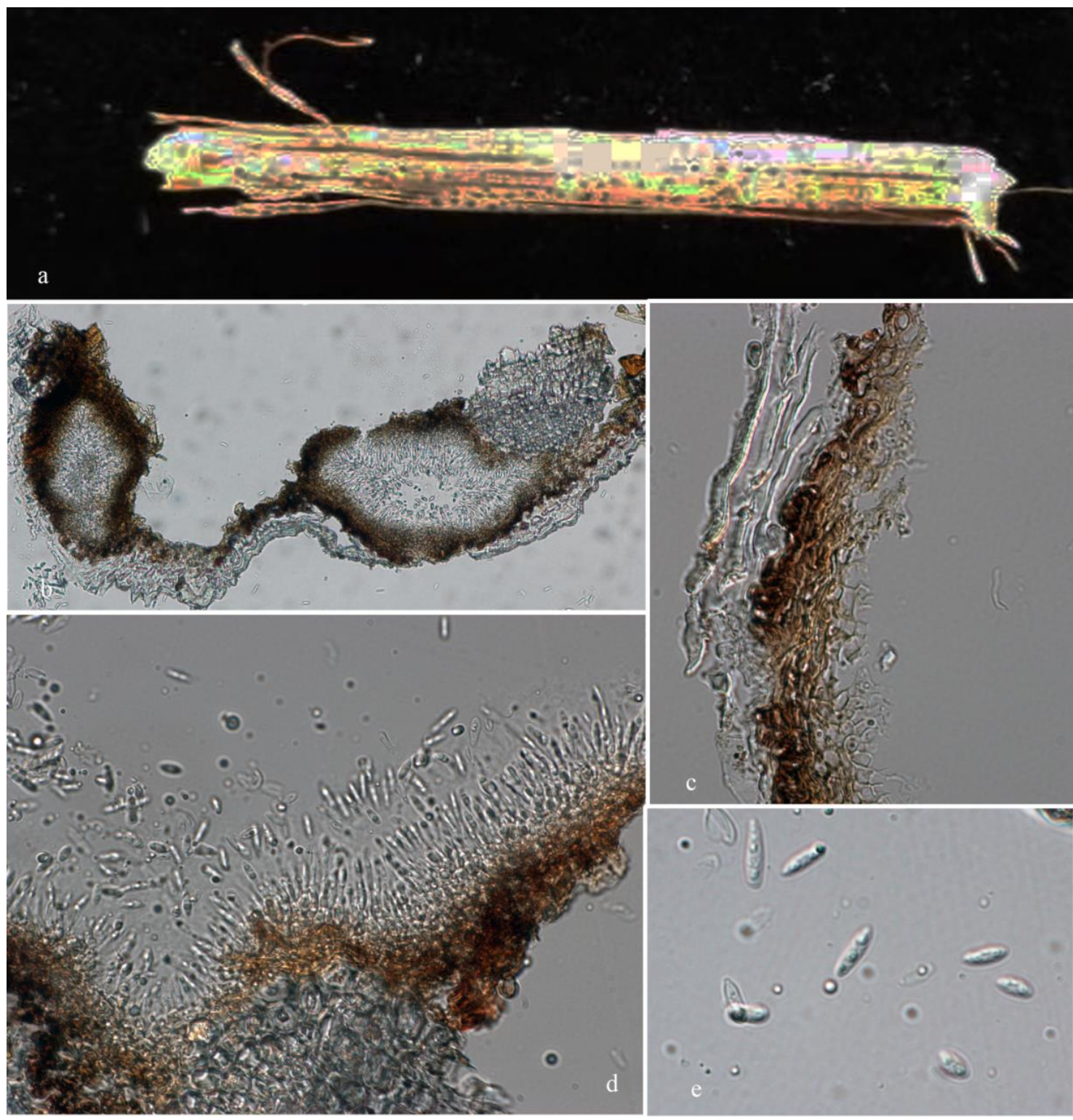

Fig. 35 - Ascochyta herbicola (JZB 380047, new host record). a Appearance of fruiting bodies on host substrate. $\mathrm{b}$ Section through the fruiting body. c Peridium cell wall. $\mathrm{d}$ Immature and mature conidia attached to conidiogenous cells. e Conidia. Scale bars: $\mathrm{a}=200 \mu \mathrm{m}, \mathrm{e}=10 \mu \mathrm{m}$.

Culture characters - Colonies on OA $4-5 \mathrm{~cm}$ diameter after 7 days, regular, colorless to dark concentric zones, aerial mycelium, fluffy, white, reverse colorless.

Known distribution (based on molecular data) - Italy, Forlì-Cesena Province, United States, Wyoming (Chen et al. 2015, this study).

Known hosts (based on molecular data) - Orobanche sp., Water, stems of Syntheris dissecta

Material examined - Italy, Province of Forlì-Cesena [FC], Forlì - Via Pietro Nenni, on dead aerial stem of Orobanche sp., (Orobanchaceae) 9 March 2017, E. Camporesii, IT 3850 (MFLU 380047), living culture, JZB380047.

GenBank number - ITS: MN989422.

Notes - The collection obtained from dead aerial stem of Orobanche sp. was identified as Ascochyta herbicola with support from morphology and phylogeny. Our isolate clustered with the reference strain of the Ascochyta herbicola (CBS 629.97 7) in the combined LSU, ITS, RPB2 and TUB sequence phylogeny (Fig. 34). We could not obtain RPB2 and TUB sequence data from our isolate, and were unable to perform further analysis. This is the first record of Ascochyta herbicola from Orobanche sp. in Italy. 


\section{Calophoma Qian Chen \& L. Cai}

Chen et al. (2015) introduced Calophoma clematidina as the type species and there are ten epithets recorded under Calophoma (Chen et al. 2015). All species have been reported with sexual and asexual morphs. Calophoma clematidina was reported from the stem of Clematis sp. in the Netherlands (Woudenberg et al. 2009). Calophoma is characterised by solitary, pycnidial conidiomata which have conspicuously papillate ostioles. The pycnidial wall comprises several layers of oblong to isodiametric cells. Conidiogenous cells are phialidic, hyaline, smooth, and ampulliform to doliiform. Conidia are cylindrical to ellipsoidal, smooth and thin, and aseptate or 1septate. Chlamydospores are tan to dark brown. In this study, we introduce a novel species to this genus with support from combined LSU and ITS sequence data.

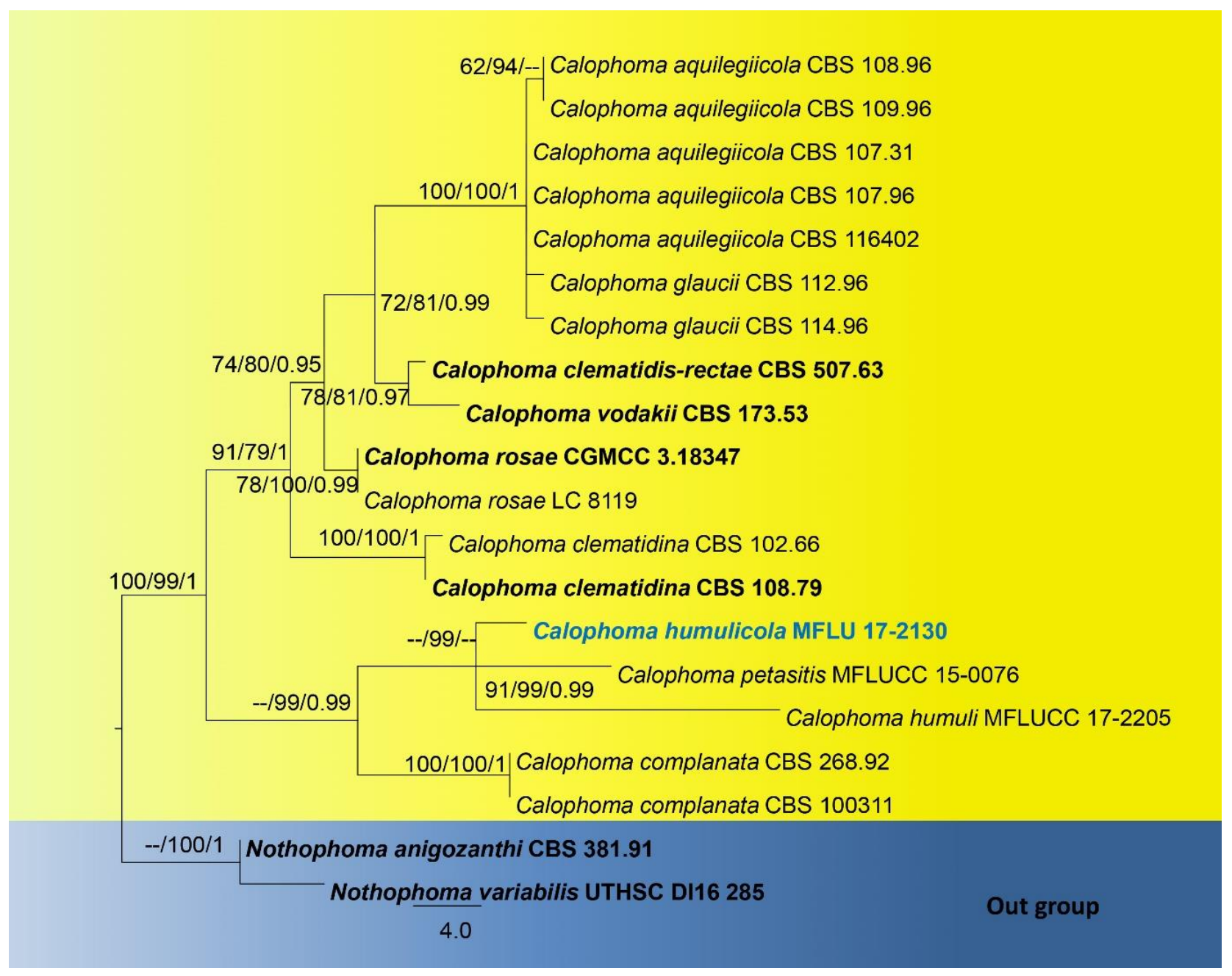

Fig. 36 - Phylogram generated from maximum likelihood analysis based on combined LSU and ITS sequence data. Twenty-two strains are included in the combined gene analyses comprising 1,358 characters after alignment (888 characters for LSU, 470 characters for ITS). Didymella macropodii CBS100191 is used as the outgroup taxon. The tree topology derived from the Bayesian analysis was similar to that derived from the maximum likelihood analysis. The best RaxML tree with a final likelihood value of - 2481.811840 is presented. The matrix had 66 distinct alignment patterns, with $0.63 \%$ undetermined characters or gaps. Estimated base frequencies were as follows: $\mathrm{A}=0.246751, \mathrm{C}=0.220789, \mathrm{G}=0.275533, \mathrm{~T}=0.256927$; substitution rates $\mathrm{AC}=$ 6.461538, $\mathrm{AG}=18.966230, \mathrm{AT}=9.278572, \mathrm{CG}=2.883910, \mathrm{CT}=46.053729, \mathrm{GT}=1.000000$; gamma distribution shape parameter $\alpha=0.020000$. Bootstrap values for maximum likelihood equal to or greater than 50 and Bayesian posterior probabilities equal or greater than 0.95 are placed above or below the branches. Ex-type strains are in bold and black. The newly generated sequence is indicated in blue. 
Calophoma humulicola Chaiwan, T.C Bulgakov, Jayaward \& K.D. Hyde, sp. nov.

Fig. 37

Index Fungorum number: IF557012; Facesoffungi number: 06956 lupulus"

Etymology - The specific epithet "humulicola" refers to the meaning "borne on humulus

Holotype - MFLU 17-2130

Saprobic on dead wood of humulus lupulus. Sexual morph: Undetermined. Asexual morph: Conidiomata pycnidial, solitary, globose to subglobose, mostly with some hyphal outgrows, produced on the agar surface or immersed, (120-)135-165 × 85-130 $\mu \mathrm{m}$. Ostioles 1(-3), conspicuously papillate. Pycnidial wall pseudoparenchymatous, 2-4-layered, 13-21 $\mu \mathrm{m}$ thick, composed of oblong to isodiametric cells. Conidiogenous cells phialidic, hyaline, smooth, ampulliform to doliiform, 5.5-7.5 × 4-7 $\mu \mathrm{m}$. Conidia ellipsoidal to cylindrical, smooth- and thinwalled, aseptate or occasionally 1-septate, 4.5-7 $\times 2-3 \mu \mathrm{m}$, with $(0-) 2-4(-8)$ polar guttules. Conidial matrix pale pink. Chlamydospores usually scanty, uni- or multi-cellular, 1-celled, intercalary, guttulate, thick-walled, green brown, 8-10 $\mu \mathrm{m}$ diameter, multi-cellular irregular dictyo/phragmosporous, somewhat botryoid and in combination with 1-celled chlamydospores, $\tan$ to dark brown, $3-50 \times 12-25 \mu \mathrm{m}$.

Culture characteristics - Conidia germinating on PDA within 24 hours. Germ tubes produced around conidia. Colonies on PDA entire edge, velvety, medium dense, flat or effuse, brownish grey. Mycelium 1.5-3.8 $\mu \mathrm{m}$ broad, partly superficial, partly immersed, white, septate.

Material examined - Russia, on dead wood of Humulus lupulus (Cannabaceae), 11 May, T.S. Bulgakov, T-1828 (MFLU 17-2130, holotype).

GenBank numbers - ITS: MN796328, LSU: MN796329.
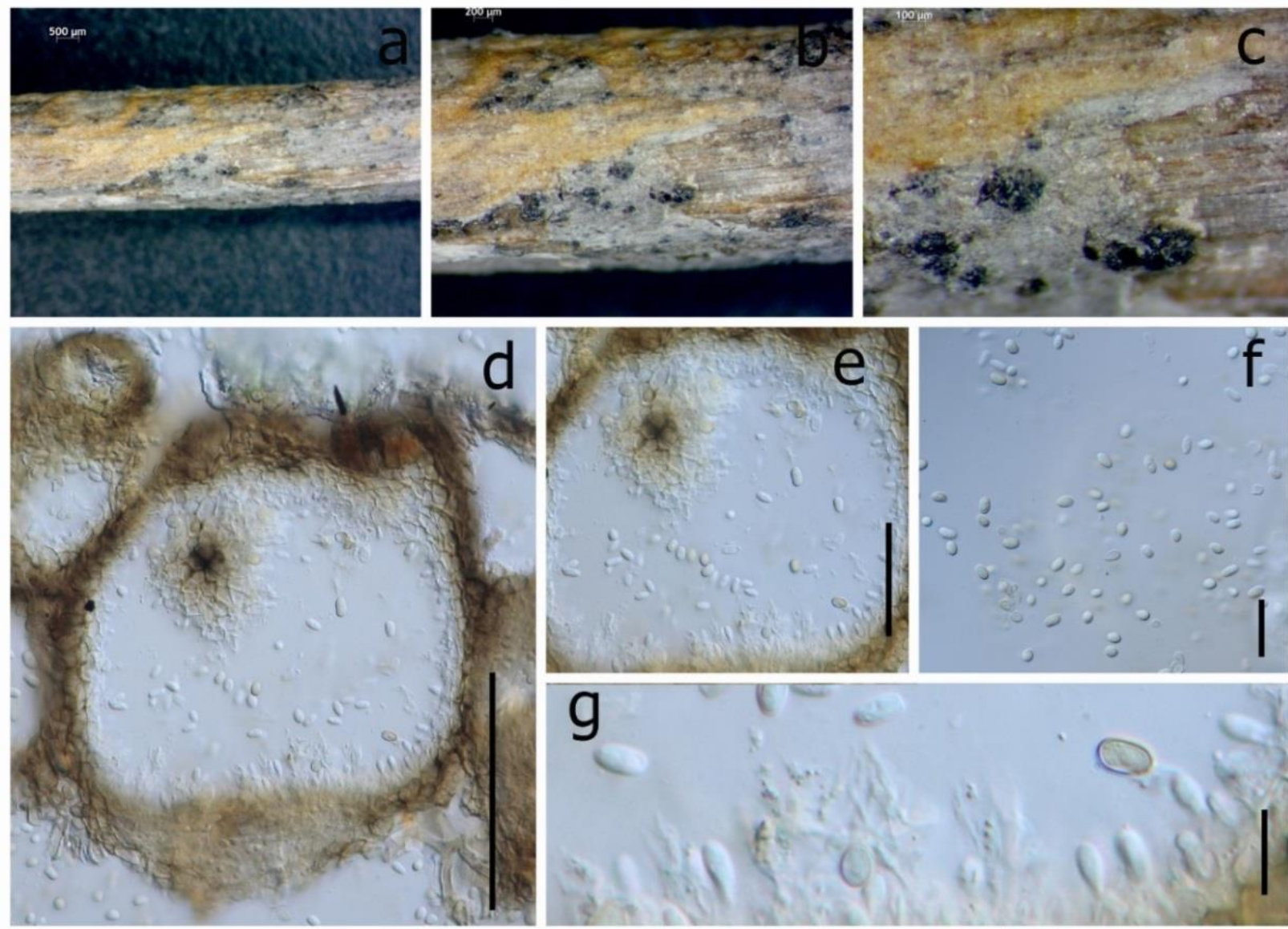

Fig. 37 - Calophoma humulicola )MFLU 17-2130, holotype(. a-c Conidia observed on host substrate. $\mathrm{d}$ Conidiomata. e pycnidial wall. $\mathrm{f}$ Conidia. g Conidiodenous cell. Scale bars: $\mathrm{a}=500$ $\mu \mathrm{m}, \mathrm{b}=200 \mu \mathrm{m}, \mathrm{c}=100 \mu \mathrm{m}, \mathrm{d}, \mathrm{e}=50 \mu \mathrm{m}, \mathrm{f}=20 \mu \mathrm{m}, \mathrm{g}=10 \mu \mathrm{m}$. 


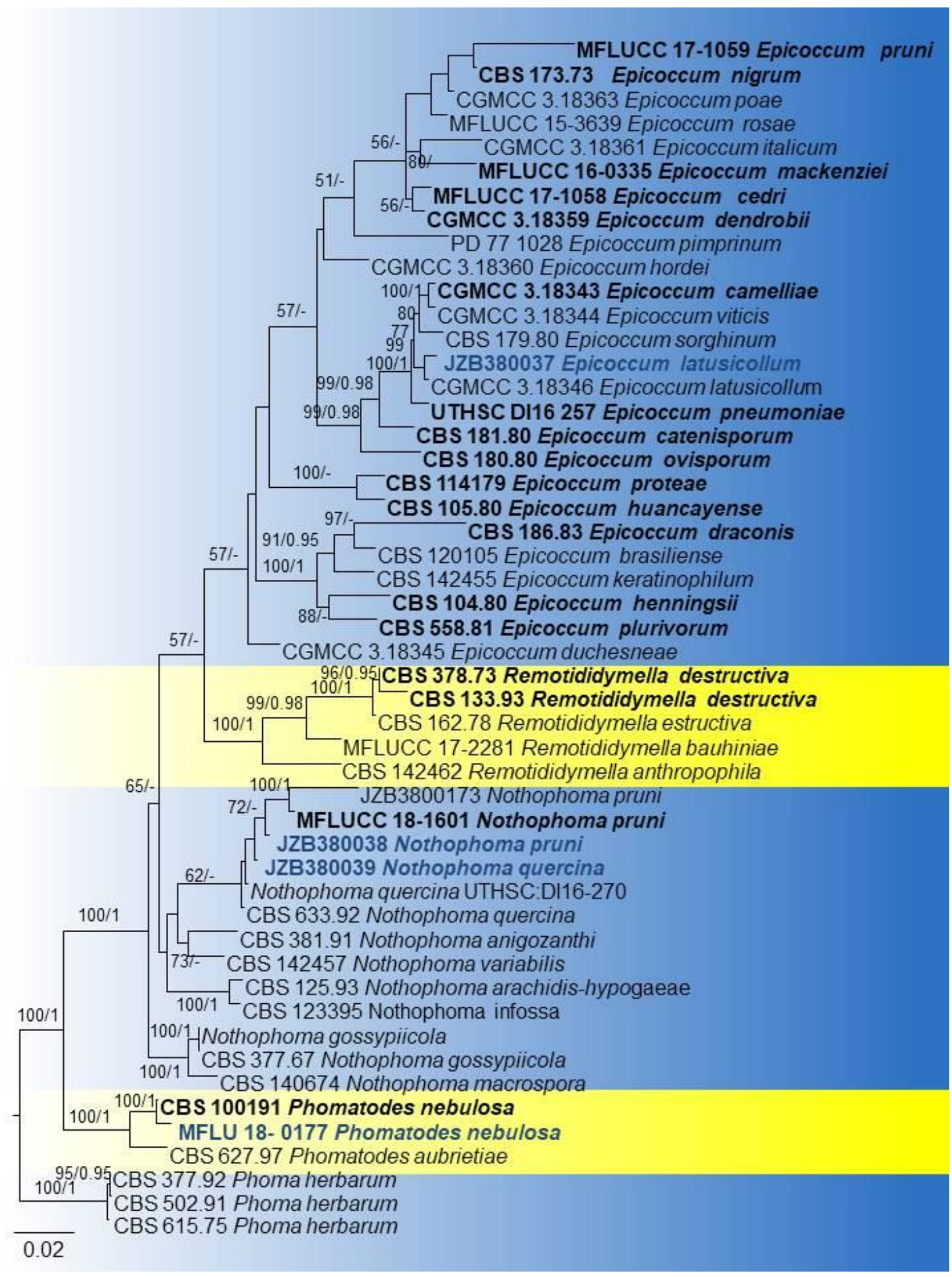

Fig. 38 - Phylogram generated from maximum likelihood analysis based on combined LSU, ITS, and RPB2 and TUB sequence data. Twenty-three strains are included in the combined gene analyses comprising 2331 characters after alignment. Phoma herbarum (CBS 377.92, 502.91 and 615.75 ) is used as the outgroup taxa. The tree topology derived from the Bayesian analysis was similar to that derived from the maximum likelihood analysis. The best RaxML tree with a final likelihood value of -10101.832594 is presented. The matrix had 465 distinct alignment patterns, with $6.60 \%$ undetermined characters or gaps. Estimated base frequencies were as follows: $\mathrm{A}=$ $0.237653, \mathrm{C}=0.243234, \mathrm{G}=0.276939, \mathrm{~T}=0.242174$; substitution rates $\mathrm{AC}=1.371854, \mathrm{AG}=$ 5.338217, $\mathrm{AT}=1.639327, \mathrm{CG}=0.874686, \mathrm{CT}=12.527823, \mathrm{GT}=1.000000$; gamma distribution shape parameter $\alpha=0.656489$. Bootstrap values for maximum likelihood and parsimony equal to or greater than 50 are placed above or below the branches. Ex-type strains are in bold and black. The newly generated sequences are indicated in blue. 
Notes - Calophoma humulicola is morphologically similar to Calophoma humuli (Hyde et al. 2019), but phylogenetically distinct and constitutes an independent lineage basal to an unidentified species of Calophoma (CBS 186.55). The new taxon groups with C. petasitis with 99\% ML statistical support (Fig. 36), which has been reported from a dead stem of Petasites sp. (Asteraceae), near Campigna-Santa Sofia, Province of Forlı'-Cesena, Italy, on 9 June 2014 (Tibpromma et al. 2017).

A comparison of LSU and ITS with these two strains reveals 3/960 (0.31\%) and 5/522 $(0.95 \%)$ base pair differences following the guidelines of Jeewon and Hyde (2016). Therefore, we introduce our collection as a new species in Calophoma.

\section{Epicoccum Link}

Epicoccum was introduced by Chen et al. (2015). Species belonging to this genus are characterized with epicoccoid, subcylindrical conidia, and irregular pycnidial conidiomata. The most recent taxonomic treatments to this family are Jayasiri et al. (2017), Valenzuela-Lopez et al. (2018), Wanasinghe et al. (2018a) and Chethana et al. (2019).

Epicoccum latusicollum Qian Chen, Crous \& L. Cai, Stud. Mycol. 87: 144 (2017)

Fig. 39

Facesoffungi number: FoF06580

Saprotrophic or pathogenic on Phragmites australis leaves. Sexual morph: Undetermined. Asexual morph: Growing well on PDA. Conidiomata pycnidial, mostly solitary, sometimes aggregated, globose to subglobose or pyriform, glabrous, produced on the agar surface, ostiolate, sometimes elongate with a short, slightly papillate neck. Pycnidial wall pseudoparenchymatous, composed of oblong to isodiametric cells, with 2-3 cell layers of which outer layers are brown. Conidiogenous cells phialidic, hyaline, smooth, ampulliform to doliiform. Conidia ellipsoidal to oblong, smooth and thin-walled, hyaline, aseptate, 4.5-6 $\times 2-3 \mu \mathrm{m}(\bar{x}=5 \times 2.5 \mu \mathrm{m}, \mathrm{n}=40)$. Chlamydospores aseptate, light brown, smooth and thin-walled, in chains or single, ovoid.

Culture characteristics - Colonies on PDA reach $80-85 \mathrm{~mm}$ diameter after $7 \mathrm{~d}$, margin regular, floccose aerial mycelia covering the whole colony, dense, white to grey, forming several white mycelial pellets; reverse white.

Known distribution (based on molecular data) - China and Japan (Chen et al. 2017, Valenzuela-Lopez et al. 2018), China (this study).

Known hosts (based on molecular data) - Acer palmatum, Camellia sinensis, Podocarpus macrophyllus, Sorghum bicolor, Vitex negundo (Chen et al. 2017, Valenzuela-Lopez et al. 2018), Phragmites australis (this study).

Material examined - China, Shandong, Yellow River Park, on leaf spots of Phragmites australis (Poaceae), 7 October 2017, Y.Y. Hao (JZBH380037, new host record) living culture, JZB380037.

GenBank submissions - ITS: MN533797, LSU MN533800.

Notes - Epicoccum latusicollum was introduced by Chen et al (2017). In our phylogenetic analysis, this isolate clusters with the type (CGMCC 3.18346) with 100\% ML bootstrap support and 1.0 BYPP (Fig. 38). The collection shares similar colony morphology, conidial structure and sizes with the protologue (Chen et al (2017). Pairwise comparison of nucleotide sequences in four gene regions reveals the following: LSU is $100 \%$ similar; ITS less than $1 \%$ dissimilar; proteincoding regions (BT and RPB2) less than 5\% dissimilar. Based on these facts isolate (JZBH380037) is considered to be Epicoccum latusicollum. This species has been reported on Acer palmatum, Camellia sinensis, Podocarpus macrophyllus, Sorghum bicolor and Vitex negundo (Chen et al. 2017). In the present study, we collected this taxon associated with leaf spots on Phragmites australis for the first time (Farr \& Rossman 2020).

Nothophoma Qian Chen \& L. Cai

Nothophoma was introduced by Chen et al. (2015) to accommodate five species. This genus is comprised of important plant pathogens. Currently, there are ten species accepted in this 
genus. Most recent treatments of this genus are Chethana et al. (2019) and Jayasiri et al. (2019).

Nothophoma pruni Chethana, J.Y. Yan, X.H. Li \& K.D. Hyde, Mycosphere 10(1): 520 (2019)

Facesoffungi number: FoF 04917

Fig. 40

Saprobic on diseased leaves of Castanea mollissima. Sexual morph: Undetermined. Asexual morph: Pycnidia on the PDA surface, $0.21-0.45 \mathrm{~mm}(\bar{x}=0.29 \mathrm{~mm}, \mathrm{n}=10)$ diameter, solitary, scattered, globose to irregularly shaped, black, ostiolate. Conidiogenous cells phialidic, hyaline, simple, doliiform to ampulliform, variable in size. Conidia 4.7-8.6 $\times 2.5-3.5 \mu \mathrm{m}(\bar{x}=6 \times 3.2 \mu \mathrm{m}, \mathrm{n}$ $=40$ ), cylindrical to obovoid or oblong, hyaline, aseptate, smooth-walled. Conidial exudates hyaline to buff. study).

Known distribution (based on molecular data) - China, Beijing (Chethana et al. 2019 and this

Known hosts (based on molecular data) - Prunus avium (Chethana et al. 2019), Castanea mollissima (this study)

Culture characteristics - Colonies on PDA reach $80 \mathrm{~mm}$ diameter after 7 days at $25{ }^{\circ} \mathrm{C}$, with regular margin, dull white aerial mycelium surface floccose, with reverse pale vinaceous.

Material examined - China, Shandong, Yellow River Park, on leaf spots of Castanea mollissima (Fagaceae), 7 October 2017, Y.Y. Hao (JZBH380038, new host record), living culture, JZB380038.

GenBank numbers - ITS: MN533798, LSU: MN533801, TUB: MN991303, RPB2: MN991306.

Notes - Nothophoma pruni was introduced by Chethana et al. (2019) as a leaf spot causing microfungus associated with Prunus avium. The taxon collected in this study fits well within Didymellaceae with its morphological characteristics. In our combined phylogenetic analysis of the LSU, ITS, TUB and RPB2 gene regions, our isolate clusters with the $N$. pruni type strain (MFLUCC 18-1601) with 100\% ML bootstrap and 1.0 BYPP. Conidial dimensions and shape are similar to the original description. Nucleotide sequences were compared in MFLUCC 18-1601 and JZBH380038. LSU and RPB2 sequences are 100\% similar and ITS has less than 1\% dissimilarity. Despite some nucleotide differences in the TUB gene region we identify JZBH380038 as Nothophoma pruni. This is the first report of $N$. pruni from China, associated with Castanea mollissima worldwide (Farr \& Rossman 2020).

Nothophoma quercina (Syd. \& P. Syd.) Qian Chen \& L. Cai, Stud. Mycol. 82: 213 (2015)

Fig. 41

Facesoffungi number: FoF06581

Saprobic on diseased leaves of Rosa multiflora. Sexual morph: Undetermined. Asexual morph: Pycnidia growing well on PDA, solitary, scattered, globose to irregularly shaped, black, ostiolate. Pycnidial wall multi-layered, composed of pale brown, pseudoparenchymatous cells, with thicker outer layer and thinner inner layer. Conidiogenous cells phialidic, hyaline, simple, doliiform to ampulliform, variable in size. Conidia 5-7 $\times 3-4.5 \mu \mathrm{m}(\bar{x}=5.5 \times 4 \mu \mathrm{m}, \mathrm{n}=40)$, variable in size and shape, subglobose to oval or obtuse, initially hyaline, becoming brown at maturity, aseptate, smooth-walled. Conidial exudates hyaline to buff.

Culture characteristics - Colonies on PDA reach $80 \mathrm{~mm}$ diameter after 7 days at $25{ }^{\circ} \mathrm{C}$, with regular margin, dull white aerial mycelium surface floccose to wooly, with greenish- olivaceous to olivaceous near the centre and reverse dark ochreous in the centre and white in the margin.

Known distribution (based on molecular data) - USA, Arizona, China, Korea, Spain, Tunisia and Ukraine (Chen et al. 2017, Moral et al. 2017, Liu et al. 2018b, Chethana et al. 2019, Farr \& Rossman 2020).

Known hosts - Chaenomeles sinensis, Malus micromalus, Microsphaera alphitoides, Olea europaea, Phellodendron amurense, Pistacia vera, Prunus avium, Rosa multiflora (this study), 
Quercus sp. and Ziziphus jujuba (Jianyu et al. 2016, Moral et al. 2017, Chethana et al. 2019, Farr \& Rossman 2020).
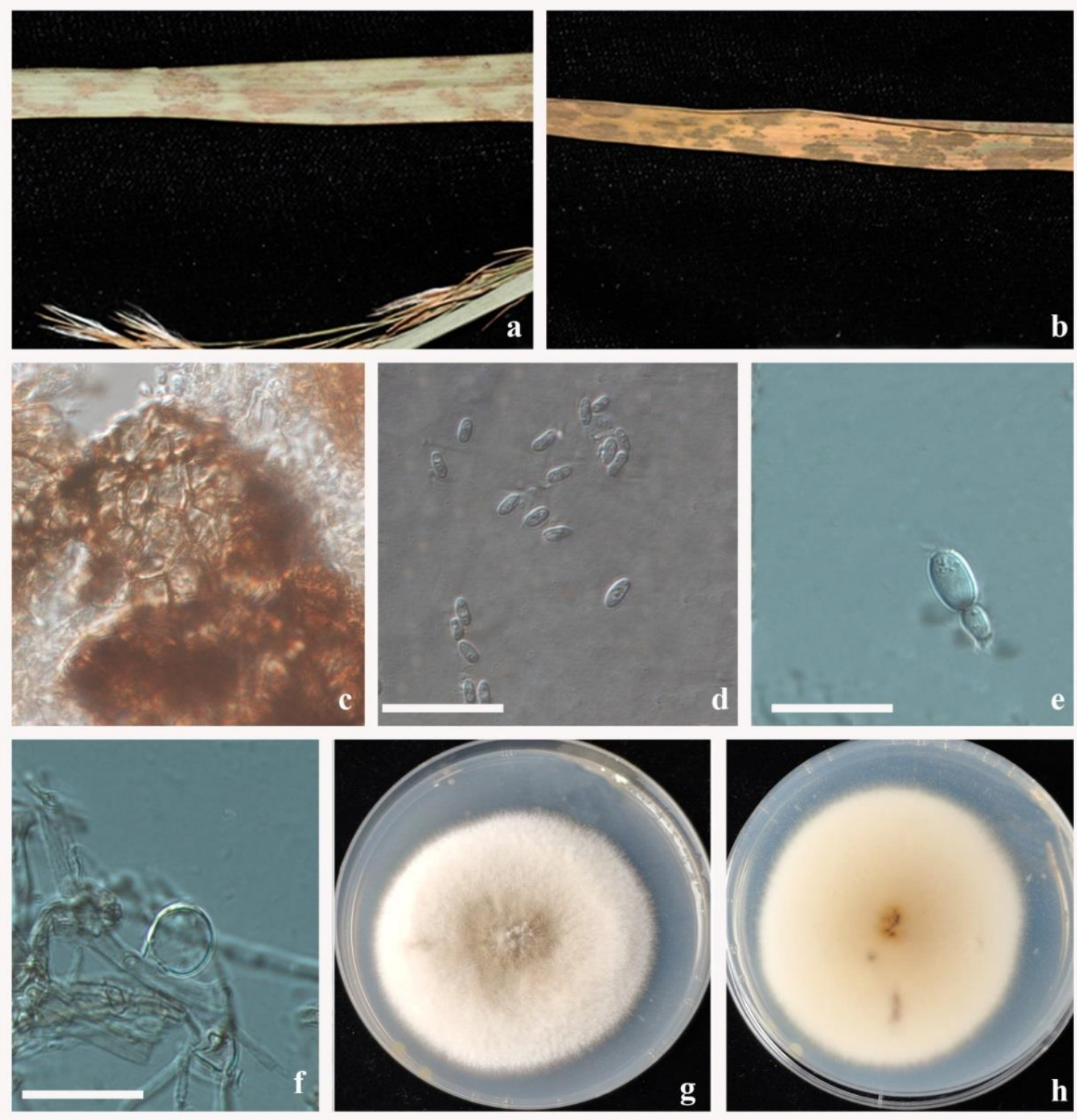

Fig. 39 - Epicoccum latusicollum )JZB380037, new host record(. a, b Material examined. c Pycnidial wall. d Conidia on PDA. e Developing chlamydospores. f Chlamydospores g Culture on PDA (upper view). h culture on PDA (reverse view). Scale bars: $d-f=20 \mu \mathrm{m}$.

Material examined - China, Shandong, Yellow River Park, on leaf spots of Rosa multiflora (Rosaceae), 7 October 2017, Y.Y. Hao (JZBH380039, new host record, living culture, JZB380039.

GenBank numbers - ITS: MN533799, LSU: MN533802, TUB: MN991304, RPB2: MN991307.

Notes - Our Nothophoma quercina isolate investigated in this study clusters with other Nothophoma quercina strains (UTHSC:DI16-270 and CBS 633.92; type) in the multiple gene phylogenies with 63\% ML bootstrap value. The morphological features of our collection are also identical to those published (Chen et al. 2013). Pairwise comparison of nucleotide sequences in the four gene regions, LSU, ITS and RPB2 of both the type and our taxon also reveal a high percentage of similarity that confirms strain JZBH380039 as Nothophoma quercina. Nothophoma quercina is a pathogenic species in Didymellaceae (Chen et al. 2013). Previously this species was known as Ampelomyces quercinus and Phoma fungicola until it was synonymised with this current name by 
Chen et al. (2013). This is the first report of N. quercina from Rosa multiflora (Farr \& Rossman 2020).
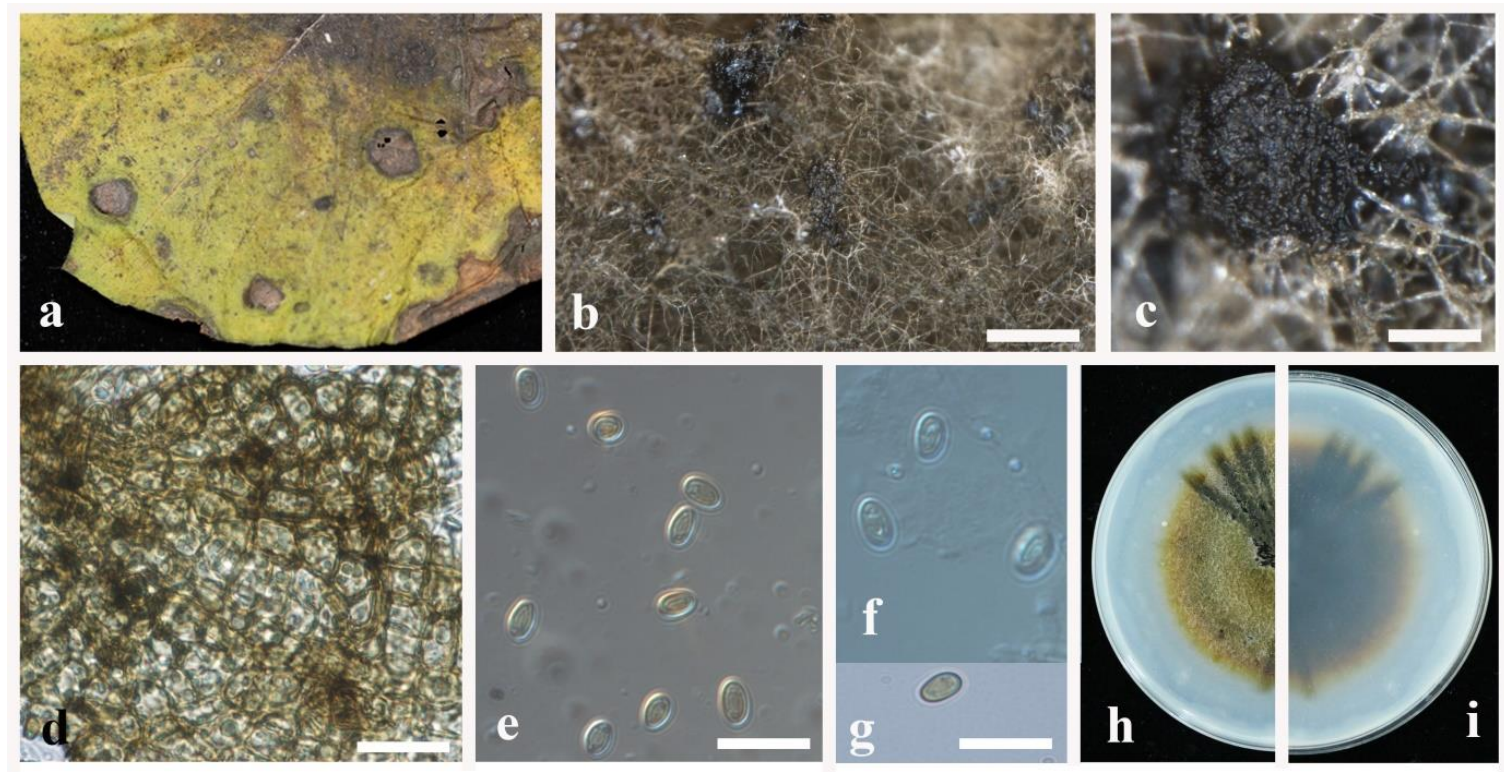

Fig. 40 - Nothophoma pruni )JZB380038, new host record(. a Material examined. b-c Pycnidia. d Pycnidial wall. e-g Conidia on PDA. h Culture on PDA (upper view). i Culture on PDA (reverse view). Scale bars: $b=100 \mu \mathrm{m}, \mathrm{c}=100 \mu \mathrm{m}, \mathrm{d}-\mathrm{f}=20 \mu \mathrm{m}, \mathrm{g}=10 \mu \mathrm{m}$.

Phomatodes Qian Chen \& L. Cai, Stud. Mycol. 82: 191 (2015)

Phomatodes was introduced by Chen et al (2015) to accommodate phoma-like taxa. The type species of this genus is Phomatodes aubrietiae which is characterized by pycnidial, globose to subglobose, ostiolate conidiomata, solitary or confluent, with a 3-5-layered, pigmented pseudoparenchymatous pycnidial wall, phialidic, hyaline, smooth, ampulliform to doliiform conidiogenous cells and cylindrical to allantoid, hyaline, thin-walled, smooth, aseptate, guttulate conidia (Chen et al 2015). In this study, we introduce a new host record of P. nebulosa.

Phomatodes nebulosa (Pers.) Qian Chen \& L. Cai, Stud. Mycol. 82: 191 (2015)

Fig. 42

Facesoffungi number: FoF06803

Saprobic or necrotrophic on Urtica spp. Sexual morph: Undetermined. Asexual morph Conidiomata pycnidial, solitary, globose to subglobose, glabrous, semi-immersed or immersed. Ostiole single, slightly papillate. Pycnidial walls pseudoparenchymatous, 4-6-layered, thick, composed of isodiametric cells. Conidiophores reduced to conidiogenous cells. Conidiogenous cells phialidic, hyaline, smooth, ampulliform to dolliform. Conidia 5-7 $\times 1.5-2.5 \mu \mathrm{m}(\bar{x}=2 \times 5.5$ $\mu \mathrm{m}, \mathrm{n}=30$ ), ellipsoidal to cylindrical, smooth- and thin-walled, aseptate.

Culture characteristics - Colonies on PDA, 35-40 mm diameter after 7 days at $25^{\circ} \mathrm{C}$, margin regular, floccose, initially yellowish white and becoming smoke-grey, with immersed thin mycelia mat, reverse dark olivaceous with a yellow entire margin.

Known distribution (based on molecular data) - Netherlands, Poland, Uzbekistan (Farr \& Rossman 2020), Italy (this study)

Known hosts (based on molecular data) - Armoracia rusticana, Datisca cannabina, Mercurialis perennis, Thlaspi arvense (Farr \& Rossman 2020), Urtica sp. (this study).

Material examined - Italy, Province of Forlì-Cesena, Fiumana di Predappio, on dead aerial branch of Urtica spp. (Urticaceae), 15 December 2015, E. Camporesi, IT 3808 (JZBH380041, new host record, living culture, JZB380041.

GenBank numbers - ITS: MN648212, LSU: MN640407. 
Notes - In the present study, Phomatodes species was collected from dead and dying twigs and branches of Urtica dioica in Forlì- Cesena, Italy. The taxon clustered together with the Phomatodes nebulosa type (CBS 100191) with 100\% ML bootstrap and 1.0 BYPP support (Fig 38). Spores and colony characters are similar to the type species (Chen et al 2015). Therefore, we confirm the taxon collected in the present study as Phomatodes nebulosa. Currently, there are only two species associated with this genus, viz. P. aubrietiae and P. nebulosa. This is the first report of Phomatodes associated with Urtica sp. (Farr \& Rossman 2020).
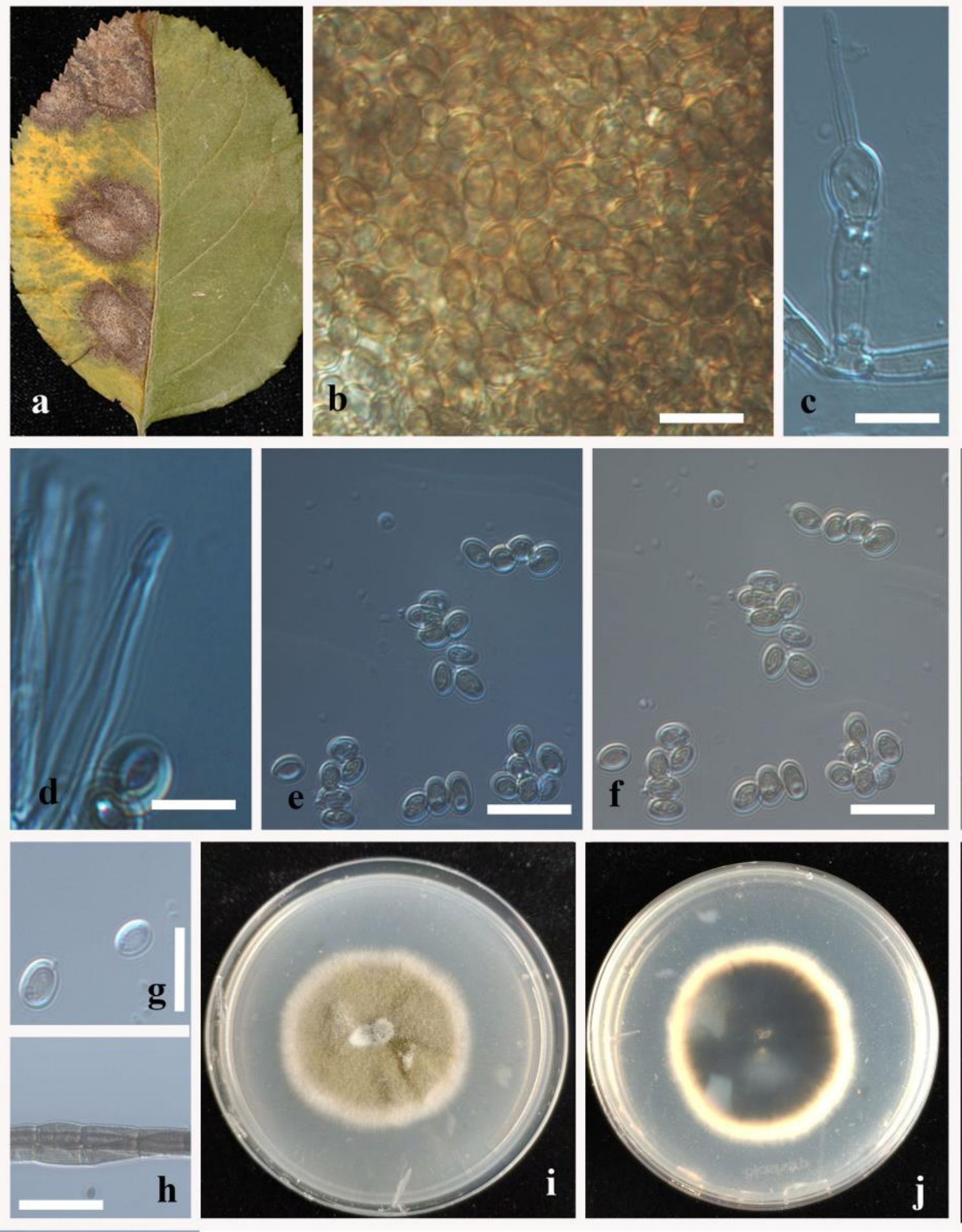

Fig. 41 - Nothophoma quercina )JZB380039, new host record(. a Material examined. b Pycnidia. $\mathrm{c}-\mathrm{d}$ Conidiogenus cell. e-g Conidia on PDA. h Mycelia. i Culture on PDA (upper view). j Culture on PDA (reverse view). Scale bars: $b-d=20 \mu \mathrm{m}, \mathrm{e}-\mathrm{h}=10 \mu \mathrm{m}$. 
Didymosphaeriaceae Munk = Montagnulaceae M.E. Barr, Mycotaxon 77: 194 (2001)

We follow the latest treatments and updated accounts of Didymosphaeriaceae presented in Ariyawansa et al. (2014a), Wanasinghe et al. (2018b) and Tibpromma et al. (2018b). This family comprises 26 accepted genera (Wijayawardene et al. 2018).
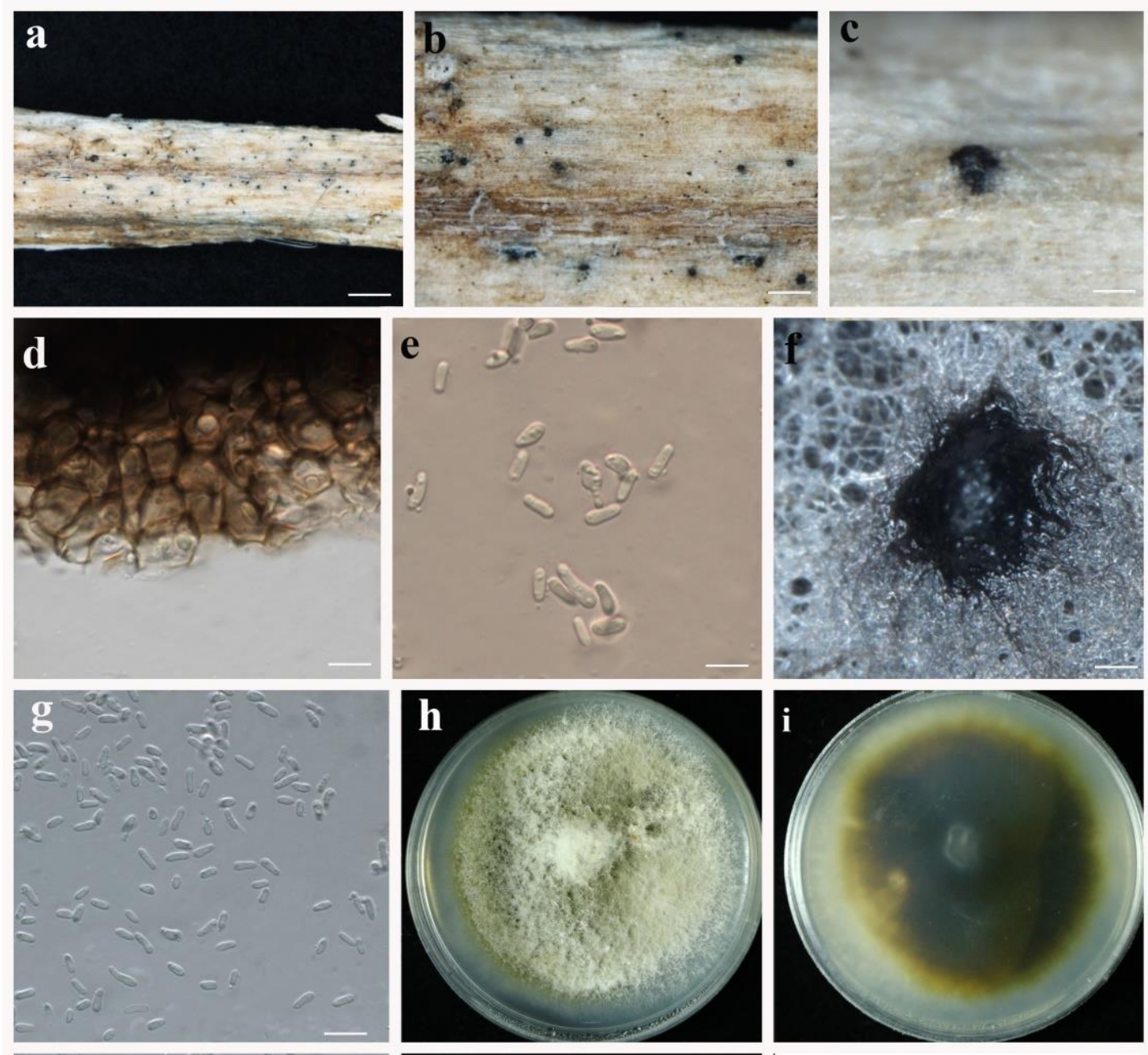

Fig. 42 - Phomatodes nebulosa (JZBH380041, new host record). a-c Appearance of conidiomata on host surface. d Pycnidial wall. e Conidia on host. f Conidioma on PDA. g Conidia on PDA. $\mathrm{h}$ Upper view of colony on PDA. $\mathrm{i}$ reverse view of colony on PDA. Scale bars: $\mathrm{a}=500 \mu \mathrm{m}, \mathrm{c}-\mathrm{f}=$ $20 \mu \mathrm{m}$.

Neokalmusia Ariyaw. \& K.D. Hyde

Neokalmusia currently comprises five species and is typified with $N$. brevispora. Previously $N$. brevispora was categorized under Phaeosphaeria as Phaeosphaeria brevispora by Hedjaroude (1968). Studies by Ariyawansa et al. (2014c) revealed that placement of Phaeosphaeria brevispora and Kalmusia scabrispora were not acceptable and hence Neokalmusia was erected to accommodate those two species.

Neokalmusia didymospora D.Q. Dai \& K.D. Hyde, Sydowia 68: 20 (2016)

Fig. 44

Facesoffungi number: FoF00061

Saprobic on dead culms of Microstegium sp. Sexual morph: Ascomata 420-460 $\mu \mathrm{m}$ high, 140-220 $\mu \mathrm{m}$ diameter, immersed and raising host tissue, becoming erumpent, solitary, scattered, globose, brown to dark brown, ostiolate, wide, brown to reddish brown, smooth. Peridium 20-35 $\mu \mathrm{m}$, wider at the apex and thinner at the base, composed of 4-6-layers of dark brown, cells of textura angularis, cells towards the inside lighter, at the outside, darker, fused with the host tissues. 
Hamathecium comprising numerous, $1.5-1.8 \mu \mathrm{m}$ wide, filamentous, branched, septate, pseudoparaphyses. Asci 135-140 × 8-9.5 $\mu \mathrm{m}, 8$-spored, bitunicate, fissitunicate, elongate-clavate to short cylindrical, long pedicellate, thick-walled at the apex, with a minute ocular chamber. Ascospores 12-13.5 $\times 5-5.5 \mu \mathrm{m}$, overlapping 1-2-seriate, hyaline to light brown when immature, becoming brown to blackish brown when mature, ellipsoidal, unequally 1-septate and strongly constricted at the septum, with the upper cell wider and pointed and lower cell longer and rounded, smooth-walled, thick-walled, without a mucilaginous sheath.

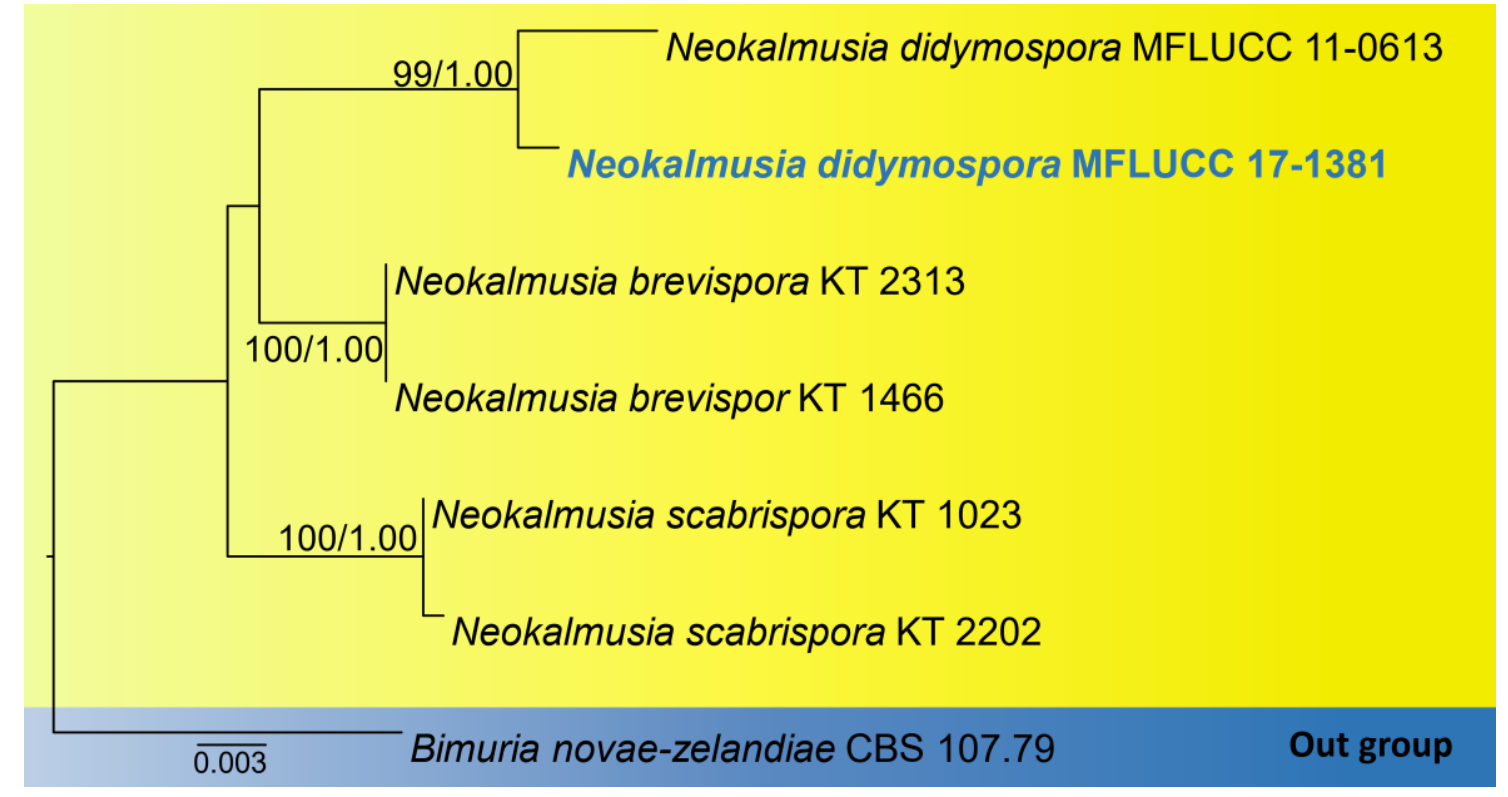

Fig. 43 - Phylogenetic tree generated from maximum likelihood (ML) based on LSU and SSU sequences. Bootstrap support (BS) values above 50\% and Bayesian posterior probabilities (BYPP) equal or greater than 0.90 are placed above the branches are shown at nodes. The tree is rooted with Bimuria novae-zelandiae (CBS 107.79). The best RaxML tree with a final likelihood value of 3717.445755 is presented. The matrix had 80 distinct alignment patterns, with $12.08 \%$ undetermined characters or gaps. Estimated base frequencies were as follows: $\mathrm{A}=0.255042, \mathrm{C}=$ $0.219029, \mathrm{G}=0.282988, \mathrm{~T}=0.242942$; substitution rates $\mathrm{AC}=2.742405, \mathrm{AG}=5.807474, \mathrm{AT}=$ 3.101080, $\mathrm{CG}=0.711167, \mathrm{CT}=15.431181, \mathrm{GT}=1.000000$; gamma distribution shape parameter $\alpha=97.241967$.

Known distribution (based on molecular data) -Thailand, Chiang Rai (Dai et al. 2015), China, Yunnan, Kunming (this study).

Known hosts (based on molecular data) - Bamboo (Dai et al. 2015), Microstegium sp. (Poaceae) (this study)

Material examined - China, Kunming, KIB premises, on decaying stems of Microstegium sp. (Poaceae), 20 October 2016, A. Karunarathna, AKKIB 48 (MFLU 17-0371, KUN 97364, new host and geographic record); living culture, MFLUCC 17-381; KUMCC 16-0233.

GenBank number - LSU: MN989184.

Notes - Our strain is phylogenetically related to $N$. didymospora (Fig. 43) and both are morphologically similar. Neokalmusia didymospora is a new host and geographic record.

\section{Paracamarosporium Wijayaw. \& K.D. Hyde}

Paracamarosporium was introduced by Wijayawardene et al. (2014) to accommodate Camarosporium psoraleae Crous \& M.J. Wingf. Both Camarosporium and Coniothyrium-like species are morphologically similar and group in this genus, hence taxa in this genus are difficult to be distinguished. Currently, there are seven species epithets under Paracamarosporium (Index Fungorum 2020). 
Paracamarosporium fagi Crous \& R.K. Schumach., Sydowia 67: 109 (2015)

Fig. 46

Facesoffungi number: FoF 06223

Saprobic on twigs of Ziziphus jujuba. Sexual morph: Undetermined. Asexual morph: Conidiomata 336-449 $\times 84-277 \mu \mathrm{m}(\bar{x}=381 \times 238.2 \mu \mathrm{m}, \mathrm{n}=10)$ solitary, immersed to erumpent, globose with central ostiole. Peridium comprising 2-3 layers of brown textura angularis. Conidiophores reduced to conidiogenous cells or with a supporting cell lining the inner cavity. Conidiogenous cells 1.8-3.1 $\times 4.7-6.9 \mu \mathrm{m}(\bar{x}=2.22 \times 5.8 \mu \mathrm{m}, \mathrm{n}=10)$, annellidic, hyaline, smooth, ampulliform, apex with prominent periclinal thickening, or with percurrent proliferation. Conidia 8-10 $\times 3-4 \mu \mathrm{m}(\bar{x}=10.1 \times 4.3 \mu \mathrm{m}, \mathrm{n}=10)$ solitary, subcylindrical with obtuse ends, initially hyaline, smooth, aseptate, becoming brown, medially 1-septate and surface becoming warty when mature.

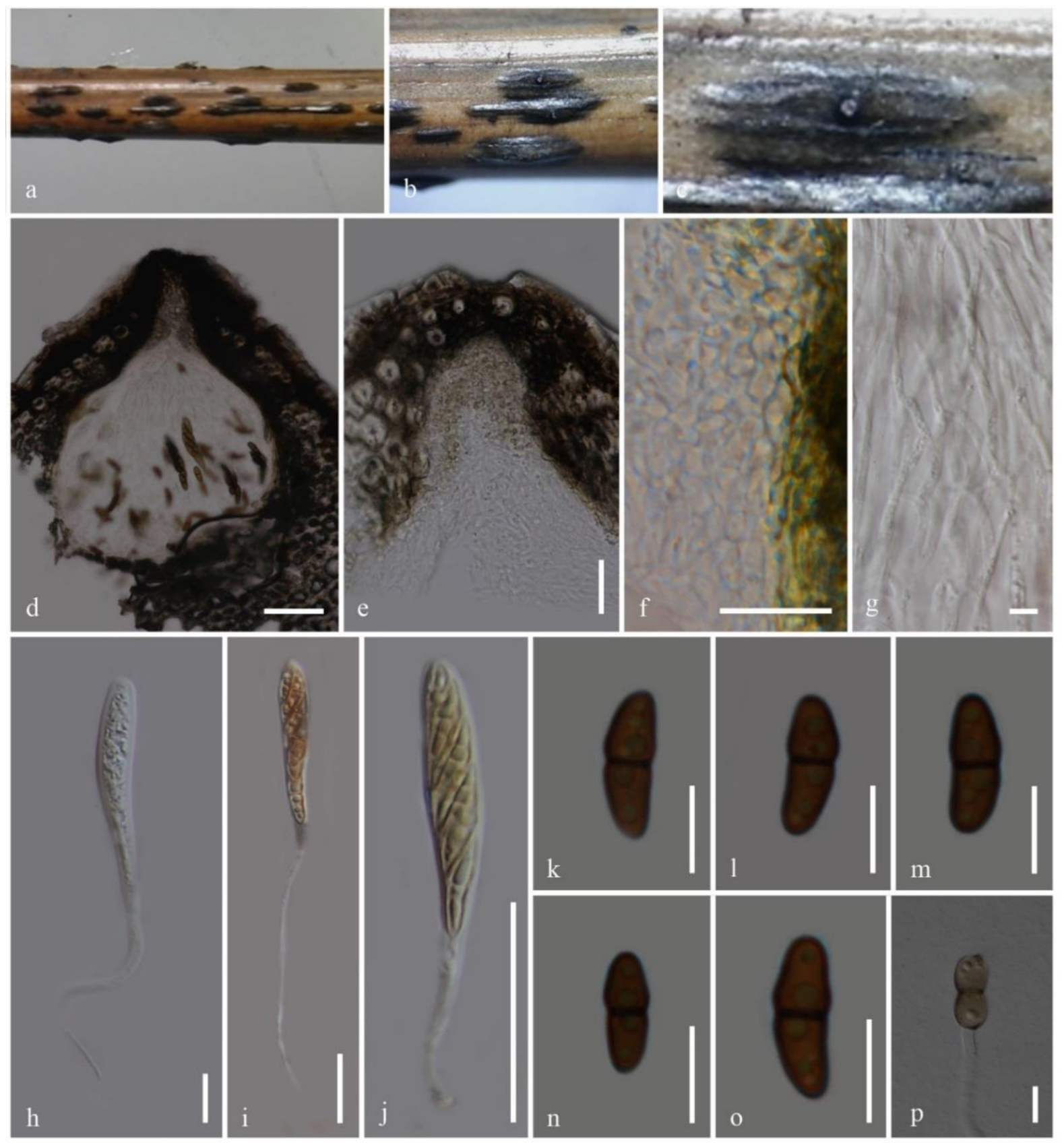

Fig. 44 - Neokalmusia didymospora (MFLU 17-0371, new host and geographic record). a-c Appearance of ascoma on host d Longitudinal section of ascoma e Longitudinal section of ostiole $\mathrm{f}$ Peridium g Pseudoparaphyses $\mathrm{h}-\mathrm{j}$ Asci $\mathrm{k}-\mathrm{o}$ Ascospores $\mathrm{p}$ Germinating ascospore. Scale bars: $\mathrm{d}=50 \mu \mathrm{m}, \mathrm{e}=50 \mu \mathrm{m}, \mathrm{f}=10 \mu \mathrm{m}, \mathrm{g}=5 \mu \mathrm{m}, \mathrm{h}-\mathrm{j}=20 \mu \mathrm{m}, \mathrm{k}-\mathrm{p}=10 \mu \mathrm{m}$. 
Culture characteristics - Colony on MEA slow growing, with sparse to moderate aerial mycelium and irregular edge, hairy, above umber colour and whitish in the middle, reverse rust to umber.

Known distribution (based on molecular data) - Germany, Republic of Latvia (Crous et al. 2015b), Ukraine (this study).

Known hosts (based on molecular data) - Betula pendula (Betulaceae), Elaeagnus rhamnoides (Elaeagnaceae), Fagus sylvatica (Fagaceae) (Crous et al. 2015b), Ziziphus jujuba (this study).

Material examined - Ukraine, Donetsk region, Donetsk City, Donetsk Botanical Garden, arboretum, on Ziziphus jujuba Mill. (Rhamnaceae), 18 May 2017, Timur Bulgakov (MFLU 172476, new host and geographic record), living cultures MFLUCC 18-0778.

GenBank numbers - LSU: MN244202, SSU: MN244179, ITS: MN244221.

Notes - Our isolate MFLUCC 18-0778 clustered with the ex-type strain of Paracamarosporium fagi (Fig. 45), formerly described by Crous et al. (2015b) from Fagus sylvatica (Fagaceae) in Germany. However, it differs from the type strain of Paracamarosporium fagi in having larger conidiomata (336-449 $\times 84-277 \mu \mathrm{m}$ vs. $200 \mu \mathrm{m}$ diameter). By considering the phylogenetic results, morphology and the host, we consider our collection as a new record on Ziziphus jujuba from Ukraine.

\section{Paraconiothyrium Verkley}

Paraconiothyrium was established by Verkley et al. (2004) to accommodate Parac. estuarinum (type species), Parac. brasiliense, Parac. cyclothyrioides and Parac. fungicola. However, subsequent molecular studies resulted in the transfer of a few species from this genus to other genera. Currently, Paraconiothyrium includes 24 accepted species as listed in Index Fungorum (2020).

Paraconiothyrium cyclothyrioides Verkley, da Silva, Wicklow \& Crous, Stud. Mycol. 50(2): 330 (2004)

Fig. 48

Facesoffungi number: FoF06572

Saprobic on decaying mangrove wood. Sexual morph: Ascomata 200-455 $\mu \mathrm{m}$ high, 150-300 $\mu \mathrm{m}$ diameter $(\bar{x}=345 \times 210 \mu \mathrm{m}, \mathrm{n}=10)$, lenticular-subglobose, immersed to semi-immersed, mostly covered by the epidermis and perforating the cortex with a small ostiole, thin-walled, black. Necks 170-230 $\mu \mathrm{m}$ long, 45-80 $\mu \mathrm{m}$ wide $(\bar{x}=285 \times 297 \mu \mathrm{m}, \mathrm{n}=10)$, cylindrical, dark-colored. Peridium 15-35 $\mu \mathrm{m}(\bar{x}=25 \mu \mathrm{m}, \mathrm{n}=10)$, composed of 3-5 layers of brown to dark brown cells of pseudoparenchymatous cells, arranged in a textura angularis type. Hamathecium comprising 1.5$2.5 \mu \mathrm{m}$ wide, filamentous, broad, cellular branched, pseudoparaphyses, anastomosing between and above the asci, embedded in a mucilaginous matrix. Asci 55-120 $\times 5-10 \mu \mathrm{m}(\bar{x}=78 \times 8.4 \mu \mathrm{m}, \mathrm{n}=$ 10), 8-spored, bitunicate, cylindrical-clavate, obtusely rounded at the apex, tapering to a short pedicel at the base, thick-walled. Ascospores 10-17 $\times 2.5-5 \mu \mathrm{m}(\bar{x}=12 \times 3.8 \mu \mathrm{m}, \mathrm{n}=10)$, biseriate, dirty olive-brown, cylindrical to ellipsoidal, obtusely rounded at both ends, 3-septate, constricted at the septa, second cell from the top largest. Asexual morph: Undetermined.

Culture characteristics - Ascospores germinating on 50\% sea water agar within 24 hours, with germ tubes developed from both ends of ascospores. Colonies on MEA fast growing, reaching 55-70 mm diameter after 15 days of incubation at $25^{\circ} \mathrm{C}$, surface olivaceous to pale olivaceous grey with a hyaline margin, reverse pale brown, floccose, irregular.

Known distribution (based on molecular data) - Papua New Guinea, Central Province, tropical countries (Verkley et al. 2004, Guégan et al. 2016), India (this study).

Known hosts - Soil sample, humans (Verkley et al. 2004, Gordon et al. 2012, Guégan et al. 2016), Suaeda monoica (this study).

Material examined - India, Tamil Nadu, Tiruvarur, Muthupet mangroves $\left(10.4^{\circ} \mathrm{N} 79.5^{\circ} \mathrm{E}\right)$, on decaying wood of Suaeda monoica (Amaranthaceae) 30 March 2016, B. Devadatha (AMH-10014, new host record), living culture, NFCCI-4387. 
GenBank numbers - ITS: MN242780, LSU: MN241143, SSU: MN241147.

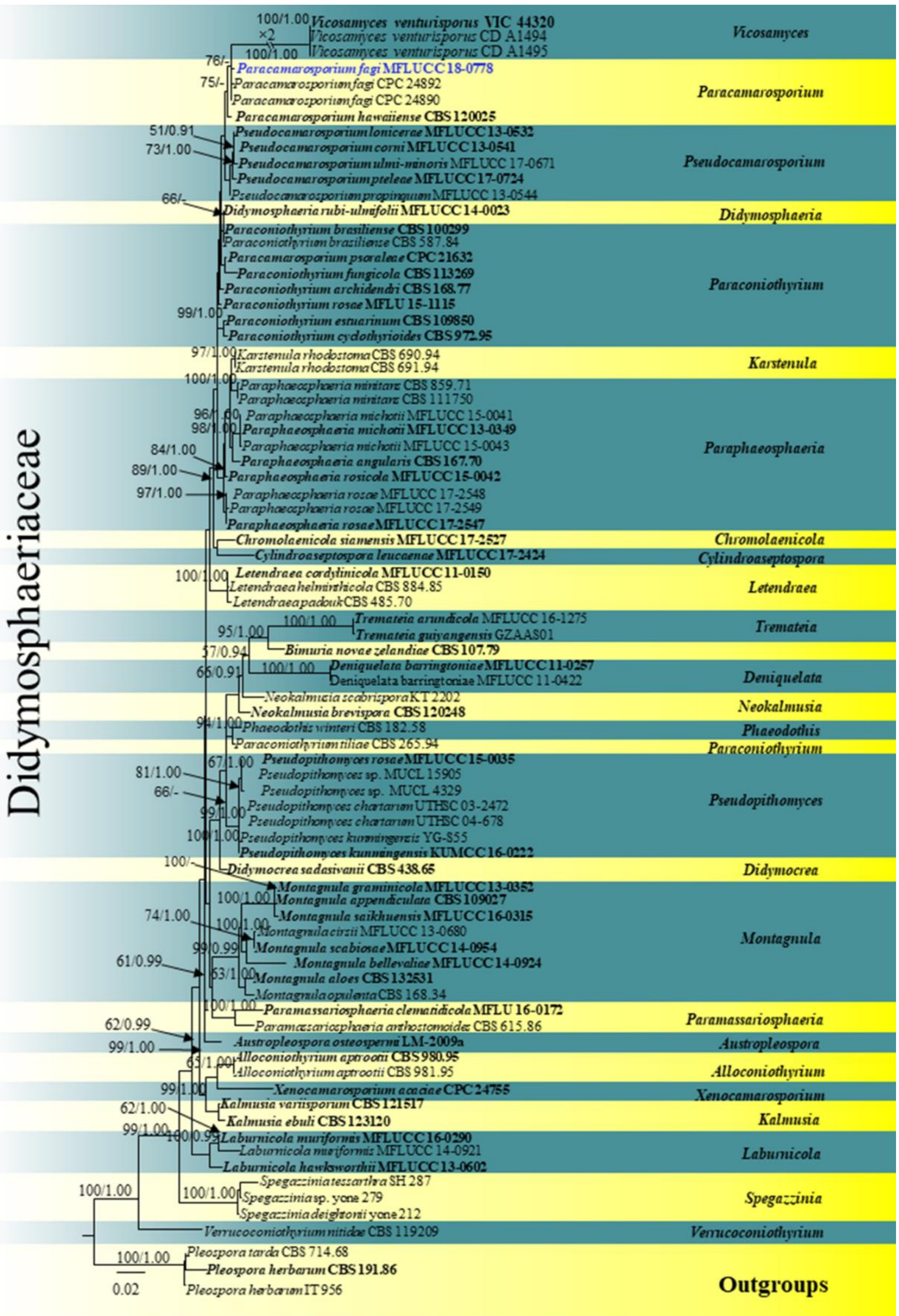

Fig. 45 - Phylogram generated from maximum likelihood analysis based on combined LSU, SSU and ITS sequence data. Eighty-two strains are included in the combined gene analyses comprising 
2306 characters after alignment (857 characters for LSU, 980 characters for SSU and 467 characters for ITS). Pleospora tarda (CBS 714.67), Pleospora herbarum (CBS 191.856) and Pleospora herbarum (IT 956) are used as the outgroup taxa. The tree topology derived from the Bayesian analysis was similar to that derived from the maximum likelihood analysis. The best RaxML tree with a final likelihood value of -11598.386568 is presented. The matrix had 816 distinct alignment patterns, with $23.53 \%$ undetermined characters or gaps. Estimated base frequencies were as follows: $\mathrm{A}=0.247509, \mathrm{C}=0.225025, \mathrm{G}=0.279478, \mathrm{~T}=0.247988$; substitution rates $\mathrm{AC}=1.541963, \mathrm{AG}=2.727864, \mathrm{AT}=1.472638, \mathrm{CG}=1.108201, \mathrm{CT}=$ $6.541487, \mathrm{GT}=1.000000$; gamma distribution shape parameter $\alpha=0.568674$. Bootstrap values for maximum likelihood equal to or greater than 50 and Bayesian posterior probabilities equal or greater than 0.90 are placed above or below the branches. Ex-type strains are in bold and black. The newly generated sequences are indicated in blue.

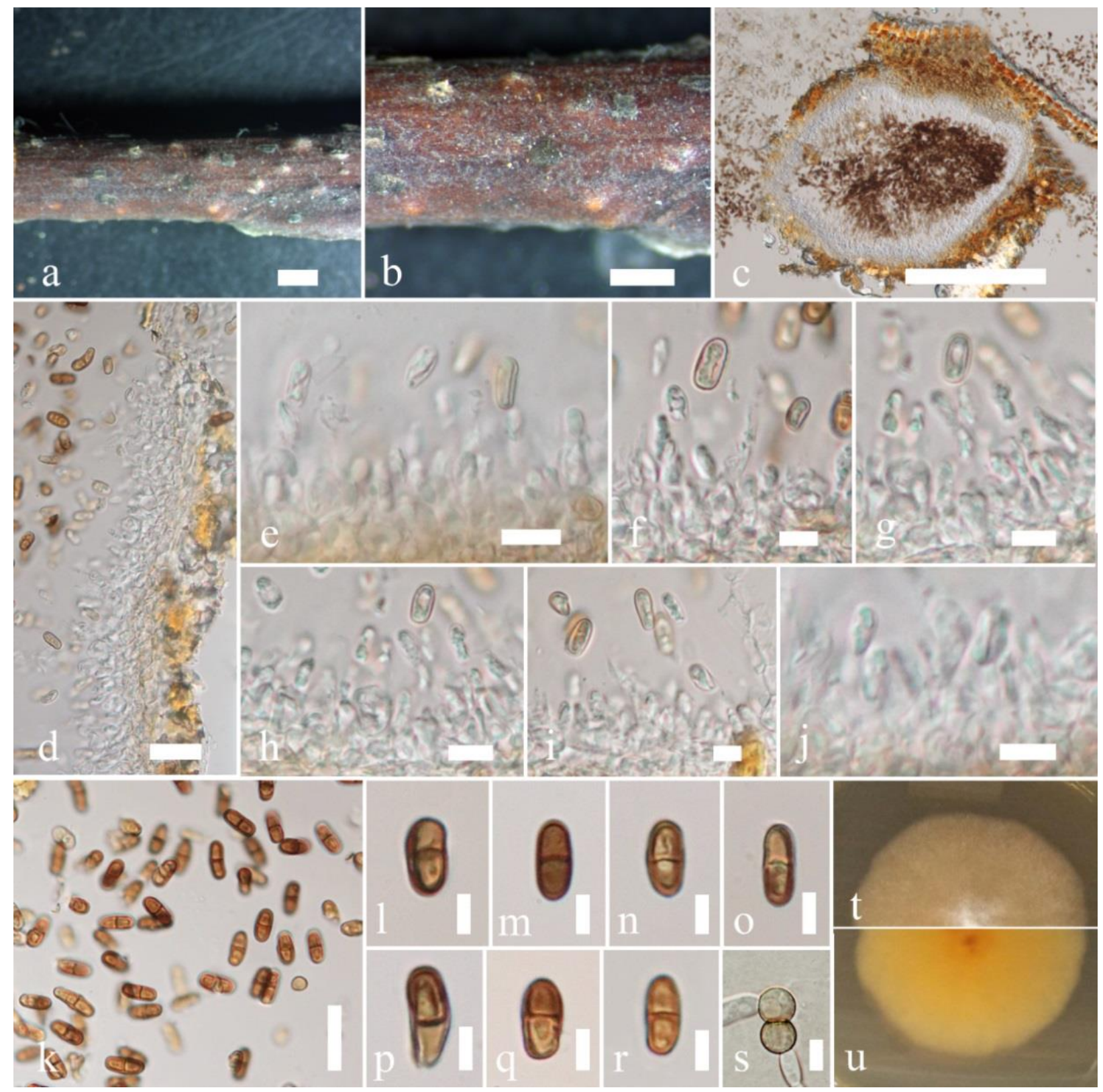

Fig. 46 - Paracamarosporium fagi (MFLU 17-2476, new host and geographic record). $\mathrm{a}, \mathrm{b}$ Appearance of conidiomata on host surface. c Vertical section through the conidioma. $\mathrm{d}$ Peridium $\mathrm{e}-\mathrm{j}$ Conidiogenesis. $\mathrm{k}-\mathrm{r}$ Conidia s Germinating conidium $\mathrm{t}, \mathrm{u}$ Culture characteristics on MEA ( $\mathrm{t}$ : above view; $\mathrm{u}$ : reverse view) Scale bars: $\mathrm{a}=500 \mu \mathrm{m}, \mathrm{b}=1000 \mu \mathrm{m}, \mathrm{c}=50 \mu \mathrm{m}, \mathrm{d}=25 \mu \mathrm{m}$, $\mathrm{e}=10 \mu \mathrm{m}, \mathrm{f}, \mathrm{g}=5 \mu \mathrm{m}, \mathrm{h}=10 \mu \mathrm{m}, \mathrm{j}=5 \mu \mathrm{m}, \mathrm{k}=20 \mu \mathrm{m}, \mathrm{l}=5 \mu \mathrm{m}, \mathrm{m}-\mathrm{r}=5 \mu \mathrm{m}, \mathrm{s}=10 \mu \mathrm{m}$. 


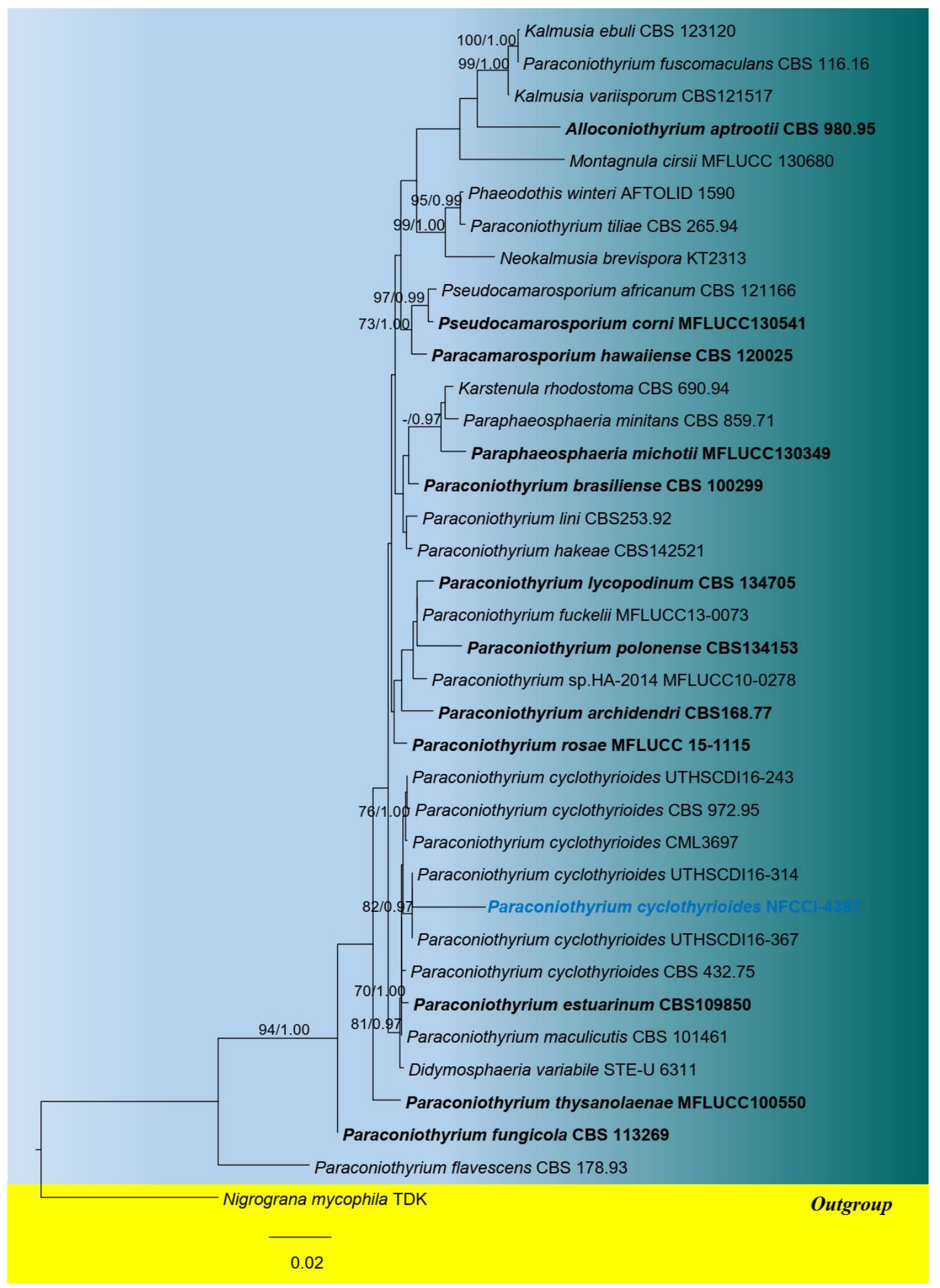

Fig. 47 - Phylogram generated from maximum likelihood analysis based on combined LSU, SSU and ITS sequence data. Thirty-seven strains are included in the combined gene analyses comprising 2457 characters after alignment (872 characters for LSU, 1035 characters for SSU, 550 characters for ITS). Nigrograna mycophila TDK is used as the outgroup taxon. The tree topology derived from the Bayesian analysis was similar to that derived from the maximum likelihood analysis. The best RaxML tree with a final likelihood value of -7749.585520 is presented. The matrix had 937 distinct alignment patterns, with $18.57 \%$ undetermined characters or gaps. Estimated base frequencies were as follows: $\mathrm{A}=0.246023, \mathrm{C}=0.226607, \mathrm{G}=0.278252, \mathrm{~T}=0.249118$; substitution rates $\mathrm{AC}=1.565451, \mathrm{AG}=1.781597, \mathrm{AT}=1.275177, \mathrm{CG}=0.882883, \mathrm{CT}=5.629469$, 
$\mathrm{GT}=1.000000$; gamma distribution shape parameter $\alpha=0.647852$. Bootstrap values for maximum likelihood equal to or greater than 75 and Bayesian posterior probabilities equal or greater than 0.95 are placed above or below the branches. Ex-type strains are in bold and black. The newly generated sequences are indicated in blue.

Notes - BLAST search analyses of LSU and ITS sequence data revealed that our taxon is $100 \%$ and $99.79 \%$ similar to Paraconiothyrium cyclothyrioides. Further phylogenetic analyses based on combined LSU, SSU and ITS showed that our taxon is nested within a monophyletic clade with other Paraconiothyrium cyclothyrioides strains with significant support from ML 82\%, MP 76\%, and 0.97 BYPP (Fig. 47). However, our collection of Paraconiothyrium cyclothyrioides (NFCCI-4387) did not sporulate even after 2 months' incubation and hence could not be compared with the asexual morph of Parac. cyclothyrioides (CBS 972.95T) (Verkley et al. 2004). Base pair differences of Paraconiothyrium cyclothyrioides (NFCCI-4387) and Parac. cyclothyrioides (UTHSC: DI16-367) are 1 out of 563bp (0.1\%) of ITS gene region which indicates both are similar species. Recent reports support that Paraconiothyrium cyclothyrioides is an emerging opportunistic human pathogen in immunocompromised patients known to cause cutaneous phaeohyphomycosis in renal transplant cases mainly from tropical regions (Gordon et al. 2012, Colombier et al. 2015, Guégan et al. 2016, Valenzuela-Lopez et al. 2017, Garcia-Hermoso et al. 2019). Our sexual morph of Paraconiothyrium cyclothyrioides resembles Parac. thysanolaenae in having cylindrical asci and ascospores that are phragmosporous, ellipsoidal, 3-septate, lack mucilaginous sheaths and constricted at the septa. However, the former is distinct from the latter in having lenticularsubglobose, uniloculate ascomata while the latter produces pseudostromata with uni- to multilocules (Liu et al. 2015). Paraconiothyrium fuckeli, Parac. magnoliae and Parac. cyclothyrioides (NFCCI-4387) share similar ascomata and ascal characters with Parac. cyclothyrioides (NFCCI4387) (Ariyawansa et al. 2014c, Liu et al. 2015). However, Paraconiothyrium fuckeli differs from Parac. cyclothyrioides (NFCCI-4387) in having narrowly ovoid to clavate ascospores (Ariyawansa et al. 2014c). Paraconiothyrium magnoliae is clearly distinguished from Parac. cyclothyrioides (NFCCI-4387) in having ascospores that are yellowish-brown, with bipolar appendages and a gelatinous sheath (Ariyawansa et al. 2014c).

Paraconiothyrium cyclothyrioides (AMH-10014) shares closer morphological characters with Leptosphaeria peruviana in having immersed ascomata, cylindrical-clavate asci and cylindrical to ellipsoidal, 3-septate, dirty olive-brown ascospores (Pang et al. 2011). However, our taxon is distinct from Leptosphaeria peruviana in having significantly larger subglobose ascomata and asci and narrower ascospores. Leptosphaeria peruviana is a marine species which was described from Salicornia ambigua $(=S$. peruviana) by Spegazzini (1881), but has also been reported from mangrove wood (Pang et al. 2011). Leptosphaeria peruviana was poorly described and subsequently Kohlmeyer \& Kohlmeyer (1979) examined the scarce type material and its placement under Leptosphaeria (Spegazzini 1881) remains extremely doubtful (Kohlmeyer \& Kohlmeyer 1979). This species is very rare and has not been isolated or sequenced.

This is the first report of this taxon as a saprobe and a first report of it being reported from a decaying plant substrate, as otherwise it is widely known as an opportunistic human pathogen. This is also the first report on a sexual morph of this species. This is the first time that this taxon is reported from marine/mangrove environment and its collection from India extends its geographical range.

\section{Leptosphaeriaceae}

Leptosphaeriaceae species are saprobes, hemibiotrophs or parasites on stems and leaves of herbaceous or woody plants in terrestrial habitats, as well as possibly in aquatic habitats (Hyde et al. 2013, Ariyawansa et al. 2015, Liu et al. 2015, Tennakoon et al. 2017). Barr (1987) introduced Leptosphaeriaceae, and designated Leptosphaeria Ces. \& De Not. as the type of the family (Hyde et al. 2013, Wanasinghe et al. 2016). Leptosphaeriaceae species are characterized by immersed or erumpent, perithecial ascomata with single papillate ostioles, fissitunicate, cylindrical asci and 
hyaline to brown, transversely septate ascospores (Hyde et al. 2013, Ariyawansa et al. 2015). The asexual morph of taxa in Leptosphaeriaceae can be either coelomycetes or hyphomycetes (Alves et al. 2013, de Gruyter et al. 2013, Hyde et al. 2013, Wanasinghe et al. 2016, Tennakoon et al. 2017). There are 12 genera within Leptosphaeriaceae (Wijayawardene et al. 2020).

\section{Plenodomus Nees}

Plenodomus Preuss was introduced by Preuss (1851) with P. rabenhorstii Preuss, as the type species (de Gruyter et al. 2013, Ariyawansa et al. 2015). The type species of Plenodomus was replaced by P. lingam (Tode) Höhn. (sexual morph: Leptosphaeria maculans (Desm.) Ces. \& De Not.) by Boerema \& Kesteren (1964) due to the type material of P. rabenhorstii being lost during World War II (Torres et al. 2005, Ariyawansa et al. 2015). The connection between L. maculans (sexual morph) and $P$. lingam (asexual morph) has been confirmed by single spore isolation (Boerema \& Kesteren 1964). Boerema et al. (1997) classified Phoma species based on their morphological characters in nine sections including Plenodomus and each of the sections were systematically illustrated in Boerema et al. (2004). Recently, de Gruyter et al. (2013) reclassified the Phoma section of Plenodomus with supporting molecular phylogeny. We describe the sexual morph of Plenodomus collinsoniae for the first time.

Plenodomus collinsoniae (Dearn. \& House) Gruyter, Aveskamp \& Verkley, Stud. Mycol. 75: 21 (2012)

Facesoffungi number: FoF07379

Fig. 50

Saprobic on dead branch of an unidentified host. Sexual morph: Ascomata 245-260 $\mu \mathrm{m}$ high, 360-390 $\mu \mathrm{m}$ diameter $(\bar{x}=255 \times 380 \mu \mathrm{m}, \mathrm{n}=20)$, solitary, scattered, appearing as small, raised black dots on the host surface, superficial or semi immersed, globose to sub globose, smooth, with a flattened top and base and, brownish black, ostiolate, papillate. Papilla central circular. Peridium 45-55 $\mu \mathrm{m}(\bar{x}=52 \mu \mathrm{m}, \mathrm{n}=10)$ at sides, $20-30 \mu \mathrm{m}(\bar{x}=28 \mu \mathrm{m}, \mathrm{n}=10)$ at the base, slightly thin at the base, composed of three layers of scleroplectenchymatous cells, inner layer comprising 2-3 cell layers of flattened, light brown cells, arranged in a textura angularis, middle layer comprising several layers of subhyaline cells arranged in a textura globulosa, outer layer composed of heavily pigmented, thick-walled, dark brown cells, textura angularis. Hamathecium comprising 1.5-2.5 $\mu \mathrm{m}$ $(\bar{x}=2.2 \mu \mathrm{m}, \mathrm{n}=10)$ wide, septate, cellular pseudoparaphyses, branching between the asci, embedded in a gelatinous matrix. Asci $105-210 \times 15-25 \mu \mathrm{m}(\bar{x}=170 \times 23 \mu \mathrm{m}, \mathrm{n}=20), 8$-spored, bitunicate, fissitunicate, cylindrical, short pedicel, apically rounded, with a distinct ocular chamber. Ascospores $40-70 \times 6-10 \mu \mathrm{m}(\bar{x}=65 \times 9 \mu \mathrm{m}, \mathrm{n}=20)$, overlapping uni- or bi-seriate, olivaceous to yellowish, cylindrical to subfusoid, with obtuse ends, 6-7 euseptate, not constricted at each septum, cell above central septum slightly wider, guttulate, thick and smooth-walled, with mucilaginous globoid-shaped apical and basal appendages. Asexual morph: Undetermined.

Known distribution (based on molecular data) - China, Japan (De Gruyter et al. 2013, MarinFelix et al. 2017b), China (this study).

Known hosts - Collinsonia canadensis, Vitis coignetiae, Unidentified dead wood (De Gruyter et al. 2013, Marin-Felix et al. 2017b).

Material examined - China, Yunnan Province. Kunming, dead branch of an unidentified host, 15 December 2018, V. Thyagaraja, DXH 015 (MFLU 19-2279, new sexual morph record).

GenBank accessions - LSU MN982862, SSU MN982863, ITS MN982858.

Notes - Plenodomus collinsoniae is only known from its phoma-like asexual morph (De Gruyter et al. 2013). In our phylogram, new strain (MFLU 19-2279) grouped together with $P$. collinsoniae (CBS 120227) with high statistical support (99 \% ML, 1.00 PP) (Fig. 49). Furthermore, DNA sequences of SSU, LSU and ITS of our strain (MFLU 19-2279) are almost similar to P. collinsoniae (CBS 120227). Therefore, it is confirmed that our new strain is the asexual morph of $P$. collinsoniae. Plenodomus collinsoniae showed close morphological and phylogenetic similarities to the type of the recently introduced $P$. sinensis. However, they are morphologically different by their asci (105-210 × 15-25 vs. 80-100 × 10-12 $\mu \mathrm{m})$ and ascospore 
sizes $(40-70 \times 6-10$ vs. $27-40 \times 3.8-4.4 \mu \mathrm{m})$. Additionally, 9 base pairs of the ITS sequence of Plenodomus collinsoniae and $P$. sinensis are different out of $536 \mathrm{bp}$ without gaps $(1.7 \%)$.

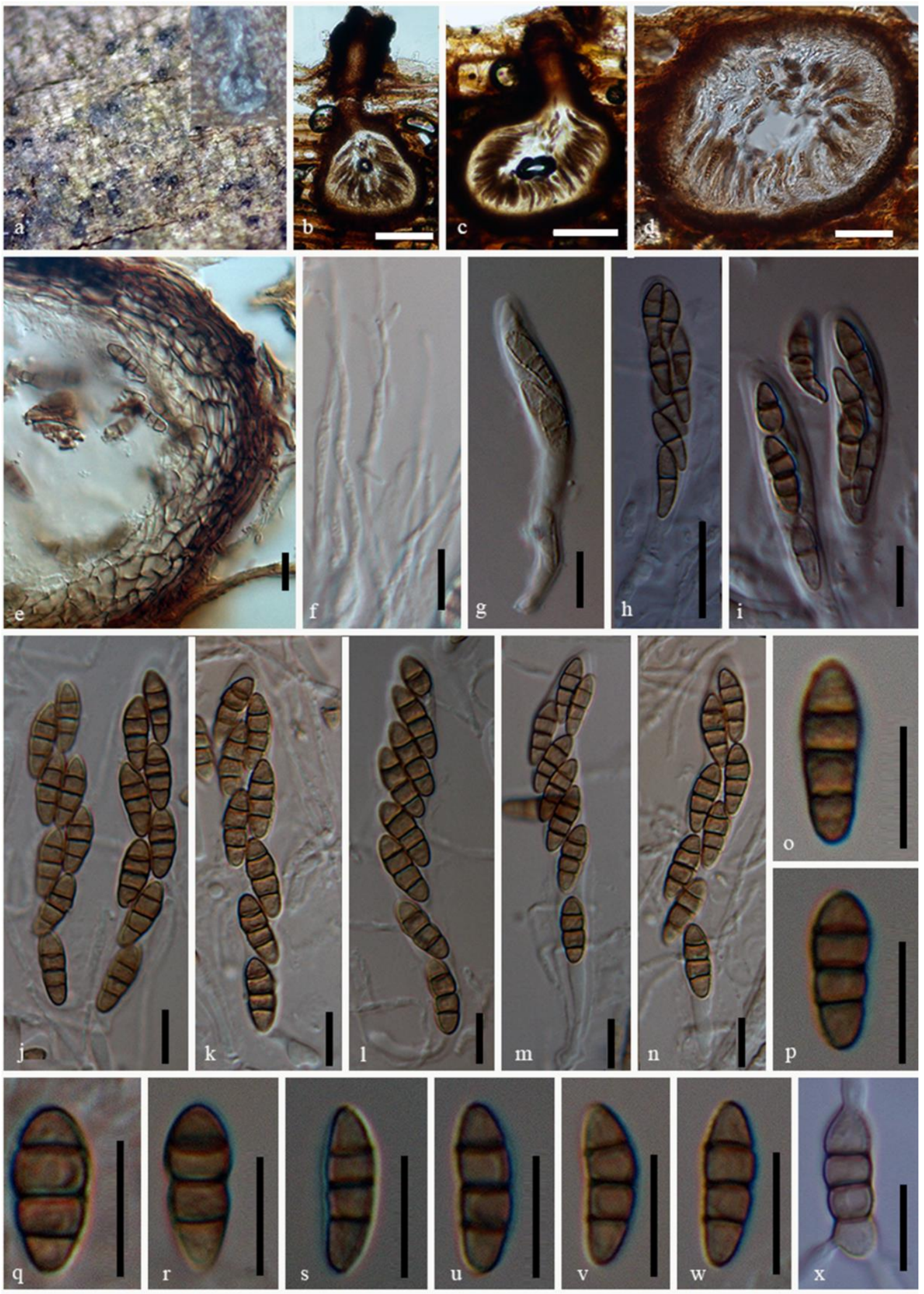

Fig. 48 - Paraconiothyrium cyclothyrioides (AMH-10014, new host record). a Ascomata semiimmersed on decaying wood. b-d Longitudinal sections of ascomata d. Peridial wall layers e Filamentous pseudoparaphyses. g-n Immature and mature asci. 1-n Immature and mature ascospores. $\mathrm{o}, \mathrm{q}-\mathrm{v}$ Ascospores. $\mathrm{x}$ Germinating ascospore. Scale bars: $\mathrm{b}-\mathrm{c}=100 \mu \mathrm{m}, \mathrm{f}, \mathrm{b}, \mathrm{d}, \mathrm{f}-\mathrm{h}=$ $50 \mu \mathrm{m}, \mathrm{c}, \mathrm{e}, \mathrm{i}-\mathrm{O}=10 \mu \mathrm{m}$. 


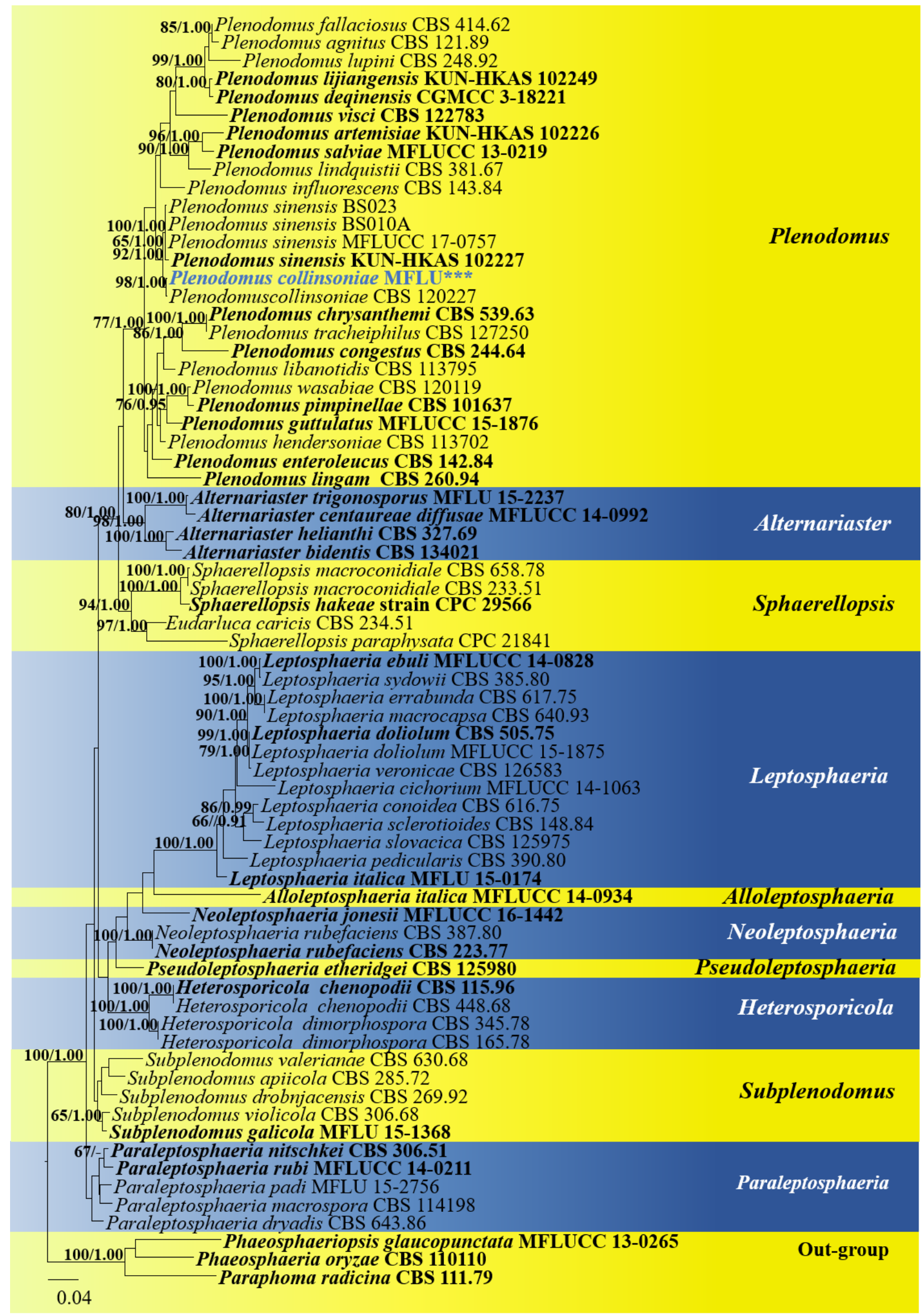

Fig. 49 - Maximum likelihood analysis with 1000 bootstrap replicates yielded a best tree with the likelihood value of -8974.532790 . The combined LSU, SSU and ITS sequence dataset comprised 79 strains with Phaeosphaeria oryzae (CBS 110110) and Phaeosphaeriopsis glaucopunctata (MFLUCC 13-0265) and Paraphoma radicina (CBS 111.79) as the outgroup taxa. The tree topology derived from the Bayesian analysis was similar to that derived from the maximum 
likelihood analysis. The matrix had 403 distinct alignment patterns, with $10.72 \%$ of undetermined characters or gaps. Estimated base frequencies were as follows; $\mathrm{A}=0.232805, \mathrm{C}=0.250707, \mathrm{G}=$ $0.235491, \mathrm{~T}=0.280998$; substitution rates $\mathrm{AC}=1.078239, \mathrm{AG}=3.114105, \mathrm{AT}=1.506841, \mathrm{CG}=$ $0.917572, \mathrm{CT}=3.843879, \mathrm{GT}=1.000000$; gamma distribution shape parameter $\alpha=0.753063$. Maximum likelihood bootstrap (ML) values > 65\% and Bayesian posterior probabilities (PP) > $0.80 \%$ are given above the nodes. The scale bar indicates 0.04 changes. The ex-type strains are in bold and the new isolate in blue bold.

\section{Lophiostomataceae Sacc.}

This family currently comprises 18 genera (Wijayawardene et al. 2018). The latest treatments of Lophiostomataceae is by Hyde et al. (2019) and a new record Vaginatispora nypae is reported here.

\section{Vaginatispora K.D. Hyde}

The genus comprises ten species and its members are commonly found on dead wood in aquatic and terrestrial habitats (Hyde et al. 2019, Jayasiri et al. 2019). This genus is characterized by immersed, globose to subglobose, dark ascomata, cylindric-clavate, bitunicate asci and fusiform, septate, guttulate, hyaline ascospores, with a distinctively and thickened mucilaginous sheath. An updated phylogenetic tree is provided here.

Vaginatispora nypae Jayasiri, E.B.G. Jones \& K.D. Hyde, Mycosphere 10(1): 84 (2019)

Fig. 52

Facesoffungi number: FoF05264

Saprobic on dried twigs of unidentified host. Sexual morph: Perithecia 305-332 $\mu \mathrm{m}$ high $\times$ 269-359 $\mu \mathrm{m}$ diameter $(\bar{x}=317 \times 332 \mu \mathrm{m}, \mathrm{n}=3)$, immersed, semi-erumpent, solitary, scattered globose to subglobose, dark brown. Peridium 42-96 $\mu \mathrm{m}$ wide, 5-8 layers of dark brown cells of textura angularis, almost black at outside. Hamathecium comprising $3 \mu \mathrm{m}$ wide, anastomosing, septate, branched, hyaline, pseudoparaphyses. Asci $62-102 \times 9-14 \mu \mathrm{m}(\bar{x}=87 \times 11.5 \mu \mathrm{m}, \mathrm{n}=20), 8$ spored, bitunicate, fissitunicate, cylindric-clavate, sessile or with short pedicel, apically rounded, with minutely an ocular chamber. Ascospores 23-33 $\times 4-7 \mu \mathrm{m}(\bar{x}=28 \times 6 \mu \mathrm{m}, \mathrm{n}=20)$, overlapping 1-2-seriate, hyaline, fusiform with narrow, acute ends, 1-septate, constricted at the septum, with large guttules, with a distinctively and thickened mucilaginous sheath, smooth-walled. Asexual morph: Undetermined.

Culture characteristics - Ascospores germinating on MEA within $24 \mathrm{~h}$ at room temperature and germ tubes produced from the ends of the ascospore. Colonies on MEA reaching $2.4 \mathrm{~cm}$ diameter after 4 weeks at $25^{\circ} \mathrm{C}$. Initially aerial mycelium white, slightly raised, in old cultures dark green to black, flattened on surface, crenate, green to dark green from below, light green margin.

Known distribution (based on molecular data) - Thailand (Jayasiri et al. 2019, this study).

Known hosts (based on molecular data) - On fallen fruit pericarp of Nypa fruticans (Jayasiri et al. 2019), on submerged decaying twigs (this study).

Material examined - Thailand, Phitsanulok, Noen Maprang District, Chomphu, Wat Pa Nong Thap Ruea, submerged decaying twigs, 30 December 2018, S. Boonmee PSL16-1 (MFLU 192440, new geographical record); living culture MFLUCC 19-0484.

GenBank numbers - ITS: MN608549, LSU: MN577418, SSU: MN577428, tef1: MN612112.

Notes - Our new isolate Vaginatispora nypae (MFLUCC 19-0484) is phylogenetically related to Vaginatispora nypae (MFLUCC 18-1543) with good statistical support (100\% ML/1.00 BYPP, Fig. 51). This new collection shares similarities in ascomata, asci and ascospores with Vaginatispora nypae (MFLUCC 18-1543, see Fig. 68 in Jayasiri et al. 2019). However, these two strains are from different substrates and geographical locations. Vaginatispora nypae (MFLUCC 18-1543) is described from fallen fruit pericarp of Nypa fruticans (Arecaceae) from Krabi Province, while our strain is described from dried twigs from Phitsanulok Province. Therefore, 
based on morphology and phylogenetic affinity, we identify our strain MFLUCC 19-0484 as Vaginatispora nypae and it is reported here as a new geographical record.
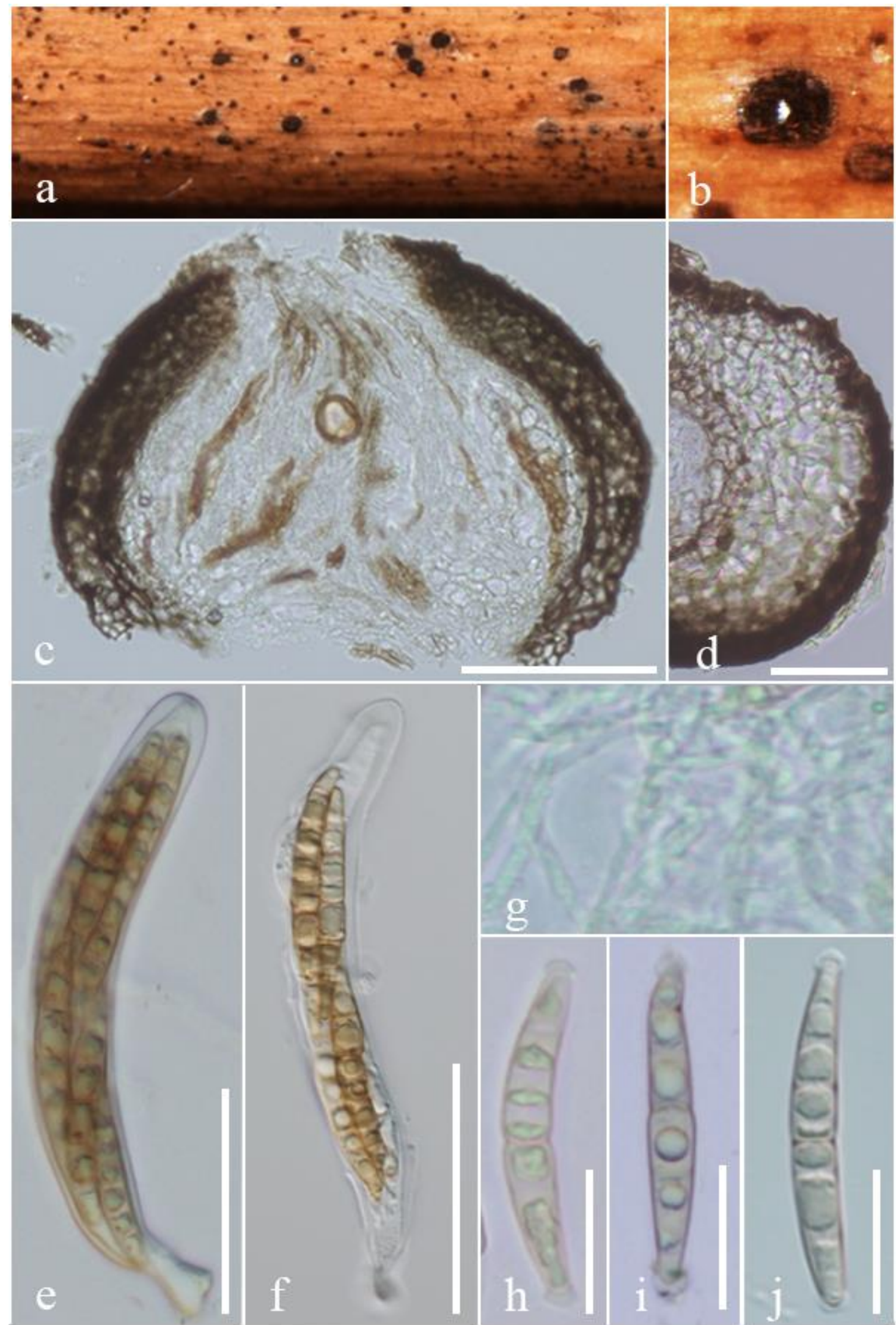

Fig. 50 - Plenodomus collinsoniae (MFLU 19-2279, new sexual morph record). a-b Ascomata on host. c Vertical section of ascoma. d Section of peridium. e-f Asci. g Pseudoparaphyses. $\mathrm{h}-\mathrm{j}$ Ascospores. Scale bars: $\mathrm{b}-\mathrm{c}=50 \mu \mathrm{m}, \mathrm{d}-\mathrm{j}=20 \mu \mathrm{m}, \mathrm{k}-\mathrm{o}=5 \mu \mathrm{m}$. 


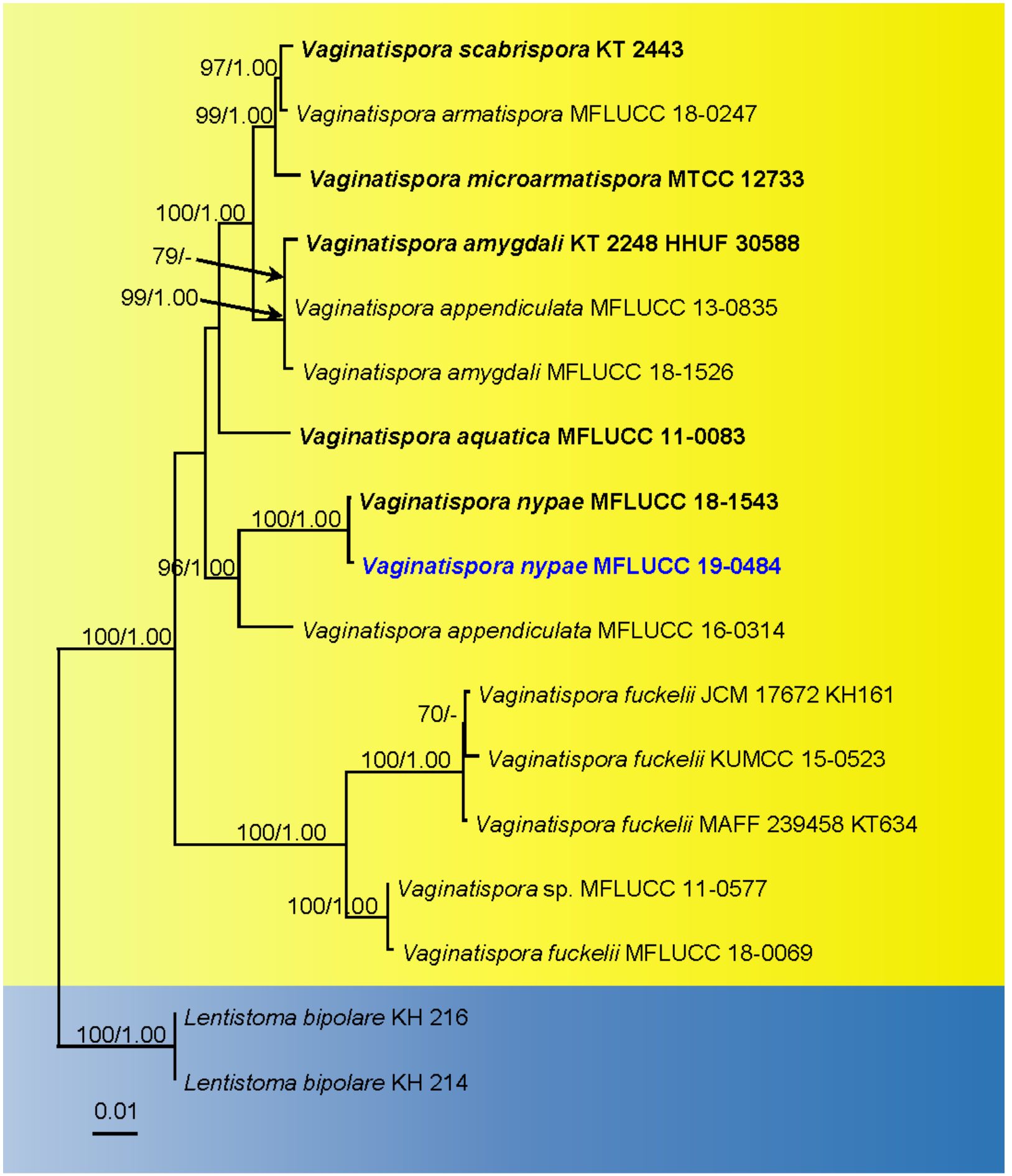

Fig. 51 - Phylogram generated from maximum likelihood analysis based on combined LSU, SSU, ITS and tefl sequence data. Twenty-one taxa are included in the combined gene analyses comprising 3311 characters after alignment (848 characters for LSU, 984 characters for SSU, 585 characters for ITS and 893 characters for tef1). Lentistoma bipolare (strains KH 216 and KH 214) are used as the outgroup taxa. The best RaxML tree with a final likelihood value of -8141.591393 is presented. The matrix had 450 distinct alignment patterns, with $13.29 \%$ undetermined characters or gaps. Estimated base frequencies were as follows: $\mathrm{A}=0.247229, \mathrm{C}=0.244566, \mathrm{G}=0.270729, \mathrm{~T}$ $=0.237477$; substitution rates $\mathrm{AC}=1.560615, \mathrm{AG}=2.113756, \mathrm{AT}=0.863778, \mathrm{CG}=1.118506$, $\mathrm{CT}=6.049102, \mathrm{GT}=1.000000$; gamma distribution shape parameter $\alpha=0.020000$. Bootstrap values for maximum likelihood equal to or greater than 50 and Bayesian posterior probabilities equal or greater than 0.95 are placed above or below the branches. Ex-type strains are in bold and black. The newly generated sequence is indicated in blue. 

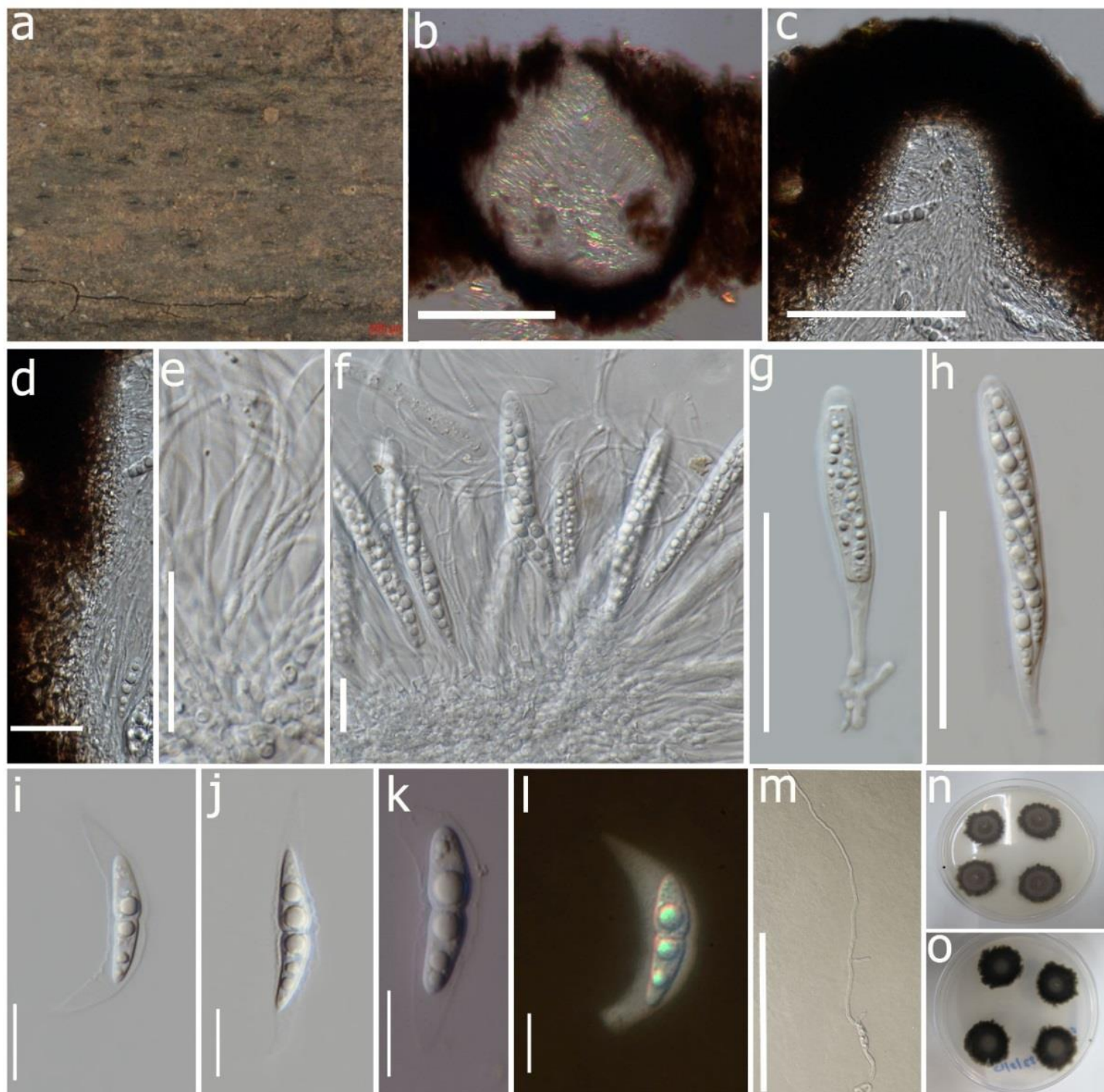

Fig. 52 - Vaginatispora nypae (MFLU 19-2440, new geographical record). a Appearance of ascomata on woody substrate. b Section of ascoma. c Closed up apical ostiole. d Peridium. e Pseudoparaphyses. f-h Asci. i-1 Ascospores (Fig. k stained in nigrosin reagent and Fig. 1 stained in India ink reagent). $m$ Germinated spore. $\mathrm{n}-\mathrm{o}$ Colonies on PDA from surface and reverse at 30 days. Scale bars: $\mathrm{b}=50 \mu \mathrm{m}, \mathrm{c}=25 \mu \mathrm{m}, \mathrm{d}=25 \mu \mathrm{m}, \mathrm{e}=50 \mu \mathrm{m}, \mathrm{f}=20 \mu \mathrm{m}, \mathrm{g}-\mathrm{h}=50 \mu \mathrm{m}, \mathrm{i}-\mathrm{l}=20$ $\mu \mathrm{m}, \mathrm{m}=50 \mu \mathrm{m}$.

Lophiotremataceae K. Hiray. \& Kaz. Tanaka

Lophiotremataceae was introduced by Hirayama \& Tanaka (2011), with Lophiotrema as the type genus. The taxonomic placement of the family was re-evaluated by Hashimoto et al. (2017). Until now, there are six genera accommodated in the family viz. Atrocalyx, Crassimassarina, Cryptoclypeus, Galeaticarpa, Lophiotrema and Pseudocryptoclypeus (Wijayawardene et al. 2018, Jayasiri et al. 2019).

Atrocalyx A. Hashim. \& Kaz. Tanaka

Atrocalyx was introduced by Hashimoto et al. (2017), with A. acutisporus as the type species. Until now, there are seven species in this genus reported from Belgium, China, Japan, Spain and Thailand (Hashimoto et al. 2017, de Silva et al. 2018, Jaklitsch et al. 2018, Jayasiri et al. 2019). 
Atrocalyx bambusae (Phookamsak, S.C. Karunarathna \& K.D. Hyde) N.I. de Silva \& K.D. Hyde, Phytotaxa 333: 204 (2018)

Facesoffungi number: FoF06536

Fig. 54

Saprobic on dead bamboo culms, forming wedged-shaped, blackened perithecia. Sexual morph: Ascomata 345-465 $\mu \mathrm{m}$ diameter, 130-200 $\mu \mathrm{m}$ high, scattered, solitary, gregarious, immersed to semi-immersed in host cortex, dark brown to black, conical to subglobose, uniloculate, coriaceous. Peridium composed of host and fungal tissues; laterally 25-35 $\mu \mathrm{m}$ thick, composed of several layers of dark brown to hyaline cells of textura angularis; basal part, with 50$80 \mu \mathrm{m}$ thick sides, comprising dark brown to hyaline cells, arranged in textura angularis to textura epidermoidea. Hamathecium comprising 1-1.3 $\mu \mathrm{m}$ broad, septate, branched, anastomosing, hyaline, filiform, pseudoparaphyses. Asci 70-95 × 7.5-10 $\mu \mathrm{m}(\bar{x}=82.5 \times 8.7 \mu \mathrm{m}, \mathrm{n}=20)$, 8-spored, bitunicate, fissitunicate, cylindric-clavate, curved, with short furcate pedicel, apical rounded with well-developed ocular chamber. Ascospores 15-23 $\times 3.5-4.5 \mu \mathrm{m}(\bar{x}=19 \times 4 \mu \mathrm{m}, \mathrm{n}=20)$, 2-seriate, hyaline, fusiform, 1- or 3-septate, constricted at the septa, straight to curved, smooth-walled, guttulate. Asexual morph: Undetermined.

Culture characteristics - Colonies on PDA reaching 20-25 mm diameter after 2 weeks at room temperature $\left(20-30^{\circ} \mathrm{C}\right)$, dark brown to black in the centre, brown, radiated at the margin from above and reverse; medium dense, woolly, irregularly flattened, slightly raised at the middle; not producing pigment in agar.

Known distribution (based on molecular data) - Thailand, Chiang Rai (Hyde et al. 2016), China, Yunnan (this study).

Known hosts (based on molecular data) - dead bamboo culms (Hyde et al. 2016; this study).

Material examined - China, Yunnan Province, Southwest Forestry University, Bamboo Garden, on dead bamboo culms (Poaceae), 8 August 2016, H.B. Jiang, XNLD001 (KUN-HKAS 101764, new geographic record), living culture KUMCC 18-0117.

GenBank numbers - ITS: MN511733, SSU: MN511734, tefl: MN525566.

Notes - Lophiotrema bambusae was introduced by Hyde et al. (2016) based on morphological and phylogenetic analyses. However, the species was transferred to Atrocalyx and became Atrocalyx bambusae based on combined SSU, ITS, LSU and tefl sequences analyses (de Silva et al. 2018). Morphological characteristics of our collection are similar to Atrocalyx bambusae. Based on a nucleotide comparison of ITS and tefl pairwise (Jeewon \& Hyde 2016), the new isolate has consistent with the base pairs with the type strain of Atrocalyx bambusae (Fig. 53). Thus, we identify the new collection as Atrocalyx bambusae. Because the record of A. bambusae was so far only reported from Thailand (Hyde et al. 2016), we report this species from China for the first time.

Lophiotrema Sacc., Michelia 1 (3): 338 (1878)

Lophiotrema established by Saccardo is a group that comprised of lophiostomataceous taxa, characterised by hyaline, multi-septate ascospores (Saccardo 1883). However, it was later treated as a synonym of Lophiostoma as they pointed out that both ascospore colour and number of transverse septa are not sufficient for generic distinctions (Chesters \& Bell 1970). However, Hirayama \& Tanaka (2011) separated Lophiotrema from Lophiostoma based on morphological traits and phylogenetic analyses, and established Lophiotremataceae to accommodate Lophiotrema.

Lophiotrema neohysterioides M.E. Barr, Mycotaxon 45: 208, 1992

Fig. 56

Facesoffungi number: FoF 05217

Saprobic on dead wood of herbaceous plant. Ascomata 250-300 $\mu \mathrm{m}$ high $\times 270-320 \mu \mathrm{m}$ diameter $(\overline{\mathrm{x}}=298 \times 311 \mu \mathrm{m}, \mathrm{n}=10)$, dark brown to black, scattered, gregarious, immersed to erumpent throughout the host tissue, globose to subglobose, uni-loculate, glabrous, coriaceous. Ostiole central, carbonaceous, with a crest-like opening. Peridium 15-20 $\mu \mathrm{m}$ thick, comprising several layers, an inner stratum with hyaline to light brown cells of textura angularis and an outer stratum with light brown to dark brown cells of textura angularis fusing with the host tissues. 
Hamathecium comprising 1.5-2 $\mu \mathrm{m}$ wide, branched, cellular pseudoparaphyses, anastomosing among and between the asci. Asci 70-90 $\times 7-10 \mu \mathrm{m}(\bar{x}=75 \times 10 \mu \mathrm{m}, \mathrm{n}=20), 8$-spored, bitunicate, fissitunicate, cylindric-clavate, short pedicellate, apically rounded with a minute ocular chamber. Ascospores $20.5-25 \times 3-5 \mu \mathrm{m}(\bar{x}=23 \times 4 \mu \mathrm{m}, \mathrm{n}=20)$, hyaline, fusiform, 3-septate, with the primary septum median, the second cell from the apex slightly enlarged downward and shortest although longer than wide, with guttules, smooth, no sheath or appendages detected.

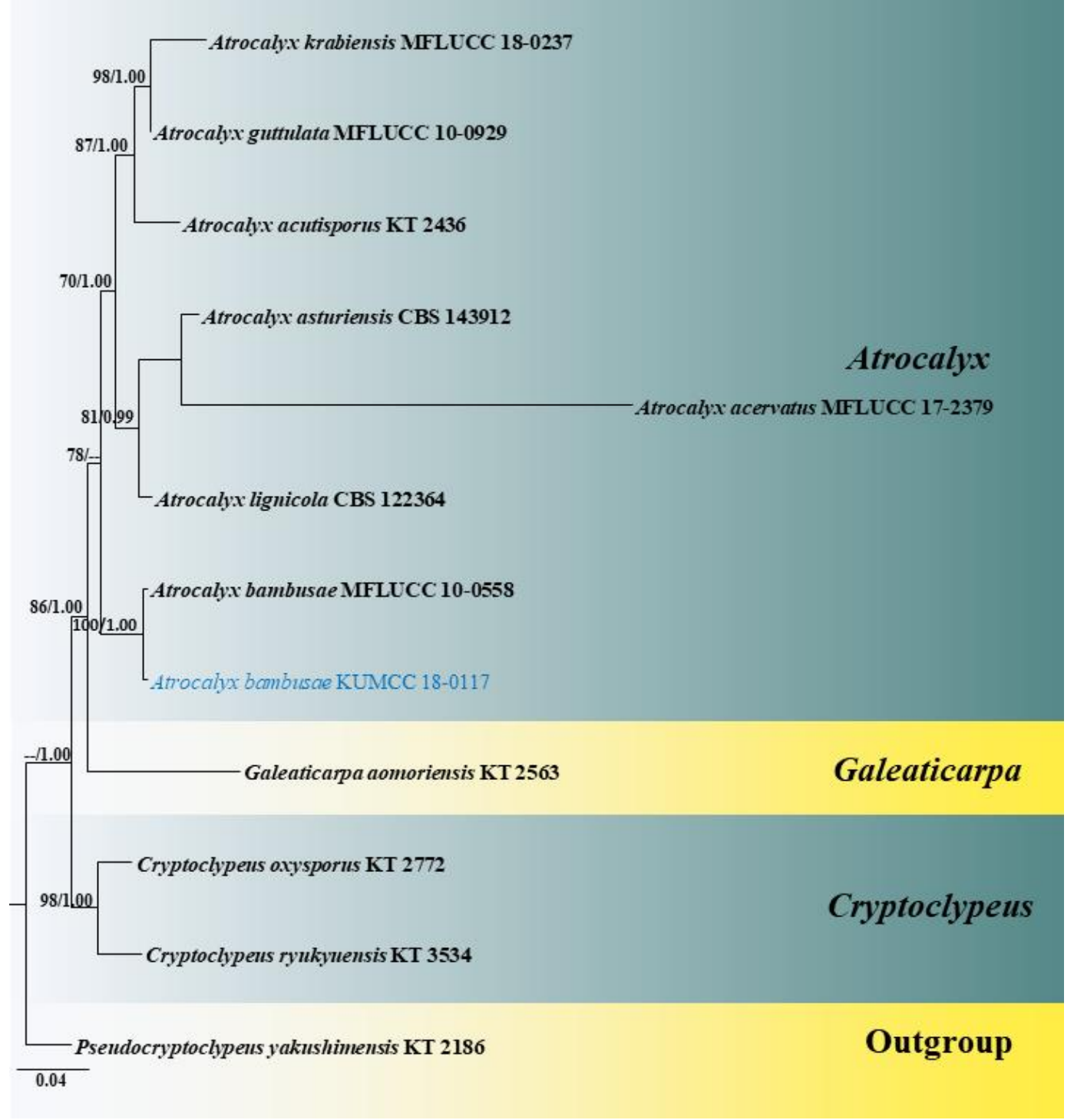

Fig. 53 - Phylogram generated from maximum likelihood analysis based on a combined ITS, LSU, SSU and tefl sequence dataset. Twelve strains are included in the combined gene analyses comprising 3,354 total characters including gaps (ITS: 1-566 bp, LSU: 567-1426 bp, SSU: 1427$2453 \mathrm{bp}$, tef1: 2454-3354 bp). The best scoring of the ML tree is selected to represent the phylogenetic relationships of Atrocalyx species, with the final ML optimization likelihood: 8153.910961. The matrix had 366 distinct alignment patterns, with $16.26 \%$ undetermined characters or gaps. Estimated base frequencies were as follows: $\mathrm{A}=0.242968, \mathrm{C}=0.259079, \mathrm{G}=$ $0.267179, \mathrm{~T}=0.230774$; substitution rates $\mathrm{AC}=1.994630, \mathrm{AG}=3.374777, \mathrm{AT}=1.799513, \mathrm{CG}=$ $1.818183, \mathrm{CT}=10.748211, \mathrm{GT}=1.000000$; Tree-Length $=0.634901 ;$ gamma distribution shape parameter $\alpha=0.585697$; The proportion of invariable sites $\mathrm{I}=0.547872$. Bayesian posterior probabilities (BYPP) from MCMC were evaluated with a final average standard deviation of split frequencies of 0.002895. Bootstrap support for maximum likelihood (MLBP) greater than $70 \%$ and Bayesian posterior probabilities (BYPP) greater than 0.95 are defined above the nodes as MLBP/BYPP. The tree is rooted to Pseudocryptoclypeus yakushimensis (KT 2186). The type 
strains are indicated in bold and newly generated sequence is shown in blue.
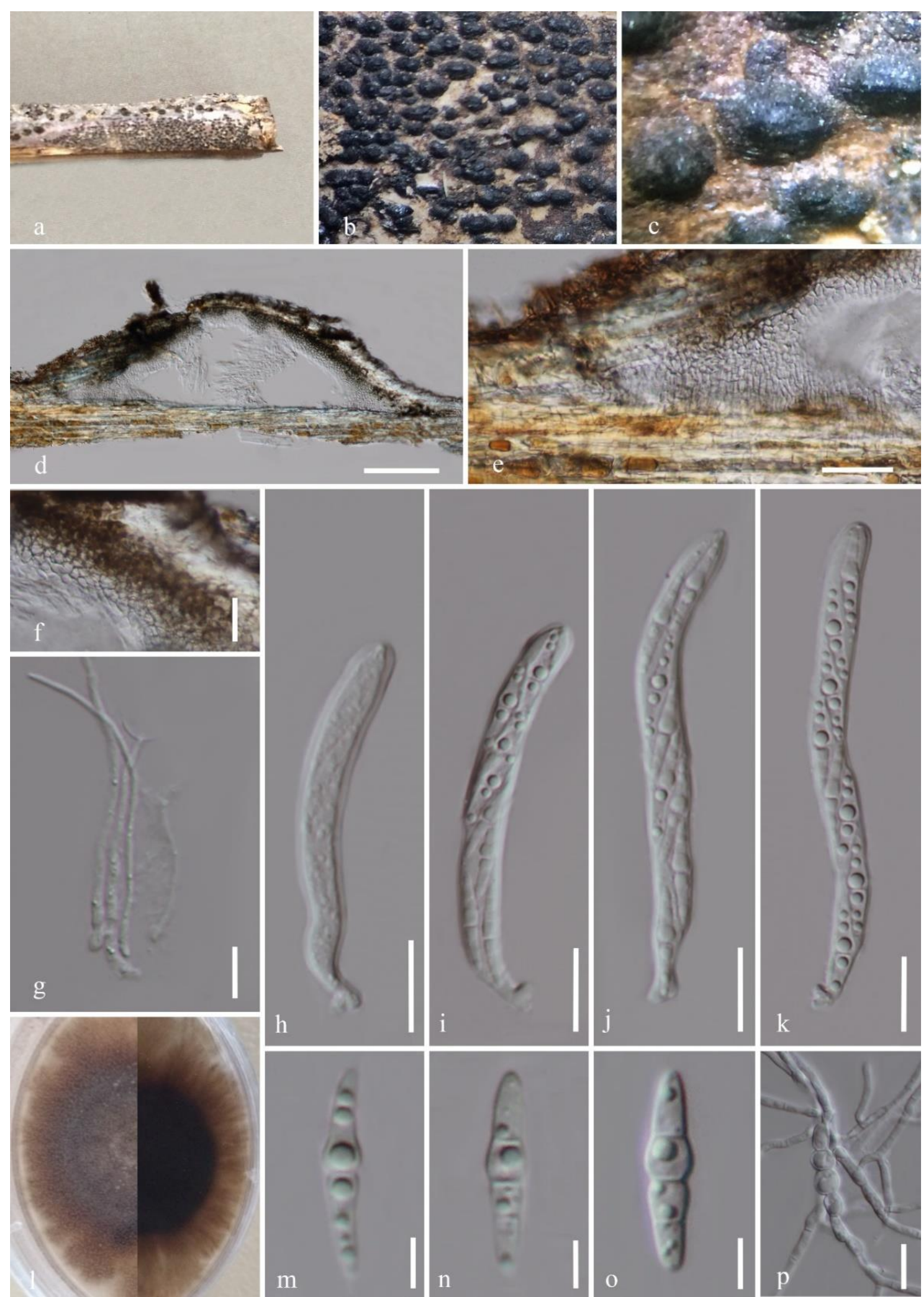

Fig. 54 - Atrocalyx bambusae (KUN-HKAS 101764, new geographic record). a-c Ascomata on bamboo host. d Vertical section of ascoma. e, f Peridium. g Pseudoparaphyses. h-k Asci. 1 Culture characteristics. $\mathrm{m}-\mathrm{O}$ Ascospores. $\mathrm{p}$ Germinating ascospore. Scale bars: $\mathrm{d}=100 \mu \mathrm{m}, \mathrm{e}=30 \mu \mathrm{m}$, $\mathrm{f}, \mathrm{h}-\mathrm{k}, \mathrm{p}=15 \mu \mathrm{m}, \mathrm{g}=10 \mu \mathrm{m}, \mathrm{m}-\mathrm{o}=5 \mu \mathrm{m}$. 


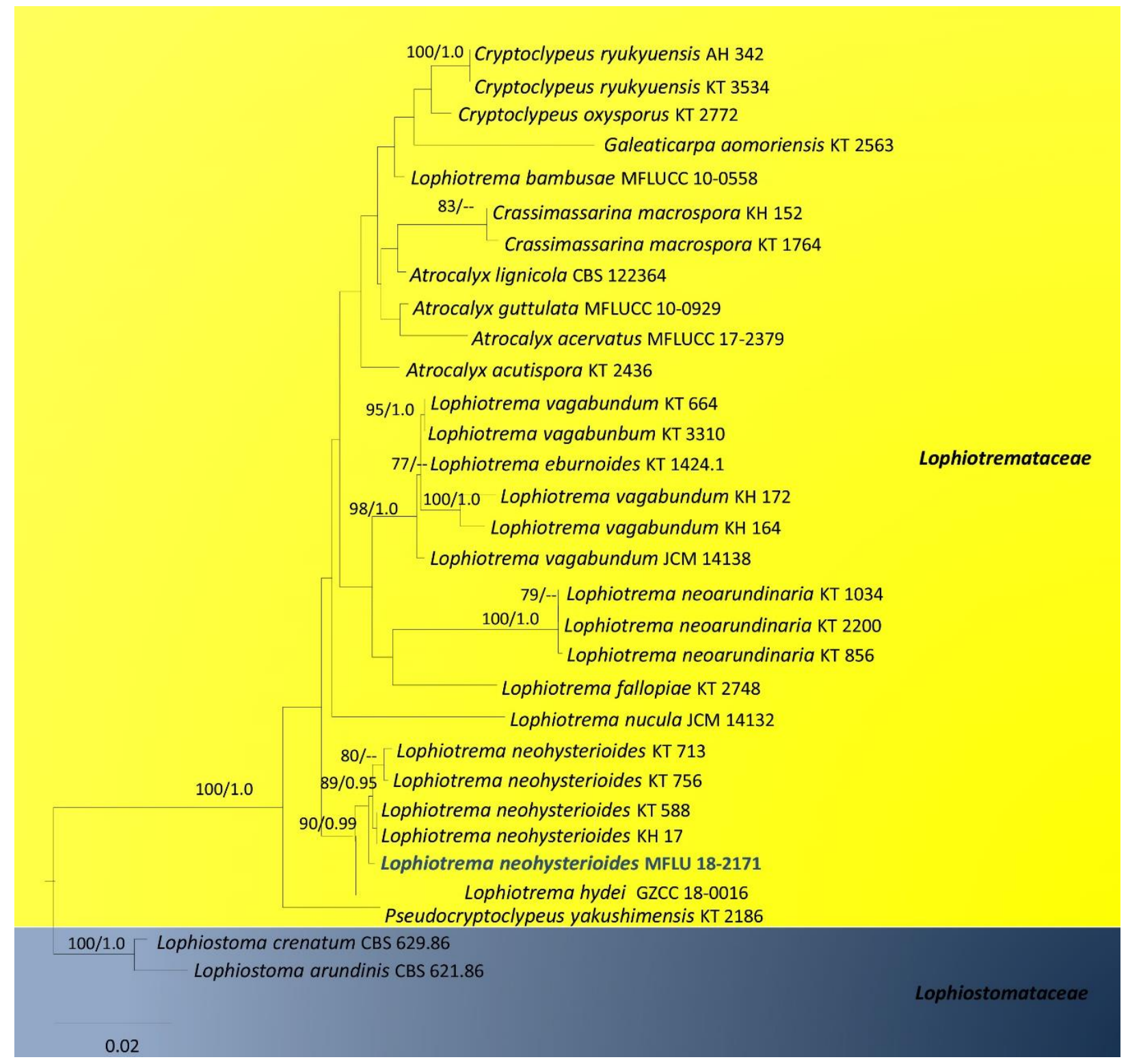

Fig. 55 - Phylogram generated from maximum likelihood analysis based on combined SSU, ITS, LSU and tef1 partial sequence data. Thirty one strains were included in the sequence analysis, which comprised 2545 characters including alignment gaps. Lophiostoma spp. (Lophiostomataceae) were used as the outgroup taxa. The tree topology derived from the Bayesian analysis was similar to that derived from the maximum likelihood analysis. The best scoring RAxML tree with a final likelihood value of -34728.193523 is presented. The matrix had 286 distinct alignment patterns, with $4.84 \%$ of undetermined characters or gaps. Estimated base frequencies were as follows; $\mathrm{A}=0.253516, \mathrm{C}=0.226561, \mathrm{G}=0.278443, \mathrm{~T}=0.241480$; substitution rates $\mathrm{AC}=1.845417, \mathrm{AG}=3.129372, \mathrm{AT}=1.390649, \mathrm{CG}=1.720102, \mathrm{CT}=$ 9.650566, GT $=1.000000$. ML bootstrap support (first set) equal or greater than $70 \%$ and Bayesian posterior probabilities equal or greater than 0.95 are given near to each branch. The new strain is in blue.

Known distribution (based on molecular data) - Japan (Hashimoto et al. 2017), China (Zhang et al. 2018).

Known hosts (based on molecular data) - Phyllostachys bambusoides, Robinia pseudoacacia (Hashimoto et al. 2017, Zhang et al. 2018), Pinus sp. (this study).

Material examined - China, Yunnan, Kunming Institute of Botany, on decaying wood of Pinus sp. (Pinaceae), 25 June 2018, A. Ekanayaka, C 467 (MFLU 18-2171, new host record). 


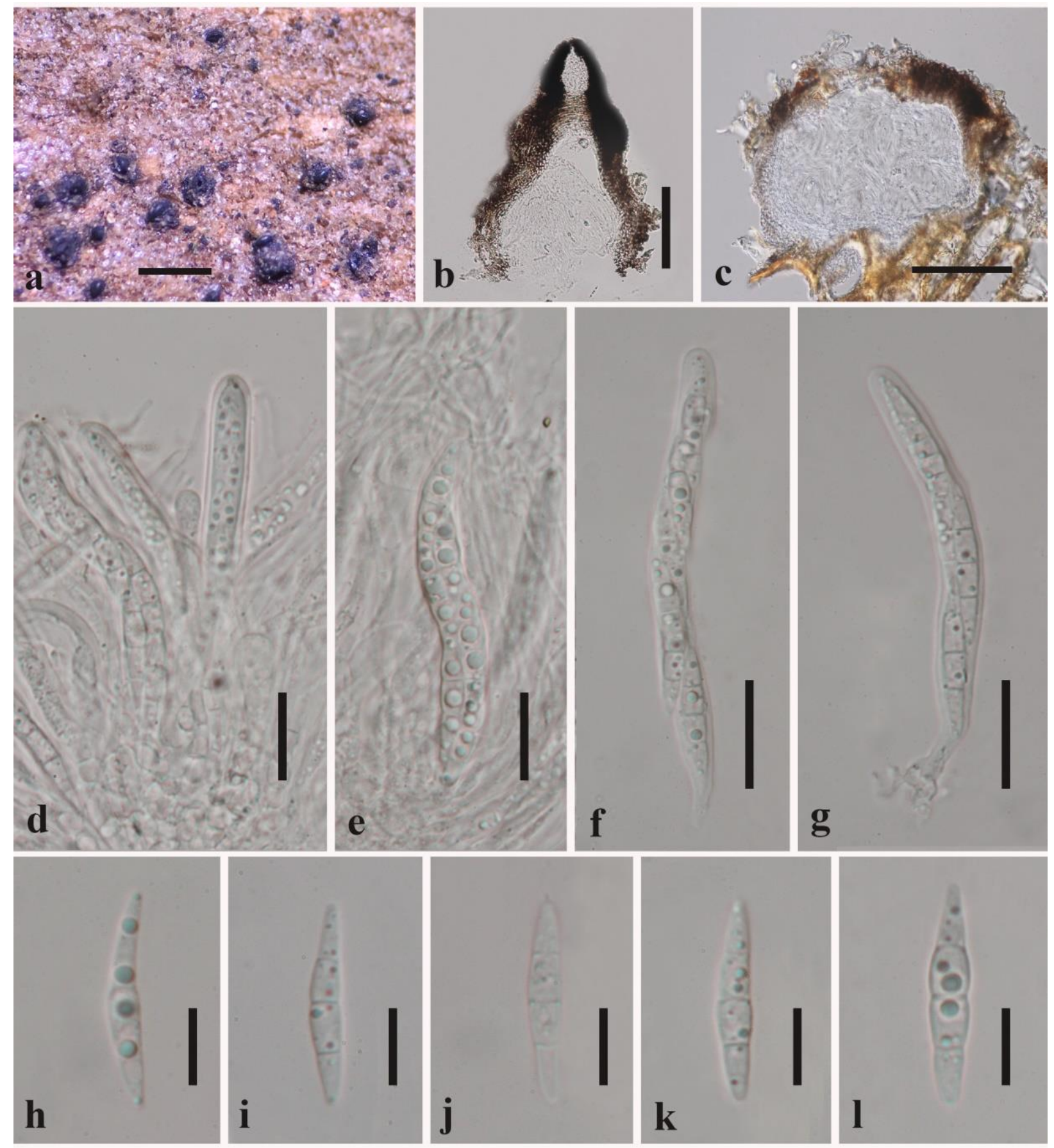

Fig. 56 - Lophiotrema neohysterioides (MFLU 18-2171, new host record). a Ascomata on host substrate. b, c Vertical section through ascomata. d-g Asci h-i Ascospores. Scale bars: $a=500 \mu \mathrm{m}$, $\mathrm{b}-\mathrm{c}=100 \mu \mathrm{m}, \mathrm{d}-\mathrm{g}=20 \mu \mathrm{m}, \mathrm{h}-\mathrm{l}=10 \mu \mathrm{m}$.

GenBank numbers - ITS: MN862638, LSU: MN862641, SSU: MN862640, tef1: MN862639.

Notes - The new collection shares similar morphological characters and DNA sequence data with Lophiotrema neohysterioides. Our collection is morphologically similar to the latter in having clavate asci and hyaline, fusiform, three equidistant septate ascospores as described in Tanaka \& Harada (2003). A comparison of the SSU, ITS, LSU and tefl nucleotides of these strains revealed less than $\leq 1.5 \%$ nucleotide differences which indicates that our isolate is a new record of Lophiotrema neohysterioides (Jeewon \& Hyde 2016). This is the second record of Lophiotrema neohysterioides from China, with a previous record which was collected from wood of an unidentified plant (Zhang et al. 2018). 


\section{Massariaceae Nitschke}

Massariaceae was introduced by Nitschke (1869) to accommodate the type genus Massaria and is a well-resolved family in Pleosporales. The species of Massariaceae have been reported as hemibiotrophs, saprotrophs or weak pathogens. Consequently, another monotypic genus Neomassaria was introduced by Hyde et al. (2016). The family is characterized by relatively large pseudothecia, central or eccentric ostioles, relatively wide, firm, opaque compressed cells of textura angularis, darkly pigmented peridium, branching and anastomosing pseudoparaphyses, 4-8-spored, bitunicate, fissitunicate asci and relatively large, oblong, cylindrical, ellipsoidal or fusoid brown ascospores. Huanraluek et al. (2018) introduced a new genus Massarioramusculicola based on phylogenetic analysis of large subunit (LSU) rRNA sequence data and morphological studies. The asexual morph is coelomycetous where known. Species of Massariaceae are distributed on a wide range of hosts namely, Aceraceae, Fabaceae, Moraceae, Rosaceae, Rutaceae, Ulmaceae and Viburnaceae worldwide especially in North America and Europe.

\section{Massaria De Not.}

Massaria was introduced by De Notaris with Sphaeria inquinans (= Massaria inquinans as type species. The genus is characterized by large subglobose, pyriform to strongly depressed pseudothecia, central or eccentric, short or long ostioles, a wide, firm, opaque pseudothecial wall composed of numerous rows of thick-walled cells of textura angularis, numerous persistent, indistinctly septate anastomosing pseudoparaphyses, bitunicate, fissitunicate, cylindrical or fusoid asci and large oblong, cylindrical, ellipsoidal or fusoid, hyaline, light to dark brown ascospores, rounded or tapered to subacute ends. Voglmayr \& Jaklitsch (2011) studied 17 species (with seven new species) and also reported their occurrence predominantly on Acer and Rosaceae hosts. Currently, 31 species are listed under Massaria. The asexual morph is coelomycetous.

Massaria anomia (Fr.) Petr., Annls Mycol. 21:114 (1923)

Fig. 58

Facesoffungi number: FoF 06536

Saprobic on dead branch of Robinia pseudoacacia. Sexual morph: Ascomata (pseudothecia) relatively large 500-1500 $\mu \mathrm{m}$ wide, $800-1400 \mu \mathrm{m}$ wide, scattered or clustered, pyriform to strongly depressed, immersed in bark and typically in pseudostromatic tissues intermixed with substrate cells, with blackened zones, clypeate, and ostiolate. Ostioles 200-325 $\mu \mathrm{m}$ long, 250-400 $\mu \mathrm{m}$ wide, central, long, solitary projecting through the bark, stout papillate, surmounted by peaks of stromatic tissues that form coarsely sulcate tips above the bark surface. Peridium 29-43 $\mu \mathrm{m}$ relatively wide, firm, opaque, composed of numerous rows of thin-walled, darkly pigmented, compressed cells of textura angularis. Hamathecium comprising 1-2 $\mu \mathrm{m}$ of numerous persistent, indistinctly septate, branching and anastomosing pseudoparaphyses embedded in a gelatinous matrix. Asci 115-194 $\times$ 18-24 $\mu \mathrm{m}(\bar{x}=150.7 \times 21.4 \mu \mathrm{m}, \mathrm{n}=20), 4$-spored, bitunicate, fissitunicate, basal and peripheral, oblong, cylindrical or fusoid, pedicellate, apically with wide ocular chamber $(2-5 \mu \mathrm{m})$ and refractive ring. Ascospores 46-49 × 13-14 $\mu \mathrm{m}(\bar{x}=47.9 \times 13.9 \mu \mathrm{m}, \mathrm{n}=20), 1-2$-seriate, dark brown, oblong, cylindrical, ellipsoidal or fusoid, rounded or tapered to subacute ends, hyaline or light to dark brown, slightly inequilateral, symmetric, biconoid and symmetrically 1-euseptate in early states, becoming 3-disto- septate at maturity, slightly constricted at the septa, secondary septa usually closer to primary septum than to ends of ascospore wall, smooth, without mucilaginous sheath, dark-brown lumina rhomboid or lenticular in the central cells, conoid in the end cells. Asexual morph: Undetermined.

Culture characters - Colonies growing on MEA, reaching $7 \mathrm{~mm}$ diameter after $7 \mathrm{~d} \mathrm{at} 16^{\circ} \mathrm{C}$, circular to slightly irregular, flat to slightly raised, dense, surface white, middle dark-grey, reverse saffron to dark-brown, white at the margin, smooth surface with entire to slightly undulate edge.

Known distribution (based on molecular data) - Austria, France, Northern Hemisphere (North America, Europe) (Voglmayr \& Jaklitsch 2011), Italy (this study).

Known hosts (based on molecular data) - on woody host of Fabaceae (Amorpha, Gleditsia, Laburnum) (Voglmayr \& Jaklitsch 2011), Robinia pseudacacia (Fabaceae) (this study). 


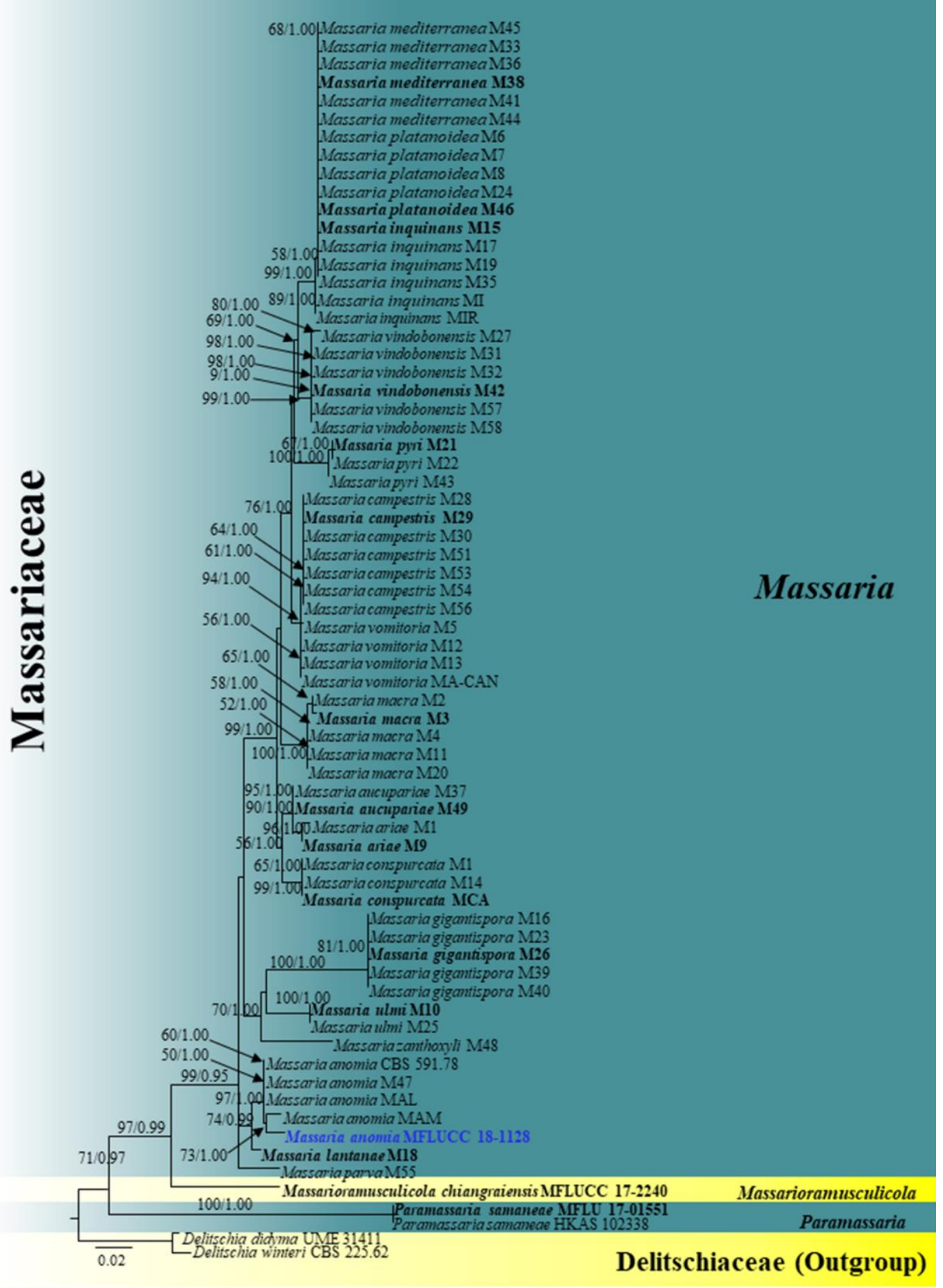

Fig. 57 - Phylogram generated from maximum likelihood analysis based on combined LSU and SSU sequence data. Sixty-nine strains are included in the combined gene analyses comprising 1939 characters after alignment (906 characters for LSU and 1035 characters for SSU). Delitschia didyma (UME 31411) and Delitschia winteri (CBS 225.62) are used as the outgroup taxa. The tree topology derived from the Bayesian analysis was similar to that derived from the maximum likelihood analysis. The best RaxML tree with a final likelihood value of -5560.059600 is presented. The matrix had 298 distinct alignment patterns, with $32.99 \%$ undetermined characters or gaps. Estimated base frequencies were as follows: $\mathrm{A}=0.262352, \mathrm{C}=0.201082, \mathrm{G}=0.289067, \mathrm{~T}=$ 
0.247499; substitution rates $\mathrm{AC}=0.654092, \mathrm{AG}=2.038393, \mathrm{AT}=0.701260, \mathrm{CG}=0.403849, \mathrm{CT}$ $=7.065520, \mathrm{GT}=1.000000$; gamma distribution shape parameter $\alpha=0.568674$. Bootstrap values for maximum likelihood equal to or greater than 50 and Bayesian posterior probabilities equal or greater than 0.90 are placed above or below the branches. Ex-type strains are in bold and black. The newly generated sequence is indicated in blue.

Material examined - Italy, Province of Forlì-Cesena [FC], near Predappio, on dead terrestrial branch of Robinia pseudoacacia (Fabaceae), 11 February 2017, E. Camporesi (MFLU 17-0490, new geographical record); living culture MFLUCC 18-1128.

GenBank numbers - LSU: MN244203, SSU: MN244180.

Notes - Our taxon is morphologically similar and phylogenetically related to Massaria anomia, but collected in different geographical locations. Both are associated with the same host, Robinia pseudoacacia (Fabaceae). Massaria anomia was recorded in Austria, France and is widely distributed in the Northern Hemisphere (Voglmayr \& Jaklitsch 2011) while our collection is from Italy. The phylogenetic placement of our strain (MFLUCC 18-1128) is shown in Fig. 57.

\section{Massarinaceae Munk}

Massarinaceae was introduced by Munk (1956) with Massarina as the type genus. The morphological characters of this family include immersed, flattened or spherical ascomata, cellular pseudoparaphyses, clavate to cylindro-clavate asci, and hyaline, fusiform to narrowly fusiform, 1 to 3-septate ascospores with or without a mucilaginous sheath. Barr (1987) treated Massarinaceae as a synonym of Lophiostomataceae. These two families are now recognized as different lineages based on morphological and molecular data (Liew et al. 2002, Zhang et al. 2009). Species of Massarinaceae are distributed in terrestrial habitats and are saprobic on wood or twigs (Hyde et al. 2013). Massarina was established to separate taxa with hyaline ascospores based on Massarina, Keissleriella, Metasphaeria, Pseudotrichia and Trichometasphaeria (Munk 1956, Hyde et al. 2013). Following its introduction, many studies have been conducted on the above mentioned genera, with the exception of Massarina and have been transferred to other families (Suetrong et al. 2009, Zhang et al. 2012, Hyde et al. 2013, Wijayawardene et al. 2014, Boonmee et al. 2017). Currently, nine genera are accommodated in Massarinaceae namely, Bambusistroma, Helminthosporium, Longiostiolum, Massarina, Pseudodidymosphaeria, Pseudosplanchnonema, Semifissispora, Stagonospora and Suttonomyces.

\section{Pseudosplanchnonema Chethana \& K.D. Hyde}

The monotypic genus Pseudosplanchnonema was introduced by Chethana et al. (2015) to accommodate Pseudosplanchnonema phorcioides, a new combination proposed by the authors earlier described as Massaria phorcioides. The genus is characterized by immersed, perithecial ascomata, short papillate ostioles, filiform pseudoparaphyses embedded in a gelatinous matrix, bitunicate cylindrical to clavate short pedicellate asci and brown ascospores with pseudosepta surrounded by a mucilaginous sheath. The asexual morph is coelomycetous. Pseudosplanchnonema phorcioides has been reported on dead branch of Morus sp. in Italy. In this study, we illustrate a new record of Pseudosplanchnonema phorcioides on Morus sp in Russia.

Pseudosplanchnonema phorcioides (I. Miyake) Chethana, Camporesi \& K.D. Hyde, Phytotaxa 231(2): 139 (2015)

Facesoffungi number: FoF06225

Fig. 60

Saprobic on dead branch of Prunus tomentosa. Sexual morph: Pseudostromata 51-102 $\mu \mathrm{m}$ diameter 56-80 $\mu \mathrm{m}$ high, immersed to semi immersed ascomata, scattered or clustered, globose, conical globose, papillate. Ostiole $25-27 \mu \mathrm{m}$ high, 49-52 $\mu \mathrm{m}$ diameter $(\bar{x}=25.2 \times 50.6 \mu \mathrm{m}, \mathrm{n}=$ 10), short, papillate, opening to exterior through bark. Peridium thin $43-63 \mu \mathrm{m}(\bar{x}=52.3 \mu \mathrm{m})$, wide at side walls, up to $85 \mu \mathrm{m}$ wide near the apex, and $47 \mu \mathrm{m}$ wide at the base comprising a few layers of brown compressed cells, outer layer composed of 3-4 layers of dark brown cells of textura 
angularis, middle layer comprising 2-3 layers cells of textura angularis and inner cells comprising pale brown cells of textura prismatica. Hamathecium comprising broad, filiform $2-3(\bar{x}=3.2) \mu \mathrm{m}$ wide, septate pseudoparaphyses, embedded in a gelatinous matrix. Asci 201-255 $\times 41-48 \mu \mathrm{m}(\bar{x}=$ $232.3 \times 43.9 \mu \mathrm{m}, \mathrm{n}=10), 8$-spored, bitunicate, fissitunicate, cylindrical to clavate, with a short pedicel with an ocular chamber. Ascospores 40-48 $\times 15-17 \mu \mathrm{m}(\bar{x}=51.1 \times 16.6 \mu \mathrm{m}, \mathrm{n}=15)$, overlapping biseriate, hyaline to pale brown when young, dark brown at maturity, fusiform to ellipsoidal, widest near the centre, with acute rounded ends, sometimes slightly curved, 1submedian septate, constricted at the septum, 5-6-guttulate, pseudosepta between the guttules, smooth-walled, surrounded by a mucilaginous sheath. Asexual morph: Undetermined.

Culture characters - Ascospores germinating on MEA within $24 \mathrm{~h}$ and the germ tubes of 2.5$3 \mu \mathrm{m}$ diameter produced near the septum. Colonies reaching $4 \mathrm{~mm}$ diameter after 10 days at $25^{\circ} \mathrm{C}$, entire edge, greenish black in the centre, greenish grey towards rim, white at the margin with a circular cottony mycelium on the surface and reverse black in the centre and grey towards the ends of the mycelium.

Known distribution (based on molecular data) - Russia, Austria, France, Northern Hemisphere (Chethana et al. 2015), Russia (this study).

Known hosts (based on molecular data) - Morus sp. (Chethana et al. 2015), Prunus tomentosa (this study).

Material examined - Russia, Rostov Region, Shakhty City, urban artificial forest, on dying twigs of Prunus tomentosa (Rosaceae), 26 May 2017, Timur Bulgakov (MFLU 17-2062, new host record), living cultures MFLUCC 18-1135; DSM 109792.

GenBank accession numbers LSU: MN244204, SSU: MN244181.

Notes - Our isolate MFLUCC 18-1135 clustered with the ex-type strains of Pseudosplanchnonema phorcioides (Fig. 59) originally described by Chethana et al. (2015) and collected from Italy. However, it differs from the type strain of $P$. phorcioides in having smaller ascomata $(51-102 \mu \mathrm{m}$ versus $159-483 \mu \mathrm{m}$ diameter) and shorter ascospores $(40-48 \times 15-17 \mu \mathrm{m}$ versus 50-66 × 14-20 $\mu \mathrm{m})$. By considering the molecular data and the host relationship, we illustrate our collection as a new record on Prunus tomentosa from Russia.

Melanommataceae G. Winter.

We follow the latest treatment and updated account of Melanommataceae in Tian et al. (2015), Li et al. (2016d, 2017a), Hyde et al. (2018) and Wanasinghe et al. (2018a). Melanommataceae comprises 33 genera (Wijayawardene et al. 2020).

\section{Byssosphaeria Cooke.}

Byssosphaeria was introduced by Cooke (1879) and is typified by B. keithii. The genus is widespread on hosts and distribution, occurring on various hosts as a saprobe worldwide (Farr \& Rossman 2020). Recently, there are 46 epithets listed in Index Fungorum (2020), however, only nine species have their phylogenetic affinities investigated within Melanommataceae (Tennakoon et al. 2018). We follow the updated accounts of Byssosphaeria in Tennakoon et al. (2018). Byssosphaeria musae is reported from decaying frond of a palm in Yunnan Province, China for the first time.

Byssosphaeria musae Phookamsak \& K.D. Hyde, Fungal Diversity 72: 119 (2015)

Fig. 62

Facesoffungi number: FoF00436

Saprobic on decaying frond of palm. Sexual morph: Ascomata 525-600 $\mu \mathrm{m}$ high, 560-640 $\mu \mathrm{m}$ diameter, black, superficial, solitary, gregarious, uni-loculate, subglobose, setose, with dark brown to black, filiform, filamentous setae, tapering towards the apex, apapillate, ostiole central with porelike opening, producing yellowish to orange pigmented around the ostiole at maturity. Peridium 45-60 $\mu \mathrm{m}$ wide at side, 75-100 $\mu \mathrm{m}$ wide at base, 70-90 $\mu \mathrm{m}$ wide near ostiole, thick-walled, composed of dark brown to black pseudoparenchymatous cells, arranged in textura angularis to textura prismatica. 

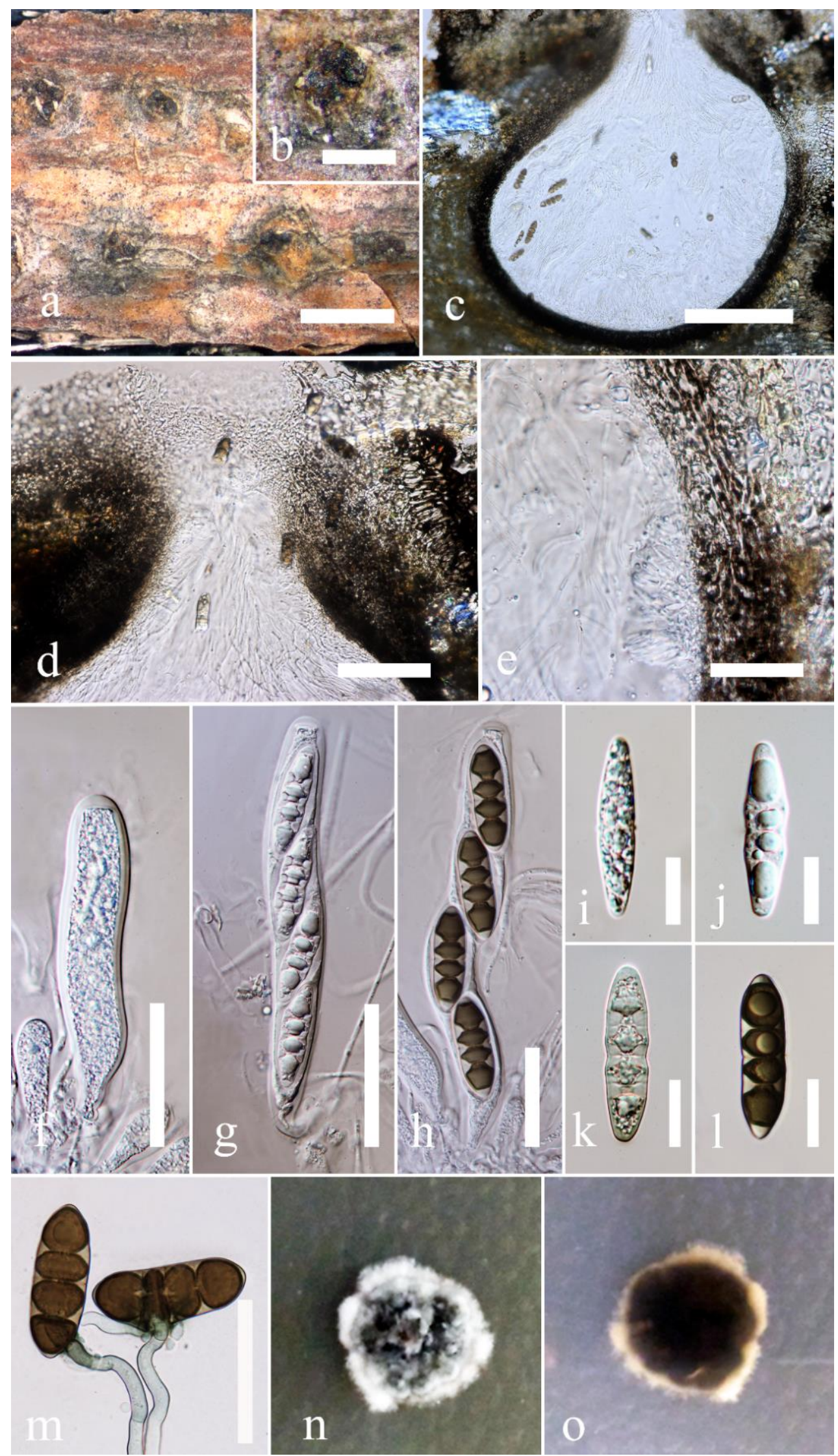

Fig. 58 - Massaria anomia (MFLU 17-0490, new geographical record). a, b Appearance of ascomata on host surface. c Vertical section through the ascomata. d Ostiole. e Peridium $\mathrm{f}-\mathrm{h}$ Asci. i-1 Ascospores m Germinating ascospores n, o Culture characteristics on MEA (n: above view, o: reverse view). Scale bars: $\mathrm{a}=400 \mu \mathrm{m}, \mathrm{b}=500 \mu \mathrm{m}, \mathrm{c}=50 \mu \mathrm{m}, \mathrm{d}=30 \mu \mathrm{m}, \mathrm{e}=50 \mu \mathrm{m}, \mathrm{f}=40 \mu \mathrm{m}, \mathrm{g}$, $\mathrm{h}=50 \mu \mathrm{m}, \mathrm{i}-\mathrm{m}=20 \mu \mathrm{m}$. 


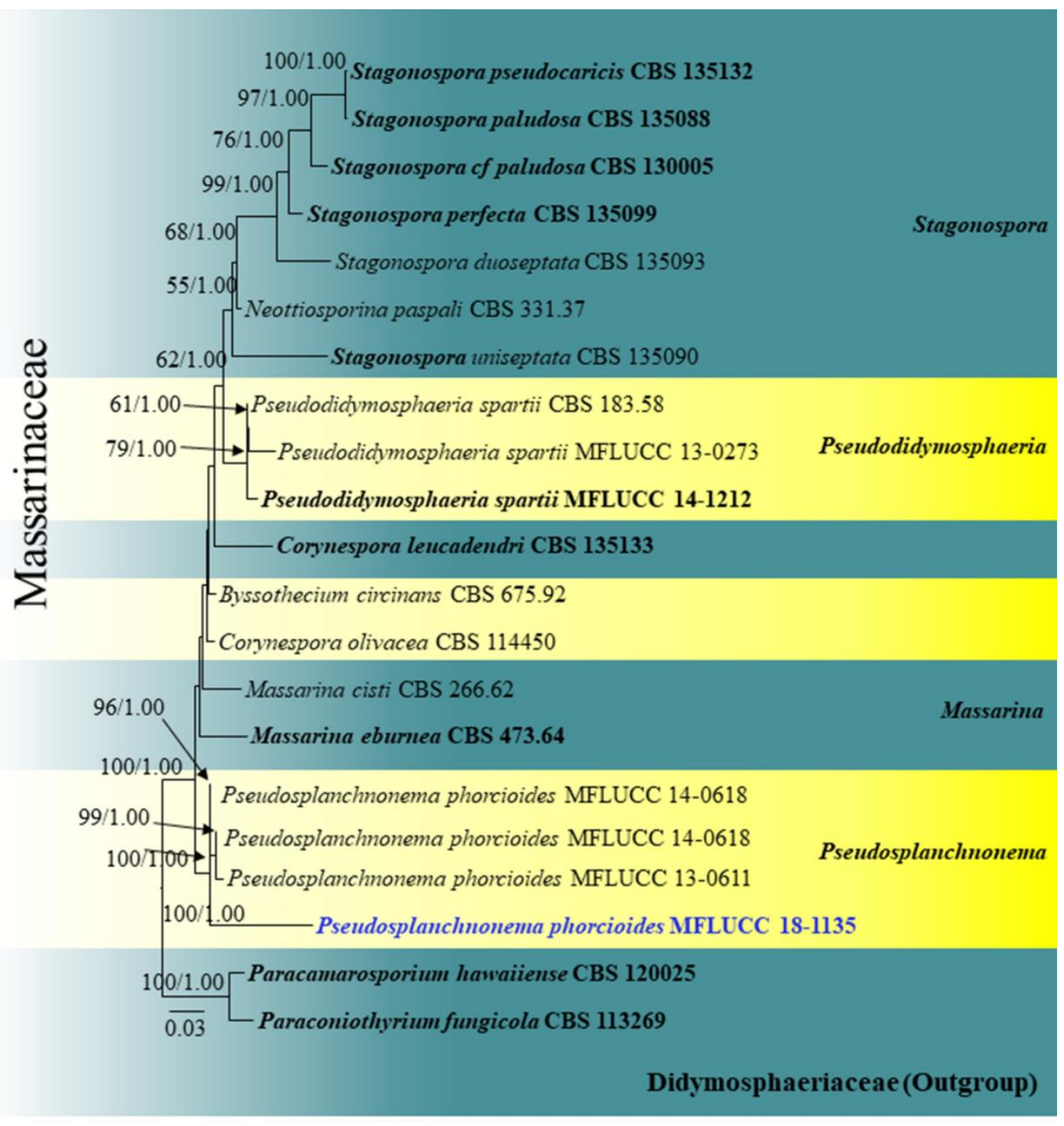

Fig. 59 - Phylogram generated from maximum likelihood analysis based on combined LSU and SSU sequence data. Twenty-one strains are included in the combined gene analyses comprising 2327 characters after alignment (856 characters for LSU, 1058 characters for SSU and 411 characters for TUB). Paracamarosporium hawaiiense (CBS 120025) and Paraconiothyrium fungicola (CBS 113269) are used as the outgroup taxa. The tree topology derived from the Bayesian analysis was similar to that derived from the maximum likelihood analysis. The best RaxML tree with a final likelihood value of -7593.818950 is presented. The matrix had 535 distinct alignment patterns, with $30.91 \%$ undetermined characters or gaps. Estimated base frequencies were as follows: $\mathrm{A}=0.254460, \mathrm{C}=0.214516, \mathrm{G}=0.284400, \mathrm{~T}=0.246625$; substitution rates $\mathrm{AC}=$ 1.248767, $\mathrm{AG}=2.513663, \mathrm{AT}=1.269748, \mathrm{CG}=0.839642, \mathrm{CT}=4.977442, \mathrm{GT}=1.000000$; gamma distribution shape parameter $\alpha=0.917153$. Bootstrap values for maximum likelihood equal to or greater than 50 and Bayesian posterior probabilities equal or greater than 0.95 are placed above or below the branches. Ex-type strains are in bold and black. The newly generated sequence is indicated in blue.

Hamathecium comprising dense, 1-2.7 $\mu \mathrm{m}$ wide, septate, filiform, anastomosed pseudoparaphyses, embedded in a gelatinous matrix. Asci 126-147 $\times 11-15.5 \mu \mathrm{m}(\bar{x}=136.8 \times 13$ $\mu \mathrm{m}, \mathrm{n}=20$ ), 8 -spored, bitunicate, cylindric-clavate, rounded at the apex with ocular chamber, with a long pedicel (45-65 $\mu \mathrm{m}$ long). Ascospores 33-37 $\times 7-9 \mu \mathrm{m}(\bar{x}=35.5 \times 7.8 \mu \mathrm{m}, \mathrm{n}=20)$, overlapping 1-2-seriate, initially hyaline, light brown at maturity, fusiform, 1-septate, slightly 
curved, obviously constricted at the septum, guttulate, surrounded by a thin gelatinous sheath. Asexual morph: Undetermined.
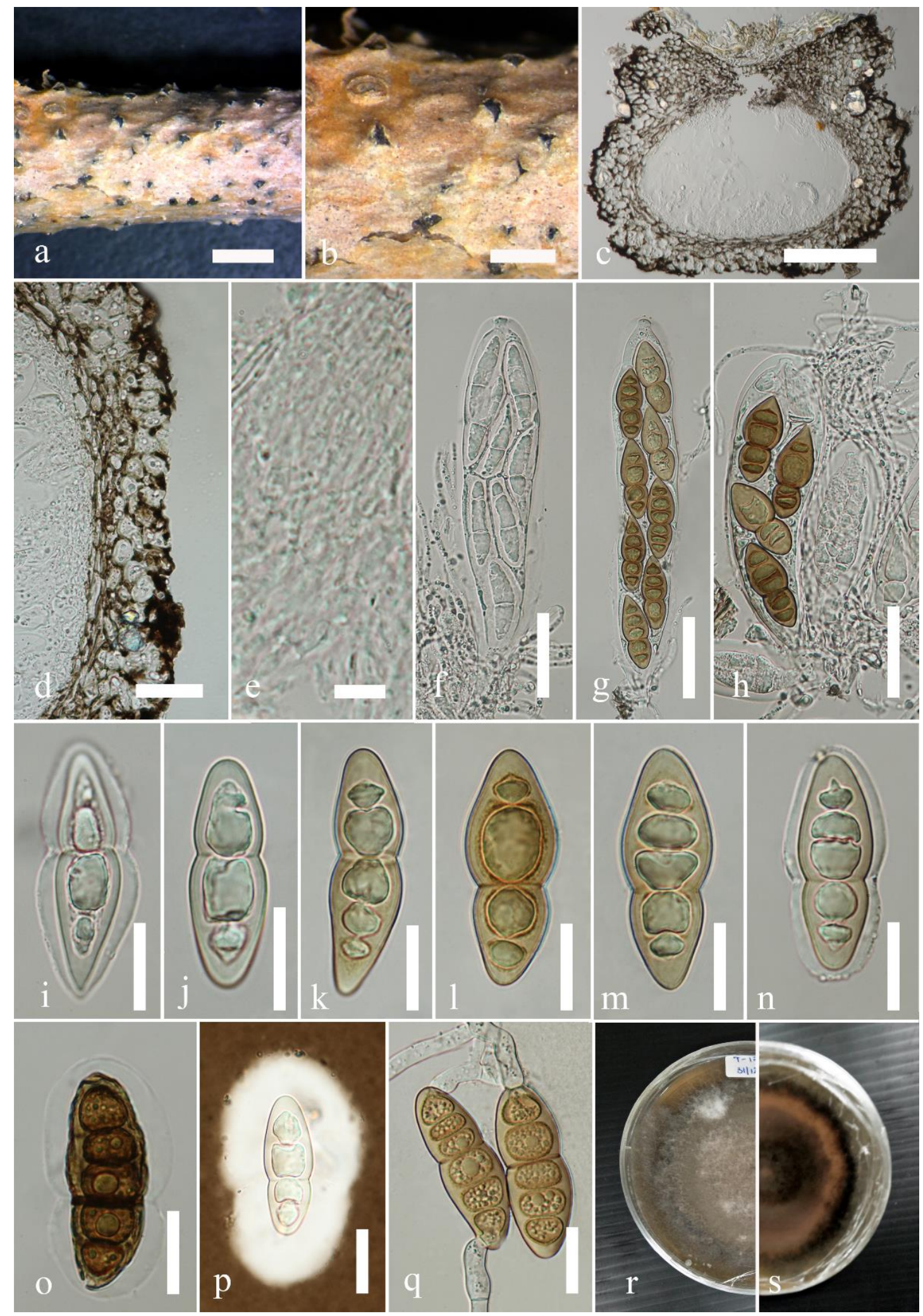

Fig. 60 - Pseudosplanchnonema phorcioides (MFLU 17-2062, new host record). a, b Appearance of ascomata on host surface. c Vertical section through the ascoma. d Peridium. e Hamathecium. $\mathrm{f}-\mathrm{h}$ Asci $\mathrm{i}-\mathrm{p}$ Ascospores (p: Indian ink showing surrounding mucilaginous sheath). q Germinating ascospores. $r$, $s$ Culture characteristics on MEA (q: above view; $r$ : reverse view). Scale bars: $\mathrm{a}=$ $200 \mu \mathrm{m}, \mathrm{b}, \mathrm{c}=100 \mu \mathrm{m}, \mathrm{d}=5 \mu \mathrm{m}, \mathrm{e}-\mathrm{g}=10 \mu \mathrm{m}, \mathrm{h}=20 \mu \mathrm{m}, \mathrm{i}-\mathrm{m}=10 \mu \mathrm{m}$. 
Culture characteristics - Colonies on PDA reaching $18 \mathrm{~mm}$ diameter after 1 week at room temperature $20-25^{\circ} \mathrm{C}$, circular, convex, effuse, velvety to fluffy, light yellow to orange from the above; yellowish-brown at the centre, yellowish brown to orange at the margin from the reverse; not producing pigments in PDA.

Known distribution (based on molecular data) - Thailand, Chiang Rai (Liu et al. 2015), China, Yunnan (this study).

Known hosts (based on molecular data) - Musa sp. (Musaceae) (Liu et al. 2015), Palm (this study).

Material examined - China, Yunnan Province, Kunming, Dianchi Lake, on decaying frond of a palm, 25 January 2019, E.F. Yang, DY1 (KUN-HKAS 102550, new host record), living culture, KUMCC 20-0003.

GenBank numbers - LSU: MT000759, SSU: MT000958, tef1: MT005775.

Notes - Byssosphaeria musae Phookamsak \& K.D. Hyde was introduced by Liu et al. (2015). The taxon was collected from Musa sp. (Musaceae) in Chiang Rai Province, Thailand. Our new collection was collected from a palm nearby Lake Dianchi of Kunming, Yunnan Province, China. Morphologically of our collection is similar to B. musae. Phylogenetic analyses demonstrate our new collection is conspecific with the type of B. musae (Fig. 61). A comparison of the tefl nucleotides of these two strains reveals less than $1.5 \%$ nucleotide differences (Jeewon \& Hyde 2016). Based on known distribution and host of B. musae (Liu et al. 2015), the new collection is reported as a saprobe on palm in Yunnan, China for the first time.

\section{Morosphaeriaceae Suetrong, Sakay., E.B.G. Jones \& C.L. Schoch}

This family comprises four saprobic genera namely Aquilomyces, Clypeoloculus, Helicascus and Morosphaeria and are commonly found from both freshwater and marine habitats (Suetrong et al. 2009, Devadatha et al. 2018, Wijayawardene et al. 2018, Zeng et al. 2018). The family is characterized by subglobose to lenticular, erumpent ascomata, cylindric-clavate asci with an apical ring and fusiform to ellipsoidal, hyaline to pigmented ascospores, surrounded by thick gelatinous sheath.

\section{Helicascus Kohlm.}

The genus currently comprises twelve species (Kohlmeyer 1969, Zeng et al. 2018, Index Fungorum 2020). Its members are saprobic on dead or decaying wood in aquatic habitats from freshwater and marine environments. They share common characters such as immersed to erumpent with uni- to multi-loculate ascomata, bitunicate asci and septate, pigmented ascospores, with or without mucilaginous sheath (Luo et al. 2016, Zeng et al. 2018). An updated tree is provided here.

Helicascus elaterascus (Shearer) H. Zhang \& K.D. Hyde, Cryptog. Mycol. 33: 158 (2012)

Facesoffungi number: FoF02018

Fig. 64

Saprobic on decaying, submerged wood in freshwater. Sexual morph: Ascomata 200-300 $\mu \mathrm{m}$ high $\times 165-180 \mu \mathrm{m}$ diameter, immersed, solitary, aggregated, subglobose to ellipsoidal, black, periphysate ostiolar neck, papillate. Peridium 26-55 $\mu \mathrm{m}$, composed of several layers of brown to dark brown cells of textura angularis, outer layer dark brown, inner layer of hyaline, flattened cells of textura angularis, $2-3 \mu \mathrm{m}$ wide. Asci $88-130 \times 18-23 \mu \mathrm{m}(\bar{x}=101 \times 20, \mathrm{n}=15)$, 8-spored, bitunicate, cylindric-clavate, long pedicellate, form a long tail-like extension, apically rounded. Ascospores 20-27 $\times 8-12 \mu \mathrm{m}(\bar{x}=25 \times 10, \mathrm{n}=20), 2$-seriate partially overlapping, hyaline when young and brown when mature, ellipsoid-fusiform, upper cell wider, tapering towards the narrow end, 1-septate, constricted at the septum, refractively guttulate, smooth-walled, with or without mucilaginous sheath. Asexual morph: Undetermined. 


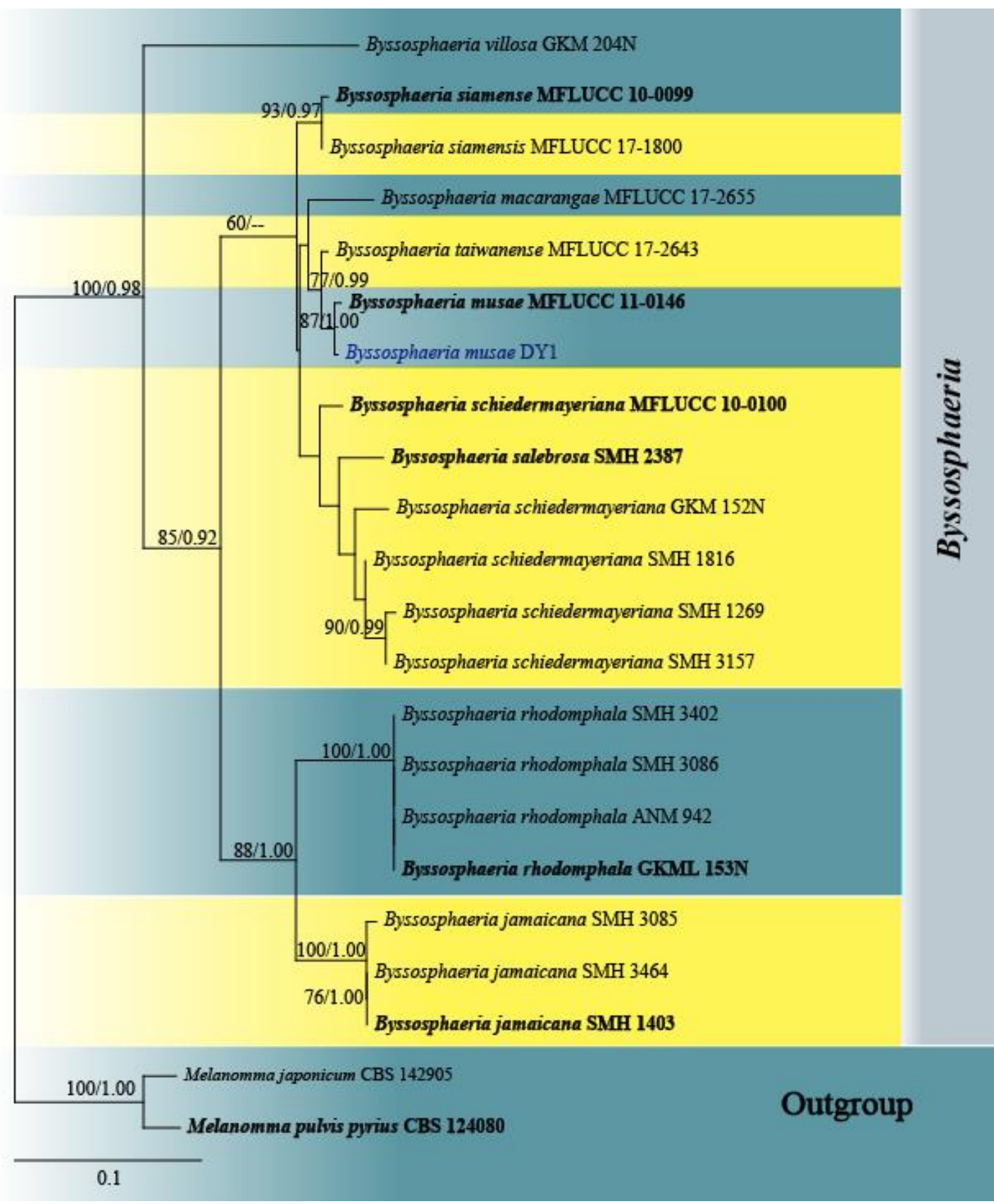

Fig. 61 - Phylogram generated from maximum likelihood analysis based on a combined LSU, tef1 sequence dataset. Twenty-two strains are included in the combined gene analyses comprising 1731 total characters including gaps (LSU: 1-847 bp, tefl: 848-1731 bp). The tree topology derived from the Bayesian analysis was similar to that derived from the maximum likelihood analysis. The best scoring of the ML tree is selected to represent the phylogenetic relationships of taxa in Byssosphaeria, with the final ML optimization likelihood: -4023.863653. The matrix had 225 distinct alignment patterns, with $22.61 \%$ undetermined characters or gaps. Estimated base frequencies were as follows: $\mathrm{A}=0.239041, \mathrm{C}=0.253223, \mathrm{G}=0.291157, \mathrm{~T}=0.216579$; substitution rates $\mathrm{AC}=18.961935, \mathrm{AG}=44.431399, \mathrm{AT}=17.632231, \mathrm{CG}=23.885049, \mathrm{CT}=$ 290.21010184, GT = 1.000000; Tree-length $=0.230788$; gamma distribution shape parameter $\alpha=$ 1.795248. Bayesian posterior probabilities (BYPP) from MCMC were evaluated with a final average standard deviation of split frequencies of 0.002700. Bootstrap support for maximum likelihood (ML) greater than 60\% and Bayesian posterior probabilities (PP) greater than 0.90 are defined above the nodes as ML/PP. The tree is rooted to Melanomma japonicum (CBS 142905) and M. pulvis-pyrius (CBS 124080). The type strains are indicated in bold and the newly generated sequence is shown in blue. 

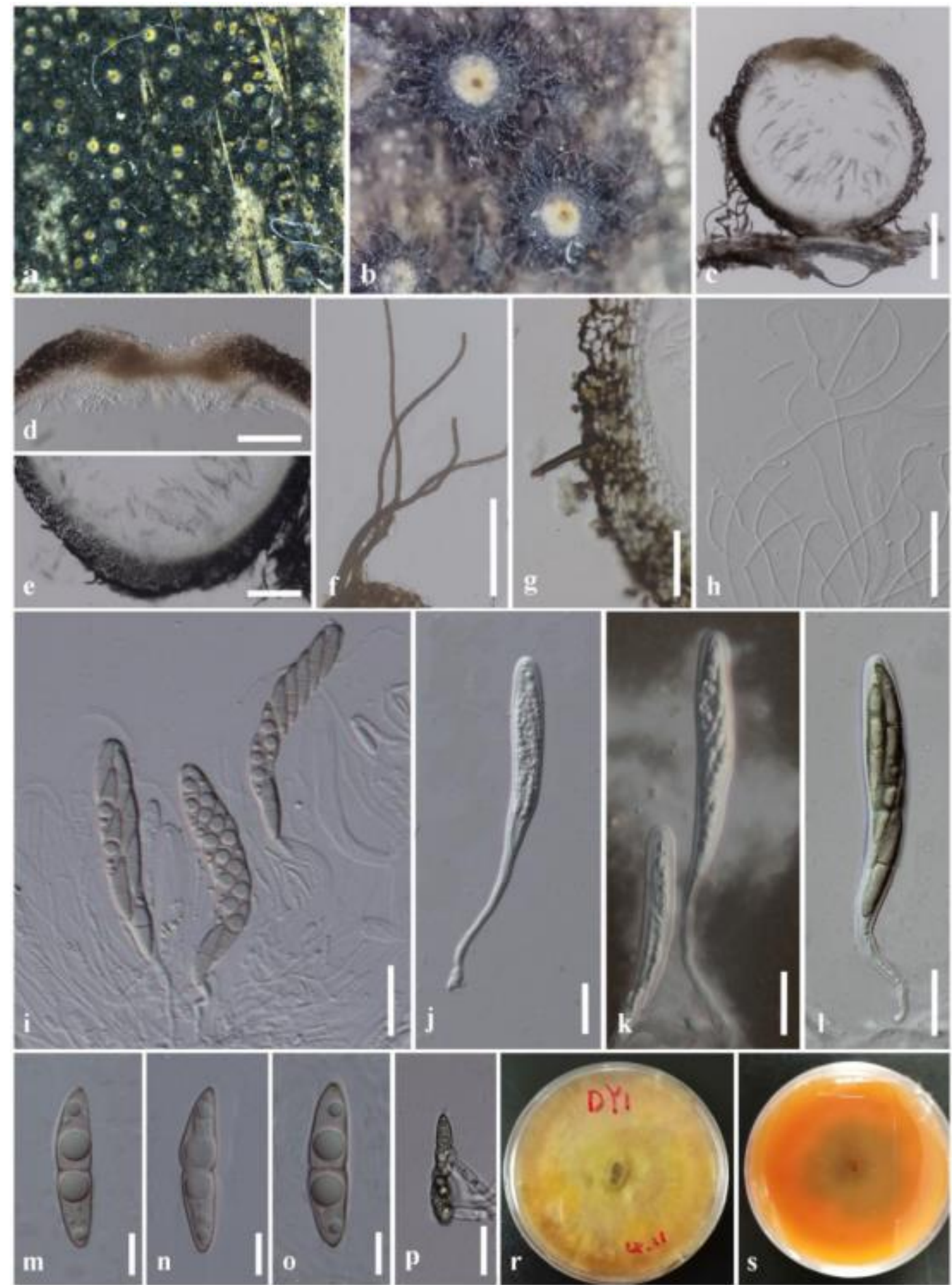

Fig. 62 - Byssosphaeria musae (KUN-HKAS 102550, new host record). a Ascomata on host. b Close-up of ascomata. c Section of ascoma. d Ostiole. e Peridium at the base. f Setae. g Section of peridium at side. $\mathrm{h}$ Pseudoparaphyses. $\mathrm{i}-1$ Asci ( $\mathrm{k}=$ stained in Indian ink). $\mathrm{m}-\mathrm{o}$ Ascospores. $\mathrm{p}$ Germinated ascospore. $\mathrm{r}$ Colony from above. $\mathrm{s}$ Colony from below. Scale bars: $\mathrm{c}=200 \mu \mathrm{m}, \mathrm{d}-\mathrm{f}=$ $100 \mu \mathrm{m}, \mathrm{g}, \mathrm{h}=50 \mu \mathrm{m}, \mathrm{i}=30 \mu \mathrm{m}, \mathrm{j}-1, \mathrm{p}=20 \mu \mathrm{m}, \mathrm{m}-\mathrm{o}=10 \mu \mathrm{m}$.

Culture characteristics - Ascospores germinated on WA within 24 hours. Colonies on MEA, circular, flat surface, wavy margin, reaching $0.2-0.5 \mathrm{~cm}$ diameter in 5 days at $25^{\circ} \mathrm{C}$, at first white to pale yellow, later becoming brown, white and brown at the edge and dark brown at reverse side. 


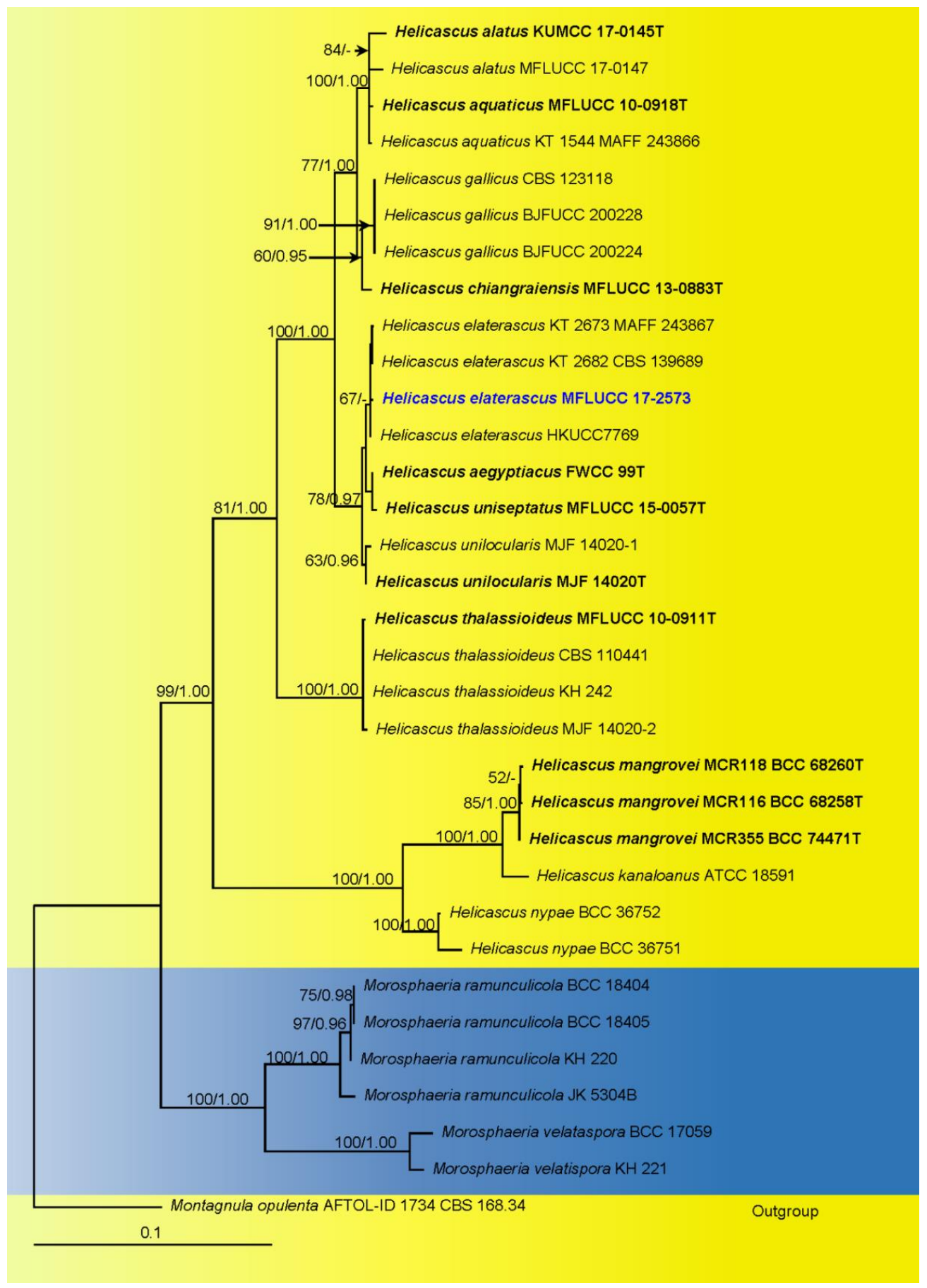

Fig. 63 - Phylogram generated from maximum likelihood analysis based on combined LSU, ITS and tefl sequence data. Thirty-three taxa are included in the combined gene analyses comprising 3022 characters after alignment (1340 characters for LSU, 722 characters for ITS, 960 characters for tef1). Montagnula opulenta (CBS 168.34) is used as the outgroup taxon. The tree topology derived from the Bayesian analysis was similar to that derived from the maximum likelihood analysis. The best RaxML tree with a final likelihood value of -9903.231098 is presented. The matrix had 861 distinct alignment patterns, with $35.34 \%$ undetermined characters or gaps. Estimated base frequencies were as follows: $\mathrm{A}=0.246736, \mathrm{C}=0.232999, \mathrm{G}=0.271140, \mathrm{~T}=$ 0.249124; substitution rates $\mathrm{AC}=0.687317, \mathrm{AG}=1.868367, \mathrm{AT}=1.195520, \mathrm{CG}=0.682068, \mathrm{CT}$ $=5.607541, \mathrm{GT}=1.000000$; gamma distribution shape parameter $\alpha=0.238117$. Bootstrap values for maximum likelihood equal to or greater than 50 and Bayesian posterior probabilities equal or greater than 0.95 are placed above or below the branches. Ex-type strains and/or reference specimens are in bold and black. The newly generated sequence is indicated in blue. 

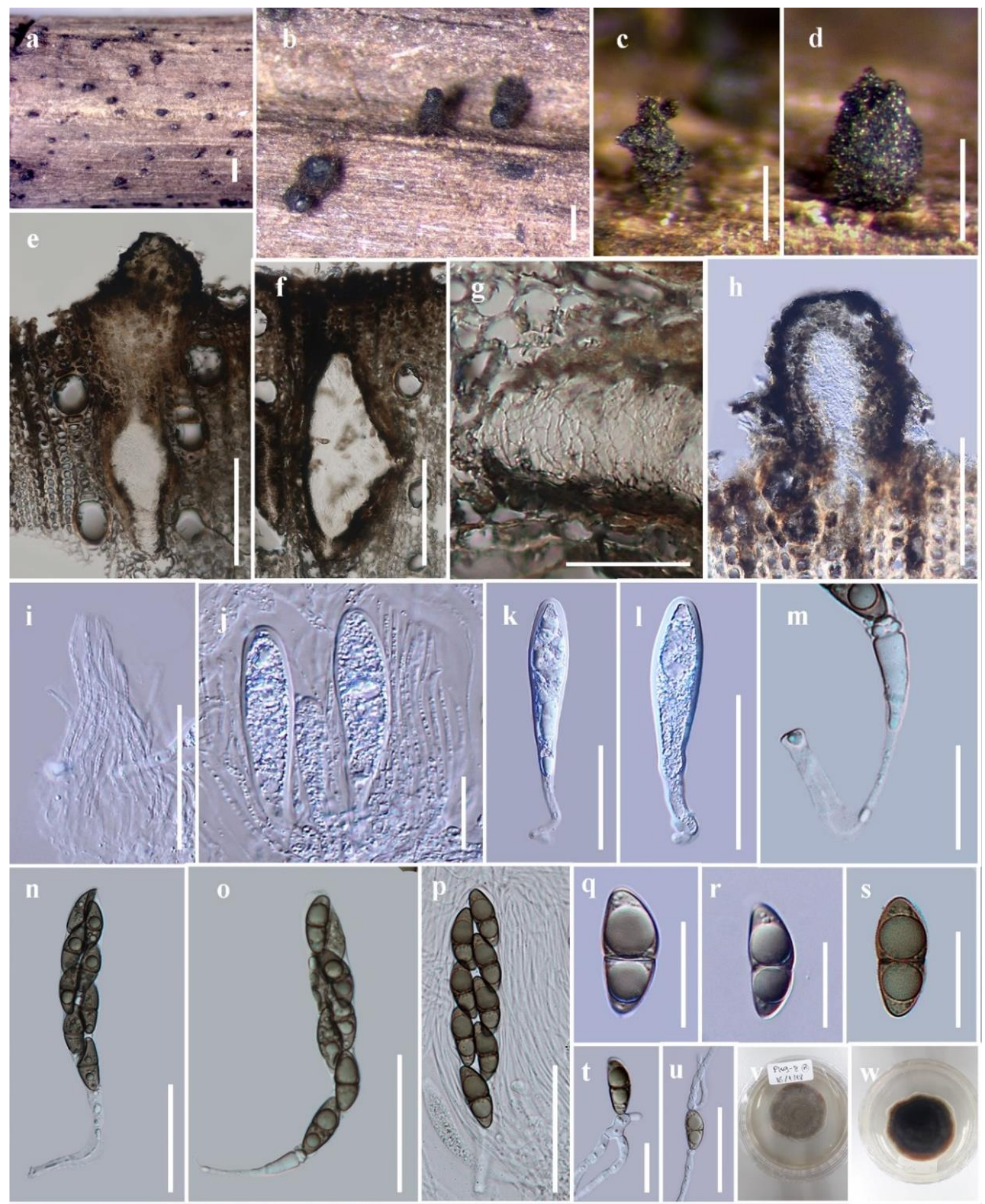

Fig. 64 - Helicascus elaterascus (MFLU 18-1089, new geographical record). a-d Appearance of masses of ascospores on host surface. e, f Section of ascoma. g Peridium, h Ostiole section. i Pseudoparaphyses. j-p Asci. m, o Exotunica of asci showing 'jack-in-a-box' action. q-s Ascospores. $\mathrm{t}, \mathrm{u}$ Ascospore germination. $\mathrm{v}, \mathrm{w}$ Culture on MEA from surface and reverse after 3 weeks. Scale bar: $\mathrm{a}=400 \mu \mathrm{m}, \mathrm{b}-\mathrm{d}, \mathrm{f}=200 \mu \mathrm{m}, \mathrm{e}, \mathrm{g}, \mathrm{i}, \mathrm{k}, \mathrm{l}, \mathrm{n}-\mathrm{p}, \mathrm{u}=50 \mu \mathrm{m}, \mathrm{h}=100 \mu \mathrm{m}, \mathrm{j}, \mathrm{m}, \mathrm{q}-\mathrm{t}=$ $20 \mu \mathrm{m}$.

Known distribution (based on molecular data) - Hong Kong (Kodsueb et al. 2004), Japan (Tanaka et al. 2015), Thailand (this study).

Known substrates (based on molecular data) - On dead wood and submerged twigs of woody plant of unidentified hosts (Tanaka et al. 2015), on submerged wood (Kodsueb et al. 2004, this study). 
Material examined - Thailand, Phayao, on submerged wood in a freshwater stream, 11 September 2017, S. Boonmee, PK98 (MFLU 18-1089, new geographical record); living culture (MFLUCC 17-2573).

GenBank numbers - ITS: MN608548, LSU: MN577417, tef1: MN612111.

Notes - Helicascus elaterascus (MFLUCC 17-2573) was collected from decaying submerged wood in freshwater stream in Phayao Province, Thailand. Our strain shares similarities in ascomata, asci and ascospores with Helicascus elaterascus strains KT 2673, KT 2682 and HKUCC 7769. Multi-gene phylogenetic analysis placed our strain among H. elaterascus strains with 67\% ML support (Fig. 63).

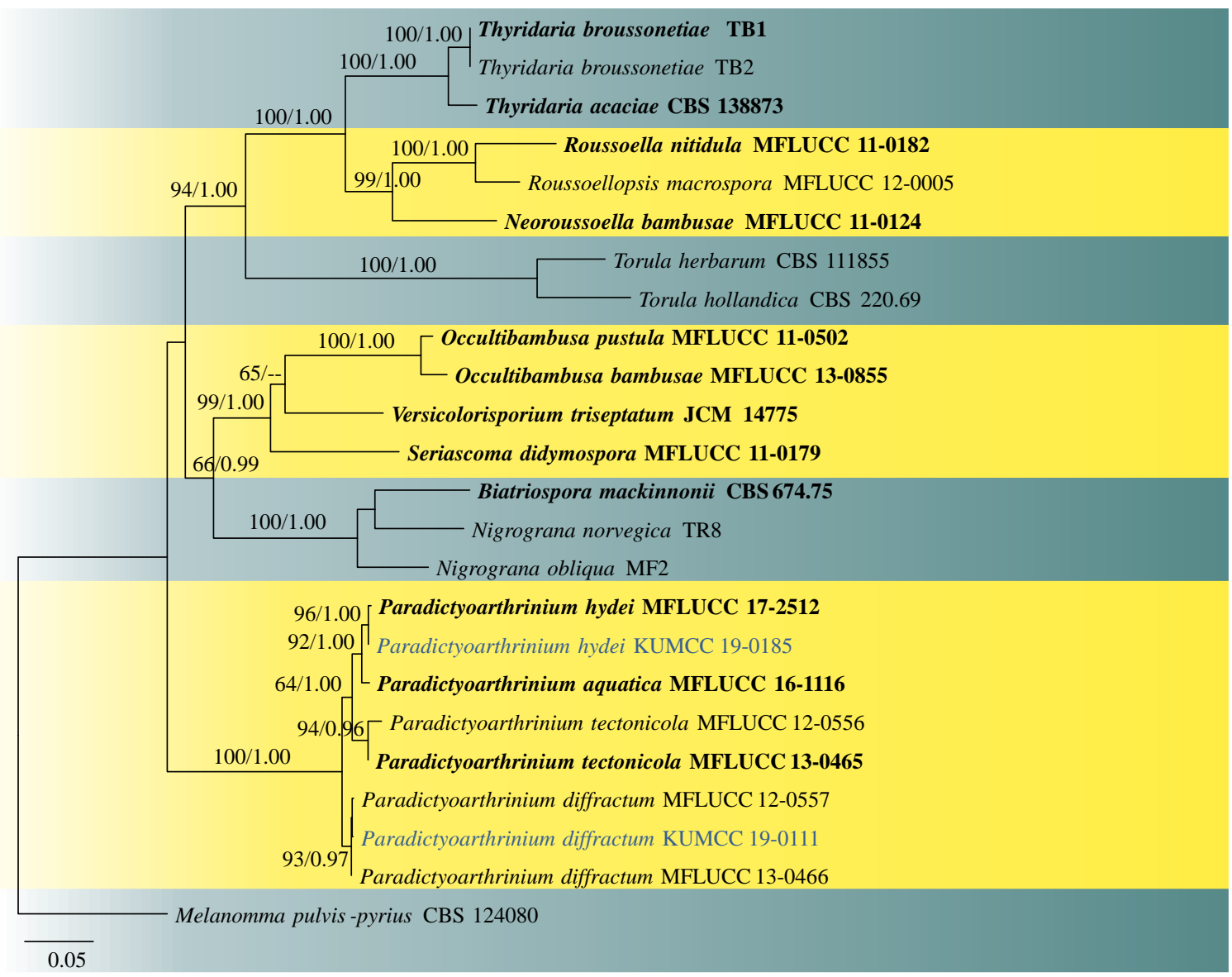

Fig. 65 - Phylogram generated from maximum likelihood analysis based on combined LSU, ITS, and RPB2 sequence data. Twenty-four strains are included in the combined gene analyses comprising 2428 characters after alignment (842 characters for LSU, 633 characters for ITS, 953 characters for RPB2). Melanomma pulvis-pyrius (CBS 124080) is used as the outgroup taxon. The tree topology derived from the Bayesian analysis was similar to that derived from the maximum likelihood analysis. The best RaxML tree with a final likelihood value of -13896.135660 is presented. The matrix had 936 distinct alignment patterns, with $19.07 \%$ undetermined characters or gaps. Estimated base frequencies were as follows: $\mathrm{A}=0.249814, \mathrm{C}=0.243135, \mathrm{G}=0.282173, \mathrm{~T}$ $=0.224878$; substitution rates $\mathrm{AC}=1.382955, \mathrm{AG}=3.921767, \mathrm{AT}=1.529942, \mathrm{CG}=0.881236$, $\mathrm{CT}=8.046891, \mathrm{GT}=1.000000$; gamma distribution shape parameter $\alpha=0.204652$. Bootstrap values for maximum likelihood equal to or greater than 50 and Bayesian posterior probabilities equal or greater than 0.95 are placed above or below the branches. Ex-type strains are in bold and black. The newly generated sequences are indicated in blue.

This species has been reported from submerged wood in a freshwater habitat from Japan and Hong Kong (Tanaka et al. 2015, Kodsueb et al. 2004). In this study, we, therefore, report our strain Helicascus elaterascus (MFLUCC 17-2573) as a new geographical record from northern Thailand. 
Paradictyoarthriniaceae Doilom, Jian K. Liu \& K.D. Hyde

Paradictyoarthriniaceae was established by Liu et al. (2015) to accommodate a single genus Paradictyoarthrinium. Wanasinghe et al. (2018a) introduced another genus Xenomassariosphaeria Jayasiri, Wanas. \& K.D. Hyde which was on dead aerial spines of Rosa canina (Rosaceae).

\section{Paradictyoarthrinium Matsush.}

Paradictyoarthrinium was typified by $P$. diffractum and accommodated in Paradictyoarthriniaceae by Liu et al. (2015). Paradictyoarthrinium is a hyphomycetous genus and includes four species in Index Fungorum (2020) with molecular data confirmed. Paradictyoarthrinium aquatica and $P$. diffractum were collected on submerged wood in freshwater habitat, while $P$. hydei and P. tectonicola were from decaying wood in terrestrial habitats (Liu et al. 2015, 2018a). Until now, there is still no sexual morphs reported in the genus.

Paradictyoarthrinium diffractum Matsush., Matsush. Mycol. Mem. 9:18 (1996)

Fig. 66

Facesoffungi number: FoF01854

Saprobic on dead wood of Mangifera indica. Sexual morph: Undetermined. Asexual morph: Colonies on natural substrate, superficial, black, gregarious, scattered, powdery. Conidiophores up to $25 \mu \mathrm{m}$ long, 2-6 $\mu \mathrm{m}$ wide, macronematous, rarely micronematous, erect to slightly curved, dark brown, slightly constricted at the septa, septate, branched to unbranched, arising from hyphae. Conidiogenous cells holoblastic, integrated, terminal, indeterminate. Conidia 13-28 $\times 9-17(\bar{x}=20$ $\times 13 \mu \mathrm{m}, \mathrm{n}=30$ ), muriform, initially globose to subglobose, brown to dark brown when young, ellipsoidal to irregular in shape, brown to black at maturity, solitary or developing in branched chains, with 1-2-spored short chains, verrucose, very variable in size and shape; circular to irregular with a protruding basal cell; rounded to truncate at the base.

Culture characteristics - Conidia germinating on PDA within $24 \mathrm{~h}$. Germ tubes produced around conidia. Colonies on PDA edge entire, velvety, medium dense, flat or effuse, brownish grey. Mycelium 1.5-3.8 $\mu \mathrm{m}$ broad, partly superficial, partly immersed, pale brown to dark brown, septate. Conidiophores up to $19 \mu \mathrm{m}$ long, 3.5-5.5 $\mu \mathrm{m}$ wide, micronematous, erect to slightly curved, dark brown, constricted at the septa, septate, branched to unbranched, mostly unbranched, arising from laterally and terminally on hyphae. Conidiogenous cells holoblastic, terminal. Conidia $7-24 \times 7-21(\bar{x}=14 \times 13.5 \mu \mathrm{m}, \mathrm{n}=30)$, initially globose to subglobose, ellipsoidal to irregular shape, muriform, brown to dark brown, mostly with 1-spored chains, very variable in size and shape; circular to irregular with a protruding basal cell; rounded to truncate at the base.

Known distribution (based on molecular data) - India, Goa State (Prabhugaonkar and Bhat 2011), Thailand, Chiang Rai Province (Doilom et al. 2017b), China, Yunnan Province (this study).

Known hosts (based on molecular data) - Cocos nucifera (Prabhugaonkar and Bhat 2011), Tectona grandis (Doilom et al. 2017b), Mangifera indica (this study).

Material examined - China, Yunnan Province, Yuxi Prefecture, on dead wood of Mangifera indica (Anacardiaceae), 29 March 2019, M. Doilom (MFLU 19-2366, new host record and new geographical record), living culture (KUMCC 19-0111).

GenBank numbers - ITS: MN582741, LSU: MN582756, RPB2: MN643158.

Notes - Paradictyoarthrinium species are hard to distinguish based on morphological characters. This is particularly true of conidial size which is not a comparative character due to the large variation among species. Species can however, be differentiated by DNA sequence data (Liu et al. 2018). Phylogenetic analysis of combined LSU, ITS and RPB2 sequence data confirmed that our isolate (KUMCC 19-0111) is P. diffractum (Fig. 65). Paradictyoarthrinium diffractum has been reported on a dead twig in a stream from South Africa (holotype), a dead decaying spathe of Cocos nucifera from India, and dead stumps and stems of Tectona grandis in Thailand (Matsushima 1996, Prabhugaonkar and Bhat 2011, Doilom et al. 2017b). This is new host record and new geographical record of $P$. diffractum on Mangifera indica in China. 

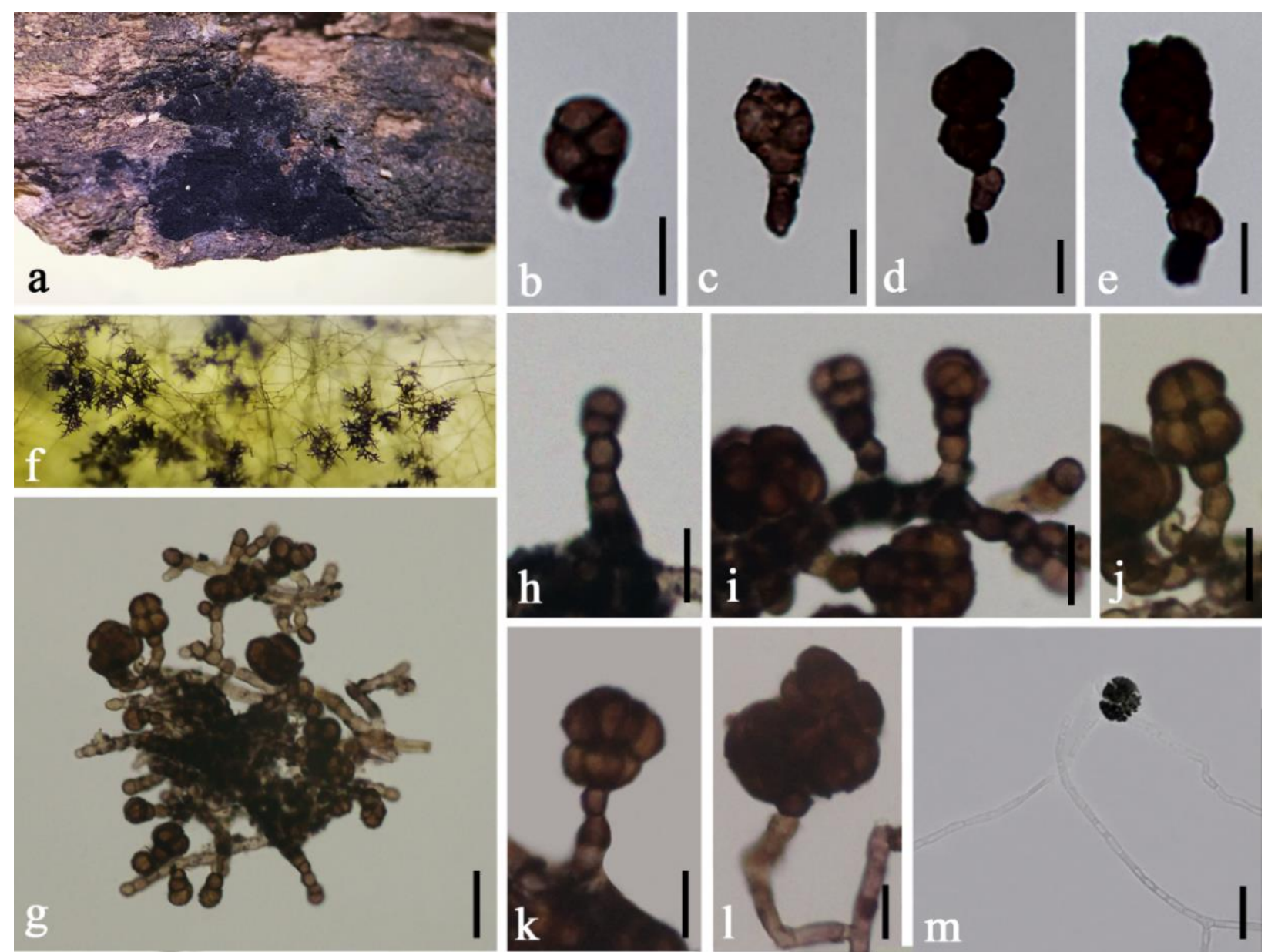

Fig. 66 - Paradictyoarthrinium diffractum )MFLU 19-2366, new host record and new geographical record(. a Conidia observed on host substrate. b-e Conidia on host substrate. f Conidia arising from hyphae on PDA. g-1 Conidia observed on PDA. m Germinated conidium. Scale bars: b-e, h-1 = 10 $\mu \mathrm{m}, \mathrm{g}, \mathrm{m}=20 \mu \mathrm{m}$.

Paradictyoarthrinium hydei N.G. Liu \& J.K. Liu, Phytotaxa 338(3): 290 (2018)

Fig. 67

Facesoffungi number: FoF 03933

Saprobic on dead inner branch of Quercus variabilis. Sexual morph: Undetermined. Asexual morph: Colonies on natural substrate, superficial, black, gregarious, powdery. Mycelium mostly immersed, composed of pale brown to brown, septate, branched hyphae. Conidiophores 3-6 $\mu \mathrm{m}$ diameter, macronematous, rarely micronematous, short or reduced, dark, straight to slightly curved, branched or unbranched, slightly constricted at the septa, uneven, thick-walled. Conidiogenous cells holoblastic, monoblastic, integrated, determinate, terminal, dark. Conidia 14.5-25 $\times 12.5-20$ $\mu \mathrm{m}(\bar{x}=18.5 \times 16 \mu \mathrm{m}, \mathrm{n}=15)$, solitary or developing in chains, muriform, subglobose or irregular, septate, constricted at the septa, dark brown to black, verrucose.

Culture characteristics - Conidia germinating on PDA within 24 hours. Germ tubes produced around conidia. Colonies on PDA edge entire, velvety, dense, effuse, brown to dark grey. Mycelium 1.5-2.5 $\mu \mathrm{m}$ wide, partly superficial, pale brown to dark brown, septate. Conidiophores up to $70 \mu \mathrm{m}$ long, 3-5 $\mu \mathrm{m}$ wide, micronematous, straight or flexuous, olive when mounted in water, redish brown when mounted in lactic acid, septate, constricted at the septa, mostly unbranched, rarely branched, arising from laterally and terminaly on hyphae. Conidiogenous cells monoblastic, terminal. Conidia 9.5-24 ×8-20 ( $\overline{\mathrm{x}}=14 \times 13 \mu \mathrm{m}, \mathrm{n}=20)$, initially subglobose, becoming irregular at maturity, muriform, septate, constricted at the septa, olive when mounted in water, reddish brown when mounted in lactic acid, verrucose. 

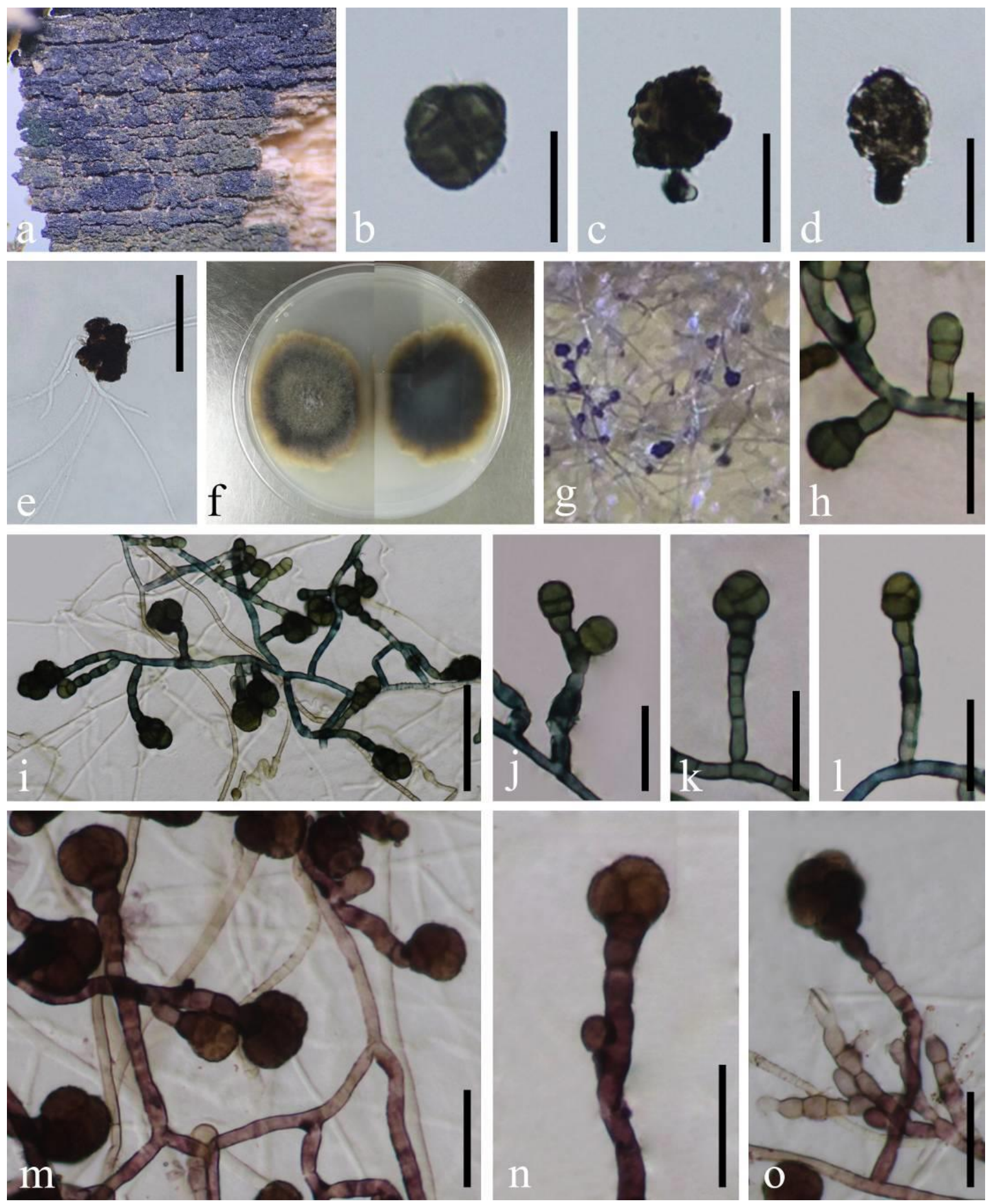

Fig. 67 - Paradictyoarthrinium hydei )MFLU 19-2365, new host record and new geographical record(. a Conidia observed on host substrate. b-d Conidia from host substrate. e Germinated conidium. f Colony on PDA (above and below views). g Conidia arising from hyphae on PDA. $\mathrm{h}-1$ Conidia from culture mounted in water. $\mathrm{m}-\mathrm{o}$ Conidia from culture mounted in $60 \%$ lactic acid. Scale bars: $b=15 \mu \mathrm{m}, \mathrm{c}, \mathrm{d}, \mathrm{h}, \mathrm{j}-\mathrm{O}=20 \mu \mathrm{m}, \mathrm{e}, \mathrm{i}=50 \mu \mathrm{m}$.

Known distribution (based on molecular data) - Thailand, Chiang Mai Province (Liu et al. 2018a), China, Yunnan Province (this study).

Known hosts (based on molecular data) - Quercus variabilis (this study)

Material examined - China, Yunnan Province, Kunming, on dead inner branch of Quercus variabilis (Fagaceae), 10 June 2019, M. Doilom (MFLU 19-2365), living culture (KUMCC 190185). 
GenBank numbers - ITS: MN582742, RPB2: MN643159.

Notes - Our isolate (KUMCC 19-0185) is identified as Paradictyoarthrinium hydei based on the phylogenetic analysis of combined LSU, ITS and RPB2 sequence data (Fig. 5). Paradictyoarthrinium hydei was reported on decaying wood from Thailand (Liu et al. 2018a). This is new host record and new geographical record of $P$. hydei on Quercus variabilis in China. The conidia were observed in the PDA culture and showed two different pigmentations when mounted in water (olive, Figs. h-1) and lactic acid (reddish brown, Figs. m-o).

\section{Phaeosphaeriaceae M.E. Barr}

We follow the latest treatment and updated accounts of Phaeosphaeriaceae in Hyde et al. (2019) and Phookamsak et al. (2019). Eighty-three genera are accepted in this family (Bakhshi et al. 2019, Hyde et al. 2019, Maharachchikumbura et al. 2019, Phookamsak et al. 2019, Yang et al. 2019, Zhang et al. 2019a, Hongsanan et al. 2020).

\section{Leptospora Rabenh.}

Leptospora was introduced by Rabenhorst (1857) and is typified by L. porphyrogona. Rabenhorst (1857) established the genus to accommodate two species, previously described as Sphaeria porphyrogona and $S$. rubella. Recently, 36 species were accepted in this genus (Index Fungorum 2020) but, phylogeny have been investigated for only four species in Phaeosphaeriaceae (Hyde et al. 2016, Zhang et al. 2019a). We follow the latest treatments and updated accounts of Leptospora in Hyde et al. (2016) and Zhang et al. (2019a). Based on molecular analysis, L. rubella is reported from Dipsacus sp. (Caprifoliaceae) in Italy for the first time.

Leptospora rubella (Pers.) Rabenh., Klotzschii Herb. Viv. Mycol., Edn 2: no. 532 (1857)

Fig. 69

Facesoffungi number: FoF02442

Saprobic on Dipsacus sp. Sexual morph: Ascomata 340-395 $\mu$ m high (excluding neck), 175$275 \mu \mathrm{m}$ diameter, scattered, solitary, visible as black spots with reddish brown area around ascomata on host surface, semi-immersed, subglobose to ampulliform, uni-loculate, glabrous, dark brown to black, with a long neck. Neck 170-200 $\mu \mathrm{m}$ high, 80-100 $\mu \mathrm{m}$ diameter, subcarbonaceous, easily broken, cylindrical, slightly narrower towards the apex, ostiole central, filled with hyaline periphyses, producing red pigment at the apex. Peridium 10-30 $\mu \mathrm{m}$ wide, of equal thickness, slightly thin, composed of 3-5 cell layers, of dark brown to black pseudoparenchymatous cells, paler towards the inner layer, arranged in textura angularis to textura prismatica. Hamathecium comprising dense, 2-3 $\mu \mathrm{m}$ wide, hyaline, filamentous, pseudoparaphyses, septate, constricted at the septa, anastomosed above the asci, embedded in a hyaline gelatinous matrix. Asci 144-184.5 × 4.5$6 \mu \mathrm{m}(\bar{x}=162.2 \times 5.2 \mu \mathrm{m}, \mathrm{n}=20), 8$-spored, bitunicate, fissitunicate, numerous, cylindrical to subcylindric-clavate, subsessile to short pedicellate, apically rounded, with well-developed ocular chamber, clearly seen at immature state. Ascospores $155-182 \times 1-3 \mu \mathrm{m}(\bar{x}=168.6 \times 1.5 \mu \mathrm{m}, \mathrm{n}=$ 10), spiraled or twisted in the ascus, pale brown to yellowish brown, filiform, curved, indistinctly multi-septate, smooth-walled, with guttules, tapering to the lower end cell, lacking a mucilaginous sheath. Asexual morph: Undetermined.

Known distribution (based on molecular data) - Colombia (Crous et al. 2006b), UK (Hyde et al. 2016, Vu et al. 2019), France (Vu et al. 2019), Korea (Eo et al. 2014), Slovenia (Hauptman et al. 2013), Italy (this study).

Known hosts (based on molecular data) - Eucalyptus sp. (Myrtaceae), (Crous et al. 2006b), submerged wood in freshwater (Hyde et al. 2016), Ginseng (Eo et al. 2014), Fraxinus excelsior (Hauptman et al. 2013), Dipsacus sp. (Caprifoliaceae) (this study). 


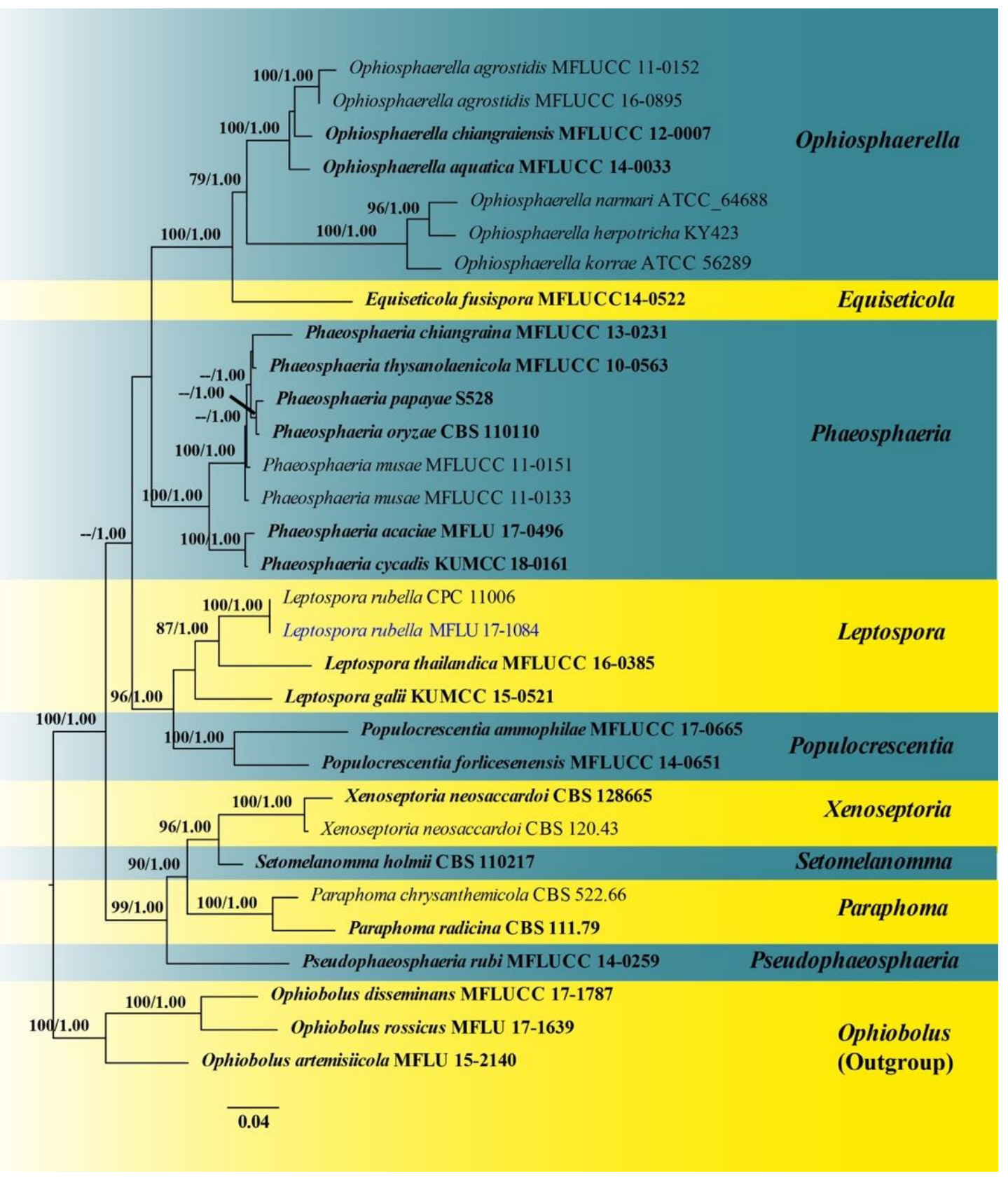

Fig. 68 - Phylogram generated from maximum likelihood analysis based on a combined LSU, SSU, tefl and ITS sequence dataset. Thirty-one strains are included in the combined gene analyses comprising 3,248 total characters including gaps (LSU: 1-820 bp, SSU: 821-1803 bp, tef1: 1804$2677 \mathrm{bp}$, ITS: 2678-3248 bp). The tree topology derived from the Bayesian analysis was similar to that derived from the maximum likelihood analysis. The best scoring of the ML tree is selected to represent the phylogenetic relationships of Leptospora with other related genera in Phaeosphaeriaceae, with the final ML optimization likelihood: -11371.252016. The matrix had 614 distinct alignment patterns, with $15.84 \%$ undetermined characters or gaps. Estimated base frequencies were as follows: $\mathrm{A}=0.244665, \mathrm{C}=0.235963, \mathrm{G}=0.262539, \mathrm{~T}=0.256833$; substitution rates $\mathrm{AC}=1.412087, \mathrm{AG}=3.425246, \mathrm{AT}=3.364101, \mathrm{CG}=0.789417, \mathrm{CT}=8.829065$, $\mathrm{GT}=1.000000$; Tree Length $=1.875762$; gamma distribution shape parameter $\alpha=0.626011$. Bayesian posterior probabilities (BYPP) from MCMC were evaluated with a final average standard deviation of split frequencies of 0.009183. Bootstrap support for maximum likelihood (ML) greater than $70 \%$ and Bayesian posterior probabilities (PP) greater than 0.95 are defined above the nodes as ML/PP. The tree is rooted to Ophiobolus artemisiicola (MFLU 15-2140), O. disseminans (MFLUCC 17-1787) and O. rossicus (MFLU 17-1639). The type strain is indicated in bold and newly generated sequence is shown in blue. 


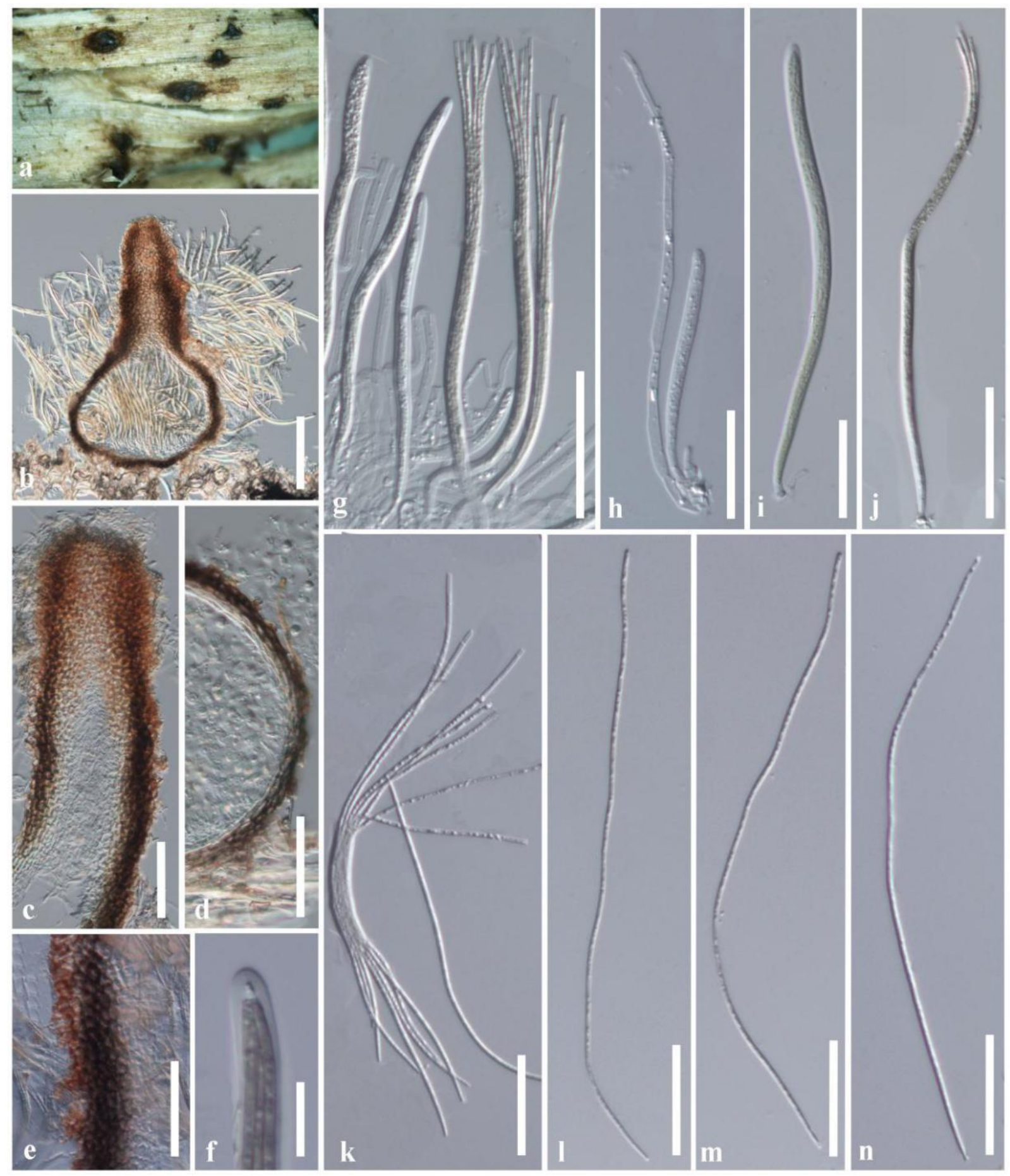

Fig. 69 - Leptospora rubella (MFLU 17-1084, new host record). a Appearance of ascomata on host. b Section of ascoma. c Ostiolar neck. d, e. Peridium. f Conical ocular chamber. g, i, j Asci ( $\mathrm{j}=$ fissitunicate). h Septate pseudoparaphysate. $k-n$ ascospores. Scale bars: $b=100 \mu \mathrm{m}, \mathrm{c}-\mathrm{e}=50 \mu \mathrm{m}$, $\mathrm{f}=10 \mu \mathrm{m}, \mathrm{g}-\mathrm{n}=30 \mu \mathrm{m}$.

Material examined - Italy, Province of Forlì-Cesena [FC], Lago Pontini-Bagno di Romagna, on dead land stem of Dipsacus sp. (Caprifoliaceae), 7 June 2017, E. Camporesi, IT3376 (MFLU 17-1084, new host record).

GenBank numbers - ITS: MN994333, LSU: MN994335, SSU: MN994336, tef1: MT005778.

Notes - Leptospora rubella (Pers.) Rabenh. has been reported from various hosts in European and North American countries as well as India (Shoemaker 1976, Farr \& Rossman 2020) and the species can be easily recognized on the host with the red-purple staining of the host substrate 
(Shoemaker 1976, Hyde et al. 2016, Zhang et al 2019a). However, these studies did not include phylogeny based on DNA sequence data. Hyde et al. (2016) designated the reference specimen of $L$. rubella from decaying submerged wood in the UK. Our collection is similar to L. rubella in having semi-immersed ascomata with short to long necks, producing red pigment on the host substrate, bitunicate, cylindrical asci and pale brown to yellowish brown, filiform, multi-septate ascospores twisted in the ascus (Crous et al. 2006b, Hyde et al. 2016). In the NCBI BLASTn search of ITS sequences, our strain MFLU 17-1084 matches L. rubella (strains CPC 11006 and MFLU 16-0965) with $99.81 \%$ similarity. Phylogenetic analyses of a combined LSU, SSU, tefl and ITS sequence dataset also indicated that our collection belongs to L. rubella (CPC 11006) with high support (100\% ML and 1.00 PP, Fig. 68). We hence, identify our collection as L. rubella, collected from Dipsacus sp. in Italy for the first time.

Pseudoophiobolus Phookamsak, Wanas. \& K.D. Hyde

Pseudoophiobolus was introduced to accommodate ophiobolus-like taxa (Phookamsak et al. 2017). This genus is morphologically and phylogenetically distant from Ophiobolus sensu stricto. Currently, there are eight species (Index Fungorum 2020).

Pseudoophiobolus pseudoitalicus Senan., \& K.D. Hyde sp. nov.

Fig. 71

Index Fungorum number: IF556963; Facesoffungi number: FoF06901 italicus.

Etymology - Species epithets based on its morphological similarity to Pseudoophiobolus

Holotype - MFLU 14-0840

Saprobic on stem of Galium sp. Sexual morph: Ascomata 200-300 $\mu \mathrm{m}$ high (excluding papilla), 300-400 $\mu \mathrm{m}$ diameter, dark brown to black, scattered or rarely clustered, immersed or erumpent through host tissue, visible as raised, small, black dots on the host surface, uni-loculate, subglobose to ampulliform, coriaceous, ostiolate, papillate. Papilla 100-150 ×60-120 $\mu \mathrm{m}$, central, rounded to truncate at the apex, ostiole canal periphysate. Peridium 25-35 $\mu \mathrm{m}$ wide, composed of dark brown to black, thick-walled, cells of textura angularis. Hamathecium comprising numerous, 2-3 $\mu \mathrm{m}$ wide, filamentous, broadly cellular pseudoparaphyses, aseptate, guttulate, embedded in a mucilaginous matrix, anastomosing at the apex. Asci 150-200 $\times 9-13 \mu \mathrm{m}(\bar{x}=180 \times 11 \mu \mathrm{m}, \mathrm{n}=$ 20), 8-spored, bitunicate, cylindrical to cylindric-clavate, short pedicellate, apically rounded with indistinct ocular chamber. Ascospores 115-145 $\times 2-4 \mu \mathrm{m}(\bar{x}=135 \times 3 \mu \mathrm{m}, \mathrm{n}=20)$, fasciculate, parallel, hyaline to olivaceous, scolecosporous, filiform, with or without a swollen cell at the centre, multi-septate, not constricted at the septa, smooth-walled, with small guttules. Asexual morph: Undetermined.

Material examined - Italy, Province of Forlì-Cesena, Fiumicello di Premilcuore, dead aerial stem of Galium sp., E. Camporesi, 20 March 2014, IT1771 (MFLU 14-0840, holotype ex-type living culture MFLUCC 14-0840).

GenBank Numbers - ITS: MN720058, LSU: MN720057.

Notes - The blast results of ITS of our Pseudoophiobolus strain gives $90.86 \%$ similarity with Pseudoophiobolus rosae and $91.4 \%$ similarity with $P$. galii and blast results of LSU gives $99.55 \%$ similarity with Pseudoophiobolus italicus. There are 14 unique base pair differences in the ITS locus. However combined ITS, LSU, SSU and tefl sequence data analysis in this study (Fig. 70) shows that our Pseudoophiobolus strain forms an independent lineage basal to Pseudoophiobolus italicus and $P$. mathieui with moderate support. This taxon also exhibits some similar characters to Pseudoophiobolus italicus (Phookamsak et al. 2017). However it is differs from P. italicus in having globose to subglobose conidiomata and 14-15-septate ascospores swollen at the $6^{\text {th }}$ cell near the basal septum. Therefore, based on morphology and phylogeny, we introduce and describe our strain as a new species, Pseudoophiobolus pseudoitalicus.

Pleosporaceae Nitschke, Verh.

A family of saprotrophic, opportunistic human and plant pathogenic fungi within 
Pleosporales (Ariyawansa et al. 2015). This family was introduced by Nitschke (1869) and is the largest family in Pleosporales which includes more than 16 genera (Wijayawardene et al. 2017).

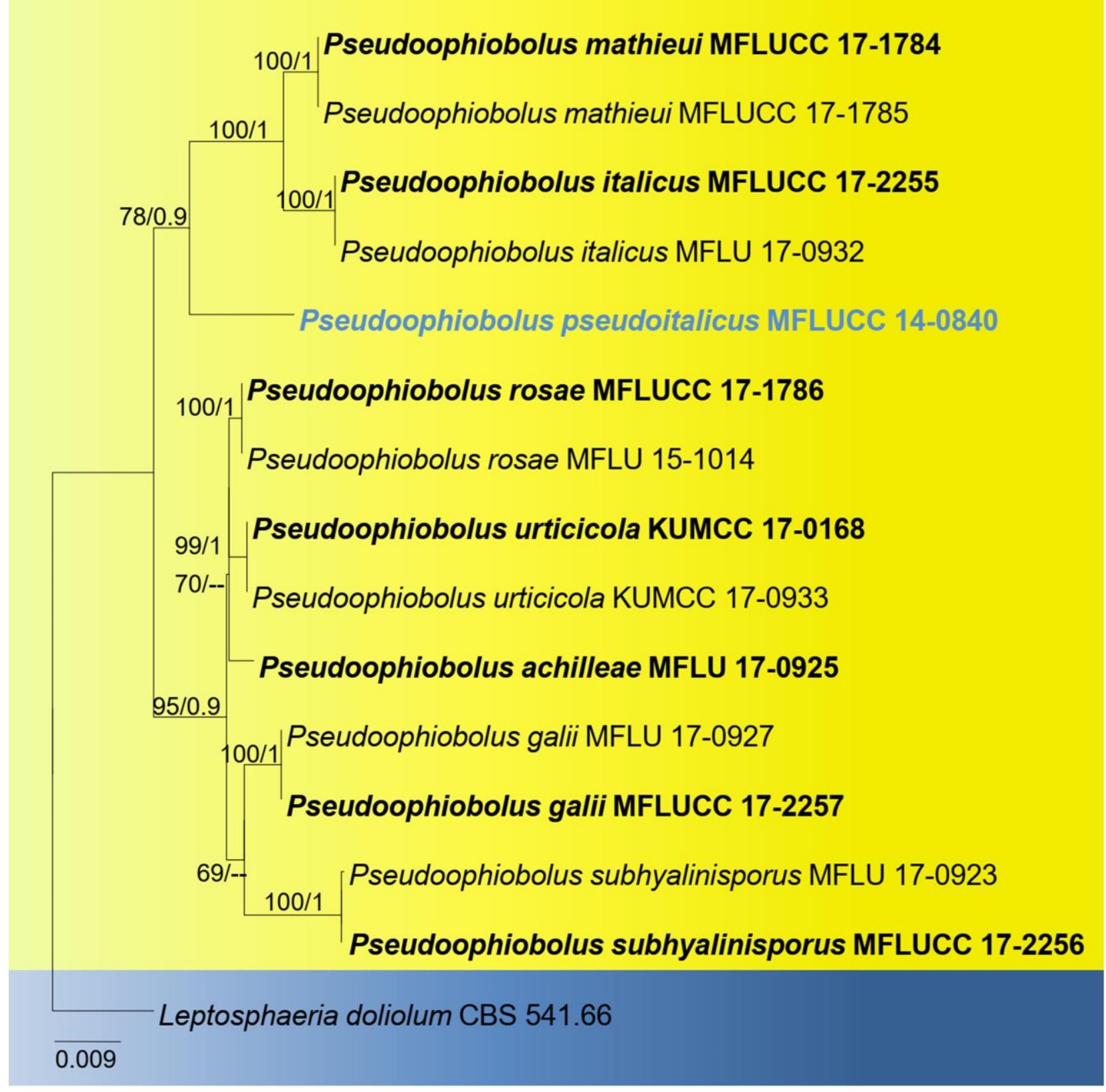

Fig. 70 - Phylogram generated from maximum likelihood analysis based on combined ITS, LSU, SSU and tef1 sequence data. Bootstrap support for ML greater than 50\% and Bayesian posterior probabilities greater than 0.90 are given near nodes. The tree is rooted with Leptospheria doliolum (CBS 541.66). Ex-type strains are in black bold and the newly generated sequence is indicated in blue bold.

Bipolaris Shoemaker

Bipolaris was introduced by Shoemaker (1959). Its sexual morph, Cochliobolus typified by C. heterostrophus, is now linked with the type species of Bipolaris, B. maydis (Rossman et al. 2013). The genus comprises 132 species )Index Fungorum 2020(. 


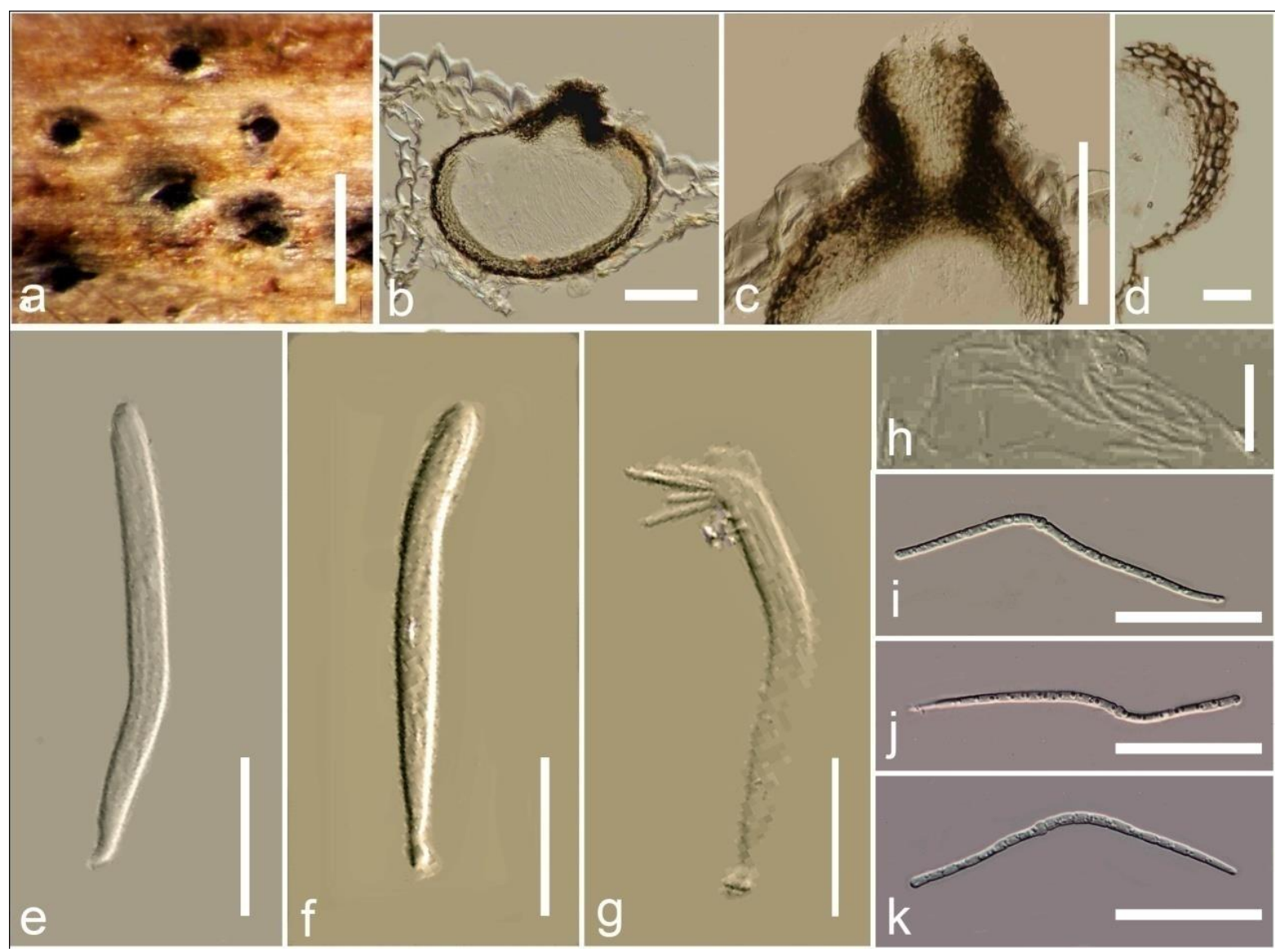

Fig. 71 - Pseudoophiobolus pseudoitalicus (MFLU 14-0840, holotype). a Appearance of ascomata on the host surface. b Section through ascoma. c Section through peridium. $\mathrm{d}-\mathrm{j}$ Asci. k Pseudoparaphyses. 1-r Ascospores. Scale bars: $b=100 \mu \mathrm{m}, \mathrm{c}-\mathrm{e}=50 \mu \mathrm{m}, \mathrm{f}-\mathrm{j}=20 \mu \mathrm{m}$.

Bipolaris yamadae (Y. Nisik.) Shoemaker, Can. J. Bot. 37(5): 884 (1959)

Fig. 73

Facesoffungi number: FoF07377

Leaf spots on Setaria palmiforlia (Poaceae): Small, indefinite, dark brown, eye spots. Asexual morph: Conidiophores 65-170 × 6-8 $\mu \mathrm{m}(\mathrm{n}=20)$, macronematous, mononematous, arising singly or in small groups, simple, septate, sometimes geniculate at upper part, with a swollen basal cell, dark brown. Conidiogenous cells mono- or polytretic, terminal or sympodial Conidia 65-105 × 15-20 $\mathrm{m}(\mathrm{n}=30)$, usually curved, sometimes straight, elliptical, sometimes obclavate, widest at or just below middle, tapering towards the ends, pale brown to dark brown, distoseptate. Sometimes end cells of conidia swell to form more or less global vesicles from which germ tubes originate.

Known distribution (based on molecular data) - Japan (Manamgoda et al. 2014), Taiwan, Chia Yi (this study).

Known hosts (based on molecular data) - Panicum capillare (Manamgoda et al. 2014), Setaria palmiforlia (Poaceae) (this study)

Material examined - Taiwan, Chia Yi Province, Kwang Hwa, Setaria palmiforlia (Poaceae), 27 April 2018, A. Karunarathna AKTW 21 (MFLU 19-2697, new host and geographical record), living culture (NCYUCC 19-0368).

GenBank numbers - ITS - MN982855

Notes - Herein, we provide a new host and geographical record based on morphology and phylogeny. Our strain is morphologically similar to extant $B$. yamadae and phylogeny reveals a close association with B. yamadae CBS 202.29 (Fig. 72). 


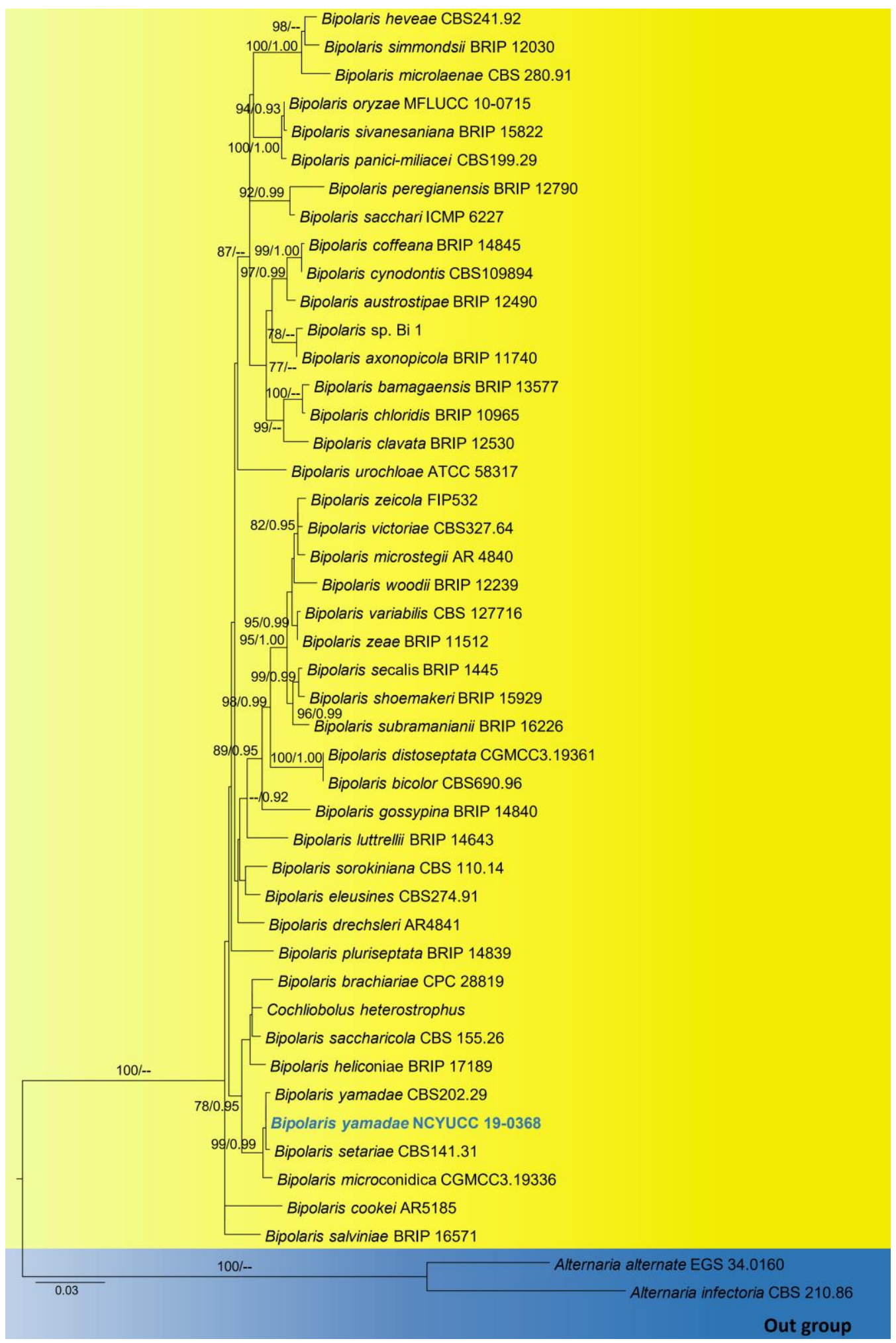

Fig. 72 - Phylogenetic tree generated from maximum likelihood (ML) based on ITS and TUB sequences. Bootstrap support (BS) values above $50 \%$ and Bayesian posterior probabilities equal or greater than 0.90 are shown above the branches at nodes. The tree is rooted with Alternaria alternate and Alternaria infectoria. The best RaxML tree with a final likelihood value of -5166.414243 is presented. The matrix had 415 distinct alignment patterns, with $12.79 \%$ undetermined characters or gaps. Estimated base frequencies were as follows: $\mathrm{A}=0.244996, \mathrm{C}=$ $0.276409, \mathrm{G}=0.226712, \mathrm{~T}=0.251883$; substitution rates $\mathrm{AC}=0.801095, \mathrm{AG}=2.393119, \mathrm{AT}=$ $0.892562, \mathrm{CG}=0.630462, \mathrm{CT}=4.085231, \mathrm{GT}=1.000000$; gamma distribution shape parameter $\alpha$ $=0.865731$. Newly generated sequence is indicated in blue bold. 
Curvularia Boedijn, Bull. Jard.

Curvularia was introduced by Boedijn (1933) and species belong to this genus are pathogenic or saprobes on plant or humans (Manamgoda et al. 2014). Species of Curvularia are characterized by sympodial conidiophores, intercalary and terminal conidiogenous cell (Berbee et al. 1999, Manamgoda et al. 2014, 2015). Presently, more than 100 species are accepted in this genus (Heidari et al. 2018, Tibpromma et al. 2018b).
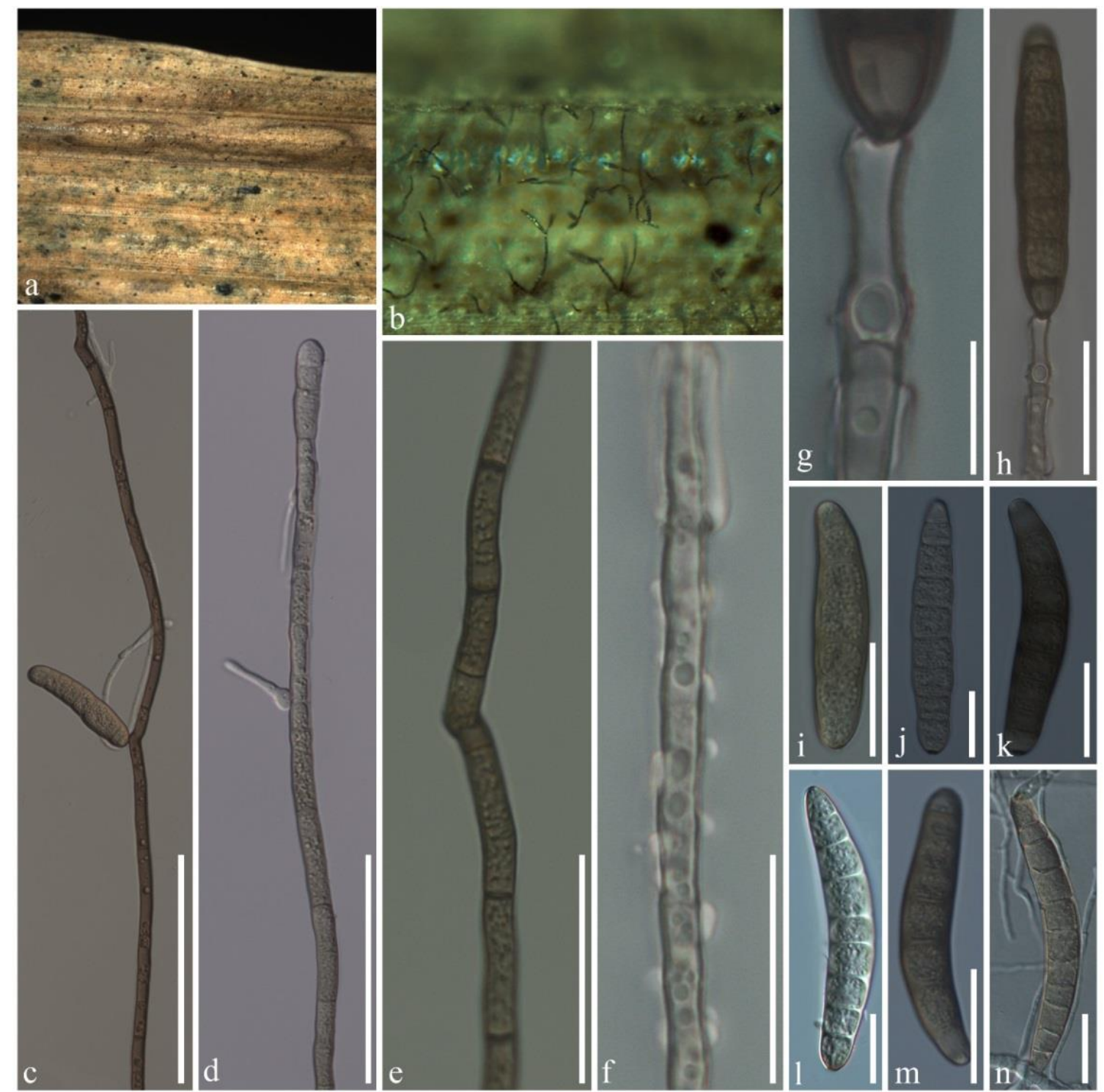

Fig. 73 - Bipolaris yamadae (MFLU 19-2697, new host and geographical record). a Appearance of the host tissue. b Appearance of conidial structures on host. $\mathrm{c}-\mathrm{f}$ Conidiophores. $\mathrm{g}-\mathrm{h}$ Conidial attachments. $\mathrm{i}-\mathrm{m}$ Conidia. $\mathrm{n}$ Germinating conidia. Scale bars: $\mathrm{c}-\mathrm{f}=50 \mu \mathrm{m}, \mathrm{g}-\mathrm{n}=20 \mu \mathrm{m}$.

Curvularia hawaiiensis (Bugnic. ex M.B. Ellis) Manamgoda, L. Cai \& K.D. Hyde, Fungal Diversity 56(1): 141 (2012)

Facesoffungi number: FoF 07051

Fig. 75

Saprobic or pathogenic on plant. Sexual morph: see Manamgoda et al. (2015). Asexual morph: Colonies on PDA fast growing, funiculose to cottony, grey, with a fimbriate margin. Conidiophores $80-160 \times 2-6 \mu \mathrm{m}(\bar{x}=120, \mathrm{n}=10)$, single, flexuous, septate, geniculate, pale to mid brown. Conidiogenous nodes dark brown, polytretic, sympodial. 


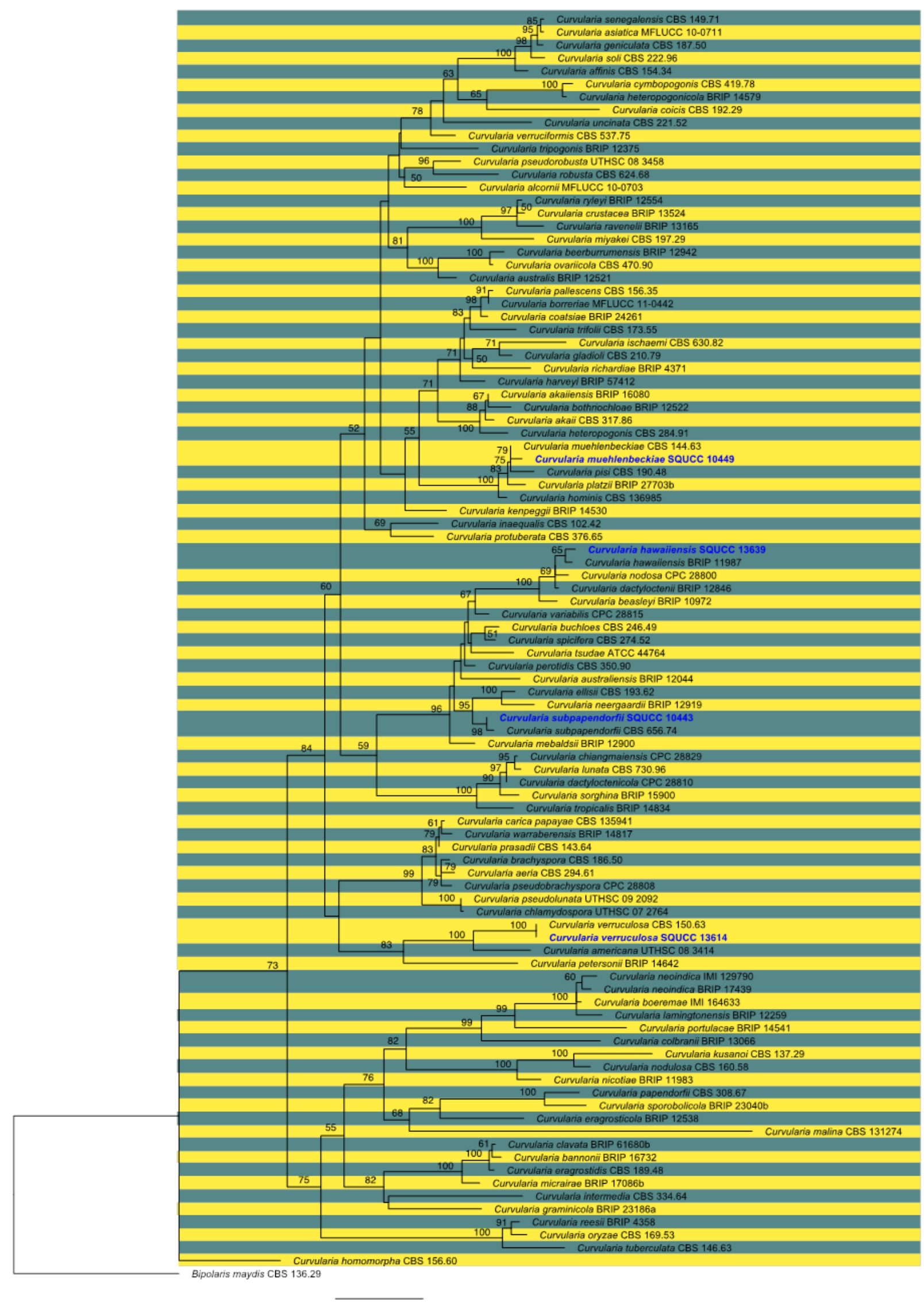

0.04

Fig. 74 - Phylogram generated from maximum likelihood analysis based on combined ITS and GPDH sequence data. Ninety-eight strains are included in the combined gene analyses comprising 1083 characters after alignment (544 characters for ITS, 539 characters for GPDH). Bipolaris 
maydis (CBS 136.29) is used as the outgroup taxon. The newly generated sequences are indicated in blue bold.

Conidia 19-29 × 7.5-10 $\mu \mathrm{m}(\bar{x}=22.5 \times 9, \mathrm{n}=30)$, straight, oblong or cylindrical, rounded at ends, pale to mid brown, 4-7-distoseptate, end cells paler and smooth-walled.

Known distribution - Widely distributed (see Gadallah et al. 1995, Manamgoda et al. 2015, Marin-Felix et al. 2017a, Heidari et al. 2018), Oman (this study). study).

Known hosts - Wide host range (see Manamgoda et al. 2015), Saccharum griffithii (this

Material examined - Oman, Muscat Governorate, Wadi Hoqain, on root of Saccharum griffithii (Poaceae), 12 March 2017, F Al-AbdulSalam, living culture (SQUCC 13639, new host record and geographical record).

GenBank numbers - ITS: MN758886, GPDH: MN781207.

Notes - The morphology of our collection (SQUCC 13639) is similar to the type of Curvularia hawaiiensis. Furthermore, our collection shares a close phylogenetic affinity to $C$. hawaiiensis (BRIP 11987) in the combined ITS and GPDH sequence analyses. This is a new host record and new geographical record of Curvularia hawaiiensis on Saccharum griffithii in Oman.
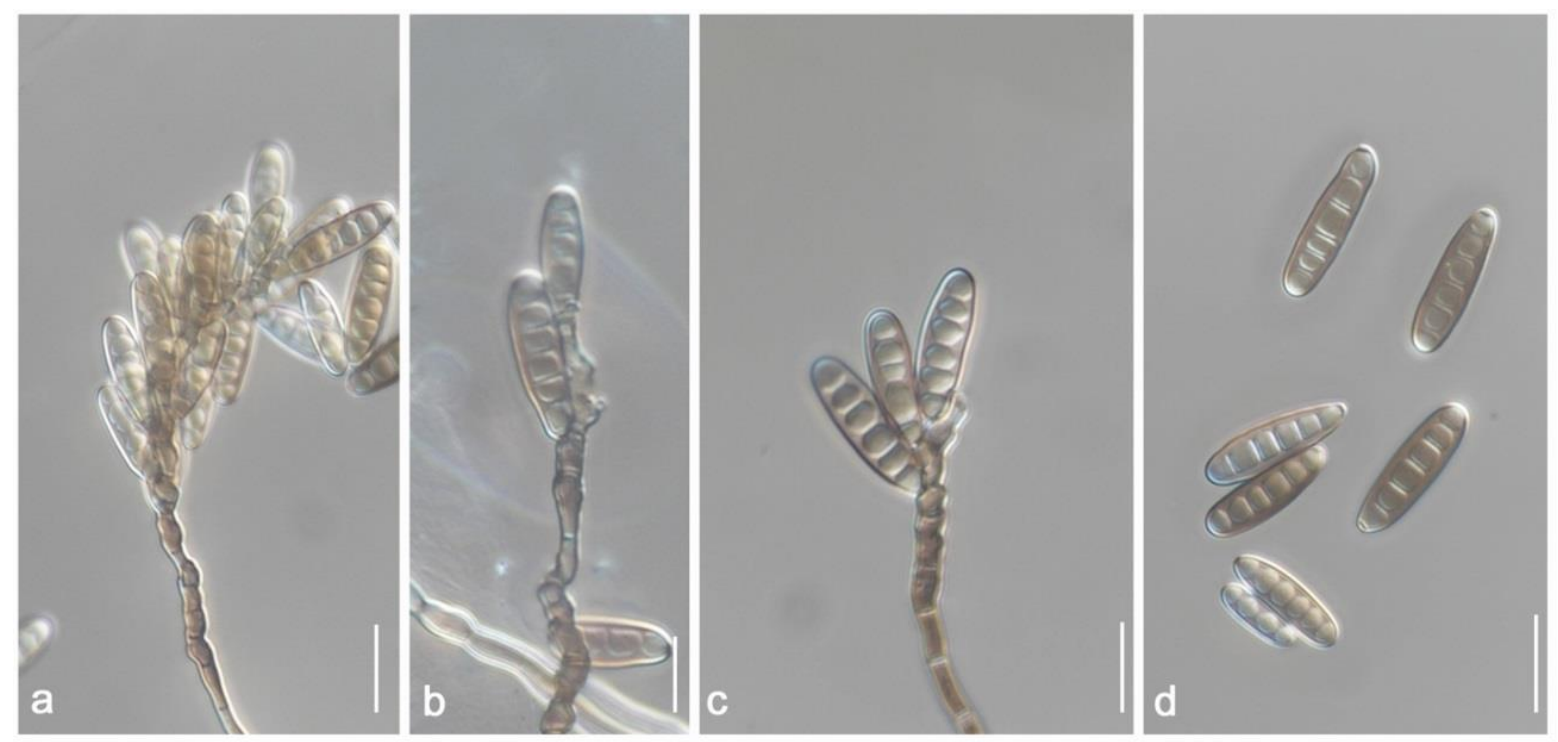

Fig. 75 - Curvularia hawaiiensis (SQUCC 13639, new host record and geographical record(. $\mathrm{a}-\mathrm{c}$ Conidia and Conidiophores on PDA. d Conidia on PDA. Scale bars: $\mathrm{a}-\mathrm{b}=20 \mu \mathrm{m}$

Curvularia muehlenbeckiae Madrid, K.C. Cunha, Gené, Guarro \& Crous, Persoonia 33: 56 (2014)

Fig. 76

Facesoffungi number: FoF07050

Human pathogens or saprobic or pathogenic on plant. Sexual morph: Undetermined. Asexual morph: Colonies on PDA cottony, pale grey at the centre. Hyphae smooth, subhyaline to brown, septate, branched. Conidiophores mononematous, macronematous, septate, simple or rarely branched, straight or curved, geniculate near the apex, pale brown, subnodulose and nodulose intercalary swellings 4-10 $\mu \mathrm{m}$ wide. Conidiogenous cells integrated, mono- or polytretic, terminal, sympodial, light brown. Conidia straight or slightly curved, 3-distoseptate, rounded at both ends; with third cell larger than other cells, distinctly verruculose, $15-25 \mu \mathrm{m}(\overline{\mathrm{x}}=21 \mu \mathrm{m}, \mathrm{n}=30)$ long, 8 $13 \mu \mathrm{m}(\overline{\mathrm{x}}=10.5 \mu \mathrm{m}, \mathrm{n}=30)$ wide; end cells paler and smooth-walled.

Known distribution - Australia, India, Oman, USA (Madrid et al. 2014), Oman (this study).

Known hosts - Muehlenbeckia sp., Oryza sp., culture from human chest (Madrid et al. 2014), Citrus $\times$ aurantiifolia (this study). 
Material examined - Oman, Muscat Governorate, Al-Seeb, Al-Sahwa park, on root of Citrus $\times$ aurantiifolia (Rutaceae), January 2016, HHA Al-Nadabi, living culture (SQUCC 10447, new host record and geographical record).

GenBank numbers - ITS: MN758887, GPDH: MN781208

Notes - Curvularia muehlenbeckiae was recently introduced by Madrid et al. (2014) from the leaf of Muehlenbeckia sp. collected in India. Our collection from Citrus $\times$ aurantiifolia has a similar morphology to the holotype of Curvularia muehlenbeckiae. Furthermore, the multiphylogenetic analysis based on the combined sequence data of ITS and GPDH depicts that our strain (SQUCC 10447) clusters with the ex-type of C. muehlenbeckiae with moderate bootstrap support (79\% ML). Therefore, we confirm our collection as $C$. muehlenbeckiae, and this is the first record of $C$. muehlenbeckiae on Citrus $\times$ aurantiifolia and from Oman.
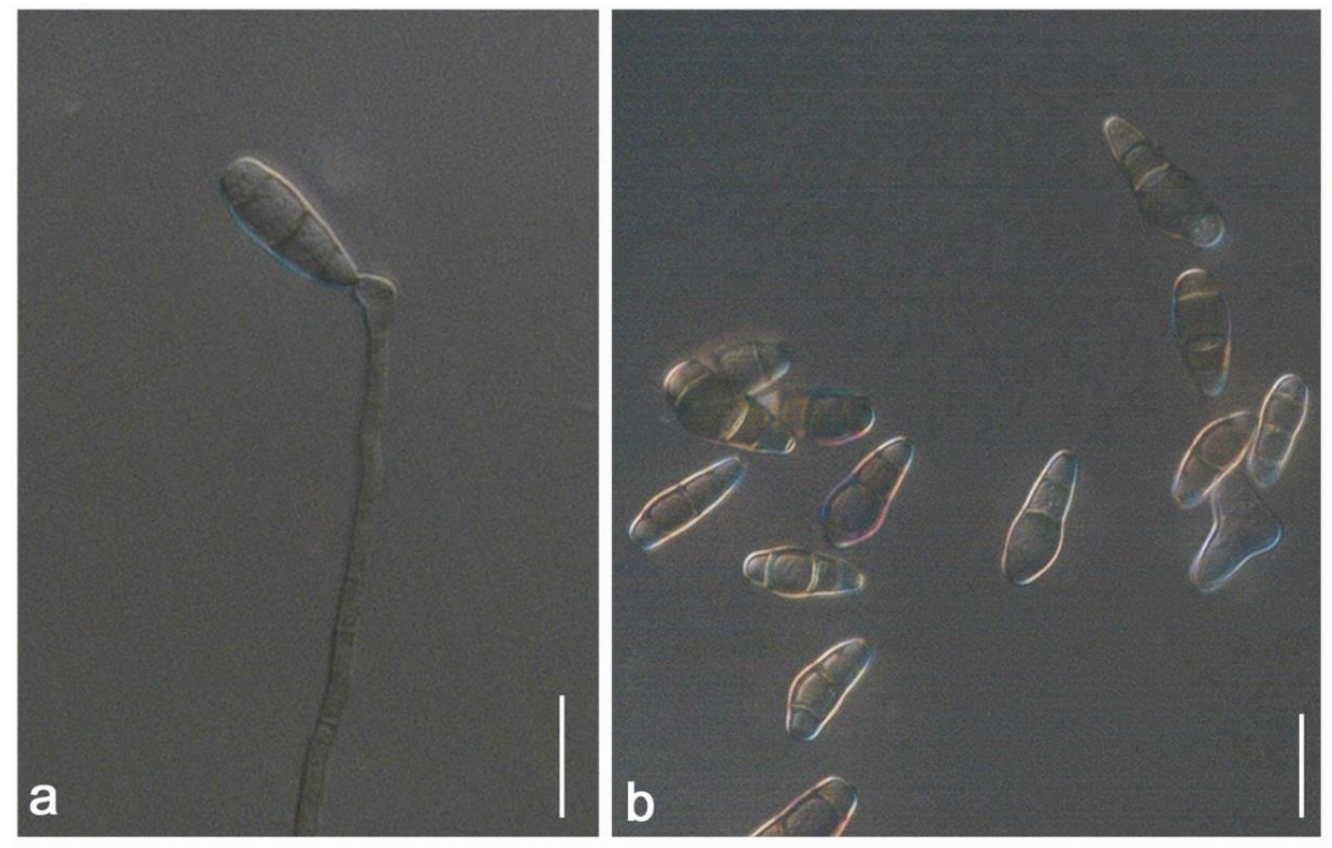

Fig. 76 - Curvularia muehlenbeckiae (SQUCC 10447, new host record and geographical record(. a Conidia and Conidiophores on PDA. $b$ Conidia on PDA. Scale bars: $a-b=20 \mu \mathrm{m}$.

Curvularia subpapendorfii (Mouch.) Manamgoda, Rossman \& K.D. Hyde, Stud. Mycol. 79: 282 (2014)

Facesoffungi number: FoF07052

Fig. 77

Saprobic on plant or soil Sexual morph: Undetermined. Asexual morph: Colonies on PDA fast-growing, funiculose, grey, margin fimbriate. Conidiophores 160-275 $\times 4-8 \mu \mathrm{m}(\bar{x}=159 \times 15$, $\mathrm{n}=15$ ), arising singly, simple or branched, flexuous, septate, geniculate at spore bearing part, dark brown, paler towards apex. Conidiogenous nodes polytretic, sympodial, dark brown, smooth, with cicatrized pores. Conidia 17-25 × 10-14 $(\bar{x}=21 \times 12, \mathrm{n}=25)$, smooth-walled, obpyriform, tapering towards rounded ends, pale brown to dark reddish brown, 3-septate. Hilum usually inconspicuous or sometimes slightly protuberant.

Known distribution - India (Manamgoda et al. 2015), Oman (this study).

Known hosts - Desert soil, Phaseolus aconitifolius (Manamgoda et al. 2015), Citrus $\times$ aurantiifolia (this study).

Material examined - Oman, Muscat Governorate, Al-Seeb, Al-Sahwa park, on root of Citrus $\times$ aurantiifolia (Rutaceae), January 2016, HHA Al-Nadabi, living culture (SQUCC 10443, new host record and geographical record).

GenBank numbers - ITS: MN758885, GPDH: MN781206.

Notes - Manamgoda et al. (2014) designated a lectotype for Curvularia subpapendorfii for a sample reported from desert soil in Egypt (ex-type culture CBS 656.74). Our collection from the 
root of Citrus $\times$ aurantiifolia (SQUCC 10443) is closely related to the ex-type culture (CBS 656.74) of $C$. subpapendorfii. In addition, conidiophores and conidial size of our specimen are similar to the description of the type material. Thus we identified our collection as $C$. subpapendorfii, and this is the first report of C. subpapendorfii on Citrus $\times$ aurantiifolia in Oman.
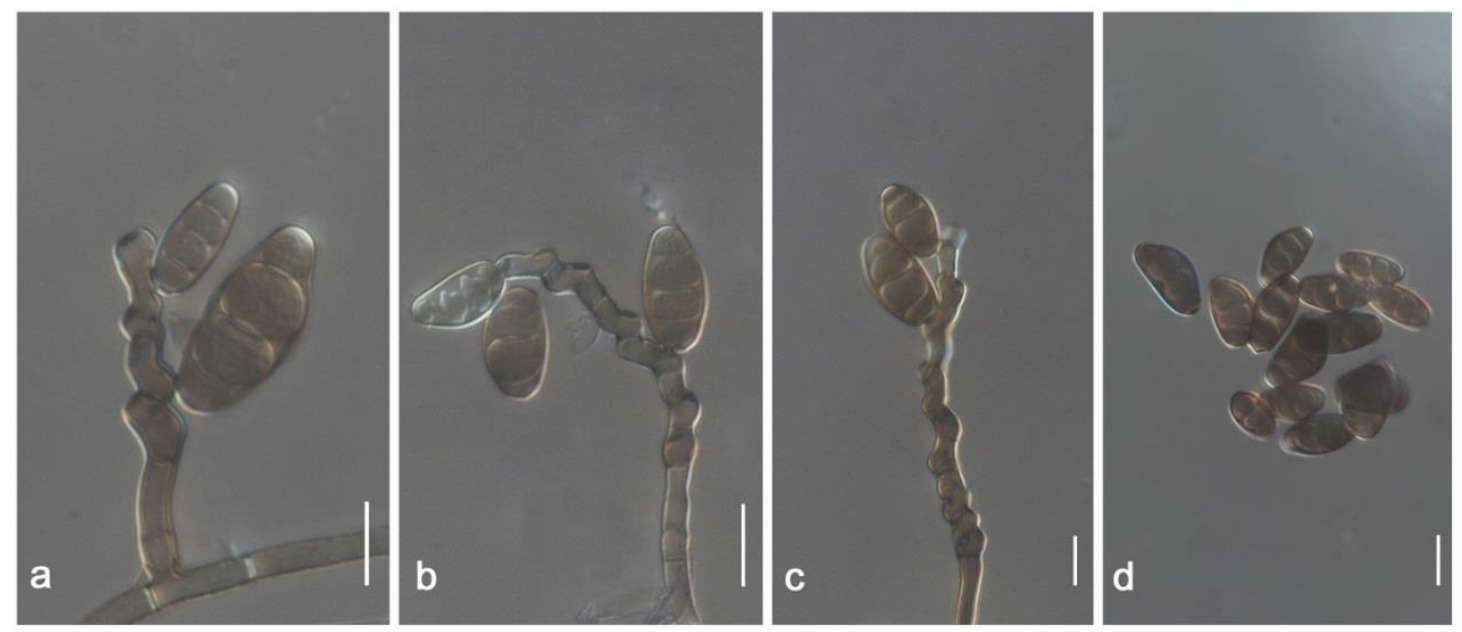

Fig. 77 - Curvularia subpapendorfii (SQUCC 10443, new host record and geographical record(. $\mathrm{a}-\mathrm{c}$ Conidia and Conidiophores on PDA. $\mathrm{d}$ Conidia on PDA. Scale bars: $\mathrm{a}-\mathrm{b}=20 \mu \mathrm{m}$.

Curvularia verruculosa Tandon \& Bilgrami ex M.B. Ellis, Mycol. Pap. 106: 20 (1966)

Facesoffungi number: FoF 00571

Fig. 78

Human pathogens or saprobic or pathogenic on Poaceae. Sexual morph: Undetermined. Asexual morph: Colonies on PDA superficial, effuse, scattered, dark brown. Hyphae smooth, subhyaline, septate, branched. Conidiophores mononematous, macronematous, septate, simple or rarely branched, straight or curved, geniculate near the apex, pale brown, 7-9-septate, nodose, 115$230 \mu \mathrm{m}(\bar{x}=150 \mu \mathrm{m}, \mathrm{n}=15)$ long, 4-8 $\mu \mathrm{m}(\bar{x}=6 \mu \mathrm{m}, \mathrm{n}=15)$ wide. Conidiogenous cells terminal or intercalary, polytretic, sympodial, light brown, Conidia straight or slightly curved, 3distoseptate, rounded at both ends; with third cell larger than other cells, distinctly verruculose, 20 $32 \mu \mathrm{m}(\overline{\mathrm{x}}=25 \mu \mathrm{m}, \mathrm{n}=30)$ long, $10-16 \mu \mathrm{m}(\bar{x}=13.5 \mu \mathrm{m}, \mathrm{n}=30)$ wide, pale to dark brown.

Known distribution - Australia, China, India, Nigeria, Oman, Pakistan, Thailand (Madrid et al. 2014, Manamgoda et al. 2015, Su et al. 2015), Oman (this study).

Known hosts - Various monocotyledons and dicotyledons plants (Manamgoda et al. 2015), grass (this study).

Material examined - Oman, Muscat Governorate, Al-Seeb, Al-Sahwa park, on unidentified dead grass (Poaceae), 12 March, 2017, SSN Maharachchikumbura, living culture (SQUCC 13614, new geographical record).

GenBank numbers - ITS: MN758884, GPDH: MN781205.

Notes - Our collection SQUCC 13614 morphologically resembles the type description of the Curvularia verruculosa. Furthermore, phylogenetically our collection forms a strongly supported clade with the epitype CBS 150.63 of $C$. verruculosa (Fig. 74), which was collected from leaves of Punica granatum in India. This is the first report of $C$. verruculosa from the Arabian Peninsula.

\section{Pyrenophora Fr.}

Pyrenophora typified by Pyrenophora phaeocomes was introduced by Rebentisch (1804). The absence of pseudoparaphyses and smaller ascospore dimensions distinguishes Pyrenophora from other genera of Pleosporaceae (Sivanesan 1984, Ariyawansa et al. 2014b). The asexual morph is Drechslera (Zhang \& Berbee 2001, Ariyawansa et al. 2014b, Marin-Felix et al. 2019). Several Pyrenophora species are considered severe pathogens (Tekauz 1983, Lamari \& Bernier 1989, 
Kingsland 1991, Gupta \& Loughman 2001, Ariyawansa et al. 2014b). Owing to its widespread pathogenicity, the development of molecular genetic markers for rapid identification of Pyrenophora species have been detailed in Moreno et al. (2011). Most recently Marin-Felix et al. (2019) accepted 27 Pyrenophora species with molecular data based on a combined ITS, LSU, GAPDH and RPB2 gene sequence data analysis.
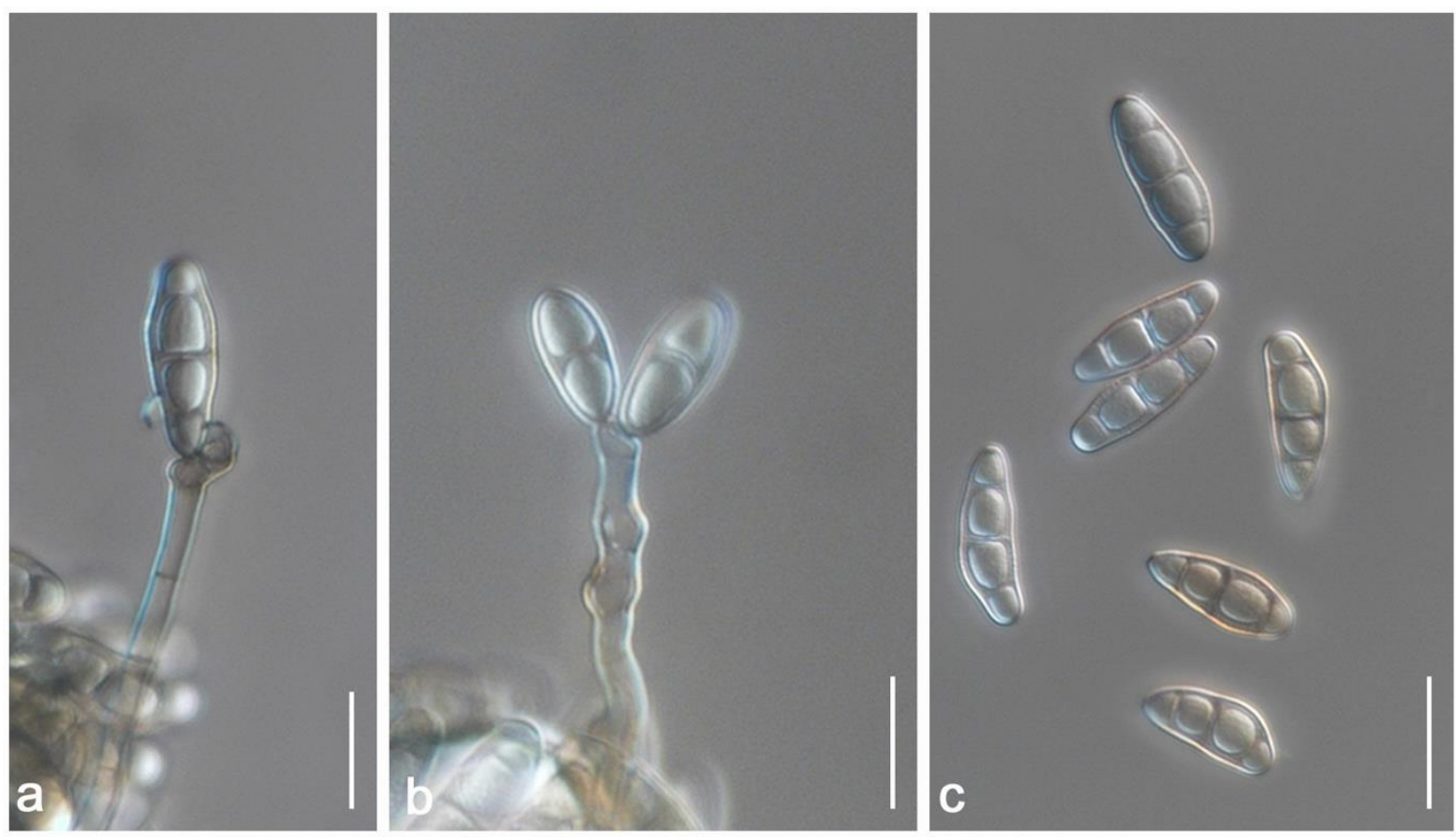

Fig. 78 - Curvularia verruculosa (SQUCC 13614, new geographical record(. a-b Conidia and Conidiophores on PDA. c Conidia on PDA. Scale bars: $\mathrm{a}-\mathrm{c}=20 \mu \mathrm{m}$.

Pyrenophora trichostoma (Fr.) Fr., Jb. nassau. Ver. Naturk. 23-24: 215 (1870) [1869-70]

Fig. 80

Facesoffungi number: FoF06984

Saprobic or biotrophic on dead stems and leaves of Bothriochloa ischaemum. Sexual morph: Ascomata 350-450 $\mu \mathrm{m}$ high 250-300 $\mu \mathrm{m}$ diameter $(\mathrm{n}=10)$, black, immersed to semi-immersed or superficial, solitary or in small groups, uniloculate, globose to subglobose, visible as black dome shaped structures on the host surface, conspicuous, surrounded by dark brown setae, glabrous, without papillate, black. Ostiole short, rounded, immersed in ascomata, with a pore-like opening. Peridium 10-17 $\mu \mathrm{m}$ wide, thin, 3-5-layered of cells of outer dark brown textura angularis, and inner pale brown flattened textura angularis to textura prismatica. Hamathecium consisting of irregular-shaped cellular matter, pseudoparaphyses absent. Asci 40-102 ×6-15 $\mu \mathrm{m}(\bar{x}=70 \times 10$ $\mu \mathrm{m}, \mathrm{n}=15), 8$-spored, bitunicate, fissitunicate, broadly cylindric-clavate, slightly curved, with a bilobed, short pedicel, apex rounded with a well-distinct ocular chamber, smooth-walled. Ascospores 45-85 $\times 15-35 \mu \mathrm{m},(\bar{x}=55 \times 23 \mu \mathrm{m}, \mathrm{n}=40)$, overlapping biseriate, initially hyaline, becoming pale to golden brown at maturity, muriform, mostly ellipsoidal, with 3 transverse septa, with 1-2 longitudinal septa, constricted at the septa, asymmetrical, end cells slightly lighter, conical and broadly to narrowly rounded at the ends, surrounded by a thick mucilaginous sheath. Asexual morph: Undetermined.

Culture characteristics - Colonies on PDA reaching approximately $2.5 \mathrm{~cm}$ diameter after 7 days at $16-18^{\circ} \mathrm{C}$, pinkish-white, dense mycelium, circular, flat, with smooth margins, becoming orangish-red and reverse brown. 


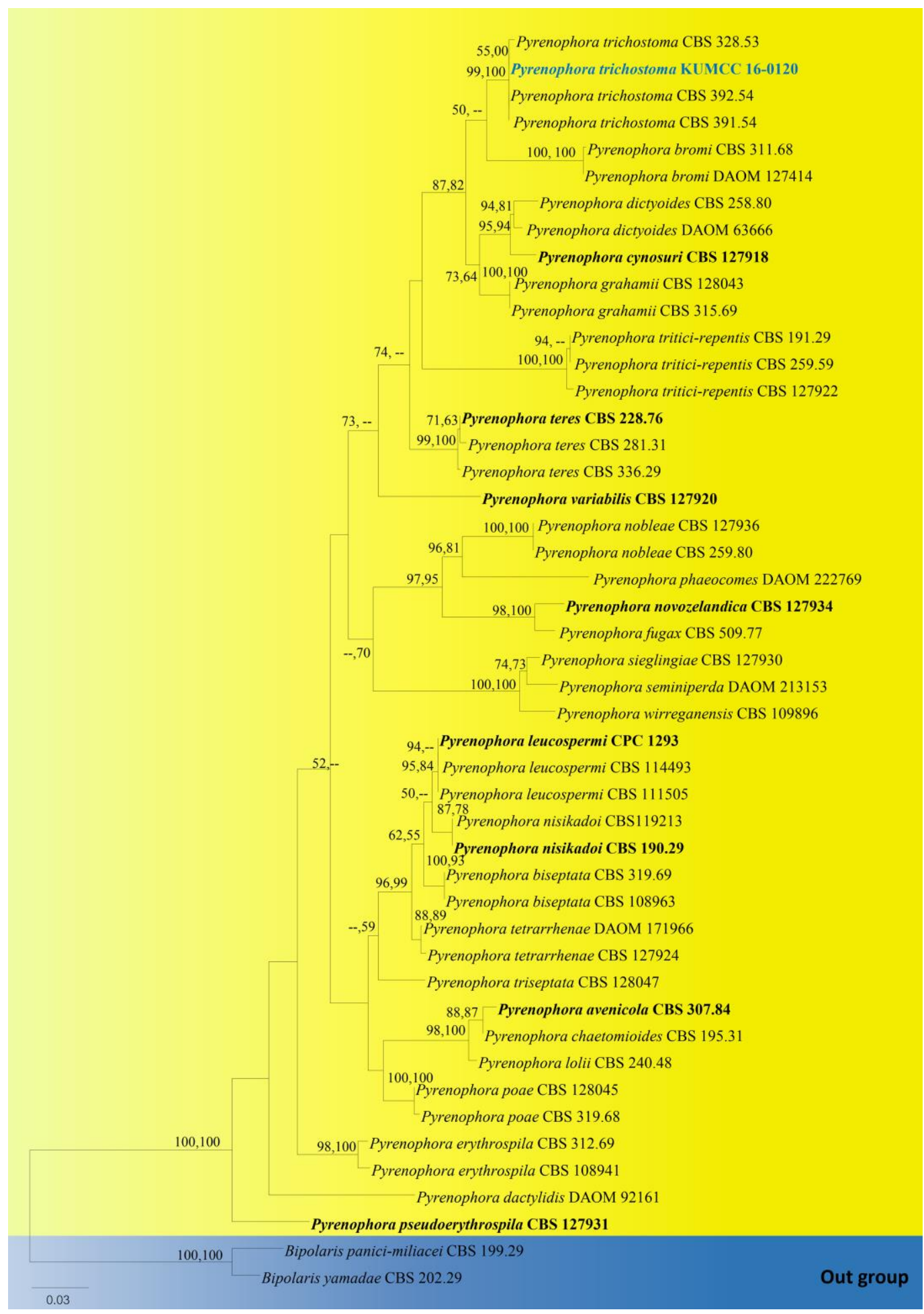

Fig. 79 - Phylogram generated from maximum likelihood analysis based on combined ITS and LSU sequence data. Forty-seven strains are included in the combined gene analyses comprising 1779 characters after alignment (868 characters for ITS, 911 characters for LSU). Bipolaris panicimiliacei (CBS 202.29) and Bipolaris yamadae (CBS 199.29) are used as outgroup taxa. The best RaxML tree with a final likelihood value of -7099.358315 is presented. The matrix had 466 distinct alignment patterns, with $13.76 \%$ undetermined characters or gaps. Estimated base frequencies were as follows: $\mathrm{A}=0.245308, \mathrm{C}=0.229294, \mathrm{G}=0.279135, \mathrm{~T}=0.246263$; substitution rates $\mathrm{AC}$ $=1.382376, \mathrm{AG}=1.398715, \mathrm{AT}=1.182859, \mathrm{CG}=0.745659, \mathrm{CT}=3.139154, \mathrm{GT}=1.000000 ;$ gamma distribution shape parameter $\alpha=0.108634$. Bootstrap values for ML and MP equal to or greater than 50 are placed above or below the branches. Ex-type strains are in bold and black. The newly generated sequence is indicated in blue. 
Known distribution (based on molecular data) - USA, Kansas State (Vu et al. 2019), Russia (this study).

Known hosts (based on molecular data) - Bothriochloa ischaemum (this study).

Material examined - Russia, Rostov region, Shakhty City, 20th anniversary of Red Army microdistrict, Solyonaya Balka (Salty gully), on dying stems and leaves of Bothriochloa ischaemum (L.) Keng (Poaceae), 21 May 2015, Timur S. Bulgakov, T-336 (MFLU 15-2040, new host record), living culture KUMCC 16-0120.

GenBank numbers - LSU MN733192, ITS MN736436.

Notes - Pyrenophora species are characterized by immersed to semi-immersed ascomata with necks surrounded by brown to reddish-brown setae, absence of pseudoparaphyses, clavate to saccate asci with large apical rings, and muriform, terete ascospores (Ariyawansa et al. 2014b, Marin-Felix et al. 2019). Fuckel (1870) described P. trichostoma as having $330 \mu \mathrm{m}$ long, $40 \mu \mathrm{m}$ wide, 8-spored, asci, with broad-oblong, yellow, asymmetric, muriform ascospores that are 4-6 septate, constricted at the mid septum, $52 \times 20 \mu \mathrm{m}$ (translated from Latin). In the specimen illustrated here, we observed $300 \times 54 \mu \mathrm{m}$, broadly cylindric-clavate and slightly curved asci with asymmetric, broad, muriform, $55 \times 23 \mu \mathrm{m}$ ascospores. Therefore, we conclude that our species is morphologically similar to $P$. trichostoma. Pyrenophora trichostoma was once synonymized under $P$. tritici-repentis based on their morphological similarities (Ciuffetti \& Tuori 1999) such as producing ascospores with mucilaginous sheaths (Sivanesan 1984). However, in their phylogenetic analysis, Kodsueb et al. (2006) discussed that despite their phylogenetic affinities, there were several base pair differences in the sequences of these two species. In our phylogenetic analysis we have included three strains CBS. 328.53, CBS 391.54 and CBS 392.54 identified as P. trichostoma following Marin-Felix et al. (2019). Our isolate clustered together with these strains with high bootstrap support (99\% ML, 100\% MP) (Fig. 79).

Hosford (1970) reported a form of P. trichostoma which severely infected wheat in North Dakota in 1968 and 1969. Disease symptoms on leaves included light-brown lesions with yellow halos and the presence of many ascostromata on wheat stubble. The pathogenicity of the taxon varied, as well as the resistance of different wheat cultivars towards the pathogen varied, depending on the duration of free moisture on leaves (Hosford 1970). Pyrenophora trichostoma has been reported worldwide on a range of cereal crops, from Bolivia, Brazil, China, Europe (Austria, Italy, England, Poland, Portugal, Spain) to North America (Farr \& Rossman 2020), however, no molecular data in GenBank are given in these reports.

Roussoellaceae Jian K. Liu, Phook., D.Q. Dai \& K.D. Hyde

Liu et al. (2014) erected the Roussoellaceae to accommodate Neoroussoella, Roussoella and Roussoellopsis. Jaklitsch \& Voglmayr (2016b) treated Roussoellaceae as a synonym of Thyridariaceae. Tibpromma et al. (2017), Wanasinghe et al. (2018b), Wijayawardene et al. (2018) and Phookamsak et al. (2019) accepted Roussoellaceae as a stable family.

Pseudoneoconiothyrium Wanas., Phukhams., Camporesi \& K.D. Hyde

Wanasinghe et al. (2018b) introduced Pseudoneoconiothyrium Wanas et al. to accommodate Roussoella-like taxa in Thyridariaceae (Phookamsak et al. 2019). However, in multi gene (LSU, SSU, tef1, ITS and RPB2) analysis conducted by (Phookamsak et al. 2019) Pseudoneoconiothyrium clustered within Roussoellaceae.

Pseudoneoconiothyrium rosae (Phukhams., Camporesi \& K.D. Hyde) Phukhams., Camporesi \& K.D. Hyde, Index Fungorum 357: 1 (2018)

Fig. 82

Facesoffungi number: FoF 04055

Biotrophic on living branch of Lonicera sp. Sexual morph: Undetermined. Asexual morph: Conidiomata 200-320 $\mu \mathrm{m}$ diameter $\times 280-350 \mu \mathrm{m} \mu \mathrm{m}$ high $(\bar{x}=265.74 \times 341.3 \mu \mathrm{m}, \mathrm{n}=5)$, pycnidial, semi-immersed, erumpent from the substrate, solitary-scattered, globose to subglobose, dark brown to black, unilocular, ostiolate. Ostiole central, single, apapillate, filled with dark brown 
to light brown cells. Pycnidial wall 20-35 $\mu \mathrm{m}$ thick, 5-7 layered, composed of cells of textura angularis, cells in outer layer brown-walled, cells in inner layer light brown to hyaline. Conidiogenous cells ampulliform, enteroblastic, annellidic, with distinct percurrent proliferations, integrated, discrete, smooth, and hyaline. Conidia 8-12 $\times 6-9 \mu \mathrm{m}(\bar{x}=10.41 \times 7.98 \mu \mathrm{m}, \mathrm{n}=35)$, initially hyaline, smooth and thin-walled, light brown to dark brown and rough-walled when mature, globose to ellipsoid, aseptate, guttulate.

Culture characteristics - Conidia germinating on WA within two days. Colonies on PDA reaching 5-10 mm diameter after one week at $16^{\circ} \mathrm{C}$, entire edge, flat or effuse, greyish white, with dense, flat mycelium on the surface, dark green in reverse.
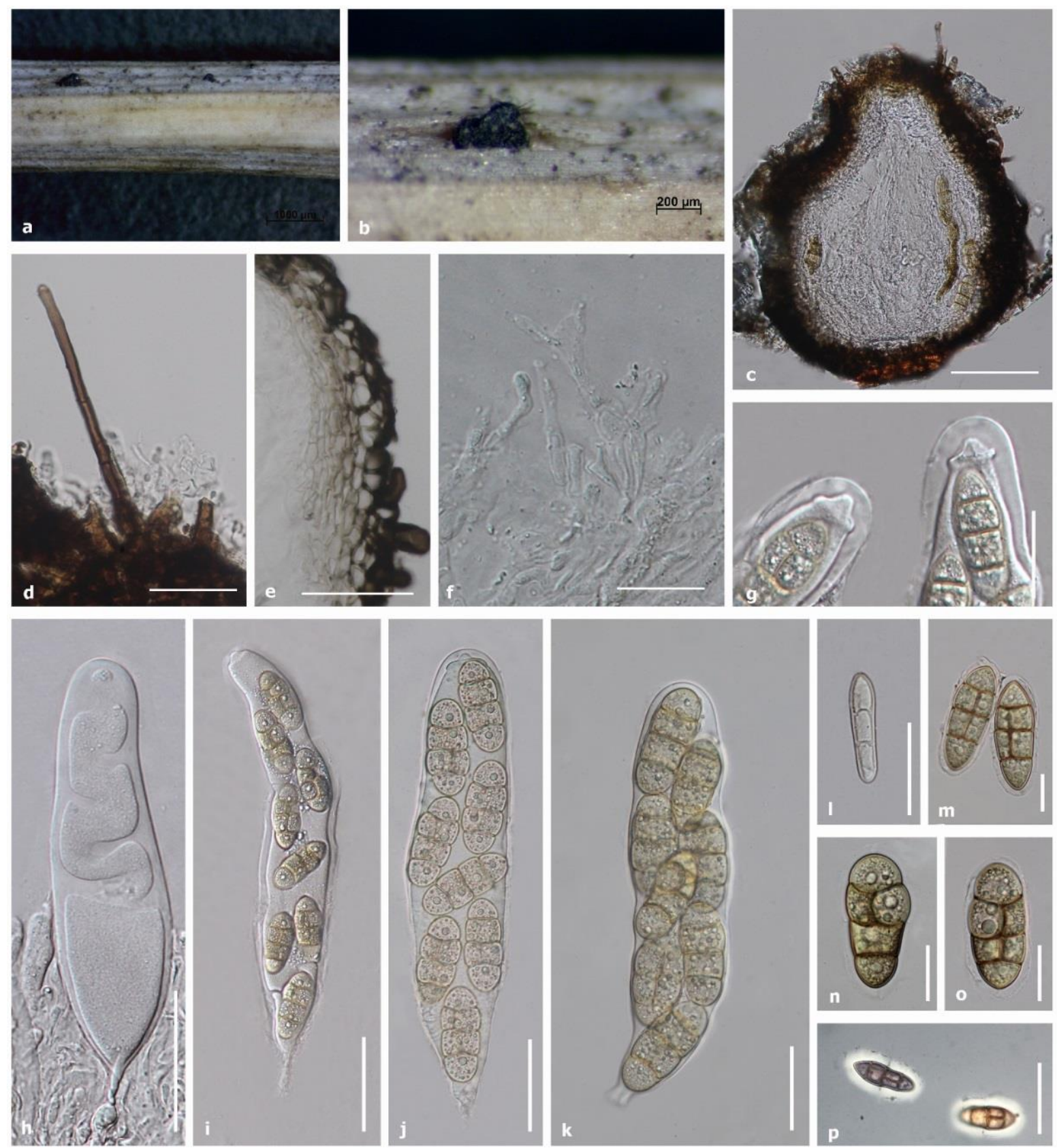

Fig. 80 - Pyrenophora trichostoma (MFLU 15-2040, new host record and geographical record). a Appearance of ascomata on host. b Close up of ascoma. c Section of ascoma. d Setae. e Peridium. $\mathrm{f}$ Hypha-like matter in hamathecium. g Ocular chambers in asci. $\mathrm{h}-\mathrm{k}$ Immature to mature asci. $1-\mathrm{o}$ Ascospores. $\mathrm{p}$ Sheath surrounding the ascospores. Scale bars: $\mathrm{a}=500 \mu \mathrm{m}, \mathrm{b}=200 \mu \mathrm{m}, \mathrm{c}=100$ $\mu \mathrm{m}, \mathrm{d}, \mathrm{f}, \mathrm{g}=20 \mu \mathrm{m}, \mathrm{e}, \mathrm{h}-\mathrm{m}=10 \mu \mathrm{m}$. 


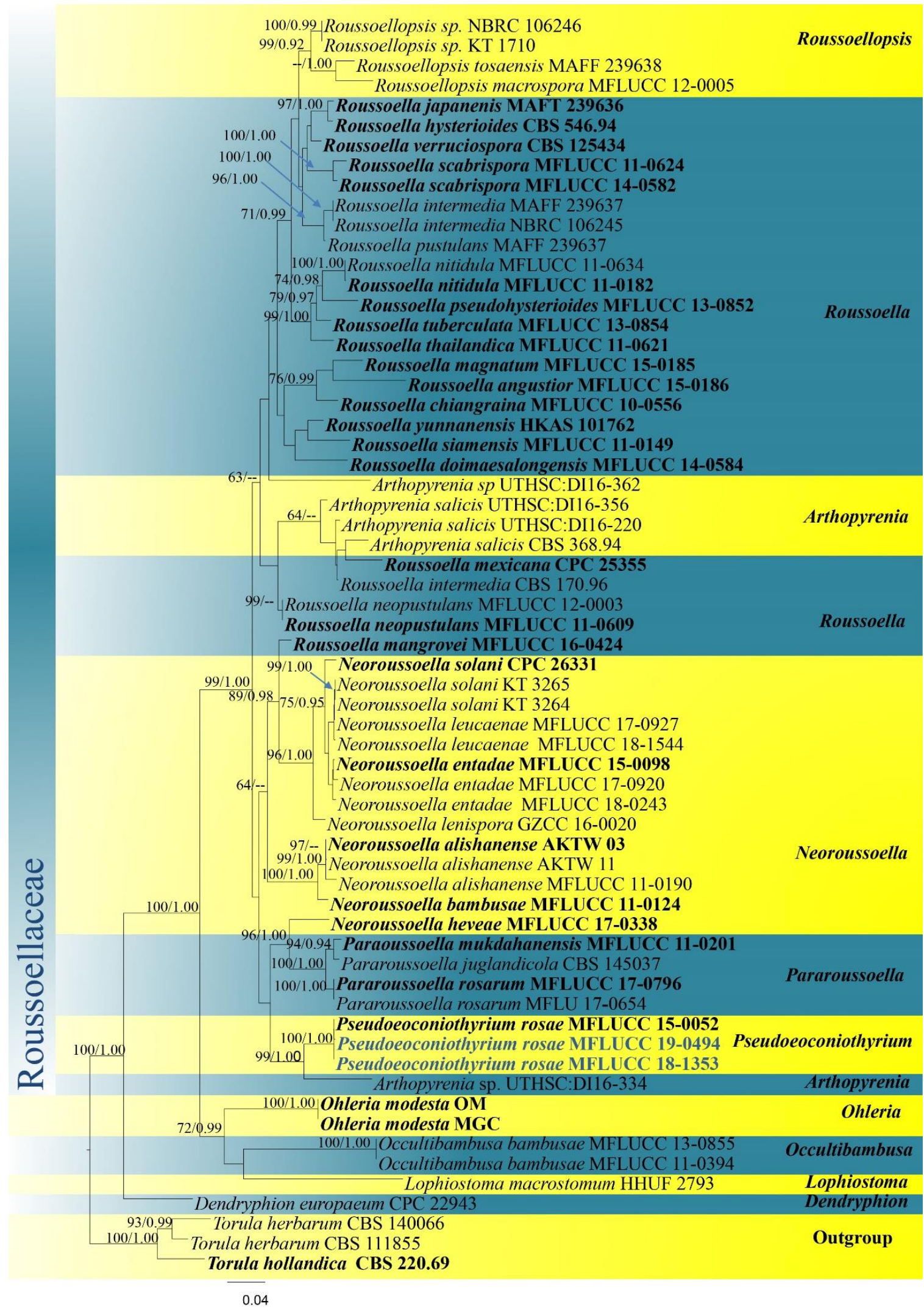

Fig. 81 - Phylogram generated from maximum likelihood analysis (RAxML) of Roussoellaceae based on ITS, LSU, SSU, tef1 and RPB2 sequence data. The tree is rooted to Torula herbarum (CBS 140066, CBS 111855) and Torula hollandica (CBS 220.69). The tree topology derived from the Bayesian analysis was similar to that derived from the maximum likelihood analysis. The final optimization likelihood value is -22735.444576 of the best RaxML tree with 1227 distinct alignment patterns and $37.75 \%$ undetermined characters or gaps. Estimated base frequencies are as 
follows: $\mathrm{A}=0.245625, \mathrm{C}=0.250937, \mathrm{G}=0.271866, \mathrm{~T}=0.231572$; substitution rates $\mathrm{AC}=$ $1.253105, \mathrm{AG}=3.802122, \mathrm{AT}=1.885811, \mathrm{CG}=0.990899, \mathrm{CT}=8.801862, \mathrm{GT}=1.000000$; gamma distribution shape parameter $\alpha=0.158691$. Maximum likelihood bootstrap values equal or above $60 \%$ and the Bayesian posterior probabilities equal or above 0.90 (ML/PP) are given at the nodes. The ex-type strains are indicated in black-bold and the newly generated strains are in bluebold.

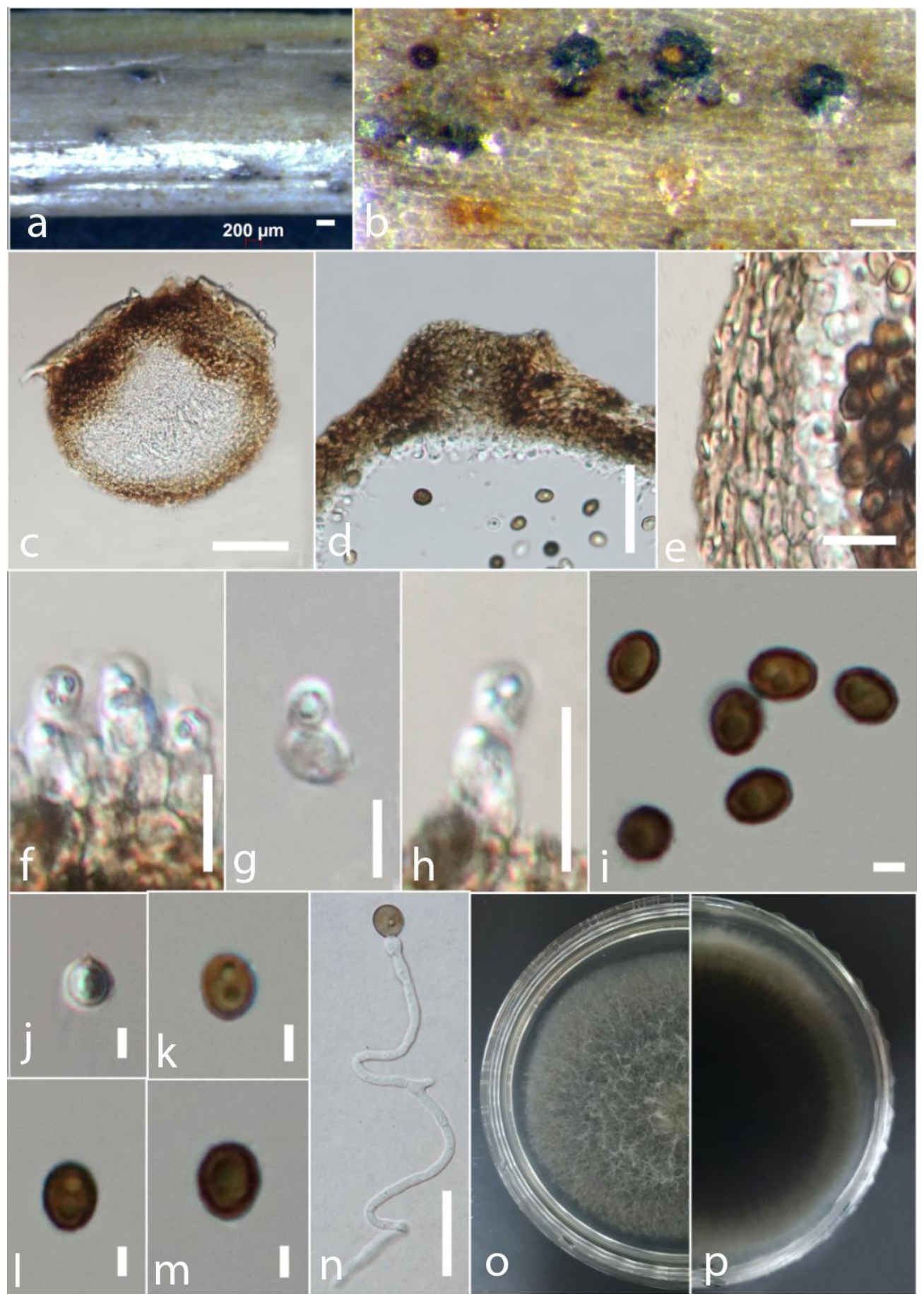

Fig. 82 - Pseudoneoconiothyrium rosae (MFLU 18-0930, new host record). a Conidiomata on living host surface of Lonicera sp. b Enlarged conidiomata on host. c Longitudinal section of conidioma. d Longitudinal section of ostiole. e Longitudinal section of a pycnidial wall. $\mathrm{f}-\mathrm{h}$ Conidiogenous cells with developing conidia. $\mathrm{i}-\mathrm{m}$ Conidia. $\mathrm{n}$ Germinating conidium. $\mathrm{o}-\mathrm{p}$ Colonies on PDA (o upper, o lower). Scale bars: $\mathrm{a}=200 \mu \mathrm{m}, \mathrm{b}-\mathrm{c}=100 \mu \mathrm{m}, \mathrm{d}, \mathrm{n}=50 \mu \mathrm{m}$, $\mathrm{e}-\mathrm{f}, \mathrm{h}=20 \mu \mathrm{m}, \mathrm{g}=10 \mu \mathrm{m}, \mathrm{i}-\mathrm{m}=5 \mu \mathrm{m}$. 
Known distribution (based on molecular data) - Italy, Province of Forlì-Cesena (Wanasinghe 2018b, Phookamsak et al. 2019, this study).

Known hosts (based on molecular data) - dead aerial spines of Rosa canina (Rosaceae) (Wanasinghe 2018b, Phookamsak et al. 2019), lives on a live stem of Lonicera sp. (Caprifoliaceae) (this study).

Material examined - Italy, Province of Forlì-Cesena, Rocca delle Caminate - Predappio, on a live stem of Lonicera sp. (Caprifoliaceae), 4 April 2018, E. Camporesi (MFLU 18-0930, new host record), culture (MFLUCC 18-1353).

GenBank numbers - ITS: MN783333, LSU: MN783330, RPB2: MN814846, SSU: MN783332.

Notes - In the multi-gene phylogeny (LSU, ITS, SSU and RPB2) of our study, our novel strain (MFLUCC 18-1353) and the ex-type strain of Pseudoneoconiothyrium rosae (MFLUCC 150052) clustered with relatively high support (100\% ML/ 1.00 PP) (Fig. 81). These isolates were collected from different hosts, but in the same locality. The characters of our species overlap with the holotype (Wanasinghe et al. 2018b, Phookamsak et al. 2019). Neoconiothyrium rosae (MFLU 18-0117, holotype) was synonymized under Pseudoneoconiothyrium rosae (Phookamsak et al. 2019). Both species have many similar characters with solitary-scattered and erumpent, globose to subglobose, dark brown to black, unilocular ascomata and single, central, ostioles. The pycnidial wall is composed of cells of textura angularis and cells in the outer layer are brown-walled, cells in inner layer light brown to hyaline. Conidia are also globose to ellipsoid shape aseptate and guttulate. However, the size of ascomata, conidia and the number of pycnidial wall cell layers of our new collection is comparatively larger than the ex-type strain. According to the guidelines of Jeewon and Hyde (2016), we have analyzed nucleotide differences within the rRNA gene region for further clarifications. When comparing the ITS region (ITS1-5.8S-ITS2) from 436 nucleotides and LSU rDNA region from 799 bp, there are zero bp (0 \%) differences between MFLUCC 181353 and MFLUCC 15-00520 strains. Considering the morpho-molecular data analysis, we conclude that our new collection is another record of Pseudoneoconiothyrium rosae and a new host record on Lonicera sp. (Caprifoliaceae) in Italy.

Pseudoneoconiothyrium rosae (Phukhams., Camporesi \& K.D. Hyde) Phukhams., Camporesi \& K.D. Hyde, Index Fungorum 357: 1 (2018)

Fig. 83

Facesoffungi number: FoF 04055

Saprobic on dead and hanging branch of Galium sp. Sexual morph: Undetermined. Asexual morph: Conidiomata 300-320 $\mu \mathrm{m}$ diameter $\times 240-360 \mu \mathrm{m}$ high $(\bar{x}=311.6 \times 295 \mu \mathrm{m}, \mathrm{n}=5)$, pycnidial, semi immersed, erumpent from the substrate, solitary-scattered, globose to subglobose, dark brown to black, unilocular, ostiolate. Ostiole single, circular. Pycnidial wall 30-50 $\mu \mathrm{m}$ thick, 5-8 layered, composed of cells of textura angularis, cells in outer layer brown walled, cells in inner layer light brown to hyaline. Conidiogenous cells ampulliform, enteroblastic, annellidic with distinct percurrent proliferations, sometimes cylindrical, elongate neck, integrated, discrete, smooth and hyaline. Conidia 7-10 $\times 4-8 \mu \mathrm{m}(\bar{x}=8.45 \times 6.22 \mu \mathrm{m}, \mathrm{n}=20)$, initially hyaline, smooth and thin-walled, light brown to dark brown and rough-walled when mature, globose to ellipsoid, aseptate, guttulate.

Culture characteristics - Conidia germinating on MEA within three days. Colonies on MEA reaching 5-10 mm diameter after one week at $16{ }^{\circ} \mathrm{C}$, undulate, white, with dense, flat mycelium on the surface, pale yellow in reverse.

Known distribution (based on molecular data) - Italy, Province of Forlì-Cesena (Wanasinghe et al. 2018b, Phookamsak et al. 2019, this study).

Known hosts (based on molecular data) - dead aerial spines of Rosa canina (Rosaceae) (Wanasinghe et al. 2018b, Phookamsak et al. 2019), lives on a dead hanging stem of Galium sp. (Rubiaceae) (this study). 
Material examined - Italy, Province of Forlì-Cesena, Rocca delle Caminate - Predappio, on a dead hanging stem of Galium sp. (Rubiaceae), 4 April 2018, E. Camporesi (MFLU 18-0927, new host record), culture (MFLUCC19-0494).

GenBank numbers - ITS: MN783331, LSU, MN783329, tef1: MN794051.
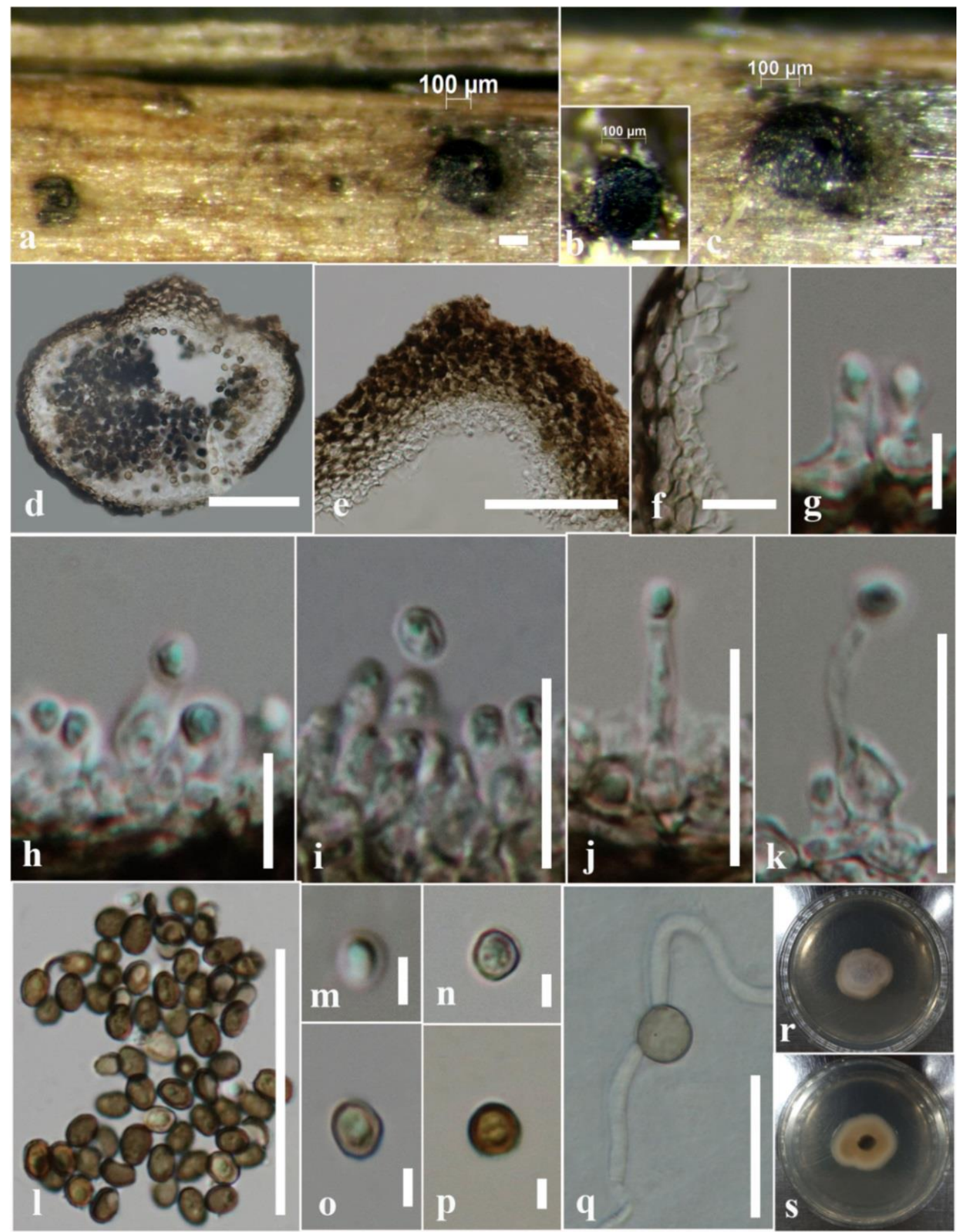

Fig. 83 - Pseudoneoconiothyrium rosae (MFLU 18-0927, new host record). a Conidiomata on dead branch of Galium sp. b-c Enlarged conidiomata on host surface. d Longitudinal section of a conidioma with ostiole. e Longitudinal section of ostiole. $f$ Longitudinal section of a conidioma wall. $\mathrm{g}-\mathrm{k}$ Conidiogenous cells with developing conidia; $1-\mathrm{p}$ Conidia. $\mathrm{q}$ Germinating conidium. $\mathrm{r}-\mathrm{s}$ Colonies on PDA $(\mathrm{r}=$ upper, $\mathrm{s}=$ lower). Scale bars: $\mathrm{a}, \mathrm{e}, \mathrm{l}=100 \mu \mathrm{m}, \mathrm{q}=50 \mu \mathrm{m}, \mathrm{j}-\mathrm{k}=30 \mu \mathrm{m}$, $\mathrm{f}-\mathrm{i}=20 \mu \mathrm{m}, \mathrm{g}-\mathrm{h}=10 \mu \mathrm{m}, \mathrm{m}-\mathrm{p}=5 \mu \mathrm{m}$. 
Notes - In the multi-gene phylogeny (LSU, ITS, SSU, RPB2 and tef1) of our study, our novel strain (MFLUCC 19-0494) and the ex-type strain of Pseudoneoconiothyrium rosae (MFLUCC 150052) clustered with relatively high support (100\% ML/ 1.00 PP) (Fig. 81). These collections are from different hosts, but in the same locality. When morphological characters of our species were examined, they overlap with the holotype from dead spines of Rosa canina (Rosaceae) in Italy which was described by Wanasinghe et al. (2018b). Neoconiothyrium rosae (MFLU 18-0117, holotype) was synonymized under Pseudoneoconiothyrium rosae (Phookamsak et al. 2019). Both species have similar morphological characters with solitary-scattered, globose to subglobose, dark brown to black, uniloculate and erumpent ascomata, with a single, central, ostiole. Conidia are also globose to ellipsoid, aseptate and guttulate. However, the size of ascomata and thickness of pycnidial wall of our new collection are comparatively larger than the ex-type strain. Also, some conidiogenous cells have cylindrical and elongate neck. According to the guidelines of Jeewon \& Hyde (2016) we have analyzed nucleotide differences within the rRNA gene region for further clarifications. In comparison of ITS regions (ITS1-5.8S-ITS2) from 436 nucleotides and LSU rDNA region from $799 \mathrm{bp}$, there are zero bp (0\%) differences between MFLUCC 18-1353 and MFLUCC 15-00520 strains. Considering the morpho-molecular data analysis, we conclude that our new collection is a record of Pseudoneoconiothyrium rosae and also a new host record on Galium sp. (Rubiaceae) in Italy.

\section{Roussoella Sacc.}

Roussoella was introduced with the type species $R$. nitidula Sacc. \& Paol. The ambiguous placement of Roussoella was later on resolved by Liu et al. (2014). The latest treatment for Roussoella was by Phookamsak et al. (2019).

Roussoella siamensis Phook., Phook., Jian K. Liu \& K.D. Hyde, Phytotaxa 181(1): 18 (2014)

Facesoffungi number: FoF01984

Fig. 85

Saprobic on Phragmites australis. Asexual morph: Coelomycetous. Conidiomata 140-150 $\mu \mathrm{m}$ high $\times 220-225 \mu \mathrm{m}$ diameter $(\bar{x}=145 \times 223 \mu \mathrm{m}, \mathrm{n}=5)$, pycnidial, immersed in host epidermis, raised, hemisphaerical to subconical, coriaceous, scattered or clustered, solitary or gregarious, uniloculate, glabrous, centrally ostiolate, with minutely pore-like opening. Pycnidial wall 6-8 $\mu \mathrm{m}$ wide, composed of several layers of brown to dark brown pseudoparenchymatous cells of textura angularis, flattened at the base, thick at side towards the apex, outer layer fused with the host tissue. Conidiophores reduced to conidiogenous cells. Conidiogenous cells $8-13.5 \times 1.5-3 \mu \mathrm{m}(\bar{x}=$ $11 \mu \mathrm{m} \times 2 \mu \mathrm{m}, \mathrm{n}=20$ ), enteroblastic, phialidic, unbranched, cylindrical to cylindric-clavate, or ampulliform, hyaline, aseptate, thin-walled, smooth-walled, arising from the basal cavity around the conidiomata. Conidia $3-4 \times 1.5-2.5 \mu \mathrm{m}(\bar{x}=3.7 \times 2 \mu \mathrm{m}, \mathrm{n}=40)$, oblong to ellipsoidal, hyaline, aseptate with two guttules, smooth-walled.

Culture characteristics - Conidia germinated on PDA within $12 \mathrm{~h}$ at $20-25^{\circ} \mathrm{C}$, with germ tubes produced from a one end. Colonies on PDA moderate growing, dense, irregular in shape, flattened to raised, slightly rough-walled with lobate edge, and uneven margin, velvety, colony from above dark green to dark brown, from below black; sometimes white to pale pinkish, radiated with yellowish-brown to dark brown brown at the center and white at the margin, not producing pigmentation on agar medium.

Known distribution (based on molecular data) - Thailand, Chiang Rai Province (Liu et al. 2014), China, Dali (this study).

Known hosts (based on molecular data) - Bambusa (Liu et al. 2014), Phragmites australis (this study)

Material examined - China, Kunming, Lake Dianchi, on decaying stems of Phragmites australis (Poaceae), 5 November 2016, K.D. Hyde, AKD 04 (MFLU 17-0351, new host and geographic record); ex-type living culture, MFLUCC 17-1352; KUMCC 16-0207.

GenBank number - LSU: MN989183. 


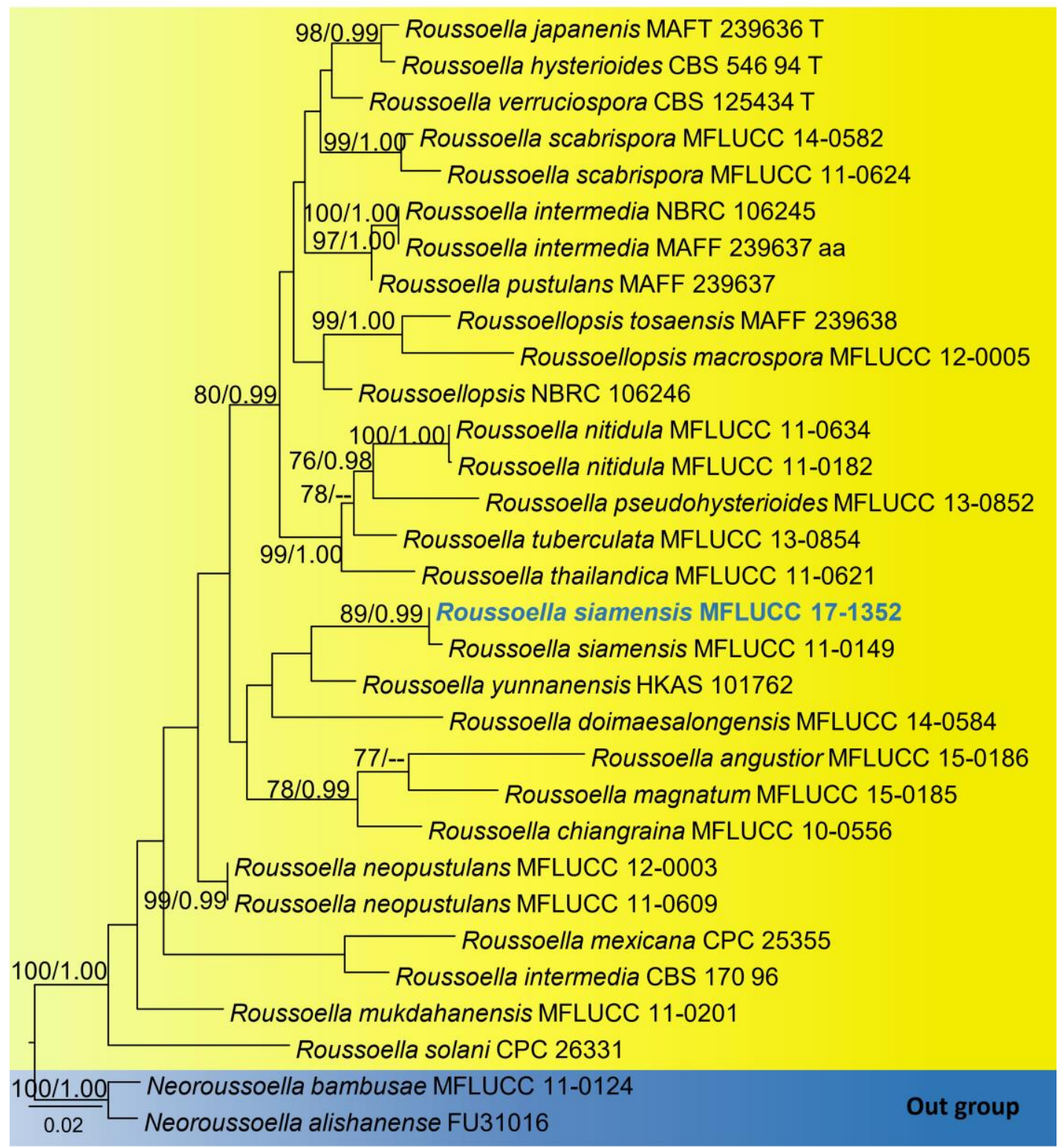

Fig. 84 - Phylogenetic tree generated from maximum likelihood (ML) based on LSU, SSU, ITS, tef1 and RPB2 sequences. Bootstrap support (BS) values above 50\% and Bayesian posterior probabilities equal or greater than 0.90 are shown above the branches at nodes. The tree is rooted with Neoroussoella bambusae and $N$. alishanense. The best RaxML tree with a final likelihood value of -13900.126681 is presented. The matrix had 823 distinct alignment patterns, with $44.82 \%$ undetermined characters or gaps. Estimated base frequencies were as follows: $\mathrm{A}=0.244559, \mathrm{C}=$ $0.251803, \mathrm{G}=0.271754, \mathrm{~T}=0.231883$; substitution rates $\mathrm{AC}=1.179055, \mathrm{AG}=4.071030, \mathrm{AT}=$ $1.977458, \mathrm{CG}=0.917006, \mathrm{CT}=10.347006, \mathrm{GT}=1.000000$; gamma distribution shape parameter $\alpha=0.763580$. The newly generated sequence is indicated in blue.

Notes - This is the first record of the asexual morph of Roussoella siamensis. The species is supported by phylogeny. Herein, we provide the new host and geographic record for the Roussoella siamensis. In our phylogenetic analysis, the strain MFLUCC 17-1352 clusters with MFLU 11-0149 (Fig. 84).

\section{Sporormiaceae Munk}

Sporormiaceae is classified in Pleosporales, in Dothideomycetes and was established by Munk (1957) and is typified by Sporormia (De Notaris 1844), with the type species Sporormia fimetaria (Phukhamsakda et al. 2016). This type genus is characterized by ostiolate, globose 
pseudothecia and spores joined in bundlse by a common gelatinous sheath (Ahmed \& Cain 1972, Dissing 1992). Even though absent in the type species, germ slits are also considered as a common character in this family (Kruys et al. 2006). Sporormiaceae is the largest coprophilous ascomycetes family and most members are cosmopolitan on a vast variety of animal dung types and have also been reported on other substrates like decaying plant debris, decaying textiles, rotten vegetation, soil and wood, or as endophytes and saprobes in plants (Kruys et al. 2006, Sue et al. 2014). Sporormiaceae comprises approximately 100 species classified in ten genera, including the recently described genus, Sporormurispora by Wanasinghe et al. (2018b) and Chaetopreussia, Forliomyces, Pleophragmia, Preussia, Sparticola, Sporormia, Sporormiella, Spororminula, and Westerdykella (Karunarathna et al. 2017, Phukhamsakda et al. 2016).

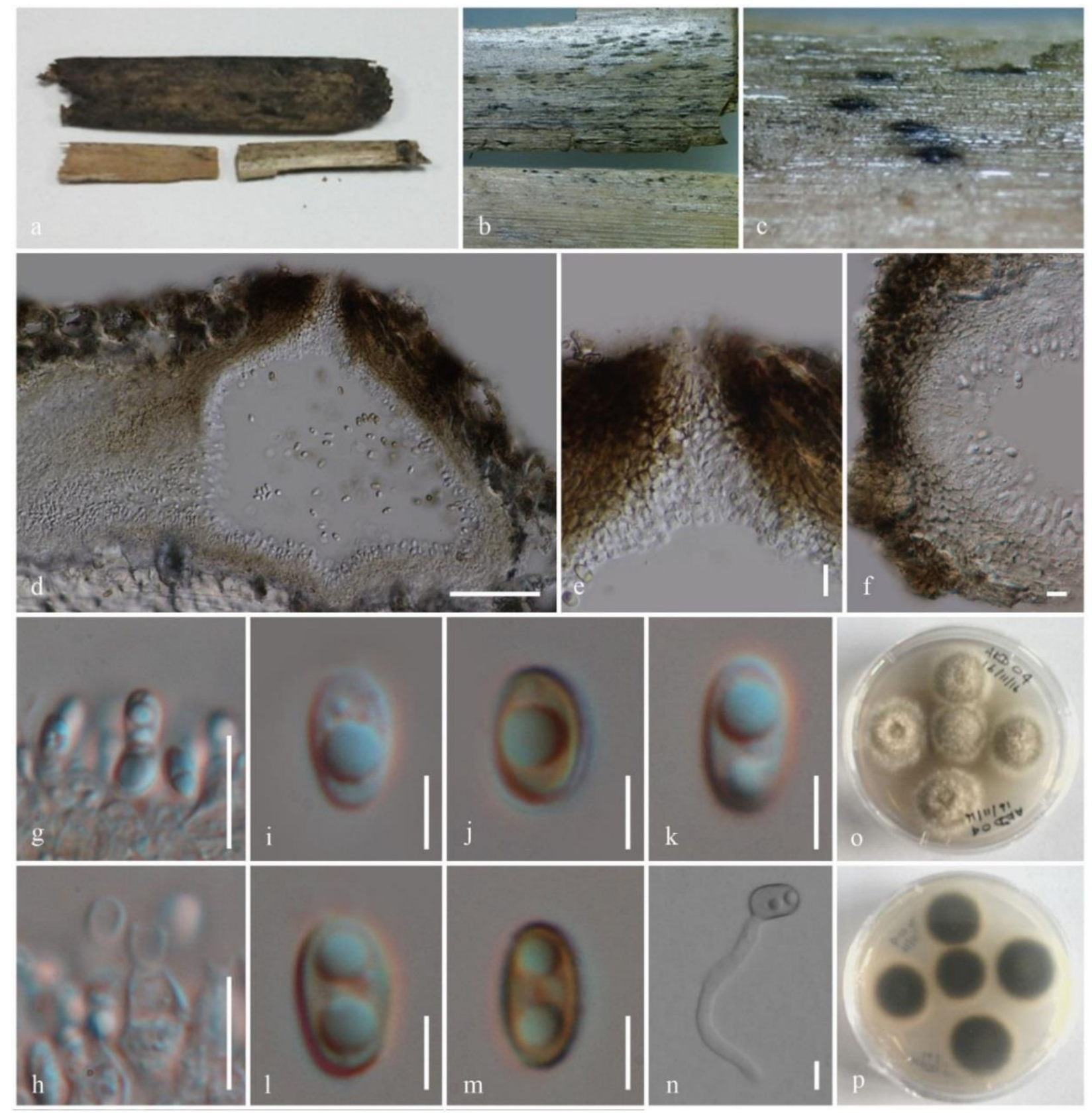

Fig. 85 - Roussoella siamensis (MFLU 17-0351, new host and geographic record). a Appearance of the host. $b-c$ Colonies on host substrate. $d$ Longitudinal section of conidioma. e Longitudinal section of ostiole. f Peridium. g-h Conidiogenous cells and developing conidia. $\mathrm{i}-\mathrm{m}$ Conidia. n Germinated conidium. o-p Culture characteristics. Scale bars: $d=50 \mu \mathrm{m}, \mathrm{e}=10 \mu \mathrm{m}, \mathrm{f}=10 \mu \mathrm{m}$, $\mathrm{g}-\mathrm{h}=10 \mu \mathrm{m}, \mathrm{i}-\mathrm{n}=5 \mu \mathrm{m}$. 
Forliomyces Phukhams.,Camporesi \& K.D. Hyde

Forliomyces is a monotypic genus in Sporormiaceae introduced by Phukhamsakda et al. (2016) with Forliomyces uniseptatus as the type species. Forliomyces is characterized by immersed, pycnidial, subglobose, unilocular conidiomata with oblong to subobovoid, slightly curved, septate, conidia with abscission scars (Phukhamsakda et al. 2016). Sexual connections are still unclear for Forliomyces.

Forliomyces uniseptatus Phukhams., Camporesi \& K.D. Hyde, Cryptog. Mycol. 37(1): 84 (2016)

Facesoffungi number: FoF07381

Saprobic on dead aerial branches of Hippocrepis emerus. Conidiomata visible as round dark brown to black dots on the host surface. Sexual morph Undetermined. Asexual morph

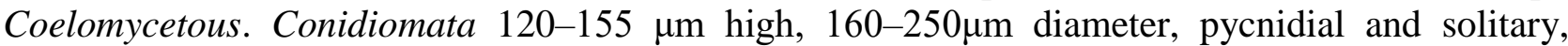
scattered, globose, semi-immersed to immersed, unilocular, thin walled. Conidioma wall composed of 3-4 layers, similarly dense 15-21 $\mu \mathrm{m}$ wide, outer and inner layers light brown to brown, with thin walled cells of textura angularis. Conidiophores reduced to conidiogenous cells. Conidiogenous cells formed from the cells lining the inner wall of conidiomata 2-8 $\mu \mathrm{m}$ long $\times 1-3$ $\mu \mathrm{m}$ wide, hyaline, enteroblastic, phialidic, determinate, discrete, and oblong to clavate. Conidia 8 $13 \times 4-7 \mu \mathrm{m}$ wide $(\bar{x}=10 \times 5 \mu \mathrm{m}, \mathrm{n}=20)$, oblong, sub oboviod and some are ellipsoidal to cylindrical, initially hyaline to light brown, brown to dark brown at maturity, slightly bent at the middle, septate, thick, smooth-walled, guttulate.

Culture characteristics - Colonies on PDA reaching $15 \mathrm{~mm}$ diameter after 2 weeks at $25{ }^{\circ} \mathrm{C}$, circular colony, concentrically zonate, flat, smooth, with pinkish to orange diffusible pigments into agar. Colonies from above light creamy orange at the margin and pinkish red with whitish in middle, reverse colony color deep red in center, pinkish to red in middle and creamy orange at the edge.

Known distribution (based on molecular data) - Italy (Phukhamsakda et al. 2016) and (this study).

Known hosts (based on molecular data) - On Salvia (Phukhamsakda et al. 2016), on dead aerial branch of Hippocrepis emerus (this study)

Material examined - Italy, Province of Forlì-Cesena [FC], Galeata, Strada San Zeno, on dead aerial branch of Hippocrepis emerus (Fabaceae), 25 April 2017, E. Camporesi, IT 3331 (JZBH 3390001, new host record), living culture: JZB: 3390001.

GenBank numbers - LSU: MK066913, SSU: MK066909, ITS: MK066911.

Notes - The collection obtained from dead aerial branches of Hippocrepis emerus was identified as Forliomyces uniseptatus with support from morphology and phylogeny. Our isolate clustered with the type species, Forliomyces uniseptatus (MFLUCC 15-0765), in the combined LSU, SSU, ITS, tef1 and RPB2 sequence phylogeny (Fig. 86). In addition, our isolate showed $100 \%, 98 \%$ and $100 \%$ base pair similarity with Forliomyces uniseptatus (MFLUCC 15-0765) in LSU, ITS and tefl gene regions. This is the first record of Forliomyces uniseptatus species reported from Hippocrepis emerus from Italy.

Tetraplosphaeriaceae Kaz. Tanaka \& K. Hiray.

Tetraplosphaeriaceae was introduced by Tanaka et al. (2009) and typified by Tetraploa with T. aristata as the type species (Berkeley \& Broome 1850, Hyde et al. 2013). Recently, seven genera were accommodated in this family viz. Ernakulamia, Polyplosphaeria, Pseudotetraploa, Quadricrura, Shrungabeeja, Tetraploa and Triplosphaeria (Berkeley \& Broome 1850, Rao \& Reddy 1981, Subramanian 1994, Tanaka et al. 2009, Hyde et al. 2013, Wijayawardene et al. 2018). 


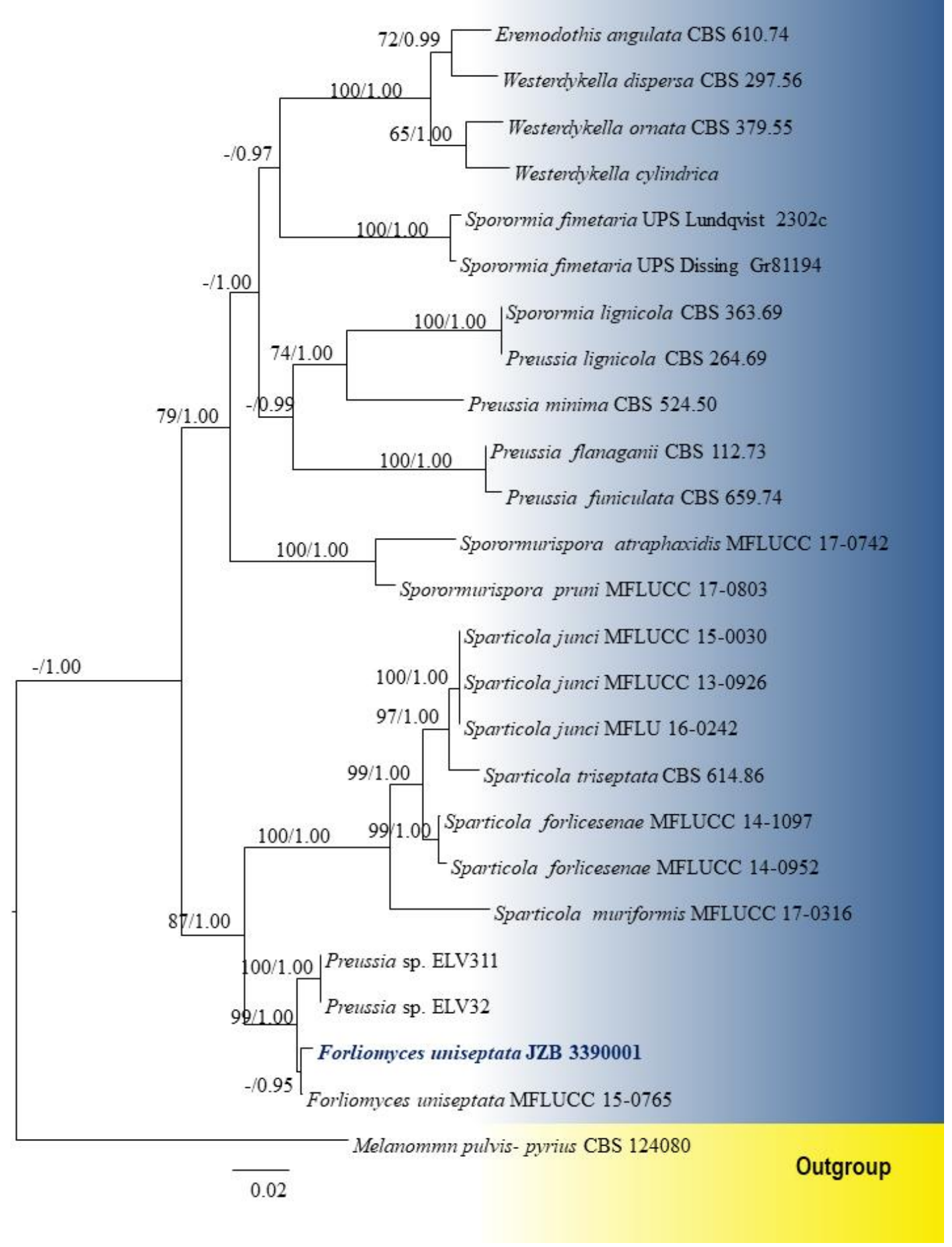

Fig. 86 - Phylogram generated from maximum likelihood analysis based on combined LSU, SSU, ITS, tef1 and RPB2 sequence data. Twenty-four strains are included in the combined gene analyses and Melanomma pulvis-pyrius (CBS 124080) is used as the out-group taxon. The tree topology derived from the Bayesian analysis was similar to that derived from the maximum likelihood analysis. The best RaxML tree with a final likelihood value of -13932.036268 is presented. The matrix had 1020 distinct alignment patterns, with $45.20 \%$ undetermined characters or gaps. Estimated base frequencies were as follows: $\mathrm{A}=0.246751, \mathrm{C}=0.248031, \mathrm{G}=0.276957, \mathrm{~T}=$ 0.228261 ; substitution rates $\mathrm{AC}=1.342154, \mathrm{AG}=2.568856, \mathrm{AT}=1.620900, \mathrm{CG}=1.189329, \mathrm{CT}$ $=7.343609, \mathrm{GT}=1.000000$; gamma distribution shape parameter $\alpha=0.669698$. Bootstrap values for maximum likelihood equal to or greater than 60 and Bayesian posterior probabilities equal or greater than 0.90 are placed above or below the branches. Ex-type strains are in bold and black. The newly generated sequence is indicated in blue. 
Polyplosphaeria Kaz. Tanaka \& K. Hiray.

Polyplosphaeria was introduced by Tanaka et al. (2009), with $P$. fusca as the type. Until now, there are five species accommodated in the genus viz. $P$. fusca, $P$. nabanheensis, $P$. pandanicola, $P$. thailandica, $P$. xishuangbannaensis, however, only $P$. fusca has been reported as a holomorph, while in the other four species only the asexual morph is known (Tanaka et al. 2009, Li et al. 2016a, Tibpromma et al. 2018b). The sexual morph of the genus is characterized by globose, black to sometimes reddish-brown ascomata, 8-spored, fissitunicate, clavate asci with 1(-3)-septate, hyaline to pale olive-brown, fusiform ascospores (Tanaka et al. 2009). The asexual morph produces globose to subglobose, brown to dark brown conidia with setose appendages (Tanaka et al. 2009, Li et al. 2016a, Tibpromma et al. 2018b).

Polyplosphaeria fusca Kaz. Tanaka \& K. Hiray., Studies in Mycology 64: 193 (2009)

Fig. 89

Facesoffungi number: FoF 06806

Saprobic on stem of dead bamboo branches. Sexual morph: Ascomata 200-300 $\mu \mathrm{m}$ high, 300-500 $\mu \mathrm{m}$ diameter, immersed under the host, solitary, scattered, 2-3-gregarious, dark brown, conical to subglobose, with ostiole vertically lined with hyaline periphyses, uni-loculate, mostly associated with reddish pigments. Peridium 20-30 $\mu \mathrm{m}$ wide, comprising several layers, with dark brown to brown parenchymatous cells arranged in textura angularis. Hamathecium of hyaline, septate, branched, 1-1.7 $\mu \mathrm{m}$ broad pseudoparaphyses, anastomosing at the top. Asci 115-140 $\times 23-$ $32 \mu \mathrm{m}(\bar{x}=127.5 \times 27.5 \mu \mathrm{m}, \mathrm{n}=20), 8$-spored, fissitunicate, broadly cylindric-clavate, pedicellate with slightly furcate ends, apically rounded. Ascospores 46-58 $\times 9-10 \mu \mathrm{m}(\bar{x}=52 \times 9.5 \mu \mathrm{m}, \mathrm{n}=$ 20), 1-3-seriate, hyaline to pale olive-brown, fusiform, slightly curved, 1-septate, constricted at the septum, smooth-walled, with guttules. Asexual morph: Refer to Tanaka et al. (2009).

Culture characteristics - Ascospores germinating on PDA within $24 \mathrm{~h}$. Colonies on PDA reaching $17-20 \mathrm{~mm}$ diameter after 10 days at room temperature $\left(20-30^{\circ} \mathrm{C}\right)$; from above, raised, convex, brown to dark brown at the centre, white at the margin, circular, velvety; from reverse, yellowish.

Known distribution (based on molecular data) - Japan, Aomori, Nagasaki, Shizuoka, Tochigi (Tanaka et al. 2009), China, Yunnan (this study).

Known hosts (based on molecular data) - Chimonobambusa marmorea (Gramineae), Phyllostachys bambusoides (Gramineae), Pleioblastus chino (Gramineae), Sasa kurilensis (Gramineae) (Tanaka et al. 2009), bamboo (Tanaka et al. 2009; this study).

Material examined - China, Yunnan Province, Honghe Prefecture, Pingbian County, nearby Tuanpo Reservoir, on stem of dead bamboo (Poaceae), 21 September 2017, H.B. Jiang, Pb002 (KUN-HKAS 101757, new geographic record), living culture KUMCC 18-0110.

GenBank numbers - ITS: MN629458, LSU: MN629359, SSU: MN629360.

Notes - The new collection is similar to Polyplosphaeria fusca and based on multi-gene phylogenetic analyses, it clustered with $P$. fusca (KT1616 and KT2124), but separated from $P$. fusca (KT1043 and KT1640) (Fig. 88). These two groups can be distinguished based on the characteristics, such as ascomata in these specimens are "almost superficial without associated pigmentation" and "immersed with reddish pigments", respectively, as reported by Tanaka et al. (2009). A comparison of the ITS, LSU and SSU nucleotides of the type strain of $P$. fusca (KT1616) and the new strain revealed $\leq 1.5 \%$ nucleotide differences which confirm our new strain as $P$. fusca (Jeewon \& Hyde 2016). Polyplosphaeria fusca was reported from Japan (Tanaka et al. 2009); our collection is a new geographic record for China.

Tetraploa Berk. \& Broome

Tetraploa was given preference over Tetraplosphaeria as discussed by Hyde et al. (2013) and Wijayawardene et al. (2014). Among the 20 epithets in Index Fungorum (2020), molecular data is available for only for seven species. Tetraploa species are mostly found on monocotyledons (Tanaka et al. 2009, Farr \& Rossman 2020). 

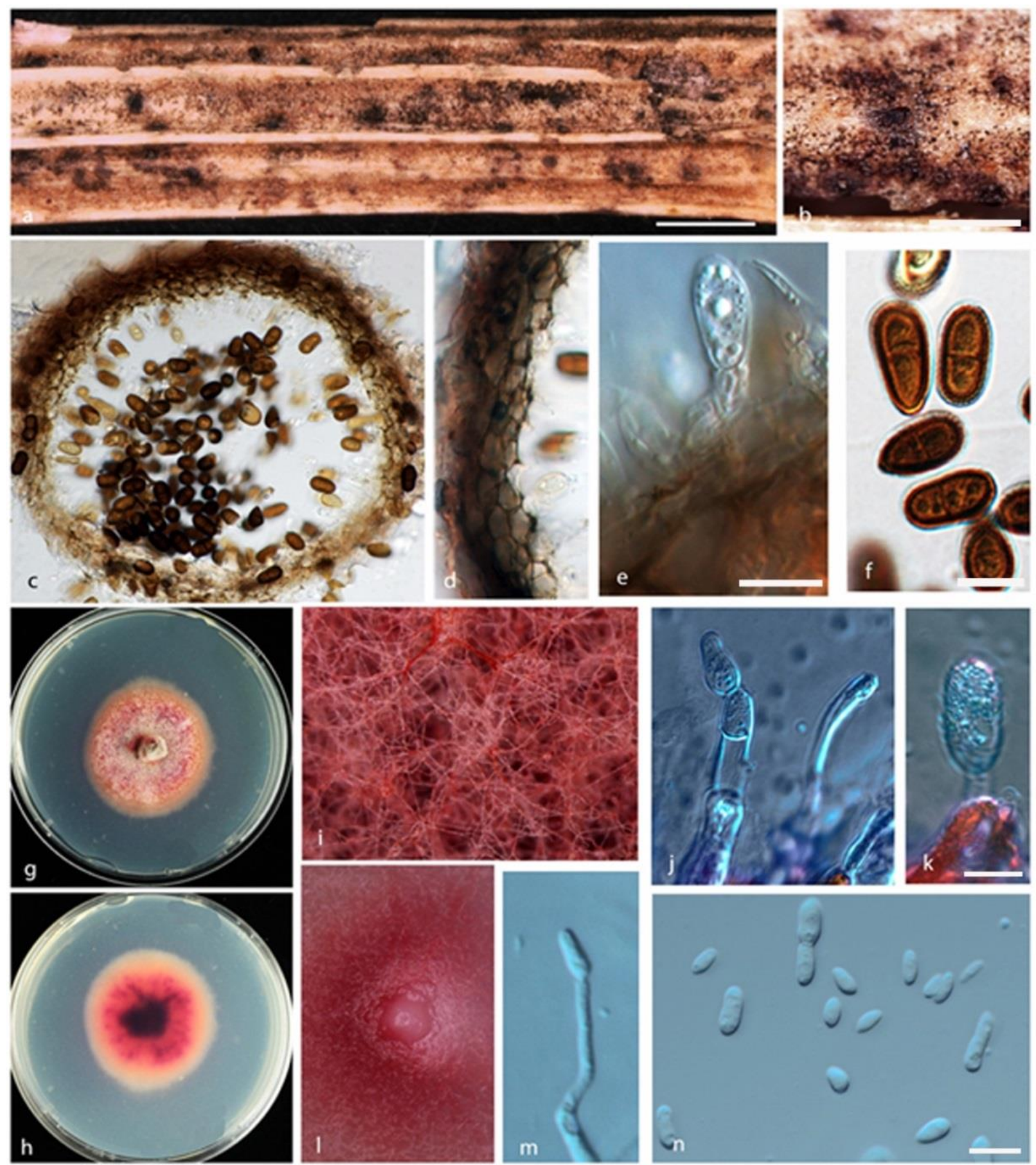

Fig. 87 - Forliomyces uniseptatus (JZB 3390001, new host record). a Appearance of conidiomata on the host. b Close up view of conidiomata. c Section through the conidioma. d Conidioma wall. e Immature conidia attached to conidiogenous cell. f Mature conidia. $\mathrm{g}-\mathrm{h}$ Colony on PDA after 2 weeks ( $\mathrm{g}=$ from above, $\mathrm{h}=$ from below). i Pigmented mycelium. $\mathrm{j}-\mathrm{k}$ Clamydospores formation from hyphae. 1 Conidiomata in culture. $\mathrm{m}$ Conidiogenous cell. $\mathrm{n}$ Conidia. Scale bars: $\mathrm{a}, \mathrm{b}=200 \mu \mathrm{m}$, $\mathrm{e}, \mathrm{f}, \mathrm{k}=20 \mu \mathrm{m}, \mathrm{n}=10 \mu \mathrm{m}$.

Tetraploa sasicola (Kaz. Tanaka \& K. Hiray.) Kaz. Tanaka \& K. Hiray.

Fig. 91

Facesoffungi number: FoF 01984

Saprobic on decaying culms of Phragmites australis. Mycelia dark brown to black, effuse, branched, septate, partly superficial. Asexual morph: Conidiogenous cells micronematous, dark brown, monoblastic, integrated, short, usually undistinguishable from superficial hyphae. Conidia short cylindrical, conidial body four columnar, coarsely verruculose, 35-45 × 25-30 $\mu \mathrm{m}$, with four 
apical setose appendages, 90-120 $\mu \mathrm{m}$, partly split conidia, where the two columns of the upper part of the main conidial body is totally split along with the two apical appendage but connected at the base.

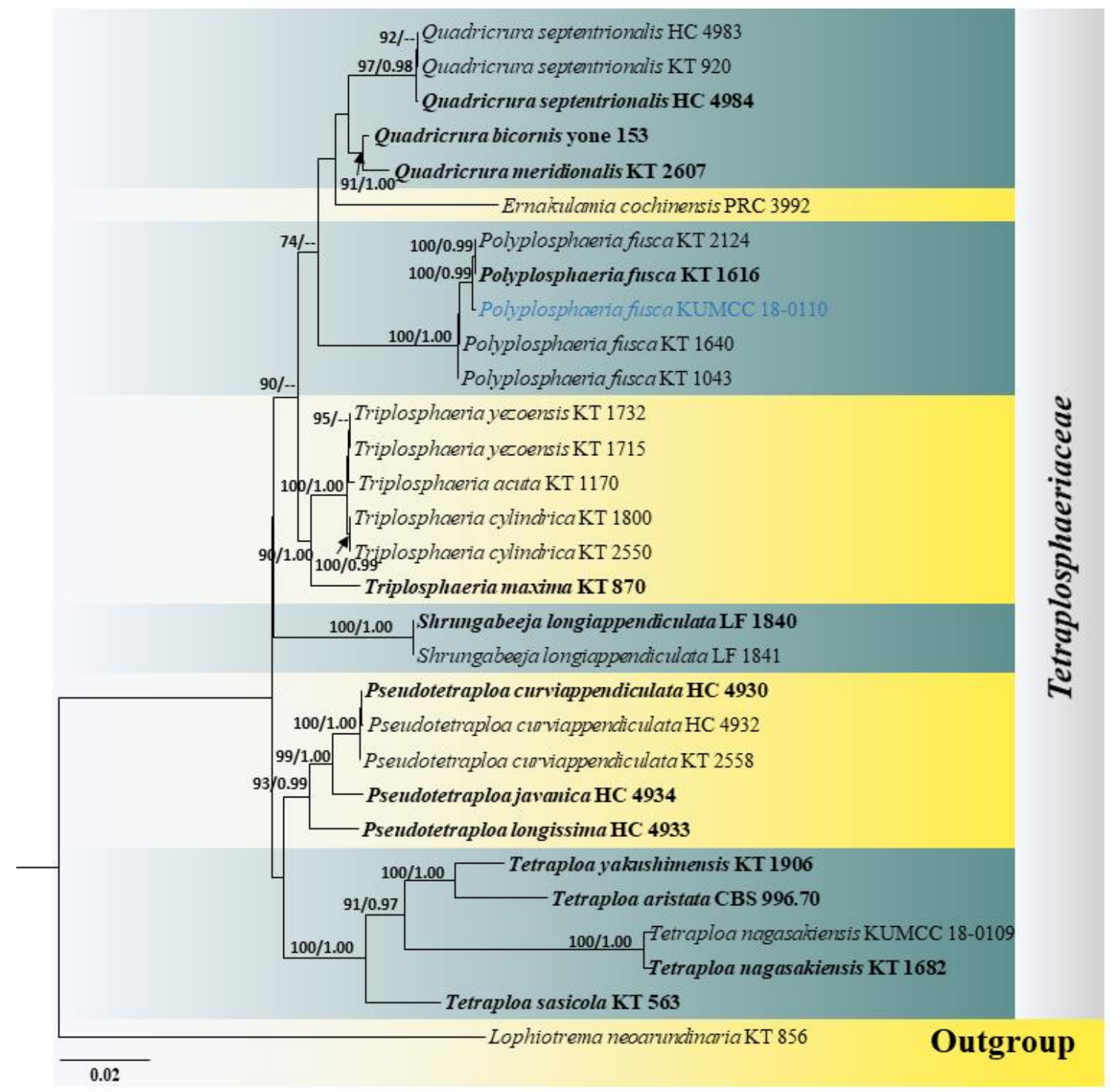

Fig. 88 - Phylogram generated from maximum likelihood analysis based on a combined ITS, LSU and SSU sequence dataset. Thirty strains are included in the combined gene analyses comprising 3,219 total characters including gaps (ITS: 1-579 bp, LSU: 580-1877 bp, SSU: 1878-3219 bp). The tree topology derived from the Bayesian analysis was similar to that derived from the maximum likelihood analysis. The best scoring of the ML tree is selected to represent the phylogenetic relationships of Polyplosphaeria species, with the final ML optimization likelihood: 8728.061069. The matrix had 434 distinct alignment patterns, with $16.08 \%$ undetermined characters or gaps. Estimated base frequencies were as follows: $\mathrm{A}=0.251610, \mathrm{C}=0.234539, \mathrm{G}=$ $0.277180, \mathrm{~T}=0.236670$; substitution rates $\mathrm{AC}=4.456817, \mathrm{AG}=4.305638, \mathrm{AT}=2.601496, \mathrm{CG}=$ 1.500369, CT $=14.507453, \mathrm{GT}=1.000000$; Tree-Length $=0.450936$; gamma distribution shape parameter $\alpha=0.497321$; The proportion of invariable sites $\mathrm{I}=0.712404$. Bayesian posterior probabilities (BYPP) from MCMC were evaluated with a final average standard deviation of split frequencies of 0.009756. Bootstrap support for maximum likelihood (MLBP) greater than $70 \%$ and Bayesian posterior probabilities (BYPP) greater than 0.95 are defined above the nodes as MLBP/BYPP. The tree is rooted to Lophiotrema neoarundinaria (KT 856). The type strain is indicated in bold and newly generated sequence is shown in blue. 

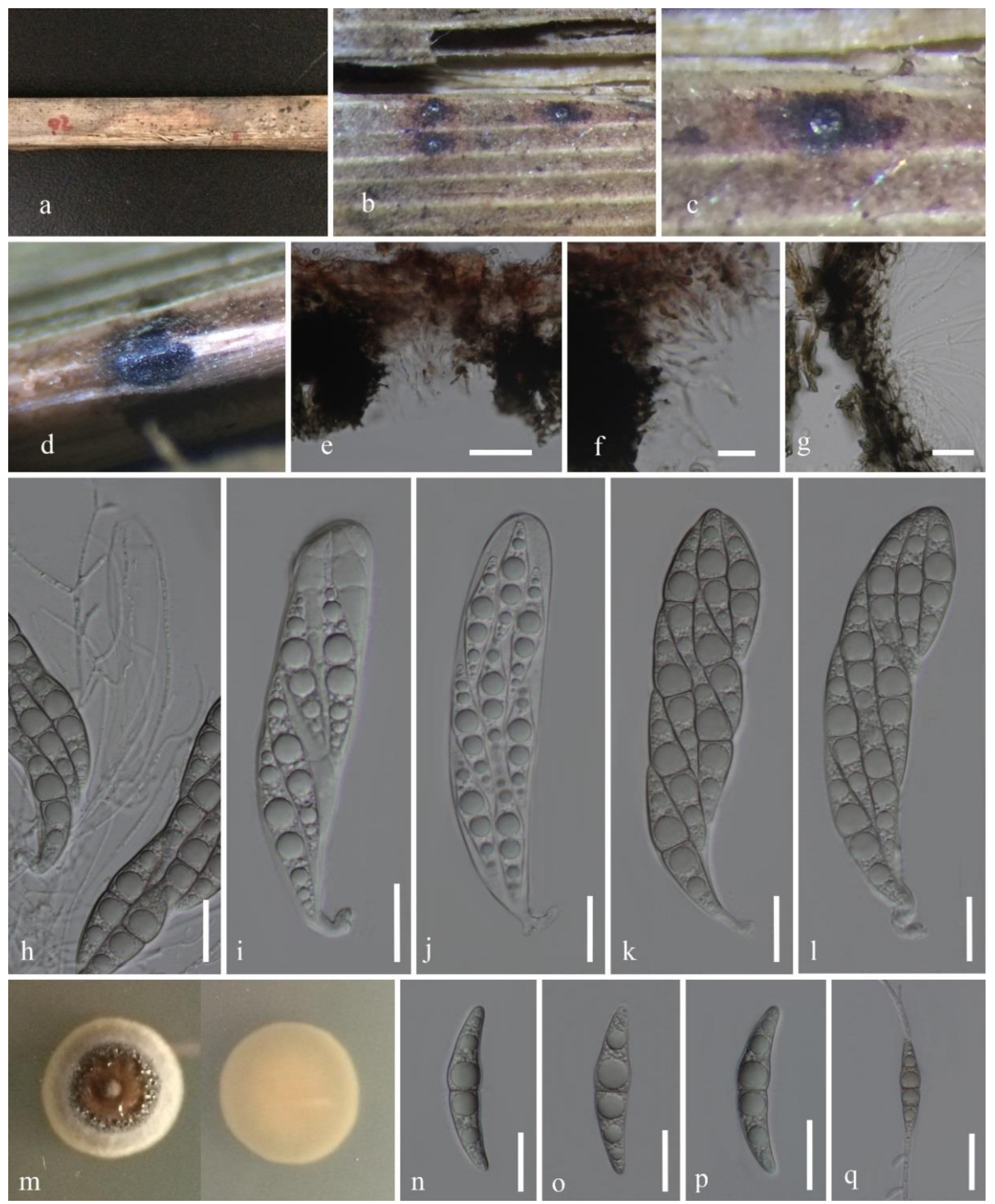

Fig. 89 - Polyplosphaeria fusca (HKAS 101757, new geographic record). a-c Ascomata on bamboo host. d Ascoma from lateral view. e Ostiole. f Periphyses. g Peridium. h Pseudoparaphyses. i-1 Asci. $m$ Culture characteristics. $n-p$ Ascospores. $q$ Germinating ascospore. Scale bars: e, $q=30$ $\mu \mathrm{m}, \mathrm{g}-1, \mathrm{n}-\mathrm{p}=20 \mu \mathrm{m}, \mathrm{f}=10 \mu \mathrm{m}$.

Culture characteristics - Conidia germinated on PDA within $12 \mathrm{~h}$ at $20-25^{\circ} \mathrm{C}$, with germ tubes produced from a one end. Colonies on PDA moderate growing, dense, irregular in shape, flattened to raised, rough with entire edge, velutinous to floccose, greenish grey in the center and to dull green towards margin, mycelia white, thin, regular. Colony reverse grey to brownish grey. Soluble pigments and exudates are absent.

Known distribution (based on molecular data) - JAPAN, Hokkaido, Yoichi, Sawamachi (Tanaka et al. 2009), China, Kunming (this study). 
Known hosts (based on molecular data) - Sasa senanensis (Tanaka et al. 2009), Phragmites australis (this study).

Material examined - China, Kunming, Lake Dianchi, on decaying stems of Phragmites australis (Poaceae), 28 Nov 2016, A. Karunarathna, AKKIB 57 (MFLU 17-0377, new host and geographical record); living culture, MFLUCC 17-1387; KUMCC 16-0240.

GenBank number - LSU: MN989185.

Notes - Here we report our collection from a new host and geographical record for Tetraploa sasicola based on phylogeny and taxonomy (Fig. 90).

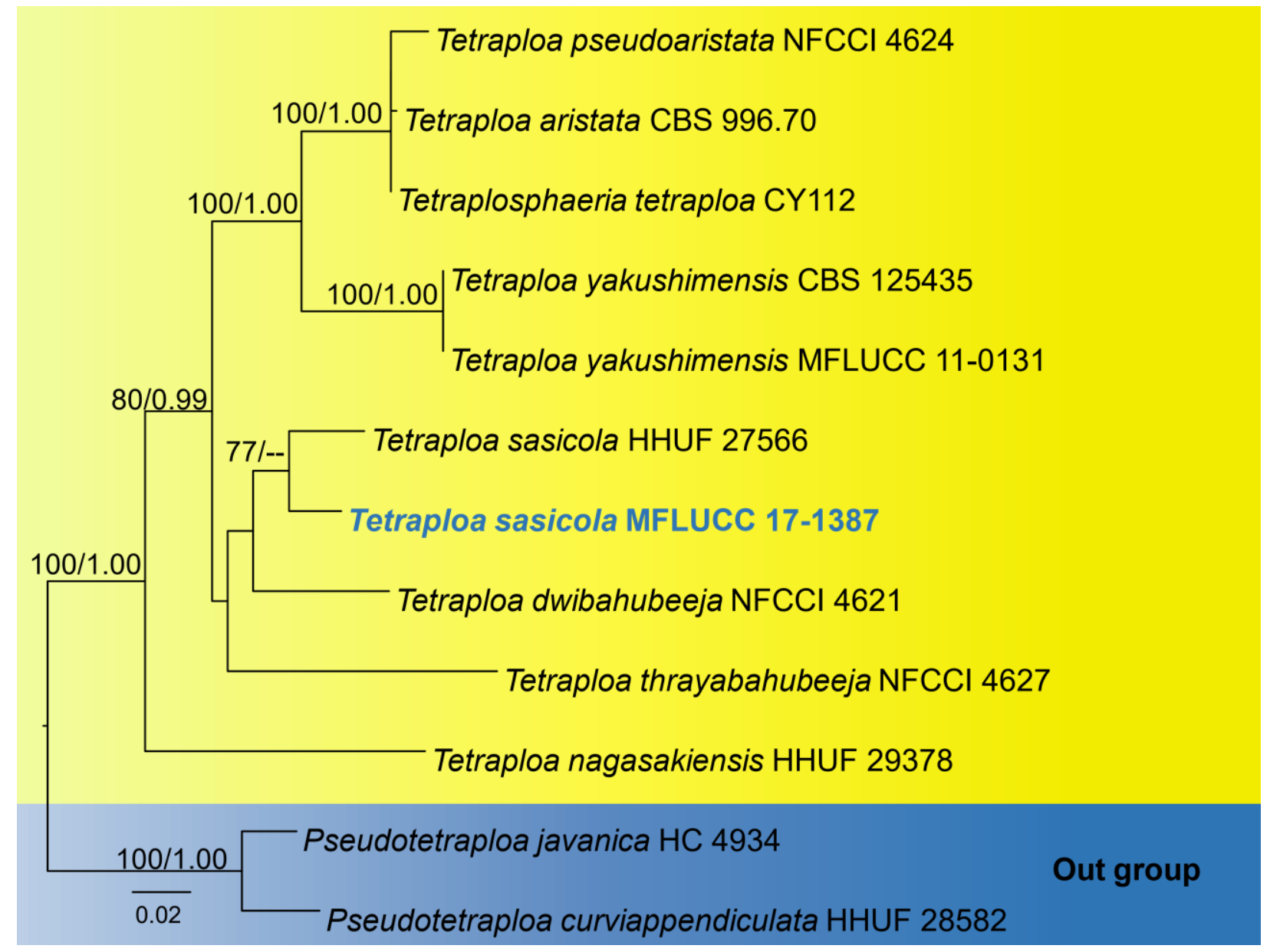

Fig. 90 - Phylogenetic tree generated from maximum likelihood (ML) based on LSU, ITS and RPB2 sequences. Bootstrap support (BS) values above 50\% and Bayesian posterior probabilities equal or greater than 0.90 are shown above the branches at nodes. The tree is rooted with Pseudotetraploa javanica and P. curviappendiculata. The best RaxML tree with a final likelihood value of -6537.602092 is presented. The matrix had 461 distinct alignment patterns, with $24.41 \%$ undetermined characters or gaps. Estimated base frequencies were as follows: $\mathrm{A}=0.238681, \mathrm{C}=$ $0.271326, \mathrm{G}=0.277534, \mathrm{~T}=0.212459$; substitution rates $\mathrm{AC}=3.351614, \mathrm{AG}=2.810733, \mathrm{AT}=$ 2.048449, $\mathrm{CG}=1.295686, \mathrm{CT}=8.198345, \mathrm{GT}=1.000000$; gamma distribution shape parameter $\alpha$ $=0.563647$. Newly generated sequence is in blue-bold.

\section{Torulaceae Corda}

Torulaceae was established by Sturm (1829) based on the type genus Torula Pers. Sexual morphs are undetermined in this family. Currently, six genera are accepted in Torulaceae (Li et al. 2016c, Su et al. 2016b, Su et al. 2018, Hyde et al. 2020).

Dendryphion Wallr.

Dendryphion was introduced by Wallroth (1833), typified by D. comosum. Seventy seven 
epithets and eight varieties are listed in Index Fungorum (2020), but sequence data are available for only few species.

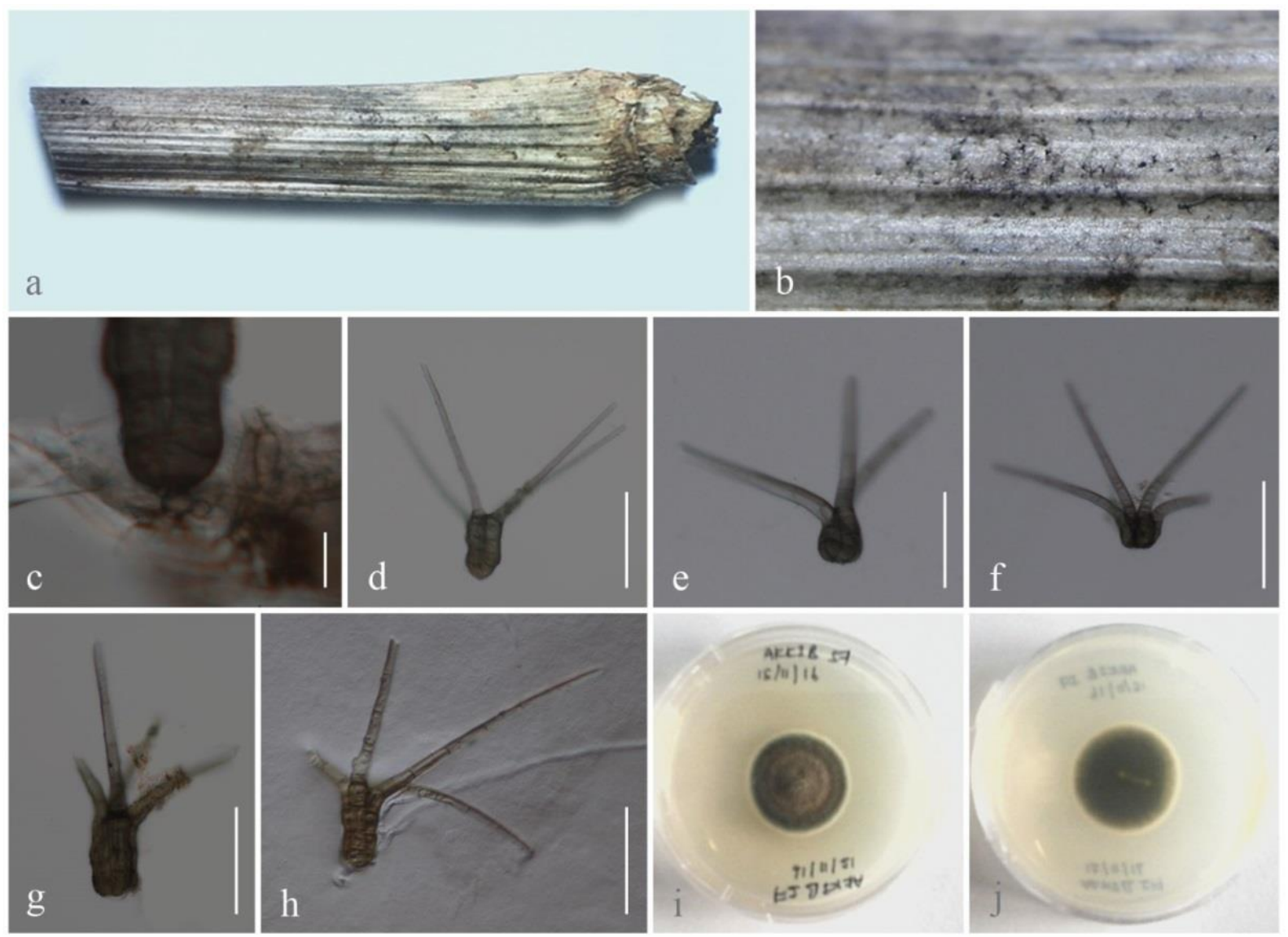

Fig. 91 - Tetraploa sasicola (MFLU 17-0377, new host and geographical record). a Appearance of the host. b Colonies on host substrate. c Attachment of conidia to the conidiophore. $\mathrm{d}-\mathrm{g}$ Conidia. $\mathrm{h}$ Germinated conidium. $\mathrm{i}-\mathrm{j}$ Culture characteristics. Scale bars: $\mathrm{c}=50 \mu \mathrm{m} \mathrm{d}-\mathrm{h}=50 \mu \mathrm{m}$.

Dendryphion nanum (Nees) S. Hughes, Canadian Journal of Botany 36 (6): 761 (1958) Fig. 93 Facesoffungi number: FoF06714

Saprobic on dead stems of Sambucus ebulus. Sexual morph: Undetermined. Asexual morph: Hyphomycetous. Colonies on natural substrate effuse, dark brown or black, velvety. Mycelium immersed, composed of hyaline, septate, branched hyphae. Conidiophores macronematous, mononematous, erect, straight to broadly curved, septate, thick-walled, smooth, dark brown, branched in the upper half. Conidiogenous cells polytretic, terminal and intercalary, integrated, discrete, cylindrical, with raised brown large scars appear when the conidia detached. Conidia 40$70 \mu \mathrm{m}$ long, 8-14 $\mu \mathrm{m}$ in the broadest part, solitary or in chains, dry, obclavate always tapered somewhat at the ends, rounded or with a scar at the apex; truncate with a well-defined dark brown scar at the base, septate, slightly constricted at the septa, median brown when immature, dark brown except for the end cells which are median brown, verrucose.

Culture characteristics - Conidia germinating on water agar within $24 \mathrm{~h}$. Germ tubes produced from one or both ends. Mycelia superficial, circular, sparse, decumbent, with entire edge, white from above and below.

Known distribution (based on molecular data) - Germany (Farr et al. 2000), Canada (Corredor 2011), Sweden (Grudzinska-Sterno et al. 2016) China, Yunan Province (Su et al. 2016b), Su et al. 2018), Italy (this study).

Known hosts (based on molecular data) - Soil (Farr et al. 2000), Salix sp. (Corredor 2011), Winter wheat (Grudzinska-Sterno et al. 2016), Submerged decaying wood (Su et al. 2016b, Su et 
al. 2018), Sambucus ebulus (this study).

Material examined - Italy, on a dead stem of Sambucus ebulus, E. Camporesi, I3077 (GZAAS 20-0004, new geogrophical record), living culture, GZCC 20-0003.

GenBank numbers - ITS: MN999921; LSU: MN999926.

Notes - Dendryphion nanum was introduced by Hughes (1958). These species are common on dead stems of herbaceous plants, also on roots and cut tree stumps (Ellis 1971). Su et al. (2016b) reported D. nanum on submerged decaying wood from Yunnan Province, China and designated the reference specimen (HKAS 84012). Our collection has slightly smaller conidia (40-70 $\mu \mathrm{m}$ long) compared with those of reference specimen (56.5-74.5 $\mu \mathrm{m}$ long). Molecular data confirmed that our collection is D. nanum (Fig. 92). This is a new geographical record of D. nanum in Italy.

\section{Torula Pers.}

Torula is an asexual genus, which is characterized by dark brown to black, effuse, powdery colonies, with terminal or lateral, monoblastic or polyblastic conidiogenous cells. The conidiogenous cells are thickened, with heavily melanized walls at the base, becoming coronate at the apex, cupulate, brown, smooth to verruculose, with the terminal cell of conidia often becoming a conidiogenous cell. Conidia are acrogenous, in short to long branched chains, pigmented, phragmosporous to scolecosporous, moniliform, smooth to verrucose ( $\mathrm{Su}$ et al. 2016b, $\mathrm{Li}$ et al. 2017b, 2020, Hyde et al. 2019, 2020). Currently, more than 500 epithets are listed in Index Fungorum (2020), although, only 16 species have molecular data to confirm their phylogenetic affinities in Torulaceae (Crous et al. 2015a, Su et al. 2016b, 2018, Li et al. 2017b, Hyde et al. 2017, 2019, 2020, Tibpromma et al. 2017). We followed the latest treatments and updated accounts of Torula in Hyde et al. (2019, 2020). In this study, Torula chromolaenae is reported from dead branch of a herbaceous plant in Yunnan, China for the first time.

Torula chromolaenae J.F. Li, Phookamsak, A. Mapook \& K.D. Hyde, Mycol Progress 16:447-461 (2017)

Fig. 94

Facesoffungi number: FoF 02713

Saprobic on herbaceous litter. Sexual morph: Undetermined. Asexual morph: Hyphomycetous, visible as black, powdery, dense to effuse colony on the natural substrate. Mycelium 1.8-3.5 $\mu \mathrm{m}$ wide, immersed to superficial, composed of septate, branched, smooth, pale brown hyphae, becoming black closer to fertile region. Conidiophores $(2-) 5-8(-13) \times 2-5 \mu \mathrm{m}(\bar{x}=7.2 \times 3.7 \mu \mathrm{m}, \mathrm{n}$ = 30) macronematous, mononematous, erect, solitary, brown, oblong to cylindrical, smooth, straight or slightly flexuous, 0-1-septate, without apical branches, occasionally reduced to conidiogenous cells. Conidiogenous cells $4-8(-11) \times 4-6.5 \mu \mathrm{m}(\bar{x}=5.5 \times 5.5 \mu \mathrm{m}, \mathrm{n}=30)$, polyblastic, integrated, terminal, brown to dark brown, cupulate to subglobose, with terminal cell of conidia become conidiogenous cell, smooth, thick-walled, doliiform. Conidia (9-)10-16(-22) $\times 5-$ $7 \mu \mathrm{m}(\bar{x}=13 \times 6.1 \mu \mathrm{m}, \mathrm{n}=50)$, catenate, in branch chains, dry, acrogenous, brown to dark brown, oblong to cylindrical, 2-3-septate, constricted at septa, rounded at apex, with truncate base, smooth to minutely verruculose.

Known distribution (based on molecular data) - Thailand (Li et al. 2017b), Yunnan, China (Tibpromma et al. 2018b, this study).

Known hosts (based on molecular data) - Chromolaena odorata (Asteraceae; Li et al. 2017b), on fallen dead and decaying leaves of Pandanus tectorius (Tibpromma et al. 2018b), on herbaceous litter (this study).

Material examined - China, Yunnan Province, Xishaungbanna, Jinghong, the Nabanhe National Nature Reserve, on herbaceous litter, 25 November 2015, R. Phookamsak, XB012 (MFLU 20-0140, new host record). 


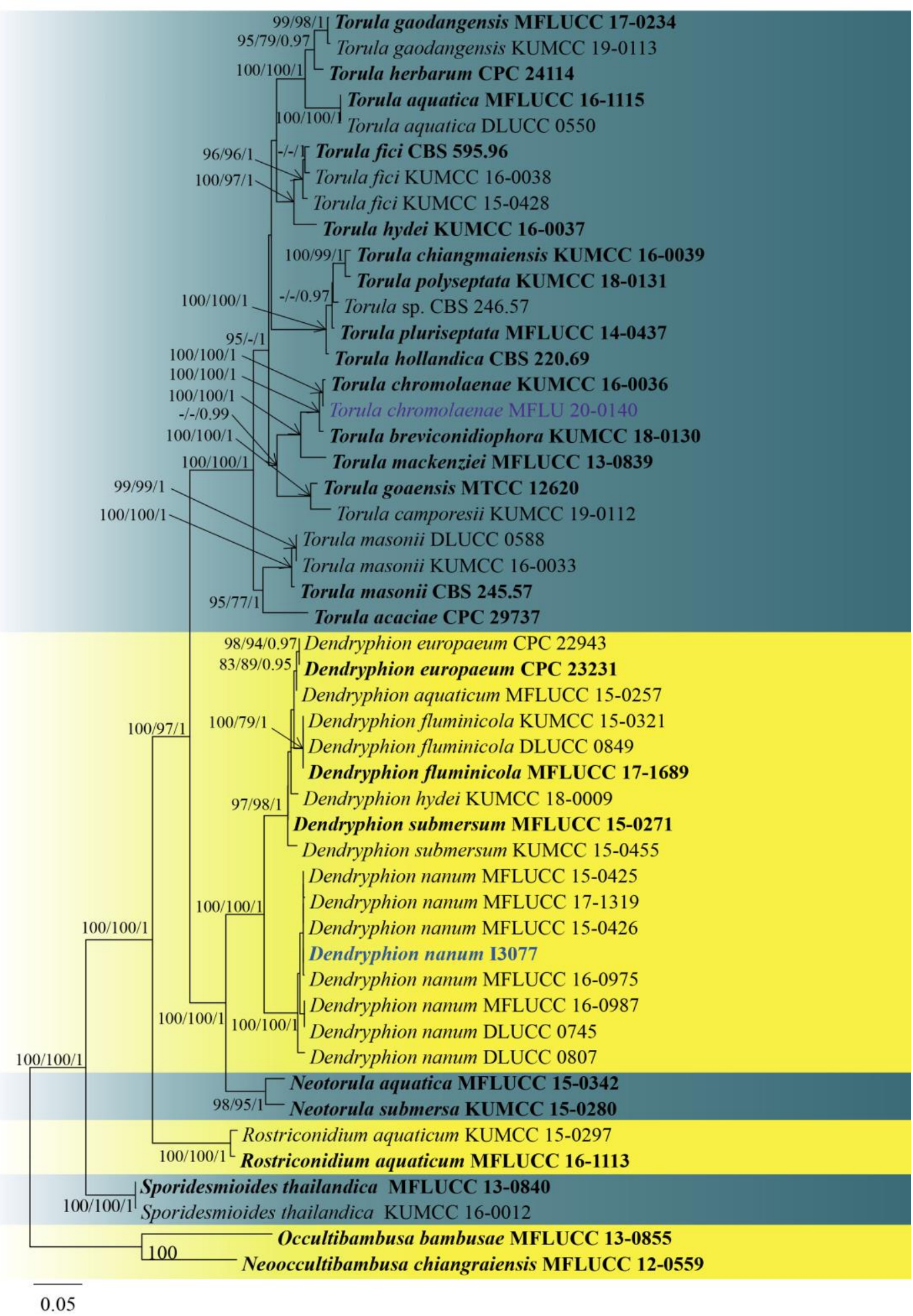

Fig. 92 - Phylogram generated from maximum likelihood analysis based on combined LSU, ITS, SSU, tef1 and RPB2 sequence data. Forty-nine strains are included in the combined gene analyses comprising 4003 characters after alignment (841 characters for LSU, 592 characters for ITS, 934 characters for SSU, 787 characters for tef1, 849 characters for RPB2). Neooccultibambusa chiangraiensis (MFLUCC 12-0559) and Occultibambusa bambusae (MFLUCC 13-0855) are used as the outgroup taxa. The tree topologies derived from the Bayesian analysis and maximum parsimony analysis were similar to that derived from the maximum likelihood analysis. The best RAxML tree with a final likelihood value of -18261.964665 is presented. The matrix had 1151 distinct alignment patterns, with $33.41 \%$ undetermined characters or gaps. Estimated base frequencies were as follows: $\mathrm{A}=0.245872, \mathrm{C}=0.261687, \mathrm{G}=0.270980, \mathrm{~T}=0.221461$; 
substitution rates $\mathrm{AC}=1.850933, \mathrm{AG}=4.358262, \mathrm{AT}=1.744260, \mathrm{CG}=1.264921, \mathrm{CT}=$ 9.195943, GT $=1.000000$; gamma distribution shape parameter $\alpha=0.137863$. Bootstrap values for maximum likelihood equal to or greater than 75 and Bayesian posterior probabilities equal or greater than 0.95 are placed near the nodes. Ex-type or ex-epitype strains are in bold and black. The newly generated sequences are indicated in bold and blue.

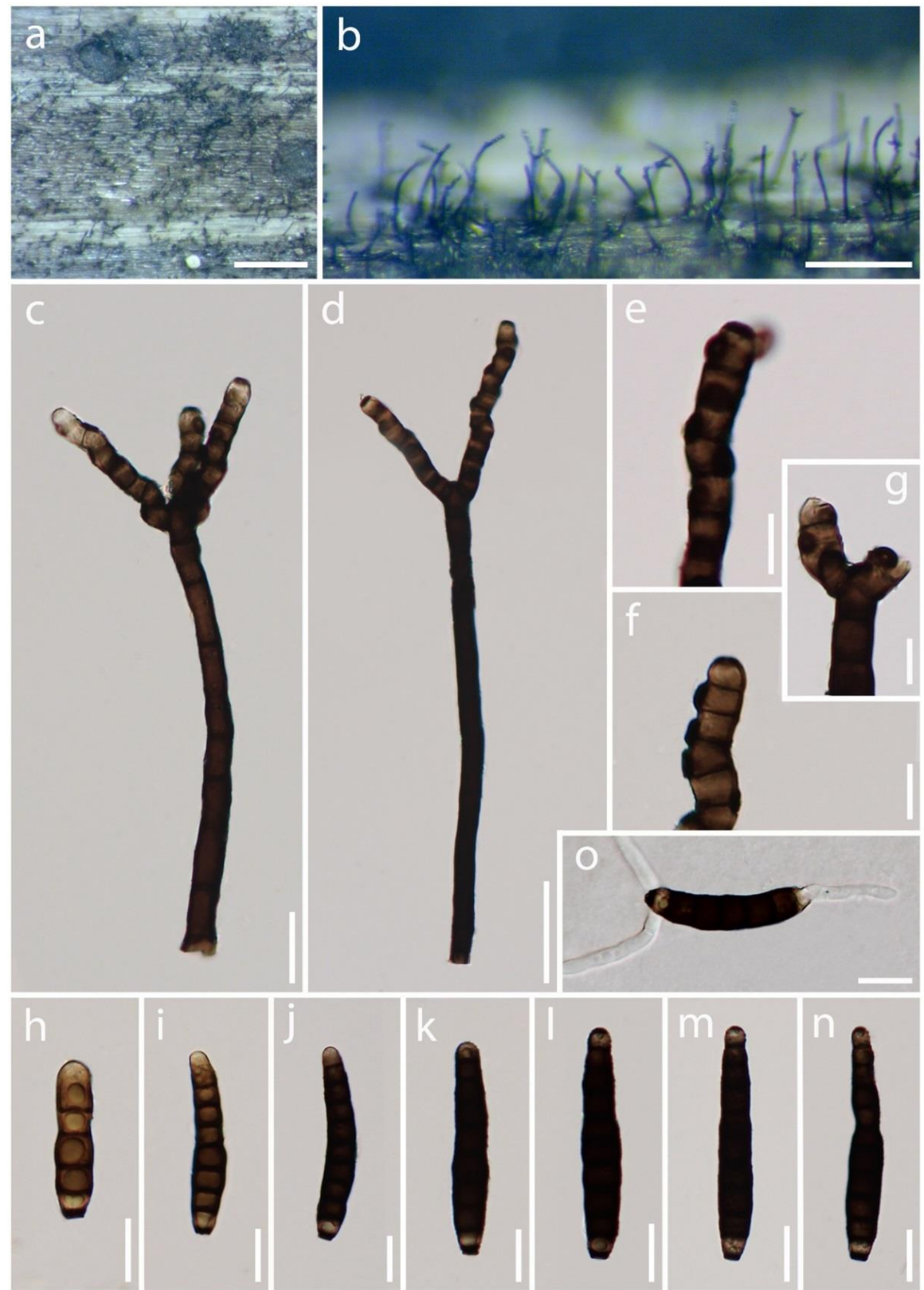

Fig. 93 - Dendryphion nanum (GZAAS 20-0004, new geogrophical record). a, b Colonies on substrate. c, d Conidiophores. e-g Conidiogenous cells. $\mathrm{h}-\mathrm{n}$ Conidia. o Geminated conidium. Scale bar: $\mathrm{a}=500 \mu \mathrm{m}, \mathrm{b}=200 \mu \mathrm{m}, \mathrm{c}=20 \mu \mathrm{m}, \mathrm{d}=50 \mu \mathrm{m}, \mathrm{e}-\mathrm{g}=10 \mu \mathrm{m}, \mathrm{h}-\mathrm{m}=15 \mu \mathrm{m}$. 


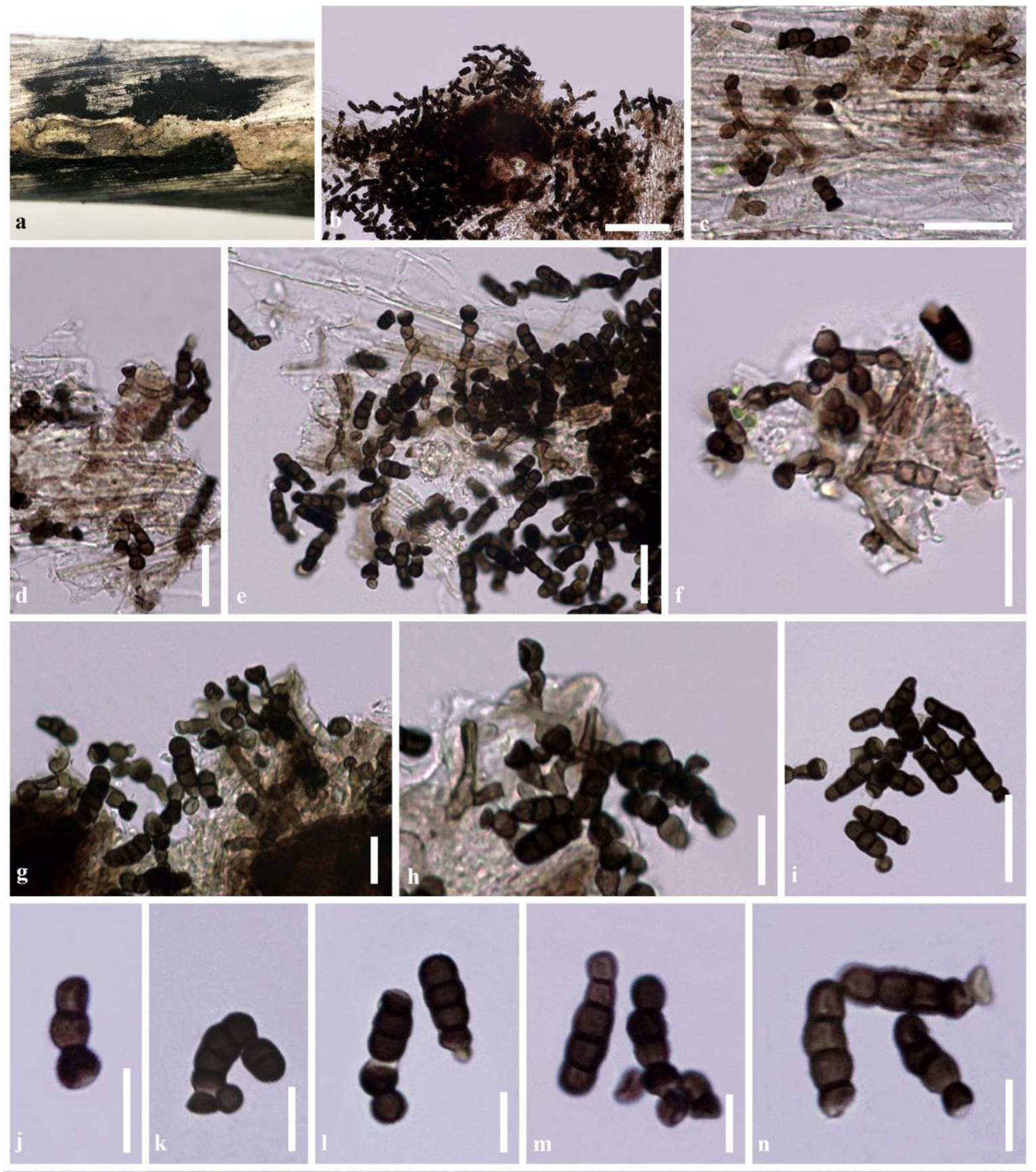

Fig. 94 - Torula chromolaenae (MFLU 20-0140, new host record). a Appearance of colonies on host substrate. b-e Colonies on host substrate showing partly immersed mycelium and its erect conidiophores attached with conidia. $\mathrm{f}-\mathrm{h}$ Conidiophores and conidiogenous cells. $\mathrm{i}-\mathrm{n}$ Conidia. Scale bars: $\mathrm{b}=50 \mu \mathrm{m}, \mathrm{c}-\mathrm{f}, \mathrm{i}=20 \mu \mathrm{m}, \mathrm{g}, \mathrm{h}, \mathrm{j}-\mathrm{n}=10 \mu \mathrm{m}$.

GenBank numbers - ITS: MT007586, LSU: MT007599, SSU: MT007598.

Notes - In NCBI BLASTn search based on ITS sequences, our strain XB012 is most similar to Torula chromolaenae strain NBHC3-4 (99.18\% similarity) and the type strain KUMCC 16-0036 (99.05\% similarity). Multi-gene phylogenetic analyses showed that our strain is sister to the type strain of T. chromolaenae with high support (99\% ML, 1.00 BYPP; Fig. 92). A comparison of ITS and tefl also showed that our strain is identical to T. chromolaenae. Our collection shares the size range of the conidiophores, conidiogenous cells and conidia with the type of $T$. chromolaenae (Li et al. 2017b). Furthermore, our collection resembles the type of T. chromolaenae in having brown, oblong to cylindrical, 2-3-septate, smooth to minutely verruculose conidia, although, our collection has a truncate base to the conidia and lacks a paler apex (Li et al. 2017b). Therefore, we identify 
our collection as T. chromolaenae. Torula chromolaenae was introduced by Li et al. (2017b) from Chromolaena odorata (Asteraceae) in Thailand. Tibpromma et al. (2018b) reported the species occurring on decaying leaves of Pandanus tectorius in Yunnan, China. Our collection is also collected from Yunnan, China. However, it was found on herbaceous litter.

Asterinales M.E. Barr ex D. Hawksw. \& O.E. Erikss.

\section{Asterinaceae Hansf.}

Phylogenetic studies have found that phylogenetic placement of Asterinaceae is unresolved with taxa occurring in two separate lineages (Zeng et al. 2019).

\section{Asterostomella Speg.}

Asterostomella is the asexual morph of Asterinaceae characterized by brown, ovoid to oblong conidia, sometimes with a hyaline band in the center (Hongsanan et al. 2014). Species distinction in the genus has largely been based on fruiting body diameter and conidia measurements (Reynolds \& Gilbert 2005). We provide the first sequence data for this genus herein and phylogenetically confirm its placement as a member of Asterinaceae.

Asterostomella grewiae Petr., Sydowia 12: 485 (1959) [1958]

Fig. 96

Facesoffungi number: FoF06899

Parasitic on living leaves. Sexual morph: Undetermined. Asexual morph: Colonies epiphyllous, densely scattered, confluent. Hyphae straight to substraight, loosely reticulate. Hyphopodia 8-11 $\times 6-9 \mu \mathrm{m}(\bar{x}=9 \times 7 \mu \mathrm{m}, \mathrm{n}=5), 2$-celled, mostly lobed. Pycnothyria $71-93 \mu \mathrm{m}$ $(\bar{x}=80 \mu \mathrm{m}, \mathrm{n}=20)$ in diameter, superficial, dark brown, seated on a thin, hyaline basal stroma with a central X-or Y-shaped dehiscence. Upper wall comprising radial arranged square cells, with meandrous marginal cells. Pycnothyriospores 12-15 $\times 10-11 \mu \mathrm{m}(\bar{x}=14 \times 10.5 \mu \mathrm{m}, \mathrm{n}=30)$, aseptate, ovoid to pyriform, 2-layered.

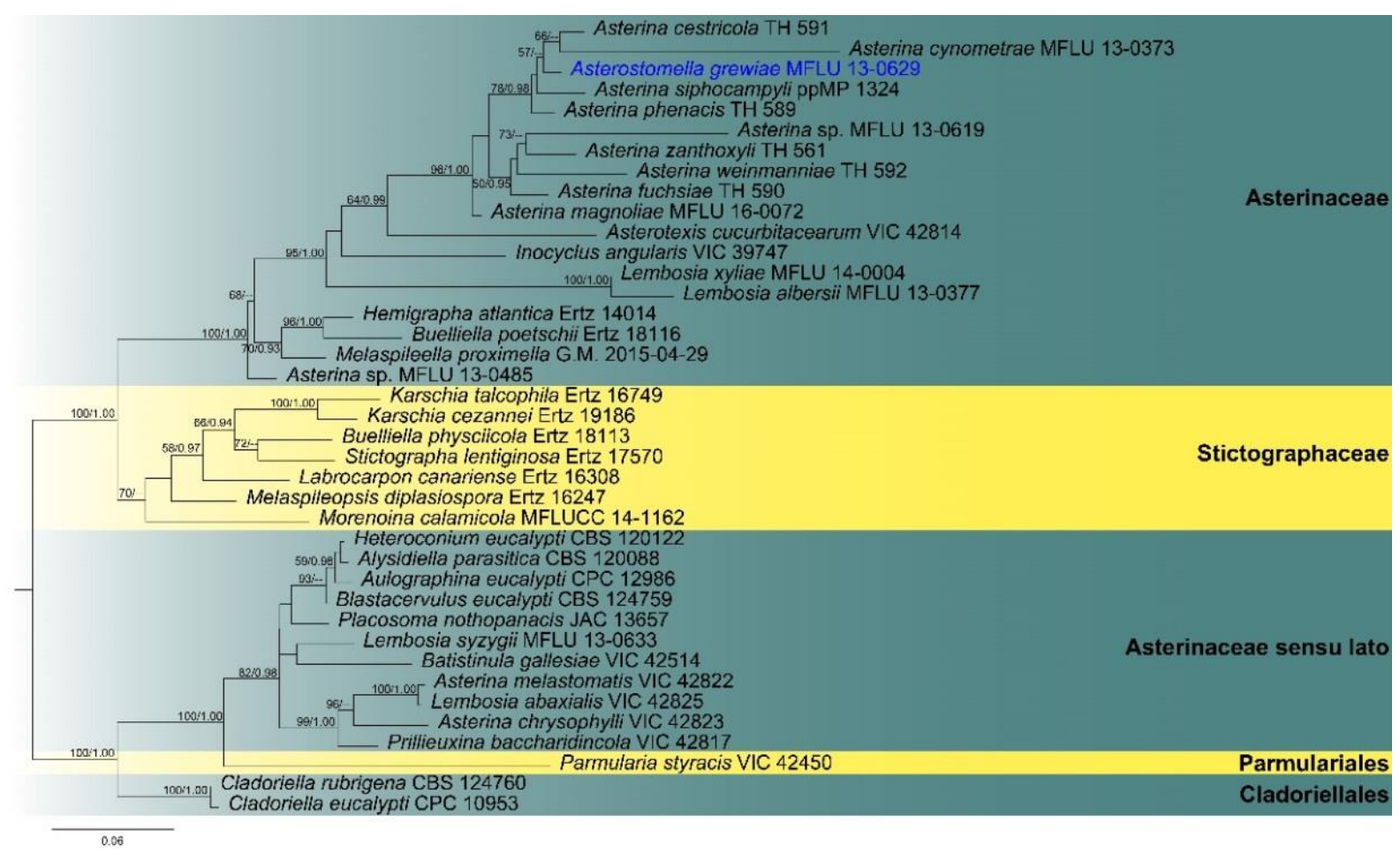

Fig. 95 - Phylogram generated from maximum likelihood analysis based on LSU sequence data. Thirty-nine strains are included in the analyses comprising 832 characters after alignment. Cladoriella rubrigena (CBS 124760) and C. eucalypti (CPC 10953) are used as out group taxa. The best RAxML tree with a final likelihood value of -6491.621315 is presented. The matrix had 453 distinct alignment patterns, with $11 \%$ undetermined characters or gaps. Estimated base frequencies 
were as follows: $\mathrm{A}=0.231783, \mathrm{C}=0.252162, \mathrm{G}=0.326987, \mathrm{~T}=0.189068$; substitution rates $\mathrm{AC}=$ $0.895393, \mathrm{AG}=2.984753, \mathrm{AT}=0.711520, \mathrm{CG}=1.133511, \mathrm{CT}=9.597818, \mathrm{GT}=1.000000$; gamma distribution shape parameter $\alpha=0.587263$. Bootstrap values for maximum likelihood equal to or greater than 50 and Bayesian posterior probabilities equal or greater than 0.95 are placed above the branches. The newly generated sequence is indicated in blue.

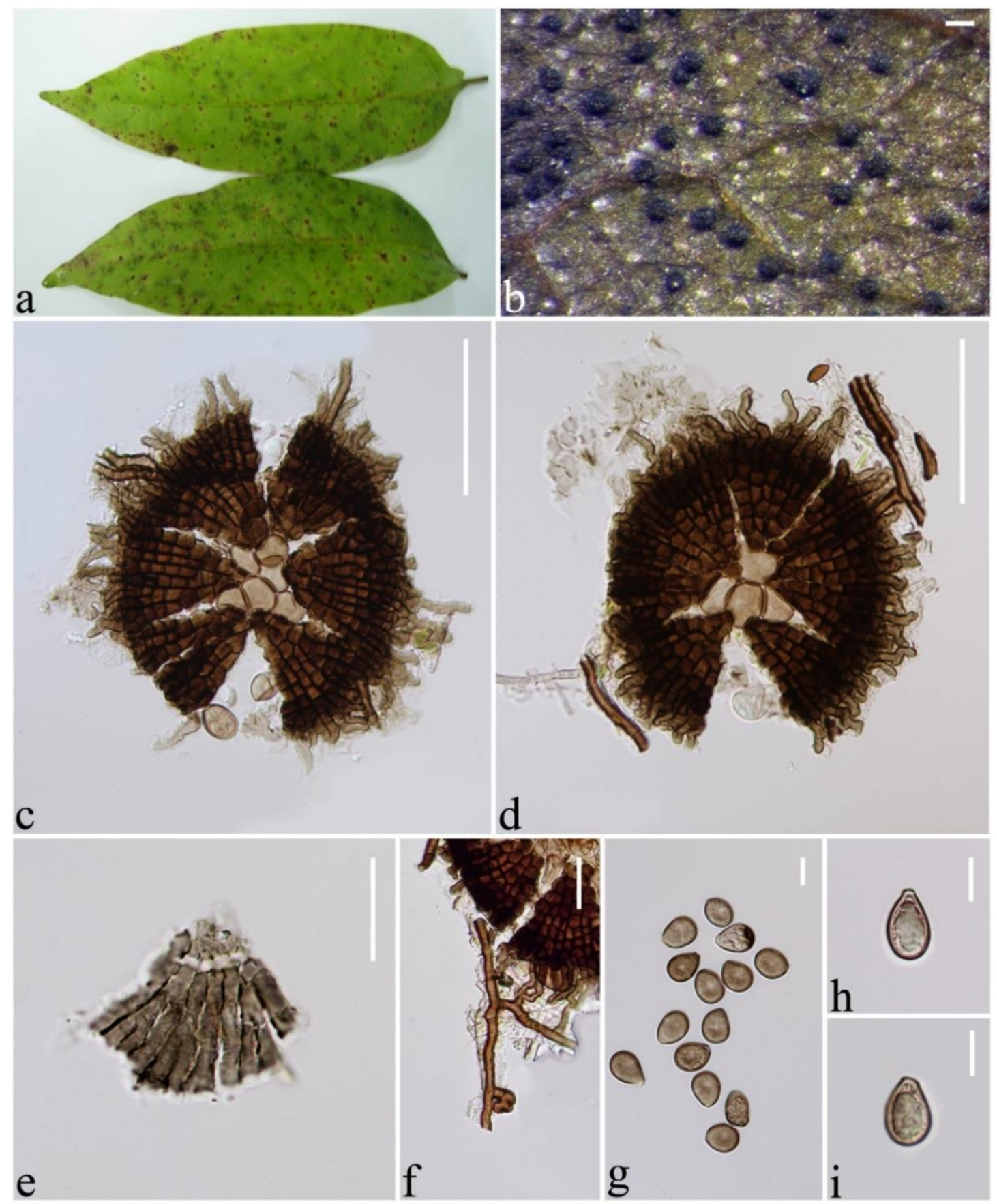

Fig. 96 - Asterostomella grewiae (MFLU 13-0629, geographical record). a Host leaves. b Colonies on leaf surface. c, d Ascomata. e Basal layer of the pycnothyrium wall. f Hyphae with hyphopodia. $\mathrm{g}-\mathrm{i}$ Conidia. Scale bars: $\mathrm{b}=100 \mu \mathrm{m}, \mathrm{c}, \mathrm{d}=50 \mu \mathrm{m}, \mathrm{e}, \mathrm{f}=20 \mu \mathrm{m}, \mathrm{g}-\mathrm{i}=10 \mu \mathrm{m}$.

Known distribution (based on molecular data) - Philippines (Petrak 1983), Thailand (this study). study).

Known hosts (based on molecular data) - Grewia multiflora (Petrak 1983), Malvaceae (this 
Material examined - Thailand, Chiang Mai, Mae Taeng, Pa Pae, Bahn Pa Deng, 128 Moo 3, Mushroom Research Centre, on the living leaves of Malvaceae, 22 November 2013, X.Y. Zeng (MFLU13-0629, new sequence data and geographical record, reference specimen designated here).

GenBank numbers - LSU: MN364645; SSU: MN364416.

Notes - Asterostomella species reported from Malvaceae include A. diplocarpa, A. grewiae, A. helicteris and $A$. isothea. Our new collection is similar to Asterostomella grewiae in the size of pycnothyria (71-93 $\mu \mathrm{m}$ vs 40-90 $\mu \mathrm{m}$ in diameter), hyphopodia $(8-11 \times 6-9 \mu \mathrm{m}$ vs 5-8 $\times 6-11 \mu \mathrm{m})$ and pycnothyriospores $(12-15 \times 10-11 \mu \mathrm{m}$ vs $10-17 \times 6-10 \mu \mathrm{m})$, as well as 4-lobed hyphopodia and the host leaves. Therefore, we designate a reference specimen herein with illustrations and sequence data (Fig. 95).

\section{Botryosphaeriales}

\section{Aplosporellaceae Slippers, Boissin \& Crous}

Aplosporellaceae was introduced by Slippers et al. (2013) and is typified by Aplosporella with $A$. chlorostroma as the type species. Recently, three genera were accommodated in this family viz. Alanomyces, Aplosporella and Bagnisiella (Dissanayake et al. 2016, Sharma et al. 2017, Wijayawardene et al. 2018).

\section{Aplosporella Speg.}

Aplosporella was introduced by Spegazzini (1880) and is characterized by uni- to multiloculate, pycnidial, stromatic conidiomata, with a communal ostiole, and aseptate, ellipsoid to subcylindrical conidia, brown to dark brown and spinulose when mature (Hyde et al. 2013, Slippers et al. 2013, Ekanayaka et al. 2016, Sharma et al. 2017). We follow the latest treatments and updated accounts of Aplosporella in Fan et al. (2015b), Dissanayake et al. (2016), Ekanayaka et al. (2016), Du et al. (2017), Dou et al. (2017a), Jia et al. (2019) and Phillips et al. (2019). Aplosporella javeedii is reported from Acer palmatum in Yunnan, China for the first time.

Aplosporella javeedii Jami, Gryzenh., Slippers \& M.J. Wingf., Fungal Biology 118(2): 174 (2013)

Facesoffungi number: FoF07383

Fig. 98

Saprobic on dead hanging twigs of Acer palmatum. Sexual morph: Undetermined. Asexual morph: Conidiomata 210-290 $\mu \mathrm{m}$ high, 270-680 $\mu \mathrm{m}$ diameter, pycnidial, rounded or valsoid, stromatic, immersed to erumpent through host epidermis, becoming raised, superficial, dark brown to black, subglobose to dome-shaped, discoid or irregular in shape, 1-3-loculate, ostiolate, with a single ostiole throughout stroma. Locules quadrilateral to subglobose, or irregular in shape, 2-3 subdivided chamber separated by invaginations with common walls, the developing invaginations frequently present. Pycnidial walls 25-60 $\mu \mathrm{m}$ wide, thick-walled, composed of two type cells, outer layers comprising several cell layers, of large, broad, thick-walled, dark brown to black cells, arranged in a textura angularis to textura globulosa, inner layers comprising 1-3 layers of small, flattened, light brown pseudoparenchymatous cells paler towards the inner region, arranged in a textura prismatica to textura angularis; interstitial walls of a locule composed of several cell layers of flattened, light brown to brown pseudoparenchymatous cells, arranged in textura prismatica or palisade-like. Paraphyses 28-111 $\mu \mathrm{m}$ long, 1.4-3.2 $\mu \mathrm{m}(\bar{x}=68.4 \times 2.1 \mu \mathrm{m}, \mathrm{n}=40)$ wide, hyaline, indistinctly septate, thin-walled, filiform, with rounded tip, filamentous, associated with blastic conidiogenesis. Conidiophores reduced to conidiogenous cells. Conidiogenous cells (5.5-)15-28($31.5) \times(2.5-) 3-5.7(-7.7) \mu \mathrm{m}(\bar{x}=19.7 \times 4.1 \mu \mathrm{m}, \mathrm{n}=30)$, raising from the inner cavity, holoblastic, monophialidic, hyaline, smooth, short cylindrical to flask-shaped. Conidia 15-27 $\times 7-12 \mu \mathrm{m}(\bar{x}=$ $21.3 \times 10 \mu \mathrm{m}, \mathrm{n}=100$ ), initial hyaline, becoming brown to dark brown when mature, ellipsoidal to oblong with rounded ends, or subcylindrical, aseptate, reticulate.

Culture characteristics - Colonies on PDA fast growing, reaching $90 \mathrm{~mm}$ diameter, after 1 week at $20-25^{\circ} \mathrm{C}$, colonies medium sparse to dense, with aerial mycelia, circular, flat, surface slightly rough with edge entire, margin well defined, cottony to fairly fluffy, with standing tufts; 
colony from above and below, white to cream at the beginning, becoming black after 4 weeks; not producing pigments on PDA.

Known distribution (based on molecular data) - China, Beijing, Gansu, Henan, Heilongjiang, Shanxi, Yunnan (Fan et al. 2015b, Dou et al. 2017a, Jia et al. 2019), South Africa, Gauteng (Jami et al. 2013).

Known hosts (based on molecular data) - Acer buergerianum (Aceraceae), Albizia julibrissin (Fabaceae), Broussonetia papyrifera (Moraceae), Celtis africana (Cannabaceae), Chaenomeles sinensis (Rosaceae), Gleditsia sinensis (Fabaceae), Juglans regia (Juglandaceae), Juniperus chinensis (Cupressaceae), Morus alba (Moraceae), Searsia lancea (Anacardiaceae), Styphnolobium japonicum (Fabaceae), Ziziphus jujuba (Rhamnaceae) (Jami et al. 2013, Fan et al. 2015b, Dou et al. 2017a, Jia et al. 2019).

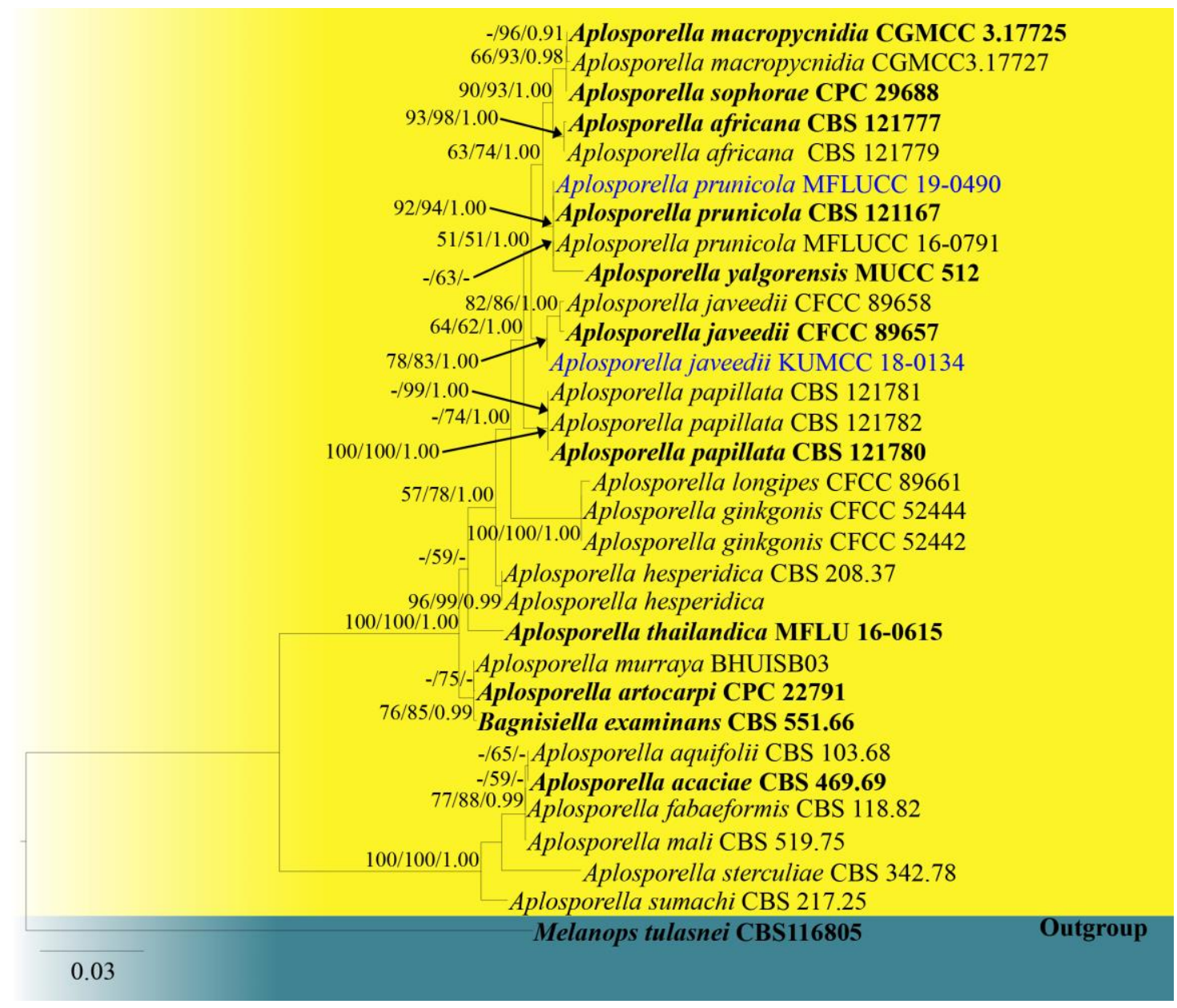

Fig. 97 - Phylogram generated from maximum likelihood analysis based on combined LSU-ITS and tefl sequence data. Thirty strains are included in the combined gene analyses comprising 1631 characters after alignment (844 characters for LSU, 621 characters for ITS, 164 characters for tef1). Melanops tulasnei (CBS 116805) is used as the outgroup taxon. The tree topology derived from the Bayesian analysis was similar to that derived from the maximum likelihood analysis. The best RAxML tree with a final likelihood value of -4527.083852 is presented. The matrix had 342 distinct alignment patterns, with $22.06 \%$ undetermined characters or gaps. Estimated base frequencies were as follows: $\mathrm{A}=0.235117, \mathrm{C}=0.239252, \mathrm{G}=0.286504, \mathrm{~T}=0.239127$; substitution rates $\mathrm{AC}=2.896203, \mathrm{AG}=3.311309, \mathrm{AT}=1.557883, \mathrm{CG}=2.969131, \mathrm{CT}=$ 7.107161, GT = 1.000000; gamma distribution shape parameter $\alpha=0.699794$. Bootstrap values for maximum parsimony and maximum likelihood equal to or greater than $50 \%$ and Bayesian posterior probabilities equal or greater than 0.95 are placed above or below the branches. Ex-type strains are in bold. The newly generated sequences are indicated in blue. 

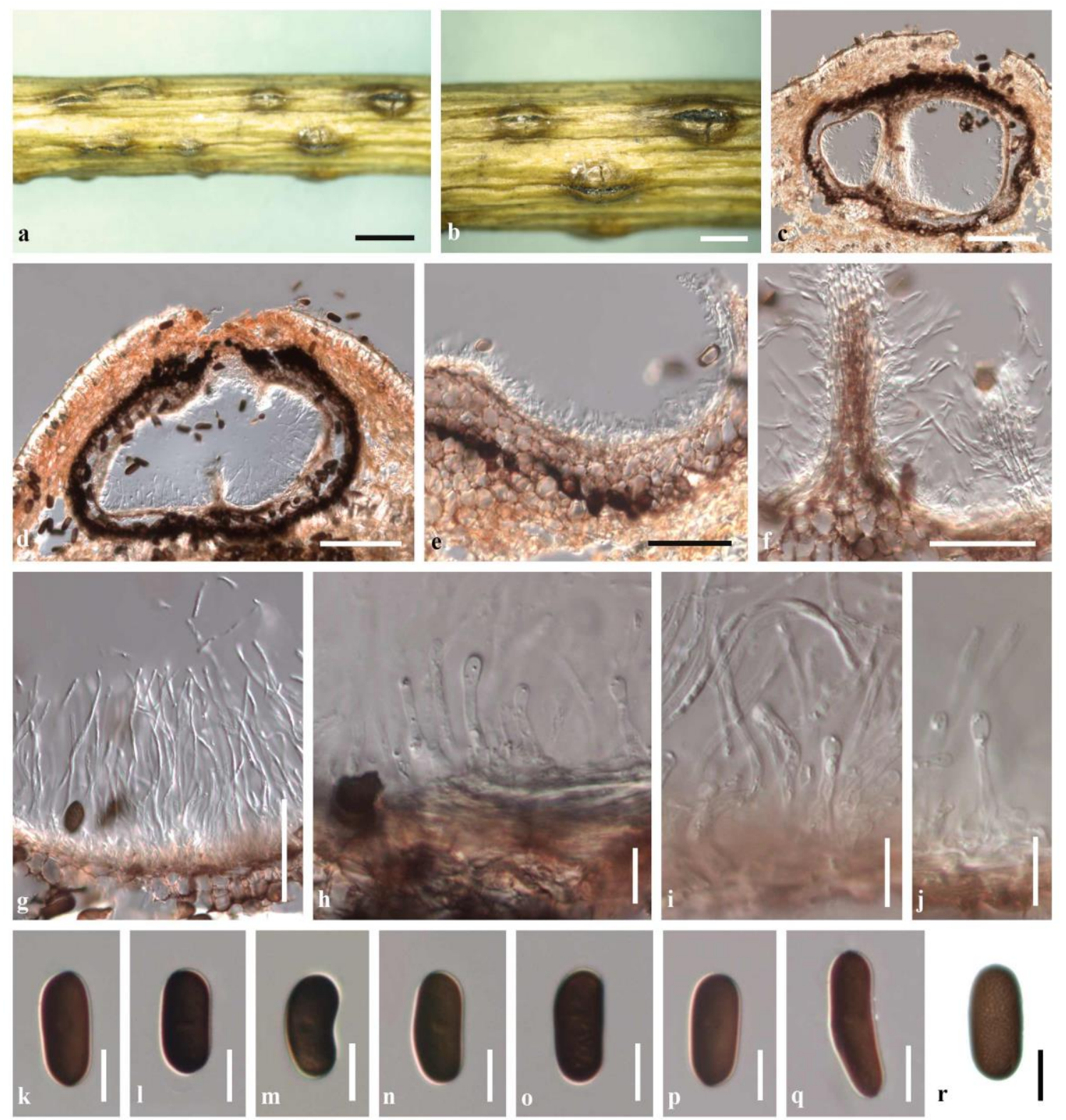

Fig. 98 - Aplosporella javeedii (KUN-HKAS 102211, new host and geographical record). a, b Appearance of conidiostromata on host substrate. c, d Section through conidiostroma. $\mathrm{e}$, f Section through pycnidial wall. g Paraphyses. $\mathrm{h}-\mathrm{j}$ Conidiogenous cells embedded in paraphyses. $\mathrm{k}-\mathrm{q}$ Variation in shape of conidia. $\mathrm{r}$ Conidia showing reticulate, rough-walled. Scale bars: $\mathrm{a}=1000 \mu \mathrm{m}, \mathrm{b}=500 \mu \mathrm{m}, \mathrm{c}, \mathrm{d}=100 \mu \mathrm{m}, \mathrm{e}-\mathrm{g}=50 \mu \mathrm{m}, \mathrm{h}-\mathrm{r}=10 \mu \mathrm{m}$.

Material examined - China, Yunnan Province, Kunming, Panlong, Ciba, on dead hanging twigs of Acer palmatum (Aceraceae), 28 November 2015, R. Phookamsak, COE008, KUN-HKAS 102211, new host and geographical record, living culture, KUMCC 18-0134.

GenBank numbers - ITS: MH974687, tef1: MT005776.

Notes - An NCBI BLASTn search of ITS sequence data shows that our strain (KUMCC 180134) is identical to Aplosporella javeedii with $100 \%$ similarity. Phylogenetic analyses of concatenated ITS and tefl sequence dataset also indicated that our taxon has a close affinity with $A$. javeedii with strong support (Fig. 97, 100\% ML and 1.00 PP). Furthermore, our collection (KUNHKAS 102211) shares a size range of conidia and conidial characters with the type and other representative specimens (Jami et al. 2013, Fan et al. 2015b). Therefore, we identify our collection as A. javeedii. This species is collected from dead hanging twigs of Acer palmatum in Yunnan, China for the first time. 
Facesoffungi number: FoF 04955

Saprobic on a dead branch of Prunus serrulata (Rosaceae). Sexual morph: Undetermined. Asexual morph: Conidiomata 650-800 × 350-420 $\mu \mathrm{m}$, pycnidial, solitary, dark brown, immersed to semi immersed, erumpent, multi-loculate, central ostiole. Conidioma wall 55-90 $\mu \mathrm{m}$ wide at the side, 35-46 $\mu \mathrm{m}$ wide at the base, composed of 6-12 layers of brown, thick-walled cells, arrange in a textura angularis. Paraphyses $26.5-60 \mu \mathrm{m}(\bar{x}=40.2 \mu \mathrm{m}, \mathrm{n}=10)$ long, $4-6.3 \mu \mathrm{m}(\bar{x}=4.5 \mu \mathrm{m}, \mathrm{n}=$ 10) wide at base, 1.6-2.7 $\mu \mathrm{m}(\bar{x}=2 \mu \mathrm{m}, \mathrm{n}=10)$ wide at upper part, hyaline, smooth-walled, septate, blunt ended. Conidiophores 7.4-13.2 $\times 4.7-6.7 \mu \mathrm{m}(\bar{x}=10.5 \times 5.6 \mu \mathrm{m}, \mathrm{n}=15)$, reduced to conidiogenous cells, monophialidic, hyaline, cylindrical to doliiform, smooth-walled. Conidia $17.5-21 \times 9.7-12.2 \mu \mathrm{m}(\bar{x}=19.5 \times 10.9 \mu \mathrm{m}, \mathrm{n}=30), \mathrm{L} / \mathrm{W} 1.8$, aseptate, initially hyaline, smoothwalled, broadly ellipsoidal to subcylindrical, with rounded ends, becoming dark brown.

Culture characteristics - Conidia germinating on PDA within $16 \mathrm{hr}$. Colonies on PDA, reaching $41.5 \mathrm{~mm}$ diameter after one week at $25-30^{\circ} \mathrm{C}$, circular, flat or effuse, dense, upper surface initially greenish, becoming blackish-green from the center within 7 days. Reverse dark green to black.

Known distribution (based on molecular data) - South Africa, Limpopo, China, Henan, Yunnan (Damm et al. 2007, Dou et al. 2017a).

Known hosts (based on molecular data) - Acacia cochlearis, Cercis chinensis $f$. chinensis, Ficus sp., Prunus persica var. nucipersica (Damm et al. 2007, Dou et al. 2017a).

Material examined - China, Guizhou Academy of Agricultural Sciences (GZAAS), Guiyang, Guizhou, on a dead branch of Prunus serrulata (Rosaceae), 12 June 2018, MC. Samarakoon, SAMC156 (MFLU 18-0774, HKAS 102387, new host record), living culture MFLUCC 19-0490.

GenBank numbers - ITS: MN473059, LSU: MN473053, SSU: MN473047, TUB: MN987001.

Notes - Our strain (MFLUCC 19-0490) collected on Prunus serrulata is similar to Aplosporella prunicola (Damm et al. 2007), which was collected from the bark of a small dead tree of $P$. persica var. nucipersica. BLASTn searches of ITS and LSU gave results of $100 \%$ similarity of bp comparisons with CBS 121167 and STE U 6326 strains of Aplosporella prunicola. The combined LSU, ITS, tefl phylogenetic analyses show the clustering of MFLUCC 19-0490 in the A. prunicola clade. Based on similar morphology and molecular data, here, we report our strain as a new host record from Prunus serrulata.

\section{Botryosphaeriaceae Theiss. \& Syd.}

The most recent treatments of Botryosphaeriaceae are by Phillips et al. (2019) and Burgess et al. (2019), and species are widespread in tropical and temperate regions, and are forest pathogens, while some may be saprobes or endophytes. Currently, 28 genera are accepted in Botryosphaeriaceae (Wijayawardene et al. 2018).

\section{Barriopsis A.J.L. Phillips, A. Alves \& Crous}

Barriopsis was introduced by Phillips et al. (2008) with B. fusca as the type species. The peculiar taxon is characterized by brown, aseptate ascospores without apiculi (Phillips et al. 2008). Currently, Barriopsis archontophoenicis, B. iraniana, B. tectonae, B. stevensiana and B. thailandica are accepted (Abdollahzadeh et al. 2009, Doilom et al. 2014, Konta et al. 2016, Tibpromma et al. 2017, Wijayawardene et al. 2017). In this study, we illustrate and describe $B$. stevensiana on Cassia sp. from Thailand, a new geographical record.

Barriopsis stevensiana A.J.L. Phillips \& Pennycook, Fungal Diversity, 86: 56 (2017)

Fig. 101

Facesoffungi number: FoF07061

Saprobic on dead twigs of Cassia sp. Sexual morph: Ascostromata individual locule 150-280 $\mu \mathrm{m}$ high $\times$ 215-300 $\mu \mathrm{m}$ diameter (ascostromata with papilla), black, convex on host tissue, appearing through cracks in bark, solitary, initially immersed, becoming erumpent, when cut 
horizontally locules visible as white contents and dark ascospore dots, uni to multi-loculate, globose to subglobose or flask-shaped, with central papilla, ostiole with periphyses. Peridium composed of two layers, outer layer composed of black to reddish brown, thick-walled cells of textura angularis, inner layer composed of hyaline, thin-walled cells of textura angularis. Hamathecium comprising 3-5.5 $\mu \mathrm{m}$ wide, hyaline, hyphae-like, numerous, septate, pseudoparaphyses, constricted at the septa. Asci 100-180 × 28-35 $\mu \mathrm{m}(\bar{x}=123 \times 32 \mu \mathrm{m}, \mathrm{n}=15)$, 8-spored, bitunicate, fissitunicate, cylindro-clavate or clavate, with a short or long pedicel, apically rounded with an ocular chamber. Ascospores 21-34 $\times 11.5-15 \mu \mathrm{m}(\bar{x}=33 \times 14 \mu \mathrm{m}, \mathrm{n}=30)$, uniseriate at the base, 2-3-seriate at the centre and end, hyaline when immature, gradually pale brown, becoming brown when mature, ellipsoidal, aseptate, straight or slightly curved, widest in the middle, thick-walled, smooth. Asexual morph: Undetermined.

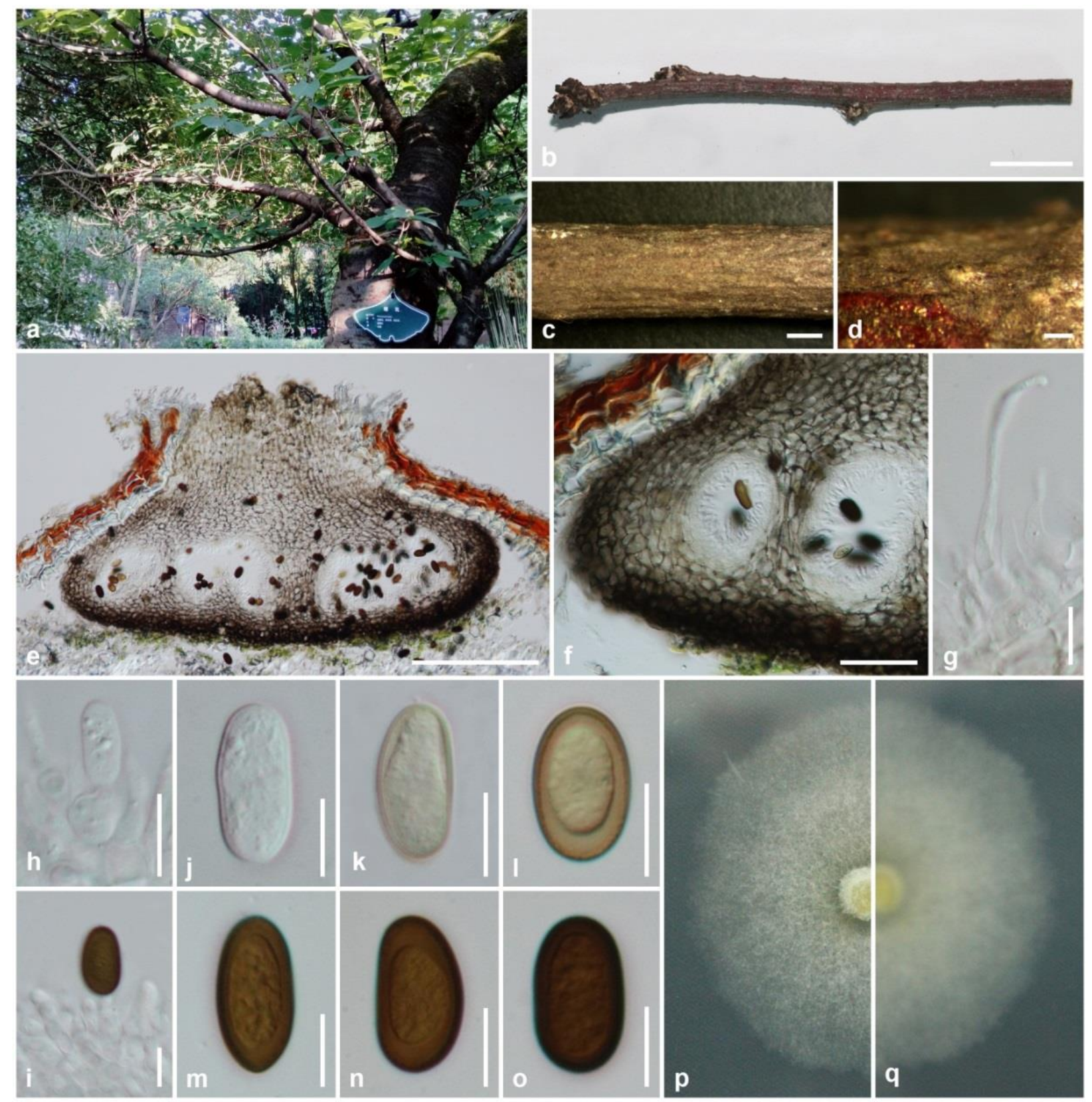

Fig. 99 - Aplosporella prunicola (MFLU 18-0774, new host record). a Host. b-d Conidiomata on the substrate. e Vertical section of conidioma. f Peridium. g Paraphyses. h, i Conidiogenous cells. $\mathrm{j}-\mathrm{o}$ Conidia. $\mathrm{p}$ Upper view of the colony. $\mathrm{q}$ Reverse view of the colony. Scale bars: $\mathrm{b}=1 \mathrm{~cm}$, $\mathrm{c}=1000 \mu \mathrm{m}, \mathrm{d}, \mathrm{e}=200 \mu \mathrm{m}, \mathrm{f}=50 \mu \mathrm{m}, \mathrm{g}-\mathrm{O}=10 \mu \mathrm{m}$. 


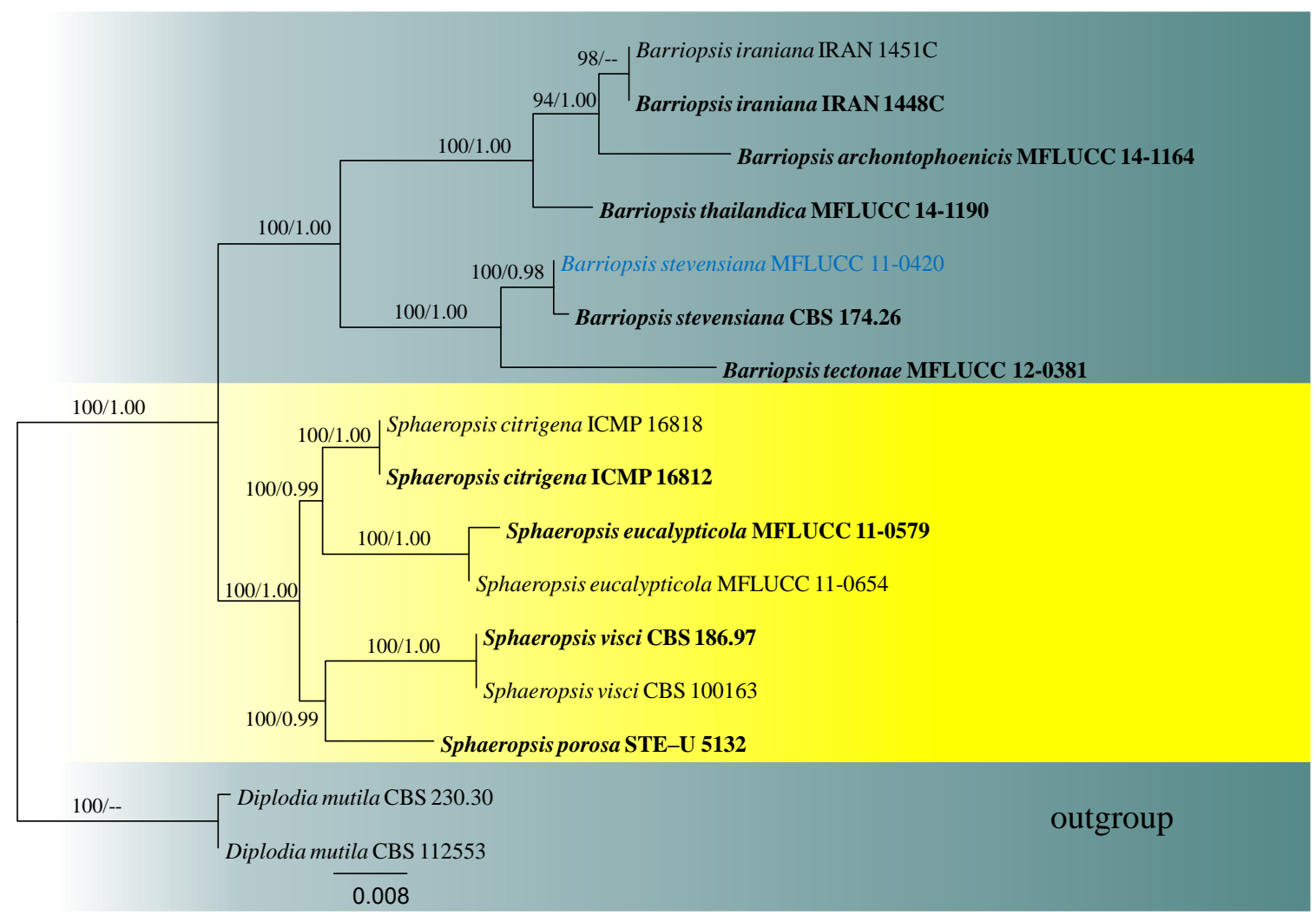

Fig. 100 - Phylogram generated from maximum likelihood analysis based on combined LSU, ITS and tefl sequence data. Sixteen strains are included in the combined gene analyses comprising 1770 characters after alignment (866 characters for LSU, 539 characters for ITS, 365 characters for tef1). Diplodia mutila (strains CBS 230.30 and CBS 112553) are used as the outgroup taxa. The tree topology derived from the Bayesian analysis was similar to that derived from the maximum likelihood analysis. The best RaxML tree with a final likelihood value of -4240.541158 is presented. The matrix had 264 distinct alignment patterns, with $18.91 \%$ undetermined characters or gaps. Estimated base frequencies were as follows: $\mathrm{A}=0.229339, \mathrm{C}=0.254463, \mathrm{G}=0.291126, \mathrm{~T}=$ 0.225072; substitution rates $\mathrm{AC}=1.116498, \mathrm{AG}=2.893632, \mathrm{AT}=0.794870, \mathrm{CG}=1.586169, \mathrm{CT}$ $=5.750768, \mathrm{GT}=1.000000$; gamma distribution shape parameter $\alpha=0.140521$. Bootstrap values for maximum likelihood equal to or greater than 70 and Bayesian posterior probabilities equal or greater than 0.95 are placed above or below the branches. Ex-type strains are in bold and black. The newly generated sequences are indicated in blue.

Known distribution (based on molecular data) - Cuba (Phillips et al. 2008) study).

Known hosts (based on molecular data) - Citrus sp. (Phillips et al. 2008), Cassia sp. (this

Material examined - Thailand, Chiang Rai Province, Muang District, Tha Sut Subdistrict, on dead twigs of Cassia sp. (Fabaceae), 30 September 2011, M. Doilom (MFLU 19-1560), living culture (MFLUCC 11-0420).

GenBank numbers - ITS: MN582740, LSU: MN582755, SSU: MN582689.

Notes - Our specimen is similar to the holotype of Barriopsis stevensiana ( $\equiv$ Barriopsis fusca) which was re-examined by Liu et al. (2012b) and Phillips et al. (2013). Based on phylogenetic analysis of combined LSU, ITS and tefl sequence data our strain (MFLUCC 110420) clusters with the ex-type strain of B. stevensiana (CBS 174.26) with high support (Fig. 100, $100 \% \mathrm{ML} / 0.98 \mathrm{PP}$ ). The holotype was collected on twigs of Citrus in Cuba (Stevens 1926, Phillips et al. 2008, Liu et al. 2012b). This is new geographical record of B. stevensiana on Cassia sp. in Thailand. 

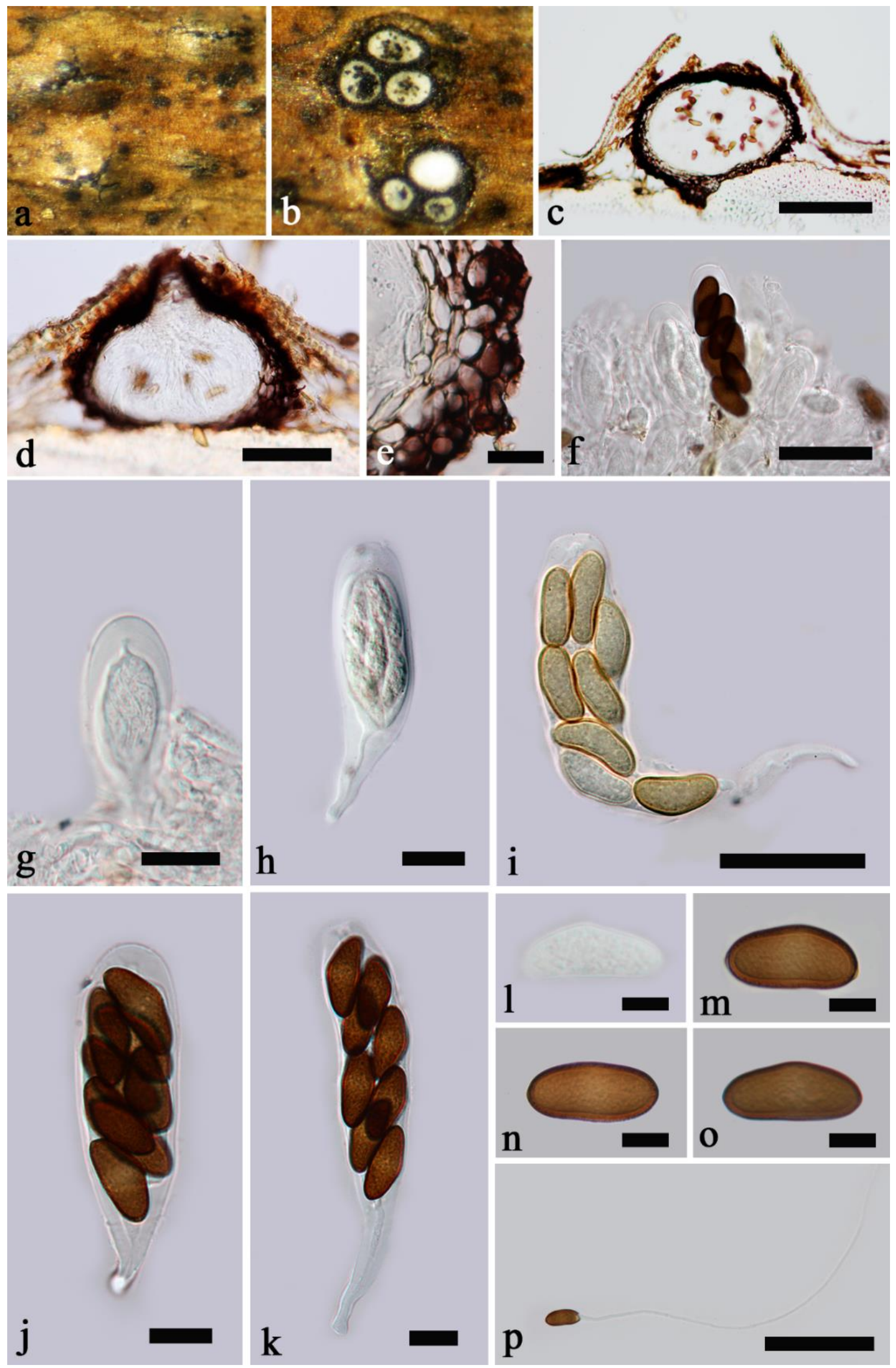

Fig. 101 - Barriopsis stevensiana )MFLU 19-1560, new geographical record(. a Ascomata on dead twigs of Cassia sp. b Ascomata cut through horizontally showing the white contents with dark spots. c, d Sections through ascomata. e Peridium. f-k Asci. 1-o Ascospores. p Germinated ascospore. Scale bars: c, d $=200 \mu \mathrm{m}, \mathrm{e}, \mathrm{g}, \mathrm{h}, \mathrm{j}, \mathrm{k}=20 \mu \mathrm{m}, \mathrm{f}, \mathrm{i}=50 \mu \mathrm{m}, \mathrm{l}-\mathrm{o}=10 \mu \mathrm{m}, \mathrm{p}=100 \mu \mathrm{m}$. 
Botryosphaeria Ces. \& De Not.

Botryosphaeria is typified with Botryosphaeria dothidea. This genus has undergone various revisions and updates over the years and the most recent taxonomic treatment is Jayawardena et al. (2019a). In this study, we collected Botryosphaeria dothidea from Torilis arvensis.

Botryosphaeria dothidea (Moug.) Ces. \& De Not., Comm. Soc. crittog. Ital. 1(fasc. 4): 212 (1863)

Fig. 103

Facesoffungi number: FoF03512

Saprotrophic on dead and aerial branches of Torilis arvensis. Sexual morph: Undetermined. Asexual morph: Colonies on natural substrate stromatic, Conidiophores hyaline, cylindrical, smooth. Conidiogenous cells phialidic, hyaline, subcylindrical. Conidia $15-10 \times 7-5 \mu \mathrm{m}(\bar{x}=13 \times$ $6 \mu \mathrm{m} \mathrm{n}=20$ ), hyaline, 1-celled, narrowly fusiform, with a subtruncate to bluntly rounded base, forming a septum before germination, smooth-walled with a granular content.

Culture characteristics - Colonies on PDA reaching $50 \mathrm{~mm}$ diameter after $4 \mathrm{~d}$ at $28{ }^{\circ} \mathrm{C}$. Mycelial mat moderately dense, margin smooth, olivaceous, becoming grey.

Known distribution (based on molecular data) - worldwide (Phillips et al. 2013, Manawasinghe et al. 2018), Italy (this study).

Known hosts (based on molecular data) - wide range of hosts from monocotyledons to dicotyledons (Phillips et al. 2013, Dissanayake et al. 2017), Torilis arvensis (this study).

Material examined - Italy, Province of Forlì-Cesena, Teodorano - Meldola, on dead branch of Torilis arvensis (Apiaceae), 30 January 2018, E. Camporesi IT 3699 (MFLU 18-0177, new host record), living culture, JZB310196.

GenBank number - ITS: MN533806.

Notes - Botryosphaeria dothidea is the type species. In this study, we isolated a Botryosphaeria strain from Italy associated with branches of Torilis arvensis. In the phylogenetic analysis, this strain clustered with the Botryosphaeria dothidea isolates with $<50 \% \mathrm{Ml}$ bootstrap and $<0.95$ BYPP values (Fig. 102). However, the characters, such as conidial shape, size and colour are similar to the type (Phillips et al. 2005, Phillips et al. 2013). Therefore, we identified our current taxon as Botryosphaeria dothidea. This species has a worldwide distribution on many economical important trees (Phillips et al. 2005, Phillips et al. 2013, Dissanayake et al. 2017). This is the first report of B. dothidea on Torilis arvensis (Farr \& Rossman 2020).

\section{Diplodia}

Diplodia was introduced by Montagne and is typified by D. mutila. Diplodia has a worldwide distribution and are known to be pathogens, endophytes and saprobes on a wide range of woody hosts (Crous et al. 2006a, Slippers \& Wingfield 2007, Phillips et al. 2008, Phillips et al. 2013, Ariyawansa et al. 2015). The genus comprises 30 species with molecular data (Hyde et al. 2018). Two types of distinct conidial morphologies are observed in the genus. The first type of conidia is initially hyaline, aseptate and later becomes pale to dark brown and 1-septate. In this type, delayed pigmentation is observed and in some species dark conidia are never observed. The second type of conidia becomes pigmented at an early stage of development where they are still enclosed inside the pycnidia and rarely become septate (Phillips et al. 2013).

Diplodia galiicola Dissanayake, Camporesi \& K.D. Hyde, Fungal Diversity, 75: 54 (2015)

Facesoffungi number: FoF00884

Fig. 105

Saprobic on dead aerial stem of Knautia sp. Sexual morph: Undetermined. Asexual morph: Conidiomata 220-225 $\mu \mathrm{m}$ high $\times 372-380 \mu \mathrm{m}$ diameter $(\bar{x}=219 \times 376 \mu \mathrm{m}, \mathrm{n}=10)$, stromatic, solitary, semi immersed in host epithelium, dark brown to black, uniloculate or biloculate, globose to subglobose. Peridium $36-54 \mu \mathrm{m}$ wide $(\bar{x}=44 \mu \mathrm{m}, \mathrm{n}=10)$, outer layer composed of dark brown cells of textura angularis, inner layers of thin-walled hyaline cells of textura angularis. Conidiogenous cells $7-12 \mu \mathrm{m}$ high $\times 3-7 \mu \mathrm{m}$ wide $(\bar{x}=10 \times 4 \mu \mathrm{m}, \mathrm{n}=15)$, holoblastic, integrated, 
hyaline, thin-walled, smooth, cylindrical, swollen at the base, producing a single conidium at the apex. Conidia $18-26 \times 8-18 \mu \mathrm{m}(\bar{x}=21 \times 11 \mu \mathrm{m}, \mathrm{n}=30)$, initially hyaline, turning dark brown with maturity, moderately thick-walled, aseptate, ovoid, widest in the center, obtuse apex, verruculose.

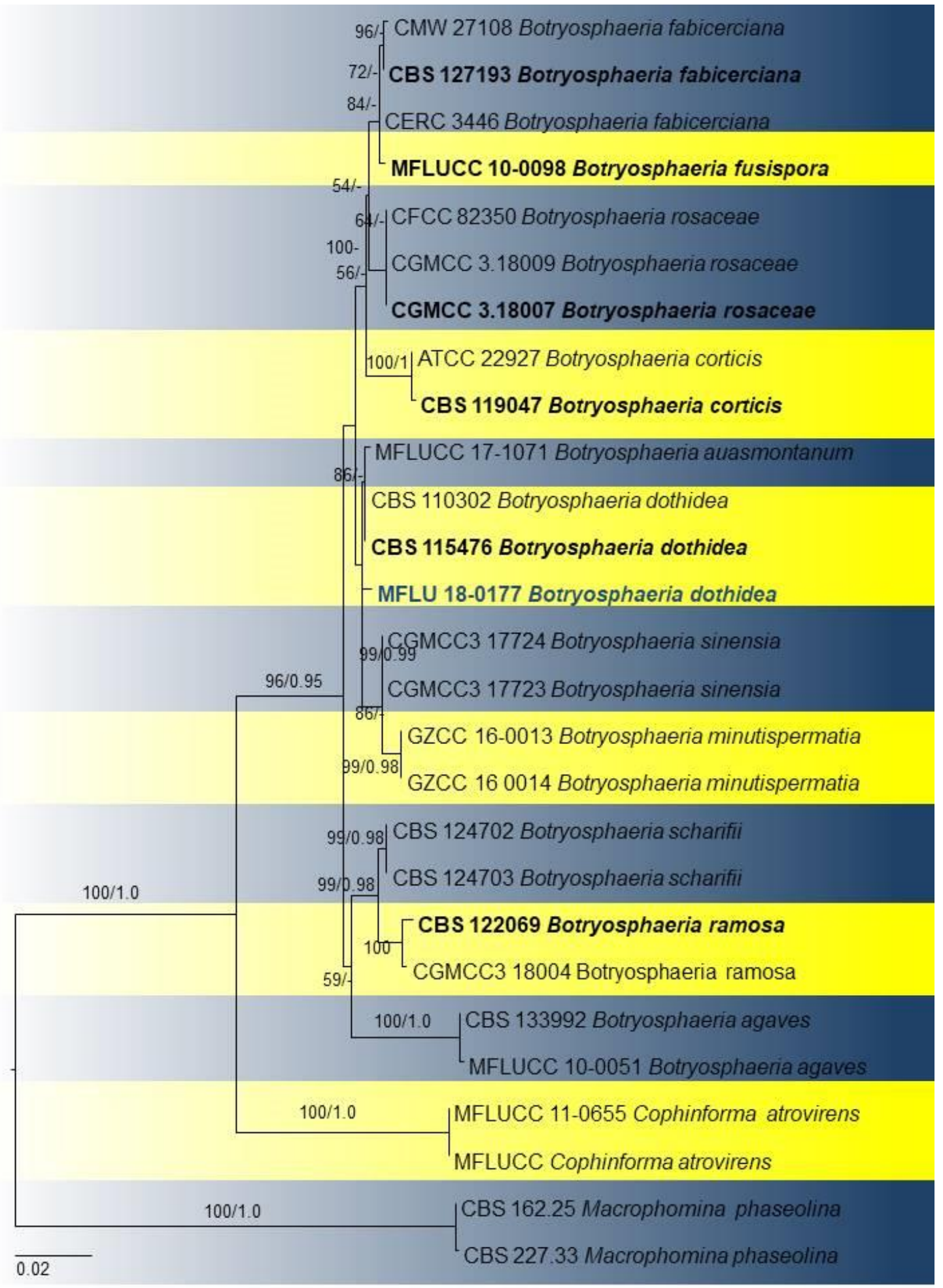

Fig. 102 - Phylogram generated from maximum likelihood analysis based on combined ITS, tefl, and TUB sequence data. Twenty-seven strains are included in the combined gene analyses comprising characters after alignment. Macrophoma phaseolina (strains CBS 162.25 and CBS 227.33) are used as the outgroup taxa. The tree topology derived from the Bayesian analysis was similar to that derived from the maximum likelihood analysis. The best RaxML tree with a final likelihood value of -3083.503236 is presented. The matrix had 224 distinct alignment patterns, 
with $14.27 \%$ undetermined characters or gaps. Estimated base frequencies were as follows: $\mathrm{A}=$ $0.210590, \mathrm{C}=0.307570, \mathrm{G}=0.251651, \mathrm{~T}=0.230189$; substitution rates $\mathrm{AC}=0.677624, \mathrm{AG}=$ $3.002265, \mathrm{AT}=1.150518, \mathrm{CG}=0.720959, \mathrm{CT}=5.263152, \mathrm{GT}=1.000000$; gamma distribution shape parameter $\alpha=1.009656$. Bootstrap values for maximum likelihood greater than 50 are placed above or below the branches. Ex-type strains are in bold and black. The newly generated sequence is indicated in blue bold.
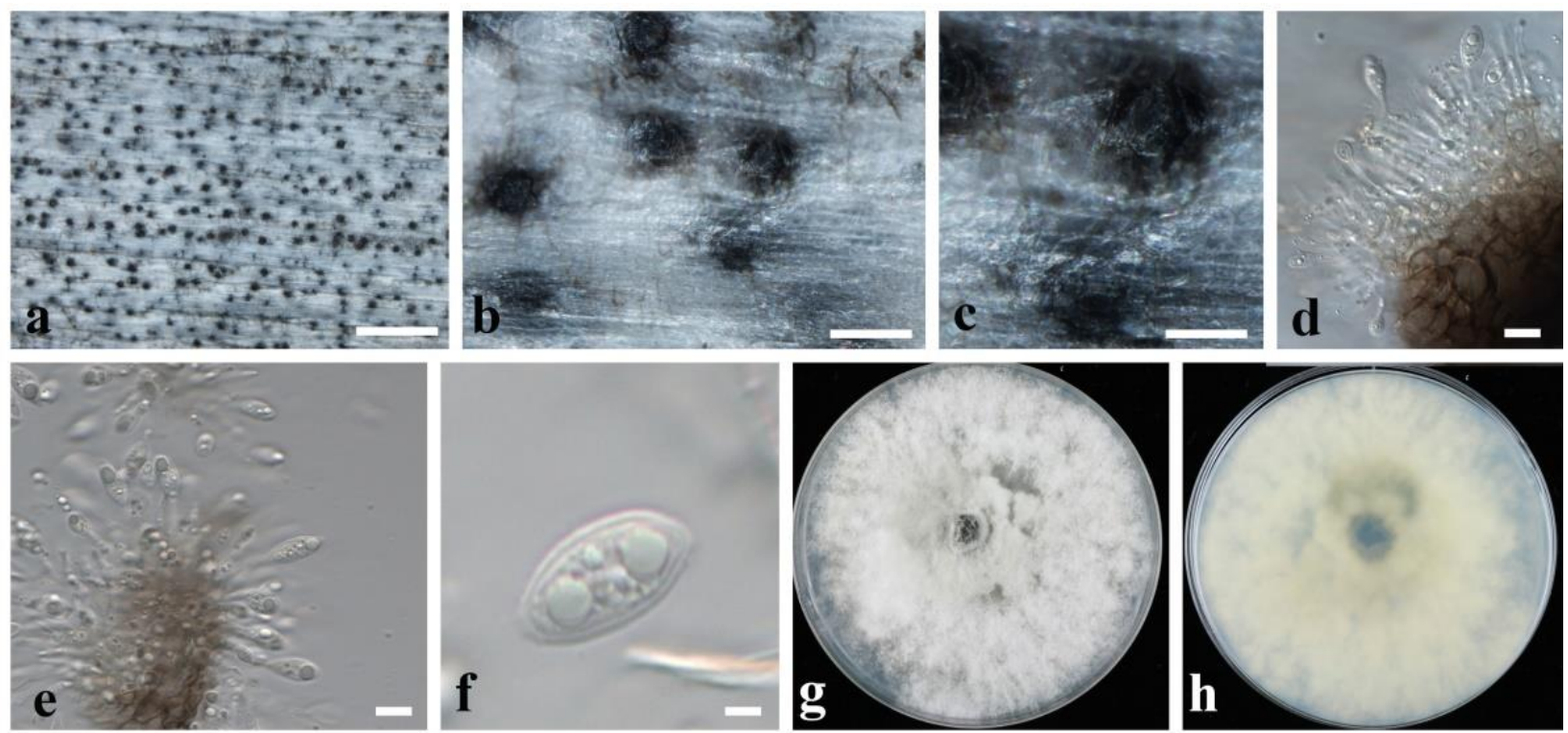

Fig. 103 - Botryosphaeria dothidea (MFLU 18-0177, new host record). a Material examined. $\mathrm{b}-\mathrm{c}$ Appearance of conidia on host surface. $\mathrm{d}$ Conidiogenous cell attached to conidia. e Conidiogenous cell. f Conidia. g Upper view of colony on PDA. h Reverse view of colony on PDA. Scale bars: $\mathrm{a}-\mathrm{c}=500 \mu \mathrm{m}, \mathrm{d}-\mathrm{f}=100 \mu \mathrm{m}$.

Known distribution (based on molecular data) - Italy (Giambra et al. 2016, Linaldeddu et al. 2016), Italy, Forli-Cesena Province, Strada San Zeno Province, Galeata Province (Dissanayake et al. 2016).

Known hosts (based on molecular data) - Galium sp. (Rubiaceae) (Ariyawansa et al. 2015), Knautia sp. (Caprifoliaceae) (this study).

Material examined - Italy, Province of Forlì-Cesena [FC], Civitella di Romagna, Buggiana, on dead aerial stem of Knautia sp. (Dipsacaceae),12 February 2018, E. Camporesi, IT 3712 (JZB 3140014, new

host record).

GenBank submissions - ITS: MN757871, tef1: MN854663.

Notes - Based on the phylogenetic analysis of combined ITS and tefl sequence data of Diplodia species (Fig. 104), our strain (JZB 3140014) was identified as D. galiicola. Diplodia galiicola is phylogenetically closely related with D. seriata (Fig. 104). The difference is observed in conidial length of $D$. galiicola being shorter as compared to $D$. seriata. The average conidial length of $D$. seriata is greater than or equal to $25 \mu \mathrm{m}$ (Phillips et al. 2013), while the conidial length of D. galiicola is less than $25 \mu \mathrm{m}$. For our strain (JZB 3140014), the culture characteristics could not be obtained since the spores did not germinate in culture (PDA) and DNA was directly extracted carefully from fruiting bodies. Base pair comparison of ITS and tefl gene regions between our strain (JZB 3140014) and reference strain of D. galiicola (MFLU 15-1310) reveal less than $1 \%$ difference. The morphological characters such as conidiogenous cells and conidial dimensions also overlap confirming that the two strains are the same species (Ariyawansa et al. 2015). Diplodia galiicola previously has only been isolated as a saprobe on Galium sp. from Italy. This is the first time D. galiicola has been collected from Knautia sp. 


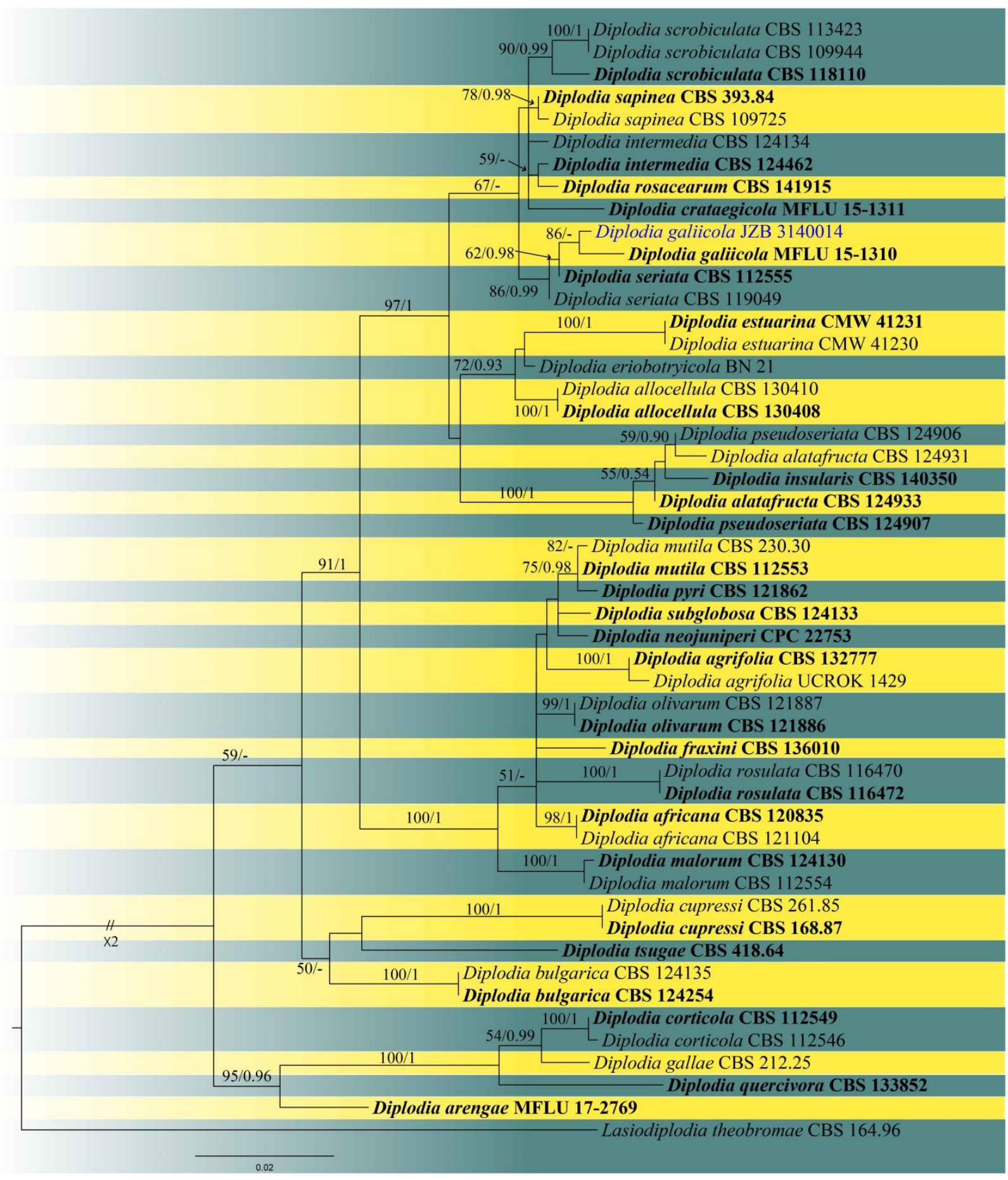

Fig. 104 - Phylogram generated from maximum likelihood analysis based on combined ITS, and tefl sequence data. Fourty seven strains are included in the combined gene analyses comprising 866 characters after alignment (578 characters for ITS, 288 characters for tef1). Lasiodiplodia theobromae CBS 164.96 is used as the outgroup taxon. The tree topology derived from the Bayesian analysis was similar to that derived from the maximum likelihood analysis. The best RaxML tree with a final likelihood value of -3349.531698 is presented. The matrix had 261 distinct alignment patterns, with $12.08 \%$ undetermined characters or gaps. Estimated base frequencies were as follows: $\mathrm{A}=0.203138, \mathrm{C}=0.302938, \mathrm{G}=0.263561, \mathrm{~T}=0.230363$; substitution rates $\mathrm{AC}$ $=0.989254, \mathrm{AG}=4.136603, \mathrm{AT}=0.730217, \mathrm{CG}=1.833881, \mathrm{CT}=4.680274, \mathrm{GT}=1.000000$; gamma distribution shape parameter $\alpha=0.775702$. Bootstrap values for maximum likelihood equal to or greater than 50 and Bayesian posterior probabilities equal or greater than 0.95 are placed above or below the branches. Ex-type strains are in bold and black. The newly generated sequence is indicated in blue. 

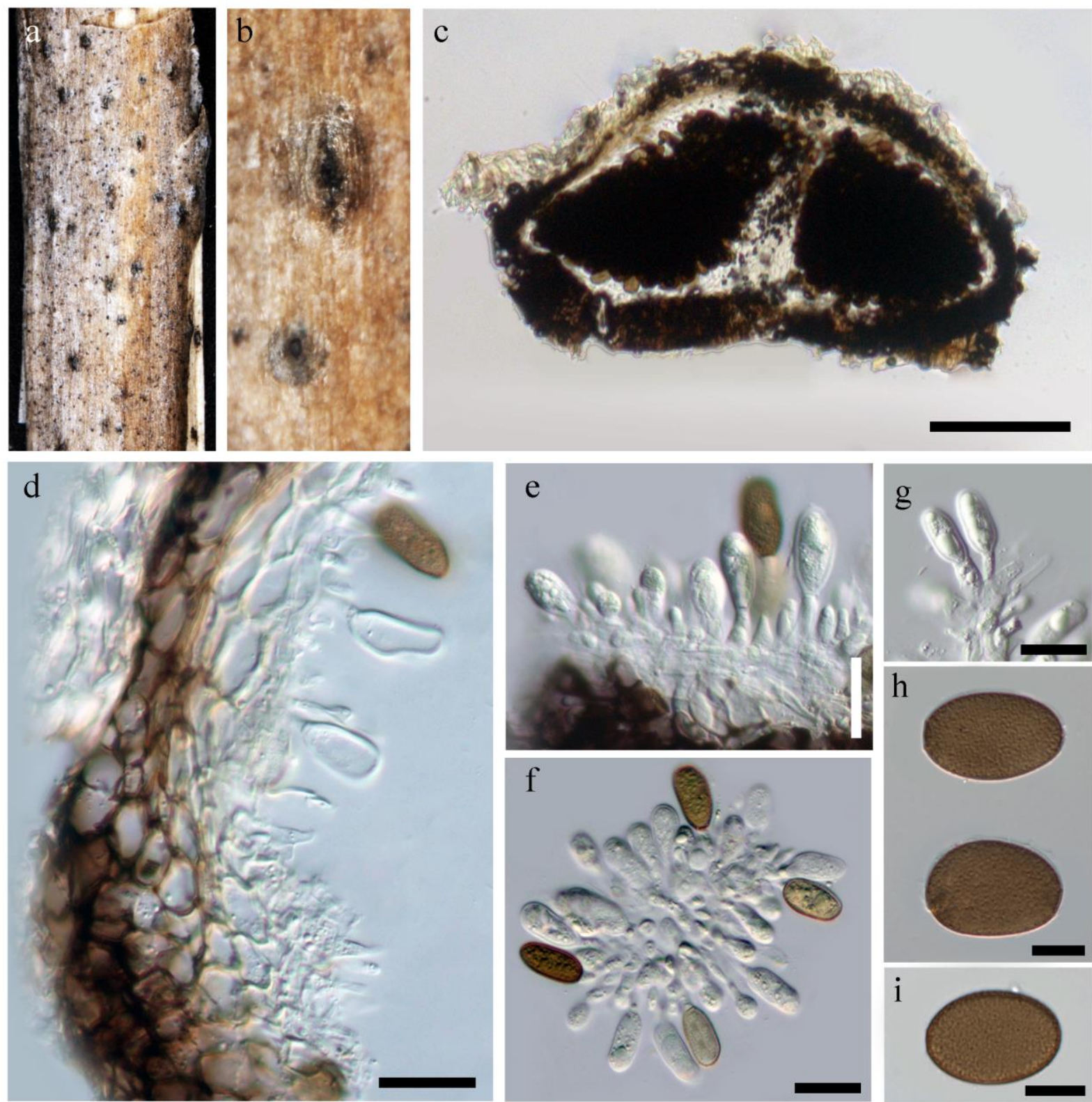

Fig. 105 - Diplodia galiicola (JZB 3140014, new host record). a, b Conidiomata on host substrate. c Cross section of conidioma. d Peridium. e, f Mature and immature conidia attached to conidiogenous cells. g Immature conidia attached to conidiogenous cells. h, i Mature conidia. Scale bars: $\mathrm{c}=100 \mu \mathrm{m}, \mathrm{d}-\mathrm{g}=20 \mu \mathrm{m}, \mathrm{h}, \mathrm{i}=10 \mu \mathrm{m}$.

\section{Dothiorella Sacc.}

We follow the latest treatment and updated accounts of Dothiorella in Yang et al. (2017), Hyde et al. (2019) and Phookamsak et al. (2019). Yang et al. (2017) treated Spencermartinsia as a synonym of Dothiorella based on molecular phylogeny and the morphological character of apiculate ascospores which were not reliable to distinguish Spencermartinsia from Dothiorella (Yang et al. 2017, Hyde et al 2019). Phookamsak et al. (2019) mentioned that Spencermartinsia alpina and S. yunnana had to be transferred to Dothiorella in further studies. In this study, we collected Spencermartinsia alpina from a different host in Yunnan Province, China. Based on molecular data and phylogenetic analyses, we thus, transfer S. alpina to Dothiorella. A new combination species, Dothiorella alpina is introduced. Furthermore, Dothiorella symphoricarpicola is also reported from Acer monspessulanum in Italy for the first time. 


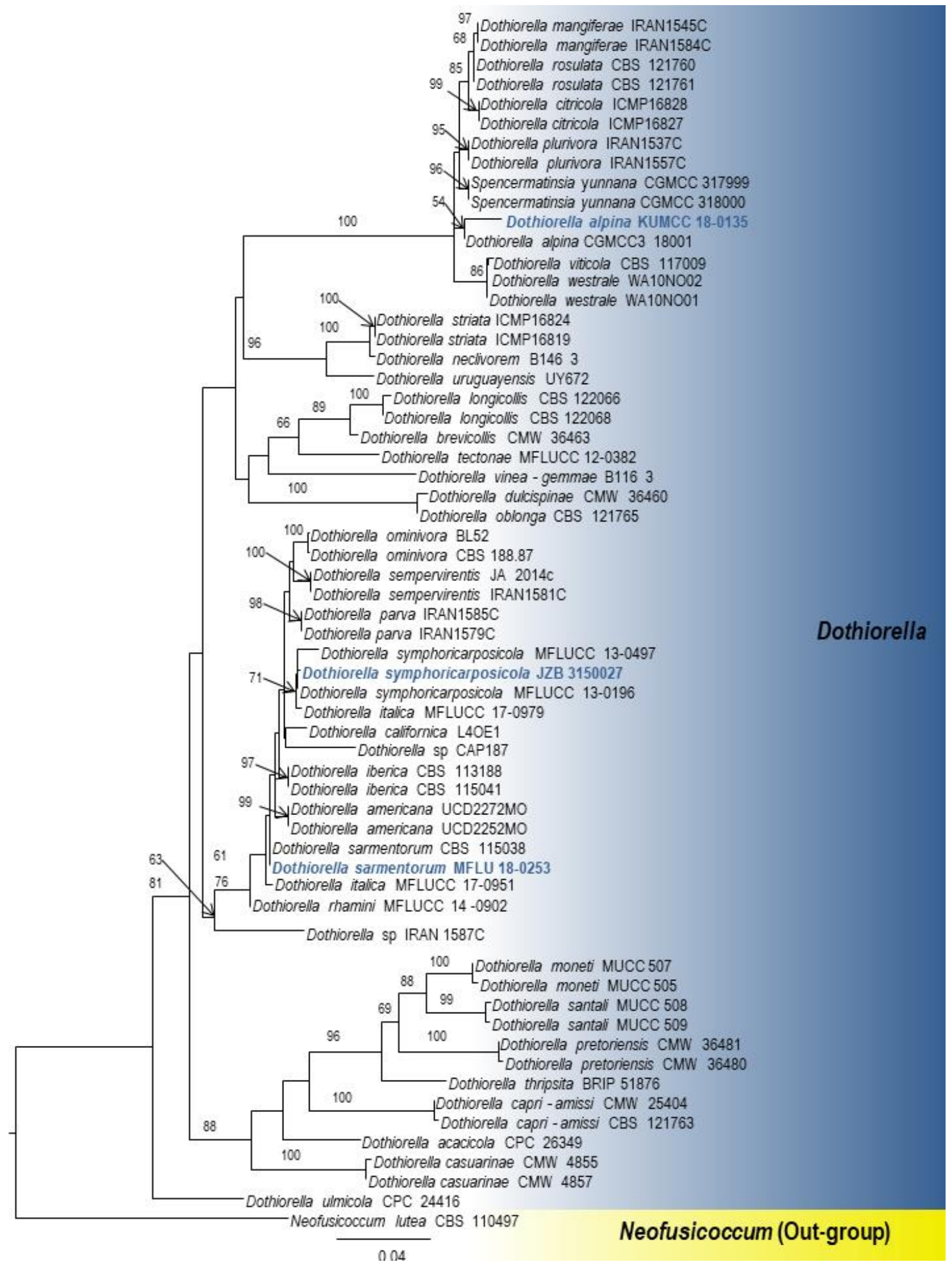

Fig. 106 - Phylogram generated from maximum likelihood analysis based on a combined ITS and tefl sequence dataset. Forty-six strains are included in the combined gene analyses comprising 740 total characters including gaps (ITS: $1-509 \mathrm{bp}$, tefl: $510-740 \mathrm{bp}$ ). The tree topology derived from the Bayesian analysis was similar to that derived from the maximum likelihood analysis. The best scoring of the ML tree is selected to represent the phylogenetic relationships of taxa in Dothiorella, with the final ML optimization likelihood: -3536.406809. The matrix had 293 distinct alignment patterns, with $18.47 \%$ undetermined characters or gaps. Estimated base frequencies were as follows: $\mathrm{A}=0.211039, \mathrm{C}=0.292321, \mathrm{G}=0.252470, \mathrm{~T}=0.244170$; substitution rates $\mathrm{AC}=$ $1.328514, \mathrm{AG}=2.488703, \mathrm{AT}=1.264091, \mathrm{CG}=0.687931, \mathrm{CT}=4.488656, \mathrm{GT}=1.000000$; TreeLength $=1.050171$; gamma distribution shape parameter $\alpha=0.517888$. Bayesian posterior 
probabilities (BYPP) from MCMC were evaluated with a final average standard deviation of split frequencies of 0.009627. Bootstrap support for maximum likelihood (ML) greater than 50\% and Bayesian posterior probabilities (PP) greater than 0.95 are defined above the nodes as ML/PP. The tree is rooted to Neofusicoccum parvum (CMW 9081). The type strains are indicated in bold and newly generated sequences are shown in blue bold.

Dothiorella alpina (Y. Zhang ter \& Ming Zhang) Phookamsak \& K.D. Hyde, comb. nov.

Fig. 107

Index Fungorum number: IF55723; Facesoffungi number: FoF07384

= Spencermartinsia alpina Y. Zhang ter \& Ming Zhang, Mycosphere 7(7): 1058 (2016)

Saprobic on dead stolon of Ipomoea sp. Sexual morph: Undetermined. Asexual morph: Conidiomata 135-245 $\mu \mathrm{m}$ high, 150-235 $\mu \mathrm{m}$ diameter, pycnidial, immersed, raised, becoming erumpent through host epidermis, gregarious, visible as black, gnarled with dark area on host, globose to subglobose, 1-loculate, occasionally developed hyaline, palisade-like invaginations, raising from basal cavity, ostiole central, with a small, narrow papilla. Pycnidial walls 15-30 $\mu \mathrm{m}$ wide, composed of 3-5 layers, of large, dark brown to black pseudoparenchymatous cells, arranged in textura angularis to textura globulosa. Conidiophores reduced to conidiogenous cells. Conidiogenous cells $7-10 \times 2-4(-5) \mu \mathrm{m}(\bar{x}=8.9 \times 3.6 \mu \mathrm{m}, \mathrm{n}=30)$, raising from the inner cavity, enteroblastic, phialidic, integrated, determinate, ampulliform to cylindrical, unbranched, aseptate, hyaline, smooth, with minute collarette, with thin periclinal wall thickening. Conidia $22-25(-28) \times$ $10-12(-13) \mu \mathrm{m}(\bar{x}=24.4 \times 11.1 \mu \mathrm{m}, \mathrm{n}=50)$, initially hyaline, becoming brown to dark brown when mature, oblong with rounded ends, occasionally irregular in shape, 1-septate, smooth-walled.

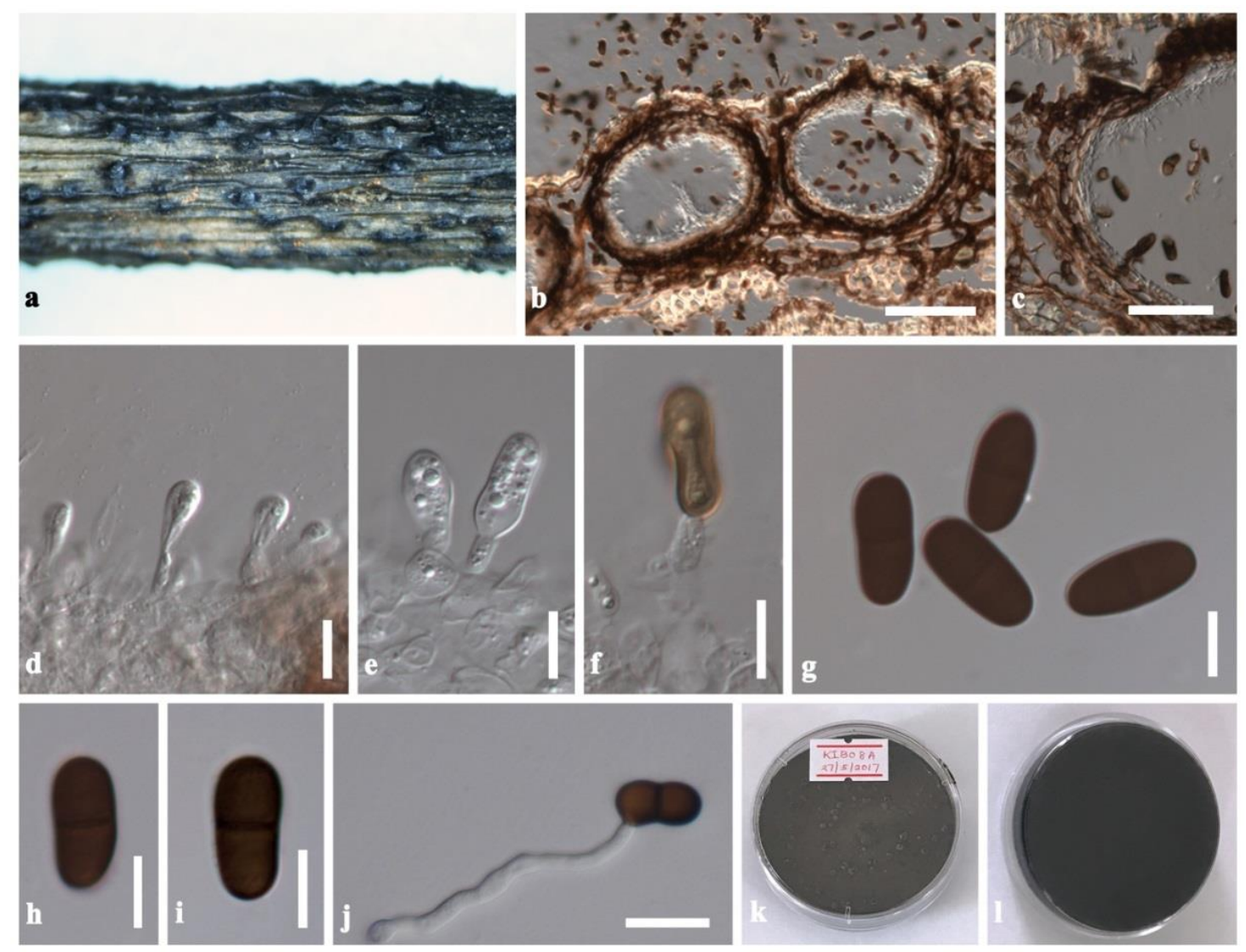

Fig. 107 - Dothiorella alpina (KUN-HKAS 102212, new combination). a Appearance of conidiomata on host substrate. b Section through conidiomata. c Section through pycnidial wall. $\mathrm{d}-\mathrm{f}$ Conidiogenous cells with the development of conidia. $\mathrm{g}-\mathrm{i}$ Conidia. $\mathrm{j}$ Germinated conidium. $\mathrm{k}, 1$ Culture characteristics on PDA ( $\mathrm{k}=$ from above, $1=$ from below). Scale bars: $\mathrm{b}=100 \mu \mathrm{m}$, $\mathrm{c}=50 \mu \mathrm{m}, \mathrm{d}-\mathrm{f}, \mathrm{j}=20 \mu \mathrm{m}, \mathrm{g}-\mathrm{i}=10 \mu \mathrm{m}$. 
Culture characteristics - Colonies on PDA fast growing, reaching $50 \mathrm{~mm}$ diameter after 1 week at $20-25^{\circ} \mathrm{C}$, colonies initially medium sparse with aerial mycelia, white grey, becoming dense, with hyphal lumps, circular, flat, surface smooth with edge entire, margin well defined, cottony to fluffy; colony from above and below, black with white to black hyphal lumps; not producing pigments on PDA.

Known distribution (based on molecular data) - China, Yunnan (Zhang et al. 2016), China, Yunnan (this study).

Known hosts (based on molecular data) - Platycladus orientalis (Cupressaceae) (Dissanayake et al. 2016, Zhang et al. 2016), Ipomoea sp. (this study).

Material examined - China, Yunnan Province, Kunming City, Kunming Institute of Botany, on dead stolon of Ipomoea sp. (hanging on the tree), 18 October 2016, R. Phookamsak, KIB008 (KUN-HKAS 102212, new host record), living culture, KUMCC 18-0135, KUMCC 18-0136.

GenBank numbers - ITS: MT002267, LSU: MT002266, SSU: MT002268, RPB2: MT005773 (KUMCC 18-0135); ITS: MT000718, LSU: MT000720, SSU: MT000725, RPB2: MT005774 (KUMCC 18-0136).

Notes - The NCBI BLASTn search of ITS sequence data shows that our strains (KUMCC 18-0135, KUMCC 18-0136) are identical to Spencermartinsia sp. 'alpina' strain CGMCC3.18001 and Spencermartinsia sp. JC-2017 strain CFCC 51564 (as Dothiorella magnoliae; You et al. 2017) with $100 \%$ similarity. Phylogenetic analyses of a concatenated ITS and tefl sequence dataset indicated that our strains have a close affinity with S. alpina (CGMCC3.18001) with strong support (97\% ML and 1.00 PP, Fig. 106). Morphological characters and phylogenetic analyses indicated that our collection is conspecific with S. alpina (Zhang et al. 2016). We, therefore, transfer $S$. alpina to Dothiorella. A new combination, Dothiorella alpina is designated and is reported from Ipomoea sp. in Yunnan, China for the first time.

Dothiorella sarmentorum (Fr.) A.J.L. Phillips, J. Luque \& A. Alves, Mycologia 97: 522 (2005)

Facesoffungi number: FoF 02148

Fig. 108

Saprobic on a wide range of hosts including Torilis arvensis. Sexual morph: Undetermined. Asexual morph: Conidiomata 162-243 $\mu \mathrm{m}$ high $\times 224-297 \mu \mathrm{m}$ diameter $(\bar{x}=199 \times 265 \mu \mathrm{m}, \mathrm{n}=5)$, stromatic, solitary or scattered, semi-immersed, uniloculate, individual, black, globose to subglobose, ostiolate. Peridium 28-43 $\mu \mathrm{m}(\bar{x}=34 \mu \mathrm{m}, \mathrm{n}=15)$, outer layers composed of thickwalled, dark brown, cells of textura angularis and inner layers composed of thin-walled, hyaline textura angularis cells. Conidiophores reduced to conidiogenous cells. Conidiogenous cells $7-9.5 \times$ 2.5-4 $\mu \mathrm{m}(\bar{x}=8 \times 3.5 \mu \mathrm{m}, \mathrm{n}=15)$, phialidic, hyaline, cylindrical to subcylindrical. Conidia 19-25 $\times 8-10 \mu \mathrm{m}(\bar{x}=21.5 \times 9 \mu \mathrm{m}, \mathrm{n}=30)$, oval to ovoid, with a broadly rounded apex and truncate base, initially hyaline, 1-celled, becoming cinnamon to sepia and 1-septate, while still attached to conidiogenous cells; detached conidia, hyaline, sepia or dark brown, 1-celled or 1-septate, moderately thick-walled, wall externally smooth, slightly constricted at the septum.

Known distribution (based on molecular data) - England, The Netherlands, Norway, Sweden, and a worldwide distribution can be observed (Phillips et al. 2005), Italy (this study).

Known hosts (based on molecular data) - Plurivorous including Acer, Malus, Menispermum, Prunus, Pyrus, Ulmus - (Phillips et al. 2005, Farr \& Rossman 2020), Torilis arvensis (this study).

Material examined - Italy, Province of Ravenna [RA], Faenza, Santa Lucia, on dead aerial stem of Torilis arvensis (Apiaceae), 15 January 2018, E. Camporesi, IT 3677 (MFLU 18-0253, new host record).

GenBank numbers - ITS: MN782001, tef1: MN854664.

Notes - Phillips et al. (2005) introduced Dothiorella sarmentorum based on the asexual morph of Botryosphaeria sarmentorum. This species has a worldwide distribution and has been recorded from 34 host species (Phillips et al. 2005, 2013). The sexual morph of D. sarmentorum is characterized by partially erumpent ascomata with papillate ostioles, 4-6(-8)-spored asci and 
oblong to ovate (0-)1-septate, finely verruculose ascospores, widest in the middle part (Phillips et al. 2013).
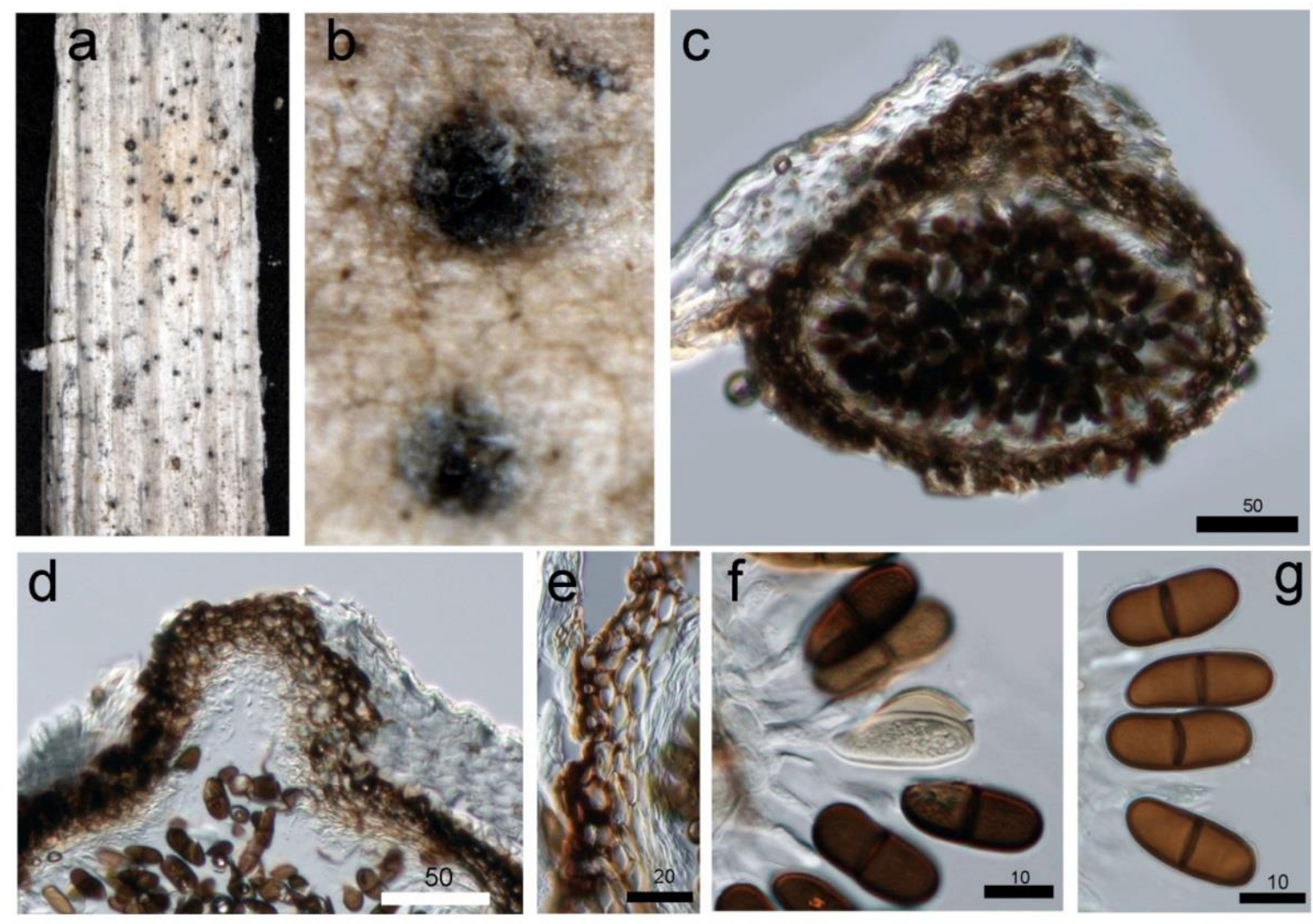

Fig. 108 - Dothiorella sarmentorum (MFLU 18-0253, new host record). a, b Appearance of conidiomata on the host. c Vertical section through conidioma. d Ostiole. e Peridium. $\mathrm{f}$ Conidia developing on conidiogenous cells. $g$ Mature dark brown conidia. Scale bars: $c, d=50 \mu \mathrm{m}, \mathrm{e}=20$ $\mu \mathrm{m}, \mathrm{f}, \mathrm{g}=20 \mu \mathrm{m}$.

Dothiorella symphoricarposicola W.J. Li, Jian K. Liu \& K.D. Hyde, Mycol. 35(3): 265 (2014)

Facesoffungi Number: FoF 06222

Fig. 109

Saprobic on dead branch of Acer opalus. Sexual morph: Undetermined. Asexual morph: Coelomycetous. Conidiomata 220-265 $\mu \mathrm{m}$ high $\times 260-380 \mu \mathrm{m}$ diameter $(\bar{x}=245 \times 340 \mu \mathrm{m}, \mathrm{n}=$ 10), pycnidial, stromatic, solitary or clustered, immersed in the host, erumpent at maturity, dark brown to black, ostiolate, apapillate. Peridium 25-35 $\mu \mathrm{m}$ wide, outer and inner layers composed of dark brown and thin-walled hyaline textura angularis. Conidiogenous cells 10-22 $\mu \mathrm{m}$ high $\times 4-6$ $\mu \mathrm{m}$ wide, phialidic, hyaline, thin-walled, smooth, cylindrical, and swollen at the base, integrated. Conidia $21-27 \times 11-16 \mu \mathrm{m}(\bar{x}=24 \times 13 \mu \mathrm{m}, \mathrm{n}=50)$, globose to subglobose, with rounded apex, initially hyaline, becoming dark brown and 1-septate at maturity, with moderately thick wall.

Culture characteristics - Colonies growing on PDA, covering the entire plate in 5 days at 28 ${ }^{\circ} \mathrm{C}$, mycelium grey to olivaceous black at the surface and olivaceous black from below.

Known distribution - Italy (Dissanayake et al. 2016, Farr \& Rossman 2020 and this study).

Known hosts (based on molecular data) - On dead aerial branch of Acer opalus (Rosaceae), Dothiorella symphoricarposicola strains have also been reported from Corylus avellana, Symphoricarpos sp., Sambucus nigra, Laurus nobilis and Laburnum alpinum in Italy (Dissanayake et al. 2016, Farr \& Rossman 2020, this study). 
Material examined - Italy, Province of Forlì-Cesena [FC], Pieve di Rivoschio - Bangno di Romagna, on dead aerial branch of Acer opalus (Rosaceae), 3 May 2017, E. Camporesi, IT 310 (MFLU 3150027, new host record), living culture: JZB: 3150027.

GenBank number - ITS: MN989423.

Notes - Our collection obtained from dead aerial branches of Acer opalus was identified as Dothiorella symphoricarposicola based on morphology and phylogeny. Our collection clustered with the reference strain of the Dothiorella symphoricarposicola (MFLUCC 13-0196), in the combined ITS and tefl sequence phylogeny with $71 \%$ statistical support. Furthermore, our isolate showed 97\% ITS similarity with Dothiorella symphoricarposicola. This is the first record of Dothiorella symphoricarposicola reported from Acer opalus from Italy.
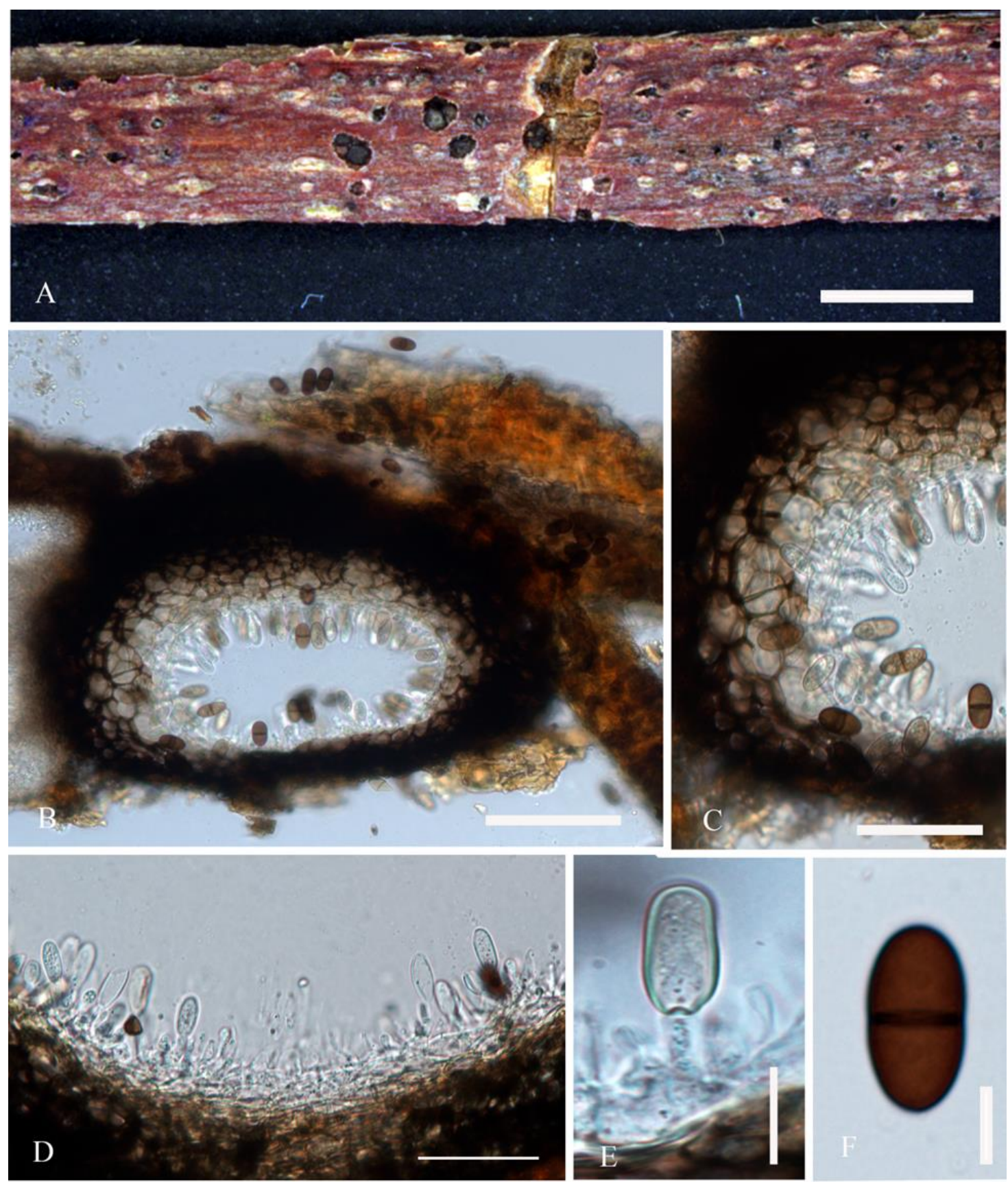

Fig. 109 - Dothiorella symphoricarposicola (MFLU 17-0729, new host record). a Appearance of conidiomata on the host. b Section through the conidioma. c Conidioma cell wall d, e Immature conidia attached to conidiogenous cell. $\mathrm{f}$ Mature conidia. Scale bars: $\mathrm{a}, \mathrm{b}=100 \mu \mathrm{m}, \mathrm{c}, \mathrm{d}=50 \mu \mathrm{m}$, $\mathrm{e}, \mathrm{f}=10 \mu \mathrm{m}$. 
Lasiodiplodia Ellis \& Everh.

Lasiodiplodia is a common genus of Botryosphaeriaceae. They are saprobes, endophytes and pathogens occur on numerous hosts with a wide geographic range (Dissanayake et al. 2016, Doilom et al. 2017b, Tibpromma et al. 2018a, Hyde et al. 2019). Lasiodiplodia is characterised by initially hyaline, aseptate and thick-walled conidia, becoming dark brown and septate with irregular longitudinal striations, and pycnidial paraphyses (Phillips et al. 2008, Liu et al. 2012b, Dou et al. 2017b). There are 67 epithets reported for Lasiodiplodia in Index Fungorum (2020). Recently many new species were introduced (Dou 2017b, Tibpromma et al. 2018a, de Silva et al. 2019).

Lasiodiplodia theobromae (Pat.) Griffon \& Maubl., Bull. Soc. Mycol. Fr. 25: 57 (1909)

Facesoffungi number: FoF 00167

Fig. 111

Saprobic on dead leaf sheath of Phoenix roebelenii. Sexual morph: not established. Asexual morph: Conidiomata pycnidial, dark brown to black, solitary or clustered, convex on host tissue, uni to multi-loculate, immersed in the host becoming erumpent when mature. Paraphyses $1.5-3.5$ $\mu \mathrm{m}$ wide, hyaline, septate, branched. Conidiogenous cells hyaline, cylindrical, phialidic. Conidia 19-25 $\times 10-16(\bar{x}=25 \times 13 \mu \mathrm{m}, \mathrm{n}=20)$, initially hyaline, aseptate, subovoid to ellipsoid-ovoid, with granular content, finally becoming dark brown, 1-septate at centre, thick-walled, upper cells wider than lower cells, truncate or rounded at the base, broadly rounded at the apex, with longitudinal striations from apex to base, guttulate.

Culture characteristics - Conidia germinating on PDA within 4-5 h. Colonies on PDA initially whitened in a few days, after 5-7 days becoming grey to black, filamentous, raised, fluffy, dense, reaching the edge of the Petri-dish after 2 days.

Known distribution (based on molecular data) - Widely distributed in Asia, Africa, America, Europe (Farr and Rossman 2020): e.g. Papua New Guinea (Phillips et al. 2005), South Africa (Gauteng) (Jami et al. 2015), (KwaZulu-Natal) (Osorio et al. 2017, Begoude et al. 2010), (Limpopo) (Mehl et al. 2017), (Western Cape) (Van Niekerk et al. 2004), Uganda (Slippers et al. 2004), USA (Arizona, California) (Inderbitzin et al. 2010), (Florida) (Mehl et al. 2017), (Hawaii) (Marincowitz et al. 2008), Thailand (Tibpromma et al. 2018a), (Phayao Province) (Doilom et al. 2015, 2017b), China (Tennakoon et al. 2016).

Known hosts (based on molecular data) - Barringtonia racemosa (Osorio et al. 2017), Eucalyptus amplifolia (Mehl et al. 2017), Leucospermum sp. (Marincowitz et al. 2008), Mangifera indica (Mehl et al. 2017), Pandanus sp. (Tibpromma et al. 2018a), Pistacia vera, Prunus domesticus (Inderbitzin et al. 2010), Tectona grandis (Doilom et al. 2015, 2017b), Terminalia catappa (Begoude et al. 2010), Vachellia karroo (Jami et al. 2015), Vitex donniana (Slippers et al. 2004), Vitis vinifera (Van Niekerk et al. 2004), Phoenix roebelenii (this study), For more information see Farr \& Rossman (2020).

Material examined - Thailand, Chiang Mai Province, Sansai District, on dead leaf sheath of Phoenix roebelenii (Arecaceae), 2 February 2012, M. Doilom \& R. Phookamsak (MFLU 19-1559, new host record), living culture (MFLUCC 12-0173).

GenBank numbers - ITS: MN582743, tef1: MN629743.

Notes - Lasiodiplodia theobromae has been recorded on numerous host plants in Thailand such as Hevea brasiliensis, Licuala longicalycata, Pandanus sp. and Tectona grandis (Pinruan et al. 2007, Seephueak et al. 2011, Doilom et al. 2015, 2016, 2017b, Tibpromma et al. 2018a, Farr \& Rossman 2020). The species is also known on several palm species such as Phoenix canariensis in Florida, $P$. dactylifera in Egypt, India, Oman, Venezuela, and P. hanceana in China and Hong Kong (Anonymous 1960, Mathur 1979, Lu et al. 2000, Zhuang 2001, El-Deeb et al. 2007, Al-Sadi et al. 2013, Li et al. 2018, Farr \& Rossman 2020). However, it has not yet been reported on Phoenix roebelenii (palm) in Thailand (Farr \& Rossman 2020). We identify specimen (MFLU 191559 ) as L. theobromae based on morphology and phylogeny. Phylogenetic analysis of the combined sequence data of ITS and tefl showed that our strain (MFLUCC 12-0173) clustered with the ex-neotype strain of $L$. theobromae (CBS 164.96) and other strains of $L$. theobromae with good 
support (Fig. 110, 69\% MP/95\% ML). This is a new host record of Lasiodiplodia theobromae on Phoenix roebelenii in Thailand.

Neofusicoccum Crous, Slippers \& A.J.L. Phillips

Neofusicoccum species have a wide geographical and host range (Lopes et al. 2016). They are endophytes, saprobes and pathogens which caused dieback, cankers and trunk diseases (Golzar \& Burgess 2011, Massonnet et al. 2017, Jami et al. 2018). Recently, many species have been introduced based on morphology and multiple-gene phylogenies by Marin-Felix et al. (2017a), Zhang et al. (2017) and Tibpromma et al. (2018a).

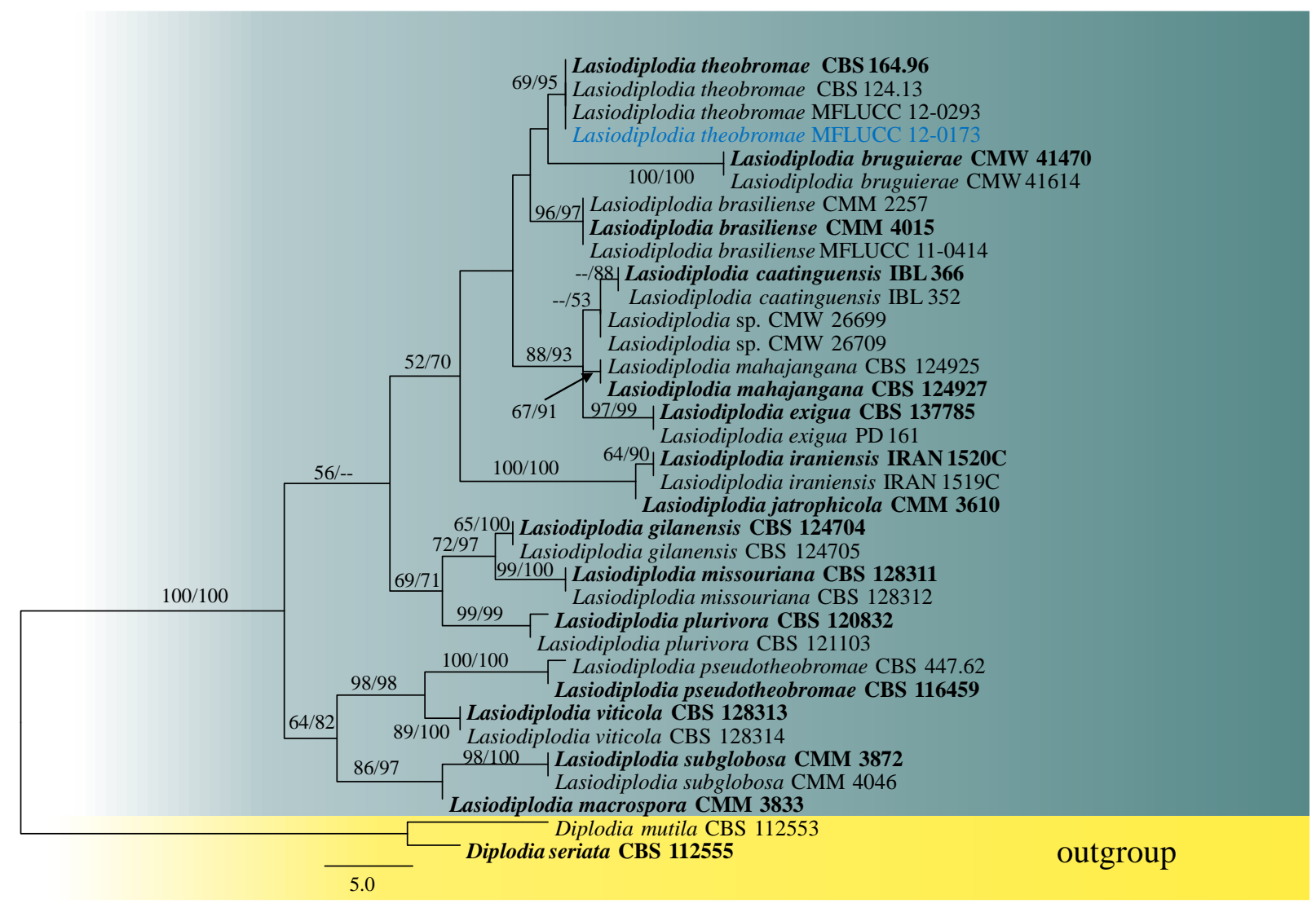

Fig. 110 - Phylogram generated from maximum parsimony analysis based on combined ITS and tef1 sequence data. Thirty-five strains are included in the combined gene analyses comprising 739 characters after alignment (482 characters for ITS, 257 characters for tef1). Diplodia mutila (CBS 112553 ) and D. seriata (CBS 112555) are used as the outgroup taxa. The tree topology derived from the Bayesian analysis was similar to that derived from the maximum likelihood analysis. The best RaxML tree with a final likelihood value of -2080.598320 is presented. The matrix had 125 distinct alignment patterns, with $6.41 \%$ undetermined characters or gaps. Estimated base frequencies were as follows: $\mathrm{A}=0.205263, \mathrm{C}=0.292383, \mathrm{G}=0.256816, \mathrm{~T}=0.245539$; substitution rates $\mathrm{AC}=0.821151, \mathrm{AG}=3.299106, \mathrm{AT}=1.173326, \mathrm{CG}=0.763958, \mathrm{CT}=$ 3.692977, GT $=1.000000$; gamma distribution shape parameter $\alpha=0.067254$. Bootstrap values for maximum parsimony and maximum likelihood equal to or greater than 50 are placed above or below the branches. Ex-type strains are in bold and black. The newly generated sequence is indicated in blue.

Neofusicoccum parvum (Pennycook \& Samuels) Crous, Slippers \& A.J.L. Phillips, Stud. Mycol. 55: 248 (2006)

Fig. 113

Facesoffungi number: FoF02411 
Saprobic on dead branch. Ascostromata 155-250 $\mu \mathrm{m}$ diameter, 115-190 $\mu \mathrm{m}$ high (including the papilla), black dots and convex on host tissue, appearing through cracks in bark, solitary or clustered, when cut horizontally locules visible as white contents and dark ascospore dots, semiimmersed to erumpent under epidermis, individually globose to subglobose, with papilla. Ostiole central, circular, papillate. Peridium 25-65 $\mu \mathrm{m}$, comprising two layers, outer layer composed of dark brown to black, thick-walled cells of textura angularis, inner layer composed of hyaline, thinwalled cells of textura angularis. Hamathecium comprising 2-4 $\mu \mathrm{m}$ wide, hyaline, hyphae-like, numerous, septate, pseudoparaphyses, slightly constricted at the septa, embedded in gelatinous matrix. Asci 55-135 × 16-23 $\mu \mathrm{m}(\bar{x}=84 \times 19 \mu \mathrm{m}, \mathrm{n}=20), 8$-spored, bitunicate, fissitunicate, clavate to cylindro-clavate, short to long pedicellate, with ocular chamber, apically rounded. Ascospores $17-25.5 \times 7-10 \mu \mathrm{m}(\bar{x}=20 \times 8 \mu \mathrm{m}, \mathrm{n}=20)$, uniseriate at the base, $2-3$-seriate at the centre and end, hyaline, ellipsoidal to fusiform, aseptate, wider in the centre, rounded or acute at the ends, thick-walled.

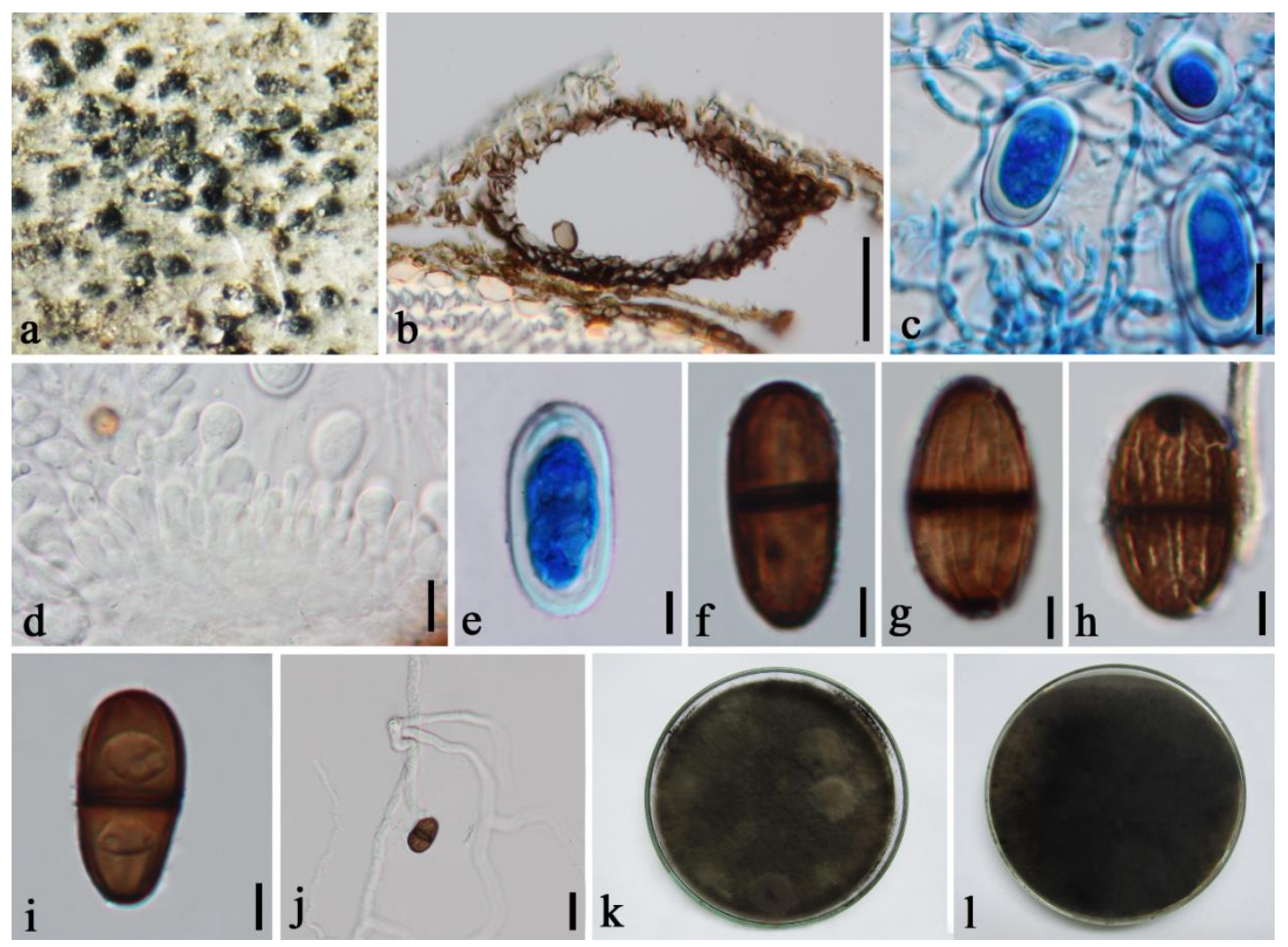

Fig. 111 - Lasiodiplodia theobromae )MFLU 19-1559, new host record(. a Conidiomata on host substrate. $\mathrm{b}$ Vertical section through conidioma. c Conidiogenous cells with paraphyses. $\mathrm{d}$ Conidia attached to conidiogenous cells. e Immature conidium. $\mathrm{f}-\mathrm{i}$ Mature conidia. $\mathrm{j}$ Germinated conidium. $\mathrm{k}, 1$ Colony on PDA ( $\mathrm{k}$ from above view, 1 from below view). Notes: c, e stained in lactophenol cotton blue. Scale bars: $b=50 \mu \mathrm{m}, c, d=10 \mu \mathrm{m}, \mathrm{e}-\mathrm{i}=5 \mu \mathrm{m}, \mathrm{j}=20 \mu \mathrm{m}$.

Culture characteristics - Colonies on PDA fluffy, fairly dense, aerial, raised, filamentous, pigments not produced, white at first, becoming gray to grey-black after one to two weeks.

Known distribution (based on molecular data) - worldwide e.g. Algeria (Berraf-Tebbal et al. 2014), Australia, Indonesia (Sumatra), Brazil, China (Sichuan), France (Van Niekerk et al. 2004), Italy (Milan) (Moricca et al. 2012), New Zealand (TePuke) (Alves et al. 2005), Portugal (Palmela) (Phillips et al. 2008), (Hogsback, Kwazulu-Natal, Mpumalanga) (Slippers et al. 2004), (e.g. Gauteng, Limpopo) (Mehl et al. 2017), USA (California) (Úrbez-Torres et al. 2007), (Hawaii) (Slippers et al. 2004), Thailand (Chiang Mai Province) (Liu et al. 2012b, Trakunyingcharoen et al. 2015), (Phayao Province) (this study), For more information see Farr \& Rossman (2020). 
Known hosts (based on molecular data) - Actinidia deliciosa, Heteropyxis natalensis, Malus sylvestris, Populus nigra, Eucalyptus sp., E. grandis, E. smithii, Ribes sp., Sequoia gigantea (Slippers et al. 2004), Acer pseudoplatanus, Quercus robur (Moricca et al. 2012), Actinidia deliciosa, A. chinensis, Populus nigra, Eucalyptus obliqua, Prunus cerasoides (Trakunyingcharoen et al. 2015), Pinus nigra, Vitis vinifera (Crous et al. 2006a, Phillips et al. 2008), Aesculus hippocastanum, Eucalyptus globulus, Ferula communis (Lopes et al. 2016), Juglans regia, Salix sp., Sclerocarya birrea subsp. caffra (Mehl et al. 2017), Avicennia marina, Bruguiera gymnorrhiza, Lumnitzera racemose, Rhizophora mucronata (Osorio et al. 2017), Linum usitatissimum (Liu et al. 2012b), Vitis vinifera (Alves et al. 2005), Mangifera indica (Mehl et al. 2017, this study), For more information see Farr \& Rossman (2020).

Material examined - Thailand, Phayao Province, Muang District, on dead branch of Mangifera indica (Anacardiaceae), 12 March 2012, M. Doilom (MFLU 19-1562, new host record), living culture (MFLUCC 12-0380).

GenBank numbers - ITS: MN582744, tef1: MN629744, TUB: MN643160.

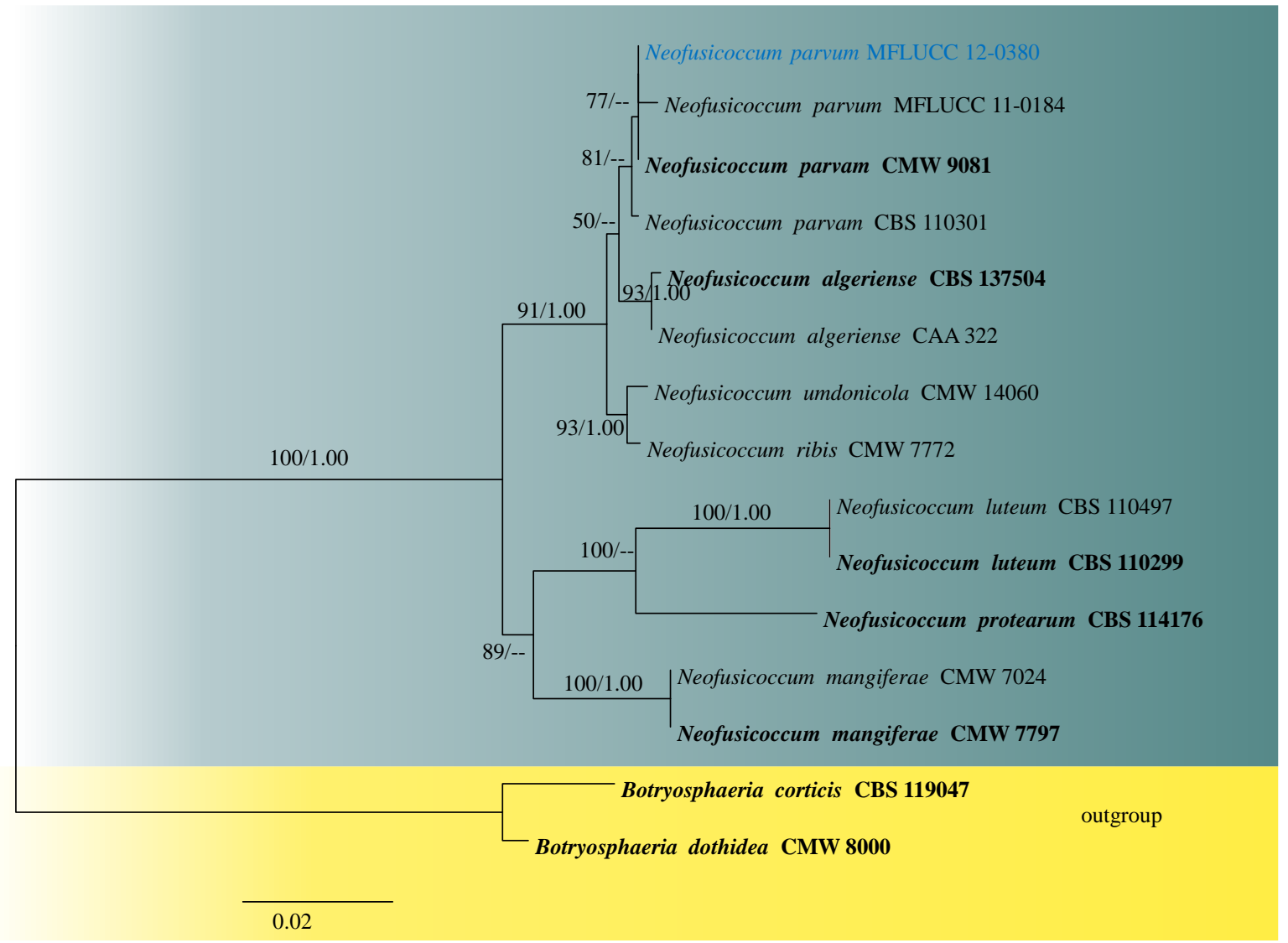

Fig. 112 - Phylogram generated from maximum likelihood analysis based on combined ITS, tef1 and TUB sequence data. Fifteen strains are included in the combined gene analyses comprising 1247 characters after alignment (499 characters for ITS, 314 characters for tefl, 434 characters for TUB). Botryosphaeria corticis (CBS 119047) and B. dothidea (CMW 8000) are used as the outgroup taxa. The tree topology derived from the Bayesian analysis was similar to that derived from the maximum likelihood analysis. The best RaxML tree with a final likelihood value of 3032.363425 is presented. The matrix had 192 distinct alignment patterns, with 6.38\% undetermined characters or gaps. Estimated base frequencies were as follows: $\mathrm{A}=0.201816, \mathrm{C}=$ $0.314088, \mathrm{G}=0.262406, \mathrm{~T}=0.221689$; substitution rates $\mathrm{AC}=1.071994, \mathrm{AG}=4.639898, \mathrm{AT}=$ 1.573574, $\mathrm{CG}=1.216826, \mathrm{CT}=6.907567, \mathrm{GT}=1.000000$; gamma distribution shape parameter $\alpha$ $=0.171757$. Bootstrap values for maximum likelihood equal to or greater than 50 and Bayesian posterior probabilities equal or greater than 0.95 are placed above or below the branches. Ex-type strains are in bold and black. The newly generated sequence is indicated in blue. 
Notes - Neofusicoccum parvum has been previously reported on Eucalyptus obliqua, Linum usitatissimum and Prunus cerasoides in Thailand (Liu et al. 2012b, Trakunyingcharoen et al. 2015). The holotype is from dead branches of Populus nigra in New Zealand (Pennycook \& Samuels 1985). Although this species is widely distributed on mango in many countries such as Australia, Brazil, Italy, México, Peru, Puerto Rico, Spain and Taiwan (Javier-Alva et al. 2009, Ni et al. 2012, Ismail 2013, Sandoval-Sanchez et al. 2013, Serrato-Diaz et al. 2013, Arjona-Girona \& LopezHerrera 2016), it has not yet been reported on Mangifera indica (mango) in Thailand (Farr \& Rossman 2020). Conidial size of our specimen is similar to the type $N$. parvum $(20 \times 8 \mu \mathrm{m}$ vs 20.8 $\times 9.2 \mu \mathrm{m})($ Pennycook \& Samuels 1985) as well as the $N$. parvum isolate identified by Liu et al. (2012b) $(20 \times 8 \mu \mathrm{m})$. The isolate MFLUCC 12-0380 clustered with the ex-type strain of $N$. parvum (CMW 9081) and $N$. parvum (MFLUCC 11-0184) (Fig. 112). Thus we identify our collection as $N$. parvum and this is the first report of N. parvum on Mangifera indica in Thailand.
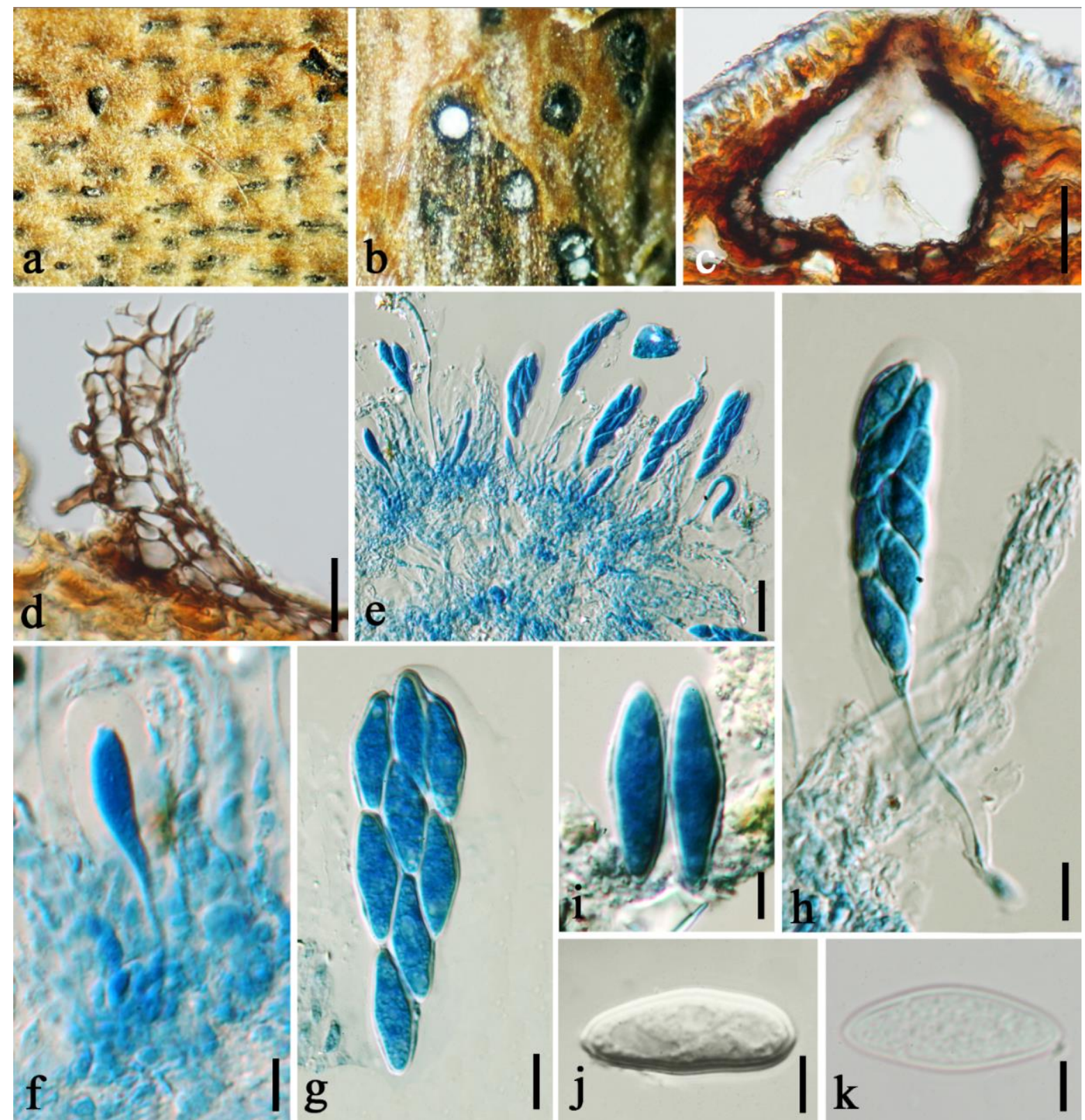

Fig. 113 - Neofusicoccum parvum (MFLU 19-1562, new host record). a Ascomata on host substrate. b Close up of ascomata on host. c Vertical section through ascoma. d Peridium. e, f Immature and mature asci with pseudoparaphyses. g, h Mature asci. i-k Ascospores. Notes: $\mathrm{e}-\mathrm{i}$ stained in lactophenol cotton blue. Scale bars: $\mathrm{c}=50 \mu \mathrm{m}, \mathrm{d}=20 \mu \mathrm{m}, \mathrm{e}=30 \mu \mathrm{m}, \mathrm{f}-\mathrm{h}=10 \mu \mathrm{m}$, $\mathrm{i}-\mathrm{k}=5 \mu \mathrm{m}$. 
Venturiales Y. Zhang ter, C.L. Schoch \& K.D. Hyde

Sympoventuriaceae Y. Zhang ter, C.L. Schoch \& K.D. Hyde

Sympoventuriaceae belongs to Venturiales (Dothideomycetes). This family is related to Verruconis and nested within the Sympoventuriaceae. In their natural habitat, fungi from this family commonly occur in soils and decaying plant material (Barron \& Busch 1962, Dwivedi 1959). Species are also known as opportunistic vertebrate pathogens (de Hoog et al. 2000, Horré et al. 1999) and can cause infections in humans and animals (Ge et al. 2012, Padhye et al. 1994, Salkin et al. 1990, Singh et al. 2006, see review in Yarita et al. 2007).

\section{Ochroconis de Hoog \& Arx}

de Hoog \& von Arx (1973) introduced Ochroconis constricta as the type species and they are characterised with sympodial conidiogenesis and septate, ellipsoidal conidia which were liberated rhexolytically. Previously, species of Ochroconis have been placed in different genera: Diplorhinotrichum (D. gallopavum; Georg et al. 1964), Dactylaria (D. humicola and D. gallopava; Bhatt \& Kendrick 1968), and Scolecobasidium (S. terreum and S. constrictum; Abbott 1927).

Ochroconis musae (G.Y. Sun \& Lu Hao) Samerp. \& de Hoog, Mycological Progress 14 (2/6): 8 (2015)

Fig. 115

Facesoffungi number: FoF06957

Saprobic on Draceana. Sexual morph: Undetermined. Asexual morph: Full descriptions of this species were given by Hao et al. )2013( and by Samerpitak et al. )2015( under its synonymous name $O$. mirabilis. Conidiophores mostly arising laterally from vegetative hyphae, erect or flexuous, cylindrical with 1-2 septa, at $25^{\circ} \mathrm{C}$. Conidia were T or Y shaped or bilobed, cylindrical, ellipsoid, clavate or fusiform.

Culture characteristics - Conidia germinated on PDA within 24 hours with germ tubes produced from one or both end cells, mostly from basal cell of conidia. Colonies on PDA reaching $25-30 \mathrm{~mm}$ in diameter after 2 weeks at $25-30^{\circ} \mathrm{C}$, colonies circular, flat, moderately expanding, smooth, dry, greyish brown to dark brown, submerged colony margin, reverse as dark brown in the central portion; not producing pigmentation in agar. Mycelium hyphae subhyaline to pale brown, smooth- and thick-walled.

Material examined - Thailand, Chiangmai Province, on dead leave of Draceana, 27 September 2017, Napalai Chaiwan, NCCM003 living culture, MFLUCC 17-2598 (new host record).

GenBank numbers - ITS: MN788641, LSU: MN788642

Notes - Our strain shares similar characters with $O$. musae which was collected from banana, Musa basjoo, in Ledong county, Haikou City, Hainan Province, China (Samerpitak et al. 2015). Phylogenetic analysis (Fig. 114) also supports the close relatedness to O. musae. The mycelium of Ochroconis consists of smooth, pale brown to medium brown. However, previously O. musae has only been reported from banana. This study provides the first report of new host record of this species from Dracaena as well as its first report from Thailand.

Class Eurotiomycetes Tehler ex O.E. Eriksson \& K. Winka

Chaetothyriales M.E. Barr

Trichomeriaceae Chomnunti \& K.D. Hyde.

Species are epiphytic on the surface of leaves associated with honey dew insect excretions and colonies are often mixed with capnodiaceous taxa. Phylogenetic data (LSU and ITS) clearly shows that Trichomeriaceae belongs in Chaetothyriales (Chomnunti et al. 2012a). The type genus is Trichomerium.

\section{Trichomerium Speg.}

Trichomerium was introduced by Spegazzini (1918) with Trichomerium coffeicolum as the type species. Species in this genus are foliar epiphytes with superficial, setiferous, uniloculate 
ascostromata surrounded by loosely interwoven mycelium, with bitunicate asci and hyaline, septate ascospores (Spegazzini 1918, Chomnunti et al. 2012a, Hongsanan et al. 2016). The asexual morph of Trichomerium gloeosporum was recorded as Tripospermum-like (Hongsanan et al. 2016). Some species of Trichomerium can be found on rocks and are slow-growing. Molecular analyses indicated that species of Trichomerium cluster separately from Capnodiaceae and Chaetothyriaceae (Chomnunti et al. 2012a, b, 2014, Yang et al. 2014, Hongsanan et al. 2016).

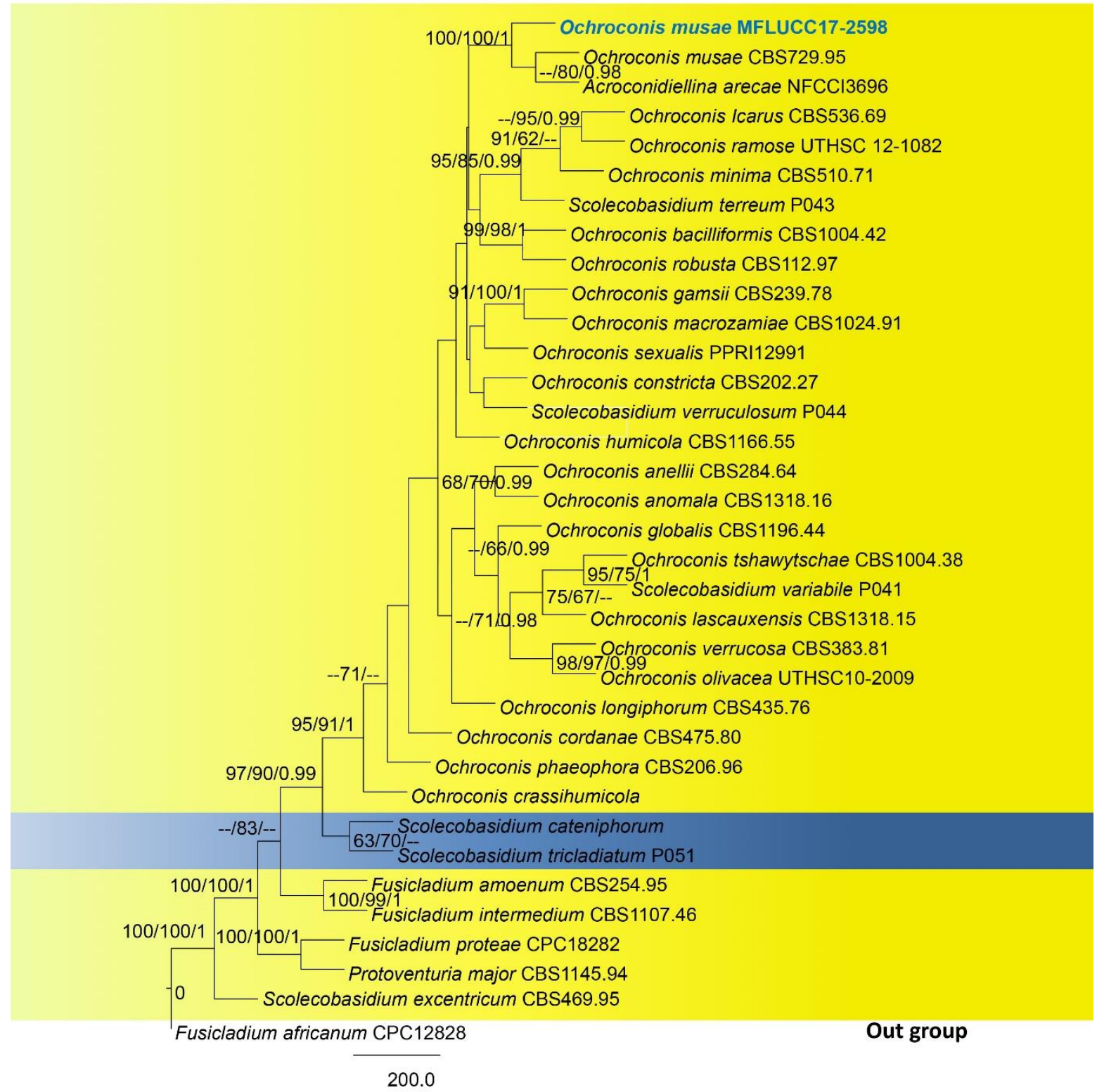

Fig. 114 - Phylogram generated from maximum likelihood analysis based on combined LSU and ITS sequence data. Thirty-five strains are included in the combined gene analyses comprising 2019 characters after alignment (972 characters for LSU, 1047 characters for ITS). Fusicladium africanum CPC12828 is used as the outgroup taxon. The tree topology derived from the Bayesian analysis was similar to that derived from the maximum likelihood analysis. The best RaxML tree with a final likelihood value of -16950.850707 is presented. The matrix had 1069 distinct alignment patterns, with $37.28 \%$ undetermined characters or gaps. Estimated base frequencies were as follows: $\mathrm{A}=0.239425, \mathrm{C}=0.234508, \mathrm{G}=0.322263, \mathrm{~T}=0.203804$; substitution rates $\mathrm{AC}=$ $0.815774, \mathrm{AG}=1.380551, \mathrm{AT}=0.895824, \mathrm{CG}=0.990791, \mathrm{CT}=3.616055, \mathrm{GT}=1.000000$; gamma distribution shape parameter $\alpha=0.259219$. Bootstrap values for maximum likelihood equal 
to or greater than 50 and Bayesian posterior probabilities equal or greater than 0.95 are placed above or below the branches. The new host record generated sequence is indicated in blue.

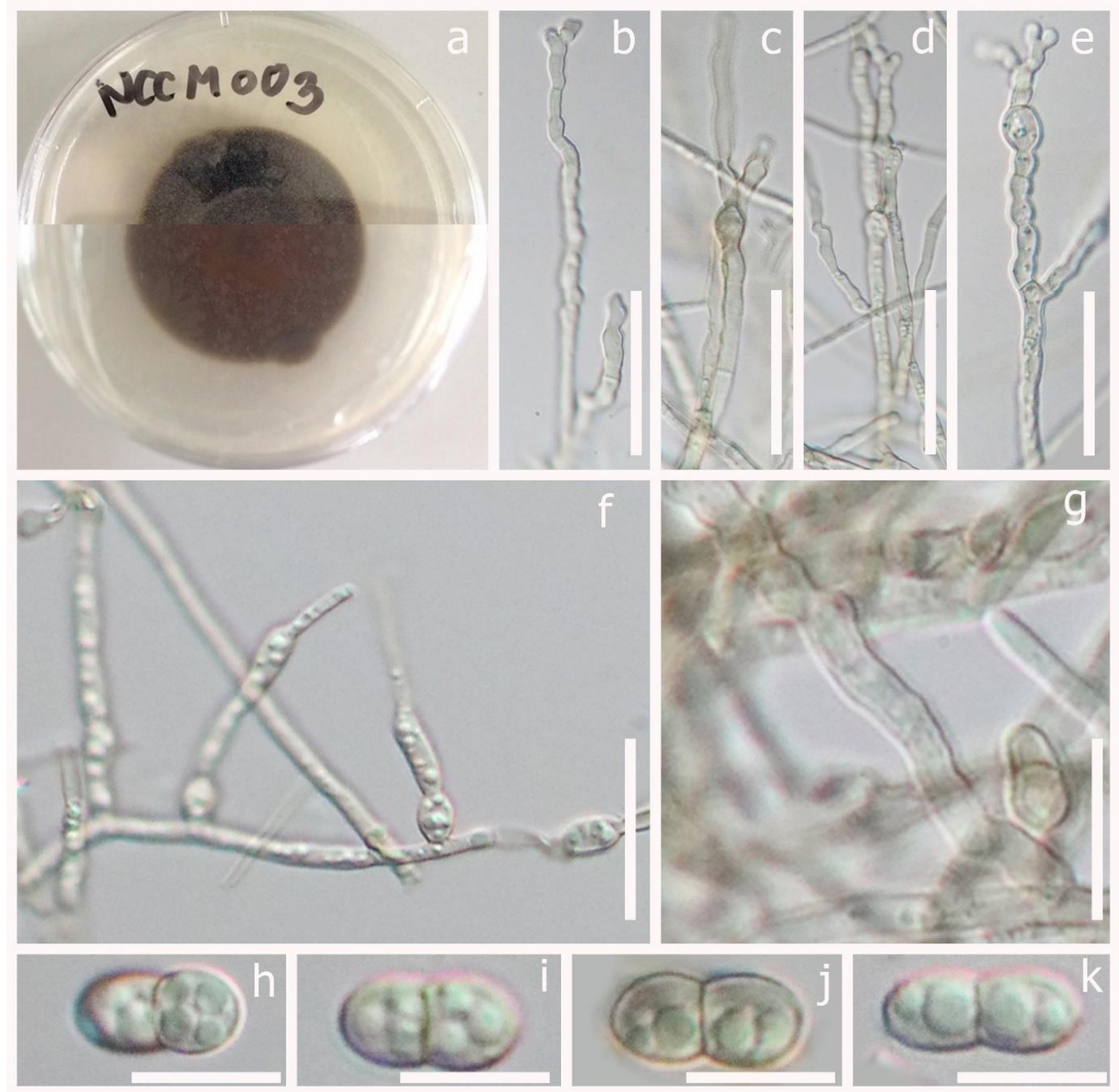

Fig. 115 - Ochroconis musae )MFLUCC 17-2598, new host record(. a Culture. b-f Conidiophore g Conidiogenous cell h-k Conidia. Scale bars: $b-g=20 \mu \mathrm{m}, \mathrm{h}-\mathrm{k}=10 \mu \mathrm{m}$.

Trichomerium camporesii Marasinghe \& K.D. Hyde sp. nov.

Fig. 117

Index Fungorum Number: IF556916; Facesoffungi number: FoF06236

Etymology - Named after Erio Camporesi, a great fungi collector

Holotype - MFLU 19-2251

Epiphytic on the surface of Psidium guajava leaves. Black sheets of mycelia cover the leaves of the host with dark brown superficial, scattered, ascostromata. Hyphae septate, cylindrical, pale brown to brown with constrictions at the septa, $4.5-6 \mu \mathrm{m}$ wide $(\bar{x}=5.5 \mu \mathrm{m}, \mathrm{n}=20)$. Ascostromata 55-65 $\times 45-55 \mu \mathrm{m}(\bar{x}=60 \times 48 \mu \mathrm{m}, \mathrm{n}=10)$ globose to subglobose, brown, covered with attached aseptate to septate, dark brown to brown setae. Ascostroma wall 70-80 $\mu \mathrm{m}$ wide $(\bar{x}=65 \mu \mathrm{m}, \mathrm{n}=10)$, thick-walled, inwardly hyaline, pale brown and brown towards the outside, comprised 2-3 layers of textura angularis. Asci 55-60 $\times 20-25 \mu \mathrm{m}(\bar{x}=57 \times 22 \mu \mathrm{m}, \mathrm{n}=10), 8$-spored, bitunicate, ellipsoid to clavate, some subglobose, with or without short pedicle. Paraphyses not observed. Ascospores 25-30 $\times 5-10 \mu \mathrm{m}(\bar{x}=28 \times 8 \mu \mathrm{m}, \mathrm{n}=10)$, fasciculate, hyaline, fusoid, ends rounded, 3 septate, with 1-2 distinct guttules, slightly curved at the middle, smooth-walled. Asexual morph: Undetermined. 
Material examined - Thailand, Chiang Rai, on living leaf of Psidium guajava (Myrtaceae), 21 June 2018, M.W.D Sandamali (MFLU 19-2251, holotype).

GenBank Numbers - LSU: MN644511, ITS: MN644590.

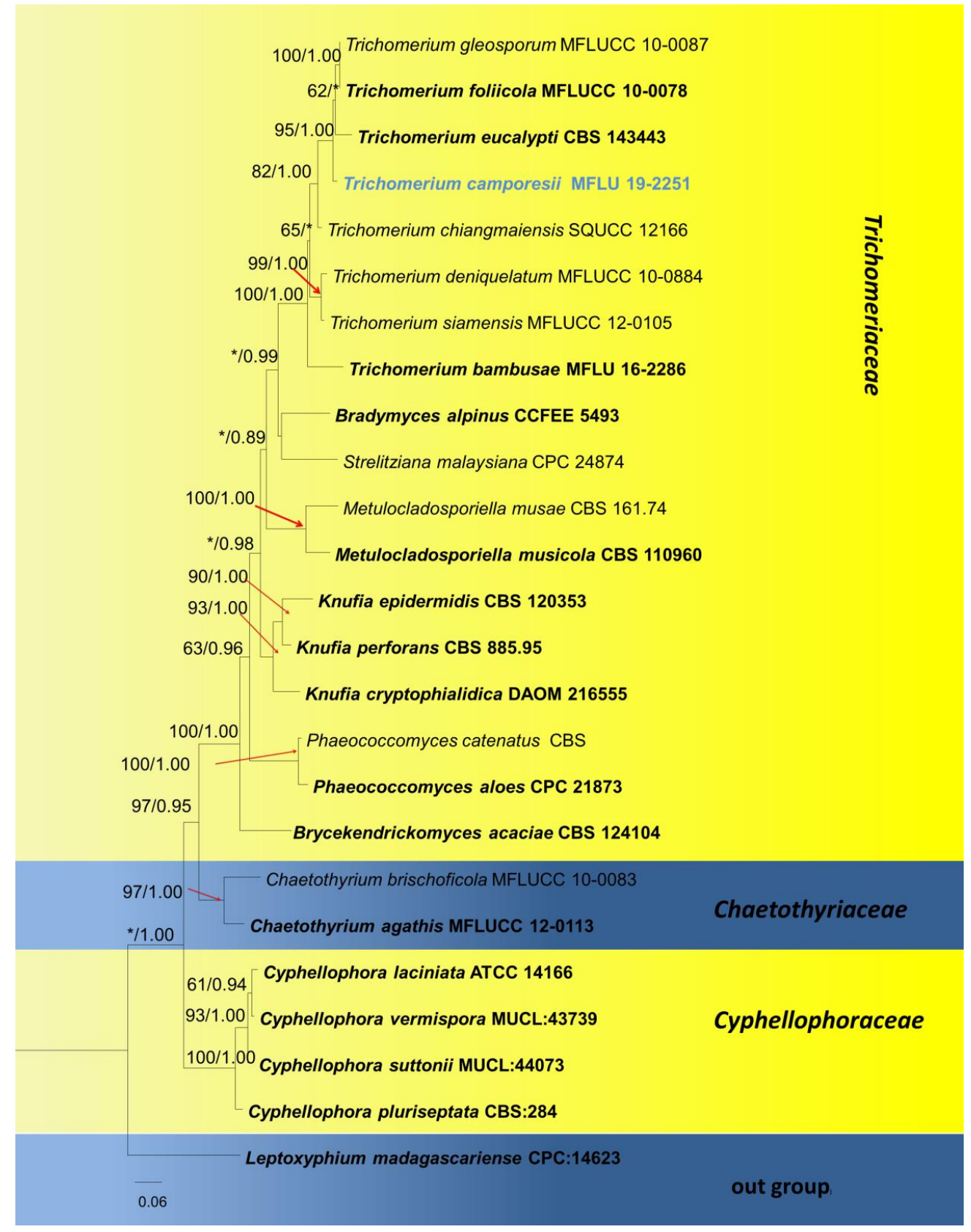

Fig. 116 - Phylogram generated from maximum likelihood analysis based on combined LSU and ITS sequence data representing Trichomeriaceae, Cyphellophoraceae, Chaetothyriaceae and the Capnodiaceae (outgroup). Related sequences are taken from Maharachchikumbura et al. (2018). Twenty five strains are included in the combined analyses which comprise 1629 characters (791 characters for LSU and 837 characters for ITS) including gaps. The tree topology derived from the Bayesian analysis was similar to that derived from the maximum likelihood analysis. The best RaxML tree with a final likelihood value of -10206.223000 is presented. The matrix had 701 distinct alignment patterns, with $20.98 \%$ undetermined characters or gaps. Estimated base frequencies were as follows: $\mathrm{A}=0.244909, \mathrm{C}=0.243885, \mathrm{G}=0.276707, \mathrm{~T}=0.234500$; substitution rates $\mathrm{AC}=1.442198, \mathrm{AG}=1.950067, \mathrm{AT}=1.512274, \mathrm{CG}=1.034625, \mathrm{CT}=$ 4.856831, GT $=1.000000$; gamma distribution shape parameter $\alpha=0.293070$. Bootstrap values for maximum likelihood (ML) equal to or greater than $60 \%$ and clade credibility values greater than 
0.80 (the rounding of values to 2 decimal proportions) from Bayesian-inference analysis labeled on the nodes. Ex-type strains are in bold and black, the new isolate is indicated in bold and blue.

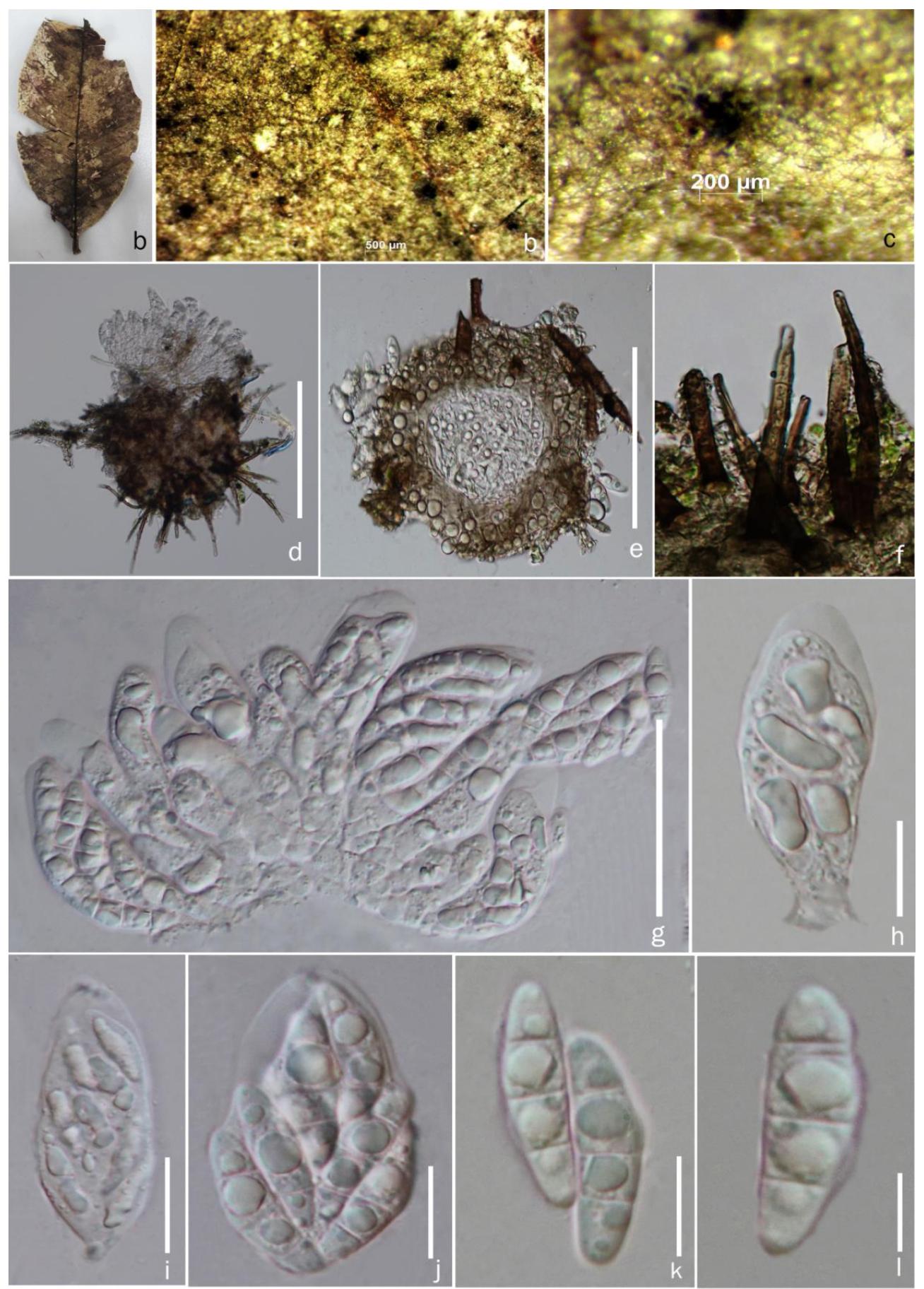

Fig. 117 - Trichomerium camporesii (MFLU 19-2251 holotype). a Dead leaf specimen. $\mathrm{b}$, c Ascostromata on the surface of leaf. d Ascostroma with setae. e Section through ascoma. f Ascoma setae. $\mathrm{g}-\mathrm{j}$ Asci. k, 1 Ascospores. Scale bars: $\mathrm{d}-\mathrm{f}=100 \mu \mathrm{m}, \mathrm{g}=50 \mu \mathrm{m}, \mathrm{h}-\mathrm{j}=20 \mu \mathrm{m}$, $\mathrm{k}, 1=10 \mu \mathrm{m}$.

Notes - Trichomerium camporesii is similar to T. foliicola, but differs in having ellipsoid to clavate asci and slightly curved ascospores with relatively large 1-4 guttules, while $T$. foliicola has cylindrical to clavate asci with relatively small 1-2-guttulate ascospores. Phylogenetic analysis based on LSU and ITS sequence data shows that $T$. camporesii constitutes an independent lineage basal to T. eucalypti (Fig. 116, $92 \%$ ML, 1.00 BYPP). 
Class Lecanoromycetes O.E. Erikss. \& Winka

Lecanoromycetidae families incertae sedis

Ostropales Nannf

Stictidaceae Fr.

The family contains both lichenized and non-lichenized fungi (Wedin et al. 2005).

\section{Stictis Pers.}

Stictis species are wide spread in tropical and temperate evergreen forests (Joshi et al. 2012). The genus is characterized by orbicular ascoma opening by a pore, periphysoids in apothecial margins that extend down the whole length, a hymenium that splits from the margin when dry, a thick crystalline layer in the ascoma margin. Stictis was monographed by Sherwood (Sherwood 1977) and Lücking et al. (2017).

Stictis urceolata (Ach.) Gilenstam (Ach.) Gilenstam, Lichenologist 37(1): 74 (2005)

Fig. 119

Facesoffungi number: FoF07378

Non-lichenized fungus, corticolous, forming a hyaline epiphloeodal hyphal felt or thallus, 30-60 $\mu \mathrm{m}$ thick. Sexual morph: Ascomata, urceolate, solitary, sometimes aggregated in two, at first immersed, opening by a pore, becoming erumpent and finally nearly superficial, $350-420 \mu \mathrm{m}$ in diameter, round, chroodiscoid; disc brownish to flesh coloured, densely pruinose, splitting away from the margin, up to $0.4 \mathrm{~mm}$ in diameter, deeply immersed; margin radiate, effigurate, lacerate, 5-6 lobed, white-pruinose, eroded in older apothecia, 60-120(-145) $\mu \mathrm{m}$ thick in cross section, hyaline to darken in older apothecia, sometimes layered, entirely encrusted in crystals. Outer exciple layer thicker; inner exciple layer brown, branched periphysoids present, forming the innermost layer of the margin, separated from the outer wall by crystals; crystals forming a dense layer along the inner margin of apothecia, 50-60 $\mu \mathrm{m}$ thick. Epihymenium indistinct, granular, hyaline to slightly brownish, usually covered by $40-55 \mu \mathrm{m}$ high crystalline layer, hymenium hyaline, interspersed, separated from the margin in dry condition, Paraphyses filiform, branched, with thickened apical cell, dense, conglutinate. Asci 50-70 $(-80) \times 3.5-5 \mu \mathrm{m}, 8$-spored, cylindrical, bitunicate. Ascospores 30-55 × 3.5-4.5 $\mu \mathrm{m}$, hyaline, cylindrical to fusiform, transversely septate, sheathed, locules broader than longer. Asexual morph: Undetermined.

Known distribution (based on molecular data) - North America, England (Tuckerman, et al. 1847), Taiwan, Chia Yi, Dahu (this study)

Known hosts (based on molecular data) - Miscanthus sp. (this study)

Material examined - Taiwan, Chia Yi, Dahu, on decaying stems of Miscanthus sp. (Poaceae), 27 April 2018, A. Karunarathna, AKTW 52 (MFLU 19-2695, new host and geographical record); ex-type living culture, NCYUCC 19-0365.

GenBank number - LSU: MN989186.

Notes - Herein, we provide the new host and geographical records for Stictis urceolata based on morphology and phylogeny (Fig. 118).

\section{Vibrisseaceae Korf}

Vibrisseaceae was introduced by Korf (1990). Five genera and about 80 species are currently accepted in this family (Zheng \& Zhuang 2017).

Phialocephala W.B. Kendr.

Kendrick (1961) introduced Phialocephala, which was previously known as Leptographium. Thirty-four species were accepted in Phialocephala (Tanney et al. 2016, Crous et al. 2017) and they occur as endophytes (Rashmi et al. 2019). However, several phylogenetic studies revealed that members of this genus are polyphyletic (Grünig et al. 2009, Wong et al. 2015, Ekanayaka et al. 2019). 
Facesoffungi number: FoF06717

Saprobic on decaying wood. Sexual morph: Undetermined. Asexual morph: Colonies on natural substate effuse, white, velvety. Mycelium mostly immersed, composed of hyaline, branched, septate and constricted at the septa, guttulate hyphae, 1.5-3 $\mu \mathrm{m}$ wide. Condiophores up to $300 \mu \mathrm{m}$ long, 5-7 $\mu \mathrm{m}$ wide at base, 3.5-4.5 $\mu \mathrm{m}$ wide at tip, macronematous, mononematous, erect, straight or broadly curved, subcylindrical, wider at base, septate, median brown, thick-walled, branched at the apex. Conidiogenous cells phialidic, discrete, determinate, terminal, clustered at the apex of conidiophores, hyaline, thin-walled, cylindrial, with inconspicuous openings, with minute collarettes. Conidia 2.5-4 $\mu \mathrm{m}$ long, 2-3.5 $\mu \mathrm{m}$ at widest $(\bar{x}=3.5 \times 2.5 \mu \mathrm{m}, \mathrm{n}=30)$, acrogenous, aggregated in slimy and white masses, acrogenous, solitary to catenate, hyaline, cuneate, rhomboid or shield-shaped, asepate, smooth.

Culture characteristics - Conidia germinated within 48h. Germ tubes produced from one angle. Mycelia superficial, decumbent, irregular, with entire edge, yellowish brown at center, pale brown at circumference from above.

Known distribution (based on molecular data) - Japan, Tokyo, USA, New Jersey (Kiyuna et al. 2012), China, Guizhou (Jie et al. 2013), Thailand, Phrae (this study).

Known hosts (based on molecular data) - Decayed tree, soil (Kiyuna et al. 2012), Decaying wood (this study)

Material examined - Thailand, Phrae Province, Rong Kwang, on decaying wood, 10 January 2018, N.G. Liu, N016 (MFLU 19-2852, new geogrophical record); living culture, GZCC 20-0005.

GenBank numbers - ITS: MN999924, LSU: MN901120, SSU: MN901150.

Notes - Jong and Davis (1972) introduced Phialocephala humicola from a soil sample collected from Cape May, New Jersey, USA. Subsequently, this species was isolated from soil in Japan (Matsushima 1975), USA (Ellis 1976), China (Matsushima 1980) and Australia (Matsushima 1989). Matsushima (1975) reported P. humicola on Quercus sp. in Nara City, Japan, and Matsushima (1980) found this species on leaves of Areca catechu in Taiwan, China. Our collection differs from $P$. humicola (ATCC 22801) in having cuneate, rhomboid or shield-shaped conidia, while the latter has ellipsoidal conidia, but the phylogenetic analysis of combined LSU and ITS sequence data confirmed that our collection is $P$. humicola. This is new geographical record of $P$. humicola in Thailand (Fig. 120).

\section{Rhytismatales genera incertae sedis}

Apiculospora Wijayaw., Camporesi, A.J.L. Phillips \& K.D. Hyde

Apiculospora is monotypic. This genus was placed in Helotiales genera incertae sedis by Wijayawardene et al. (2016). However, our phylogenetic analysis based on LSU, SSU and ITS sequence data show that Apiculospora is related to Porodiplodiaceae (Rhytismatales). The sexual morph of Apiculospora is undetermined. Future collections and DNA sequence analyses are needed to connect Apiculospora to its sexual morph.

Apiculospora spartii Wijayaw., W.J. Li, Camporesi, A.J.L. Phillips \& K.D. Hyde, Fungal Diversity:(2016)

Fig. 123

Facesoffungi number: FoF01426

Saprobic on dead twigs of Clematis vitalba, forming conspicuous rounded, black, conidiomata. Sexual morph: undetermined. Asexual morph: Conidiomata 150-300 $\mu \mathrm{m}$ diameter, 250-300 $\mu \mathrm{m}$ high, dark brown to black, acervular, solitary to gregarious, initially immersed, ultimately erumpent, globose to subglobose, unilocular. Ostiole absent, dehiscence by apical wall in the middle part. Conidioma wall composed of thin-walled, pale brown cells of textura angularis in the basal part. Conidiophores reduced to conidiogenous cells. Conidiogenous cells 7-18 $\times 2-3 \mu \mathrm{m}$, hyaline, holoblastic, cylindrical, thick-walled, smooth-walled, arising from the uppermost cells of the basal wall. Conidia 13-20 × 7-11 $\mu \mathrm{m}(\bar{x}=15 \times 8.5 \mu \mathrm{m} ; \mathrm{n}=20)$, hyaline when young, becoming dark brown with age, fusiform, obtuse at apex, narrowed and slightly truncated at base, straight or slightly curved, 1-septate, constricted at septum, thick and smooth-walled, guttulate. 
Culture characteristics - Colonies on PDA, reaching $10 \mathrm{~mm}$ diameter, after $7 \mathrm{~d}$, circular, whitened, spreading, flattened, felt-like, sparse, aerial, surface, smooth with crenate edge, filamentous; reverse yellowish in the central zone, whitened in edge.

Known distribution (based on molecular data) - Italy (Wijayawardene et al. 2016, this study

Known hosts (based on molecular data) - Spartium junceum (Wijayawardene et al. 2016), Clematis vitalba (this study)

Material examined - Italy, Province of Forlì-Cesena, Civitella di Romagna, Voltre, on dead twigs of Clematis vitalba (Ranunculaceae), 2 December 2013, E. Camporesi, IT1553-2 (MFLU 192559, new host record), living culture MFLUCC 15-0584 = CMP 21841.

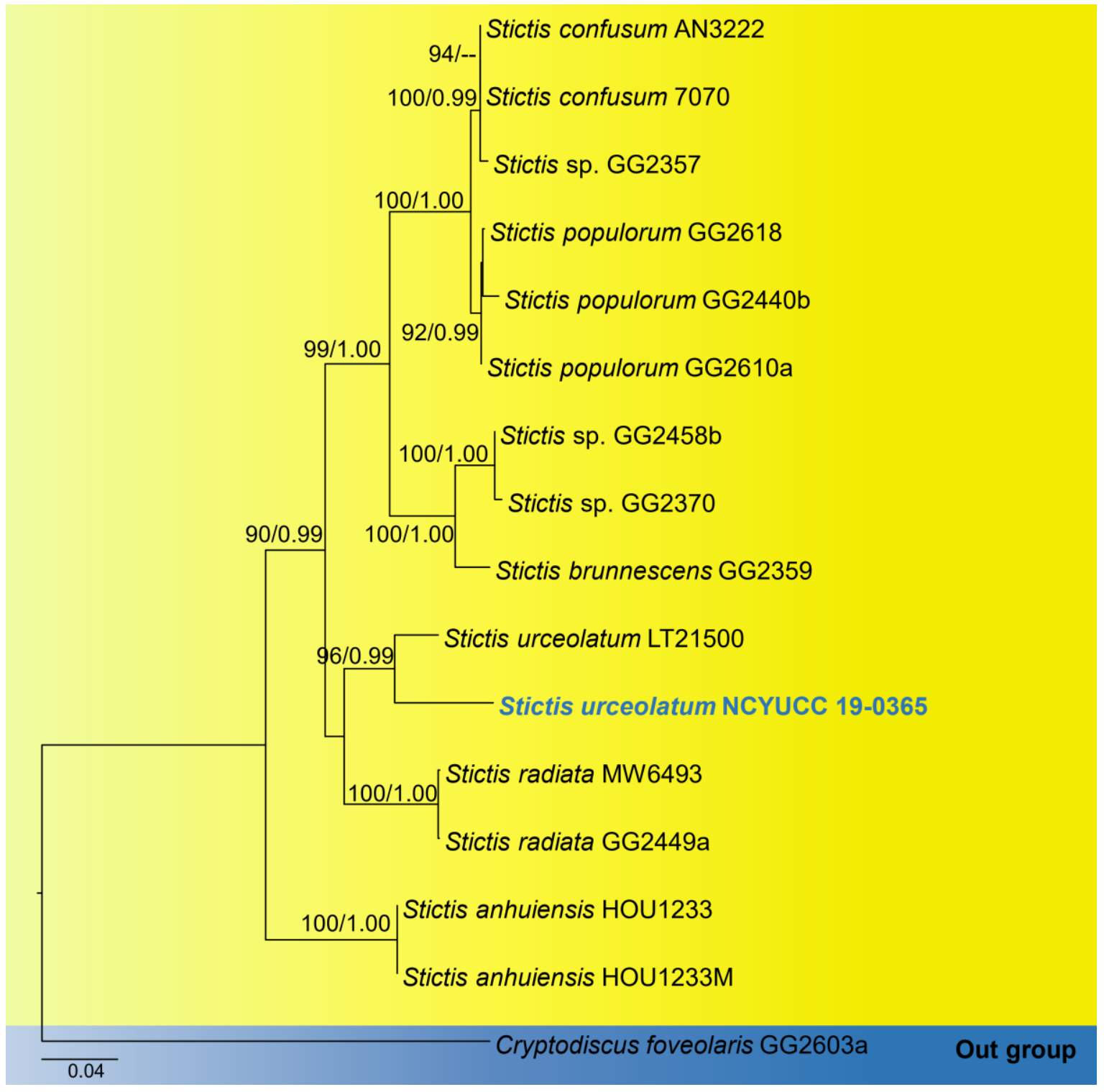

Fig. 118 - Phylogenetic tree generated from maximum likelihood (ML) based on LSU and mtSSU sequences. Bootstrap support (BS) values above 50\% and Bayesian posterior probabilities equal or greater than 0.90 are shown above the branches at nodes. The tree is rooted with Cryptodiscus foveolaris. The best RaxML tree with a final likelihood value of -4629.887606 is presented. The matrix had 356 distinct alignment patterns, with $7.44 \%$ undetermined characters or gaps. Estimated base frequencies were as follows: $\mathrm{A}=0.310275, \mathrm{C}=0.181487, \mathrm{G}=0.251212, \mathrm{~T}=0.257026$; substitution rates $\mathrm{AC}=0.679339, \mathrm{AG}=2.161324, \mathrm{AT}=1.633529, \mathrm{CG}=0.387952, \mathrm{CT}=5.104996$, $\mathrm{GT}=1.000000$; gamma distribution shape parameter $\alpha=0.637625$. Newly generate sequence is indicated in blue bold. 

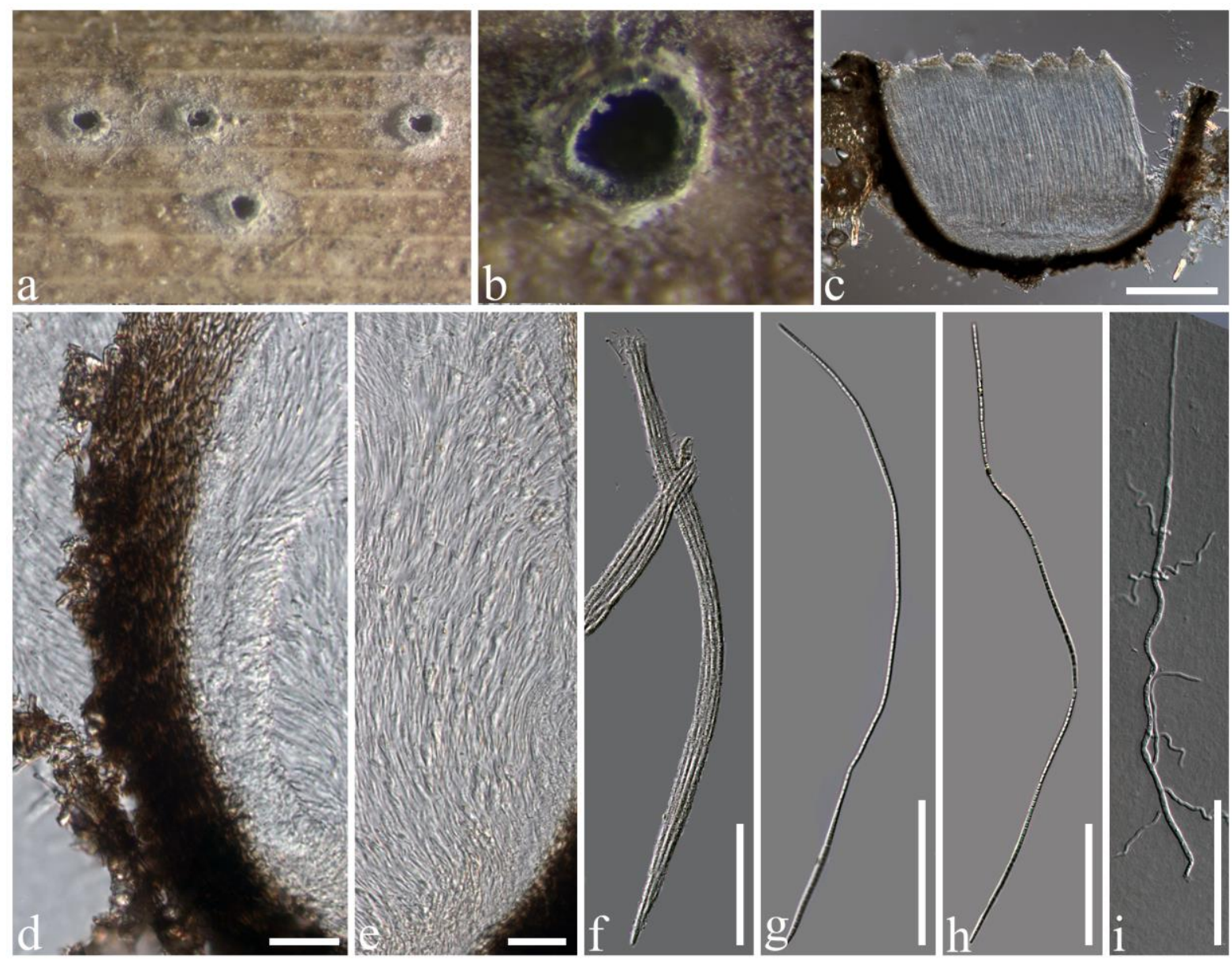

Fig. 119 - Stictis urceolata (MFLU 19-2695, new host and geographical record). a Appearance of apothecia on on host. b Apothecia. c Longitudinal section of apothecia. d Excipulum. e Paraphyses. f Asci. g, h Ascospores. i Germinating ascospore. Scale bars: $\mathrm{c}=20 \mu \mathrm{m}, \mathrm{d}-\mathrm{e}=10$ $\mu \mathrm{m}, \mathrm{f}-\mathrm{h}=10 \mu \mathrm{m}, \mathrm{i}=5 \mu \mathrm{m}$.

GenBank Numbers - (MFLUCC: 13-0400); LSU: MN660233, ITS: MN688212, SSU: MN688207, tef1: MN683863, rpb2: MN683866; (MFLUCC: 15-0584); LSU: MN688206, SSU: MN688208, tefl: MN683864.

Notes - This genus is characterized by acervular conidiomata with brown to dark brown, fusiform, 1-septate conidia (Wijayawardene et al. 2016). Our collection shares similar conidiomata, conidiogenous cells and conidia with $A$. spartii, but the latter has slightly larger conidia (17-25 $\times$ 8-11 $\mu \mathrm{m}(\overline{\mathrm{x}}=21.3 \times 9.5 \mu \mathrm{m})$. Apiculospora spartii was collected from Spartium junceum (Leguminosae), thus our collection on Clematis vitalba is regarded as a new host record (Fig. 122).

Additional notes - The sequence of Chalara sp. (voucher: MFLU 18-1812, MFLU 18-1813) submitted by Ekanayaka et al. (2019) is in fact Apiculospora spartii. The sequence similarities between MFLU 18-1812 and MFLUCC 13-0400 (type strain) are 99\% (875/877, 2 gaps) in LSU, $100 \%$ (474/474) in ITS, and 100\% (910/910) in SSU. The morphology of these two collections is almost similar to Apiculospora spartii (not shown here).

Class Sordariomycetes O.E. Erikss. \& Winka

Subclass Diaporthomycetidae Senan. et al.

Atractosporales H. Zhang et al.

Conlariaceae H. Zhang, K.D. Hyde \& Maharachch.

We follow the latest treatments and updated accounts in Zhang et al. (2017), Phookamsak et al. (2019), Xie et al. (2019) and Hyde et al. (2020). Conlarium aquaticum is reported from Thysanolaena maxima Kuntze from Yunnan, China for the first time. 


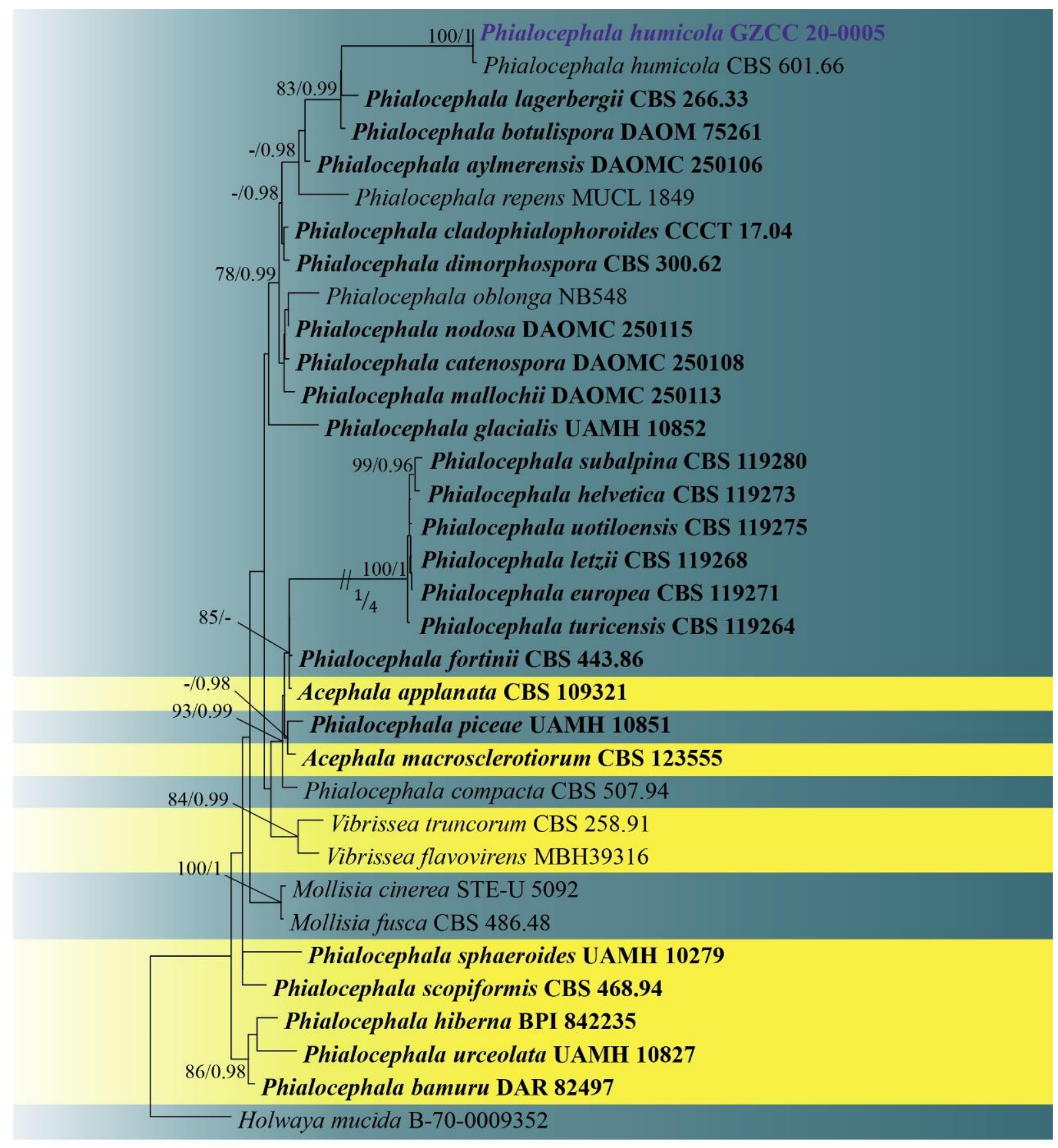

0.2

Fig. 120 - Phylogram generated from maximum likelihood analysis based on combined LSU, ITS, nad1, nat and RPB1 sequence data. Twenty-eight strains are included in the combined gene analyses comprising 4587 characters after alignment (922 characters for LSU, 609 characters for ITS, 1384 characters for nad1, 859 characters for nat, 813 characters for RPB1). Holwaya mucida (B-70-0009352) is used as the outgroup taxon. The tree topology derived from the Bayesian analysis was similar to that derived from the maximum likelihood analysis. The best RaxML tree with a final likelihood value of -14269.181523 is presented. The matrix had 795 distinct alignment patterns, with $70.07 \%$ undetermined characters or gaps. Estimated base frequencies were as follows: $\mathrm{A}=0.277515, \mathrm{C}=0.201458, \mathrm{G}=0.229770, \mathrm{~T}=0.291258$; substitution rates $\mathrm{AC}=$ 1.632078, $\mathrm{AG}=3.050144, \mathrm{AT}=1.027293, \mathrm{CG}=1.759203, \mathrm{CT}=5.265005, \mathrm{GT}=1.000000$; gamma distribution shape parameter $\alpha=0.676190$. Bootstrap values for maximum likelihood equal or greater than $75 \%$ and Bayesian posterior probabilities equal or greater than 0.95 are placed above or below the branches, respectively. Ex-type strains are in black bold. The newly generated sequence is indicated in blue bold. 


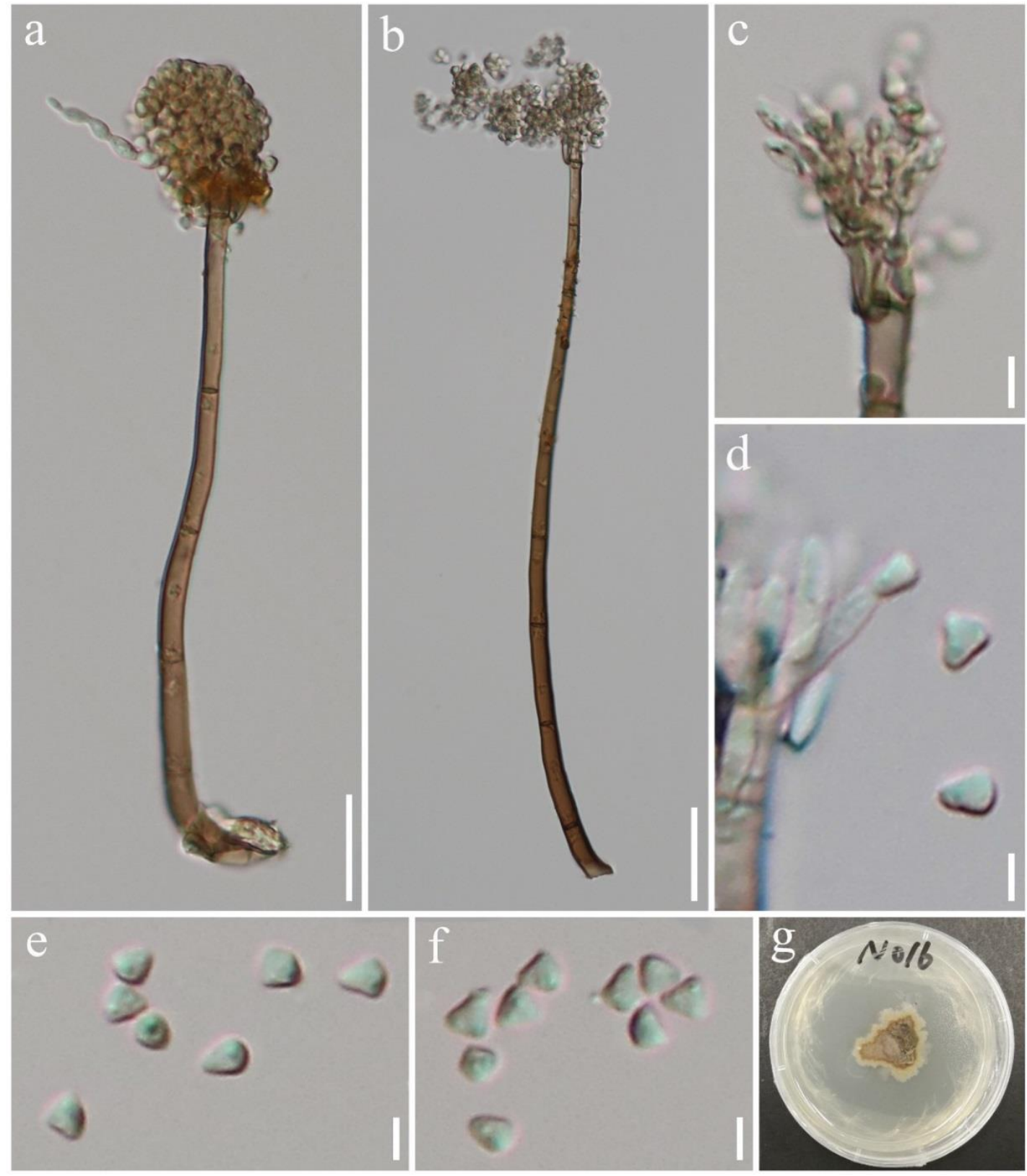

Fig. 121 - Phialocephala humicola (MFLU 19-2852, new geogrophical record). a, b Conidiophores and conidia. c, d Conidiogenous cells and conidia. e, f Conidia. g Colony on PDA media. Scale bar: $\mathrm{a}=20 \mu \mathrm{m}, \mathrm{b}=30 \mu \mathrm{m}, \mathrm{c}=5 \mu \mathrm{m}, \mathrm{d}, \mathrm{e}, \mathrm{f}=3 \mu \mathrm{m}$.

Conlarium F. Liu \& L. Cai.

Conlarium is the only genus of Conlariaceae introduced by Liu et al. (2012a) and is typified by $C$. dupliciascosporum collected from submerged wood in Guangdong Province, China. The genus is characterized by semi-immersed to superficial, dark brown to black, gregarious ascomata, with elongate necks, 8-spored, unitunicate, cylindrical asci, with a bipartite apical ring and hyaline, fusiform, aseptate to multi-septate ascospores, with or without appendages at one or each end (Liu et al. 2012a). The asexual morph of Conlarium was described as hyphomycetous, producing micronematous or semi-macronematous, mononematous, aseptate or septate, hyaline to brown conidiophores, determinate, doliiform, cylindrical, conidiogenous cells and brown, muriform, irregularly globose or subglobose conidia (Liu et al. 2012a, Zhang et al. 2017, Phookamsak et al. 2019, Xie et al. 2019). Currently, Conlarium comprises six species viz. C. aquaticum, C. baiseense, C. duplumascosporum, C. nanningense, C. sacchari and C. thailandense X (Zhang et al. 2017, Phookamsak et al. 2019, Xie et al. 2019). 


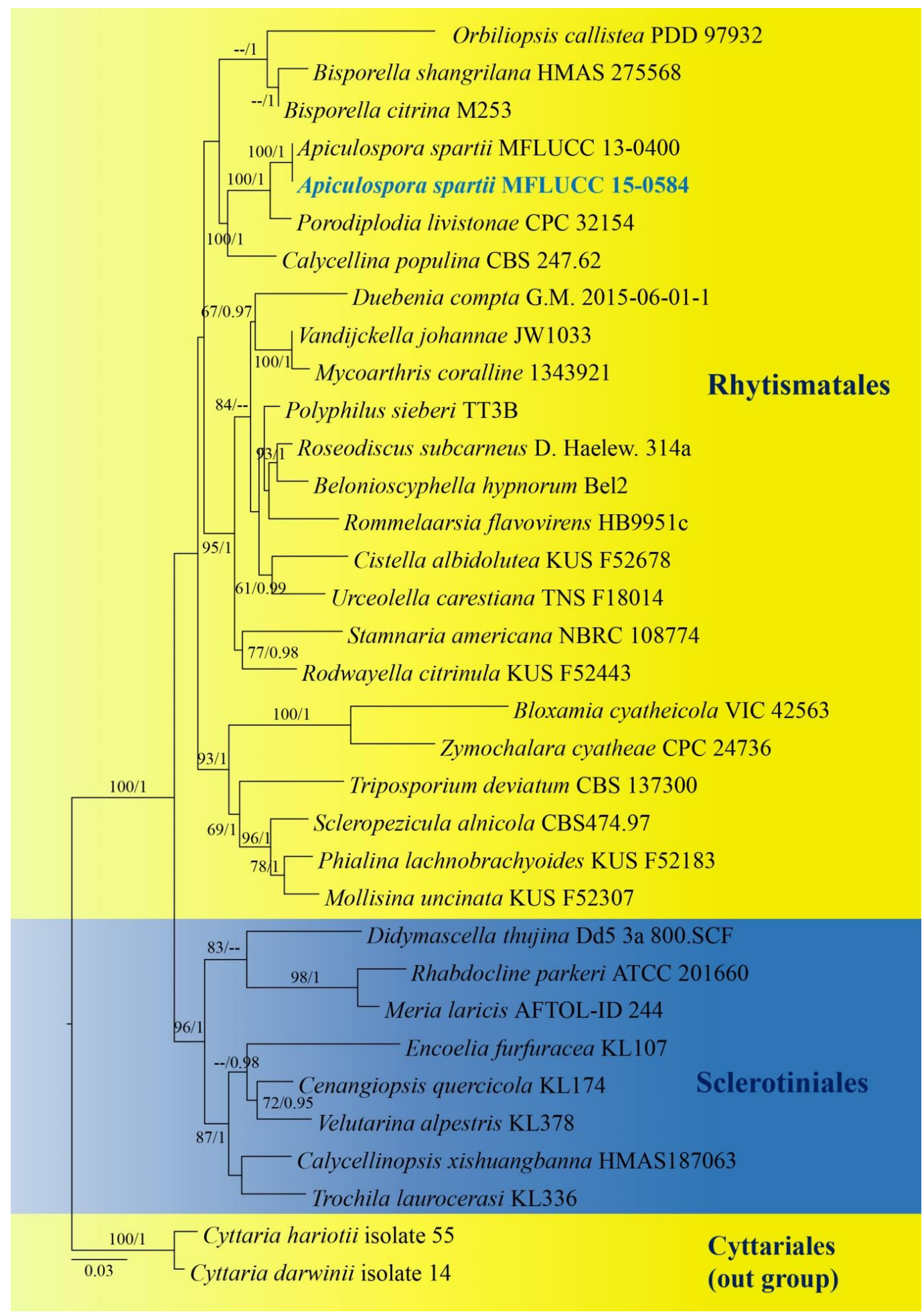

Fig. 122 - Phylogram generated from maximum likelihood analysis based on combined LSU, SSU and ITS sequence data. Related sequences are taken from Ekanayaka et al. (2019). Thirty-four strains are included in the combined analyses which comprise 2669 characters (897 characters for LSU, 1031 characters for SSU and 714 characters for ITS) after alignment. Cyttaria hariotii (isolate 55) and C. darwinii (isolate 14) in Cyttariales are used as the outgroup taxa. The tree topology derived from the Bayesian analysis was similar to that derived from the maximum likelihood analysis. The best RaxML tree with a final likelihood values of -12211.970160 is presented. The matrix had 758 distinct alignment patterns, with $44.15 \%$ undetermined characters or gaps. Estimated base frequencies were as follows: $\mathrm{A}=0.247343, \mathrm{C}=0.226463, \mathrm{G}=0.279975, \mathrm{~T}=$ 0.246219; substitution rates $\mathrm{AC}=1.333617, \mathrm{AG}=2.238128, \mathrm{AT}=1.196767, \mathrm{CG}=1.158902, \mathrm{CT}$ $=6.126029, \mathrm{GT}=1.000000$; gamma distribution shape parameter $\alpha=0.194594$. Bootstrap values 
for maximum likelihood (ML) equal to or greater than $60 \%$ and clade credibility values greater than 0.95 (the rounding of values to 2 decimal proportions) from Bayesian-inference analysis labeled on the nodes. The new isolate is indicated in bold and blue.
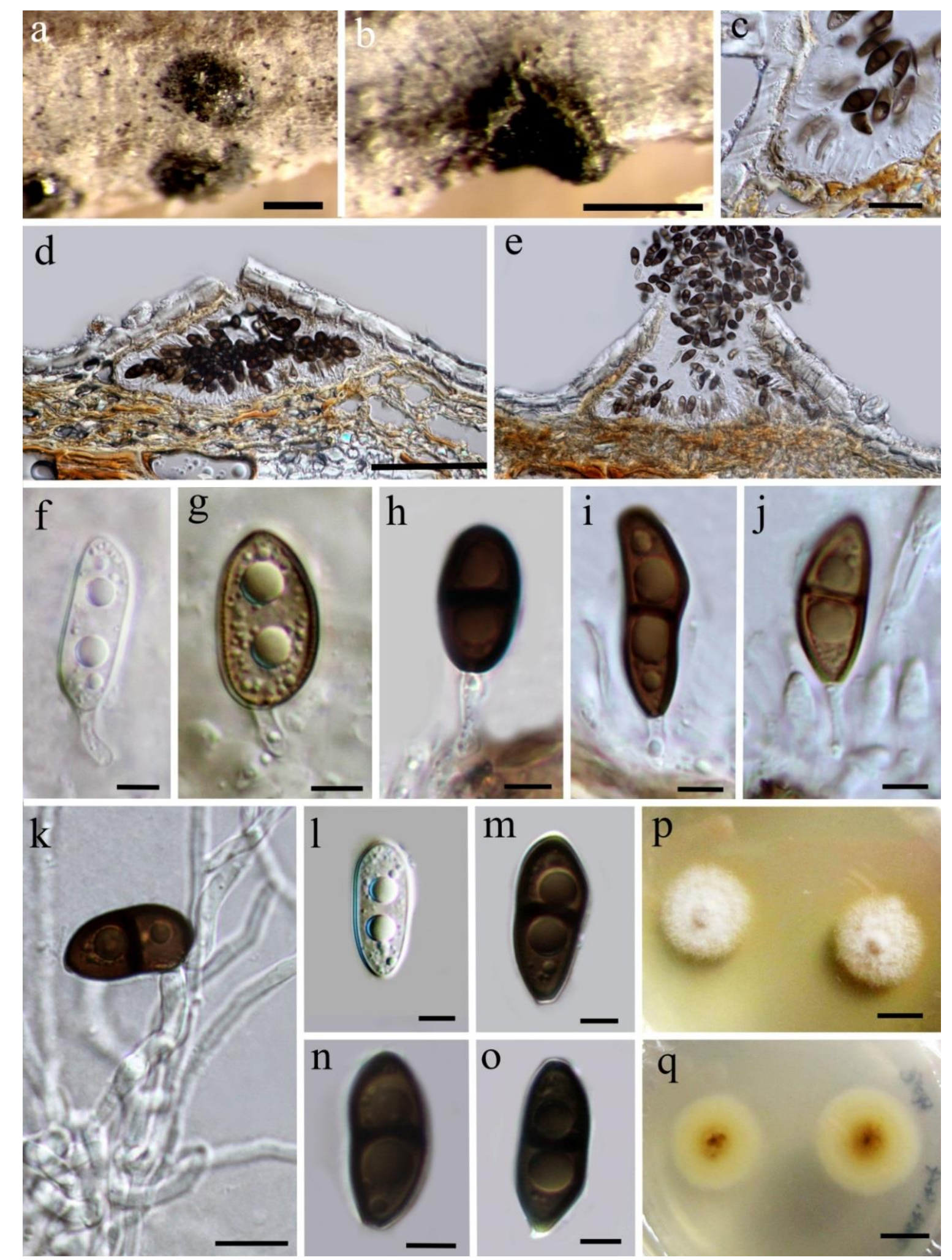

Fig. 123 - Apiculospora spartii (MFLU 19-2559, new host record). a, b Appearance of black conidiomata on the host. c Section of peridium. $d$, e Vertical sections of conidiomata. $\mathrm{f}-\mathrm{j}$ Conidiogenous cells and developing conidia. $\mathrm{k}$ Germinating conidium. $\mathrm{l}-\mathrm{o}$ Conidia. $\mathrm{p}-\mathrm{q}$ Culture on PDA. Scale bars $\mathrm{a}=500 \mu \mathrm{m}, \mathrm{b}=200 \mu \mathrm{m}, \mathrm{c}=50 \mu \mathrm{m}, \mathrm{d}-\mathrm{e}=100 \mu \mathrm{m}, \mathrm{f}-\mathrm{j}, \mathrm{l}-\mathrm{o}=5 \mu \mathrm{m}, \mathrm{k}=10 \mu \mathrm{m}$, $\mathrm{p}-\mathrm{q}=5 \mathrm{~mm}$. 
Facesoffungi number: FoF 03337

Saprobic on dead culm of Thysanolaena maxima. Sexual morph: Undetermined. Asexual morph: Colonies sporodochial, broadly punctiform, scattered to gregarious, visible as dark brown to black, velvety, shiny colony on the host surface. Mycelium mostly immersed in host substrate, hyaline to light brown, branched, septate, thin- and smooth-walled hyphae. Conidiophores 142-192 $\times$ 4.4-5.8 $\mu \mathrm{m}(\bar{x}=167.1 \times 5.1 \mu \mathrm{m}, \mathrm{n}=20)$, semi-macronematous or macronematous, mononematous, septate, unbranched, straight or flexuous, brown to dark brown. Conidiogenous Cells $15-20 \times 3.5-4.9 \mu \mathrm{m}(\bar{x}=15.6 \times 4.2 \mu \mathrm{m}, \mathrm{n}=20)$, monoblastic, holoblastic, integrated, determinate, cylindrical, hyaline to pale brown, smooth. Conidia 37-50 × 28-28.5 $\mu \mathrm{m}(\bar{x}=43.4 \times$ $33.4 \mu \mathrm{m}, \mathrm{n}=20$ ), acrogenous, dictyosporous, muriform, globose to subglobose or irregular in shape, initially hyaline, becoming brown to dark brown when mature, multi-septate, sectored, with small air bubble-like in each cell, slightly constricted at the septa. Conidia secession schizolytic.

Known distribution (based on molecular data) - Thailand (Zhang et al. 2017), China (this study).

Known hosts (based on molecular data) - Submerged wood in freshwater (Zhang et al. 2017), Thysanolaena maxima (this study).

Material examined - China, Yunnan Province, Xishuangbanna, Mengla County, Xishuangbanna Tropical Botanical Garden (XTBG), on dead stems of Thysanolaena maxima (Poaceae), 22 April 2017, R. Phookamsak, IS005 (MFLU 20-0139, new host record), living culture, KUMCC 18-0189.

GenBank numbers - ITS: MN994320, LSU: MN994322, SSU: MN994323, tef1: MT005780 (KUMCC 18-0189A); ITS: MN994328, LSU:MN994330, SSU: MN994331, tef1: MT005779 (KUMCC 18-0189B).

Notes - Our new collection has a similar morphology with the type species of Conlarium aquaticum in having brown to dark brown, muriform, multi-septate, sectored conidia (Zhang et al. 2017). However, the conidia of the new collection are slightly smaller than the type (MFLU 200139: 37-50 × 28-28.5 $\mu \mathrm{m}$ versus 45-70 × 20-57 $\mu \mathrm{m}$ : MFLU 15-2703; Zhang et al. 2017). Phylogenetic analyses of a combined LSU, SSU and ITS sequence dataset indicated that our strains form a strongly supported subclade with $C$. aquaticum (Fig. 124, MFLUCC 15-0992; 98\% ML and $1.00 \mathrm{PP})$. A comparison of the ITS sequence between these strains reveals less than $1.5 \%$ nucleotide base differences, which demonstrate that our new collection is conspecific with $C$. aquaticum (MFLU 15-2703) (Jeewon \& Hyde 2016). Therefore, we report our new collection as $C$. aquaticum which is collected from Thysanolaena maxima in Yunnan, China for the first time in this study.

Diaporthales Nannf.

Cryphonectriaceae Gryzenh. \& M.J. Wingf.

Cryphonectriaceae was established by Gryzenhout et al. (2006), which comprises mostly phytopathogens, saprobes and endophytes. Recent classification of the family accept 23 genera as Amphilogia, Aurantioporthe, Aurantiosacculus, Aurapex, Aurifilum, Celoporthe, Chromendothia, Chrysofolia, Chrysoporthe, Chrysoporthella, Cryphonectria, Cryptometrion, Diversimorbus, Endothia, Foliocryphia, Holocryphia, Immersiporthe, Latruncellus, Luteocirrhus, Mastigosporella, Microthia, Rostraureum and Ursicollum (Senanayake et al. 2017, 2018, Wijayawardene et al. 2018).

Aurifilum Begoude, Gryzenh. \& Jol. Roux

Aurifilum is a monotypic genus introduced by Begoude et al. (2010) with A. marmelostoma associated with Terminalia ivorensis as a pathogen from Cameroon. The genus is characterized by large, pulvinate to pyriform, semi-immersed ascostroma and hyaline, ellipsoidal to fusoid, 1-septate ascospores and broadly convex conidiomata, lack of conidiomatal sack and paraphyses as 
compared with other similar genera in Cryphonectriaceae with uniformly orange ascostromata (Begoude et al. 2010). Morphology and phylogenetic analyses using concatenated genes and pathogenicity tests have been confirmed the occurrence of cankers on Terminalia catappa, T. ivorensis and T. mantali in Cameroon and Taiwan (Begoude et al. 2010, Vermeulen et al. 2011, Shen et al. 2016).

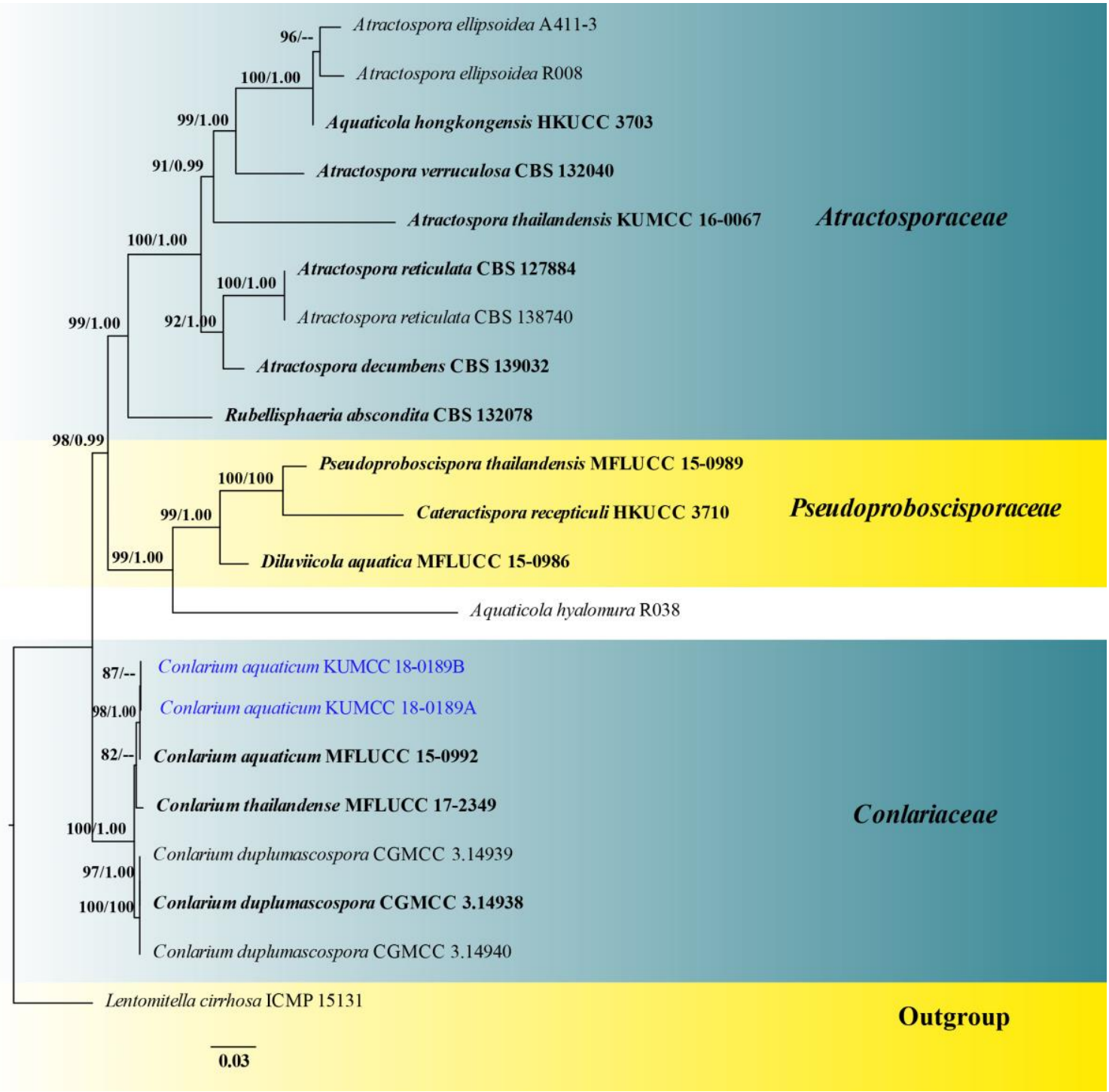

Fig. 124 - Phylogram generated from maximum likelihood analysis based on a combined LSU, SSU and ITS sequence dataset. Twenty-one strains are included in the combined gene analyses comprising 2354 total characters including gaps (LSU: 1-872 bp, SSU: 873-1824 bp, ITS: 1825$2354 \mathrm{bp}$ ). The tree topology derived from the Bayesian analysis was similar to that derived from the maximum likelihood analysis. The best scoring of the ML tree is selected to represent the phylogenetic relationships of taxa in Conlariaceae and other related families, with the final ML optimization likelihood: -9128.236780. The matrix had 631 distinct alignment patterns, with $21.35 \%$ undetermined characters or gaps. Estimated base frequencies were as follows: $\mathrm{A}=$ $0.258526, \mathrm{C}=0.222031, \mathrm{G}=0.276323, \mathrm{~T}=0.243120$; substitution rates $\mathrm{AC}=1.896853, \mathrm{AG}=$ 2.980974, $\mathrm{AT}=1.889217, \mathrm{CG}=0.816766, \mathrm{CT}=7.838961, \mathrm{GT}=1.000000 ;$ Tree-Length $=$ 1.051267; gamma distribution shape parameter $\alpha=0.518124$. Bayesian posterior probabilities (BYPP) from MCMC were evaluated with a final average standard deviation of split frequencies of 0.007152. Bootstrap support for ML greater than $70 \%$ and Bayesian posterior probabilities greater than 0.95 are defined above the nodes as ML/PP. The tree is rooted to Lentomitella cirrhosa (ICMP 15131). The type strains are indicated in bold and newly generated sequences are shown in blue. 

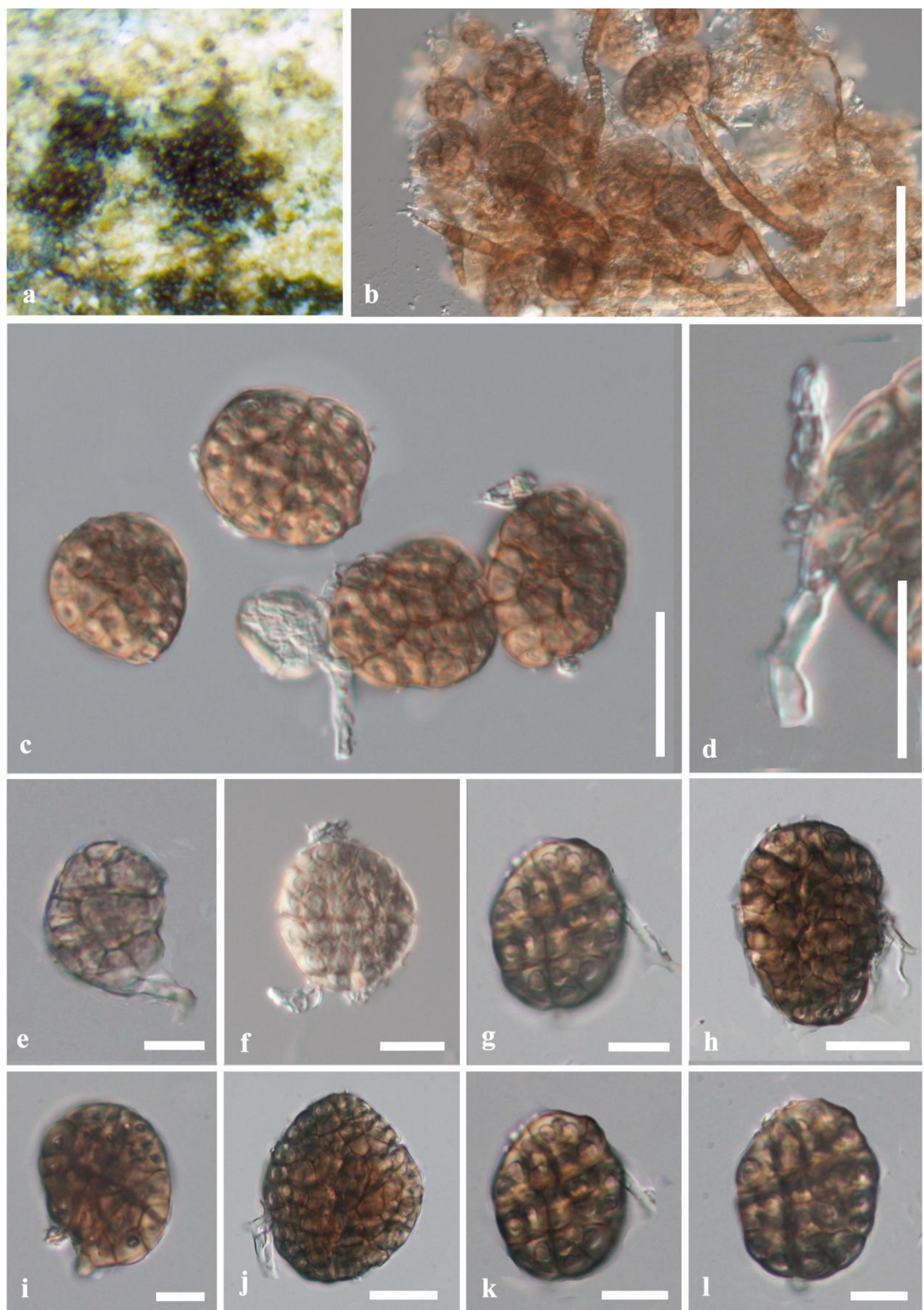

Fig. 125 - Conlarium aquaticum (MFLU 20-0139, new host record). a Colonies on host. b Squash of mycelium and conidiophores. $\mathrm{c}$, e-m Conidia. $\mathrm{d}$ Conidiogenous cell. Scale bars: $\mathrm{b}=50 \mu \mathrm{m}$, $\mathrm{c}=30 \mu \mathrm{m}, \mathrm{d}, \mathrm{f}-\mathrm{h}, \mathrm{j}, \mathrm{k}=20 \mu \mathrm{m}, \mathrm{e}, \mathrm{i}, \mathrm{l}=10 \mu \mathrm{m}$.

Aurifilum marmelostoma Begoude, Gryzenh. \& Jol. Roux, Antonie van Leeuwenhoek 98 (3): 273 (2010)

Fig. 127

Facesoffungi number: FoF 04130

Saprobic on dead branches and twigs of Terminalia ivorensis (Combretaceae). Sexual morph: Ascostromata 520-600 $\mu \mathrm{m}$ high, 540-640 $\mu \mathrm{m}$ diameter, gregarious or single, scattered, medium to 
large, usually beneath or erumpent through the bark, semi-immersed, pulvinate to pyriform, yellow to orange, black ostiole, upper region eustromatic, lower region pseudostromatic, pseudoparenchymatous to prosenchymatous tissue. Perithecia 190-330 $\mu \mathrm{m}$ diameter, valsoid, 1-6 per stroma, globose to subglobose, embedded in the stroma at irregular levels, loosely connect with the host. Peridium 7.9-13.2 $\mu \mathrm{m}(\bar{x}=9.9 \mu \mathrm{m}, \mathrm{n}=15)$ wide, composed of dark brown to reddish brown cells, 4-5 layers of textura angularis to textura epidermoidea. Perithecial necks 140-310 $\mu \mathrm{m}(\bar{x}=210 \mu \mathrm{m}, \mathrm{n}=10)$ long, 33.5-57.6 $\mu \mathrm{m}(\bar{x}=44.3 \mu \mathrm{m}, \mathrm{n}=10)$ wide, periphysate, black, emerging at stromatal surface as black ostioles, surrounded with orange stromatal tissue to form papillae, composed of with textura porrecta. Asci 47.9-51.7 $\times 8.1-12.3 \mu \mathrm{m}(\bar{x}=50.4 \times 9.8 \mu \mathrm{m}, \mathrm{n}=$ 20), 8-spored, fusoid to ellipsoidal, unitunicate with $\mathrm{J}$-, refractive apical rings, short pedicel in immature and sessile at maturity. Ascospores $8.3-10.8 \times 2.5-4 \mu \mathrm{m}(\bar{x}=9.7 \times 3.3 \mu \mathrm{m}, \mathrm{n}=40), \mathrm{L} / \mathrm{W}$ 2.9, hyaline, fusoid to ellipsoidal, 1-median septum with tapered apex. Asexual morph: Undetermined.

Culture characteristics - Colonies on PDA fast-growing, reaching $55 \mathrm{~mm}$ diameter, after one week at $25-30^{\circ} \mathrm{C}$, medium dense, circular in shape, flattened, surface smooth, with entire edge, velvety to floccose, colony from above greyish white, from below yellowish brown.

Known distribution (based on molecular data) - Cameroon, Kribi, Mbamalyo and Yaounde), Taiwan (Begoude et al. 2010, Vermeulen et al. 2011, Shen et al. 2016), Thailand (this study).

Known hosts (based on molecular data) - Terminalia catappa, T. ivorensis and T. mantaly (Begoude et al. 2010, Vermeulen et al. 2011, Shen et al. 2016), Terminalia ivorensis (this study).

Material examined - Thailand, Chiang Rai, Mae Fah Luang University, on dead branches and twigs of Terminalia ivorensis (Combretaceae), 9 July 2017, MC. Samarakoon, SAMC082 (MFLU 18-0831, HKAS 102322, new host and geographical record), living culture MFLUCC 18-0536.

GenBank numbers - ITS: MN473057, LSU: MN473051, TUB: MN987000.

Notes - Our strain is similar to the type species, Aurifilum marmelostoma in having gregarious or single, yellow ascostromata, the diameter of perithecia overlapping (190-330 $\mu \mathrm{m}$ diameter vs 190-310 $\mu \mathrm{m}$ ) and hyaline, fusoid to ellipsoidal, 1-septate ascospores with a tapered base. Our ITS-LSU based phylogenies reveal that our strain clusters with other A. marmelostoma strains with high support (Fig. 126, 100\%/100\%/0.99 PP). In addition, the ITS, LSU and BT sequences are highly similar to those from the type strain $(100 \%, 100 \%$ and $99.5 \%)$. However, there is no known record of A. marmelostoma in Thailand. This is the first record of Aurifilum marmelostoma from Terminalia ivorensis from Thailand and probably the first host record from Asia. Shen et al. (2016) discussed the pathogenicity of Aurifilum marmelostoma on Terminalia mantaly and T. catappa seedlings.

\section{Cytosporaceae Fr.}

Senanayake et al. (2017) excluded several genera and accepted only Cytospora, Pachytrype, Paravalsa, Xenotypa and Waydora within this family. Most members in this family are pathogens that cause various diseases on commercial crops and forest trees (Adams et al. 2005, 2006). Fan et al. (2015a), Jiang et al. (2020) and Shang et al. (2020) have recently introduced several new taxa to this family.

\section{Cytospora Ehrenb.}

Cytospora was introduced by Ehrenberg (1818). Currently, there are more than 100 estimated species in Cytospora (Norphanphoun et al. 2018, Species Fungorum 2020, Shang et al. 2020). Rossman et al. (2015) conserved Cytospora over Valsa giving priority to the older and commonly used name. The asexual morph of Cytospora is commonly produced in nature and has fruiting bodies that contain a single or labyrinthine of locules, filamentous conidiophores and allantoid hyaline conidia (Fan et al. 2015a). Some Cytospora species are phytopathogenic and form cankers and diebacks (Dar \& Rai 2014, Fan et al. 2015a). Some are saprobes and are involved in litter decomposition (Adams et al. 2006). 


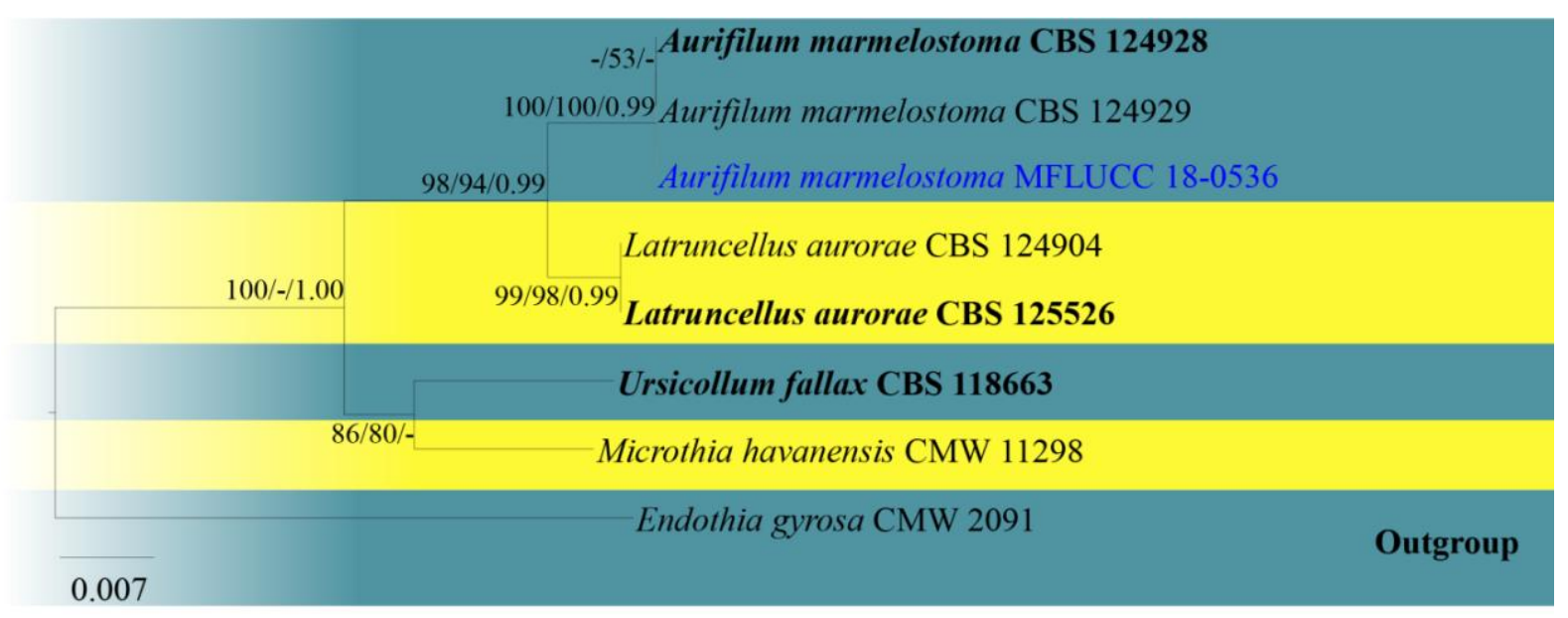

Fig. 126 - Phylogram generated from maximum likelihood analysis based on combined ITS and LSU sequence data. Eight strains are included in the combined gene analyses comprising 1165 characters after alignment (547 characters for ITS, 618 characters for LSU). Endothia gyrosa (CMW 2091) is used as the outgroup taxon. The tree topology derived from the Bayesian analysis was similar to that derived from the maximum likelihood analysis. The best RaxML tree with a final likelihood value of -2120.791979 is presented. The matrix had 94 distinct alignment patterns, with $8.42 \%$ undetermined characters or gaps. Estimated base frequencies were as follows: $\mathrm{A}=$ $0.240, \mathrm{C}=0.249, \mathrm{G}=0.281, \mathrm{~T}=0.230$; substitution rates $\mathrm{AC}=1.051005, \mathrm{AG}=1.236751, \mathrm{AT}=$ $0.728926, \mathrm{CG}=0.729107, \mathrm{CT}=5.437760, \mathrm{GT}=1.000000$; gamma distribution shape parameter $\alpha$ $=0.759635$. Bootstrap values for maximum parsimony and maximum likelihood equal to or greater than 50 and Bayesian posterior probabilities equal or greater than 0.95 are placed above or below the branches. Ex-type strains are in black bold and newly generated sequence is indicated in blue.

Cytospora cedri Syd., P. Syd. \& E.J. Butler, Annls mycol. 14(3/4): 193 (1916)

Fig. 129

Facesoffungi number: FoF06871

Saprobic on aerial branches of Lonicera sp. Sexual morph: Stromata superficial, ascomata embedded in stromatic tissues, black, irregular to oval, internally brown, pseudoparenchymatous tissues and outer, black, shiny, tightly-packed cells. Ascostromata 350-500 $\times 150-250 \mu \mathrm{m}(\bar{x}=440$ $\times 200 \mu \mathrm{m}, \mathrm{n}=20$ ), immersed in stromatic tissue, solitary or aggregated, black to dark brown, coriaceous, uniloculate, with ostiolar neck. Papilla 160-200 $\times 60-100 \mu \mathrm{m}(\bar{x}=180 \times 80 \mu \mathrm{m}, \mathrm{n}=$ 20), dark brown, internal canal covered with hyaline filamentous hyphae. Peridium comprising several layers of dark brown, thick-walled, cells of textura angularis. Hamathecium comprising cellular, hyaline paraphyses. Asci $25-35 \times 5.8-7.1 \mu \mathrm{m}(\bar{x}=30 \times 6.7 \mu \mathrm{m}, \mathrm{n}=20)$, 8-spored, unitunicate, clavate to elongate obovoid, with a J-, refractive, apical ring. Ascospores 5.5-7.5 $\times 2-$ $2.5 \mu \mathrm{m}(\bar{x}=6.4 \times 2.3 \mu \mathrm{m}, \mathrm{n}=20)$, biseriate, hyaline, elongate fusiform to allantoid, 1-celled, smooth-walled. Asexual morph: Undetermined.

Known distribution (based on molecular data) -India (Index Fungorum 2020), Italy (this study).

Known hosts (based on molecular data) - Cedrus libanus, Lonicera (Sydow et al. 1916), Lonicera sp. (this study).

Material examined - Italy, Province of Forlì-Cesena, Modigliana, Trebbio, dead aerial branch of Lonicera sp. (Caprifoliaceae), E. Camporesi, 5 April 2015, IT2434 (MFLU 15-1120 new host record).

GenBank numbers - ITS: MN764316, LSU: MN764359.

Notes - According to the ITS blast results, this collection shows a high similarity $(99.12 \%)$ with Cytospora fraxinigena, C. rosae (98.77\%) and C. cedri (99.63\%). However, combined gene analysis of ITS, LSU, Actin, and RPB2 in this study, shows that this Cytospora collection groups with Cytospora cedri (CBS 196.50), which was also collected from Italy. The holotype of Cytospora cedri was collected from Himachal Pradesh in India, on a branch of Cedrus libanus. 
However, our specimen was obtained from a branch of Lonicera species in Italy. Cytospora cedri is reported on Lonicera for the first time, but we could not obtain a culture for this specimen, therefore DNA was extracted directly from fruit-bodies. Our collection is similar to the type described by Sydow et al. (1916). Herein, we report this collection as Cytospora cedri based on morphology and phylogeny.
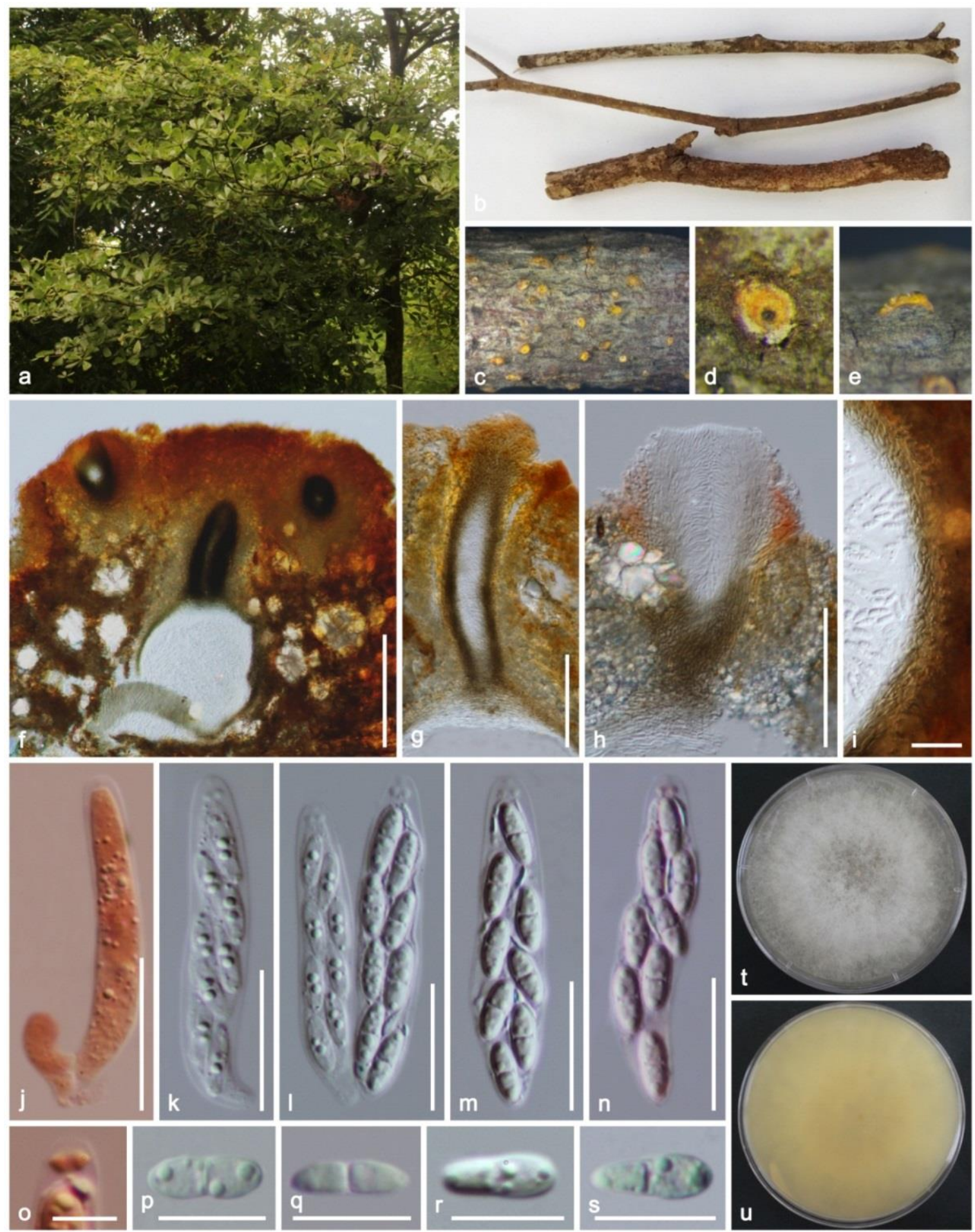

Fig. 127 - Aurifilum marmelostoma (MFLU 18-0831, new host and geographical record). a Host. b-e Ascostromata on the substrate. f Vertical section of ascostroma. g, h Ostiole section. i Peridium. $\mathrm{j}-\mathrm{n}$ Asci ( $\mathrm{j}$-in Congo Red). o Apical ring (in Congo Red). $\mathrm{p}-\mathrm{s}$ Ascospores. $\mathrm{t}$ Upper view of the colony. $u$ Reverse view of the colony. Scale bars: $f=200 \mu \mathrm{m}, \mathrm{g}, \mathrm{h}=100 \mu \mathrm{m}, \mathrm{i}-\mathrm{n}=20$ $\mu \mathrm{m}, \mathrm{p}-\mathrm{s}=10 \mu \mathrm{m}, \mathrm{o}=5 \mu \mathrm{m}$. 
Cytospora fraxiicola Chaiwan, Bulgakov, T.C., Jayaward \& K.D. Hyde, sp. nov.

Fig. 130

Facesoffungi number: FoF06958

Etymology: The specific epithet reflects the host genus Fraxinus excelsior.

Holotype: MFLU 17-2392

Saprobic on branches of Fraxinus excelsior. Sexual morph: Undetermined. Asexual morph: Pycnidia immersed on Fraxinus excelsior. Conidiomata 600-700 $\mu \mathrm{m}$ high $\times 1000-1200 \mu \mathrm{m}$ diameter $(\bar{x}=650 \times 1100 \mu \mathrm{m}, \mathrm{n}=5)$, scattered, erumpent, multi-loculate. Locules composed of numerous interconnecting, chambers arranged radially or irregularly within a continuous, mass of ectostromatic tissue, pycnidial, stromatic, solitary or clustered, immersed in the host when young, semi-erumpent at maturity, dark brown to black, globose, ostiolate. Ostioles 360-400 $\mu \mathrm{m}$ high $\times$ $380-480 \mu \mathrm{m}$ diameter $(\bar{x}=380 \times 430 \mu \mathrm{m})$. Peridium comprising a few to several layers of cells of textura angularis, with inner most layer thin, brown, outer layer dark brown to black. Conidiophores branched, reduced to conidiogenous cells. Conidiogenous cells enteroblastic, phialidic, formed from the inner most layer of pycnidial wall, hyaline, smooth-walled. Conidia 5.8$8.7 \times 1.2-2.3 \mu \mathrm{m}(\bar{x}=7.25 \times 1.75 \mu \mathrm{m}, \mathrm{n}=30)$, hyaline, elongate-allantoid, 1-celled, slightly curved, smooth-walled.

Material examined - Russia, on dead and dying branches of Fraxinus excelsior. 21 March 2017, T-2051 MFLU 17-2392 (holotype).

GenBank numbers - LSU: MN764356, ACT: MN995562.

Notes - In the multi-gene analyses, $C$. fraxiicola is distinct and forms a moderately supported lineage clade (Fig. 128). Based on multi-gene analyses, Cytospora fraxiicola is distinct from the first group of $C$. populina, $C$. fugax, C. hippophaes, $C$. gigalocus and the second group of C. cotini, $C$. ampulliformis, $C$. longispora, C. ribis, C. tanaitica, C. prunicola and C. ulmi. However, C. fraxiicola is similar to $C$. gigalocus in having multi-loculate conidiomata in their asexual morph and has larger conidia $(7.25 \times 1.75 \mu \mathrm{m})$ than C. gigalocus $(4.8 \times 1.1 \mu \mathrm{m})$ (Fan et al. 2015a. Cytospora fraxiicola differs from $C$. populina and $C$. hippophaes which was reported as a sexual morph.

Cytospora malicola Z. Urb., Česká Mykol. 10(4): 209 (1956)

Fig. 131

Facesoffungi number: FoF06902

Saprobic on branches of Salix sp. Sexual morph: Stromata immersed in bark. Ascostromata 500-900 × 150-500 $\mu \mathrm{m}$, immersed in host tissue, scattered, uni or multi-loculate, with ostiolar neck. Papilla 350-400 $\mu \mathrm{m}$, wide at the top than bottom, dark brown to black, internal canal covered with hyaline filamentous hyphae. Peridium comprising several layers of cells of textura angularis, with innermost layer thick, pale brown, outer layer dark brown to black. Hamathecium comprising long cylindrical, cellular, anastomosed paraphyses. Asci 50-65 ×9-11 $\mu \mathrm{m}(\bar{x}=58 \times 10 \mu \mathrm{m}, \mathrm{n}=15)$, 8 -spored, unitunicate, clavate to elongate obovoid, with a J-, refractive, apical ring. Ascospores $11-$ $17 \times 2.5-3 \mu \mathrm{m}(\bar{x}=15.7 \times 2.8 \mu \mathrm{m}, \mathrm{n}=20)$, biseriate to crowded, hyaline, elongate fusiform to allantoid, 1-celled, smooth-walled. Asexual morph: Undetermined.

Known distribution (based on molecular data) - Asia, Europe and North America. (Adams et al. 2006, Mehrabi et al. 2011), Italy (this study).

Known hosts (based on molecular data) - Malus sylvestris, Prunus domestica, Prunus spinosa, Crataegus oxyacantha (Hayova and Minter 1998), Salix sp (this study).

Material examined - Italy, Province of Forlì-Cesena, Santa Sofia, Corniolo, dead aerial branch of Salix sp. (Salicaceae), E. Camporesi, 16 July 2014, IT2003, MFLU 14-0831, new host record), living culture MFLUCC 14-0831.

GenBank numbers - ITS: MN764317, LSU: MN764358.

Notes - Blast results of ITS of this species show a high similarity (99.31\%) with Cytospora malicola, and C. salicina (98.81\%) (Fig. 128). However combined gene analysis of ITS, LSU, Actine, and RPB2 shows that our Cytospora species groups with Cytospora malicola (Cch92) with a moderate support. Additionally, this subclade is basal to $C$. germanica (CXY1322), $C$. kantschavelii (CXY1383), C. parakantschavelii (MFLUCC 15-0857), C. salicicola (MFLUCC 14- 


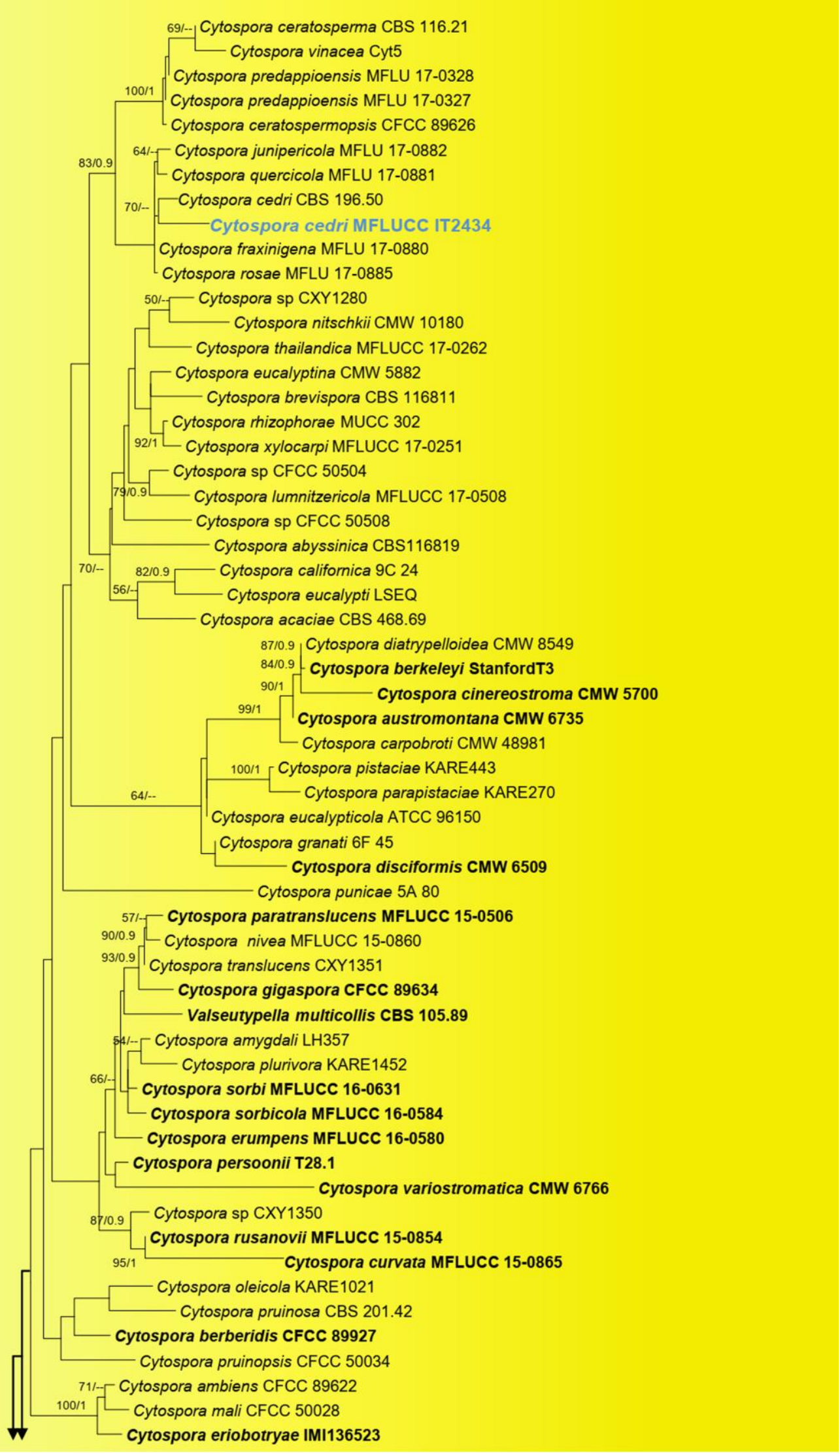

Fig. 128 - Phylogram generated from maximum likelihood analysis based on combined ITS, LSU, Actine, and RPB2 sequence data. Bootstrap support for ML greater than 50\% and Bayesian posterior probabilities greater than 0.90 are given near nodes. The tree is rooted with Diaporthe vaccinii (CBS 160.32). Ex-type strains are in black bold and the newly generated sequences are indicated in blue bold. 


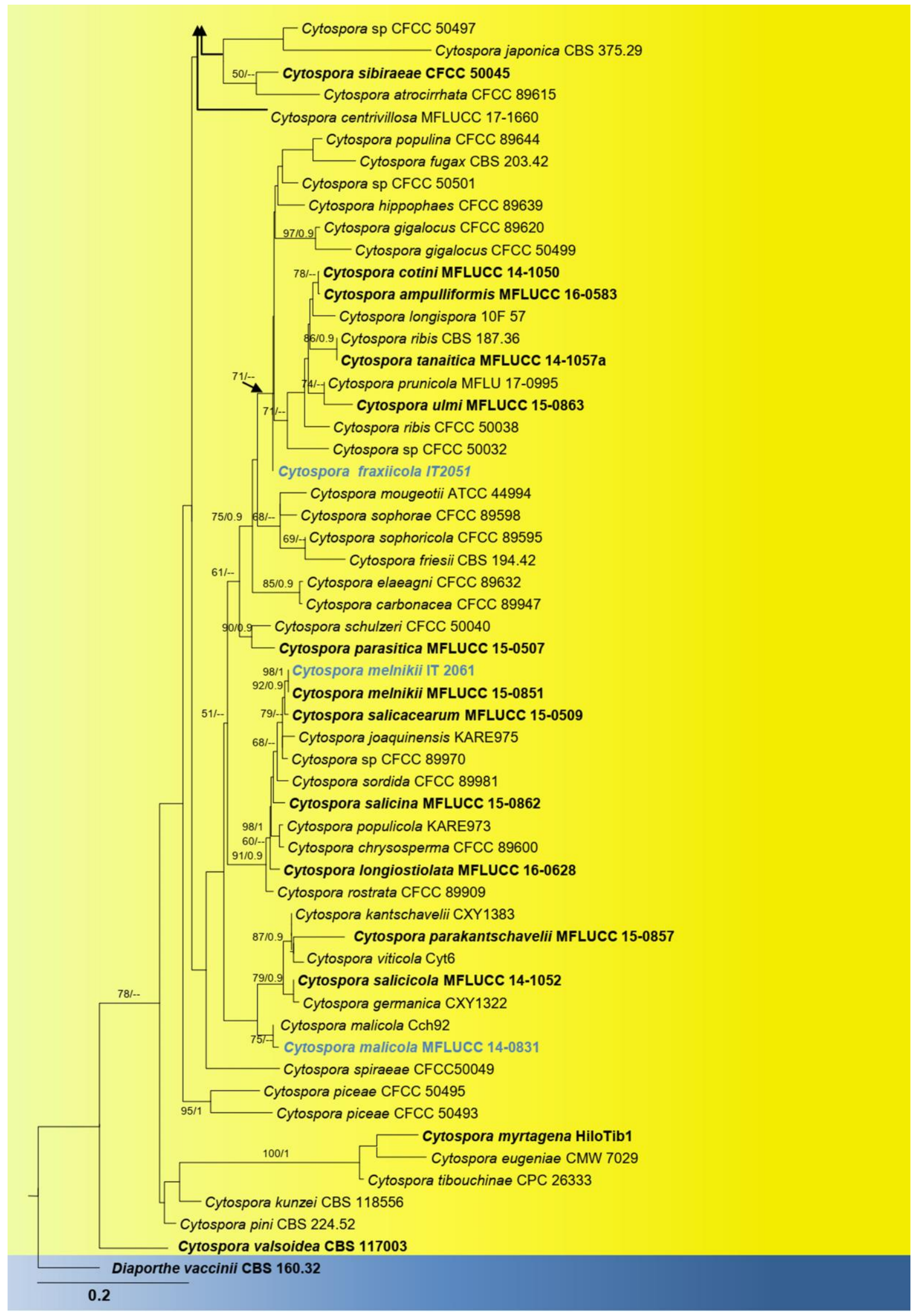

Fig. 128 - Continued.

1052) and C. viticola (Cyt6). This species was recorded from Malus sylvestris, Prunus domestica, Prunus spinosa, and Crataegus oxyacantha with both sexual and asexual morph 
(Hayova \& Minter 1998). Cytospora malicola causes dieback of Malus twigs, especially on twigs frozen, burnt, wounded, injured by insects or attacked by other pathogens (Hayova \& Minter 1998). This is the first record of $C$. malicola on Salix sp. Morphological characters and measurements of all the characters of our strain are very similar to the protologue described by Hayova \& Minter (1998). Therefore, we report this strain as Cytospora malicola based on morphology and phylogeny.

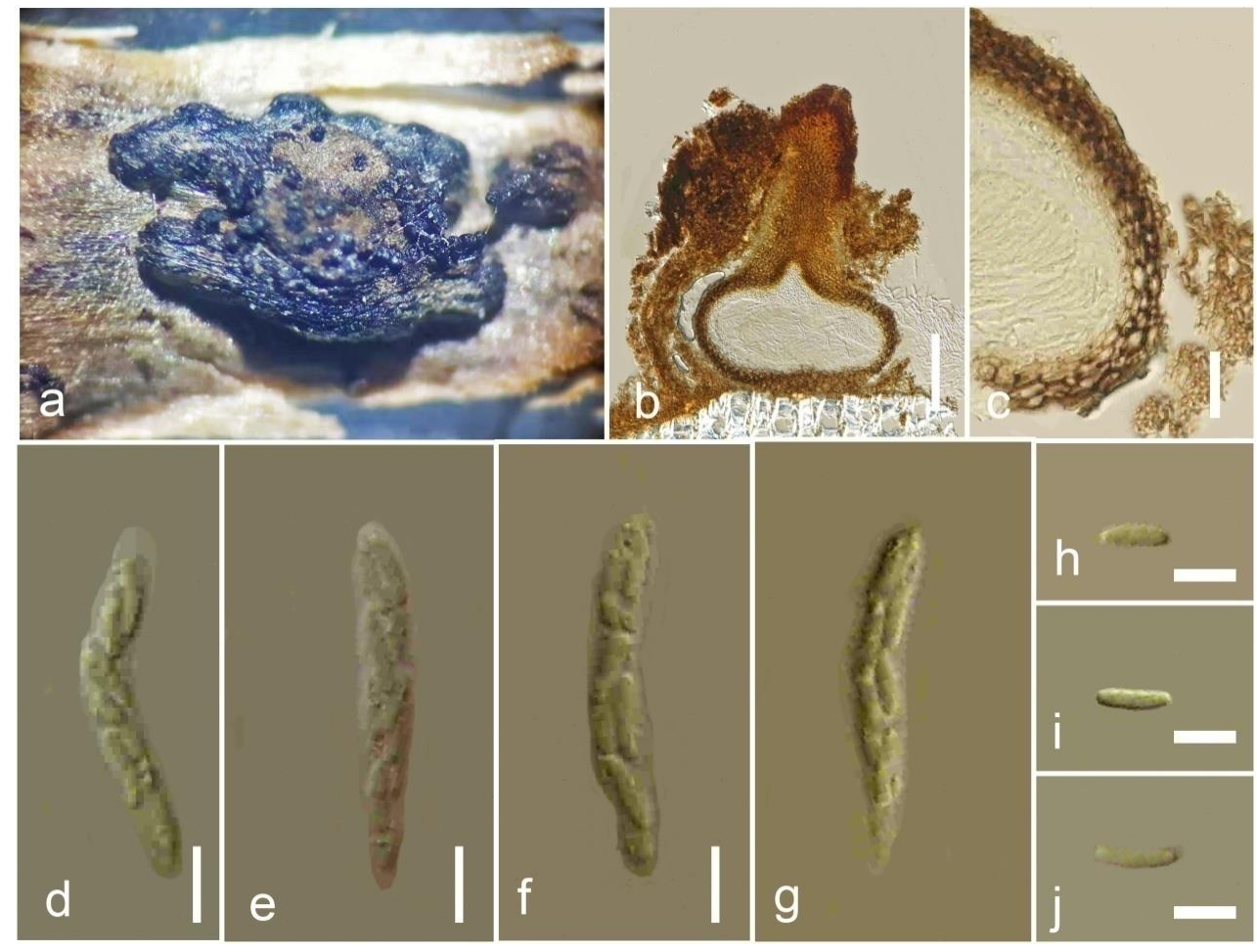

Fig. 129 - Cytospora cedri (MFLU 15-1120, new host record). a Ascostromata on substrate. b Cross section of ascoma. c Peridium. d-g Asci. $h-j$ Ascospores. Scale bars: $b=100 \mu m$, $\mathrm{c}=50 \mu \mathrm{m}, \mathrm{d}-\mathrm{g}=15 \mu \mathrm{m}, \mathrm{h}-\mathrm{j}=10 \mu \mathrm{m}$.

Cytospora melnikii Norphanphoun, Doilom, Daranagama, Phookamsak, Wen, Bulgakov \& Hyde, Mycosphere 8(1): 68 (2017)

Fig. 132

Facesoffungi number: FoF552606

Saprobic on dead branches of Salix alba. Sexual morph: Undetermined. Asexual morph: Pycnidia immersed on Salix alba. Conidiomata 500-600 $\mu \mathrm{m}$ high $\times 600-700 \mu \mathrm{m}$ diameter $(\bar{x}=550$ $\times 650 \mu \mathrm{m}, \mathrm{n}=5$ ), semi-immersed in host tissues, solitary, scattered, erumpent, discoid, circular to ovoid, unilocular, with long ostiolar necks, ostiolate. Ostioles $250-300 \mu \mathrm{m}$ high $\times 350-400 \mu \mathrm{m}$ diameter $(\bar{x}=275 \times 375 \mu \mathrm{m})$, Peridium comprising a few to several layers of cells of textura angularis, with inner most layer thin, brown, outer layer dark brown to black. Conidiophores branched, reduced to conidiogenous cells. Conidiogenous cells enteroblastic, phialidic, formed from the inner most layer of pycnidial wall, hyaline, smooth-walled. Conidia 5.5-7.5 $\times 1.5-2.5 \mu \mathrm{m}$ $(\bar{x}=6.5 \times 2 \mu \mathrm{m}, \mathrm{n}=30)$, hyaline, 1-celled, elongate-allantoid, slightly curved, smooth-walled. study)

Known distribution (based on molecular data) - Russia (Norphanphoun et al. 2017, this

Known hosts (based on molecular data) - Malus domestica Borkh, Populus nigra var. italica Münchh (Norphanphoun et al. 2017), Salix alba (this study)

Material examined - Russia, on dead and dying branches of Salix alba (Salicaceae), 25 July 2017, T-2061 (MFLU 17-2402, new host record).

GenBank numbers - ITS: MN764318, LSU: MN764355, ACT: MN995563, RPB2: MN995564. 
Notes - Norphanphoun et al. (2017) introduced Cytospora melnikii collected from Malus domestica and Populus nigra in Russia. In this study, we introduced a new record based on phylogenetic analyses. The morphological characters of our isolate is similar to $C$. melnikii as described by Norphanphoun et al. (2017). It has semi-immersed, larger conidiomata (500-600 vs $420-520 \mu \mathrm{m})$ diameter, with larger ostioles $(250-300$ vs $200-230 \mu \mathrm{m})$; and has larger conidia $(6.5$ $\times 2$ vs $4.6 \times 1.2 \mu \mathrm{m})$.

Based on multi-gene analyses, our isolate clusters with $C$. melnikii with a high bootstrap support (Fig. 128). Our BLAST search on NCBI GenBank ITS, LSU, ACT and RPB2 sequences show $100 \%$ similarity to $C$. melnikii.

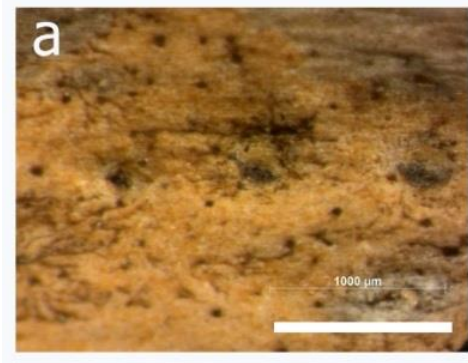

d
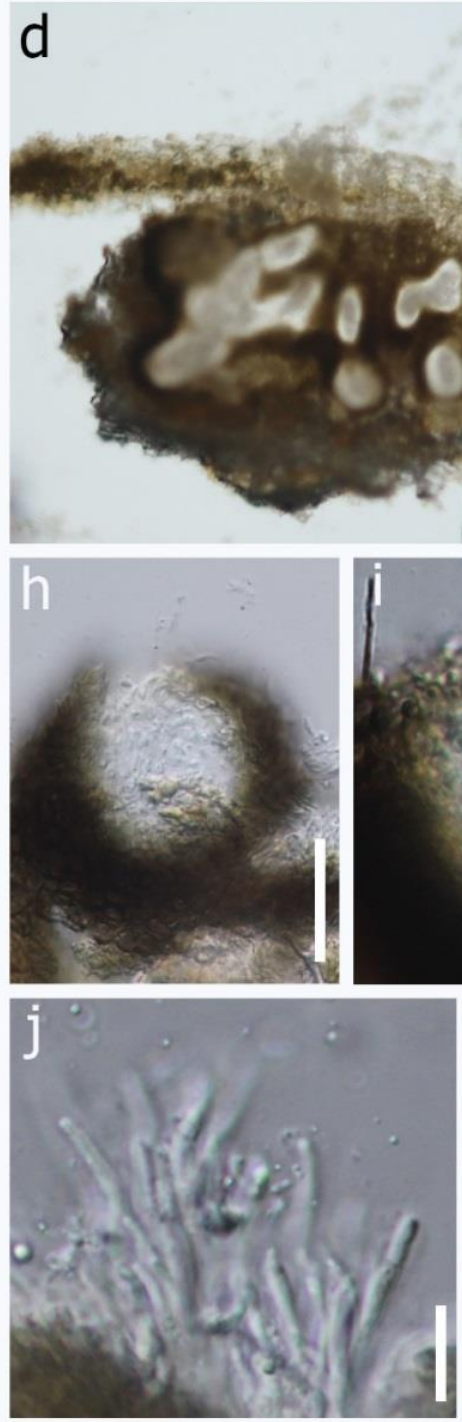
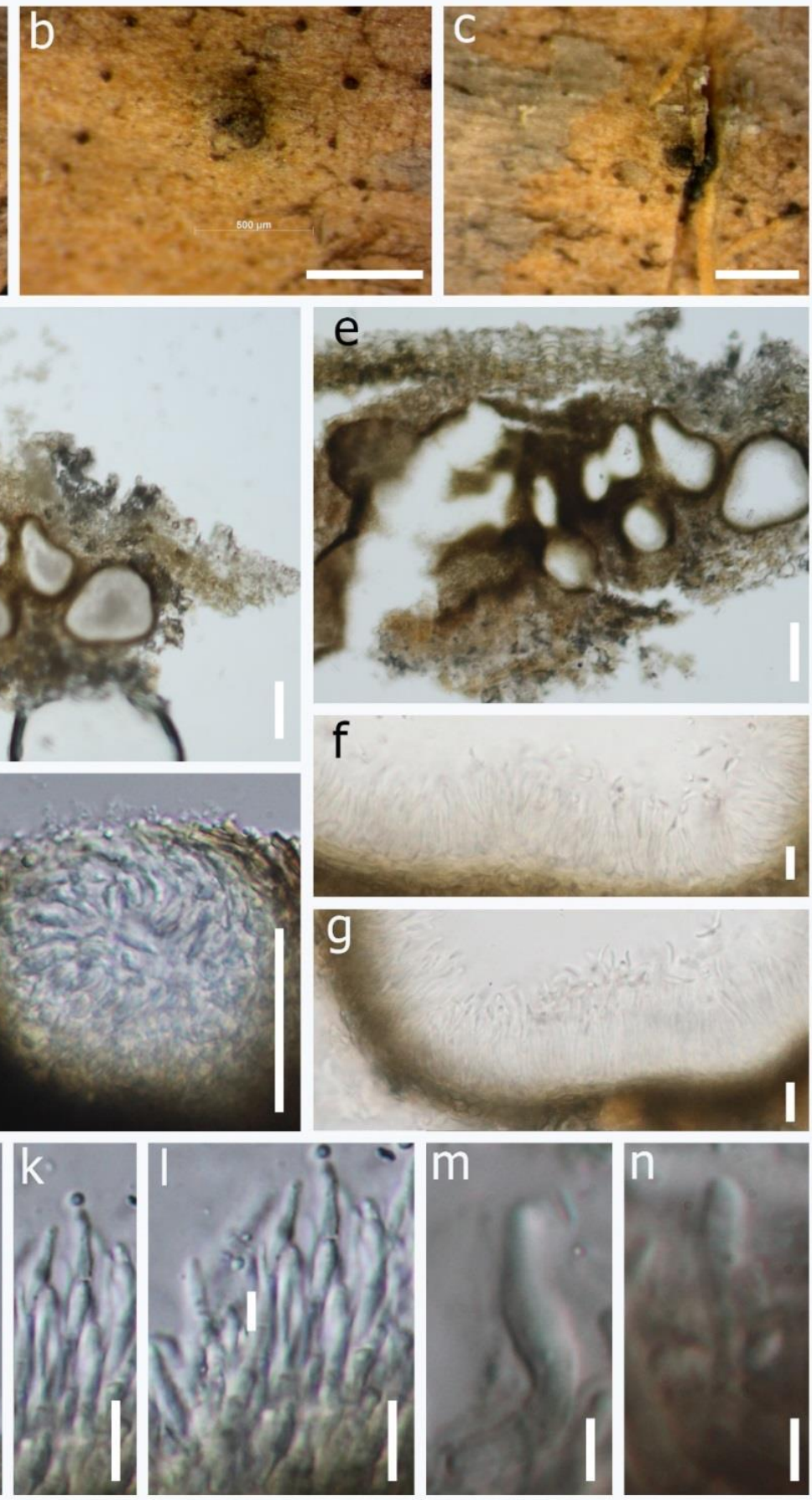

Fig. 130 - Cytospora fraxiicola (MFLU 17-2392, holotype). a-c Conidiomata on host substrate. $\mathrm{d}$, e Vertical sections of conidiomata. $\mathrm{f}-\mathrm{i}$ Conidia. $\mathrm{j}-\mathrm{n}$ Conidiogenous cells. Scale bars: $\mathrm{a}=1000$ $\mu \mathrm{m}, \mathrm{b}, \mathrm{c}=500 \mu \mathrm{m}, \mathrm{d}, \mathrm{e}=100 \mu \mathrm{m}, \mathrm{h}, \mathrm{i}=50 \mu \mathrm{m}, \mathrm{f}, \mathrm{g}, \mathrm{j}-\mathrm{n}=10 \mu \mathrm{m}$. 


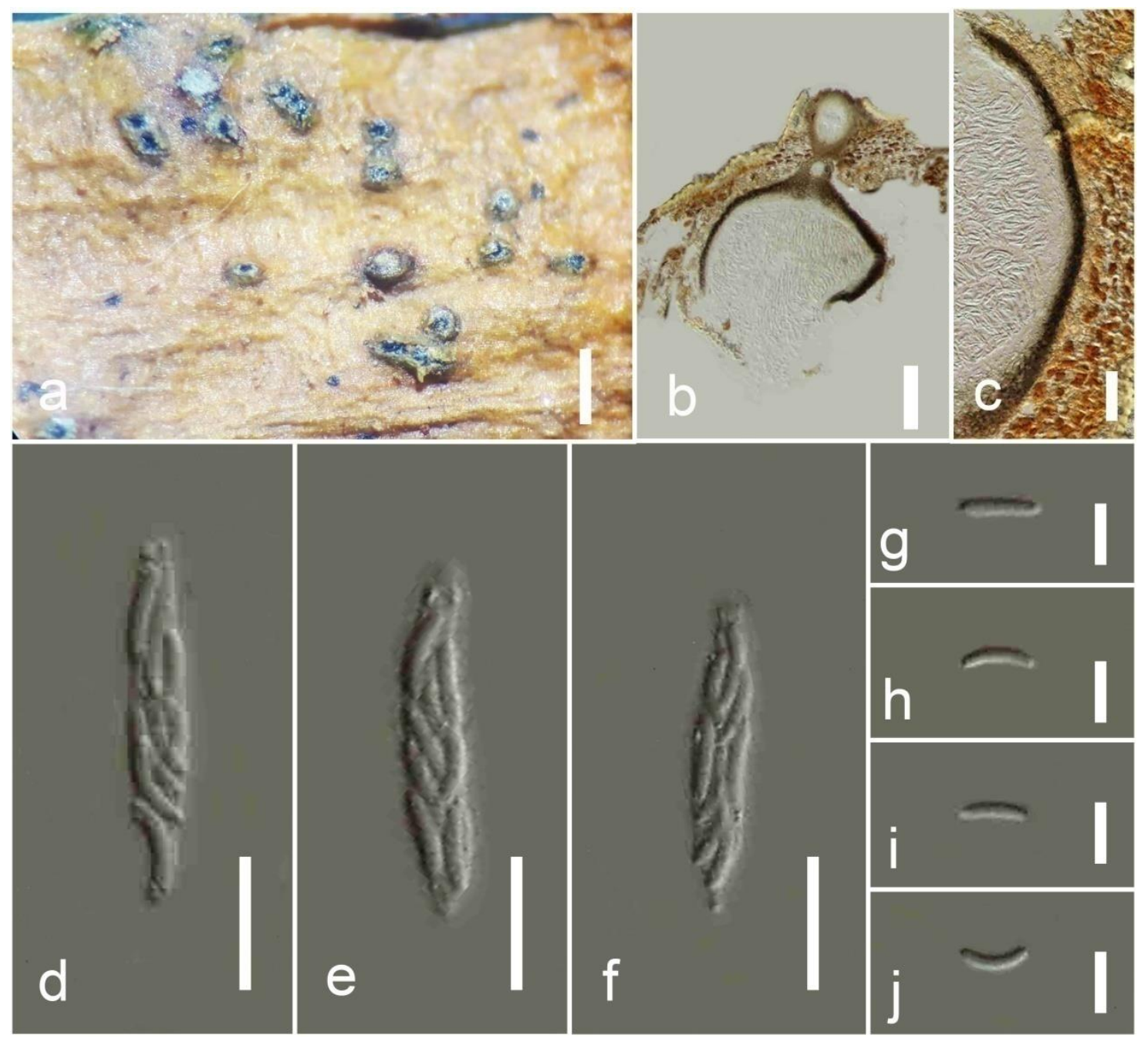

Fig. 131 - Cytospora malicola (MFLU 14-0831, new host record). a Ascostromata on substrate. b Cross section of ascoma. c Peridium. d-f Asci. g-j Ascospores. Scale bars: $a, b=100 \mu m$, $\mathrm{c}-\mathrm{j}=10 \mu \mathrm{m}$.

Diaporthales Nannf.

Diaporthaceae Höhn. ex Wehm.

The most recent taxonomic treatments of Diaporthaceae is by Senanayake et al. (2018).

Diaporthe Nitschke

Diaporthe encompasses species which are endophytic, pathogenic and saprobic on a wide range of hosts. At the same time, single species of Diaporthe can be found on diverse hosts and cooccur on the same host or lesion in different life modes (Dissanayake et al. 2017, Santos et al. 2017). Most recent treatments of this genus are from Wanasinghe et al. (2018b), Marin-Felix et al. (2019) and Manawasinghe et al. (2019).

Diaporthe ambigua Nitschke, Pyrenomyc. Germ. 2: 311 (1870)

Fig. 134

Faces of fungi number: FoF 07324

Saprobic on dead aerial stem of Iris sp. Sexual morph Undetermined. Asexual morph: Conidiomata globose,150-200 $\mu \mathrm{m}$ diameter, mostly embedded in host tissue and erumpent at maturity, up to $450 \mu \mathrm{m}$ diameter, 65-100 $\mu \mathrm{m}$ high, walls parenchymatous consisting of 3-4 layers of medium brown textura angularis. Conidiophores 7-9 $\times 1-2 \mu \mathrm{m}$, hyaline, smooth, occurring in dense clusters. Conidiogenous cells phialidic, cylindrical, terminal, with slight tapering towards apex, 0.5-1 $\mu \mathrm{m}$ diameter. Conidia $8-9 \times 2-3 \mu \mathrm{m}$, aseptate, and hyaline, smooth, ovate to ellipsoidal, biguttulate or multi-guttulate. 
Culture characteristics: colonies on PDA relatively slow growing reaching $80 \mathrm{~mm}$ diameter, after 21 days, On PDA white, fluffy aerial mycelium, and reverse with ash colour.

Known distribution -Chile, South Africa, Portugal, United Kingdom, Netherlands, Armenia, California, Uzbekistan, Canada, Cuba, Germany, China, and Spain (Farr \& Rossman 2020), Italy (this study).
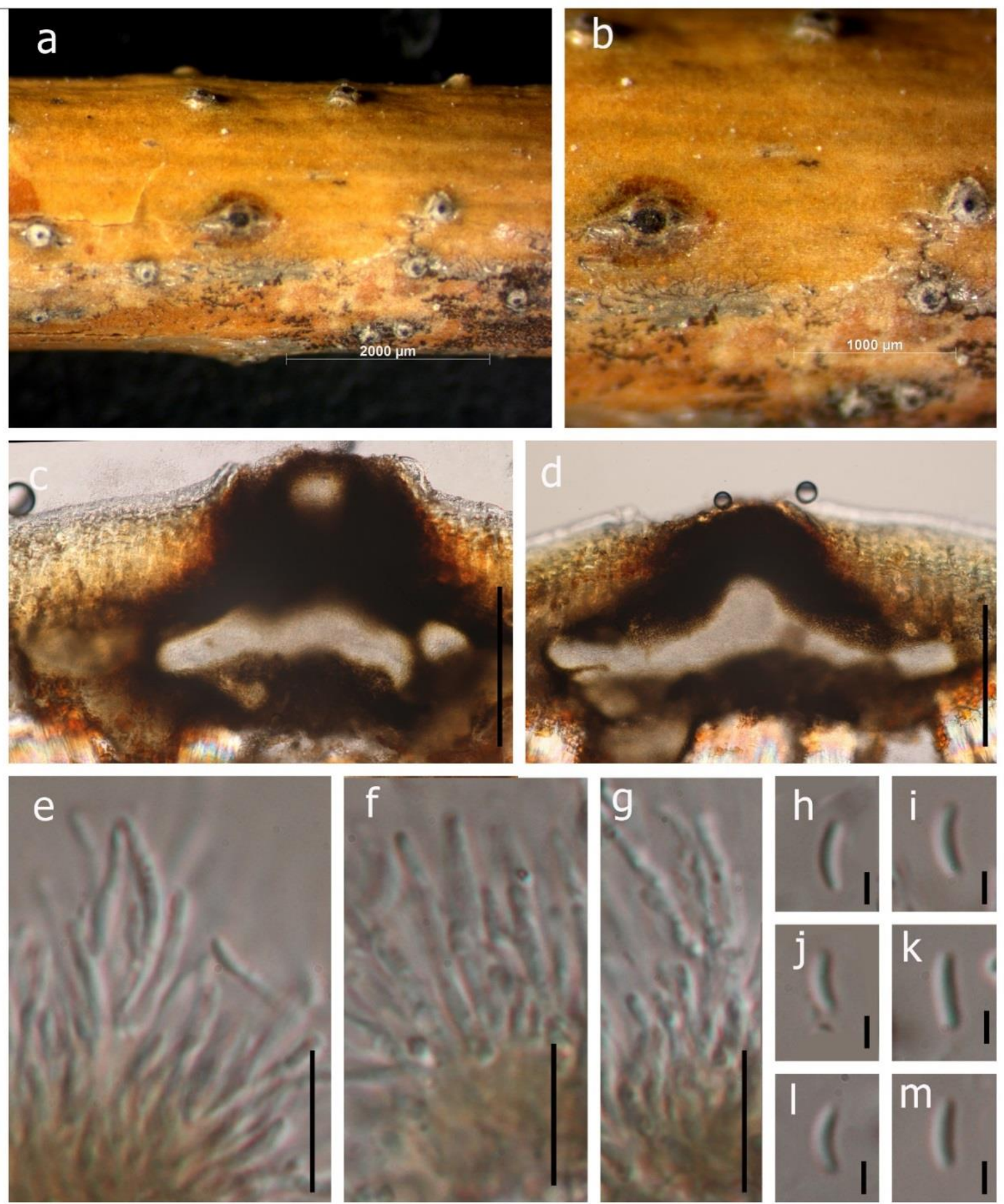

Fig. 132 - Cytospora melnikii (MFLU 17-2402, new host record). a, b Conidiomata on host substrate. c, d Vertical sections of conidiomata. e-g Conidiogenous cell. $\mathrm{h}-\mathrm{m}$ Conidia. Scale bars: $\mathrm{a}=2000 \mu \mathrm{m}, \mathrm{b}=1000 \mu \mathrm{m}, \mathrm{c}-\mathrm{g}=100 \mu \mathrm{m}, \mathrm{h}-\mathrm{m}=10 \mu \mathrm{m}$. 


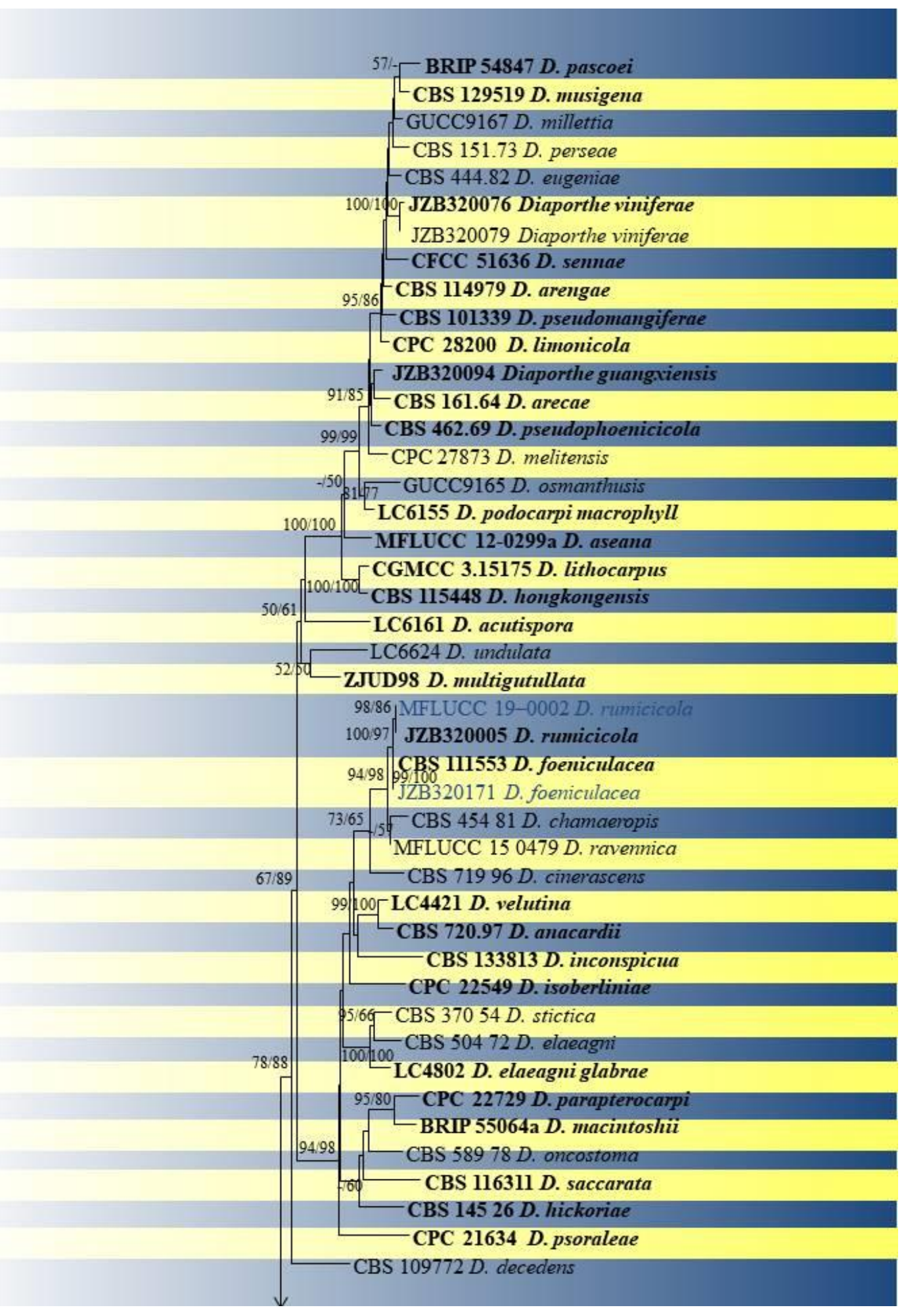

Fig. 133 - Phylogram generated from maximum likelihood analysis based on combined ITS, TUB, tefl and CAL sequence data representing the species of Diaporthe. Related sequences are obtained from Manawasinghe et al. (2019). In the combined analyses, 118 strains are included and they comprise 899 characters (558 characters for ITS, 399 characters for TUB, 353 characters for tefl, 370 characters for and CAL). The tree is rooted by Diaporthella corylina (CBS 121124) The tree topology derived from the Bayesian analysis was similar to that derived from the maximum likelihood analysis. The best RaxML tree with final likelihood values of -30526.060484 is presented. The matrix had 1055 distinct alignment patterns, with $15.59 \%$ undetermined characters or gaps. Estimated base frequencies were as follows: $\mathrm{A}=0.221027, \mathrm{C}=0.312667, \mathrm{G}=0.239910, \mathrm{~T}$ $=0.226397$; substitution rates $\mathrm{AC}=1.384376, \mathrm{AG}=3.984787, \mathrm{AT}=1.276868, \mathrm{CG}=0.999850$, 
$\mathrm{CT}=5.491949, \mathrm{GT}=1.000000 ;$ gamma distribution shape parameter $\alpha=0.956001$. The dataset consisted of 1694 characters with 713 constant characters and 758 parsimony-informative and 223 parsimony-uninformative characters. The maximum number of trees generated was 1000 , and the most parsimonious trees had a tree length of $5911(\mathrm{CI}=0.315, \mathrm{RI}=0.710, \mathrm{RC}=0.224, \mathrm{HI}=$ 0.685). Maximum likelihood (ML) and maximum parsimony (MP) equal to or greater than $50 \%$ are given as MP/ML on the respective node. Ex-type strains are in bold and black, the new host records are indicated in blue.

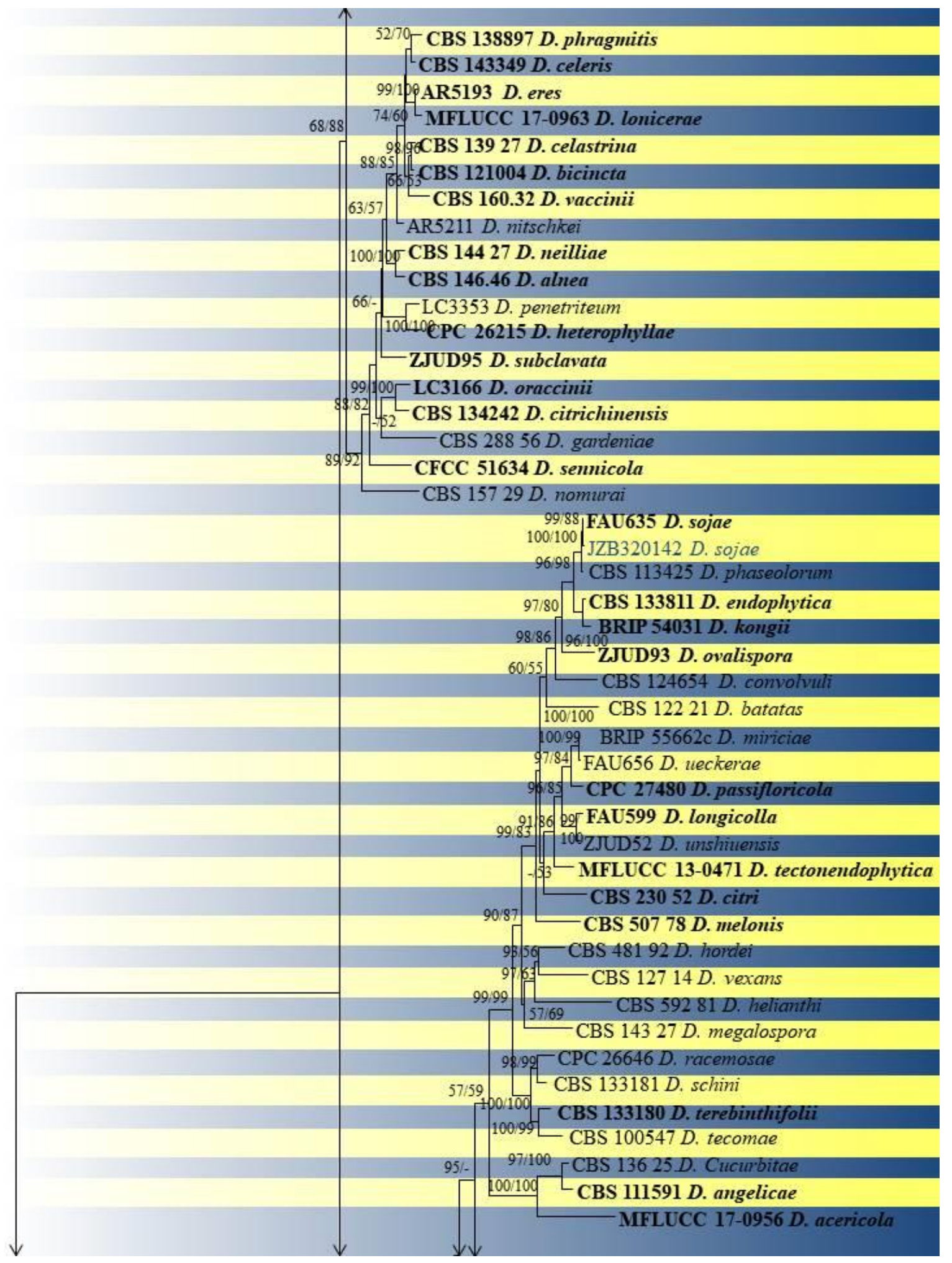

Fig. 133 - Continued. 


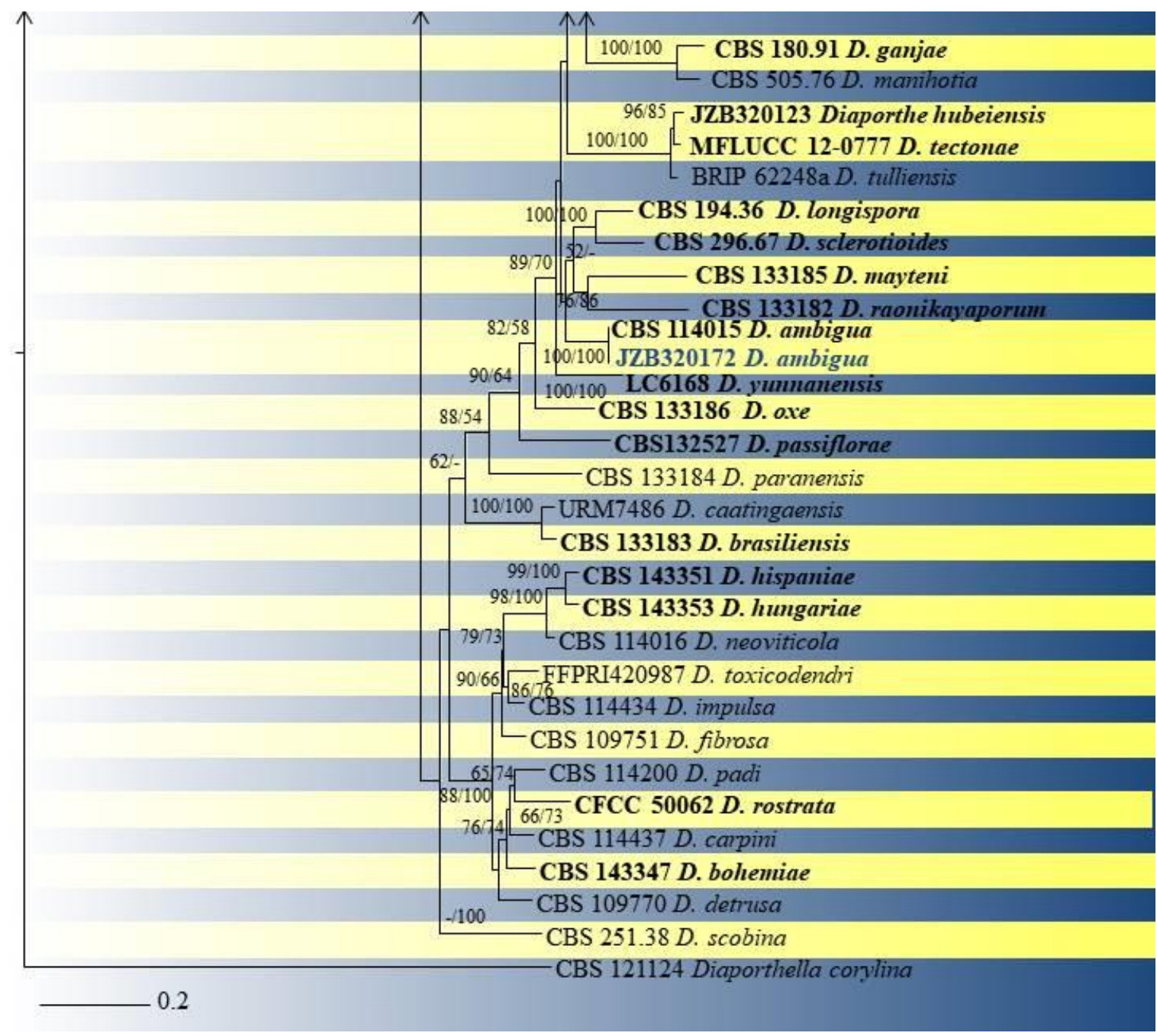

Fig. 133 - Continued.

Known hosts (based on molecular data) - Actinidia deliciosa, Aspalathus linearis, Diospyros kaki, Foeniculum vulgare, Helianthus annuus, Malus spp., Pistacia vera, Prunus spp., Pyrus spp., Vitis vinifera (Farr \& Rossman 2020), Iris sp. (this study)

Material examined - Italy, Province of Forlì-Cesena [FC], Pievequinta, on dead aerial stem of Iris sp. (Iridaceae), 5 February 2018, E. Camporesii, IT 3695, living culture, JZB320172

GenBank number - ITS: MN989426.

Notes - The collection obtained from the dead aerial stem of Iris sp. was identified as Diaporthe ambigua with support from morphology and phylogeny. Our isolate clustered with the reference strain of the Diaporthe ambigua (JZB320172) in the combined ITS, tef1, CAL, HIS and TUB sequence phylogeny with 100\% statistical support (Fig. 133). This is the first record of Diaporthe ambigua species reported from Iris sp. from Italy.

Diaporthe foeniculina Udayanga \& Castl. Persoonia 32: 95 (2014)

Fig. 135

Faces of fungi number: FoF02183

Saprobic on dead aerial stem of Artemisia vulgaris. Sexual morph Undetermined. Asexual morph: Conidiomata visible as small round to oval dark brown to black dots on the host surface. Sexual morph: not observed. Asexual morph: Coelomycetous. Conidiomata 100-200 $\mu \mathrm{m}$ high, 80$200 \mu \mathrm{m}$ diameter, pycnidial, solitary, scattered or gregarious, globose to subglobose, semiimmersed to immersed, sometimes erumpent, unilocular, thin walled. Conidioma wall composed of 3-4-layers, similarly dense at the apex and base, outer 1-2 layers dark brown to black, inner 1-2 layers hyaline, with thin walled cells of textura angularis. Conidiogenous cells $4-7 \mu \mathrm{m}$ long $\times 2-4$ 
$\mu \mathrm{m}$ wide, hyaline, phialidic, globose. Conidia 5-10 $\times 1-4 \mu \mathrm{m}$ wide $(\bar{x}=8 \times 3 \mu \mathrm{m}, \mathrm{n}=20)$, ellipsoidal to cylindrical, rounded at both ends, hyaline, straight, one septate to aseptate, thin smooth-walled, guttulate.
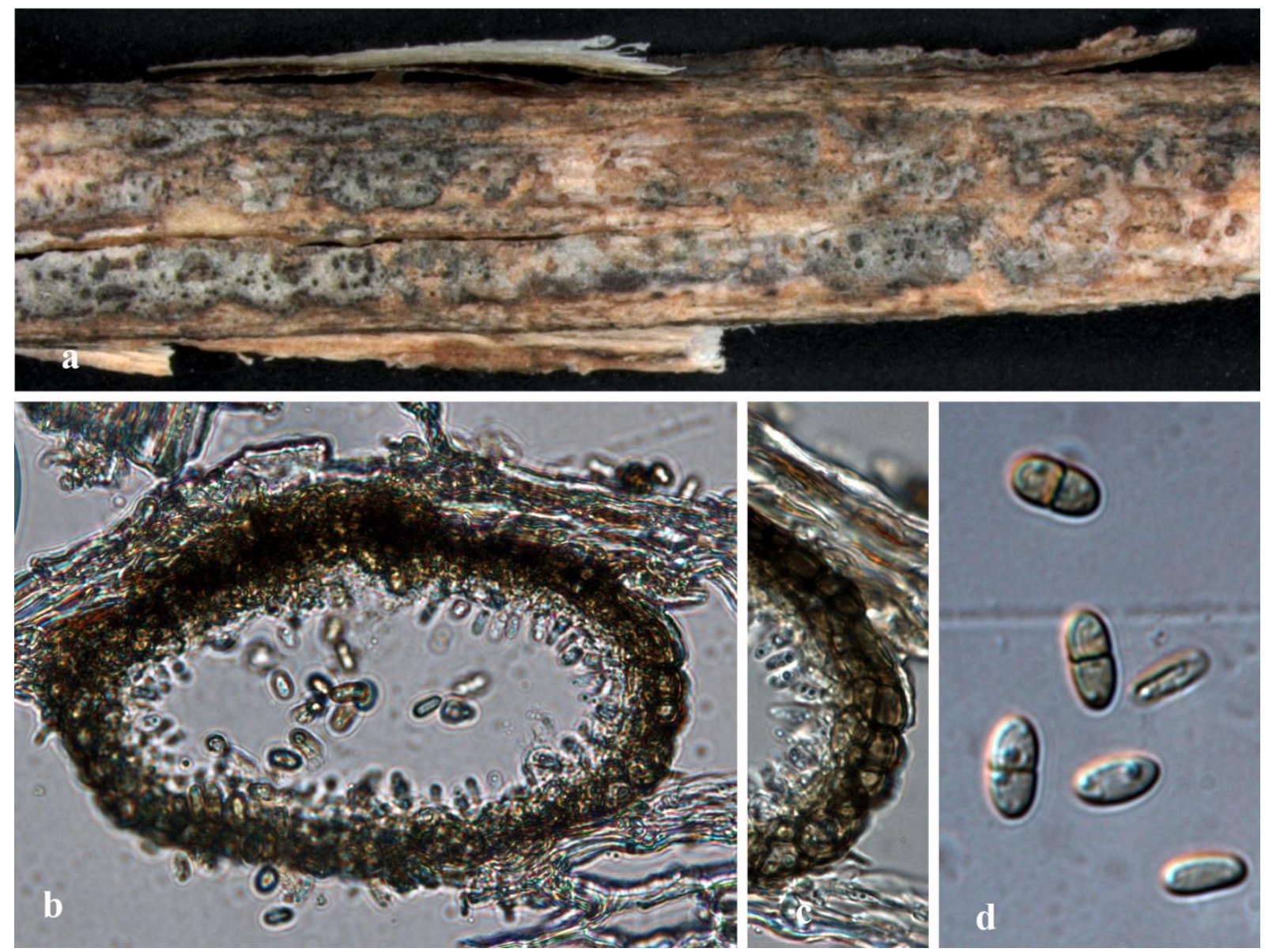

Fig. 134 - Diaporthe ambigua (JZB320172) a Appearance of conidiomata on the host. b Section through the conidioma. c Conidioma cell wall d Mature conidia.

Culture characters - Colonies on PDA reaching $80 \mathrm{~mm}$ diameter, after 14 days at $25^{\circ} \mathrm{C}$, circular colony, white, without any diffusible pigments. Pycnidia globose to subglobose. Conidia sub cylindrical, aseptate.

Known distribution (based on molecular data) - New Zealand, Greece, Malta, California, Portugal, Spain, United States, South Africa, Serbia, Uruguay (Farr \& Rossman 2020), Italy (this study).

Known hosts (based on molecular data) - Many economically important crops such as Citrus spp., Foeniculum vulgare, Glycine max, Malus domestica, Prunus, Vitis (Farr \& Rossman 2020), Artemisia vulgaris (this study).

Material examined - Italy, Province of Forlì-Cesena [FC], Marsignano - Predappio - Forlì Via Pietro Nenni, on dead aerial stem of Artemisia vulgaris (Asteraceae), 21 November 2017, E. Camporesii, IT 3578 (JZBH 320171) living culture, JZB 320171.

GenBank number - ITS: MN989424.

Notes - The collection obtained from the dead aerial stem of Artemisia vulgaris was identified as Diaporthe foeniculina with support from morphology and phylogeny. Our isolate clustered with the reference strain of the Diaporthe foeniculina (CBS 111553) in the combined ITS, tefl, TUB and CAL sequence phylogeny with 99\% (ML)/100\% (MP) statistical support. Furthermore, our isolate showed $99.45 \%$ base pair similarity with Diaporthe foeniculina in the ITS gene region. Diaporthe foeniculina has been reported from many economically important host 
plants like Citrus, Foeniculum vulgare, Glycine max, Malus domestica, Prunus and Vitis worldwide (Farr \& Rossman 2020). This is the first record of Diaporthe foeniculina on Artemisia vulgaris from Italy.
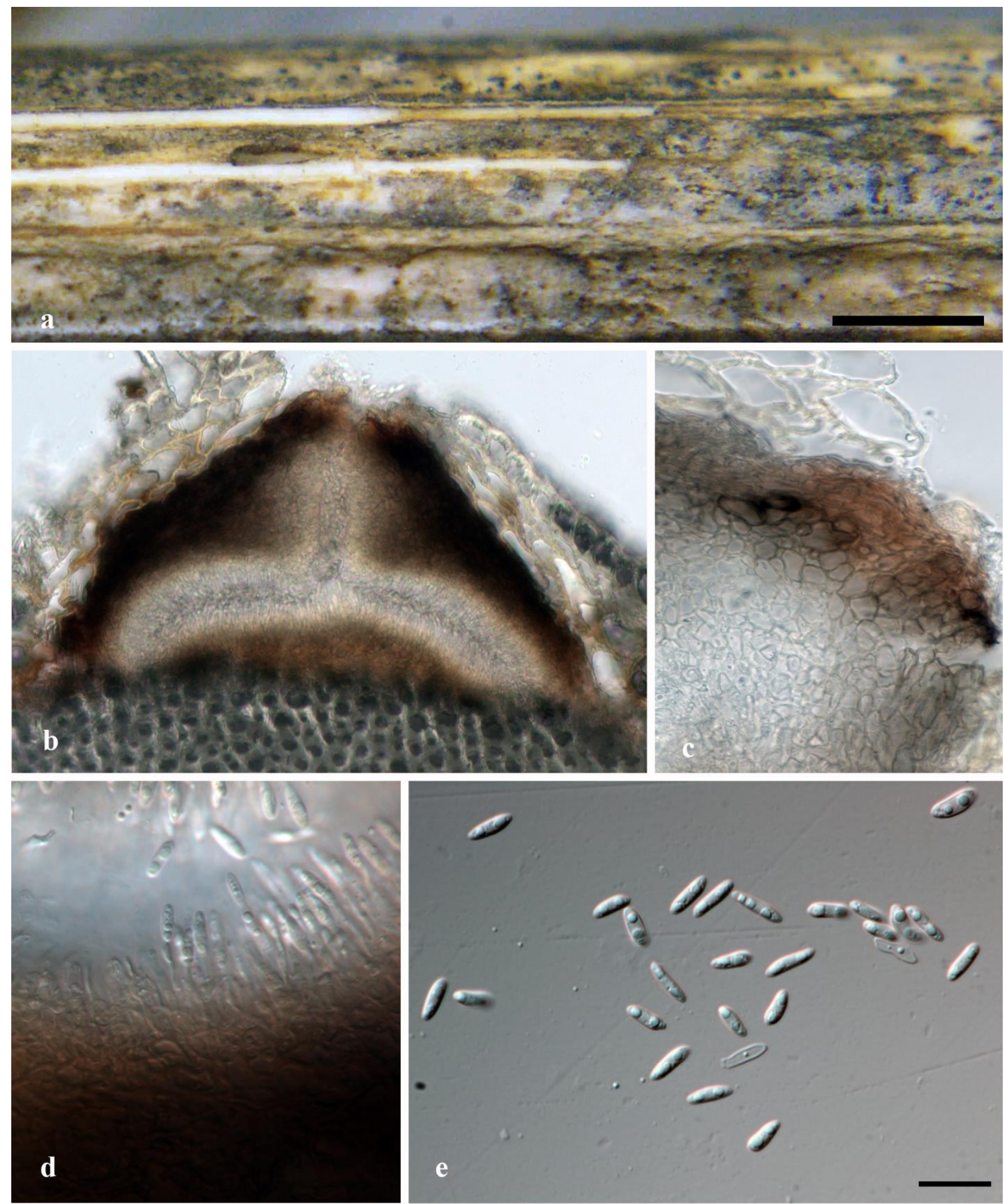

Fig. 135 - Diaporthe foeniculina (JZB 320171, new host record). a Appearance of conidiomata on the host. b Section through the conidioma. c Conidioma wall $\mathrm{d}$ Immature conidia attached to conidiogenous cell. e Mature conidia. Scale bars: $\mathrm{a}=200 \mu \mathrm{m}$, e $=10 \mu \mathrm{m}$.

Diaporthe rumicicola Manawasinghe, Camporesi \& K.D. Hyde, Fungal Diversity [138] (2019)

Facesoffungi number: FoF 04940 
Saprobic on dead aerial stems of Scrophularia canina. Sexual morph: Undetermined. Asexual morph: Conidiomata 181-202 $\mu \mathrm{m}$ high $\times 203-215 \mu \mathrm{m}$ diameter $(\bar{x}=189.2 \times 207.6 \mu \mathrm{m}, \mathrm{n}$ = 10), eustromatic, convoluted, mostly solitary, semi-immersed to immersed in the host, black, ostiolate. Ostiole 25-33 $\mu \mathrm{m}$ diameter, single, central, with a well-developed neck, thick-walled. Peridium multi-layered 15-20 $\mu \mathrm{m}$ wide at the base, $13-21 \mu \mathrm{m}$ wide in sides, comprising 6-8 layers, heavily pigmented, thick-walled, textura angularis cells. Conidiogenous cells $8.5-11.7 \mu \mathrm{m}$ high $\times$ 1.3-2.1 $\mu \mathrm{m}$ diameter $(\bar{x}=9.6 \times 1.7 \mu \mathrm{m}, \mathrm{n}=10)$, phialidic, cylindrical, terminal, with slight taper towards apex. Alpha conidia 7.5-10.7 $\times 2.4-3.5 \mu \mathrm{m}(\bar{x}=9.2 \times 2.7 \mu \mathrm{m}, \mathrm{n}=50)$, aseptate, hyaline, smooth, ellipsoidal or fusiform, with one or two guttules, rarely with subtruncate base. Beta conidia not observed.

Culture characteristics - Colonies on PDA, circular, flattened, fimbriate, crenate edged, both surfaces white, slow growing, and reach $90 \mathrm{~mm}$ diameter in 9 days at $28^{\circ} \mathrm{C}$.

Known distribution (based on molecular data) - Italy (Hyde et al. 2019, this study)

Known hosts (based on molecular data) - Rumex sp. (Polygonaceae) (Hyde et al. 2019), Scrophularia canina (this study).

Material examined - Italy, Province of Forlì-Cesena [FC], Cusercoli - Civitella di Romagna, on dead aerial stem of Scrophularia canina (Scrophulariaceae), 7 February 2018, E. Camporesi (MFLU 18-0122, new host record), living culture (MFLUCC 19-0002).

GenBank numbers - ITS: MK066126; tef1: MK078545; TUB: MK078546.

Notes -Based on multi-gene phylogenetic analysis of combined ITS, tefl, $\beta$-tubulin (TUB) and calmodulin (CAL) sequence data of Diaporthe species (Fig. 133), our strain (MFLUCC 190002) clusters with the ex-type strain of Diaporthe rumicicola (MFLUCC 18-0739), but differs in alpha conidial length $(\overline{\mathrm{x}}=9.2 \mu \mathrm{m})$ as compared to the ex-type strain $(\overline{\mathrm{x}}=3.5 \mu \mathrm{m})$, Diaporthe rumicicola (MFLUCC 18-0739).

Diaporthe sojae Lehman, Ann. Mo. bot. Gdn 10: 128 (1923)

Fig. 137

Facesoffungi number: FoF06582

Saprobic or pathogenic on diseased leaves of Rosa multiflora. Sexual morph: Undetermined. Asexual morph: Pycnidia on PDA, superficial, scattered, dark brown to black, globose, solitary in most. Conidiophores were not observed. Conidiogenous cells were not observed. Alpha conidia biguttulate, hyaline, fusiform or oval, both ends obtuse $5-7 \times 2-4(\mathrm{n}=40) \mu \mathrm{m}(\bar{x}=5.5 \times 3 \mu \mathrm{m})$. Beta conidia not observed.

Culture characteristics - Colonies on PDA reach $70 \mathrm{~mm}$ diameter after 7 days at $25^{\circ} \mathrm{C}$, producing abundant white aerial mycelia and reverse fuscous black.

Known distribution (based on molecular data) - China, Italy, Japan, Mexico, Thailand, and United States (Farr \& Rossman 2020), Italy (this study).

Known hosts (based on molecular data - Arachis hypogaea, Arctium lappa, Asparagus officinalis, Aster exilis, Caperonia palustris, Citrus limon, Citrus reticulata, Citrus unshiu, Cucumis melo, Euphorbia nutans, Glycine max, Glycine soja, Phaseolus limensi, Phaseolus vulgaris, Vitis vinifera, Stokesia laevi (Farr \& Rossman 2020), Ligustrum quihoui (this study).

Material examined - China, Shandong, Yellow River Park, on leaf spots of Ligustrum quihoui (Oleaceae), 7 October 2017, Y.Y. Hao (living culture JZB320142, new host record).

GenBank numbers - ITS: MN535308, TUB: MN561315.

Notes - In the phylogenetic analysis of combined ITS, TUB, tefl and CAL gene regions, the taxon obtained in the present study clustered together with the type species, Diaporthe sojae (FAU 635 ) with $99 \%$ ML and $88 \%$ MP bootstrap support. The taxon is also identical to the type specimen (Udayanga et al. 2015). Diaporthe sojae has a wide range of hosts. This species is a well-known pathogen associated with Glycine max in many countries including China. Previously this species was reported from Vitis and Citrus Spp. in China (Huang et al. 2015, Udayanga et al. 2015). The Ligustrum quihoui is a native plant to Korea and China and grown mainly as an ornamental shrub. This is the first report of Diaporthe sojae on L. quihoui (Farr \& Rossman 2020). 

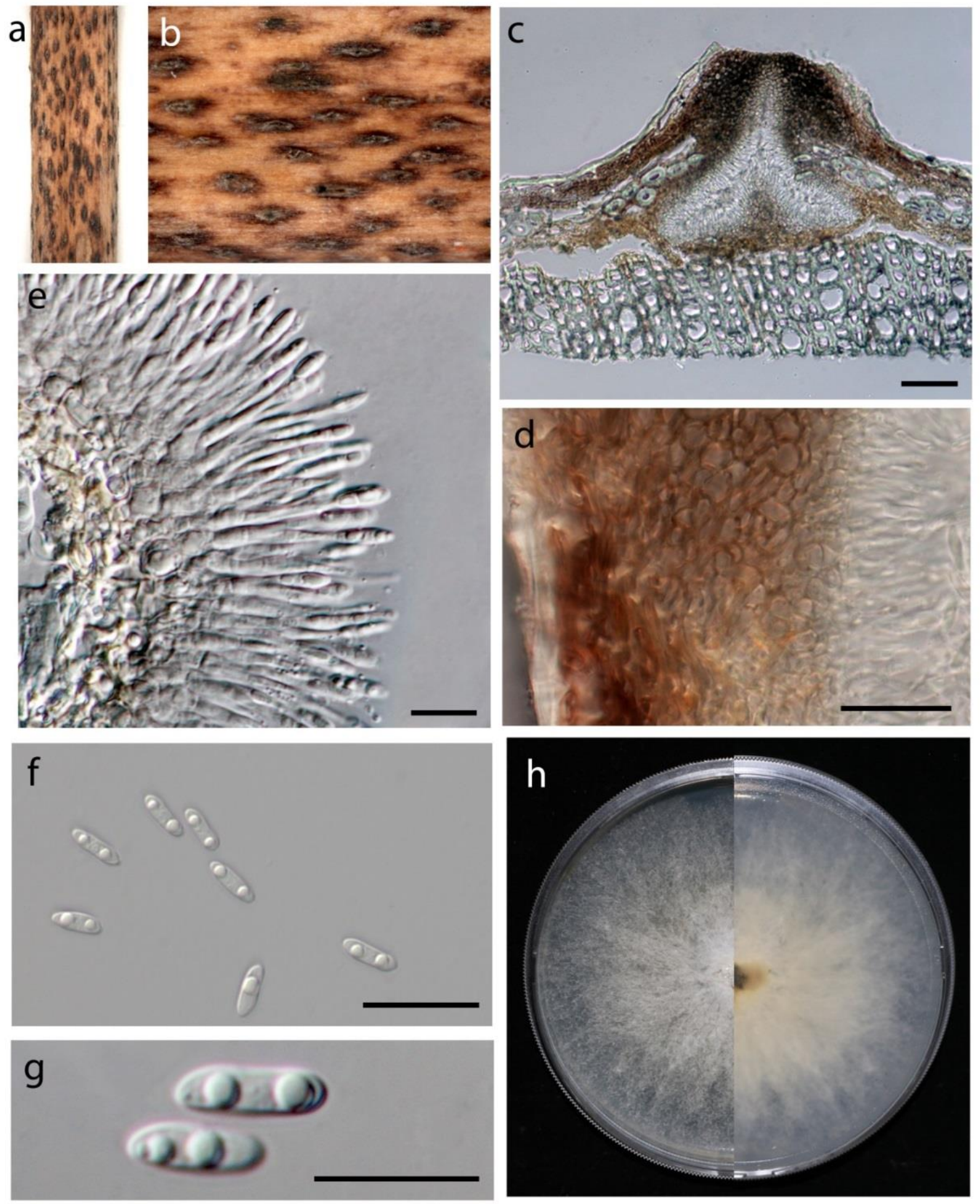

Fig. 136 - Diaporthe rumicicola (MFLU 18-0122, new host record). a, b Appearance of conidiomata on host substrate. c Vertical section of a conidioma. d Peridium of conidioma. e Immature and mature conidia attached to conidiogenous cells. f, g Mature conidia. h Culture characters on PDA. Scale bars: $\mathrm{c}=50 \mu \mathrm{m}, \mathrm{d}=20 \mu \mathrm{m}, \mathrm{e}=10 \mu \mathrm{m}, \mathrm{f}=20 \mu \mathrm{m}, \mathrm{g}=10 \mu \mathrm{m}$.

\section{Melanconidaceae G. Winter}

Melanconidaceae was introduced by Winter (1886) and is typified by Melanconis. The family is characterized by circularly arranged perithecia immersed in stromata with a central column and ostioles erumpent through an ectostromatic disc with hyaline, 1-septate ascospores; pycnidia developing before the formation of the ascomata and produce 1-celled, dark-brown conidia (Barr 
1978, Castlebury et al. 2002). Senanayake et al. (2017) restricted Melanconidaceae for Melanconis. Only the type genus, Melanconis is accommodated in the family. Species of Melanconidaceae are usually pathogens or endophytes on hardwood trees in Betulaceae. They are distributed worldwide especially in China.
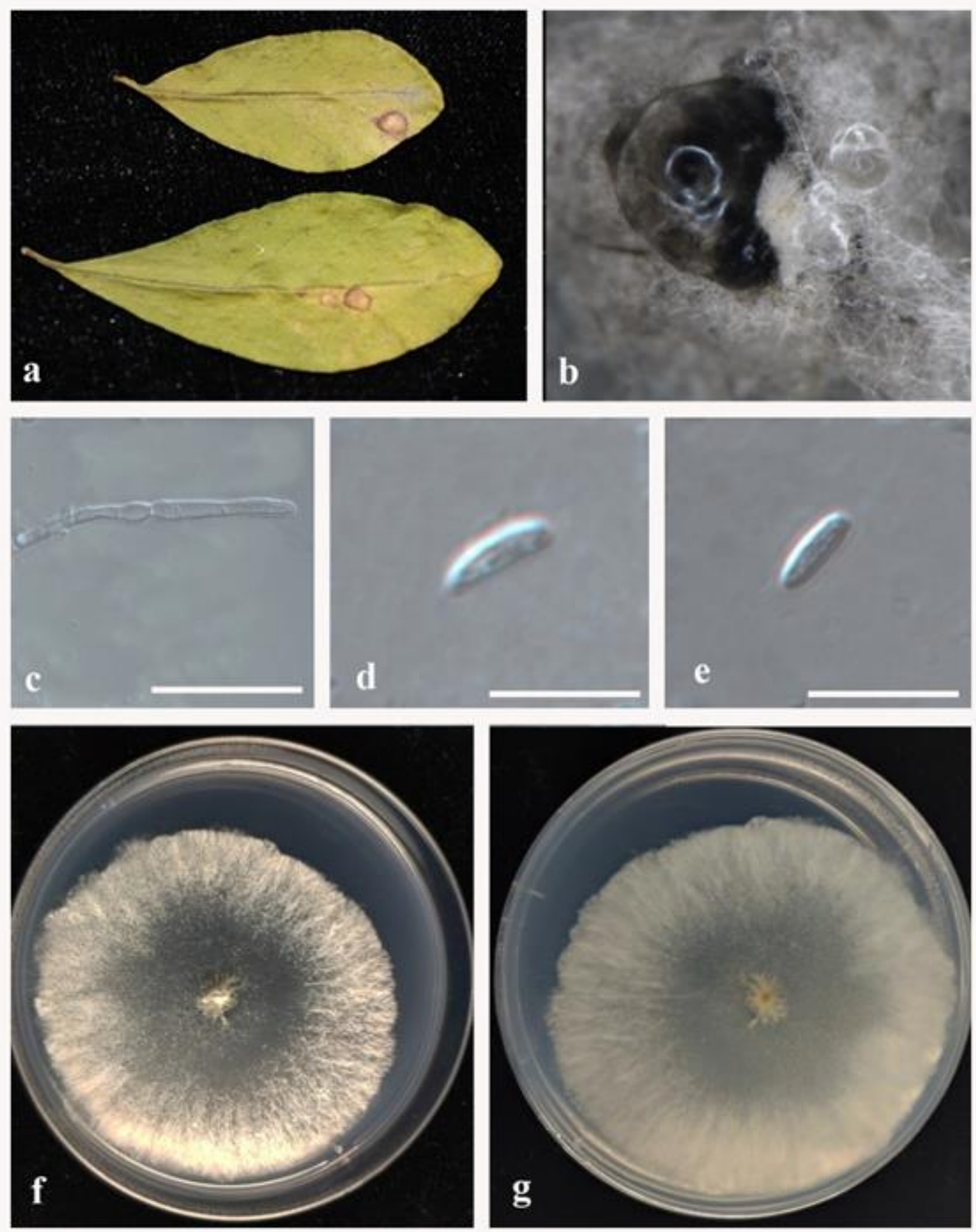

Fig. 137 - Diaporthe sojae (JZBH320142, new host record). a Material examined. b Appearance of conidia on PDA. c conidiogenus cell. d-e Conidia f Upper view of colony on PDA. g reverse view of colony on PDA. Scale bars: $\mathrm{c}-\mathrm{e}=20 \mu \mathrm{m}$. 


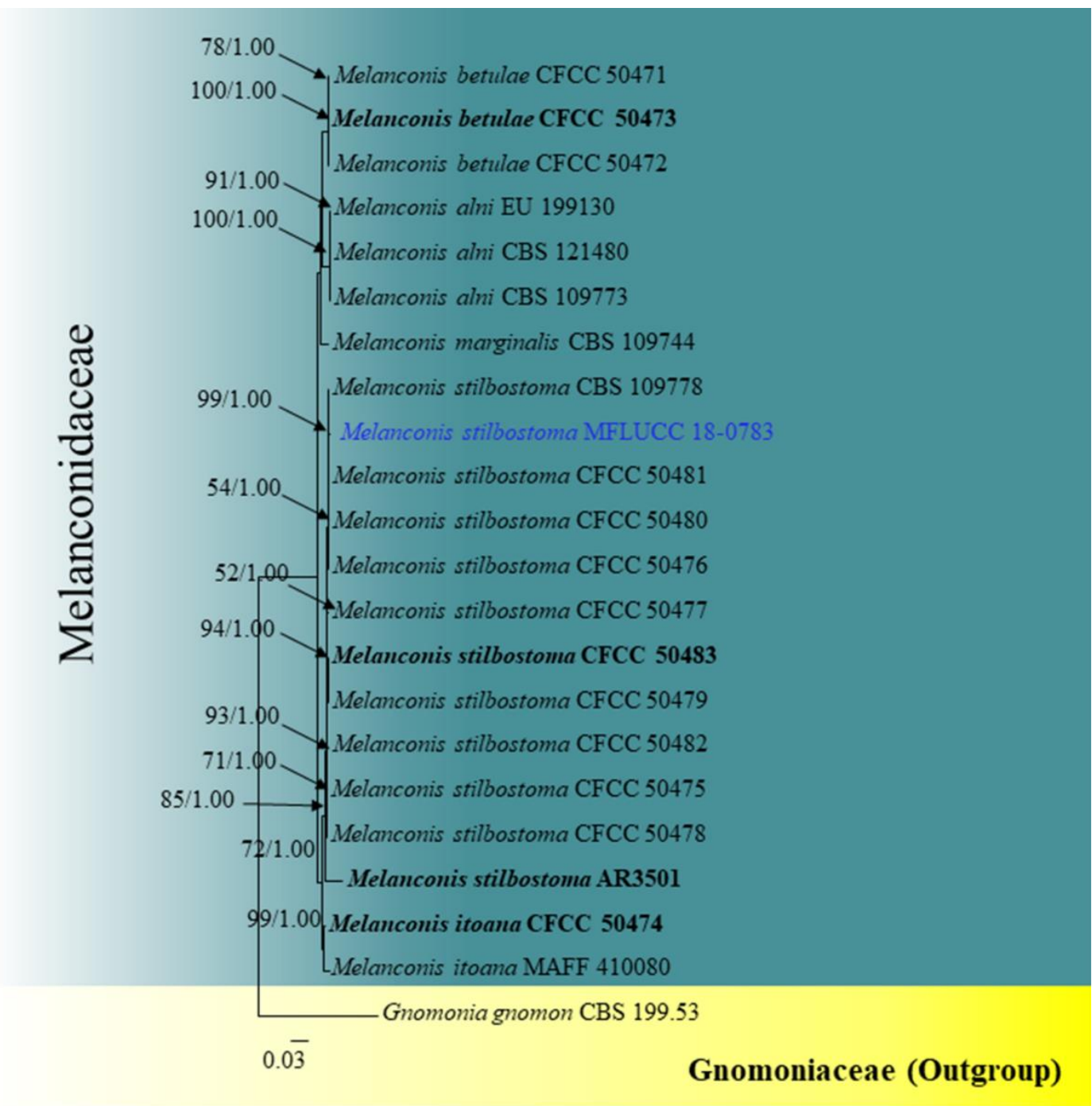

Fig. 138 - Phylogram generated from maximum likelihood analysis based on combined LSU, ITS, RPB2 and TUB sequence data. Twenty-two strains are included in the combined gene analyses comprising 2747 characters after alignment (891 characters for LSU, 572 characters for ITS, 1126 characters for RPB2 and 425 characters for TUB). Gnomonia gnomon (CBS 199.53) is used as the outgroup taxon. The tree topology derived from the Bayesian analysis was similar to that derived from the maximum likelihood analysis. The best RaxML tree with a final likelihood value of 6277.206694 is presented. The matrix had 263 distinct alignment patterns, with $17.80 \%$ undetermined characters or gaps. Estimated base frequencies were as follows: $\mathrm{A}=0.244490, \mathrm{C}=$ $0.261036, \mathrm{G}=0.265173, \mathrm{~T}=0.229301$; substitution rates $\mathrm{AC}=1.367272, \mathrm{AG}=2.659677, \mathrm{AT}=$ $1.874405, \mathrm{CG}=1.219603, \mathrm{CT}=7.861355, \mathrm{GT}=1.000000$; gamma distribution shape parameter $\alpha$ $=0.738642$. Bootstrap values for maximum likelihood equal to or greater than 50 and Bayesian posterior probabilities equal or greater than 0.95 are placed above or below the branches. Ex-type strains are in bold and black. The newly generated sequence is indicated in blue.

Melanconis Tul. \& C. Tul.

Melanconis was introduced by Tulasne \& Tulasne (1863) with Sphaeria stilbostoma Fr. as the type species and an asexual state placed in Melanconium Link. Melanconis is characterized by circularly arranged perithecia, immersed in stromata with a central column and ostioles which arise through an ectostromatic disc with hyaline, 1-septate ascospores. The asexual morph is coelomycetous often with brown aseptate conidia. There are around 100 species epithets in Melanconis. Some of them been transferred to other families such as Amphisphaeriaceae, Cucurbitariaceae, Gnomoniaceae, Pseudovalsaceae and Sydowiellaceae (Index Fungorum 2020). 
Facesoffungi number: FoF06770

Pathogenic on stems of Betula pendula Roth. Sexual morph: Undetermined. Asexual morph: Conidiomatal stromata $134-172 \times 100-159 \mu \mathrm{m}(\bar{x}=148.2 \times 128.5 \mu \mathrm{m}, \mathrm{n}=10)$ immersed or superficial in host bark, conical, black, circular to ellipsoid with an ectostromatic disc white to pale yellow, surrounded by bark. Central column beneath the disc more or less conical. Conidiophores 13-17 $\times 1.2-1.9 \mu \mathrm{m}(\bar{x}=17.2 \times 1.6 \mu \mathrm{m}, \mathrm{n}=20)$ branched at the base, elongate, hyaline to subhyaline, smooth. Conidiogenous cells monophialidic, integrated. Conidia 10-12 $\times 6-7 \mu \mathrm{m}(\bar{x}=$ $11.1 \times 7.2 \mu \mathrm{m}, \mathrm{n}=20$ ), ovoid, acute at one end and obtuse at the other, brown to olive brown, aseptate.

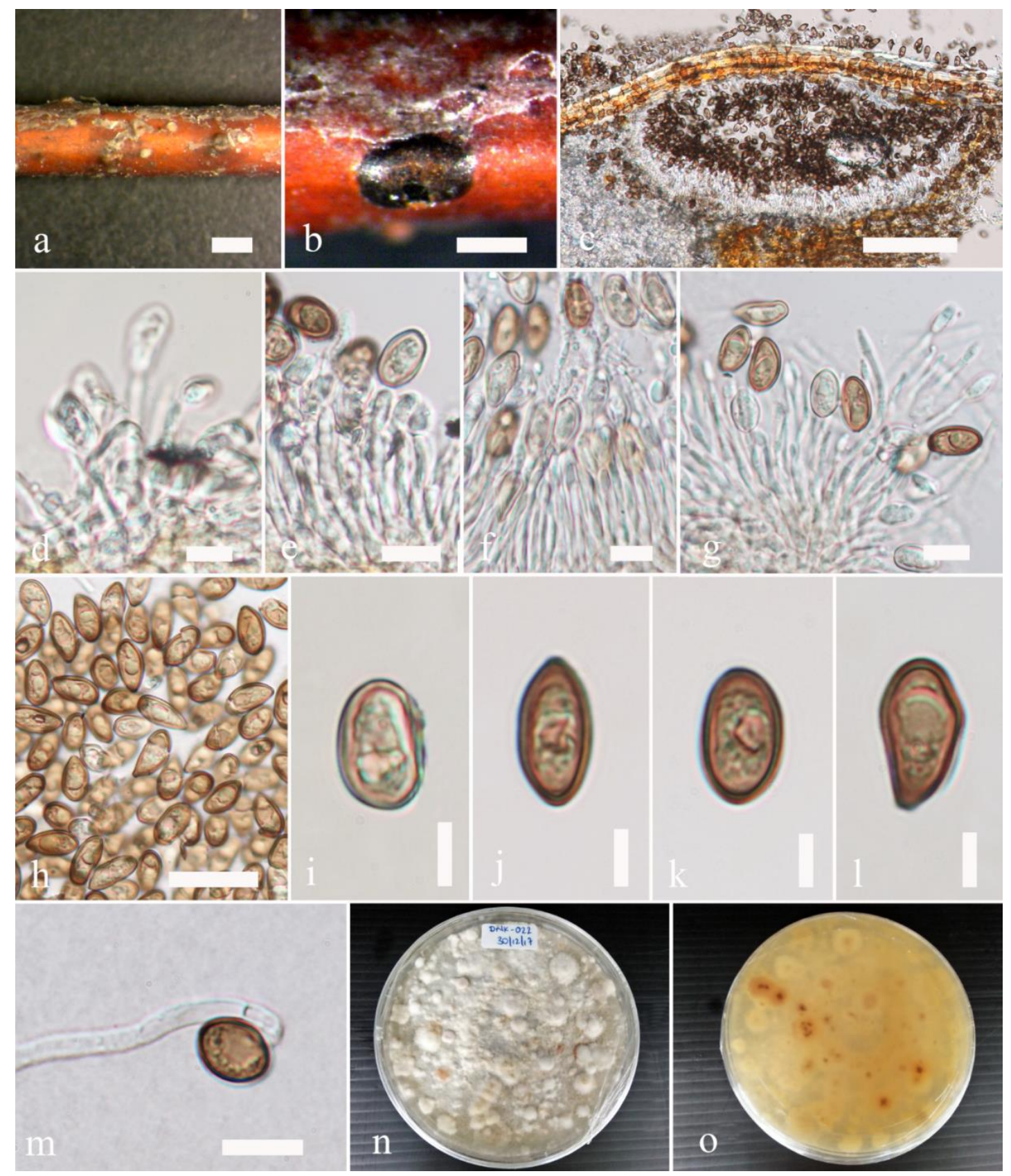

Fig 139 - Melanconis stilbostoma (MFLU 17-2444, new geographical record). a, b Appearance of conidiomata on host surface. c Vertical section through conidioma. d-g Conidiogenesis. h-1 Conidia m Germinating conidium. $\mathrm{n}$, o Culture characteristics on MEA (n: above view; o: reverse view). Scale bars: $\mathrm{a}=200 \mu \mathrm{m}, \mathrm{b}, \mathrm{c}=100 \mu \mathrm{m}, \mathrm{d}=5 \mu \mathrm{m}, \mathrm{e}-\mathrm{g}, \mathrm{i}-\mathrm{m}=10 \mu \mathrm{m}, \mathrm{h}=20 \mu \mathrm{m}$. 
Culture characteristics - Colony on MEA white at first, producing creamy white to pale yellowish brown pigment after 15 days, felty, with an irregular edge; conidiomata sparse.

Material examined - Ukraine, Donetsk region, Donetsk City, Donetsk Botanical Garden, arboretum, on Betula pendula Roth (Betulaceae), 16 May 2017, Timur Bulgakov (MFLU 17-2444, new geographical record); living cultures; MFLUCC 18-0783, DSM 109785.

Known distribution (based on molecular data) - Sweden, China (Fan et al. 2016), Russia (this study).

Known hosts (based on molecular data) - Betula spp. (B. pendula, B. rotundifolia and B. tianschanica) (Fan et al. 2016), Betula pendula (this study).

GenBank Numbers - LSU: MN244205, SSU: MN244182, ITS: MN244222.

Notes - Melanconis stilbostoma is the type species of Melanconis and mainly distributed on Betula spp. Our isolate MFLUCC 18-0783 clustered with the strains of Melanconis stilbostoma (Fig. 138), which has been reported only in Sweden and China. We, therefore report our collection as a new geographical record from Russia based on morphology and phylogenetic analyses.

\section{Schizoparmaceae Rossman}

Schizoparmaceae was introduced by Rossman et al. (2007) to accommodate Schizoparme with its asexual state Pilidella and Coniella in Diaporthales. Members of this family are pathogens that cause diseases in grapes, strawberry and other plants (Rossman et al. 2007) and saprobes in plants and soil (Alvarez et al. 2016).

\section{Coniella Höhn.}

The asexual genus Coniella was established by Von Höhnel (1918) and is typified by $C$. pulchella $(=C$. fragariae; Crous et al. 2014a). Species of Coniella are saprobes, plant pathogens and endophytes (Alvarez et al. 2016). They have a wide host range i.e., Eucalyptus, Fragaria, Hibiscus, Psidium, Punica, Terminalia and Vitis occurring on leaf litter, rotting bark, and soil (Alvarez et al. 2016).

Coniella eucalyptorum (Crous \& M.J. Wingf.) L.V. Alvarez \& Crous in Alvarez, Groenewald \& Crous, Stud. Mycol. 85: 15 (2016)

Fig. 141

Facesoffungi number: FoF07370

Saprobic on dead leaves of undetermined host. Sexual morph: Undetermined. Asexual morph: Conidiomata $90-130 \mu \mathrm{m}$ high $\times 160-200 \mu \mathrm{m}$ in diameter $(\mathrm{n}=10)$ solitary, globose, brown and dark brown from the top. Conidioma wall consisting of 2-3 layers of hyaline textura prismatica and 4-5 layers of brown textura angularis cells. Conidiophores densely aggregated, branched. Conidiogenous cells 12-15 $\times 1-2 \mu \mathrm{m}$, annellidic, narrowing at the tip, smooth and hyaline. Conidia $10-12 \times 3-6 \mu \mathrm{m}(\bar{x}=11 \times 5 \mu \mathrm{m}, \mathrm{n}=30)$, hyaline to pale brown, becoming dark brown at maturity, smooth, broadly ellipsoidal, both sides gradually tapering, smooth-walled, and multi-guttulate with one or two prominent guttules.

Culture characteristics - Colony on PDA reaching $30 \mathrm{~mm}$ diameter after 7 days at $25^{\circ} \mathrm{C}$, colony circular, margin wavy, flat, velvety appearance, colony from above: light brown; reverse: dark brown.

Known distribution (based on molecular data) - Australia, Brazil, Chile, China, Indonesia, Malaysia, Mexico, Venezuela, Vietnam (Alvarez et al. 2016), Thailand (this study).

Known hosts (based on molecular data) - Eucalyptus sp., Corymbia nesophila, Corymbia torelliana (Alvarez et al. 2016), unidentified host (this study).

Material examined - Thailand, Chiang Mai Province, Mae Taeng, Ban Pa Deng, Mushroom Research Centre, on twigs of unidentified host, 27 May 2016, N.I de Silva, NI106 (MFLU 170675), living culture, MFLUCC 17-0870.

GenBank Numbers - ITS: MN836684, LSU: MN836664

Notes - Phylogenetic analysis of combined ITS, LSU, histone and tefl sequence data confirmed that our collection is Coniella eucalyptorum with high support (Fig. 140). Coniella 
eucalyptorum was previously recorded from Australia, Brazil, Chile, Indonesia, Malaysia, Mexico, Venezuela and Vietnam (Alvarez et al. 2016). Coniella eucalyptorum was commonly recorded from Eucalyptus sp. (Alvarez et al. 2016). This is the first record of C. eucalyptorum from twigs of unidentified host in Thailand.

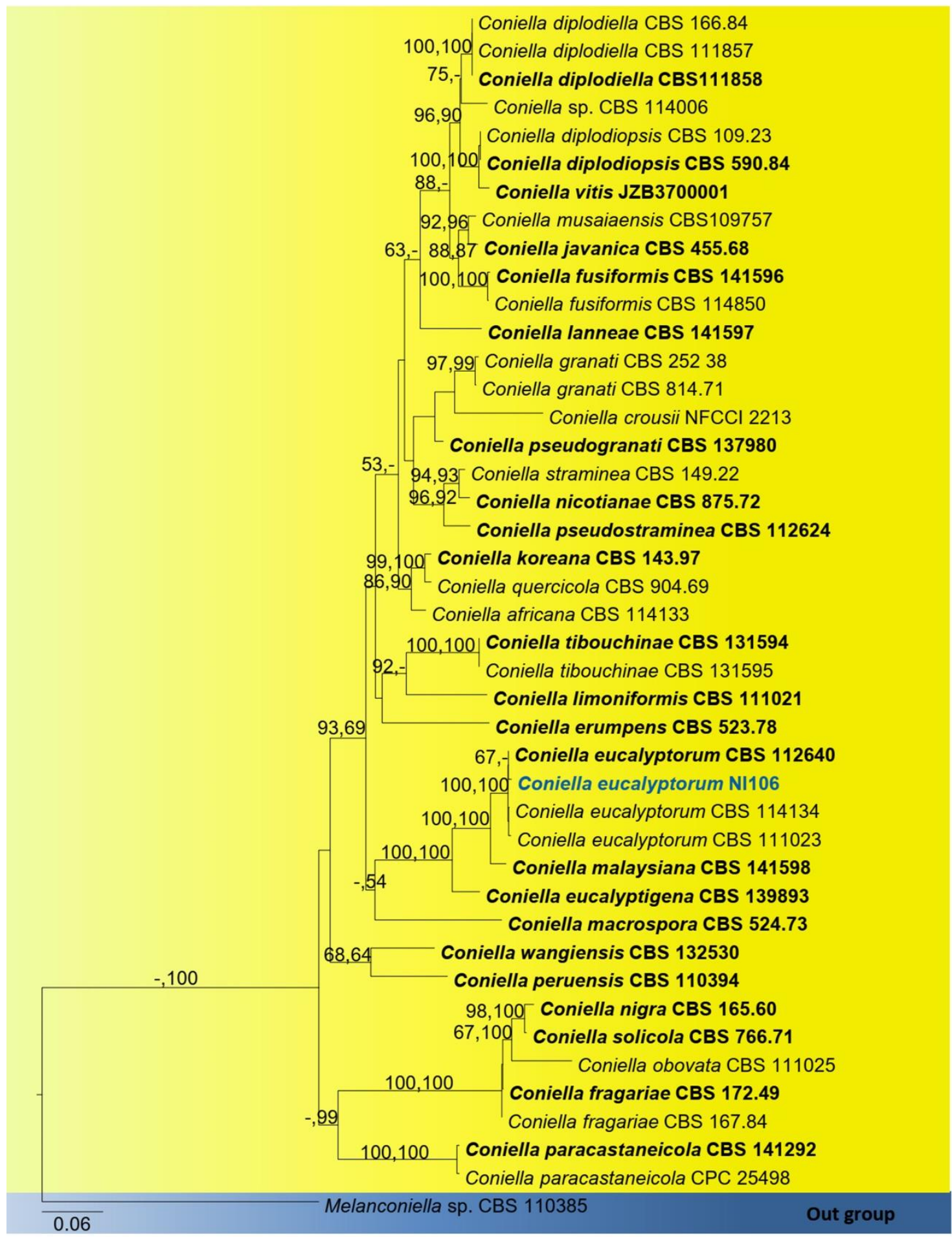

Fig. 140 - Phylogram generated from the maximum likelihood analysis based on combined ITS, LSU, histone and tefl sequence data representing genus Coniella. Related sequences are taken from Chethana et al. (2017). Forty three strains are included in the combined analyses which comprise 2866 characters (586 characters for ITS, 1180 characters for LSU, 485 characters for histone, 615 characters for tef1) after alignment. Melanconiella sp. (CBS110385) is used as the outgroup taxon. The best RaxML tree with a final likelihood values of -15179.467302 is presented. The matrix had 
796 distinct alignment patterns, with $23.91 \%$ undetermined characters or gaps. Estimated base frequencies were as follows: $\mathrm{A}=0.249525, \mathrm{C}=0.245214, \mathrm{G}=0.256307, \mathrm{~T}=0.248954$; substitution rates $\mathrm{AC}=1.240352, \mathrm{AG}=2.275249, \mathrm{AT}=1.362790, \mathrm{CG}=0.918763, \mathrm{CT}=$ 5.013442, GT $=1.000000$; gamma distribution shape parameter $\alpha=0.398515$. Bootstrap values for ML equal to or greater than 50\% (first set) and MP equal to or greater than 50\% (second set) are given above the nodes. Ex-type strains are in black bold and new strain is indicated in blue bold.
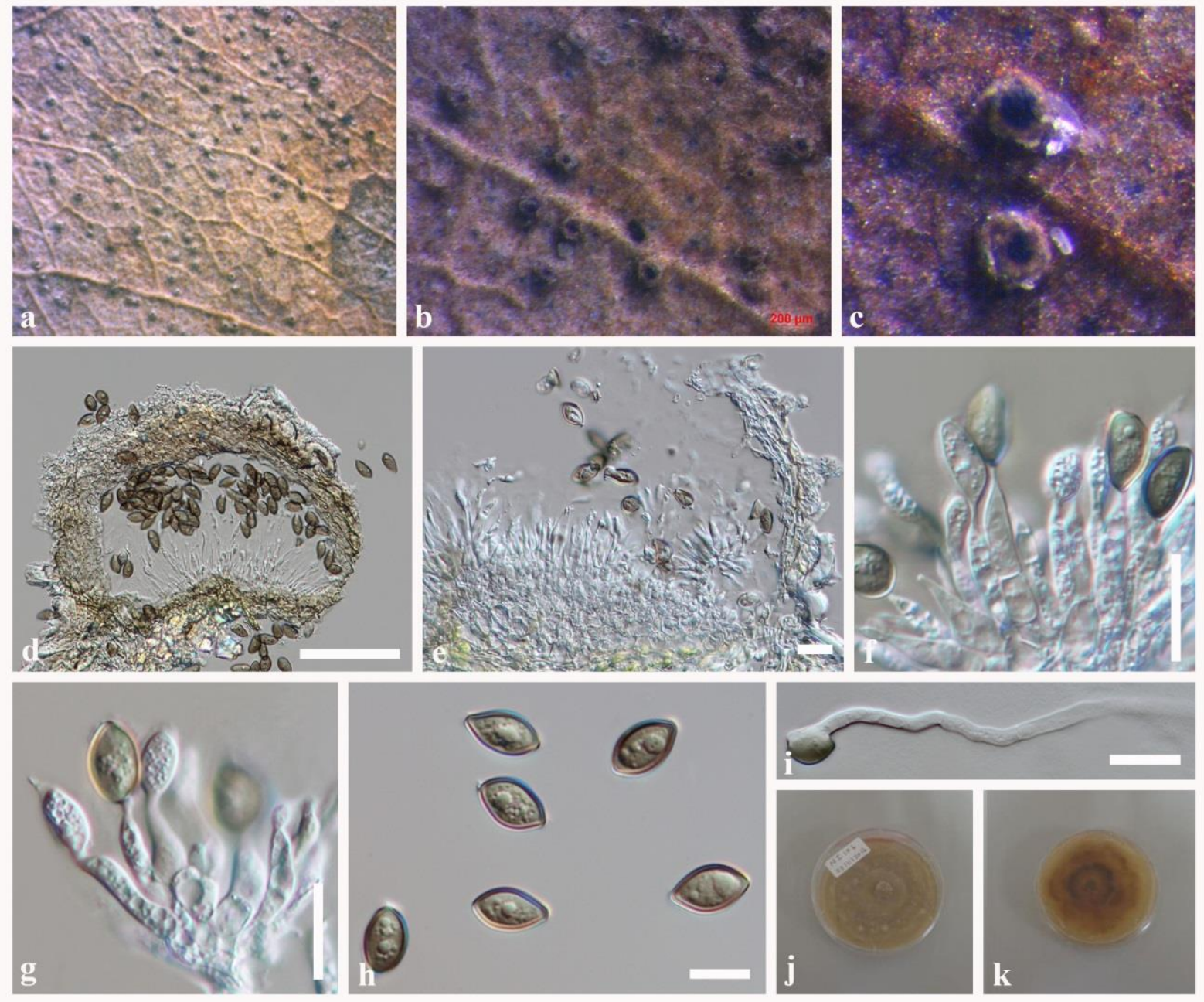

Fig. 141 - Coniella eucalyptorum (MFLU 17-0675, new geographical record). a-c Appearance of conidiomata on dead branch. d Vertical section of conidioma. e Peridium. $\mathrm{f}$, g Conidiophores. $\mathrm{h}$ Conidia. i Germinating conidia $\mathrm{j}$ Upper view of culture. $\mathrm{k}$ Lower view of culture. Scale bars: $\mathrm{d}=$ $50 \mu \mathrm{m}, \mathrm{e}-\mathrm{h}=10 \mu \mathrm{m}, \mathrm{i}=20 \mu \mathrm{m}$.

Subclass Hypocreomycetidae O.E. Erikss. \& Winka

Glomerellales Chadef.

Glomerellaceae Locq. ex Seifert \& W. Gams.

Glomerellaceae is a monotypic family mainly comprised of pathogens. This family is characterized by Colletotrichum (asexual morph) and Glomerella (sexual morph), which was synonymized under Colletotrichum (Hyde et al. 2014, Maharachchikumbura et al. 2016a).

\section{Colletotrichum Corda}

This genus was introduced by Corda (1831), for C. lineola Corda (Hyde et al. 2009). Colletotrichum comprises mainly pathogens, as well as endophytes and saprotrophs (Hyde et al. 2014, Jayawardena et al. 2016, Samarakoon et al. 2018). Kirk et al. (2001, 2008b) and Réblová et 
al. (2011) placed Colletotrichum in Glomerellaceae and the study of Maharachchikumbura et al. (2016a) further confirmed the placement of this genus.

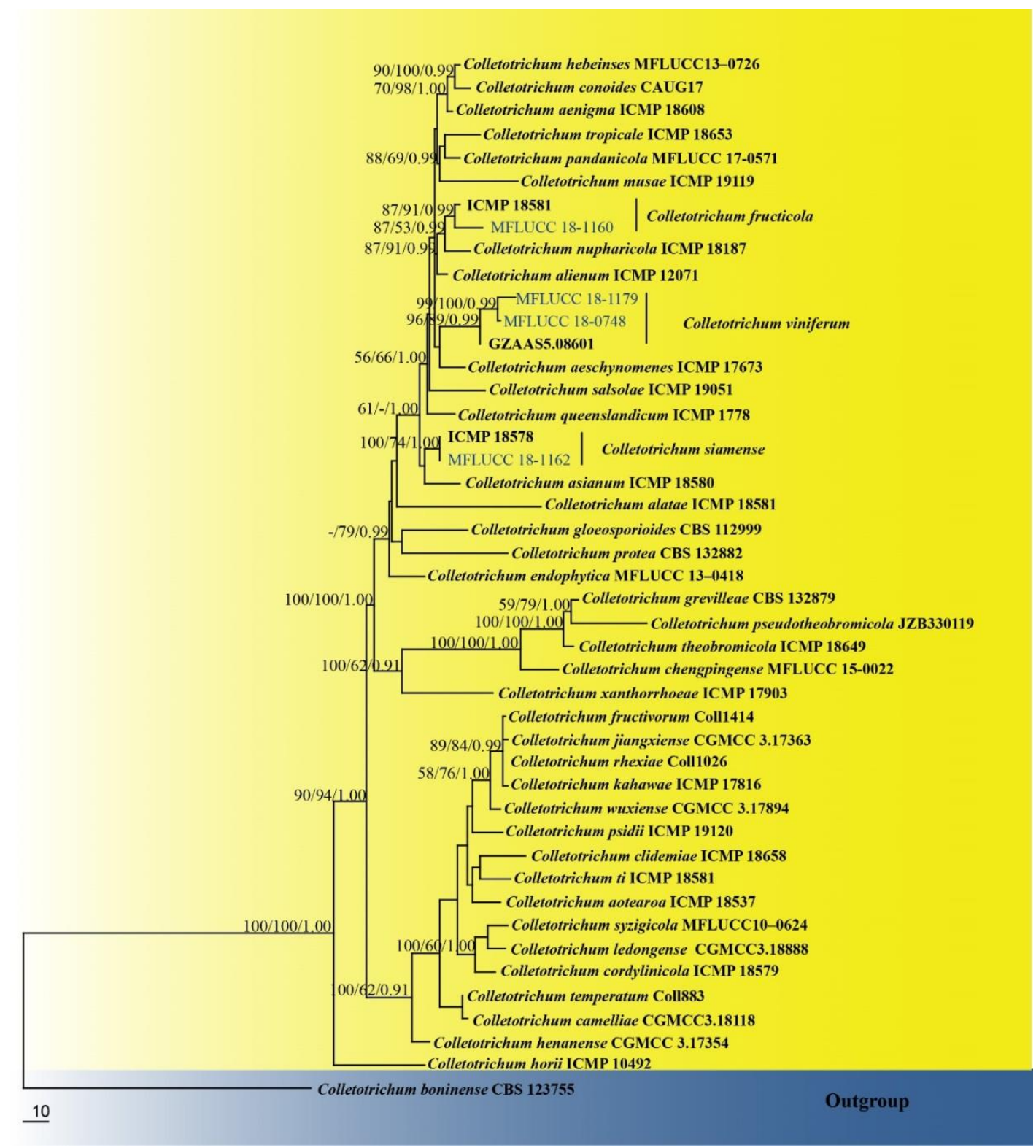

Fig. 142 - Phylogenetic tree generated by maximum parsimony analysis of combined ITS, GAPDH, CHS, ACT and TUB sequence data of Colletotrichum species. Forty-five strains are included in the analyses, which comprise 1876 characters including gaps. The tree is rooted with Colletotrichum boninense (CBS 123755). The maximum parsimonious dataset consisted of 1293 constant, 268 parsimony-informative and 315 parsimony-uninformative characters. The parsimony analysis of the data matrix resulted in the maximum of ten equally most parsimonious trees with a length of 1945 steps $(\mathrm{CI}=0.708, \mathrm{RI}=0.764, \mathrm{RC}=0.541, \mathrm{HI}=0.292)$ in the first tree. $\mathrm{MP}, \mathrm{ML}$ bootstrap values $\geq 50 \%$ and Bayesian posterior $\geq 0.90$ probability are shown near the nodes. The scale bar indicates 10 changes per site. The ex-type strains are in bold. Newly generated sequences are indicated in blue bold.

Colletotrichum fructicola Prihast., L. Cai \& K.D. Hyde, Fungal Diversity 39: 96 (2009)

Fig. 143

Facesoffungi number: FoF0767

Saprotrophic on dead leaves of Rosa hybrida. Sexual morph: Undetermined. Asexual morph: Conidiomata absent on culture. Setae numerous, base pale to dark grey, with scattered, dark brown 
setae about 50-80 $\mu \mathrm{m}$ long. Conidiophores branched at the base, elongate, hyaline, smooth. Conidiogenous cells enteroblastic, phialidic, integrated, smooth, with minute collarette at the tip. Conidia 11-14 $\times 3-5 \mu \mathrm{m}) x^{-}=12 \times 4 \mu \mathrm{m}, \mathrm{n}=40$ ( hyaline, smooth or verruculose, aseptate, guttulate, fusiform, cylindrical with obtuse to slightly rounded ends. Appressoria not observed.

Culture characteristics - Colonies on PDA reaching a maximum of $80 \mathrm{~mm}$ diameter in 7 days at $25^{\circ} \mathrm{C}$, at first white and becoming pale brownish to pinkish, reverse pale yellowish to pinkish, aerial mycelium greyish white, dense, cottony. Vegetative hyphae $2.1 \mu \mathrm{m}$ hyaline, smooth-walled, septate, branched.

Known distribution )based on molecular data( - Brazil, China, Japan, Korea, Puerto Rico, Thailand )Bhunjun et al. 2019, Cao et al. 2019, Costa et al. 2019, Hirayama et al. 2018, Veloso et al. 2018, Jayawardena et al. 2016, this study(
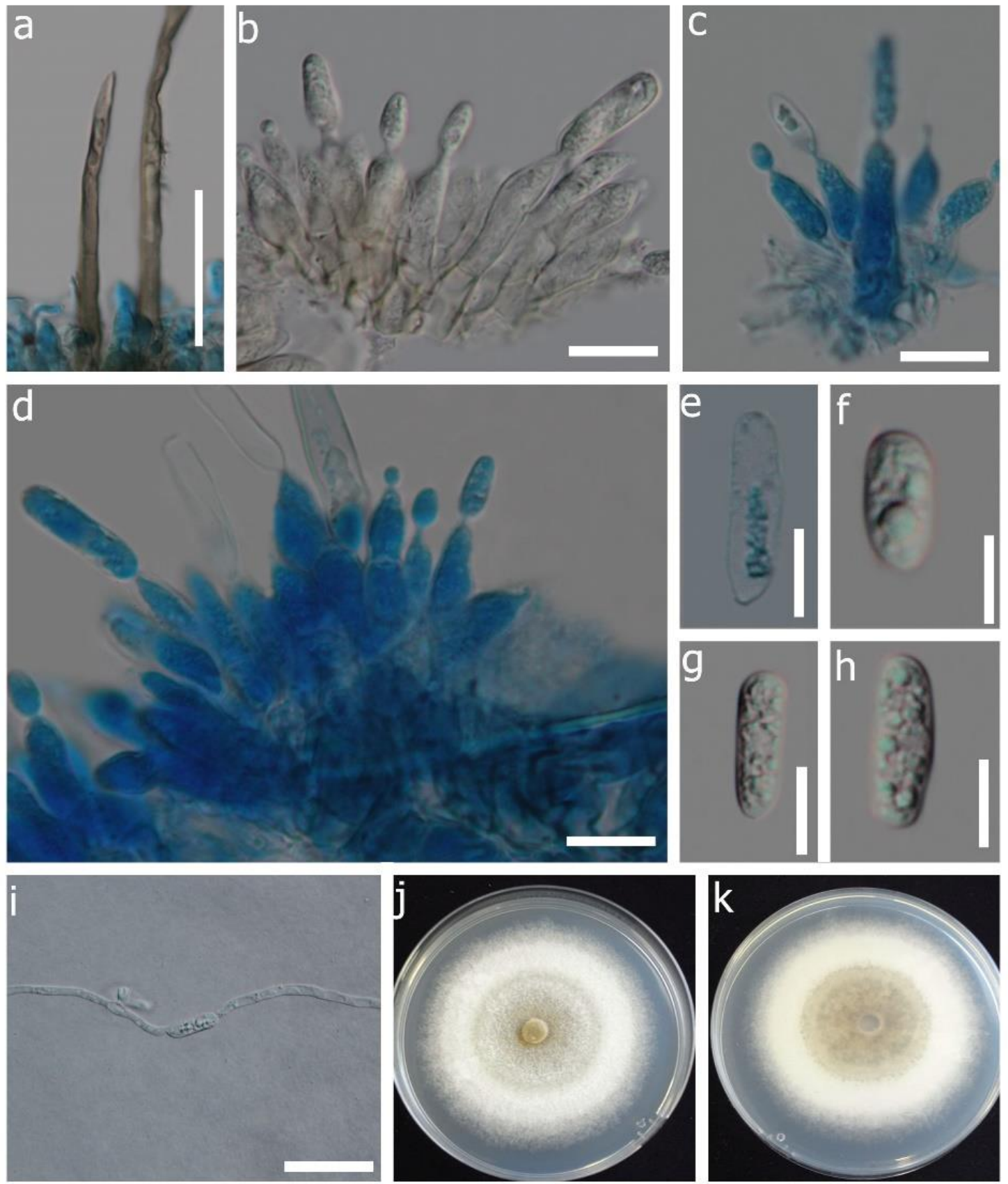

Fig. 143 - Colletotrichum fructicola (MFLU 18-1837, new host record). a Setae. b-d Conidiogenous cells. e-h Conidia. i Germinating conidium. $\mathrm{j}$ Upper view of 7 day old culture. $\mathrm{k}$ Reverse view of 7 day old culture. Scale bars: $\mathrm{a}=20 \mu \mathrm{m}, \mathrm{b}-\mathrm{i}=5 \mu \mathrm{m}$. 
Known hosts )based on molecular data( - Amaranthus blitum, Anacardium sp., Annona sp., Artocarpus sp., Camillia sp., Capsicum sp., Citrus sp., Coffea sp., Malus sp., Mangifera sp., Nephelium lappaceum, Pyrus sp., Vitis sp. )Bhunjun et al. 2019, Cao et al. 2019, Costa et al. 2019, Hirayama et al. 2018, Jayawardena et al. 2016, Veloso et al. 2018, Li et al. 2016b(, Rosa hybrida )this study(

Material examined - Thailand, Chiang Rai, on dead leaves of Rosa hybrida (Rosaceae), 15 May 2018, Ruvishika S. Jayawardena RKB5 )MFLU 18-1837, new host record(, living culture MFLUCC 18-1160.

GenBank Numbers - ITS: MN788675, GAPDH: MN995327, CHS: MN995335, ACT: MN995333

Notes - Colletotrichum fructicola has a wide host and geographical range as well as a wide distribution and is a well-known pathogen on many crops (Jayawardena et al. 2016). A strain in this study clustered with the type species of $C$. fructicola )Fig. 142(. This is the first record of $C$. fructicola as a saprotroph on Rosa hybrida in Thailand.

Colletotrichum siamense Prihast., L. Cai \& K.D. Hyde, Fungal Diversity 39: 98 )2009(

Fig. 144

Facesoffungi number: FoF 03599

Saprotrophic on dead leaves of Rosa sp. Sexual morph: Undetermined. Asexual morph: Conidiomata 70-143 $\mu \mathrm{m}$ diameter, brown to dark brown, acervulate, oval, solitary to aggregated. Setae absent. Conidiophores hyaline, cylindrical or clavate, smooth-walled, simple, to $80 \mu \mathrm{m}$ long. Conidiogenous cells 10-14 $\mu \mathrm{m}$ long, enteroblastic, phialidic, hyaline, smooth-walled, cylindrical to slightly inflated, Collarette $0.5-1 \mu \mathrm{m}$ long, periclinal thickening visible. Conidia $7-19 \times 3-4 \mu \mathrm{m}) \bar{x}$ $=11 \times 4 \mu \mathrm{m}, \mathrm{n}=40$ ( hyaline, smooth or verruculose, aseptate, guttulate, fusiform, ovoid to cylindrical or clavate with rounded apices. Appressoria not observed.

Culture characteristics - Colonies on PDA reaching a maximum of $80 \mathrm{~mm}$ diameter in 7 days at $25^{\circ} \mathrm{C}$, at first white and becoming pale brownish to pinkish, reverse pale yellowish to pinkish, aerial mycelium greyish white, dense, cottony. Vegetative hyphae $2.1 \mu \mathrm{m}$ hyaline, smooth-walled, septate, branched.

Known distribution )based on molecular data( - Australia, Brazil, China, Japan, Korea, Nigeria, Puerto Rico, South Africa, Thailand, USA, Vietnam )Yang et al. 2009, Weir et al. 2012, Jayawardena et al. 2016, Shivas et al. 2016, this study(

Known hosts )based on molecular data( - Artocarpus sp., Camillia sp., Capsicum sp., Carica papaya, Citrus sp., Coffea sp., Dioscorea rotundata, Fragaria $\times$ ananassa, Hymenocallis sp., Jasminum sambac, Malus sp., Mangifera sp., Manihot sp., Persea americana, Pistacia vera, Pyrus sp., Vitis sp. )Bhunjun et al. 2019, Cao et al. 2019, Costa et al. 2019, Hirayama et al. 2018, Veloso et al. 2018, Li et al. 2016b(, Rosa sp. )this study(.

Material examined - Thailand, Chiang Rai, on dead leaves of Rosa sp. (Rosaceae), 19 May 2018, Ruvishika S. Jayawardena RV1 )MFLU 18-1839, new host record(, living culture MFLUCC $18-1162$.

GenBank Numbers - ITS: MN788676, GAPDH: MN995328, CHS: MN995336, ACT: MN995334, TUB: MN995329

Notes - Colletotrichum siamense has a wide host range and a distribution (Jayawardena et al. 2016). A strain in this study clustered with the type species of $C$. siamense )Fig. 142(. This is the first record of $C$. siamense as a saprotroph of Rosa sp. in Thailand.

Colletotrichum viniferum Li J. Peng, L. Cai, K.D. Hyde \& Zi Y. Ying, Mycoscience 54(1): 36 (2013)

Fig. 145

Facesoffungi number: FoF 03600

Endophytic and saprotrophic on leaves of Hemerocallis and Mangifera indica. Sexual morph: Undetermined. Asexual morph: Conidiomata 48-143 $\mu \mathrm{m}$ diameter, black, acervulus, oval, solitary to aggregated. Setae absent. Conidiophores hyaline to light brown, cylindrical or clavate, 
smooth-walled, simple, wide at the base, to $46 \mu \mathrm{m}$ long, occurring in densely arranged clusters. Conidiogenous cells enteroblastic, phialidic, hyaline, smooth-walled, cylindrical to slightly inflated, collarette 0.5-1 $\mu \mathrm{m}$ long, periclinal thickening visible, Conidia 7-12 $\times 2-6 \mu \mathrm{m}) \bar{x}=9 \times 4 \mu \mathrm{m}, \mathrm{n}=$ 40(, hyaline, smooth or verruculose, aseptate, ovoid to cylindrical or clavate with rounded apices. Appressoria formed in culture 9-16 $\times 5-6 \mu \mathrm{m}) \bar{x}=13 \times 5 \mu \mathrm{m}, \mathrm{n}=10$ (, solitary to aggregated, in small groups or short chains, medium to dark brown, smooth-walled, round, oval or irregular.
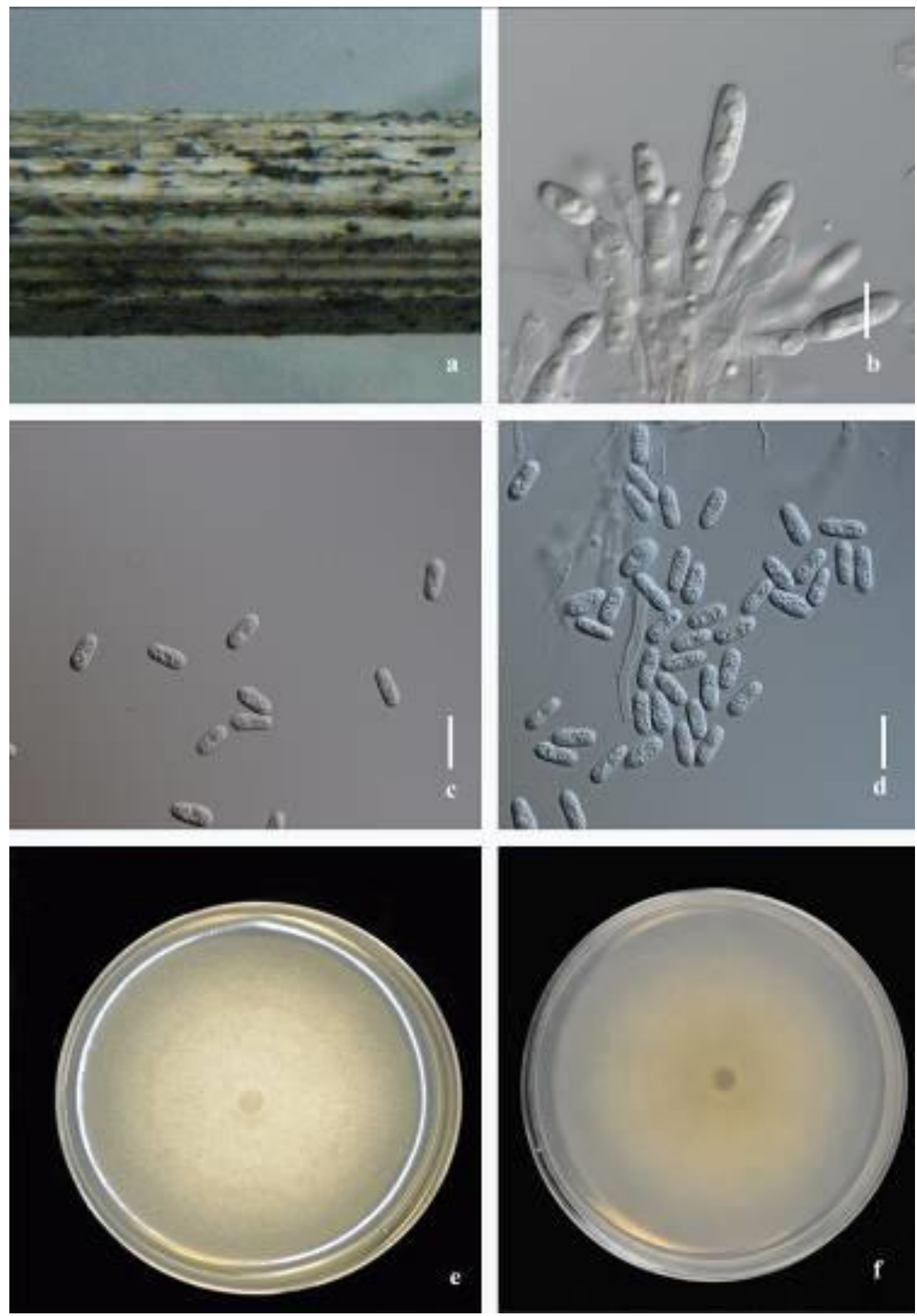

Fig 144 - Colletotrichum siamense (MFLU 18-1839, new host record). a Conidiomata on host. b Conidiophores and conidiogenous cells. c-d Conidia. e Upper view of 7 day old culture. $\mathrm{f}$ Reverse view of 7 day old culture. Scale bars: $b-d=10 \mu \mathrm{m}$. 
Culture characteristics - Colonies on PDA reaching $75 \mathrm{~mm}$ diameter in 7 day at $25^{\circ} \mathrm{C}$, at first white becoming dark grey, reverse pale yellowish, aerial mycelium greyish white, dense, cottony, vegetative hyphae $2.1 \mu \mathrm{m}$ hyaline, smooth-walled, septate, branched.

Known distribution )based on molecular data( - China, South Korea )Peng et al. 2013, Jayawardena et al. 2016(, Russia, Thailand )this study(

Known hosts )based on molecular data( - Capsicum sp., Vitis sp. )Peng et al. 2013, Jayawardena et al. 2016, Oo and Oh 2017(, Hemerocallis sp., Mangifera indica )this study(

Material examined - Russia, Rostov region, Shakhty City District, street flowerbed, on dead floriferous shoot of Hemerocallis sp. (Asphodelaceae), 20 November 2017, T. Bulgakov T2281-1 )MFLU 18-0441, new host and geographical record(, culture, MFLUCC 18-0748; Thailand, Chiang Rai, on leaves of Mangifera indica, 19 May 2018, Ruvishika S. Jayawardena MRV3 )MFLU 18-1850, new host and geographical record(, culture, MFLUCC 18-1179.

GenBank Numbers - (MFLUCC 18-0748); ITS:MN788677, TUB:MN995330 (MFLUCC 18-1179); ITS:MN788678, TUB:MN995331
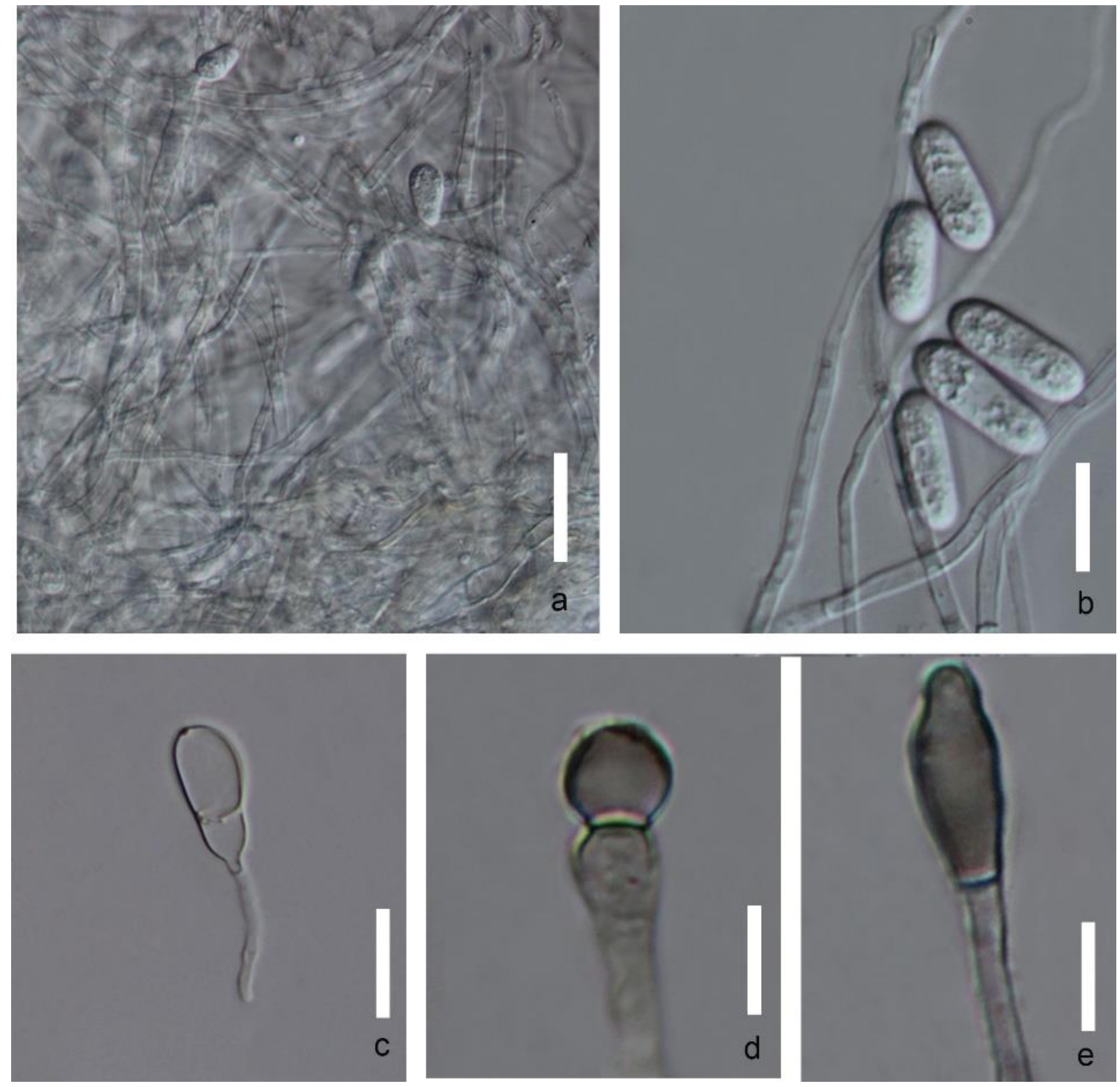

Fig. 145 - Colletotrichum viniferum (MFLU 18-1850, new host and geographical record). a Conidiogenous cells. b Mature conidia. c Germinating spore. $d-e$ Appressoria. Scale bars: $a-e=5$ $\mu \mathrm{m}$.

Notes - Colletotrichum viniferum was introduced by Peng et al. )2013(. This species is a known pathogen causing grape ripe rot in China )Yan et al. 2015(. Our strains clustered with the 
type species of $C$. viniferum with high bootstrap support )Fig. 142(. Colletotrichum asianum and $C$. gloeosporioides have been previously recorded from Mangifera spp. in Thailand (Weir et al. 2012, Jayawardena et al. 2016). This study provides both the first report of $C$. viniferum as a saprotroph from Hemerocallis sp. from Russia and as an endophyte from Mangifera indica in Thailand.

\section{Hypocreales Lindau}

Cordycipitaceae Kreisel ex G.H. Sung et al.

Cordycipitaceae was introduced by Sung et al. (2007) based on multi-gene analyses to accommodate 11 genera. Species in this family are mostly pathogenic on insects, spiders, nematodes and rust fungi, saprobic on decaying wood or occur on soil (Sung et al. 2007, Evans 2013, Nonaka et al. 2013, Mijeong et al. 2015, Zhang et al. 2017, Huang et al. 2018, Wei et al. 2018). Maharachchikumbura et al. (2016a) accepted 18 genera and provided family and generic descriptions for Cordycipitaceae and Cordyceps. Kepler et al. (2017) accepted 11 genera and rejected eight generic names in this family based on phylogenetic analyses. Wijayawardene et al. (2018) listed 17 genera while rejecting some generic names in Kepler et al. (2017), such as Granulomanus, Isaria and Microhilum. Previous research failed to account for Leptobacillium (Zare \& Gams 2016) and Samsoniella (Mongkolsamrit et al. 2018). Up to now, there are many old species in Cordycipitaceae with several genera without verification with molecular data, such as Beejasamuha, Coremiopsis and Granulomanus.

\section{Cordyceps Fr.}

Cordyceps was established by Link (1833) based on the type species Cordyceps militaris. The representative characters of this genus are fleshy, pallid or brightly pigmented stromata, superficial to completely immersed ascomata, cylindrical asci with a thickened cap and hyaline, multi-septate, disarticulating into part-spores or non-disarticulating ascospores (Sung et al. 2007, Maharachchikumbura et al. 2016a).

Cordyceps pruinosa Petch, Trans. Br. mycol. Soc. 10(1-2): 38 (1924)

Fig. 147

Facesoffungi number: FoF07468

Parasitic on limacodid pupa which resemble a plant seed. Sexual morph: Undetermined. Asexual morph: Hyphomycetous. Stroma emerging from host, single, erect, cylindrical, apically branched, red, pale yellow at the base, fleshy, with white straight hyphae and interlaced vegetative hyphae mass occurring on the surface of upper stroma. In vitro, Synnemata orange, erect, compacted below, apically branched, becoming finger-like. Hyphae 0.6-1.20 ( $\bar{x}=1, \mathrm{n}=20) \mu \mathrm{m}$, aseptate, hyaline, smooth-walled. Conidiophores macronematous, prostrate, branched, bearing solitary or 2-3 whorls of phialides. Conidiogenous cells phialidic. Phialides $11-30 \times 1.2-1.6(\bar{x}=$ $16 \times 1.3, \mathrm{n}=20) \mu \mathrm{m}$, aseptate, monophialidic, gradually tapering towards the apex, hyaline, straight. Conidia 1.8-4.8 $\times 0.9-2.7(\bar{x}=3 \times 1.5, \mathrm{n}=30) \mu \mathrm{m}$, globose, oval, cylindrical, aseptate, smooth-walled, hyaline, aggregating in imbricated slimy chains at the tip of phialides.

Culture characters - slow-growing on PDA, reaching $4 \mathrm{~cm}$ in diameter after incubating at room temperature for 26 days, yellow from upper and reverse view, floccose, dense, circular, feathery margin, with several erect, orange synnemata developing at central regions, without diffused pigment. The sporulation occurs on upper part of synnemata.

Material examined - China, Yunnan Province, Kunming City, Western hill Park. On Limacodid pupa, 27 July 2018, Deping Wei (HKAS 102537, new sequence data), living culture KUMCC 18-0340.

Known distribution (based on molecular data) - Korea (Bae et al. 2002, Moon et al. 2018); Guangdong and Guizhou in China (Wang et al. 2008, Meng et al. 2014, Zha et al. 2019); Japan (Nikoh \& Fukatsu 2001, Kepler et al. 2012).

Known hosts (based on molecular data) - Cocoon of a Limacodidae insect (Meng et al. 2014, Zha et al. 2019); Iragoides fasciata (Lepidoptera) (Spatafora et al. 2007, Sung et al. 2007).

GenBank numbers - ITS: MT012347, LSU: MT012354, SSU: MT012361, tef1:MT025053. 
Notes - In the phylogenetic tree, our collection XS2703 is closest to Cordyceps pruinosa (ARSEF 5413) and $C$. ninchuckispora with moderate support (81\% MP/ $0.99 \mathrm{PP}$ ). The strain ARSEF 5413 is not linked to any description (Kepler et al. 2017). Cordyceps pruinosa was initially described by Petch (1924) with its asexual morph linked to Mariannaea pruinosa by Liang et al. (1991) but not confirmed with molecular data. The asexual morph of Cordyceps pruinosa was characterized by verticillate conidiophores and ovoid, ellipsoidal, cylindrical, rarely septate, hyaline conidia, which adhere in imbricate chains (Liang et al. 1991). Our collection is consistent with the concept of Cordyceps pruinosa in the shape and size, as well as the arrangement of conidia. A comparison of tefl sequences between our isolates and two strains of Cordyceps ninchuckispora (EFCC 5197 and EFCC 5693) shows 17/862 bp (2\%) differences, but identical to Cordyceps pruinosa (ARSEF 5413) within 862bp. In ITS, our collection is identical to Cordyceps pruinosa (ARSEF 5413) within 355bp. We report a new collection of Cordyceps pruinosa according to the guidelines of Jeewon \& Hyde (2016).

Cordyceps rosea Kobayasi \& Shimizu, Bull. natn. Sci. Mus., Tokyo, B 8(4): 112 (1982)

Facesoffungi number: FoF07467

Fig. 148

Parasitic on Lepidoptera larva or pupa. Sexual morph: Stroma arising from insect body, stipitate, multiple or single, flexuous or straight, orange, fleshy, unbranched. Perithecia 329-846 $\times$ 343-667 $(\bar{x}=555 \times 486, \mathrm{n}=20) \mu \mathrm{m}$, superficial, born on upper region of stroma, ovoid. Peridium 22-49 ( $\bar{x}=34, \mathrm{n}=20) \mu \mathrm{m}$ wide, membranous, comprised of yellow, thick-walled cells of textura angularis. Asci $212-595 \times 2.5-5.5(\bar{x}=395 \times 4, \mathrm{n}=30) \mu \mathrm{m}, 8$-spored, unitunicate, narrowly cylindrical, with round, attenuate base and thickened, hemispherical cap. Ascospores 2-5.5 $\times 0.7-$ $1.5(\bar{x}=3 \times 1, \mathrm{n}=100) \mu \mathrm{m}$, filiform, hyaline, disarticulating into secondary spores and spraying from perithecia when mature. Secondary ascospores cylindrical, hyaline, smooth-walled, aseptate, truncated at both ends, straight, occasionally swollen spores present. Asexual morph: Undetermined.

Culture characteristics - Colonies growing on PDA, circular, slightly raised, edge entire, velvety, reaching $18 \mathrm{~mm}$ in 16 days at $25^{\circ} \mathrm{C}$, white to pale yellow in PDA medium.

Material examined - China, Yunnan Province, Honghe County, Jiayin Village, Amushan protected area, on Lepidoptera pupa, 23 October 2018, De-Ping Wei, AMS10 (HKAS 102492, reference specimen designated here), ibid; AMS06 (HKAS 102495), TSQ09B (HKAS 102493), TSQ09C (HKAS 102494), TSQ09E (HKAS 102496); KUMCC 20-0002, living culture.

Known distribution (based on molecular data) - Taiwan (Kepler et al. 2017).

Known hosts (based on molecular data) - Lepidopteran larva (Kepler et al. 2017).

GenBank numbers - ITS: TSQ09B = MT012342, TSQ09C $=$ MT012343, TSQ09E $=$ MT012344, AMS06 = MT012345, AMS10 = MT012346; LSU: TSQ09B = MT012349, TSQ09C $=$ MT012350, TSQ09E = MT012351, AMS06 = MT012352, AMS10 = MT012353; SSU: TSQ09B = MT012356, TSQ09C $=$ MT012357, TSQ09E $=$ MT012358, AMS06 $=$ MT012359, AMS10 $=$ MT012360; tefl: TSQ09B = MT025048, TSQ09C $=$ MT025049, TSQ09E $=$ MT025050, AMS06 = MT025051, AMS10 = MT025052; RPB2: TSQ09B = MT025044, TSQ09C $=$ MT025045, TSQ09E $=$ MT025046.

Notes - Cordyceps rosea was initially discovered on larva of Lepidoptera in Honshu Province, Japan and was introduced by Kobayasi \& Shimizu (1982). Its macro-morphology and molecular data were made available in Kepler et al. (2017), but the details of micro-morphology are still lacking. Our isolates resemble the reference specimen in having fleshy, orange stroma and superficial, conical perithecia. In addition, the multi-locus based phylogenetic tree shows that our isolates form a clade sister to Cordyceps rosea (spat 09-053) with high support (Fig. 146, 99\% ML/0.99 PP). The nucleotide differences between our isolates and Cordyceps rosea (spat 09-053) are lower than $1.5 \%$ for LSU and SSU sequences, and higher for tefl sequences (Table 1). Unfortunately, ITS sequences of Cordyceps rosea (spat 09-053) are not available for comparison. The ITS nucleotide sequences of our isolates TSQ09B, TSQ09C, TSQ09E, AMS10 are identical, 
but differ from AMS06 in two gaps and one base pair. To conclude, there are many differences in the tefl sequences, but the subtle differences in the ITS sequence and phylogenetic analysis as well as the macro-morphologic features support our isolates to be a new geographical record of Cordyceps rosea in mainland China. Given that the molecular data and morphological descriptions of Cordyceps rosea were untraceable, we propose a reference specimen for this species with support from phylogeny and morphological data.

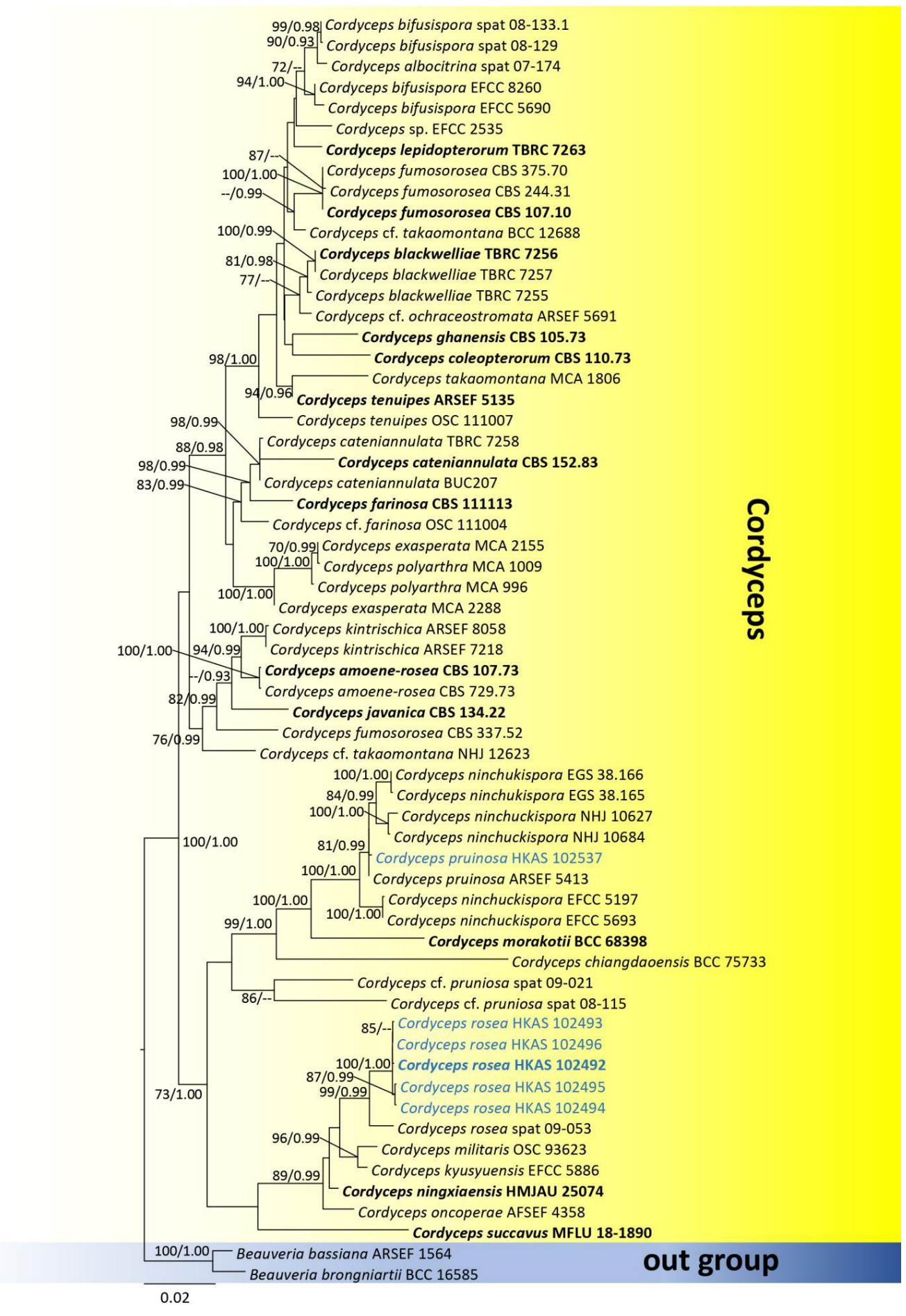

Fig. 146 - Phylogram generated from maximum likelihood analysis based on combined LSU, SSU, ITS and tefl sequence data. Sixty-one strains are included in the combined gene analyses comprising 3020 characters after alignment (802 characters for LSU, 980 characters for SSU, 375 characters for ITS, 862 characters for tef1). Beauveria bassiana (ARSEF 1564) and Beauveria brongniartii (BCC 16585) are used as the outgroup taxa. The tree topology derived from the 
Bayesian analysis was similar to that derived from the maximum likelihood analysis. The best RaxML tree with a final likelihood value of -12920.722667 is presented. The matrix had 870 distinct alignment patterns, with $20.99 \%$ undetermined characters or gaps. Estimated base frequencies were as follows: $\mathrm{A}=0.242336, \mathrm{C}=0.262549, \mathrm{G}=0.268942, \mathrm{~T}=0.226174$; substitution rates $\mathrm{AC}=1.424390, \mathrm{AG}=2.347648, \mathrm{AT}=1.121613, \mathrm{CG}=0.997130, \mathrm{CT}=$ 7.134373, GT $=1.000000$; gamma distribution shape parameter $\alpha=0.699986$. Bootstrap values for maximum likelihood equal to or greater than 70 and Bayesian posterior probabilities equal or greater than 0.90 are placed above or below the branches. The newly generated sequences are indicated in red bold.
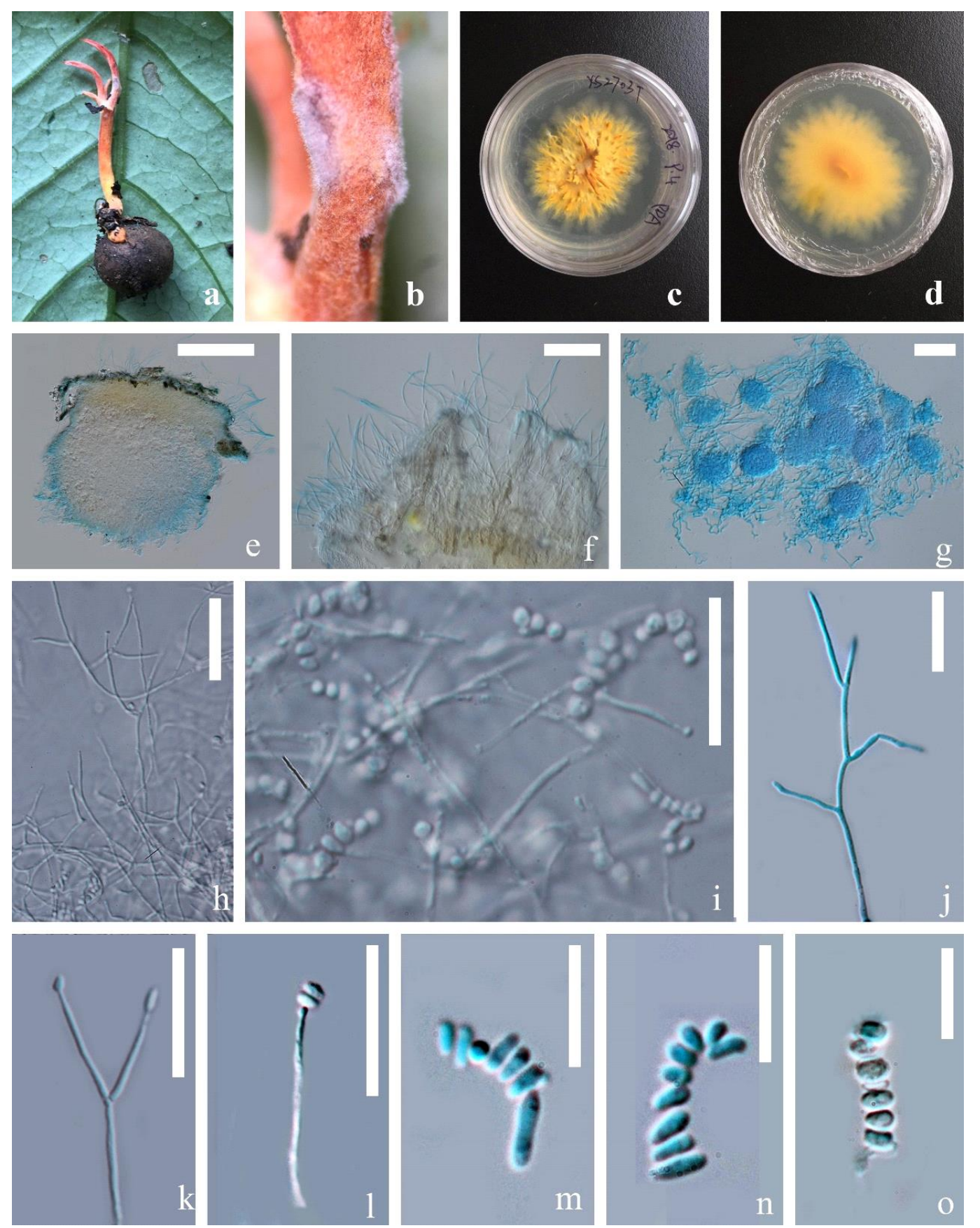

Fig. 147 - Cordyceps pruinosa (HKAS 102537, new sequence data). a Stroma emerging from host. $\mathrm{b}$, f White hypha produced on stroma. c, d Upper and reverse view of cultures on PDA after 34 days incubation. e Vertical section of stroma. g interlaced hypha occurring on upper region of stroma. h, j Conidiophore. k, 1 Phialides. i, m-o Conidia adhering in imbricated chains. Scale Bars: 
$\mathrm{e}=150 \mu \mathrm{m}, \mathrm{f}=50 \mu \mathrm{m}, \mathrm{g}, \mathrm{h}=30 \mu \mathrm{m}, \mathrm{i}-1=20 \mu \mathrm{m}, \mathrm{m}-\mathrm{o}=10 \mu \mathrm{m}$. (e-g, $\mathrm{j}-\mathrm{o}$ stained with cotton blue; $\mathrm{e}-\mathrm{g}$ observed from host and $\mathrm{h}-\mathrm{O}$ are from culture).

Microascales Luttr. ex Benny \& Kimbr

Microascaceae Luttr. ex Malloch

Luttrell (1951) proposed Microascaceae and Microascales and they were validly published with Latin descriptions by Malloch (1970), Benny \& Kimbrough (1980). Malloch (1970) reviewed the family with sexual and asexual characteristics, while Réblová et al. (2011) and Maharachchikumbura et al. (2015) investigated the phylogenetic placement of Microascaceae. Currently, this family includes 23 genera (Wijayawardene et al. 2020). Our marine fungal collection from the UK yielded Parascedosporium putredinis from submerged marine wood and this is the first record of $P$. putredinis from a marine habitat.

Table 1 A comparison of nucleotide sequences between Cordyceps rosea (spat 09-053) and our isolates.

\begin{tabular}{llrr}
\hline \multicolumn{1}{c}{ Species } & \multicolumn{1}{c}{ LSU (bp) } & SSU(bp) & tef1 (bp) \\
\hline TSQ09B & $3 / 796(0.37 \%)$ & $1 / 907(0.1 \%)$ & $24 / 834(2.87 \%)$ \\
TSQ09C & $4 / 796(0.5 \%)$ & $1 / 907(0.1 \%)$ & $26 / 834(3.11 \%)$ \\
TSQ09E & $3 / 796(0.37 \%)$ & $1 / 907(0.1 \%)$ & $24 / 834(2.87 \%)$ \\
AMS06 & $3 / 796(0.37 \%)$ & $1 / 907(0.1 \%)$ & $26 / 834(3.11 \%)$ \\
AMS10 & $3 / 796(0.37 \%)$ & $1 / 907(0.1 \%)$ & $26 / 834(3.11 \%)$ \\
\hline
\end{tabular}

Parascedosporium Gilgado, Gené, Cano \& Guarro

Parascedosporium was introduced by Gilgado et al. (2007) based on Graphium tectonae (CBS 127.84) isolated from seeds of Tectona grandis in Jamaica (Lackner \& de Hoog 2011). Graphium tectonae was synonymized as Parascedosporium tectonae by Gilgado et al. (2007). Subsequently, this species was considered as a synonym of Parascedosporium putredinis by Lackner \& de Hoog (2011) based on the analysis of ITS sequence data and examination of the extype culture (CBS 127.84). This treatment was accepted by de Beer et al. (2013). Sympodial conidia produced from denticulate conidiogenous cells are the unique features of this genus (Gilgado et al. 2007). Parascedosporium synanamorphs has solitary conidiophores that emerge from aerial mycelium and comprises of graphium-like synnemata (Gilgado et al. 2007). The sexual morphs of this genus are undetermined.

Parascedosporium putredinis (Corda) Lackner \& de Hoog

Fig. 150

Facesoffungi Number: FoF 04482

Saprobic on unidentified wood in a stream. Sexual morph Undetermined. Asexual morph Hyphomycetous. Synnemata 460-620 × 7.5-15 $\mu \mathrm{m}(\bar{x}=587 \times 8.5 \mu \mathrm{m}, \mathrm{n}=5)$, scattered but abundant, arising from the host surface. Stipes $175-223 \times 28-42 \mu \mathrm{m}$, pale to dark brown, branched and flared above. Conidiogenous cells 16-24 $\times 0.8-2.1 \mu \mathrm{m}(\bar{x}=18.5 \times 1.7 \mu \mathrm{m} ; \mathrm{n}=20)$, annellidic, pale brown, verrucose. Conidia 5.8-7.3 $\times 2.6-3.7 \mu \mathrm{m}(\bar{x}=6.2 \times 3 \mu \mathrm{m}, \mathrm{n}=30)$, aseptate, hyaline, cylindrical to obovoid, hyaline, smooth, produced in a mucilaginous mass on synnema.

Culture characteristics - Conidia germinating on PDA within $12 \mathrm{~h}$. Colonies on MEA circular, entire edge with white to cream, raised on surface media reverse gray in the center, becoming white towards the margin.

Known distribution (based on molecular data) - Australia, China, Cuba, Czech Republic, France, Italy, Jamaica, Japan, Malaysia, Madagascar, New Zealand, Nicaragua, Poland, Thailand, The Netherlands, USA (Lackner \& de Hoog 2011, Perera et al. 2018), UK (this study).

Known hosts (based on molecular data) - Human, foot mycetoma, on rotten stem of Echium sp., dried seed pod of Delonix regia (Lackner \& de Hoog 2011, Perera et al. 2018), wood (this study). 
Material examined - UK, Isle of Wight, Calbourne, on unidentified wood, stream, 7 September 2017, E.B.G. Jones, GJ429 (MFLU 19-1237, new geographical record), living culture MFLUCC 17-2483.
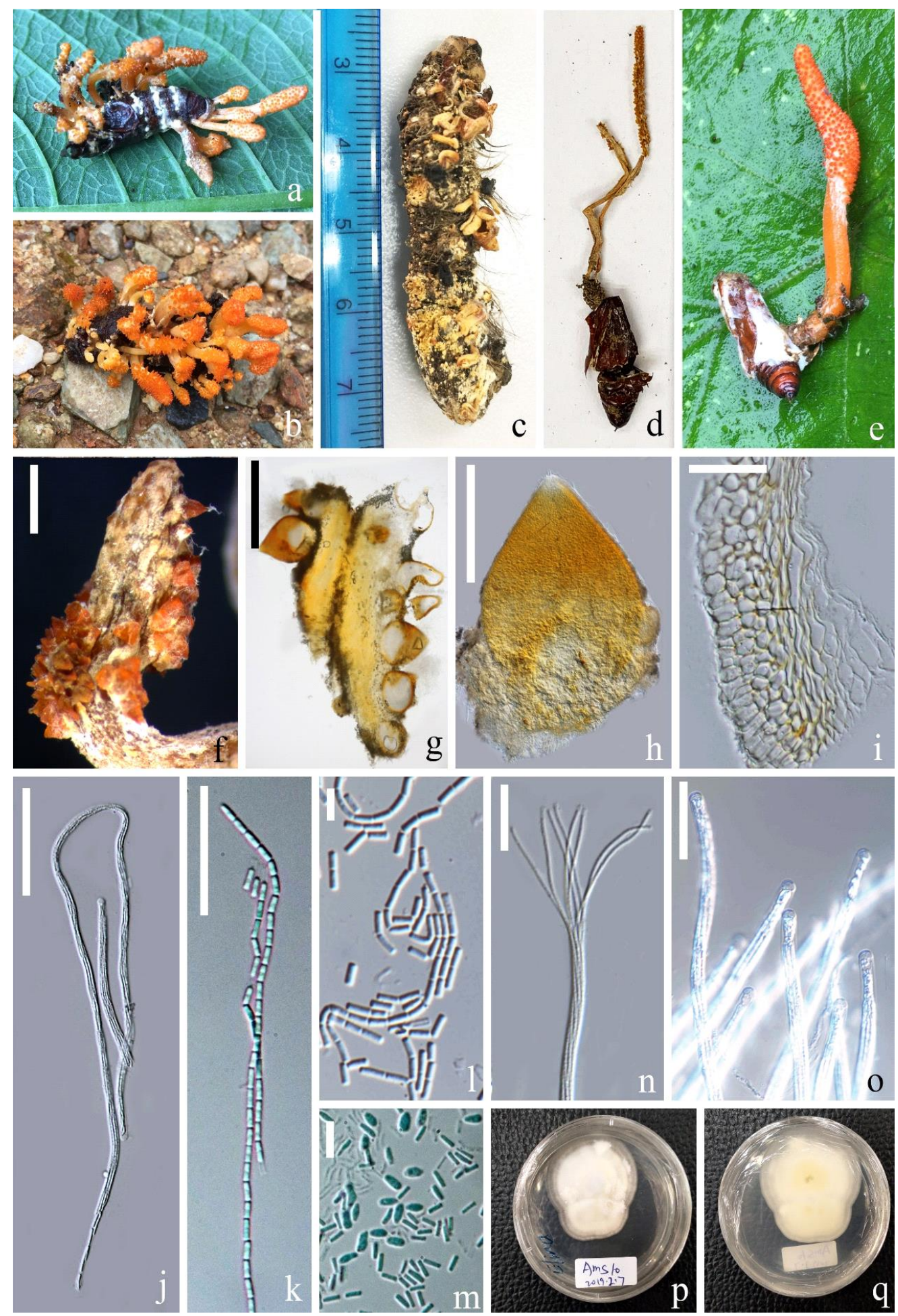

Fig. 148 - Cordyceps rosea (HKAS102492, lectotype). a-e Stroma raised from insect body. f Perithecia in stroma. g Vertical section through perithecia. h Perithecium. i Peridium. i Asci. $\mathrm{k}-\mathrm{m}$ Disarticulating ascospores. $\mathrm{n}$ Part of asci. o Apical region of asci. $\mathrm{p}, \mathrm{q}$ Upper and lower view of culture on PDA. a, f, g, h, j, k, m, p, q from HKAS102492 (neotype), b from HKAS102493, c from HKAS102494, d from HKAS102496, e, 1, n, o from HKAS102495. Scale bar: f, $g=1000$ $\mu \mathrm{m}, \mathrm{h}=500 \mu \mathrm{m}, \mathrm{i}=30 \mu \mathrm{m}, \mathrm{j}=50 \mu \mathrm{m}, \mathrm{k}, \mathrm{n}, \mathrm{o}=20 \mu \mathrm{m}, 1, \mathrm{~m}=5 \mu \mathrm{m}, \mathrm{k}=10 \mu \mathrm{m}, \mathrm{n}-\mathrm{p}=3 \mu \mathrm{m}$. (m stained with cotton blue solution). 


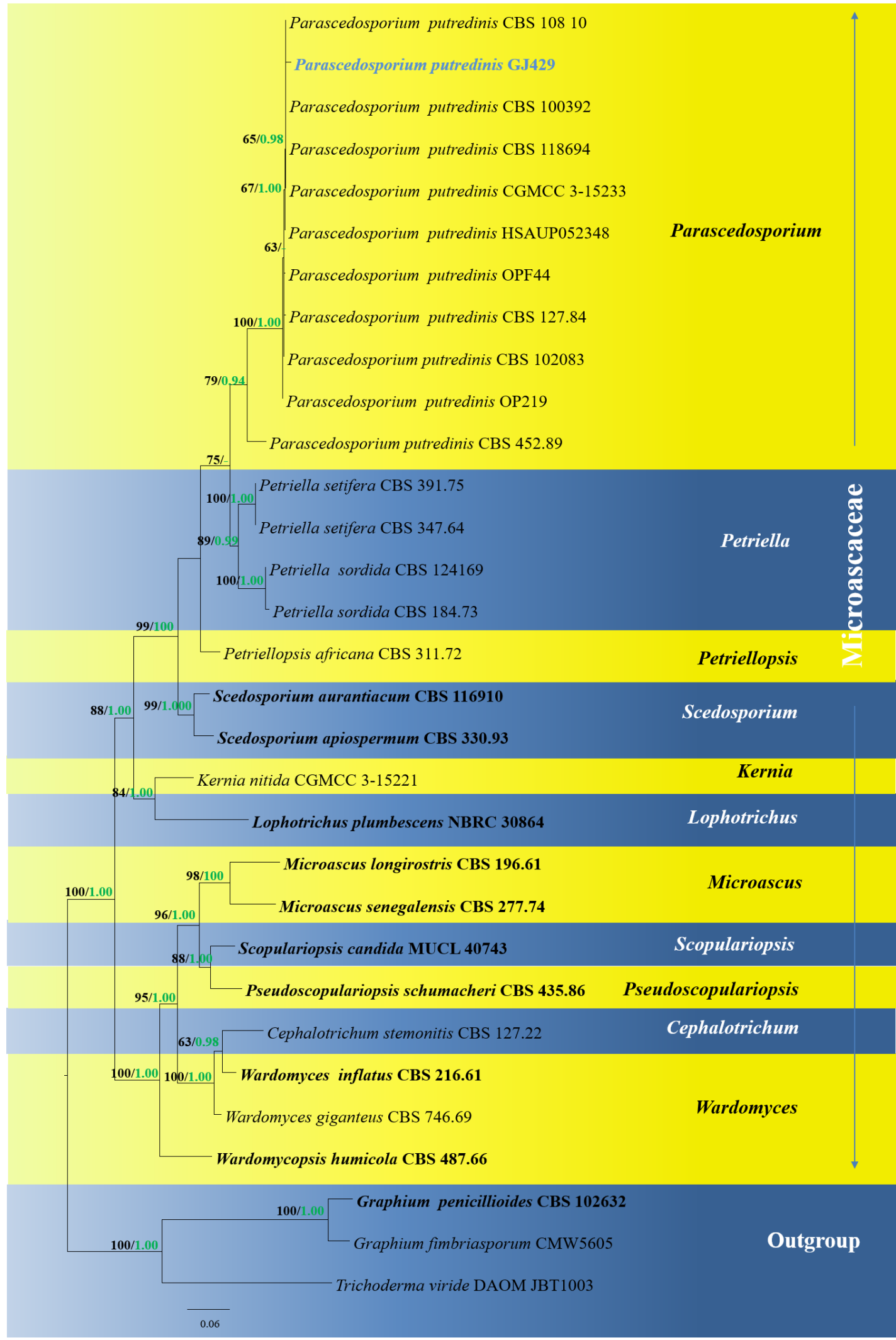

Fig. 149 - Maximum likelihood analysis with 1000 bootstrap replicates yielded a best tree with the likelihood value of -7124.535878 . The combined ITS and LSU sequence datasets comprised 32 strains of Microascales with Graphium fimbriasporum (CMW5605), G. penicillioides (CBS 102632) and Trichoderma viride (DAOM JBT1003) as the outgroup taxa. The tree topology derived from the Bayesian analysis was similar to that derived from the maximum likelihood analysis. The matrix had 564 distinct alignment patterns, with $32.45 \%$ of undetermined characters or gaps. Estimated base frequencies were as follows; $\mathrm{A}=0.239641, \mathrm{C}=0.266161, \mathrm{G}=0.273521, \mathrm{~T}$ 
$=0.220676$; substitution rates $\mathrm{AC}=2.021294, \mathrm{AG}=2.471263, \mathrm{AT}=1.983840, \mathrm{CG}=1.481755$, $\mathrm{CT}=5.565293$, GT $=1.000000$; gamma distribution shape parameter $\alpha=0.245860$. Maximum likelihood bootstrap (ML, black) values $>65 \%$ and Bayesian posterior probabilities (PP, green) > $0.80 \%$ are given above the nodes. The scale bar indicates 0.06 changes. The ex-type strains are in bold and new isolate is in blue bold.

GenBank numbers - LSU: MN017875, ITS: MN047111.

Additional GenBank number - SSU MN017934.

Notes - The morphological characters of our strain resemble $P$. putredinis (Lackner \& de Hoog 2011). Our phylogenetic analyses with combined ITS and LSU sequence data indicate that our new strain, MFLUCC 17-2483 grouped together with the Parascedosporium putredinis strains (Fig. 149). Furthermore, based on a megablast search using the ITS sequence, the closest matches of $P$. putredinis (MFLUCC 17-2483) in NCBI's GenBank nucleotide database were P. putredinis (GenBank MF782705; similarities 558/559(99\%), 1/559(0\%)), P. putredinis (GenBank MH048678; similarities 544/549(99\%), 0/549(0\%)), P. putredinis (GenBank MF782706; similarities 555/559(99\%), 1/559(0\%)) and P. putredinis (GenBank KC894850; similarities 555/559(99\%), 1/559(0\%)).

Subclass Xylariomycetidae O.E. Erikss \& Winka

Amphisphaeriales D. Hawksw. \& O.E. Erikss.

Apiosporaceae K.D. Hyde et al.

Hyde et al. (1998) re-examined the type (Apiospora montagnei Sacc.) and introduced Apiosporaceae to accommodate Apiospora (Sexual morph of Arthrinium) and Appendicospora. Subsequently, this family has been recognized Xylariales by several authors (Huhndorf et al. 2004, Zhang et al. 2006, Jaklitsch \& Voglmayr 2012, Sharma et al. 2014, Senanayake et al. 2015, Dai et al. 2016).

\section{Nigrospora Zimm.}

Nigrospora was introduced by Zimmerman (1902) for $N$. panici which was isolated as an endophyte from leaves of Panicum amphibium in Java, Indonesia. Mason (1927) transferred several black-spored hyphomycetes occurring on monocotyledonous hosts to Nigrospora and included several species in this genus. Based on its conidial characters, Nigrospora was placed in Dermateaceae (Moniliales) by Barnett \& Hunter (1998) and Kirk et al. (2008b) assigned Nigrospora and its Khuskia sexual morph to the Trichosphaeriaceae (Trichosphaeriales). Wang et al. (2017) placed Nigrospora in Apiosporaceae based on phylogenetic analyses of combined ITS, tef1$\alpha$ and TUB sequence data of 165 strains from China and Europe.

Nigrospora oryzae (Berk. \& Broome) Petch, J. Indian bot. Soc. 4: 24 (1924)

Fig. 152

Facesoffungi number: FoF06596

Saprobic on fallen dead leaves of Phyllostachys heterocycla. Sexual morph: Undetermined. Asexual morph: Hyphomycetes. Conidiophores $24-32 \times 2-3 \mu \mathrm{m}(\bar{x}=28 \times 2.5 \mu \mathrm{m}, \mathrm{n}=10)$ macronematous, mononematous, flexuous, smooth, hyaline to pale brown, branched. Conidiogenous cells 3.2-5.6 $\times 2-3 \mu \mathrm{m}(\bar{x}=4.9 \times 2.6 \mu \mathrm{m}, \mathrm{n}=20)$ holoblastic, monoblastic, solitary, hyaline to subhyaline, ampulliform to subsphaerical, discrete, smooth. Conidia 4-5.5 $\times 4.2-5 \mu \mathrm{m}$ $(\bar{x}=5.2 \times 4.8 \mu \mathrm{m}, \mathrm{n}=100)$ dry, solitary, acrogenous, simple, spherical to broadly ellipsoidal, aseptate, black, smooth, velvety.

Culture characteristics: Conidia germinating on PDA within 12 hours and germ tubes produced from all cells. Colonies growing on PDA, hairy, black, reaching $5 \mathrm{~mm}$ in 7 days at $30^{\circ} \mathrm{C}$, mycelium partly superficial, partly immersed, slightly effuse, radially striate, with irregular edge, black; Asexual spores were formed after 30 days and sexual spores not formed within 60 days.

Known distribution (based on molecular data) - Australia (Barkat et al. 2016), China (Sun et al. 2011, Chen et al. 2018, Zhang et al. 2019b), Iran (Hashemian Kalati et al. 2014), Italy 
(Lorenzini et al. 2016), Kazakstan (Eken et al. 2016), Pakistan (Alam et al. 2017b), Sri Lanka (Wang et al. 2017), Thailand (this study).
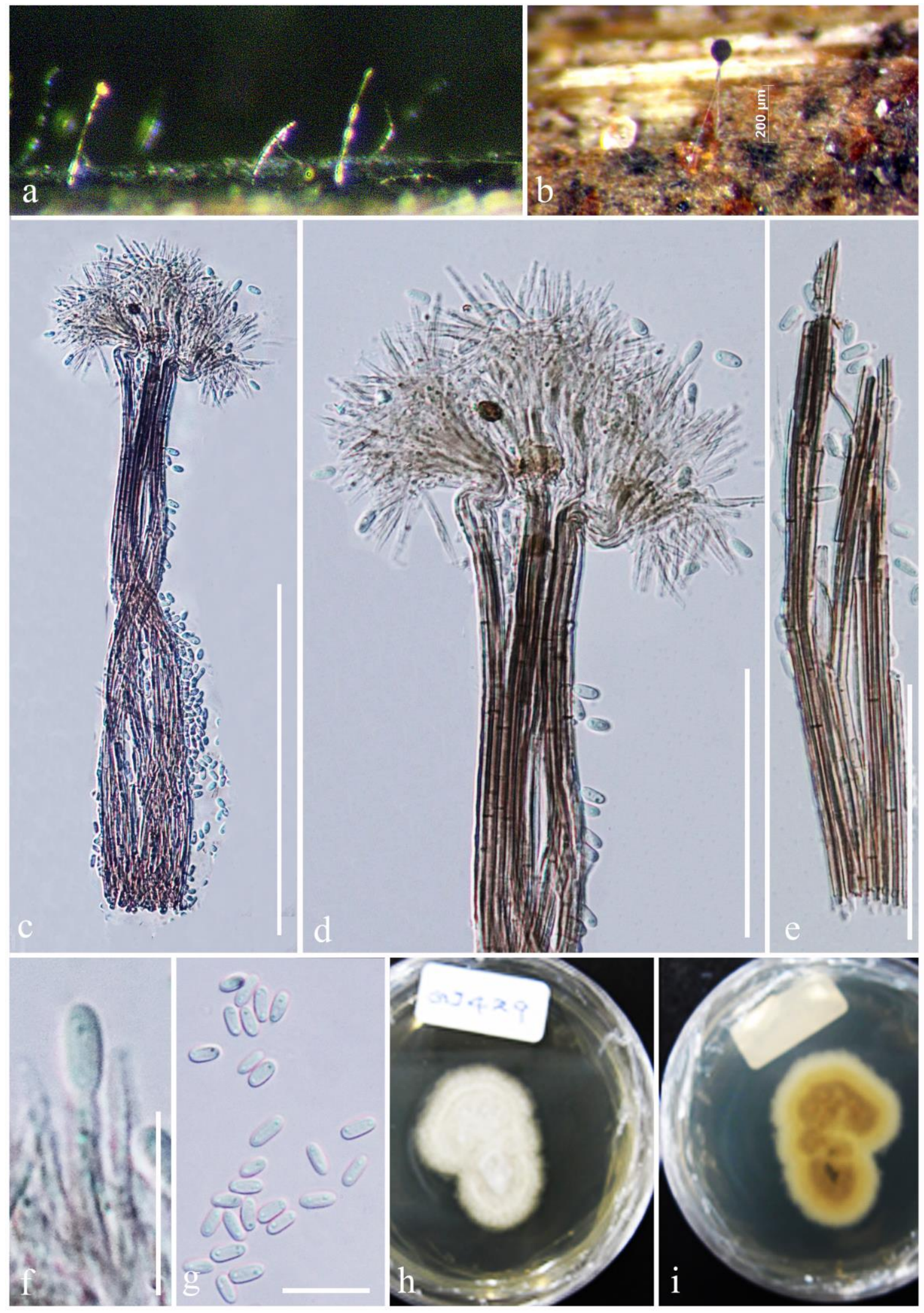

Fig. 150 - Parascedosporium putredinis (MFLU 19-1237, new geographical record). $\mathrm{a}-\mathrm{b}$ Conidiophores on host substrate. $\mathrm{c}-\mathrm{e}$ Erect synnematouse conidiophores. $\mathrm{f}$ Conidia attached to the conidiogenous cells. g Conidia. h, i Colonies on seawater MEA. (h-upper, i-lower). Scale bars: $\mathrm{c}-\mathrm{e}=100 \mu \mathrm{m}, \mathrm{f}, \mathrm{g}=10 \mu \mathrm{m}$. 
Known hosts (based on molecular data) - Acer truncatum (Sun et al. 2011), Aloe vera (Alam et al. 2017b), Castanopsis sp. (Wang et al. 2017), Cephalotaxus sinensis (Wang et al. 2017), Citrullus lanatus (Chen et al. 2018), Citrus reticulata (Wang et al. 2017), Cleyera japonica (Wang et al. 2017), Daphniphyllum macropodum (Wang et al. 2017), Daphniphyllum oldhamii (Wang et al. 2017), Gossypium hirsutum (Zhang et al. 2019b), Hamamelis mollis (Wang et al. 2017), Nelumbo nucifera (Chen et al. 2018), Nelumbo sp. (Wang et al. 2017), Neolitsea sp. (Wang et al. 2017), Oryza sativa (Wang et al. 2017), Osmanthus fragrans (Wang et al. 2017), Osmanthus sp. (Wang et al. 2017), Pennisetum americanum (Hashemian Kalati et al. 2014), Pentactina rupicola (Wang et al. 2017), Photinia serrulata (He et al. 2019a), Rhododendron simiarum (Wang et al. 2017), Rhododendron sp. (Wang et al. 2017), Rubus reflexus (Wang et al. 2017), Rubus sp. (Wang et al. 2017), Symplocos zizyphoides (Wang et al. 2017), Ternstroemia sp. (Wang et al. 2017), Triticum aestivum (Eken et al. 2016, Barkat et al. 2016), Tutcheria microcarpa (Wang et al. 2017), Vaccinium corymbosum (Zhang et al. 2019b), Vitis vinifera (Lorenzini et al. 2016), Phyllostachys heterocycla (this study).

Material examined - Thailand, Chiang Rai Province, Doi Mae Salong, on fallen dead leaves of Phyllostachys heterocycla (Poaceae), 18 May 2016, J. F Li, DMS12-2 (MFLU 17-0199, new host and geographical record), living culture at KUMCC 16-0041.

GenBank numbers - ITS: MN648321, LSU: MN64832, SSU: MN64831.

Notes - Nigrospora oryzae has a cosmopolitan distribution and a wide host range (Wang et al. 2017, Raza et al. 2019). In this study, our collection (KUMCC 16-0041) collected from Doi Mae Salong, Thailand, clustered in a single clade with $N$. oryzae (Fig. 151) and their morphological characteristics were similar to those of $N$. oryzae. Therefore, we regard this isolate (KUMCC 160041) as N. oryzae as the first report.

Nigrospora sphaerica )Sacc.( E.W. Mason, Trans. Brit. Mycol. Soc. 12: 158. )1927( Fig. 153

Facesoffungi number: FoF06599

Hyphae smooth, hyaline, branched, septate, 3-8 $\mu \mathrm{m}$ diameter Sexual morph: Undetermined. Asexual morph: Conidiophores micronematous or semi-macronematous, septate, branched, flexuous or straight, hyaline to pale brown, smooth, 3-8 $\mu \mathrm{m}$ thick; hyaline vesicles usually surrounding the septum to delimit the conidia and their conidiogenous cells. Conidiogenous cells pale brown, holoblastic, monoblastic, determinate, subsphaerical, 7-11 $\mu$ m diameter $) \bar{x}=6, \mathrm{n}=10$ (. Conidia formed abundantly, solitary, globose or subglobose, brown, black, shining, smooth, aseptate, $16-21 \mu \mathrm{m}$ diameter $) \bar{x}=18, \mathrm{n}=20$ (.

Culture characteristics - On PDA, colonies floccose, margin circular. Colonies initially white, becoming black with age, reaching $8 \mathrm{~cm}$ diameter in $7 \mathrm{~d}$ at $25^{\circ} \mathrm{C}$.

Known distribution )based on molecular data( - China and Pakistan )Alam et al. 2017a, Wang et al. 2017(, Russia )this study(.

Known hosts )based on molecular data( - Actinidia sp., Camellia sp., Citrus sp., Cleyera japonica, Deutzia sp., Harpullia longipetala, Musa paradisiaca, Nelumbo sp., Rhododendron arboreum, Rosa sp., Saccharum sp., Sesamum indicum )Zhao et al. 2014, Wang et al. 2017, Alam et al. 2017a, Cui et al. 2018(, Hemerocallis sp. )this study(.

Material examined - Russia, Rostov region, Rostov-on-Don, Zhelezhodorozhny City District, street flowerbed, on dead floriferous shoot of Hemerocallis fulva (Asphodelaceae), 11 October 2017, T. Bulgakov T2279-2 )MFLU 18-0439, new host and geographical record(, culture, MFLUCC 18-0895.

GenBank Numbers - ITS: MN788673, tef1: MN995332.

Notes - We were unable to find any report of Nigrospora sphaerica from Hemerocallis sp. )Farr \& Rossman 2020(. Therefore, herein we provide the first report of $N$. sphaerica from Hemerocallis from Russia. 


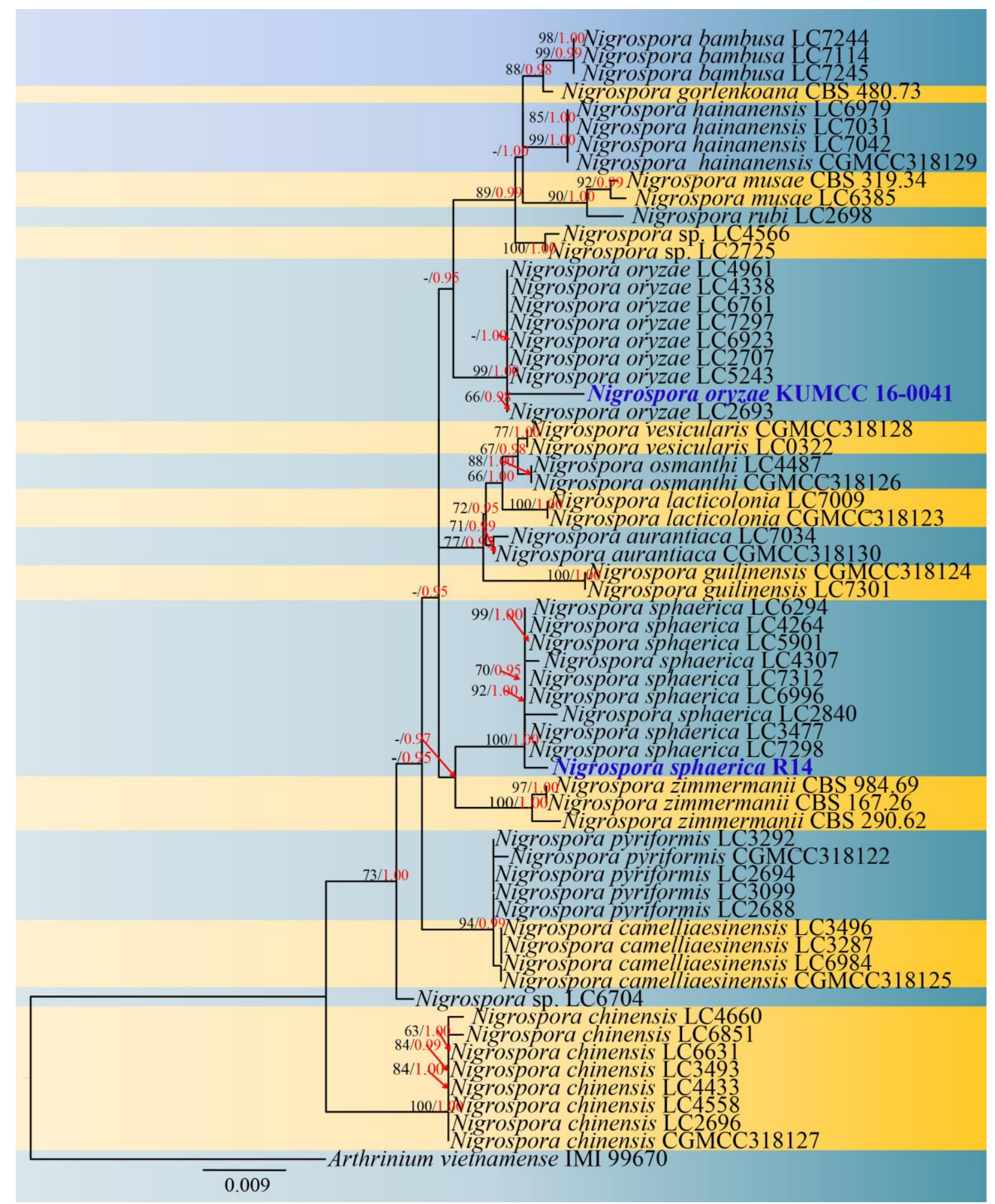

Fig. 151 - Phylogram generated from the best scoring of the RAxML tree based on combined ITS, tefl and TUB sequenced data of taxa in Nigrospora. Arthrinium vietnamense (IMI 99670) was selected as the outgroup taxon. The best RAxML tree with a final likelihood value of -3487.815676 is presented. The matrix had 227 distinct alignment patterns, with $46.27 \%$ undetermined characters or gaps. Estimated base frequencies were as follows: $\mathrm{A}=0.242574, \mathrm{C}=0.243318, \mathrm{G}=0.252046, \mathrm{~T}$ $=0.262062 ;$ substitution rates $\mathrm{AC}=1.131584, \mathrm{AG}=1.129186, \mathrm{AT}=0.645273, \mathrm{CG}=0.825588, \mathrm{CT}$ $=4.542097, \mathrm{GT}=1.000000$; gamma distribution shape parameter $\alpha=0.020014$. Bootstrap support for maximum likelihood (ML, black) equal to or greater than $60 \%$ and BYPP (red) equal to or greater than 0.95 are given above or below the nodes. Newly generated sequences are indicated in blue. 


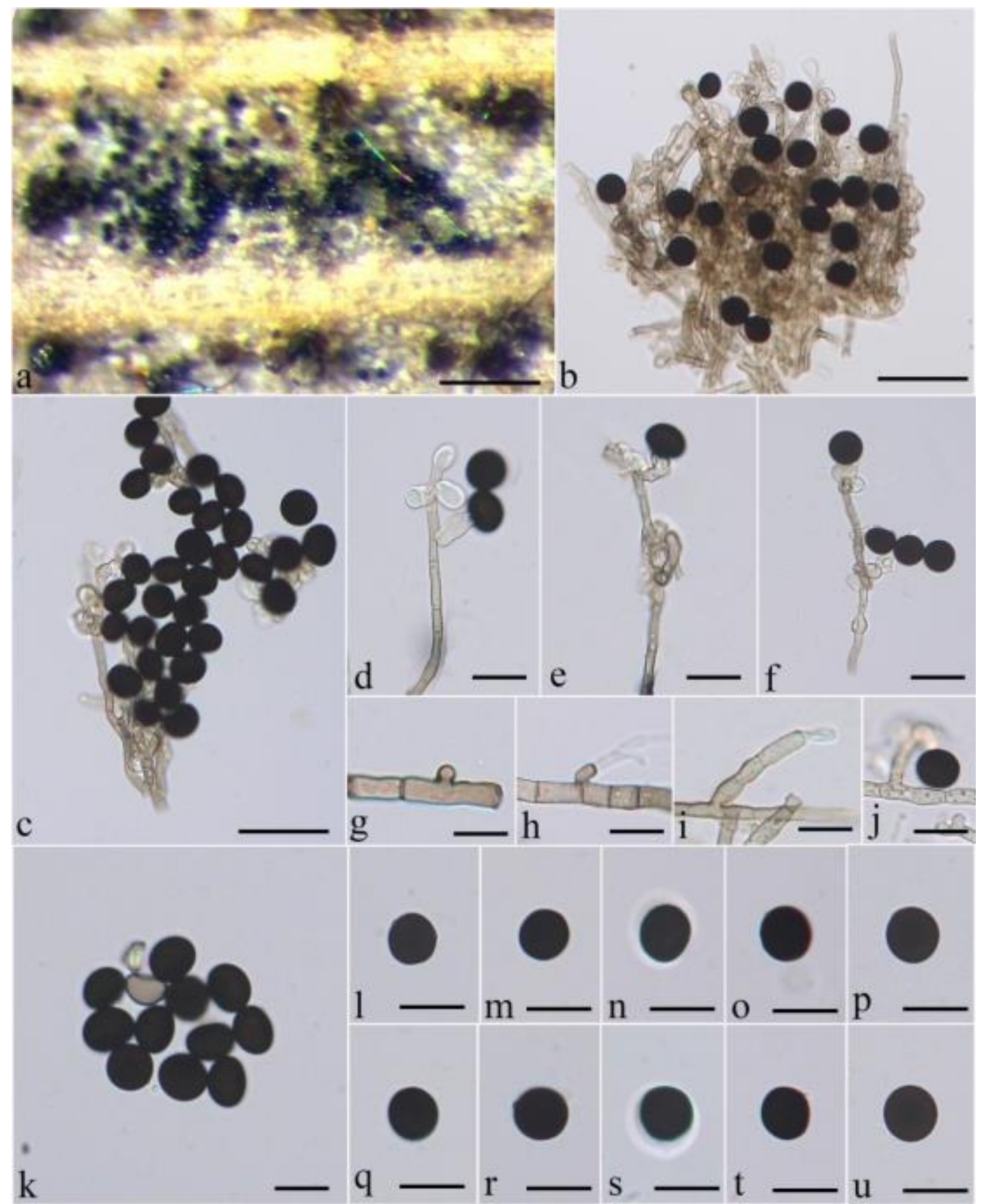

Fig. 152 - Nigrospora oryzae (MFLU 17-0199, new host and geographical record). a. Colonies on bamboo leaves. b, c. Conidia and conidiophores. $\mathrm{d}-\mathrm{j}$ Conidiophores with conidiogenous cells. $\mathrm{k}-\mathrm{u}$ Conidia. Scale bars: $\mathrm{a}=200 \mu \mathrm{m}, \mathrm{b}=50 \mu \mathrm{m}, \mathrm{c}=20 \mu \mathrm{m}, \mathrm{d}-\mathrm{f}=10 \mu \mathrm{m}, \mathrm{g}-\mathrm{u}=5 \mu \mathrm{m}$.

\section{Beltraniaceae Nann.}

Beltraniaceae is based on Beltrania and similar genera, which are characterized by biconic, lageniform to navicular conidia, with or without a hyaline band, and with or without swollen separating cells (Seifert et al. 2011, Lin et al. 2017a). Conidiophores separate, or arise from, basal cells of setae, with or without radially lobed basal cells (Seifert et al. 2011, Lin et al. 2017a). In this study, we introduce two novel species, Beltrania dushanensis and Beltraniella brevis.

Beltrania Penz., Nuovo G. bot. ital. 14(2): 72 (1882)

Fourteen species are accepted in Beltrania (Lin et al. 2017a, Tibpromma et al. 2018b). Some 
Beltrania species are plant pathogens, e.g., B. pseudorhombica was reported as the causal agent of pistachio leaf and fruit spot in Arizona, United States (Lichtemberg et al. 2019).
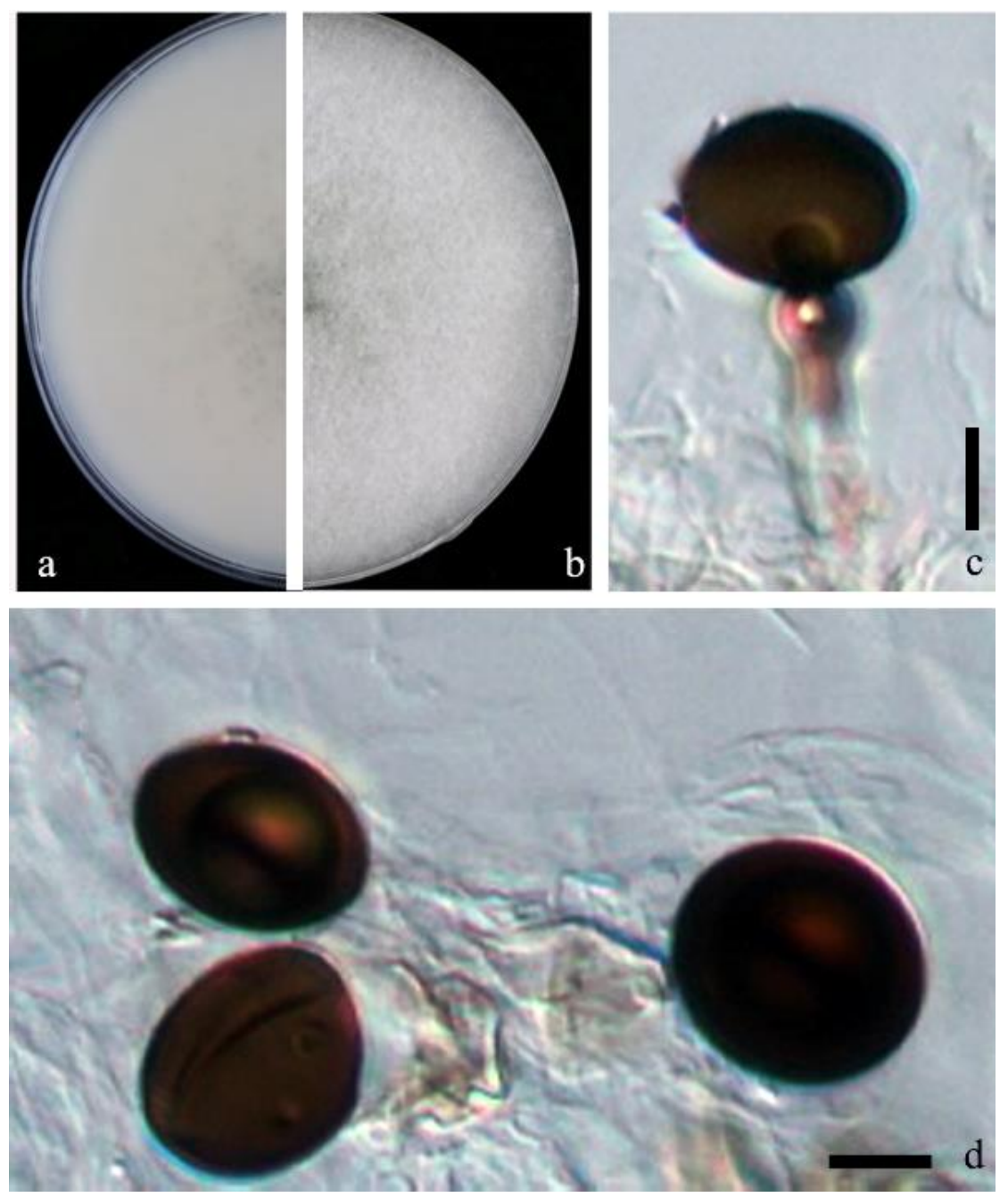

Fig. 153 - Nigrospora sphaerica (MFLU 18-0439, new host and geographical record). a-b Upper surface and reverse overview of culture on PDA. c Conidiogenous cell giving rise to conidia. $\mathrm{d}$ Conidia. Scale bars: c, $\mathrm{d}=10 \mu \mathrm{m}$.

Beltrania dushanensis C.G. Lin, Jian K. Liu \& K.D. Hyde, sp. nov.

Fig. 155

Index Fungorum Number: IF556728; Facesoffungi number: FoF06220

Etymology - Referring to Dushan, the type locality of this species

Holotype - MFLU 19-2252

Saprobic on decaying seed. Sexual morph: Undetermined. Asexual morph: Colonies on plant substrate effuse, dark brown, velutinous. Mycelium mostly immersed in the substratum. Setae numerous, erect, straight or slightly flexuous, unbranched, thick-walled, smooth, pale brown to dark brown, 180-260 $\mu \mathrm{m}$ long, $4-8 \mu \mathrm{m}$ wide at the base, tapering to a pointed apex, arising from a dark brown, swollen, radially lobed basal cell, 10-17 $\mu \mathrm{m}$ diameter Conidiophores macronematous, single or in small groups, straight or flexuous, septate, smooth, thick-walled, cylindrical or clavate, pale brown, 10-45 $\mu \mathrm{m}$ long, 4-7 $\mu \mathrm{m}$ wide, arising from basal cells of setae or from separate dark brown, swollen, radially lobed cells, $8-18.5 \mu \mathrm{m}$ diameter, often reduced to conidiogenous cells. Conidiogenous cells polyblastic, integrated, terminal, sympodial, denticulate, cylindrical, clavate, pale brown, smooth, 6.5-24 $\mu \mathrm{m}$ long, 4.3-8.5 $\mu \mathrm{m}$ wide. Separating cells obovoid, ellipsoidal, smooth, thin-walled, hyaline, 7.9-12.7 $\mu \mathrm{m}(\bar{x}=10.5 \mu \mathrm{m}, \mathrm{n}=23)$ long, 4.4-5.8 $\mu \mathrm{m}(\bar{x}=5.1 \mu \mathrm{m}, \mathrm{n}=$ 23) wide in the broadest part. Conidia arise directly from conidiogenous cells or from separating cells, acrogenous, simple, smooth, dry, straight, biconic, rostrate, appendiculate, pale brown with a 
subhyaline equatorial transverse band, 21.4-27.9 $\mu \mathrm{m}(\bar{x}=24.3 \mu \mathrm{m}, \mathrm{n}=30)$ long, 8.9-12.6 $\mu \mathrm{m}(\bar{x}=$ $10.4 \mu \mathrm{m}, \mathrm{n}=30$ ) wide in the broadest part; appendage $6.3-12.1 \mu \mathrm{m}$ long, $0.7-1.6 \mu \mathrm{m}$ wide at the base, tapering to a pointed apex.

Material examined - China, Guizhou Province, Qiannan Buyi Miao Autonomous Prefecture, Dushan County, Guizhou Zilinshan National Forest Park (Shengou District), on decaying seeds, 6 July 2018, Chuan-Gen Lin, DS 1-5 (MFLU 19-2252, holotype); ibid. (HKAS 105107, isotype); extype living culture GZCC18-0020.

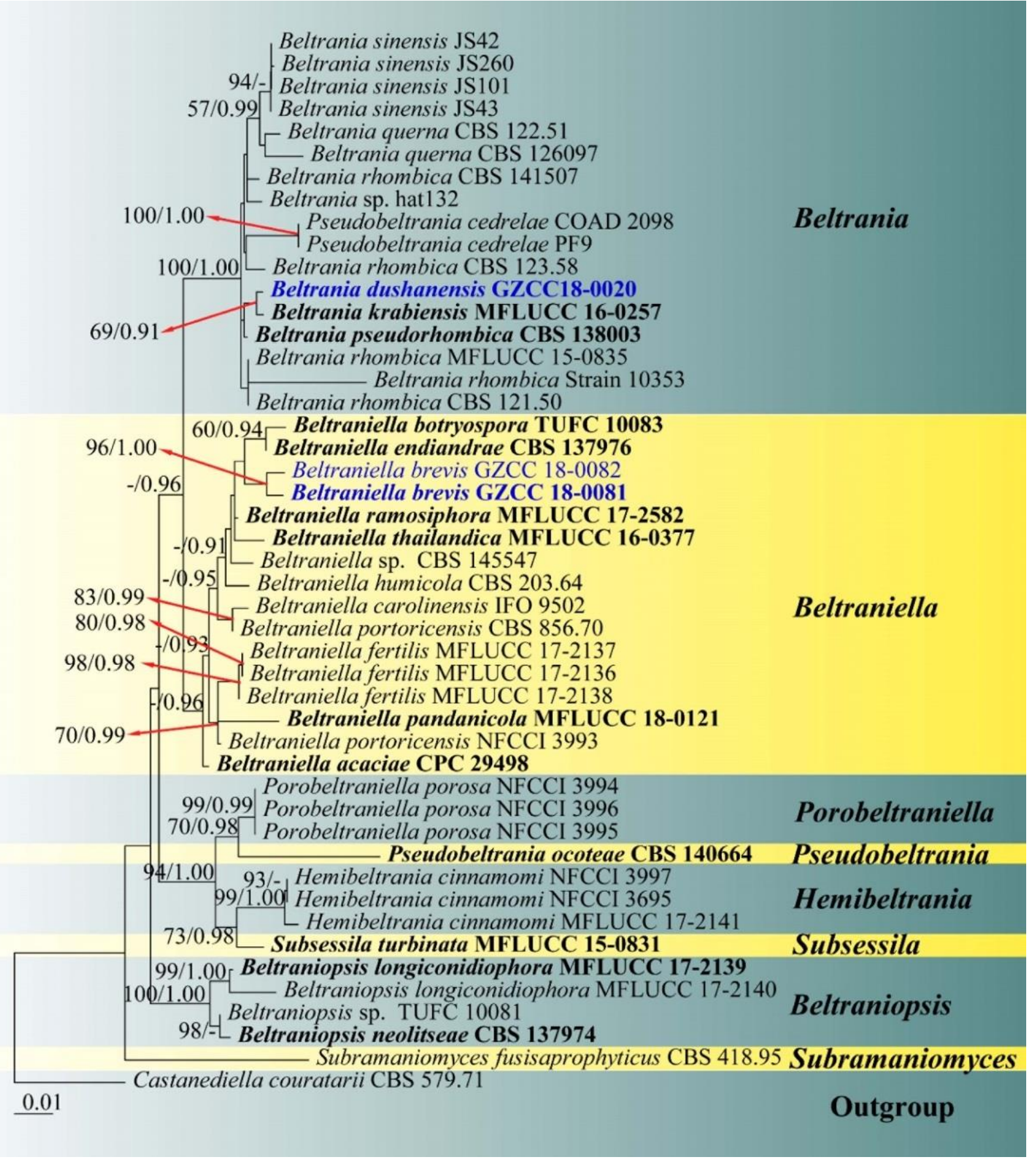

Fig. 154 - Phylogram generated from maximum likelihood analysis based on combined LSU and ITS sequence data. Forty-seven strains are included in the combined gene analyses comprising 1456 characters after alignment (856 characters for LSU, 600 characters for ITS). Castanediella couratarii (CBS 579.71) is used as the outgroup taxon. The tree topology derived from the Bayesian analysis was similar to that derived from the maximum likelihood analysis. The best RaxML tree with a final likelihood value of -4888.682999 is presented. The matrix had 384 distinct 
alignment patterns, with $11.82 \%$ undetermined characters or gaps. Estimated base frequencies were as follows: $\mathrm{A}=0.251976, \mathrm{C}=0.212516, \mathrm{G}=0.269311, \mathrm{~T}=0.266196$; substitution rates $\mathrm{AC}=$ 1.482978, $\mathrm{AG}=2.788030, \mathrm{AT}=1.703126, \mathrm{CG}=0.797716, \mathrm{CT}=6.027412, \mathrm{GT}=1.000000$; gamma distribution shape parameter $\alpha=0.610669$. Bootstrap values for maximum likelihood equal to or greater than 50 and Bayesian posterior probabilities equal or greater than 0.95 are placed near the branches. Ex-type strains are in bold and black. The newly generated sequences are indicated in blue.

GenBank numbers - ITS: MN252875, LSU: MN252882.

Notes - Beltrania dushanensis is most similar to B. rhombica Penz. in having biconic, symmetrical, light brown conidia with a V-shaped proximal end, but differs by its short conidiophores which are often reduced to conidiogenous cells (Pirozynski 1963, Morelet 2001). In addition, $B$. dushanensis and $B$. rhombica can be recognized as phylogenetically distinct lineages (Fig. 154). Phylogenetically, B. dushanensis shows a close relationship with B. krabiensis Tibpromma \& K.D. Hyde (Fig. 154) since they clustered and formed a monophyletic group, however, they can be recognized as distinct lineages. Beltrania dushanensis differs from $B$. krabiensis by its shorter conidiophores and larger conidia (Tibpromma et al. 2018b).

Beltraniella Subram., Proc. Indian Acad. Sci., Sect. B 36: 227 (1952)

Twenty-six species are accepted in Beltraniella (Crous et al. 2016b, Lin et al. 2017b, Tibpromma et al. 2018, Crous et al. 2019a). However, only 11 species of Beltraniella have molecular data. A new species of Beltraniella from China is described based on phylogenetic analyses and morphological characters.

Beltraniella brevis C.G. Lin, Jian K. Liu \& K.D. Hyde, sp. nov.

Figs 156, 157

Index Fungorum Number: IF556729; Facesoffungi number: FoF06219

Etymology - Referring to the short conidiophores

Holotype - MFLU 19-2254

Saprobic on decaying leaves. Sexual morph: Undetermined. Asexual morph: Colonies on plant substrate effuse, thin, pale brown. Mycelium mostly immersed in the substratum. Setae numerous, erect, arising from radially lobed basal cells, straight or flexuous, unbranched, single or in small groups, thick-walled, verrucose, dark brown, 89-251 $\mu \mathrm{m}$ long, 4.5-10.5 $\mu \mathrm{m}$ wide at the base, tapering to a pointed apex, arising from a dark brown, swollen, radially lobed basal cell, 15.5$27.8 \mu \mathrm{m}$ diameter, imperfect setae single, straight, septate, verrucose, thick-walled, dark brown at the base and paler at the apex, up to $310 \mu \mathrm{m}$ long, swollen at the base, slightly tapering to a pointed apex. Conidiophores macronematous, short, simple or branched, septate, sometimes reduced to conidiogenous cells, smooth-walled, swollen at the base, subhyaline to pale brown, thin-walled, arising from basal cells of setae or from separate, 6-16 $\mu \mathrm{m}$ long, 3.7-7 $\mu \mathrm{m}$ wide. Conidiogenous cells polyblastic, integrated, determinate, terminal, ampulliform, cylindrical, oblong, hyaline to subhyaline, smooth, 4.9-13.7 $\mu \mathrm{m}(\bar{x}=8.3 \mu \mathrm{m}, \mathrm{n}=29)$ long, 3.5-6.8 $\mu \mathrm{m}(\bar{x}=5.3 \mu \mathrm{m}, \mathrm{n}=29)$ wide at the base. Separating cells fusiform, thin-walled, smooth, hyaline to subhyaline, 1-denticulate at each end, 11-18 $\mu \mathrm{m}$ long, 3.4-4.1 $\mu \mathrm{m}$ wide in the broadest part. Conidia arise directly from conidiogenous cells or from separating cells, aggregated, acrogenous, simple, dry, straight, smooth, thin-walled, turbinate to pyriform, rostrate to pointed at proximal end, truncate at distal end, hyaline with a hyaline supraequatorial transverse band, 20-26.5 $\mu \mathrm{m}\left(x^{-}=22.3 \mu \mathrm{m}, \mathrm{n}=30\right)$ long, 4.5-7.2 $\mu \mathrm{m}$ $(\bar{x}=5.9 \mu \mathrm{m}, \mathrm{n}=30)$ wide in the broadest part.

Material examined - China, Guizhou Province, Qiannan Buyi Miao Autonomous Prefecture, Dushan County, Guizhou Zilinshan National Forest Park (Shengou District), unnamed road, on decaying leaves, 6 July 2018, Chuan-Gen Lin, DS 2-21 (MFLU 19-2254, holotype); ibid., HKAS 105159, isotype); ex-type living culture GZCC 18-0081; ibid, DS 2-23 (MFLU 19-2253, paratype, HKAS 105160, isoparatype); ex-paratype living culture GZCC 18-0082.

GenBank numbers - ITS: MN252876, MN252877, LSU: MN252883, MN252883. 

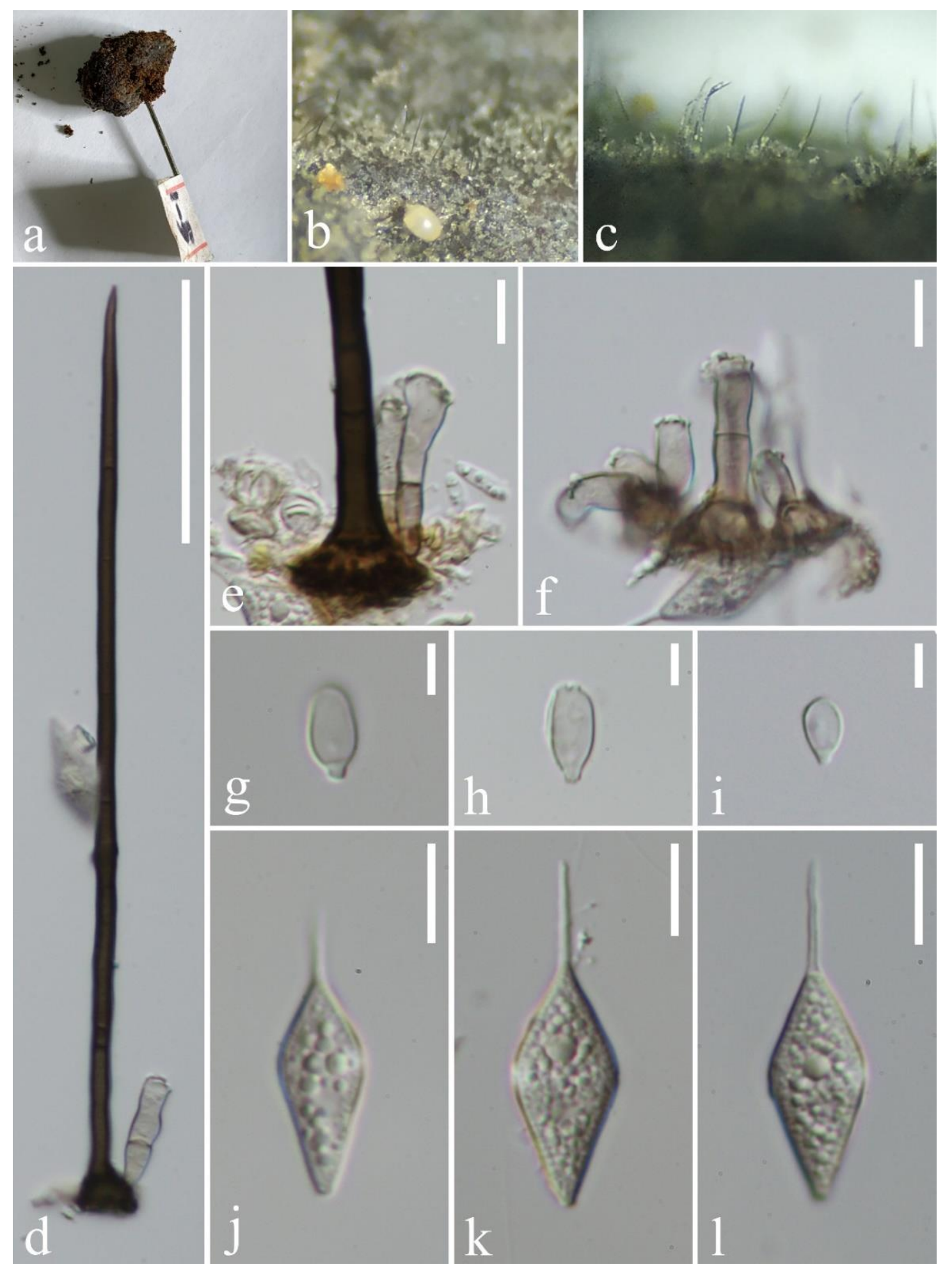

Fig. 155 - Beltrania dushanensis (MFLU 19-2252, holotype). a Host material. b, c Conidiophores on leaf surface. d Setae, conidiophores and conidiogenous cell. e, f Conidiogenous cells. $\mathrm{g}-\mathrm{i}$ Separating cells. $\mathrm{j}-1$ Conidia. Scale bars: $\mathrm{d}=50 \mu \mathrm{m}, \mathrm{e}, \mathrm{f}, \mathrm{j}-\mathrm{l}=10 \mu \mathrm{m}, \mathrm{g}-\mathrm{i}=5 \mu \mathrm{m}$.

Notes - Beltraniella brevis is most similar to Bel. portoricensis in having straight, verrucose setae and short non-setiform conidiophores arising from radially lobed basal cells, polyblastic conidiogenous cells, swollen separating cells, and turbinate, appendiculate conidia (Pirozynski 1963, Shirouzu et al. 2010). However, Bel. brevis differs from Bel. portoricensis by its hyaline conidia, whereas conidia are subhyaline to dilute yellow-olive in Bel. portoricensis (Pirozynski 1963, Shirouzu et al. 2010). In the tree generated from maximum likelihood analysis based on a 
combined LSU and ITS sequence data (Fig. 154), Bel. brevis grouped together and formed a monophyletic group with $96 \%$ ML bootstrap support and 100\% Bayesian posterior probabilities within Beltraniella.

\section{Sporocadaceae Corda}

Sporocadaceae species have acervular or pycnidial conidiomata, and hyaline, or pale brown to dark brown, cylindrical to subcylindrical or fusiform, multi-septate conidia, usually with appendages at one or both ends (Nag Raj 1993, Liu et al. 2019). The sexual morph of this family is characterized by perithecial ascomata with simple, rarely branched paraphyses, unitunicate, 8spored, cylindrical asci with $\mathrm{J}+$, apical ring, and pale, yellow to dark brown, fusiform or ellipsoidal and septate ascospores (Jaklitsch et al. 2016a, Liu et al. 2019). The taxonomy and phylogeny of Sporocadaceae species were by Jaklitsch et al. (2016a) and Liu et al. (2019). Approximately 30 well-supported monophyletic genera were included in Sporocadaceae (Liu et al. 2019).

\section{Bartalinia Tassi}

Bartalinia is characterized by pycnidial, unilocular to multi-loculate, glabrous conidiomata, and cylindrical to subcylindrical or fusiform, 3-4-euseptate conidia with a hyaline apical cell, bearing attenuated, flexuous, divergent, apical appendages with 2-4 branches and a tubular, single, unbranched, exogenous basal appendage (Nag Raj 1993, Jeewon et al. 2002). This genus has been assigned to Amphisphaeriaceae (Jeewon et al. 2003, Crous et al. 2014a) and Bartaliniaceae (Senanayake et al. 2015, Wijayawardene et al. 2016). Jaklitsch et al. (2016a) and Liu et al (2019) revived Sporocadaceae and reduced Bartaliniaceae to a synonym of this family.

Bartalinia robillardoides Tassi, Bulletin Labor. Orto Bot. de R. Univ. Siena 3: 145 (1900)

Facesoffungi number: FoF06861

Fig. 159

Saprobic on dead bark of Picea excelsa. Sexual morph: Undetermined. Asexual morph: Conidiomata 100-200 $\mu \mathrm{m}$ diameter, 100-150 $\mu \mathrm{m}$ high, brown, stromatic, pycnidial, scattered to gregarious, initially semi-immersed, ultimately erumpent, globose to subglobose, unilocular or multi-loculate, lacking an ostiole, dehiscing by an irregular break in the apical wall, glabrous. Conidioma wall 30-60 $\mu \mathrm{m}$ wide, composed of thick-walled, brown cells of textura angularis in the outer layers, gradually merging with hyaline cells in the inner layers. Conidiophores arising all around the cavity of the conidiomata, cylindrical, hyaline, branched, septate, smooth-walled. Conidiogenous cells 1-4 $\mu \mathrm{m}$ wide, hyaline, holoblastic, ampulliform to cylindrical, integrated, determinate, smooth-walled. Conidia $8-15 \times 1-2 \mu \mathrm{m}(\bar{x}=10 \times 1.5 \mu \mathrm{m} ; \mathrm{n}=50)$, cylindrical to subcylindrical, 4-septate, slightly constricted at septa, bearing appendages at both ends; basal cell pale brown, obconic with obtuse base, median cells 3, subcylindrical, thick-walled, apical cell hyaline, conical; apical appendage 5-10 $\mu \mathrm{m}$ long, hyaline, 3-branched, tubular, filiform, flexuous; basal appendage 1.5-3 $\mu \mathrm{m}$ long, single, filiform, unbranched, centric to eccentric.

Culture characteristics - Colonies fast growing, reaching $50 \mathrm{~mm}$ diameter after one week at $25{ }^{\circ} \mathrm{C}$, circular, whitened in first three days, after two weeks becoming pale grey, flattened, felt-like, dense, aerial, surface smooth with crenate edge, filamentous, reverse whitened to pale brown in a week, finally brown, sporulation after one month.

Known distribution (based on molecular data) - Australia, Italy, Netherlands, South Africa, Thailand (Crous et al. 2014a, Wijayawardene et al. 2016, Liu et al. 2019), Italy (this study)

Known hosts (based on molecular data) - Cupressus lusitanica (Cupressaceae), Eucalyptus sp. (Myrtaceae), Leptoglossus occidentalis (Coreidae), Poa sp. (Poaceae) Scadoxus puniceus (Amaryllidaceae) (Crous et al. 2014a, Wijayawardene et al. 2016, Liu et al. 2019) Picea excels (this study).

Material examined - Italy, Province of Forlì-Cesena, Santa Sofia, Spescia, on dead cone of Picea excels (Pinaceae), 3 May 2014, E. Camporesi, IT1854 (MFLU 19-2560, new host record), living culture MFLUCC 15-0133, KUN, HKAS 101685. 
GenBank Numbers - LSU: MN660234, ITS: MN688213, SSU: MN688209, tef1: MN683865, RPB2: MN683867.

Notes - Our collection fits well with the description of $B$. robillardoides. The sequence similarity between our collection and B. robillardoides (CPC 25385) is 100\% (881/881) in LSU, $99 \%(549 / 552)$ in ITS, 100\% (832/832) in RPB2 (Fig. 158). Our collection on Picea excels is regarded as a new host record.
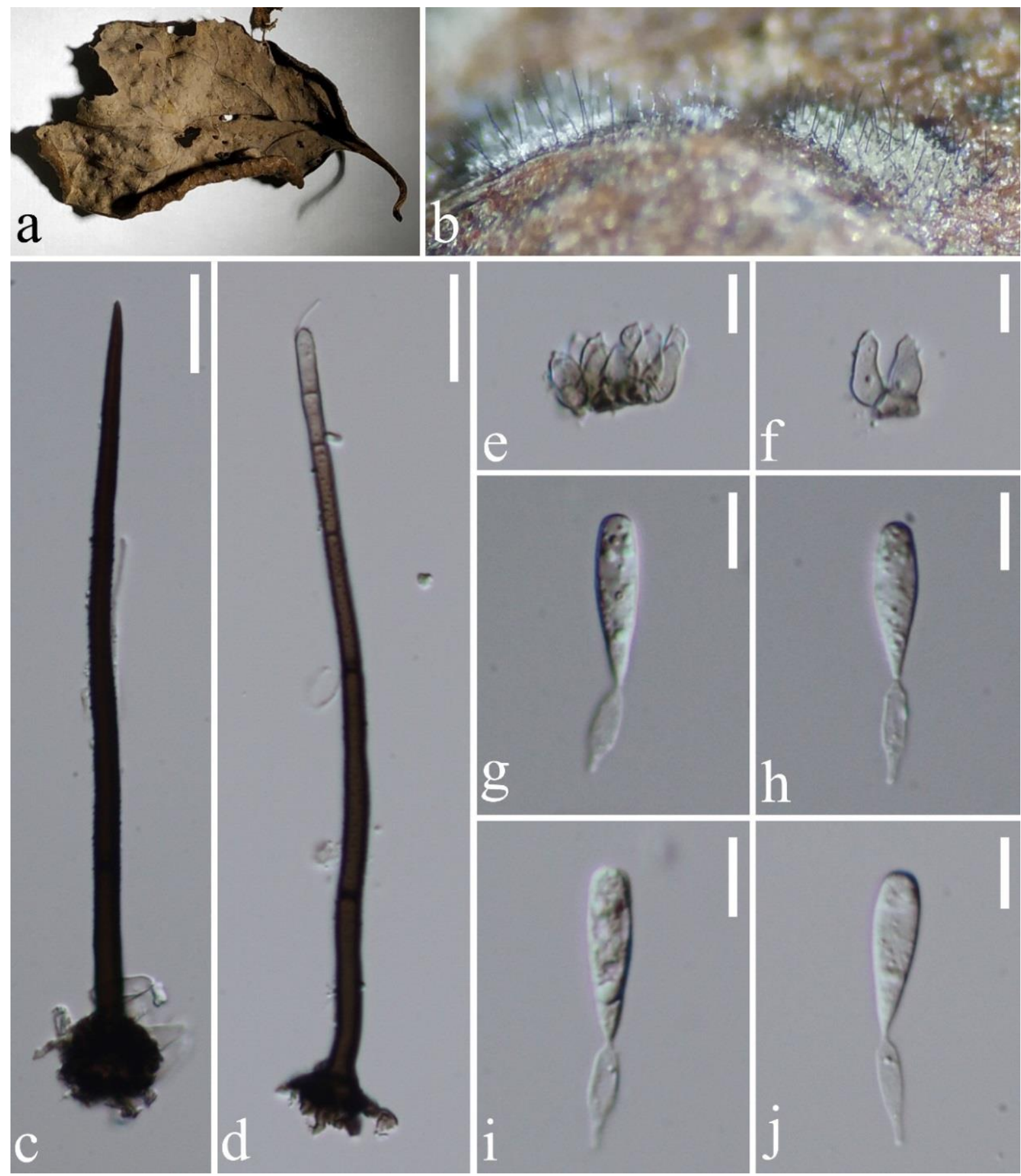

Fig. 156 - Beltraniella brevis (MFLU 19-2254, holotype). a Host material. b Conidiophores on leaf surface. c Setae with short conidiophores. d Imperfect setae. e, f Conidiogenous cells. $\mathrm{g}-\mathrm{j}$ Conidia with separating cells. Scale bars: $\mathrm{c}, \mathrm{d}=20 \mu \mathrm{m}, \mathrm{e}-\mathrm{j}=10 \mu \mathrm{m}$.

\section{Discosia Lib.}

Discosia was introduced by Libert (1837) with Discosia artocreas selected as the lectotype by Vanev (1991). Senanayake et al. (2015) introduced Discosiaceae to accommodate Adisciso, 
Discosia, Discostroma, Immersidiscosia, Sarcostroma, and Seimatosporium but Jaklitsch et al. (2016a) treated Discosiaceae as a synonym of Sporocadaceae as the family has good phylogenetic support. The generic description of Discosia, coupled with the updated morphology as well as the phylogenetic relationship based ITS sequences were reviewed by Liu et al. (2019).
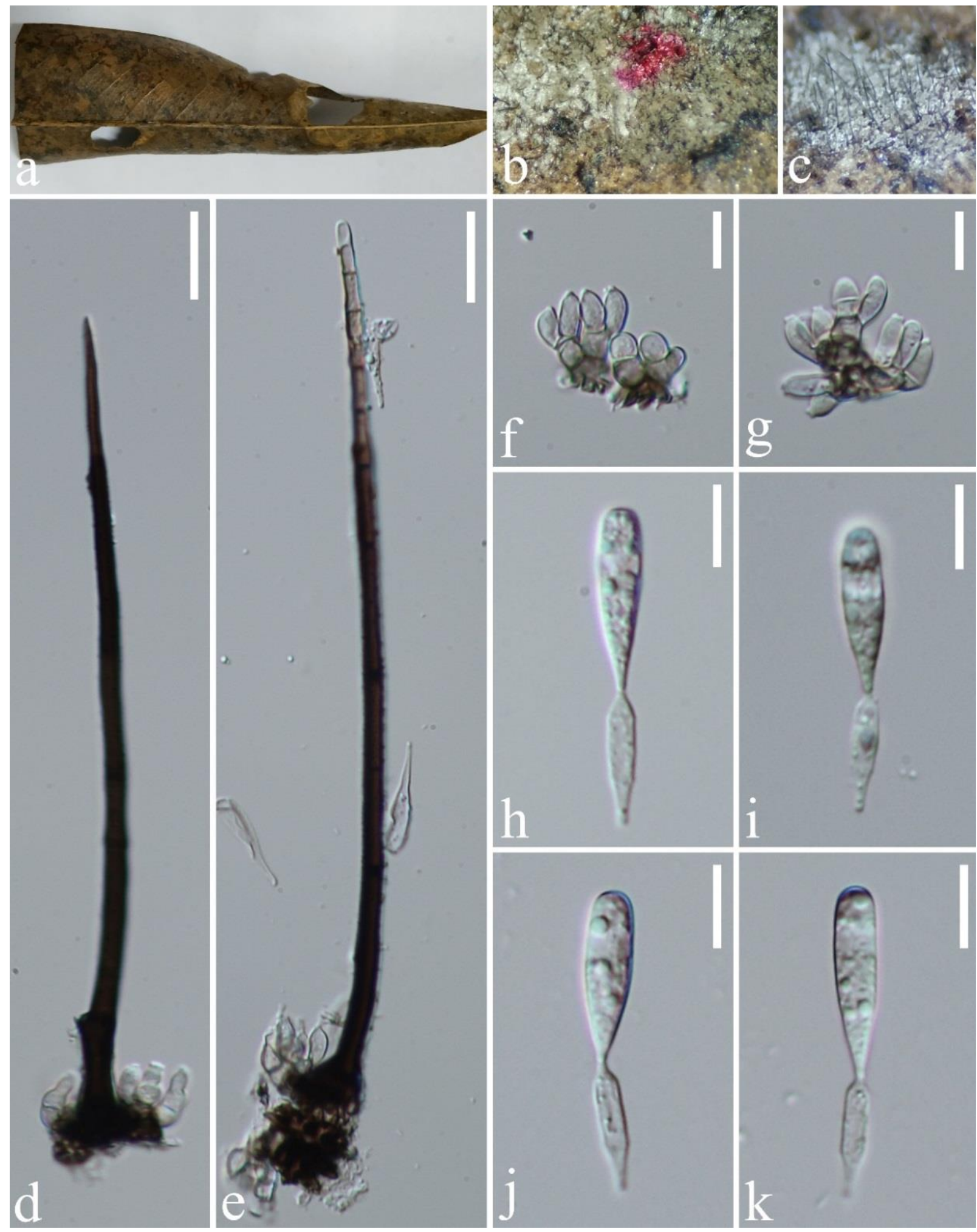

Fig. 157 - Beltraniella brevis (MFLU 19-2253, paratype). a Host material. b, c Conidiophores on leaf surface. d Setae with short conidiophores. e Imperfect setae with short conidiophores. $\mathrm{f}, \mathrm{g}$ Conidiogenous cells. $\mathrm{h}-\mathrm{k}$ Conidia with separating cells. Scale bars: $\mathrm{d}, \mathrm{e}=20 \mu \mathrm{m}, \mathrm{f}-\mathrm{k}=10 \mu \mathrm{m}$. 


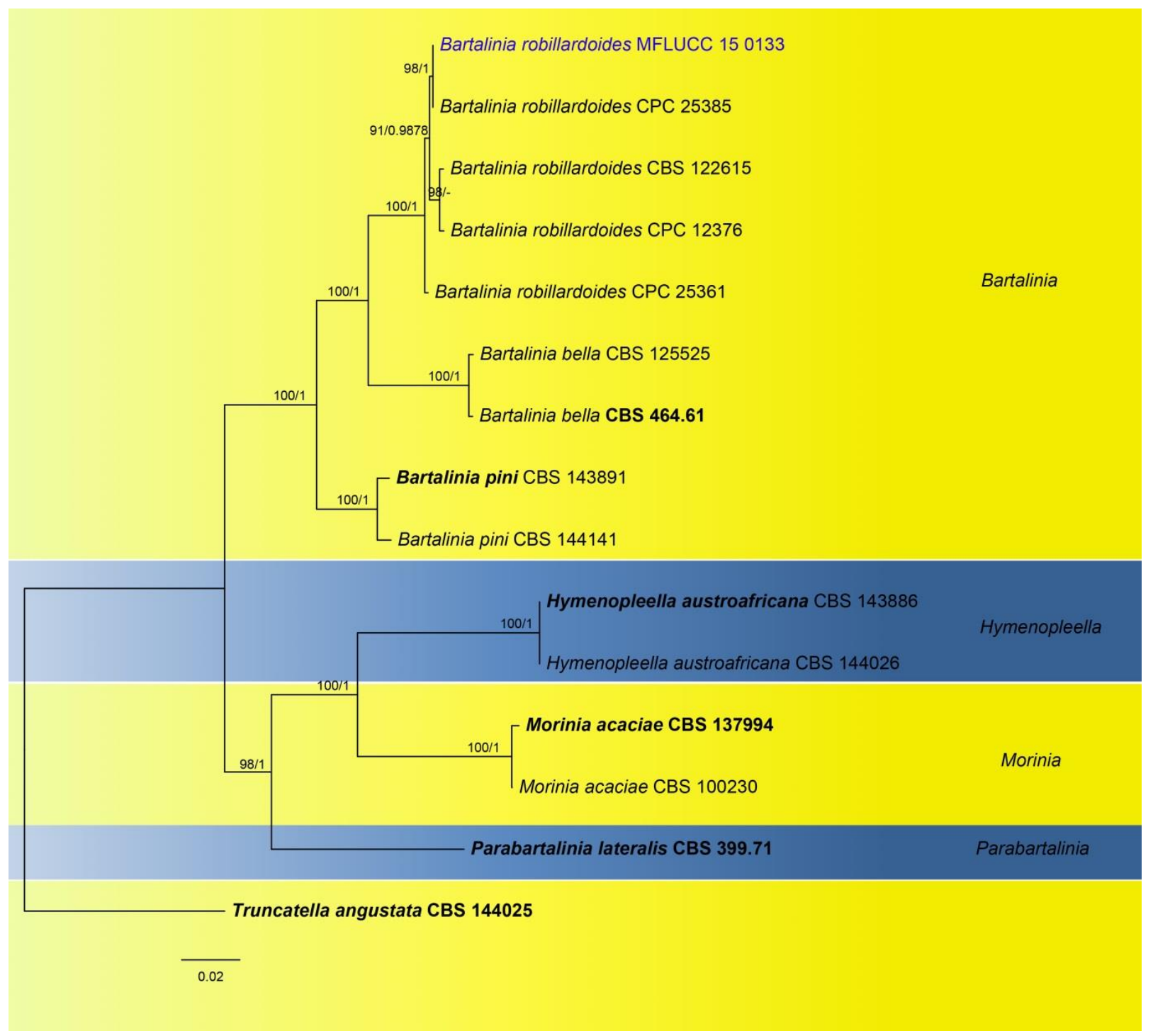

Fig. 158 - Phylogram of Bartalinia and allied genera generated from maximum likelihood analysis based on combined LSU, ITS, tef1, RPB2 sequence data. Related sequences are taken from Liu et al. (2019). Fifteen strains are included in the combined analyses which comprise 2745 characters (LSU: 1-830, ITS: 831-1349, tef1: 1350-1913, RPB2: 1914-2745) after alignment. Truncatella angustata CBS 144025 is used as the outgroup taxon. The tree topology derived from the Bayesian analysis was similar to that derived from the maximum likelihood analysis. The best RaxML tree with a final likelihood value of -8567.262112 is presented. The matrix had 512 distinct alignment patterns, with $4.60 \%$ undetermined characters or gaps. Estimated base frequencies were as follows: $\mathrm{A}=0.255888, \mathrm{C}=0.231712, \mathrm{G}=0.245946, \mathrm{~T}=0.266454$; substitution rates $\mathrm{AC}=1.331043, \mathrm{AG}$ $=3.021366, \mathrm{AT}=1.507439, \mathrm{CG}=0.983008, \mathrm{CT}=7.311485, \mathrm{GT}=1.000000 ;$ gamma distribution shape parameter $\alpha=0.131325$. Bootstrap values for maximum likelihood (ML) equal to or greater than $50 \%$ and clade credibility values greater than 0.95 from Bayesian-inference analysis labeled on the nodes. The new isolate is indicated in bold and blue.

Discosia pini Heald, Mycologia 1(5): 216 (1909)

Fig. 161

Facesoffungi number: FoF07469

Saprobic on dead fern leaves. Sexual morph: Undetermined. Asexual morph: Coelomycetous. Conidiomata 140-258 $\times 45-59(\bar{x}=195 \times 54, \mathrm{n}=5) \mu \mathrm{m}$, sparse, acervular, superficial, bilocular, which is separated by interlocular wall and there are 2-3 hyaline, vertical supporting partitions inside the bigger loculus. Peridium 14-18 $\mu \mathrm{m}$ thick, brown, comprising thick-walled cells of textura angularis. Conidiogenous cells 4-6 $61-2(\bar{x}=4.5 \times 1.5, \mathrm{n}=15) \mu \mathrm{m}$, subcylindrical, 
developing from the inner stromatic tissue, monophialidic, integrated, hyaline, smooth. Conidia 14-20 × 1.9-3 $(\bar{x}=17 \times 2.7, \mathrm{n}=50) \mu \mathrm{m}$, hyaline, fusiform with rounded ends, straight or slightly curved, 3-celled and the second cell from base is longer, with 2-appendages. Appendages 4-10 $(\bar{x}=$ $8, \mathrm{n}=50) \mu \mathrm{m}$ long, hyaline, straight, filiform, with rounded ends, with a round base, producing from the attachment between the middle cell and the terminal cell of conidia.
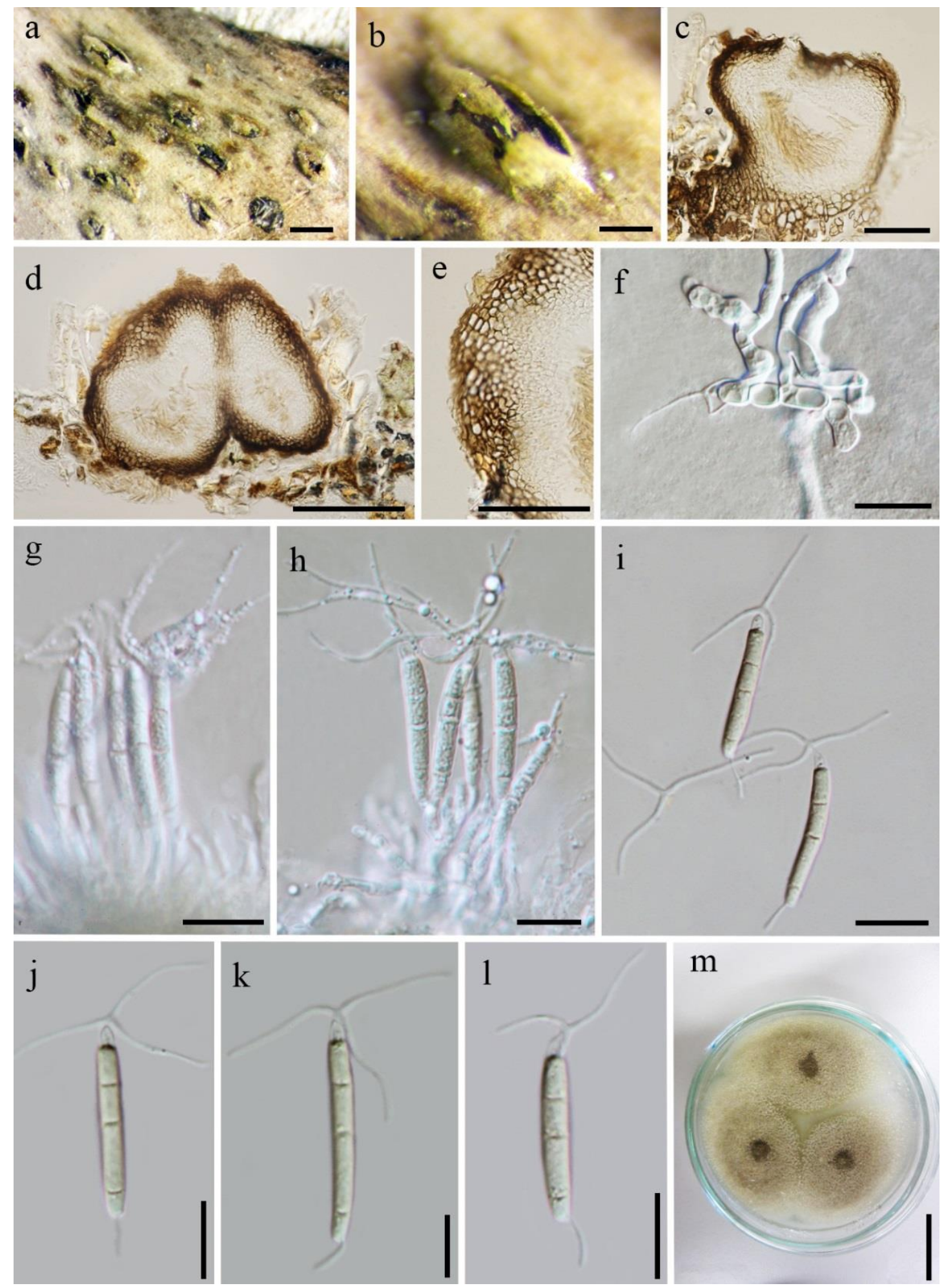

Fig. 159 - Bartalinia robillardoides (MFLU 19-2560, new host record). a, b Appearance of brown conidiomata on the host. c, d Vertical sections of conidiomata. e Section of peridium. f Germinating conidium. g, h Conidiophores, conidiogenous cells and developing conidia. $\mathrm{i}-1$ Conidia. $\mathrm{m}$ Culture on PDA. Scale bars: $\mathrm{a}=1000 \mu \mathrm{m}, \mathrm{b}-\mathrm{d}=100 \mu \mathrm{m}, \mathrm{e}=50 \mu \mathrm{m}, \mathrm{f}-\mathrm{l}=5 \mu \mathrm{m}, \mathrm{m}=25 \mathrm{~mm}$. 


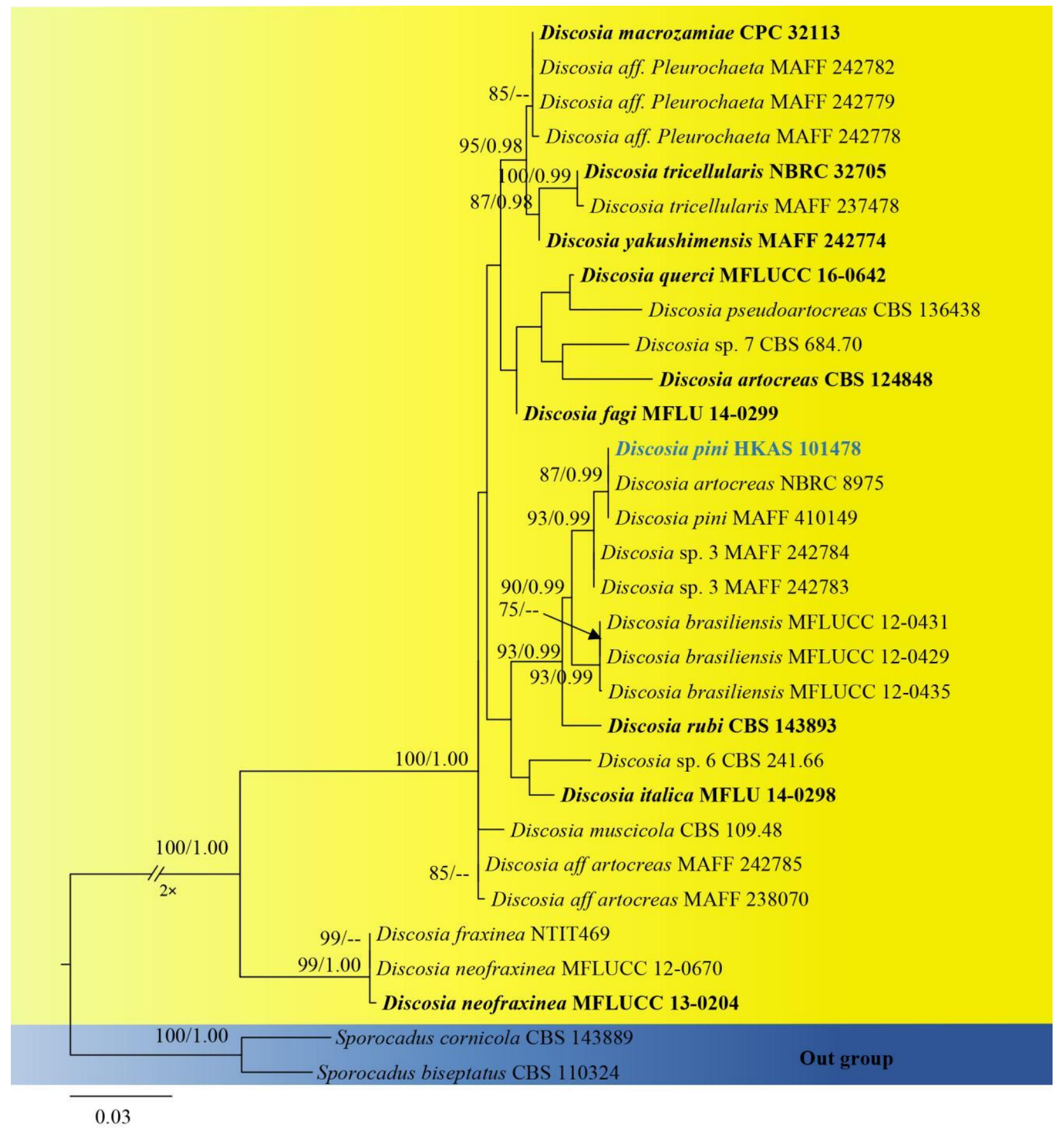

Fig. 160 - Phylogram generated from maximum likelihood analysis based on combined LSU, ITS and RPB2 sequence data. Thirty-one strains are included in the combined gene analyses comprising 2067 characters after alignment (475 characters for ITS, 784 characters for LSU, 808 characters for RPB2). Sporocadus biseptatus (CBS 110324) and Sporocadus cornicola (CBS 143889) are used as the outgroup taxa. The tree topology derived from the Bayesian analysis was similar to that derived from the maximum likelihood analysis. The best RaxML tree with a final likelihood value of 6084.123259 is presented. The matrix had 320 distinct alignment patterns, with $23.85 \%$ undetermined characters or gaps. Estimated base frequencies were as follows: $\mathrm{A}=0.251281, \mathrm{C}=$ $0.217598, \mathrm{G}=0.258884, \mathrm{~T}=0.272237$; substitution rates $\mathrm{AC}=1.723842, \mathrm{AG}=6.440596, \mathrm{AT}=$ $1.103776, \mathrm{CG}=1.039404, \mathrm{CT}=12.923260, \mathrm{GT}=1.000000$; gamma distribution shape parameter $\alpha=0.840718$. Bootstrap values for maximum likelihood equal to or greater than 70 and Bayesian posterior probabilities equal or greater than 0.90 are placed above or below the branches. The newly generated sequence is indicated in blue bold and the type strains are indicated in black bold. 

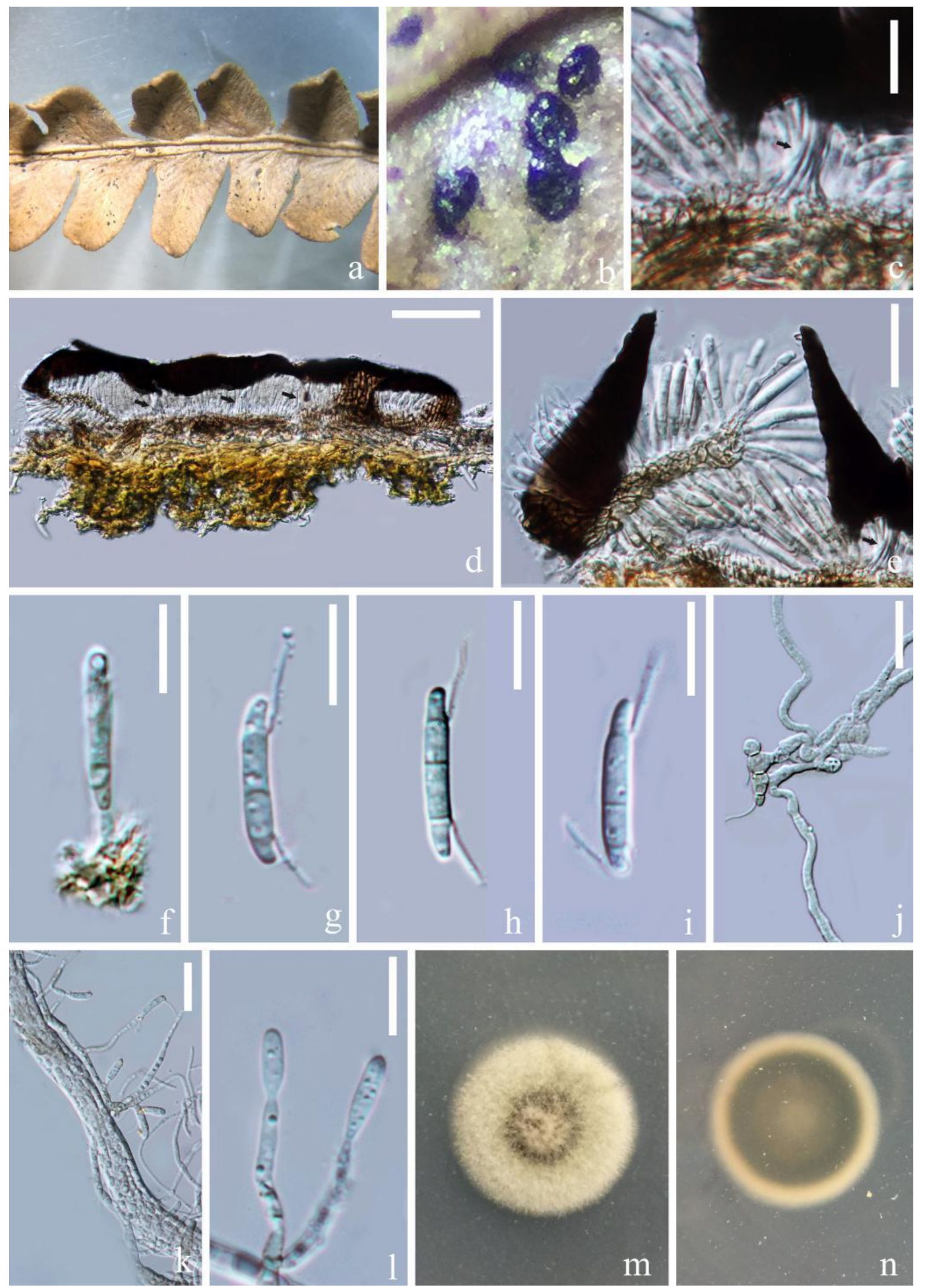

Fig. 161 - Discosia pini (HKAS 101478, new host record), a, b Conidiomata on fern leaf. c Column . d Vertical section through conidioma showing two loculi and three columns. $\mathrm{e}$, f Conidiogenous cell bearing conidia. g-i Conidia. j Germinating conidium. $\mathrm{k}, 1$ Conidiogeneous cells formed from a mycelial strand on culture. $\mathrm{m}, \mathrm{n}$ Upper and reverse review of culture after 8 days incubation on PDA. Scale bars: $d=50 \mu \mathrm{m}, \mathrm{e}, \mathrm{j}, \mathrm{k}=20 \mu \mathrm{m}$. c, $\mathrm{f}-\mathrm{i}, \mathrm{l}=10 \mu \mathrm{m}$. (The columns are indicated with black arrows, $a-i$ observed from herbarium specimen, $\mathrm{j}-\mathrm{n}$ described from culture collection). 
Culture characters - Cultures were made from germinating conidia which germinated after $1 \mathrm{~d}$ on PDA. The colonies are rapid-growing, attaining a diameter of $5 \mathrm{~cm}$ in 12 days at $22^{\circ} \mathrm{C}$. Colonies white with one grey annulation at the centre, becoming fully grey at the aging stage, velvety, circular, olive-green to grey from reverse. In vitro, the conidia form from the mycelial strand.

Material examined - China, Yunnan Province, Honghe State, Yunti County, on a decay fern leaf, 20 September 2017, De-Ping Wei, YT07 (HKAS 101478); living culture KUMCC 18-0033.

GenBank Numbers - ITS: MT012348, LSU: MT012355, RPB2: MT025047

Notes - The multiple gene tree indicates that our collection (HKAS 101478) has a close affinity to Discosia pini (MAFF 410149) and D. artocreas (NBRC 8975) with strong statistical support (87\% ML/ 0.99 PP, Fig. 160). The nucleotide comparison shows that our isolate is identical to the above two reference species within 545 bp ITS and 782 bp LSU sequences. Unfortunately, except for BT gene sequence data, other gene sequences of D. pini (MAFF 410149) and D. artocreas (NBRC 8975) are not available and we could not successfully obtain BT gene sequences for our isolate in this study. Even though the full morphology of D. pini (MAFF 410149) and D. artocreas (NBRC 8975) are not linked to any literature (Tanaka et al. 2011), our isolate is phylogenetically separated from the ex-type strain of $D$. artocreas (CBS 124848). Our collection is similar to D. artocreas (CBS 124848) in having hyaline conidia with a longer second cell from base, but differs in the position where the appendages are produced. The apical appendage of $D$. artocreas is polar and the basal appendage is located far above the conidium base (Matsushima 1975, Liu et al. 2019). However, the appendage of our collection is borne from the junction of the terminal cell and the middle cell. The type specimen of D. pini (No. 758) was originally reported on Pini ponderosae by Heald (1909), and he provided a description and hand-drawing. Our collection resembles $D$. pini in having black, glabrous, bilocular conidiomata with vertical columns inside the larger loculus and similar size of conidia (14-20 × 1.9-3 $\mu \mathrm{m}$ in our collection vs $12-20 \times 2.6 \mu \mathrm{m}$ in NEB 758 ). Our collection differs slightly from $D$. pini (NEB 758 ) however, by having round ends to the conidia and shorter appendages (4-10 $\mu \mathrm{m}$ long), while D. pini (NEB 758) has relatively pointed ends to the conidia and longer appendages (10-12 $\mu \mathrm{m}$ long). Considering our collection is most similar to the type specimen of $D$. pini (NEB 758) rather than $D$. artocreas, we report our collection as a new host record of $D$. pini from fern leaf bases based on morphological and phylogenetic evidence. However, we suggest treating the strains D. artocreas (NBRC 8975) as an undetermined species as its morphology is not known and phylogenetically it separates from its type strain. Fresh collections with further sequence data may prove this conclusion to be incorrect.

\section{Pestalotiopsis Steyaert}

Pestalotiopsis was introduced by Steyaert (1949), with the type species P. guepinii (Desm.) Steyaert, a plant pathogenic taxon, on Rhododendron. Based on molecular evidence, the genus has 66 accepted species (Norphanphoun et al. 2019, Jayawardena et al. 2019b) and 257 species named in Index Fungorum (2020).

Pestalotiopsis diploclisiae Maharachch., K.D. Hyde \& Crous, Stud. Mycol. 79: 160 (2014)

Facesoffungi number: FoF06982

Fig. 163

Isolated from asymptomatic leaf of Kandelia candel. Sexual morph: Undetermined. Asexual morph: Conidiomata pycnidial, globose, brown, semi-immersed on PDA, releasing conidia in a black, slimy, globose, glistening mass. Conidiophores indistinct. Conidiogenous cells annellidic, ampulliform to lageniform, hyaline, smooth- and thin-walled, simple, proliferating 1-2 times percurrently 5-10 × 5-8 $\mu \mathrm{m}$. Conidia $(18-) 18.5-25(-26) \times(5-) 5.5-6(-6.5) \mu \mathrm{m}(\bar{x} \pm \mathrm{SD}=21 \pm 2.2$ $\times 5.5 \pm 0.6 \mu \mathrm{m})$, fusiform to clavate, straight to slightly curved, 4-septate; basal cell obconic with a truncate base, hyaline or sometimes pale brown, thin- and smooth-walled, $(3-) 3.5-4(-5) \mu \mathrm{m}$ long $(\bar{x}$ $\pm \mathrm{SD}=3.8 \pm 0.7 \mu \mathrm{m})$; three median cells $(10-) 10.5-15(-16) \mu \mathrm{m}$ long $(\bar{x} \pm \mathrm{SD}=12.7 \pm 1.7 \mu \mathrm{m})$, brown, septa and periclinal walls darker than rest of the cell, versicolored, wall rugose; second cell from base pale brown, $(3-) 4-5(-6) \mu \mathrm{m}$ long $(\bar{x} \pm \mathrm{SD}=4.4 \pm 0.8 \mu \mathrm{m})$; third cell brown, (3.5-)4-5(- 
6) $\mu \mathrm{m}$ long $(\bar{x} \pm \mathrm{SD}=4.2 \pm 0.7 \mu \mathrm{m})$; fourth cell brown, $(3-) 4-5(-5.5) \mu \mathrm{m}$ long $(\bar{x} \pm \mathrm{SD}=4.1 \pm 0.5$ $\mu \mathrm{m})$; apical cell (4-)4.5-5(-6) $\mu \mathrm{m}$ long $(\bar{x} \pm \mathrm{SD}=4.5 \pm 0.7 \mu \mathrm{m})$, hyaline, conic to acute; with $1-3$ tubular appendages on apical cell, inserted at different loci in a crest at the apex of the apical cell, branched, flexuous, (8.5-)9.5-33(-34) $\mu \mathrm{m}$ long $(\bar{x} \pm \mathrm{SD}=20 \pm 7.6 \mu \mathrm{m})$; single basal appendage, tubular, unbranched, centric, $(3.5-) 4-9(-10) \mu \mathrm{m}$ long $(\bar{x} \pm \mathrm{SD}=6.5 \pm 1.6 \mu \mathrm{m})$.

Culture characteristics - Colonies on PDA reaching 6-7 cm diameter after $14 \mathrm{~d}$ at room temperature $\left( \pm 25^{\circ} \mathrm{C}\right)$, under light $12 \mathrm{hr} /$ dark $12 \mathrm{hr}$, colonies filamentous to circular, medium dense, aerial mycelium on surface flat or raised, fluffy, white from above and reverse.

Known distribution (based on molecular data) - China, Hong Kong (Maharachchikumbura et al. 2014), Taiwan (this study).

Known hosts (based on molecular data) - Diploclisia glaucescens (Menispermiaceae), Psychotria tutcheri (Rubiaceae) (Maharachchikumbura et al. 2014), Kandelia candle (this study).

Material examined - Taiwan, Hsinchu City, tissue isolation from asymptomatic leaf of Kandelia candel (Rhizophoraceae), 16 July 2018, Norphanphoun Chada HsE1L-1A, living cultures, NCYU19-0356 (new host record).

GenBank Numbers - TUB: MN885525, tef1: MN885527, ITS: MN887600, LSU: MN887606.

Notes - Pestalotiopsis diploclisiae was introduced by Maharachchikumbura et al. (2014) from the fruits of Diploclisia glaucescens and Psychotria tutcheri in Hong Kong. Our strain is similar to $P$. diploclisiae in spore size (in this study; $23 \pm 1.5 \times 5.5 \pm 0.5 \mu \mathrm{m}$ vs. $24 \pm 1.3 \times 5.7 \pm$ $0.4 \mu \mathrm{m}$; Maharachchikumbura et al. 2014). Based on phylogenetic analysis our strain grouped with P. diploclisiae with low bootstrap support (66\% ML, Fig. 162). Hence, we introduce our strain as $P$. diploclisiae, the first record from Kandelia candel in Taiwan.

Pestalotiopsis kenyana Maharachch., K.D. Hyde \& Crous, Stud. Mycol. 79: 166 (2014)

Facesoffungi number: FoF06981

Fig. 164

Isolated from asymptomatic leaf of Kandelia candel. Sexual morph: Undetermined. Asexual morph: Conidiomata pycnidial, globose, brown, semi-immersed on PDA, releasing conidia in a black, slimy, globose, glistening mass. Conidiophores indistinct. Conidiogenous cells holoblastic, ampulliform to lageniform, hyaline, smooth- and thin-walled, simple, 5-10 $\times 5-8 \mu \mathrm{m}$. Conidia (18) $18.5-25(-26) \times(5-) 5.5-6(-6.5) \mu \mathrm{m}(\bar{x} \pm \mathrm{SD}=21 \pm 2.2 \times 5.5 \pm 0.6 \mu \mathrm{m})$, fusiform to clavate, straight to slightly curved, 4-septate; basal cell obconic with a truncate base, hyaline or sometimes pale brown, thin- and smooth-walled, (3-)3.5-4(-5) $\mu \mathrm{m}$ long $(\bar{x} \pm \mathrm{SD}=3.8 \pm 0.7 \mu \mathrm{m})$; three median cells $(10-) 10.5-15(-16) \mu \mathrm{m}$ long $(\bar{x} \pm \mathrm{SD}=12.7 \pm 1.7 \mu \mathrm{m})$, brown, septa and periclinal walls darker than rest of the cell, versicolored, wall rugose; second cell from base pale brown, (3-)4-5(6) $\mu \mathrm{m}$ long $(\bar{x} \pm \mathrm{SD}=4.4 \pm 0.8 \mu \mathrm{m})$; third cell brown, $(3.5-) 4-5(-6) \mu \mathrm{m}$ long $(\bar{x} \pm \mathrm{SD}=4.2 \pm 0.7$ $\mu \mathrm{m})$; fourth cell brown, (3-)4-5(-5.5) $\mu \mathrm{m}$ long $(\bar{x} \pm \mathrm{SD}=4.1 \pm 0.5 \mu \mathrm{m})$; apical cell (4-)4.5-5(-6) $\mu \mathrm{m}$ long $(\bar{x} \pm \mathrm{SD}=4.5 \pm 0.7 \mu \mathrm{m})$, hyaline, conic to acute; with $1-3$ tubular appendages on apical cell, inserted at different loci in a crest at the apex of the apical cell, branched, flexuous, (8.5-)9.5$33(-34) \mu \mathrm{m}$ long $(\bar{x} \pm \mathrm{SD}=20 \pm 7.6 \mu \mathrm{m})$; single basal appendage, tubular, unbranched, centric, (3.5-)4-9(-10) $\mu \mathrm{m}$ long $(\bar{x} \pm \mathrm{SD}=6.5 \pm 1.6 \mu \mathrm{m})$.

Culture characteristics - Colonies on PDA reaching 6-7 cm diameter after $14 \mathrm{~d}$ at room temperature $\left( \pm 25^{\circ} \mathrm{C}\right)$, under light $12 \mathrm{hr} /$ dark $12 \mathrm{hr}$, colonies filamentous to circular, medium dense, aerial mycelium on surface flat or raised, fluffy, white from above and reverse.

Known distribution (based on molecular data) - China (Yunnan Province, Jiangxi Province, Chongyi County) (Liu et al. 2017a), Kenya (Maharachchikumbura et al. 2014) Kandelia candel (this study).

Known hosts (based on molecular data) - Coffea sp. (Rubiaceae) strain number CBS 442.67, Camellia sinensis (Theaceae) strain numbers LC3291, LC3633 and LC6633 (Liu et al. 2017a, Maharachchikumbura et al. 2014, Taiwan (this study). 
Material examined - Taiwan, Hsinchu City, tissue isolation from asymptomatic leaf of Kandelia candel (Rhizophoraceae), 15 July 2018, Norphanphoun Chada HsE1P, living culture NCYUCC 19-0389 (new host record).

GenBank Numbers - TUB: MN885526, ITS: MN887601, LSU: MN887607.

Notes - Pestalotiopsis kenyana was introduced by Maharachchikumbura et al. (2014) from Kenya, with the character of two basal appendages. Our strain is similar to P. kenyana in spore size $(22 \pm 2.2 \times 2.5 \pm 0.6 \mu \mathrm{m}$ vs. $25.5 \pm 1.2 \times 8 \pm 0.4 \mu \mathrm{m}$; Maharachchikumbura et al. (2014)), and conidiogenous cell size $(7-22 \times 2-5 \mu \mathrm{m}$ vs. $10-25 \times 2-5 \mu \mathrm{m}$; Maharachchikumbura et al. (2014)). Based on phylogenetic analysis our strain is related to $P$. kenyana (Fig. 162). The collection of $P$. kenyana is the first record on Kandelia candel from Taiwan.

\section{Pseudopestalotiopsis Maharachch., K.D. Hyde \& Crous}

Maharachchikumbura et al. (2014) established Pseudopestalotiopsis. It was differentiated from its two sister genera Pestalotiopsis and Neopestalotiopsis based on conidial pigmentation and molecular phylogeny. As in Pestalotiopsis, the median cells in the conidia of Pseudopestalotiopsis are concolourous, but are much darker (brown to dark brown) and bear knobbed apical appendages (Liu et al 2010, Maharachchikumbura et al. 2014). In Neopestalotiopsis the median cells are versicolorous. Pseudopestalotiopsis species have been reported from terrestrial and mangrove habitats, as saprobes or as pathogens associated with leaf spots (Maharachchikumbura et al. 2014, 2016b, Norphanphoun et al. 2019).

Pseudopestalotiopsis kubahensis Lateef, M. Sepiah \& Bolhassan, Current Research in Environmental \& Applied Mycology 5(4): 378 (2014)

Fig. 166

Facesoffungi number: FoF 01310

Endophytic or pathogenic causing irregular shaped lesions on branches of Lonicera sp. appearing as black, flattened patches. Sexual morph: Undetermined. Asexual morph: Conidiomata semi-immersed to erumpent, acervulus, exuding brown to black conidial masses, subglobose to globose with a flattened base, $250-300 \mu \mathrm{m}$ in diameter, solitary, dark brown to black. Conidioma wall composed of light brown, thin-walled, flattened cells of textura prismatica, indistinct at the base, mostly fused with host tissue. Conidiophores reduced to conidiogenous cells. Conidiogenous cells holoblastic, arising from the innermost layers of the conidiomata, discrete, hyaline, smoothwalled, cylindrical to lageniform. Conidia broad fusiform, straight to slightly curved, 4-septate, slightly constricted at septa, $22.5-26.5 \times 6.5-8.5 \mu \mathrm{m}(\bar{x}=25 \times 7.5 \mu \mathrm{m}, \mathrm{n}=40)$ basal cell conic with a truncate base, thin and smooth-walled, 2.5-3.5 $\mu \mathrm{m}$ long; three median cells 16-19.5 $\mu \mathrm{m}$ long, doliiform, concolourous, golden brown to dark brown, septa darker than the rest of the cell (second cell from base 4-6 $\mu \mathrm{m}$ long; third cell 3.5-4.5 $\mu \mathrm{m}$ long; fourth cell 5-6.5 $\mu \mathrm{m}$ long); apical cell conic, hyaline, thin and smooth-walled, 2.5-4 $\mu \mathrm{m}$ long; with 3 or occasionally 2 tubular apical appendages arising from the apical tip, flexuous, rarely branched, 15-35(40) $\mu \mathrm{m}$ long, occasionally knobbed; basal appendage not observed.

Culture characteristics - Colonies on PDA reaching approximately $7 \mathrm{~cm}$ diameter after 7 days at $16-18^{\circ} \mathrm{C}$, greyish-white, irregular, with undulate margins and black, gregarious conidiomata sporulating; reverse pale yellow.

Known distribution (based on molecular data) - Malaysia (Lateef et al. 2015), Italy (this study).

Known hosts (based on molecular data) - Macaranga sp. (Lateef et al. 2015), Lonicera sp. (this study).

Material examined - Italy, Province of Forlì-Cesena [FC], near Converselle - Castrocaro Terme e Terra del Sole, on living branch of Lonicera sp. (L.) (Caprifoliaceae), 13 December 2014, 


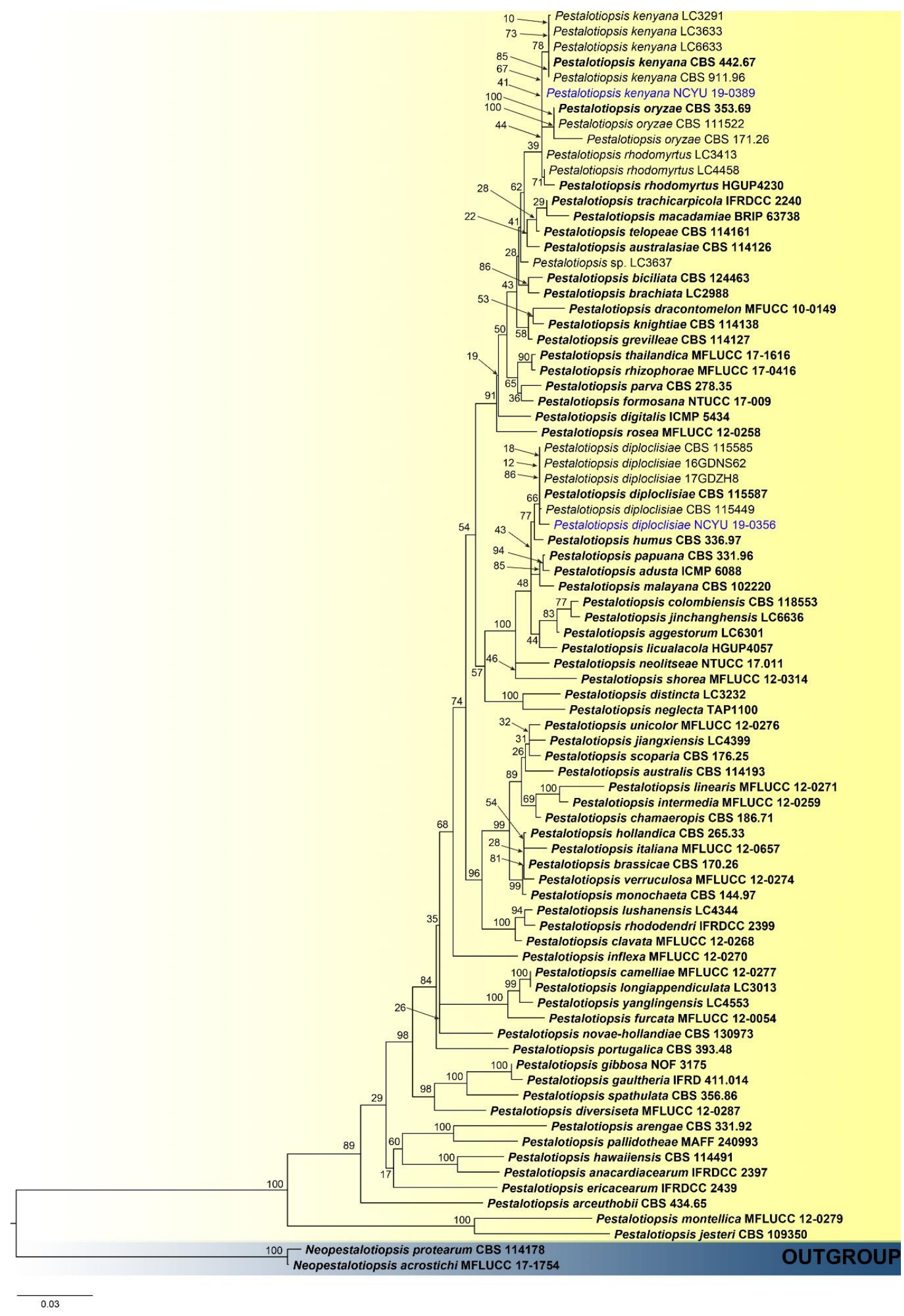

Fig. 162 - Phylogram generated from maximum likelihood analysis based on combined ITS, TUB and tefl sequence data for Pestalotiopsis. 82 strains are included in the combined gene analyses and Neopestalotiopsis protearum (CBS 114178) and N. acrostichi (MFLUCC 17-1754) are used as the out-group taxa. The best RAxML tree with a final likelihood value of -10930.743597 is presented. The matrix had 690 distinct alignment patterns, with $12.40 \%$ undetermined characters or gaps. Estimated base frequencies of Partition 0 (ITS) were as follows: $\mathrm{A}=0.235848, \mathrm{C}=0.243464, \mathrm{G}=$ $0.220143, \mathrm{~T}=0.300545$; substitution rates $\mathrm{AC}=0.277769, \mathrm{AG}=2.981361, \mathrm{AT}=0.839816, \mathrm{CG}=$ 
$0.412739, \mathrm{CT}=5.273414, \mathrm{GT}=1.000000$; gamma distribution shape parameter $\alpha=0.179684$. Estimated base frequencies of Partition 1 (TUB) were as follows: $\mathrm{A}=0.218592, \mathrm{C}=0.325639, \mathrm{G}=$ $0.225308, \mathrm{~T}=0.230461$; substitution rates $\mathrm{AC}=1.214354, \mathrm{AG}=5.267474, \mathrm{AT}=1.343109, \mathrm{CG}=$ $1.322200, \mathrm{CT}=5.809285, \mathrm{GT}=1.000000$; gamma distribution shape parameter $\alpha=0.345669$. Estimated base frequencies of Partition 2 (tefl) were as follows: $\mathrm{A}=0.261334, \mathrm{C}=0.316753, \mathrm{G}=$ $0.194433, \mathrm{~T}=0.227480$; substitution rates $\mathrm{AC}=0.938048, \mathrm{AG}=2.227476, \mathrm{AT}=1.023715, \mathrm{CG}=$ $0.645264, \mathrm{CT}=3.467662, \mathrm{GT}=1.000000$; gamma distribution shape parameter $\alpha=0.291483$. Bootstrap values for maximum likelihood equal to or greater than 60 near the branches. The newly generated sequences are indicated in blue bold.

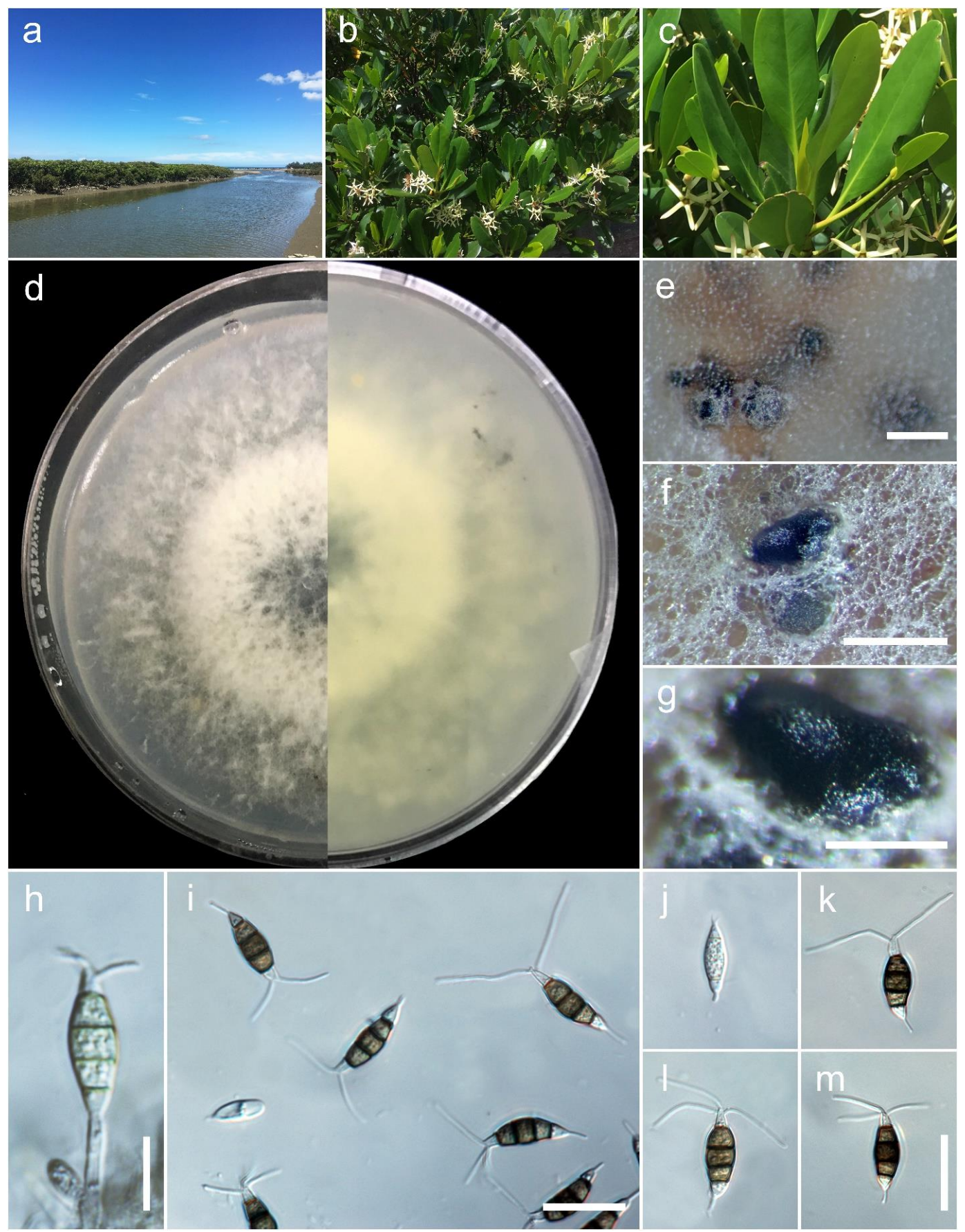

Fig. 163 - Pestalotiopsis diploclisiae (HsE1L-1A, new host record). a Habitat. b, c Kandelia candel. d Culture on PDA (leaf-above, right-reverse). $\mathrm{e}-\mathrm{g}$ Colony sporulating on PDA. $\mathrm{h}$ Conidiogenous cells giving rise to conidia. $\mathrm{i}-\mathrm{m}$ Conidia. Scale bars: $\mathrm{e}-\mathrm{f}=500 \mu \mathrm{m}, \mathrm{g}=250 \mu \mathrm{m}$, $\mathrm{h}=10 \mu \mathrm{m}, \mathrm{i}-\mathrm{m}=20 \mu \mathrm{m}$. 


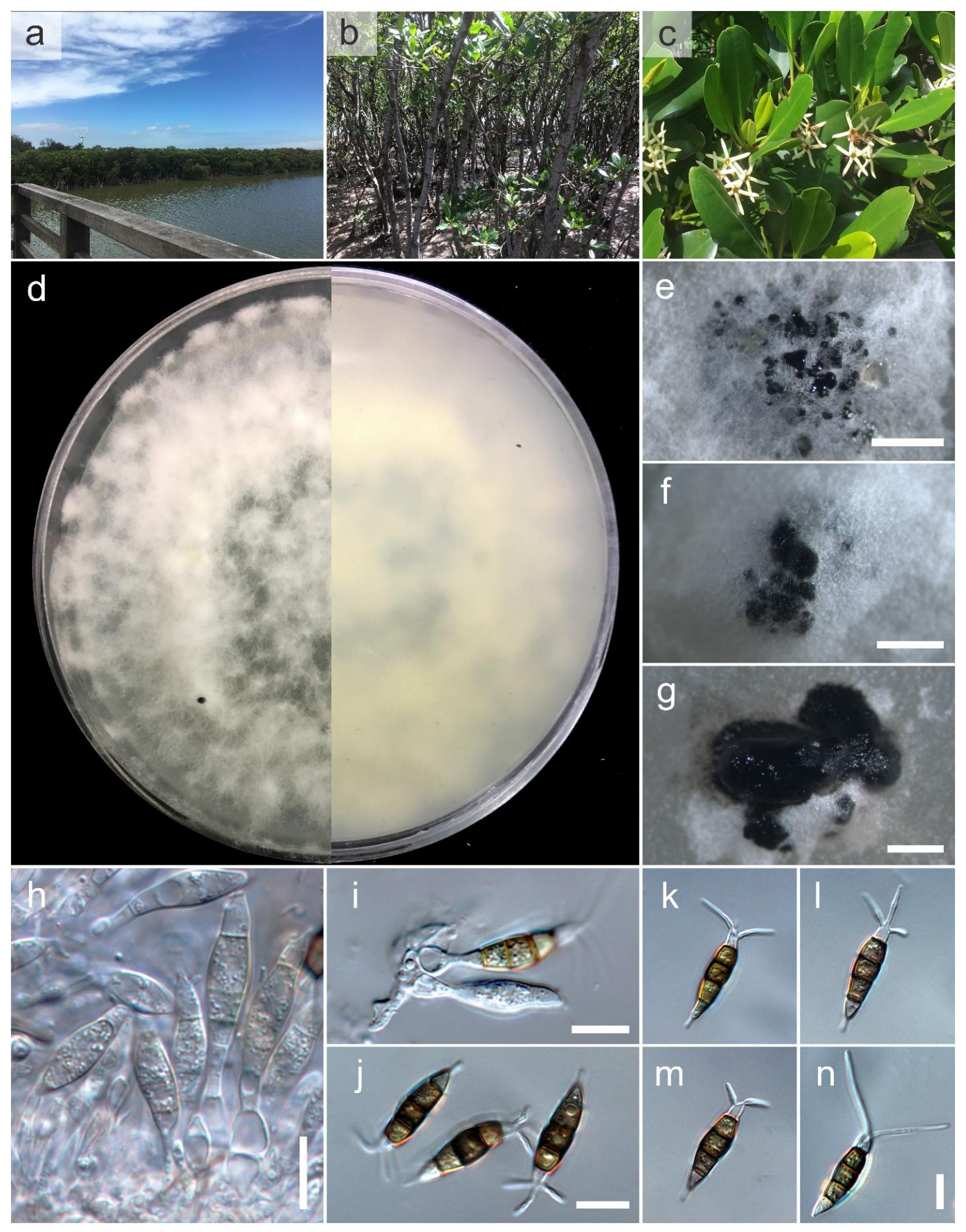

Fig. 164 - Pestalotiopsis kenyana (HsE1P, new host record). a Habitat. b, c Kandelia candel. $\mathrm{d}$ Culture on PDA (leaf-above, right-reverse). e-g Colony sporulating on PDA. $\mathrm{h}$ Conidiogenous cells giving rise to conidia. $\mathrm{i}-\mathrm{m}$ Conidia. Scale bars: $\mathrm{e}=2 \mathrm{~mm}, \mathrm{f}, \mathrm{g}=1 \mathrm{~mm}, \mathrm{~h}=250 \mu \mathrm{m}, \mathrm{i}-\mathrm{n}=10$ $\mu \mathrm{m}$.

E. Camporesi IT 2294 (MFLU 15-0844, new geographical record and host record), living culture MFLUCC 15-0565.

GenBank number - ITS: MG818971

Notes - In the phylogenetic analysis, the Pseudpestalotiopsis isolate (MFLUCC 15-0565) collected in the present study grouped with the type strain of Ps. kubahensis (UMAS KUB-P20), a species introduced from Macaranga sp. (Fig. 165). in Malaysia. Both produce spores with golden brown to dark, concolourous, median cells (Lateef et al. 2015). The conidial dimensions of the species from Malaysia is $27-30 \times 5.6-7.3 \mu \mathrm{m}$ and 22.5-26.5 $\times 6.5-8.5 \mu \mathrm{m}$ for our collection. The lengths of apical appendages for UMAS KUB-P20 and MFLUCC 15-0565 are $16-29.5 \mu \mathrm{m}$ and $15-35 \mu \mathrm{m}$ respectively. However, morphologically our collection differs from Ps. kubahensis by lacking a 
basal appendage. The relationship between the two strains is poorly resolved, as for both isolates only ITS data is available for sequence comparison. In a similar result obtained by Maharachchikumbura et al. (2016b) it was emphasized that the variation within the ITS region of Pseudopestalotiopsis species is limited, however, a combination of ITS, TUB and tefl gene data gave the best resolution as compared to any single marker for resolving Pestalotiopsis and related taxa. In the present study, we prefer to maintain our collection (MFLUCC 15-0565) as Ps. kubahensis until we have obtained more sequence data. This is the first Pseudopestalotiopsis sp. to be recorded on Lonicera sp. and is the first record from a temperate region, as all previous species introduced were from tropical regions.

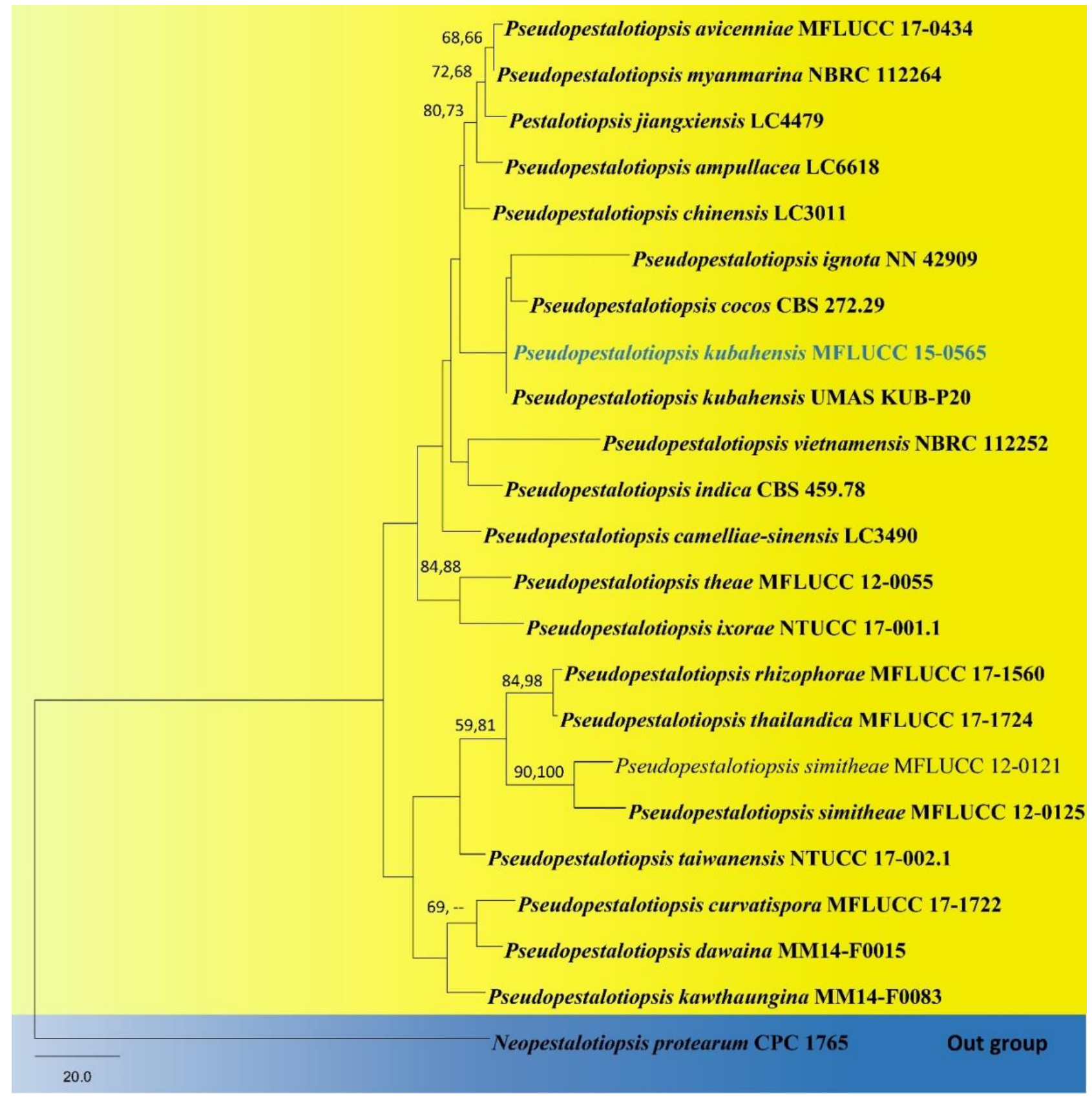

Fig. 165 - Phylogram generated from maximum parsimony (MP) analysis based on combined ITS, TUB, and tefl sequence data. Twenty-three strains are included in the combined gene analyses comprising 2323 characters after alignment (572 characters for ITS, 793 characters for TUB, 948 characters for tef1). Neopestalotiopsis protearum (CPC 1765) is used as the outgroup taxon. Bootstrap 50\% majority-rule consensus tree is presented here. The combined dataset contained 1953 constant, 270 parsimony uninformative and 100 parsimony informative characters. Gaps were 
treated as missing data. The alignment was subjected to 1000 bootstrap replicates. Statistics generated from MP analysis are as follows: $\mathrm{TL}=492, \mathrm{CI}=0.852, \mathrm{RI}=0.741, \mathrm{RC}=0.631, \mathrm{HI}=$ 0.148 . The tree topology of the maximum likelihood (ML) analysis did not differ significantly from the maximum parsimony analysis. The best RaxML tree selected had a final likelihood value of 5981.645344. Bootstrap values for MP and ML analyses equal to or greater than 50 are placed above or below the branches. Ex-type strains are in bold and black. The newly generated sequence is indicated in blue.

\section{Xylariales Nannf.}

Lopadostomataceae Daranag. \& K.D. Hyde

Lopadostomataceae was introduced by Senanayake et al. (2015) in Xylariales, to accommodate Lopadostoma and Creosphaeria. Jumillera and Whalleya were also included in the family by Daranagama et al. (2018).
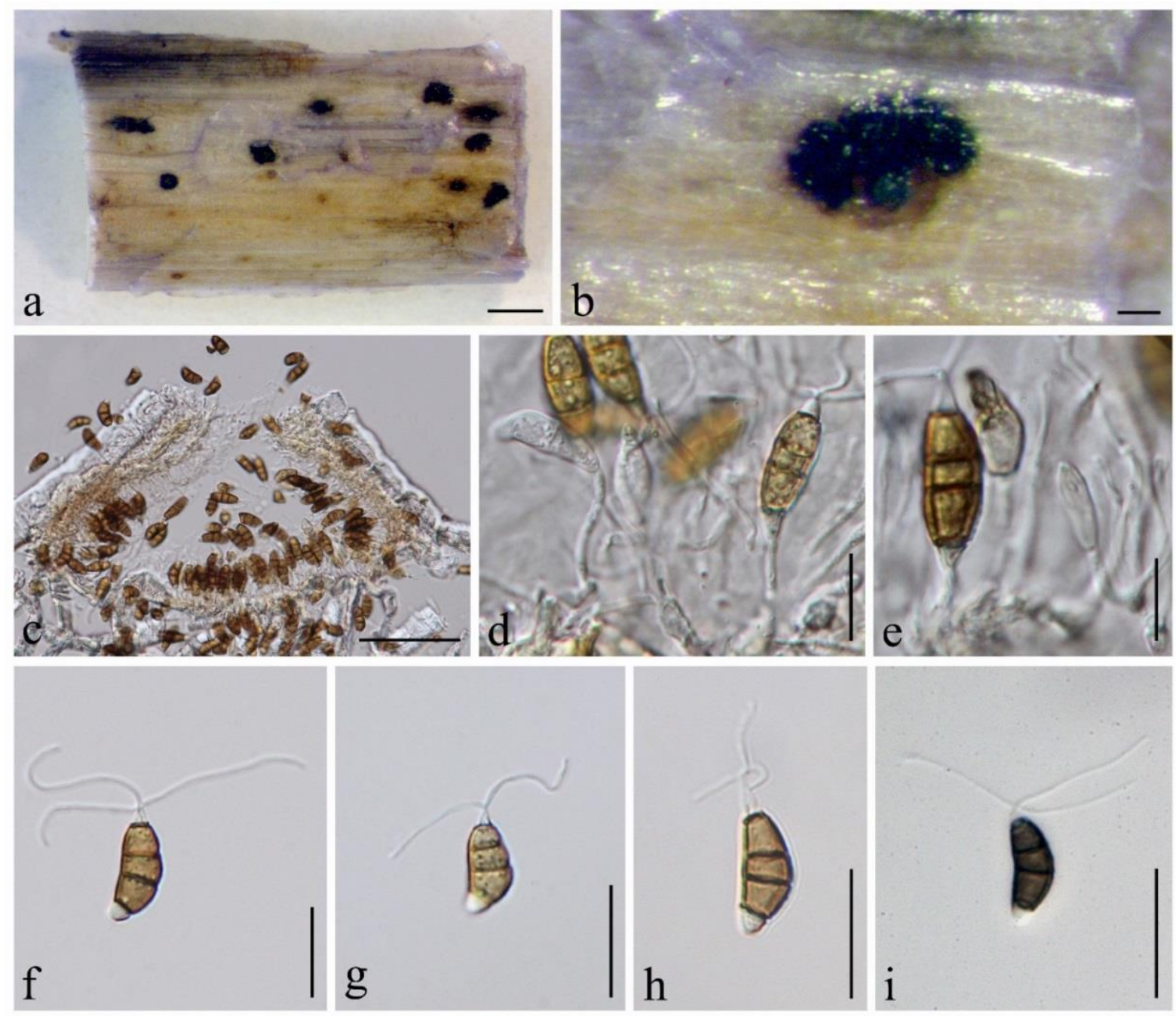

Fig. 166 - Pseudopestalotiopsis kubahensis (MFLU 15-0844, new host record and geographical record). a Appearance of conidiomata on host. b Close-up of a conidioma. c Cross section of a conidioma. $\mathrm{d}$, e Conidia arising from conidiogenous cells. $\mathrm{f}-\mathrm{i}$ Conidia. Scale bars: $\mathrm{a}=1000 \mu \mathrm{m}$, $\mathrm{b}=200 \mu \mathrm{m}, \mathrm{c}=100 \mu \mathrm{m}, \mathrm{d}, \mathrm{e}=10 \mu \mathrm{m}, \mathrm{f}-\mathrm{i}=20 \mu \mathrm{m}$.

\section{Whalleya J.D. Rogers, Y.M. Ju \& F. San Martín}

Whalleya was introduced to accommodate two species previously known in Biscogniauxia (Rogers et al. 1997). Species of Whalleya are xylariaceous in sexual morph characters (Rogers et al. 1997) and conidial characters are similar to lopadostomaceous species. Previous studies have 
placed Whalleya in Diatrypaceae (Glawe \& Rogers 1986), because of similar characters to Xylariaceae and Lopadostomaceae. Wendt et al. (2017) and Daranagama et al. (2018) have treated Whalleya as a member of family Lopadostomataceae using both phylogeny and morphology.

Whalleya microplaca (Berk. \& M.A. Curtis) J.D. Rogers, Y.M. Ju \& F. San Martín, Mycotaxon 64: 48 (1997)

Fig. 168

Faces of fungi number: FoF03015

Saprobic on woody litter of Phoebe spp. Sexual morph: Stromata applanate, solitary or confluent, bipartite, outer dehiscing layer dark brown, thin, exposing mature black surface, carbonaceous. Ascomata globose, 100-120 $\mu \mathrm{m}$ high, 213-245 $\mu \mathrm{m}$ diameter, tissue between ascomata mainly composed of fungal tissue, coriaceous, becoming brownish, tissue below the ascomatal layer inconspicuous. Ostioles umbilicate. Asci 8-spored, 33-38 $\times 2-3 \mu \mathrm{m}$, unitunicate, cylindrical, short-pedicellate, with discoid ring. Ascospores $8-9 \times 2-3 \mu \mathrm{m}$, uniseriate, light brown, ellipsoid-inequilateral, with narrowly rounded ends, 1-celled, with central prominent guttule. Asexual morph: Undetermined.

Culture characteristics - colonies on PDA white, covering entire plate in 14 days at $28{ }^{\circ} \mathrm{C}$ fluffy mycelium, and reverse white.

Known distribution - China, Yunnan, Mauritius, Louisiana, Florida, New Jersey, Philippines, and Taiwan (Farr \& Rossman 2020), China (this study).

Known hosts (based on molecular data) - Phoebe spp., Magnolia glauca, Melia sp., Persea sp., Sassafras albidum, Sassafras officinale (Farr \& Rossman 2020), Phoebe spp. (this study).

Material examined - China, Yunnan, on woody litter of Phoebe spp. (Lauraceae), 5 December 2018, D.N Wanasinghe, DW0122 (JZBH 3400001) living culture, JZB 3400001.

GenBank number - ITS: MN989425.

Notes - Whalleya is typified by W. microplaca and has sexual morph characters similar to Lopadostoma pouzarii (Daranagama et al. 2018). A collection obtained from the woody litter of Phoebe spp. was identified as Whalleya microplaca with support from morphology and phylogeny. Our isolate clustered with the reference strain of Whalleya microplaca, in the combined ITS, LSU and RPB2 sequence analysis with 100\% support (Fig. 167). This is the first record of Whalleya microplaca species reported from Phoebe sp. in China.

Xylariaceae Tul. \& Tul.

Xylariaceae was introduced by Tulasne \& Tulasne (1863) using the term "Xylariei" (Stadler et al. 2013). According to Maharachchikumbura et al. (2016a), Xylariaceae is one of the largest and most diverse families of Ascomycota. The exact number of accepted taxa varies within the family (Hyde et al. 2020). Presently, Xylariaceae comprises 44 genera and more than 1350 species (U'Ren et al. 2016, Wijayawardene et al. 2018). Xylariaceae is characterized by perithecial ascomata, embedded in a more or less well-developed, dark stromata or sometimes reduced or lack stromata, 8-spored, unitunicate, cylindrical asci with or without $\mathrm{J}+$, apical rings and pigmented ascospores with germ slits or pores. The asexual morphs produce conidia holoblastically from sympodially, or occasionally percurrently proliferating conidiogenous cells (Rogers 2000, Daranagama et al. 2018).

\section{Rosellinia De Not.}

Rosellinia was introduced by De Notaris (1844) to accommodate Rosellinia aquila (Fr.) Ces. \& De Not. as the type species. Rosellinia is a species rich genus (Petrini 1992, 2003, 2013). There are 142 accepted Rosellinia species including many type specimens based on morphological studies (Petrini 2013). Rosellinia is characterized by superficial, subglobose, semiglobose, mammate to cupulate or conical, ostiolate, uniperitheciate, brown to black stromata seated in a subiculum; cylindrical, pedicellate asci with $\mathrm{J}+$, ascal apical rings with a rounded, angular or indistinct bulge at the upper rim, the rings are usually higher than the width. The ascospores are slightly larger, 1-celled, with a germ slit in most species (Petrini 2013). The asexual morph produces conidia in the subiculum, on immature perithecia or in culture (Petrini \& Petrini 2012). 
Rosellinia species have a wide distribution as saprobes, endophytes or pathogens of various host species (Su et al. 2016a). Many Rosellinia species cause plant diseases (Whalley 1996). In our study, we introduce Rosellinia convexa as a new host record from Prunus subhirtella (Rosaceae) in China.

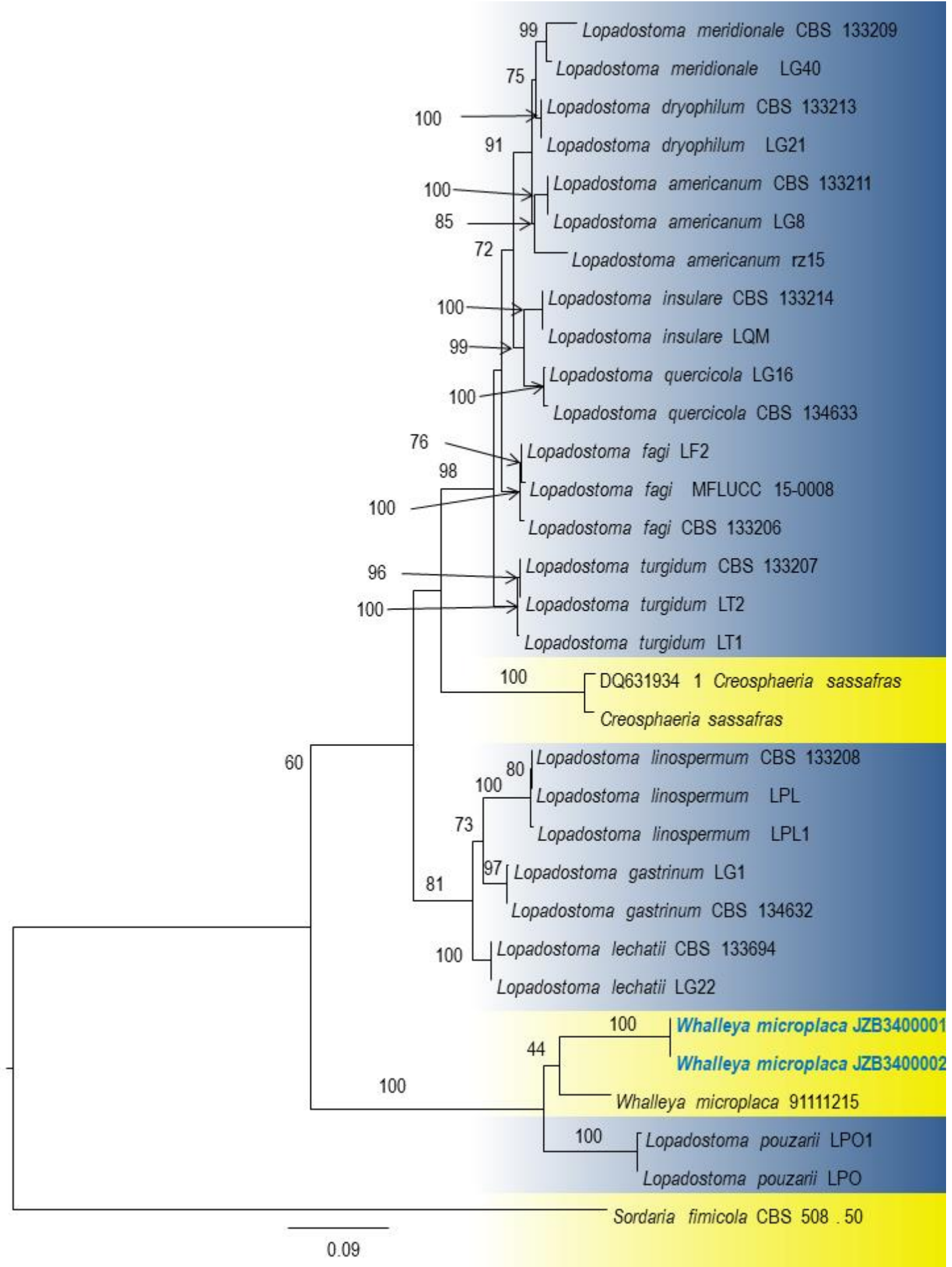

Fig. 167 - Phylogram generated from maximum likelihood analysis based on a combined ITS, LSU and RPB2 sequence dataset. Thirty-two strains are included in the combined gene analyses comprising 2319 total characters including gaps. The tree topology derived from the Bayesian analysis was similar to that derived from the maximum likelihood analysis. The best scoring of the ML tree is selected to represent the phylogenetic relationships of taxa in Lopadostomataceae, with the final ML optimization likelihood: - 3536.406809. The matrix had 293 distinct alignment patterns, with $18.47 \%$ undetermined characters or gaps. Estimated base frequencies were as 
follows: $\mathrm{A}=0.211039, \mathrm{C}=0.292321, \mathrm{G}=0.252470, \mathrm{~T}=0.244170 ;$ substitution rates $\mathrm{AC}=$ 1.328514, $\mathrm{AG}=2.488703, \mathrm{AT}=1.264091, \mathrm{CG}=0.687931, \mathrm{CT}=4.488656, \mathrm{GT}=1.000000$; TreeLength $=1.050171$; gamma distribution shape parameter $\alpha=0.517888$. The newly generated sequences are indicated in blue.
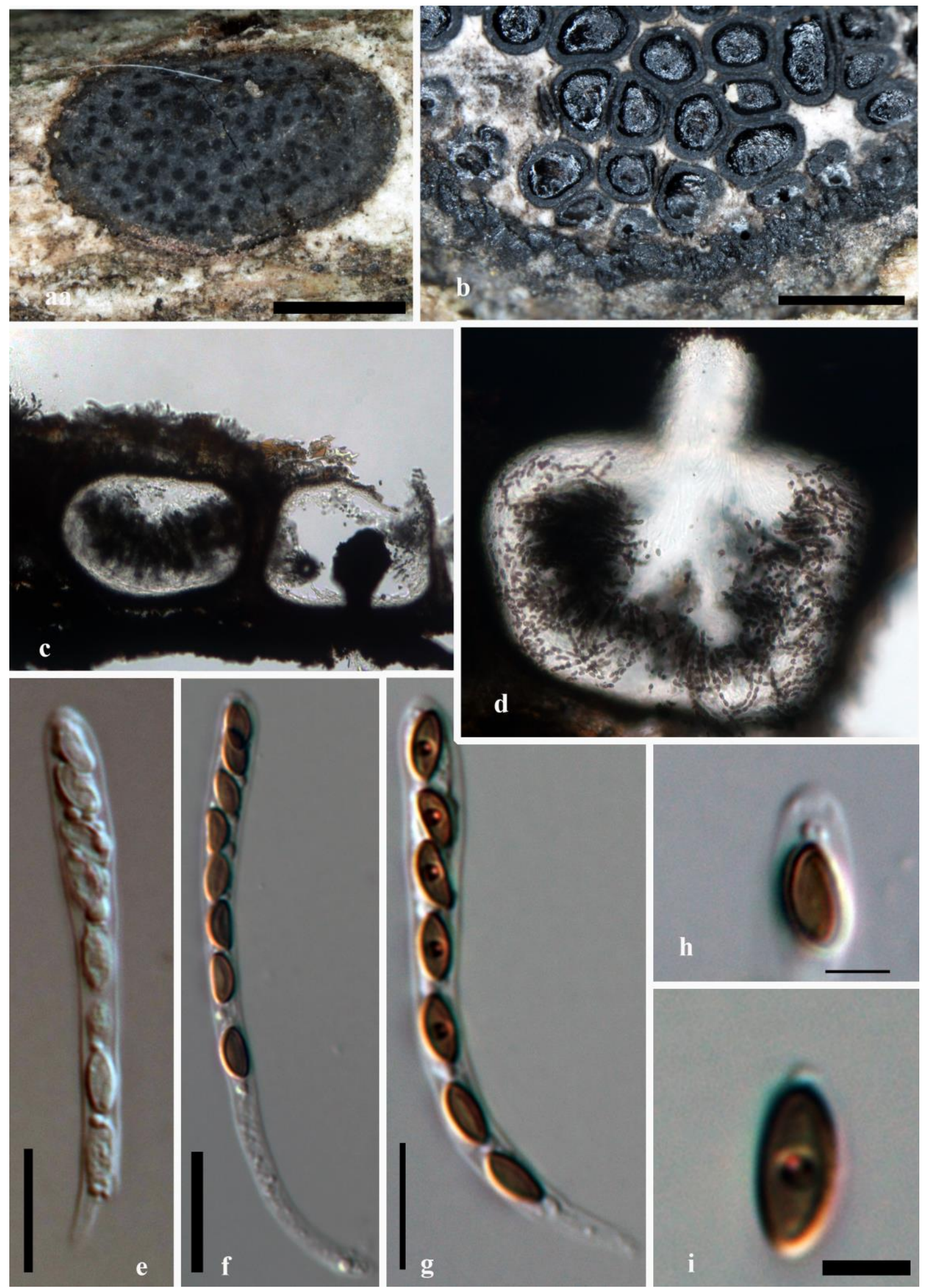

Fig. 168 - Whalleya microplaca (JZB 3400001, new host record), a, b Stromata in wood. $\mathrm{c}, \mathrm{d}$ Cross section of stroma showing ascomata encased in stromal tissue. e-g Asci. $\mathrm{h}$ Ascus tip. i Ascospores. Scale bars: $\mathrm{a}, \mathrm{b}=200 \mu \mathrm{m}, \mathrm{e}, \mathrm{f}, \mathrm{g}=10 \mu \mathrm{m} \mathrm{h}-\mathrm{i}=10 \mu \mathrm{m}$. 


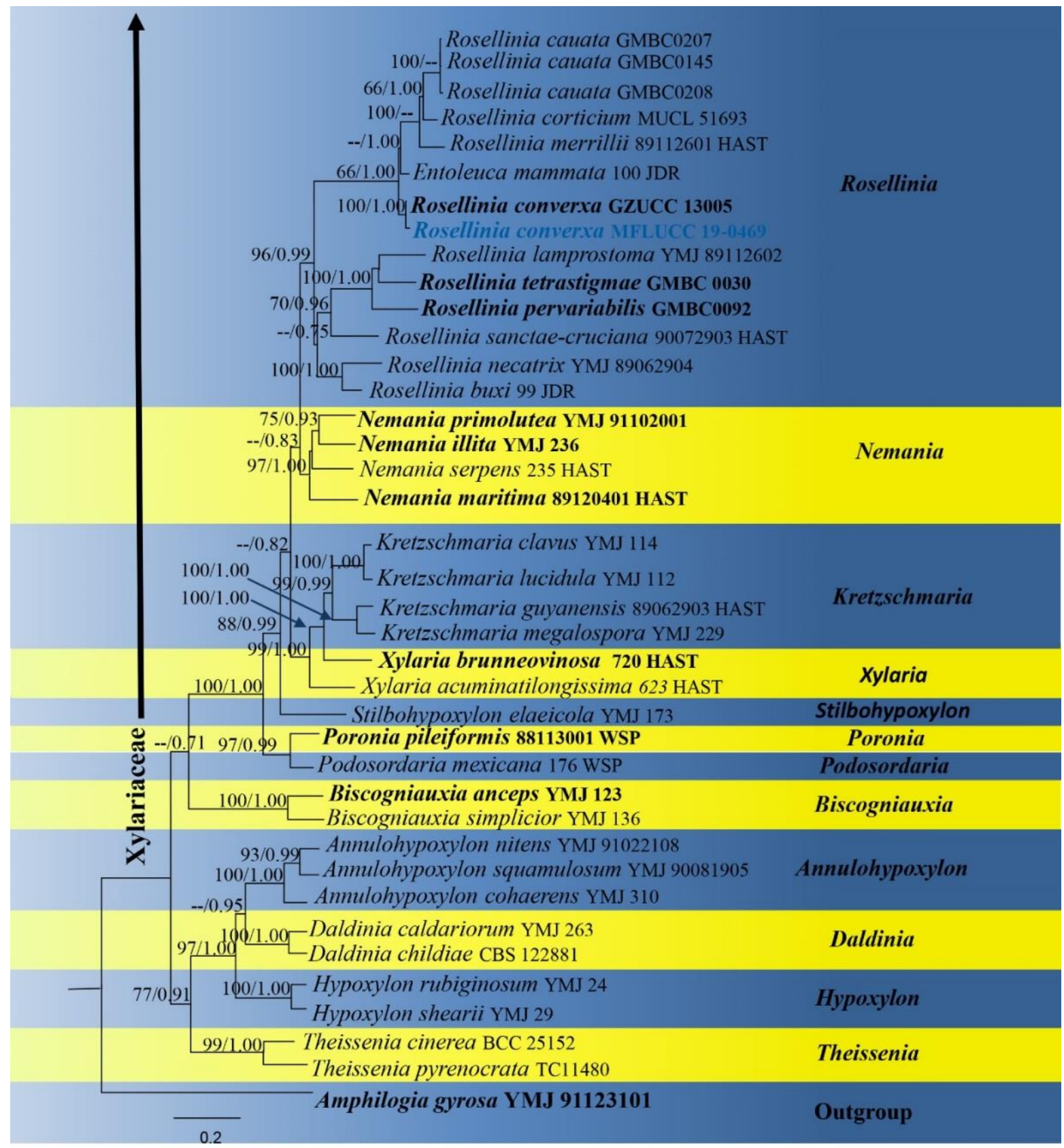

Fig. 169 - Phylogram generated from maximum likelihood analysis based on combined ITS, RPB2, ACT, and TUB sequence data. Forty strains are included in the combined gene analyses comprising 2513 characters after alignment. Amphilogia gyrosa (YMJ 91123101) is used as the outgroup taxon. The tree topology of the Bayesian analysis was similar to the maximum likelihood analysis. The best RaxML tree with a final optimization likelihood value is -34670.729423 . The matrix had 323 distinct alignment patterns, with $12.37 \%$ undetermined characters or gaps. Estimated base frequencies were as follows: $\mathrm{A}=0.240651, \mathrm{C}=0.275519, \mathrm{G}=0.236248, \mathrm{~T}=0.247582$; substitution rates $\mathrm{AC}=1.504126, \mathrm{AG}=4.316567, \mathrm{AT}=1.308364, \mathrm{CG}=1.338210, \mathrm{CT}=4.785013$, $\mathrm{GT}=1.000000$; gamma distribution shape parameter $\alpha=0.705373$. Bootstrap values for maximum likelihood equal to or greater than 65 and Bayesian posterior probabilities equal or greater than 0.95 are placed above or below the branches. The newly generated sequences are indicated in bold and blue.

Rosellinia convexa Q.R. Li \& J.C. Kang, Mycoscience 57(3): 166 (2016)

Fig. 170

Facesoffungi number: FoF 00890 
Saprobic on dead host stem of Prunus subhirtella. Sexual morph: Subiculum felty, brownish grey, persistent, appressed. Ascomata 940-1330 $\mu \mathrm{m}$ diameter $\times 760-835 \mu \mathrm{m}$ high $(\bar{x}=1165 \times 798$ $\mu \mathrm{m}, \mathrm{n}=10$ ), perithecial, scattered to gregarious, superficial with the base slightly sunken, globose to subglobose, greyish brown to black, apex with a finely conical, thick ectostroma, slightly carbonaceous, black, finely roughened, thickened at the base and spreading around the stroma base beneath the subiculum, entostroma restricted to a thin fibrous gray color tissue encasing the perithecium, perithecia not collapsed in the stroma cavity, ostiolar papilla. Paraphyses 1-2 $\mu \mathrm{m}$ wide, sparse, thin walled, tapering above asci. Asci $125-190 \times 7-13 \mu \mathrm{m}(\bar{x}=155 \times 11 \mu \mathrm{m}, \mathrm{n}=20)$ cylindrical, 8-spored, pedicel 19-40 $\mu \mathrm{m}(\bar{x}=32 \mu \mathrm{m}, \mathrm{n}=20)$ long, with apical ring, 6-7 $\times 6-9 \mu \mathrm{m}(\bar{x}$ $=8 \times 8 \mu \mathrm{m}, \mathrm{n}=20$ ), cuboid-cuneate with an upper lateral rim, J+ in Melzer's reagent. Ascospores 16-19 $\mu \mathrm{m} \times 7-10 \mu \mathrm{m}(\bar{x}=18 \times 8 \mu \mathrm{m}, \mathrm{n}=30)$, obliquely overlapping uniseriate, dark brown, narrowly to broadly ellipsoidal-equilateral with broadly rounded, with a conspicuous straight germ slit almost along entire spore-length on the ventral side, entirely surrounded by an ill-defined slimy sheath, 1.8-4.5 $\mu \mathrm{m}$ wide, visible in India ink, without appendages. Asexual morph: Undetermined.

Culture characteristics - Ascospores germinating on PDA within $24 \mathrm{~h}$, from single spore isolation. Colonies on PDA reaching $25-30 \mathrm{~mm}$ diameter after one week at $16^{\circ} \mathrm{C}$, circular, entire edge, white, with dense, convex papillate surface, brown in reverse.

Known distribution (based on molecular data) - Guizhou Province, China (Su et al, 2016a and this study).

Known hosts (based on molecular data) - Prunus subhirtella (this study).

Material examined - China, Guizhou Province, Guiyang. Lives on a dead stem of Prunus subhirtella (Rosaceae), 22 Dec. 2018, Lakmali S. Dissanayake, GHP03 (MFLU 19-0773, new host record), living culture MFLUCC 19-0469.

GenBank Numbers - ITS: MN707567, RPB2: MN987003, ACT: MN987004, TUB: MN987002.

Notes - There is a large overlap between our newly collected species (MFLU 19-0773) and Rosellinia convexa (holotype, GZUCC13005). Both species were collected in the same locality, but the holotype was collected from an unidentified host ( $\mathrm{Su}$ et al. 2016a). Both strains have globose to subglobose, scattered ascomata, papillate ostioles, 8-spored, cylindrical, uniseptate asci with an apical ring bluing in Melzer's reagent and brown ascospores with germ slits ( $\mathrm{Su}$ et al. 2016a). However, the ascospores of our new collection are different from the holotype in having narrowly to broadly ellipsoidal-equilateral ascospores with broadly rounded ends, while the holotype has lemon-shaped to podiform ascospores with convex umbilical ends. Multi-gene (ITS, RPB, ACT, TUB) analysis herein, also shows that our collection clusters with other Rosellinia convexa strains (Fig. 169). According to the guidelines of Jeewon \& Hyde (2016), we have analyzed the nucleotide differences within the rRNA gene region to further clarify the identification. In comparison of ITS regions (ITS1-5.8S-ITS2) from 738 nucleotides, there are 3 bp (0.40\%) differences between MFLU 19-0773 and GZUCC13005. We confirmed that our new collection (MFLU 19-0773) is another record of Rosellinia convexa and a new host record from dead stems of Prunus subhirtella (Rosaceae) in China.

\section{Sordariomycetes orders incertae sedis \\ Catabotryales K.D. Hyde \& Senan. \\ Catabotrydaceae Petr. ex M.E. Barr}

This family was referred to different orders including Sordariales (Barr 1990b) then transferred to Xylariales by Hyde et al. (2000), while Kirk et al. (2001) placed it in Boliniales based on its morphology. Miller and Huhndorf (2005) placed Catabotrydaceae in Diaporthales based on a multi-gene analysis. Maharachchikumbura et al. (2015, 2016a) placed it in Amplistromatales as it clustered with Amplistromataceae in phylogenetic analyses, while Hyde et al. (2020) introduced a new order to accommodate it based on divergence time estimates. 

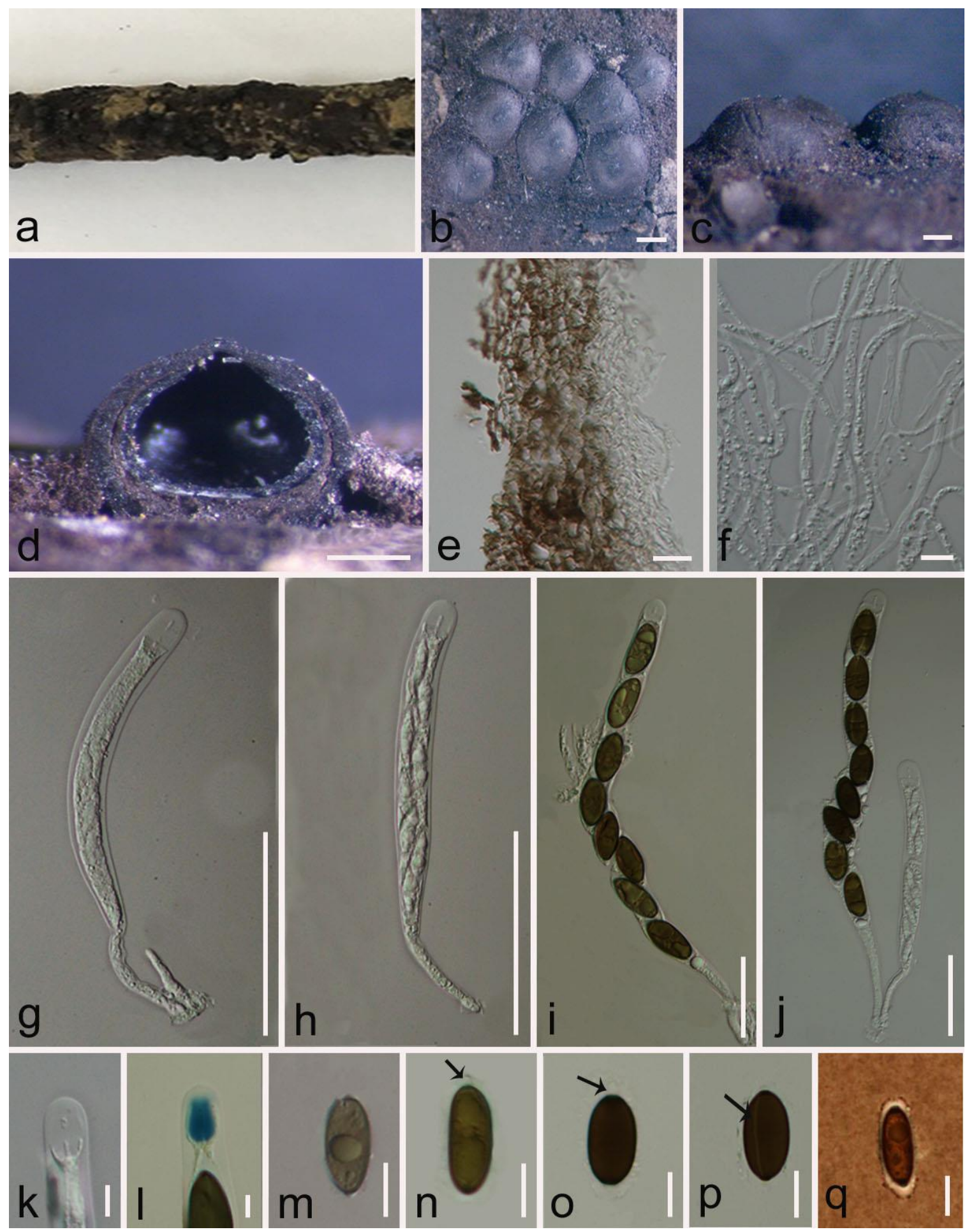

Fig. 170 - Rosellinia convexa (MFLU 19-0773, new host record). a, b Habit of stromata on host surface. c Stromata in lateral close-up showing the smooth ectostroma with small papillate ostioles. $\mathrm{d}$ Stroma in vertical section showing the thick carbonaceous ectostroma. e Peridium. $\mathrm{f}$ Paraphyses. $\mathrm{g}-\mathrm{j}$ Immature and mature asci. $\mathrm{k}$ Apical ring. 1 Ascal apical ring in Melzer's reagent. $\mathrm{m}-\mathrm{o}$ Immature and mature ascospore with cellular appendages (arrow). p Ascospore in dorso-lateral view showing a germ slit (arrows). q Ascospore with slimy sheath in Indian ink. Scale bars: $b, c=500 \mu \mathrm{m}$, $\mathrm{d}=200 \mu \mathrm{m}, \mathrm{e}, \mathrm{f}=10 \mu \mathrm{m}, \mathrm{g}-\mathrm{j}=50 \mu \mathrm{m}, \mathrm{k}, \mathrm{l}=5 \mu \mathrm{m}, \mathrm{m}-\mathrm{q}=10 \mu \mathrm{m}$.

Catabotrys Theiss. \& Syd.

The monotypic genus Catabotrys typified by Catabotrys deciduum was introduced by Theissen \& Sydow (1915). Catabotrys is characterized by a sexual morph with superficial, reddish 
brown to black, discoid to pulvinate, multi-loculate stromata, ascomata that are perithecial, globose enclosed in stromatic columns, asci that are 8-spored, unitunicate, broad cylindrical, short pedicellate, with a $\mathrm{J}-$, discoid, apical ring and ascospores that are hyaline, 1-celled, ellipsoidal to cylindrical and the asexual morph is hyphomycetous with ellipsoidal to ovoid conidia. Currently, this genus includes three species as listed in Index Fungorum (2020).

Catabotrys deciduum (Berk. \& Broome) Seaver \& Waterston, Mycologia 38(2): 184 (1946)

Facesoffungi number: FoF06530

Figs 172, 173

Saprobic on decaying wood of Rhizophora mucronata. Sexual morph: Stromata 1-3 mm high, $1-4 \mathrm{~mm}$ diameter $(\bar{x}=1.8 \times 2.3 \mathrm{~mm}, \mathrm{n}=10)$, irregularly scattered, occasionally coalescing, conspicuous, multi-loculate, superficial, with base slightly penetrating the epidermis at regular intervals, discoid to pulvinate, reddish brown to black surface, scurfy, flat or slightly convex, composed of rather thin-walled, reddish yellow cells of textura angularis, textura globosa and textura epidermoidea. Ascomata perithecial, 390-500 $\mu \mathrm{m}$ high, $180-200 \mu \mathrm{m}$ diameter $(\bar{x}=426 \times$ $197 \mu \mathrm{m}, \mathrm{n}=10$ ), deeply imbedded in stromatic columns, globose, with a long, periphysate, ostiolar neck. Ostioles 120-200 × 35-45 $\mu \mathrm{m}(\bar{x}=160 \times 40 \mu \mathrm{m}, \mathrm{n}=10)$. Peridium 40-55 $\mu \mathrm{m}(\bar{x}=45 \mu \mathrm{m}, \mathrm{n}$ $=5$ ) thick, composed of several layers of compressed, reddish brown cells of textura angularis. Paraphyses 1-3 $\mu \mathrm{m}(\bar{x}=1.7 \mu \mathrm{m}, \mathrm{n}=10)$, hypha-like, numerous, tapering towards the apex, not embedded in a gelatinous matrix. Asci 35-45 × 4-6 $\mu \mathrm{m}(\bar{x}=41 \times 5 \mu \mathrm{m}, \mathrm{n}=10)$, 8-spored, unitunicate, broad cylindrical, short pedicellate, apically rounded or truncate, with a J- discoid, refractive, apical ring. Ascospores $7-9 \times 3-4 \mu \mathrm{m}(\bar{x}=8 \times 3.5 \mu \mathrm{m}, \mathrm{n}=10)$, uniseriate to bi-seriate, hyaline, 1-celled, ellipsoidal to cylindrical, smooth-walled, lacking a mucilaginous sheath. Asexual morph: Hyphomycetous, vegetative hyphae smooth-walled, septate, hyaline, up to $3 \mu \mathrm{m}$ wide. Conidiophores micronematous, sympodial, weakly differentiated arising from vegetative hyphae. Conidiogenous cells reduced, holoblastic, polyblastic. Conidia ellipsoidal to ovoid, smooth surfaced, thick-walled, often with denticles or left over scars of detachment from conidiogenous cells, $3-5.5 \times 2-5 \mu \mathrm{m}(\bar{x}=4.3 \times 3.4 \mu \mathrm{m}, \mathrm{n}=10)$, hyaline and 1-celled.

Culture characteristics - Ascospores germinating on seawater agar within 24 hours, germ tubes arising from both ends of the ascospores. Colonies on MEA, moderately growing, reaching 25-45 $\mathrm{mm}$ diameter after 25 days of incubation at room temperature, hyaline to cream and pale yellow at center margin, reverse creamy to pale yellow, velvety, circular, surface raised.

Known distribution (based on molecular data) - Chumphon, Thailand (Hyde et al. 2019), Panama (Miller and Huhndorf 2005), Puducherry, India (this study).

Known hosts (based on molecular data) - Salacca sp, oil palms (Hyde et al. 2019), Rhizophora mucronata (this study).

Material examined - India, Puducherry, Thengaithittu mangroves $\left(11.5^{\circ} \mathrm{N} 79.5^{\circ} \mathrm{E}\right)$, on decaying wood of Rhizophora mucronata (Rhizophoraceae), 20 January 2016, B. Devadatha (AMH-10015), living culture, NFCCI-4395.

GenBank numbers - LSU: MN061355, SSU: MN173346, RPB2: MN546859, tef1: MN184789.

Notes - The monotypic genus Catabotrys is typified by $C$. deciduum and belongs to Catabotrydaceae (Petrak 1952) in Catabotryales (Hyde et al. 2020). Our collection of Catabotrys deciduum (AMH-10015) has similar morphological characters and measurements to Catabotrys deciduum (Seaver \& Waterston 1946). The nucleotide sequence data of our collection of Catabotrys deciduum (NFCCI-4395) is $100 \%$ identical in the LSU region and less than $1 \%$ nucleotide differences in protein-coding gene regions with the sequence data of $C$. deciduum (SMH3436). 


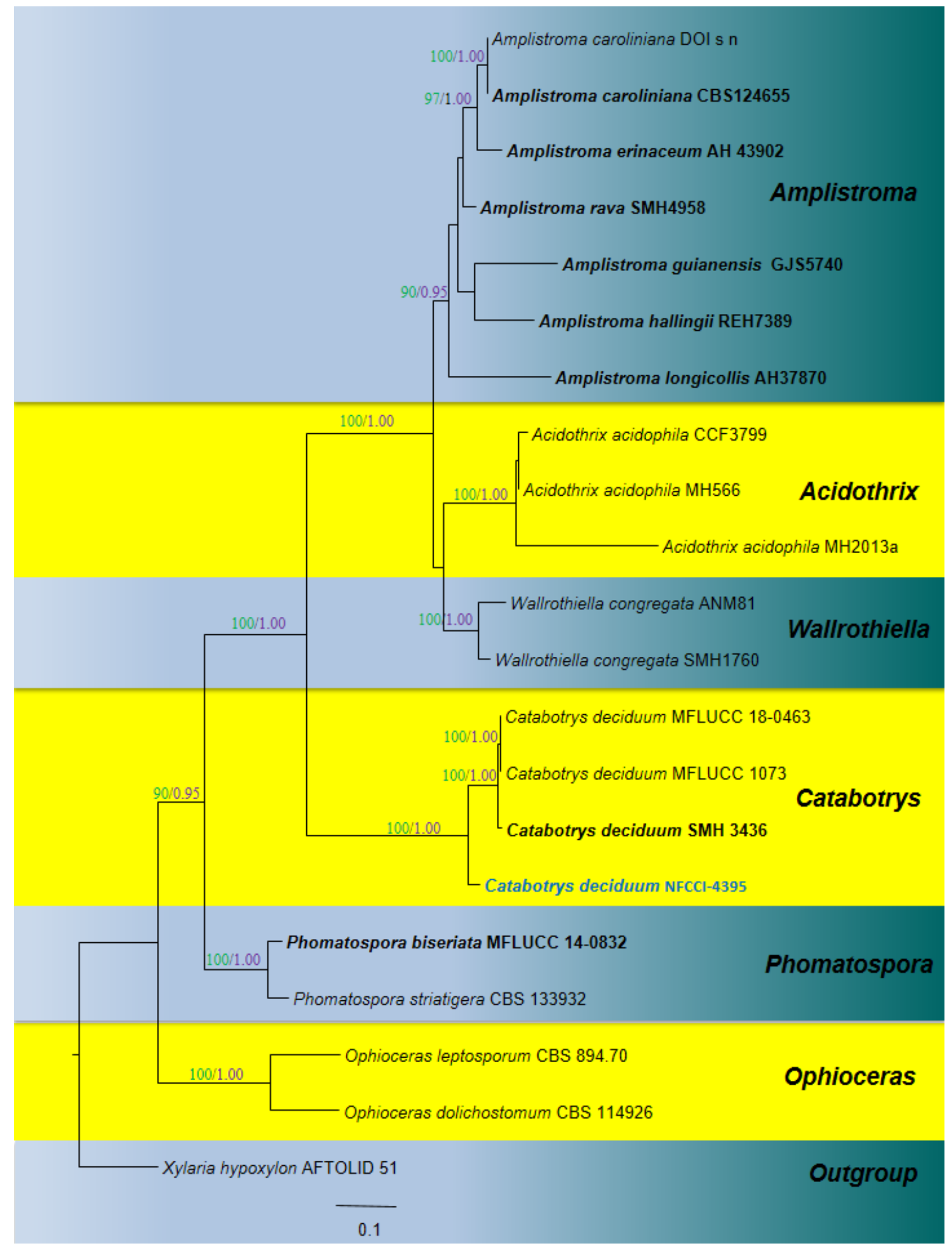

Fig. 171 - Phylogram generated from maximum likelihood analysis based on LSU, ITS, tefl and RPB2 gene regions of Amplistromatales and related genera. Bootstrap support for maximum likelihood (ML, green), equal to or greater than $75 \%$ and the values of Bayesian posterior probabilities (BIPP, purple) equal to or greater than 0.95 are given above each branch. The ex-type strains are in bold and new isolate is in blue. The tree is rooted with Xylaria hyphoxylon AFTOLID 51. The newly generated sequence is indicated in blue bold. 

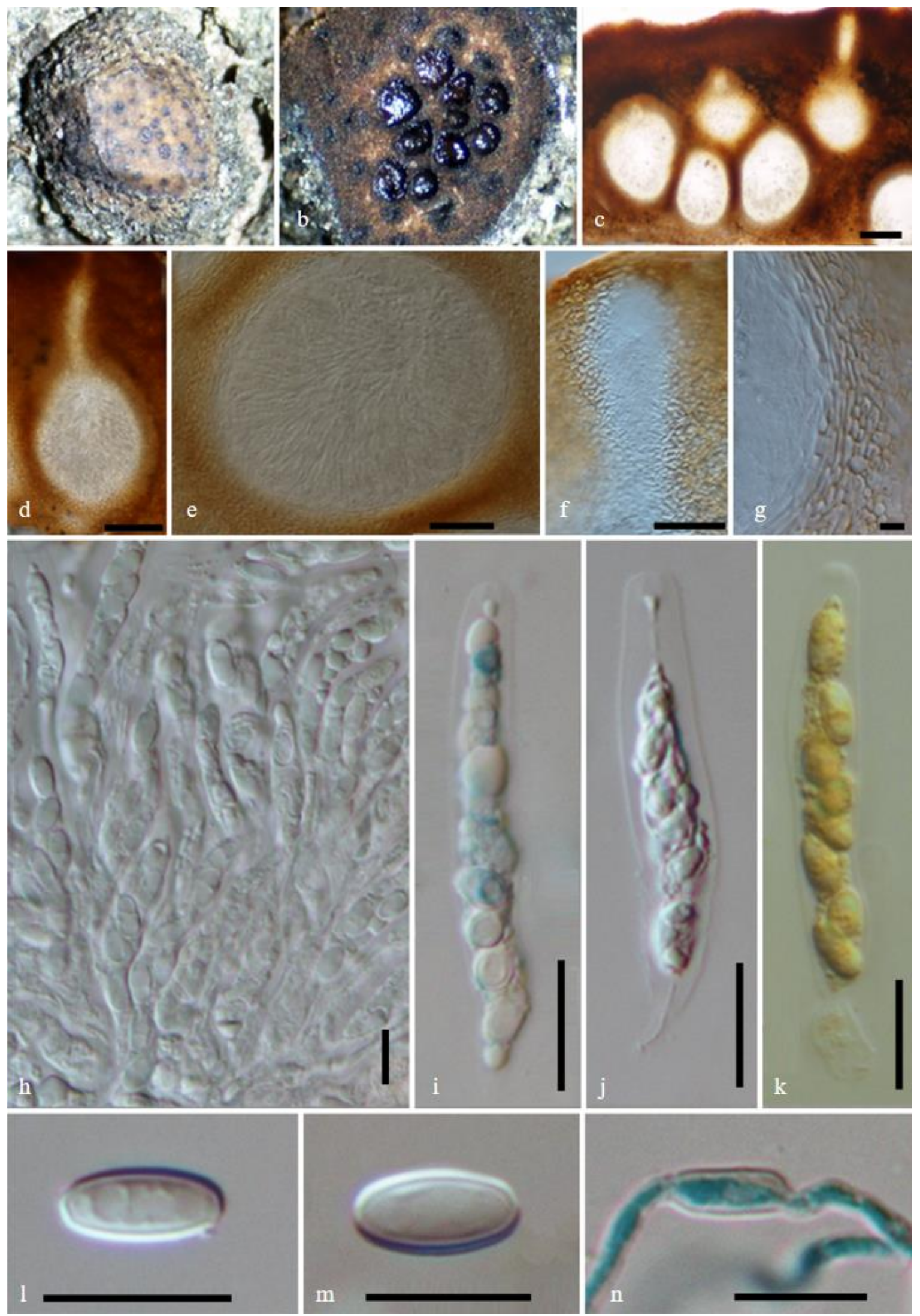

Fig. 172 - Catabotrys deciduum (AMH-10015, new geographical record). a Stromata on decaying wood of Rhizophora mucronata. b Horizontal section of stromata. c-e Longitudinal sections of ascomata. f Ostiole with periphyses. g Section of peridium comprising inner hyaline to pale brown cells of textura angularis. h Paraphyses and immature asci. $\mathrm{h}-\mathrm{j}$ Immature and mature asci. $\mathrm{k}$ Ascus in Lugol's iodine showing dextrinoid reaction. 1-m Ascospores n Germinating ascospore Scale bars: $b=100 \mu \mathrm{m}, \mathrm{c}=50 \mu \mathrm{m}, \mathrm{d}-\mathrm{n}=10 \mu \mathrm{m}$. 

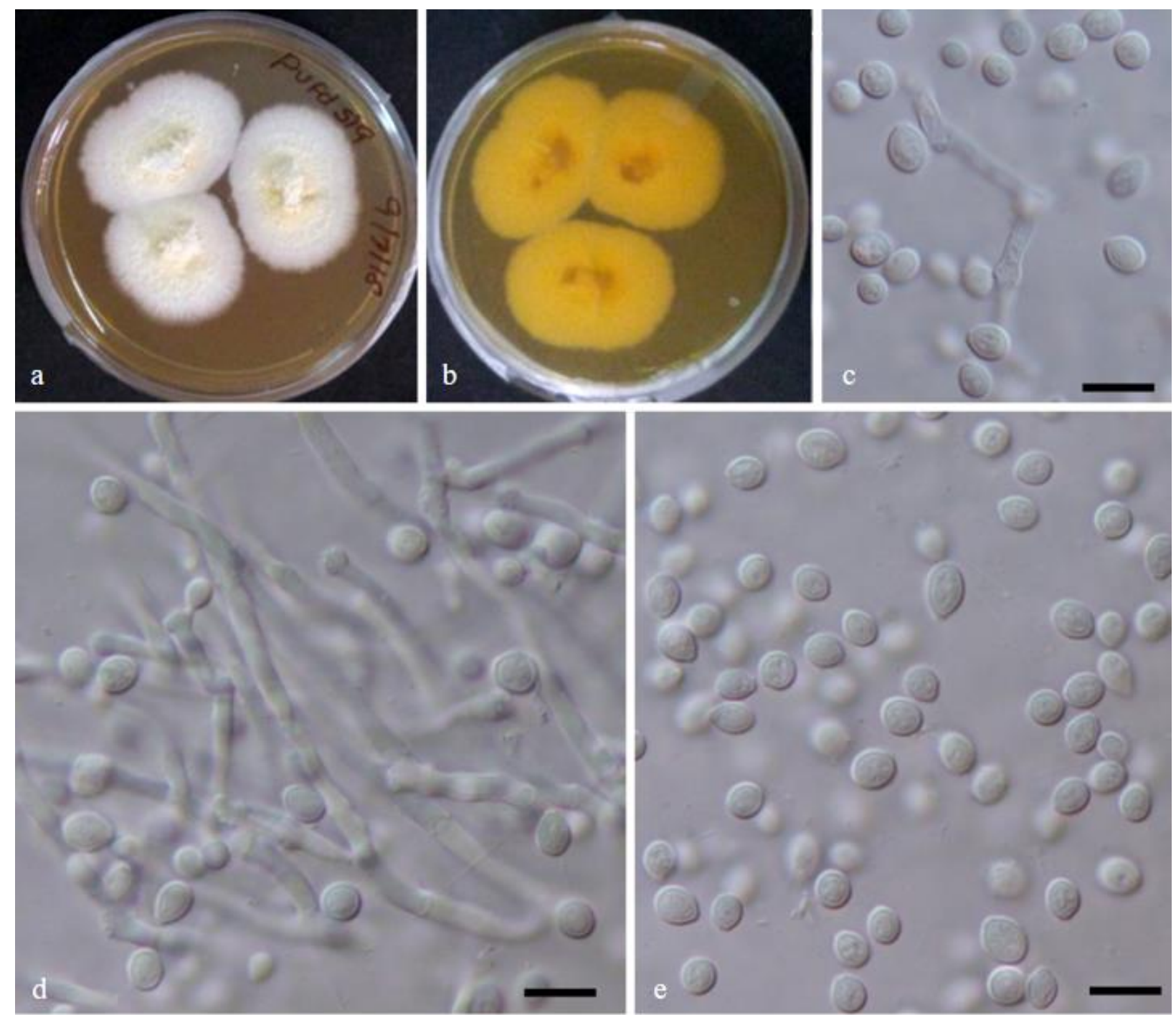

Fig. 173 - Catabotrys deciduum (NFCCI-4395, new geographical record). a, b Colonies on MEA. $(\mathrm{a}=$ from above $\mathrm{b}=$ from below $) . \mathrm{d}$ Conidiophores bearing conidia. $\mathrm{c}$, e Conidia. Scale bars: $\mathrm{c}-\mathrm{e}=$ $10 \mu \mathrm{m}$.

Further multi-gene phylogenetic analyses also showed that Catabotrys deciduum (NFCCI4395) clustered together with Catabotrys deciduum strains in a monophyletic clade with high statistical support (ML100\%, 1.00 BYPP) (Fig. 171). In the present study, we observed that Catabotrys deciduum (NFCCI-4395) produced an asexual morph with hyaline, ellipsoidal, 1-celled conidia in culture (MEA). Catabotrys deciduum is a saprobe, which commonly occurs on palms and larger monocots, with a pantropical distribution (Maharachchikumbura et al. 2016a). However, our collections of Catabotrys deciduum (AMH-10015) shows that it is frequent on intertidal decaying wood of Rhizophora mucronata thus extending its host range. Furthermore, its occurrence in Indian mangroves is a new country record and extends its geographical range.

\section{Agaricomycetes}

Agaricomycetidae Locq.

Agaricales Underw.

\section{Agaricaceae Chevall.}

Agaricaceae was established by Chevallier in 1826 (Chevallier 1826, Kirk 2008b). The classification of the family has changed considerably with the use of molecular dating results (Vellinga et al. 2004, 2011, Matheny et al. 2007, He et al. 2019b). 


\section{Lepiota (Pers.) Gray}

Lepiota is a large genus of white-spored mushrooms of Agaricaceae which is commonly distributed in tropical and temperate countries (Dennis 1952). Based on its morphology, the genus was divided into six sections (Vellinga 2001). However, DNA based studies did not support the sections (Vellinga 2003, Liang et al. 2011). There were 37 species of Lepiota recorded in Thailand (Sysouphanthong et al. 2011b, 2012, 2013, 2016, Tibpromma et al. 2017). In this study, four species of Lepiota are new records to Thailand.

Lepiota angusticystidiata J.F. Liang \& Z.L. Yang, Mycologia 110(3): 496 (2018)

Figs 175, 176

Facesoffungi number: FoF06198

Pileus 15-40 mm diameter when young conical to parabolic, expanding to hemispherical to campanulate or umbonate, finally plano-convex, with inflexed margin; when young granulose or glabrous, greyish brown to light brown (6B4-5), soon breaking up into concolorous squamules around glabrous umbo towards the margin on white background; margin with white cortinate and connected with stipe when young, when mature squamulose, fringed. Lamellae free, crowded, ventricose, 3-6 mm wide, white, with colored floccose edge. Stipe 50-75 $\times 5-8 \mathrm{~mm}$, cylindrical or slightly tapering to apex; covered with concolorous squamules as those on pileus, slightly crowded from base to annular zone, with white to yellowish white (4A2) background. Annulus an annular zone, with white fibrillose or cortinate. Context white in pileus, 2-3 $\mathrm{mm}$ wide; white to yellowish white (4A2) in stipe, hollow, with white fibrils in central cavity. Smell and Taste not observed. Spore print white.

Basidiospores 6.5-7 $\times 4-5 \mu \mathrm{m}$, avl $\times$ avw $=6.9 \times 4.6 \mu \mathrm{m}, \mathrm{Q}=1.4-1.7$, avQ $=1.5$, in sideview ellipsoid ovoid, in frontal view ellipsoid, hyaline, slightly thick-walled, dextrinoid, congophilous, cyanophilous, not metachromatic. Basidia 17-22 × 7-9 $\mu \mathrm{m}$, clavate, 4-spored, hyaline, slightly thick walled. Cheilocystidia 16-26 × 7-9 $\mu \mathrm{m}$, clavate, utriform to narrowly utriform, often fusiform, hyaline, slightly thick-walled, rarely with apical excrescence. Pileus covering a trichoderm made up of narrowly cylindrical elements with tapering to apex, 120-250 (300) $\times 10-17 \mu \mathrm{m}$, sometimes septate at base, thick-walled, with parietal pale brown pigment, with concolorous short narrowly clavate elements under these long elements, 30-50 × 13-20 $\mu \mathrm{m}$. Stipe covering of squamules a trichoderm similar to pileus covering. Clamp-connections present in all tissues.

Known distribution (based on molecular data) - China (Liang et al. 2018), northern Thailand (this study).

Known hosts (based on molecular data) - Terrestrial on soil or soil mixed with decayed leaves and wood (Liang et al. 2018, this study).

Material examined - Thailand, Chiang Mai Province, Mae Taeng District, Phadeng Village, N 190 07' 13.7", E $98^{\circ} 43^{\prime}$ 52,9", alt. 905 m., 8 October 2008. P. Sysouphanthong, PS219 (MFLU 09-0202); Chiang Mai Province, Mae Taeng District, Pongduad Village, N 1606' 16.1", E 99 43' 07,9", alt. 780-805 m., 18 July 2008. P. Sysouphanthong, PS86 (MFLU 090159); Chiangrai Province, Mae Jan District, Forest of Huay Kang Pla Village, N $20^{\circ} 05^{\prime}$ 28.1", E 99 46' 52,7", alt. 512 m., 2 July 2008. P. Sysouphanthong, PS135 (MFLU 09-0208).

GenBank numbers - ITS (MFLU 09-0159 = MN240415, MFLU 09-02020 = MN240414, MFLU 09-0208 = MN240416).

Notes - From this study, all specimens of Lepiota angusticystidiata are recognized by greyish brown to light brown squamules covering the pileus and stipe, ellipsoid ovoid basidiospores, clavate to utriform cheilocystidia, a trichodermal pileus and stipe covering made up of narrowly cylindrical elements which taper at the apex with short narrowly clavate elements and clampconnections (see Figs 175, 176). 


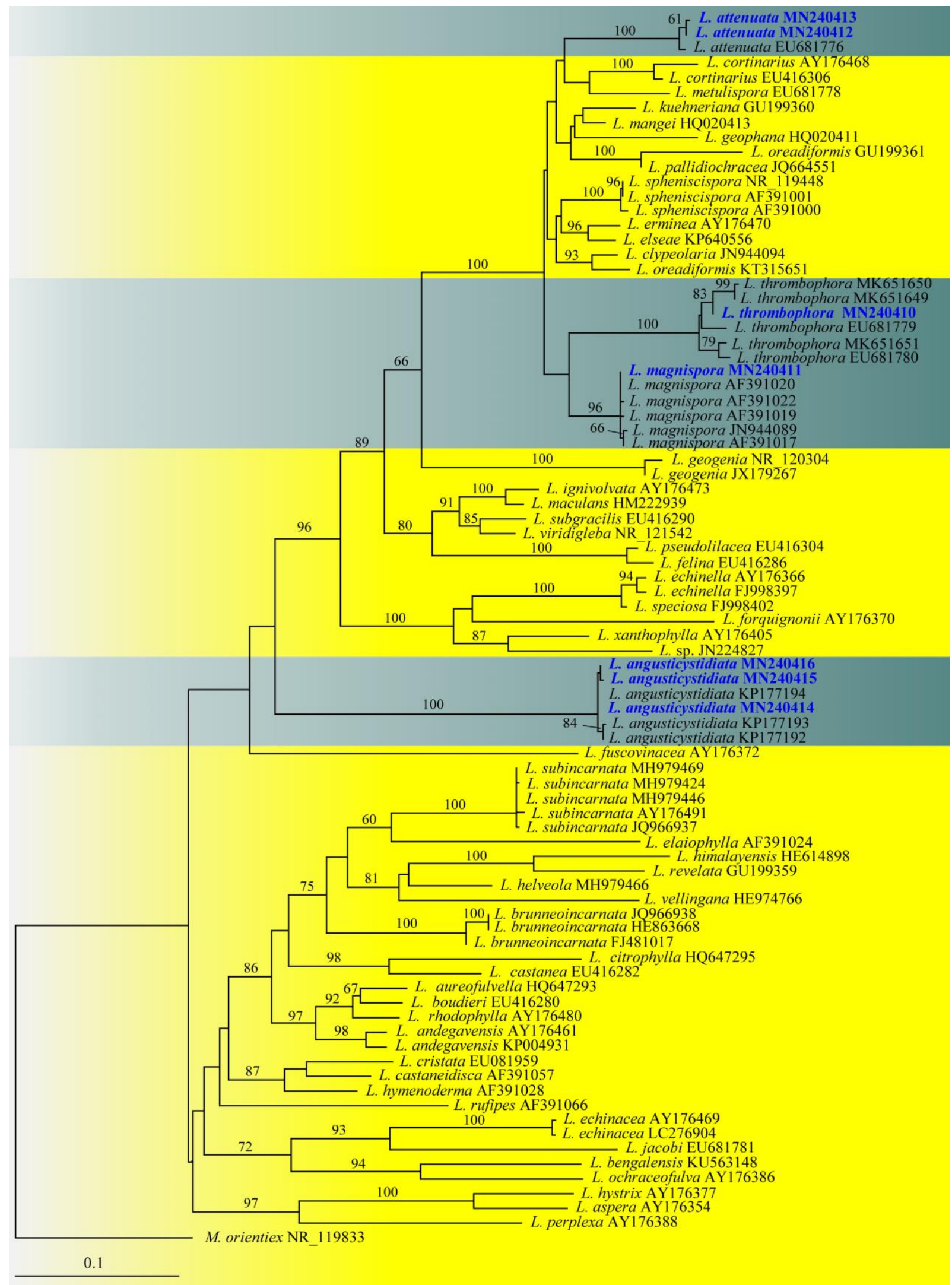

Fig. 174 - Maximum likelihood phylogenetic tree based on nrITS sequences of Lepiota species. Dataset was analysed in RAxML7.2.6 (Stamatakis et al. 2008) with GTRGAMMAI as the model of evolution, and branch support was estimated over 1000 bootstrap partitions (BP) with the rapid bootstrap option. Bootstrap support $\geq 60 \%$ are indicated at the nodes. New sequences from this study are in blue bold. The GenBank accession numbers are indicated after species name. Abbreviation $L=$ Lepiota, $M=$ Macrolepiota. The tree is rooted with Macrolepiota orienriex. 
Lepiota angusticystidiata was described from southwest China and this species belongs to section Ovisporae (J.E. Lange) Kühner (Liang et al. 2018). All Thai specimens have larger basidiomata than the type specimen from China, but all other characteristics are similar. In nrITS analysis the Thai specimens clustered with the type and other specimens from China (see Fig. 174).

Lepiota angusticystidiata is a first record for Thailand and is distributed in deciduous forest of Chiang Mai and Chiang Rai of northern Thailand with an elevation around 500-900 m.

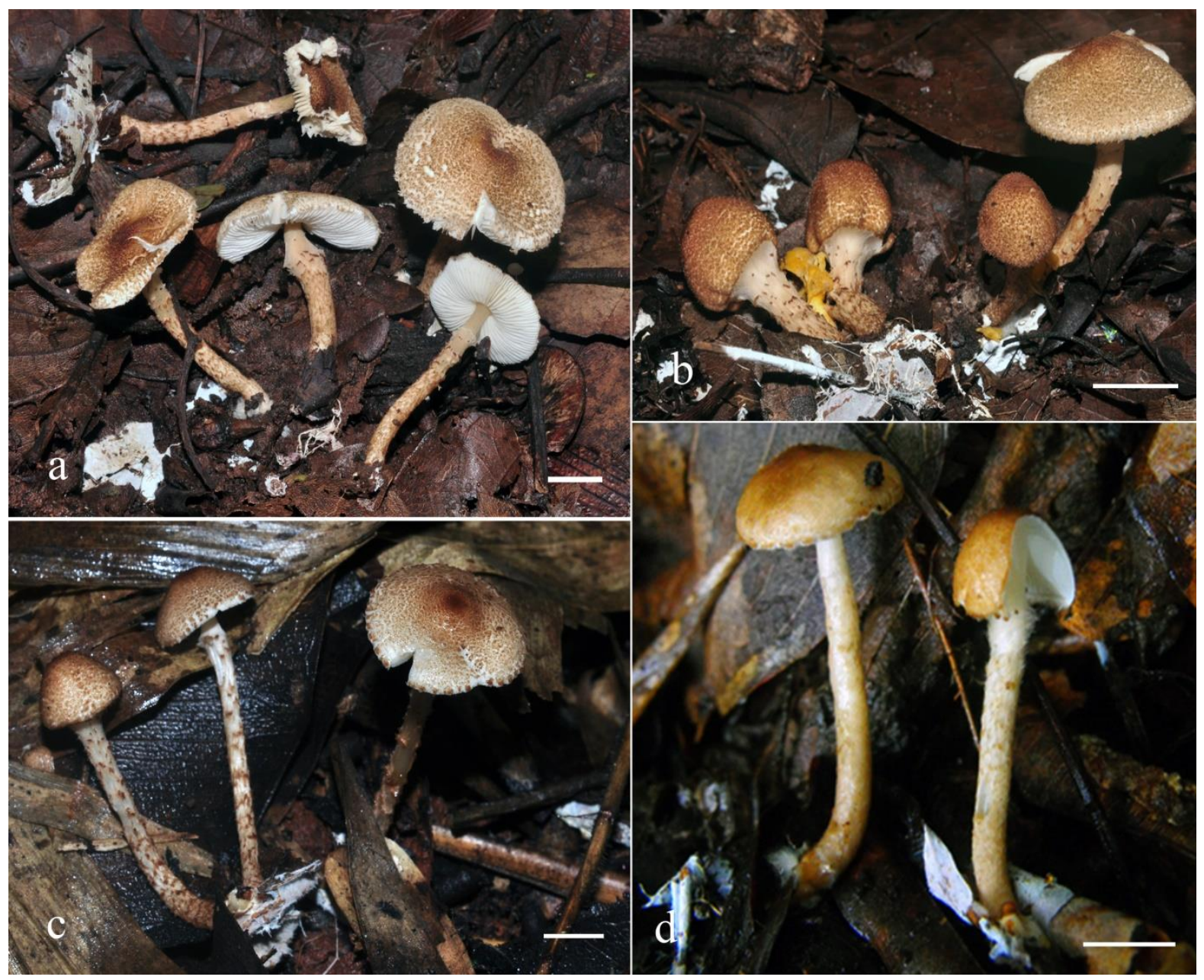

Fig. 175 - Fresh basidiomata of Lepiota angusticystidiata on field. a, b MFLU 09-0202. c MFLU 09-0159. d MFLU 09-0208. Scale bars: a-d = 10 mm.

Lepiota attenuata J.F. Liang \& Zhu L. Yang, Mycologia 103(4): 821 (2011)

Facesoffungi number: FoF06197

Figs 177,178

Pileus 40-95 mm diameter campanulate or convex, expanding to plano-convex or applanate, with straight to inflexed margin; with granulose or glabrous at center, sometime with patch-liked at center, greyish brown to brownish orange (6C2-3), with centrically concolorous squamules or fibrillose squamules around patch towards the margin, with radially reddish white fibrillose between squamules towards the margin on white background; margin striate or sulcate at marginal zone, fringed, sometime attached with white remnants and concolorous squamules. Lamellae free, crowded, broadly ventricose, 5-12 mm wide, white, with colored eroded edge. Stipe 70-110 × 6$12 \mathrm{~mm}$, cylindrical or slightly tapering to apex, covered with white to yellowish white (4A2) fibrillose on white background. Annulus attached at upper site of stipe, membranous or white cortinate with greyish brown to brownish orange (6C2-3) squamules upper part. Context white in pileus, 4-7 mm wide; white to yellowish white (4A2) in stipe, hollow, with white fibrils in central cavity. Smell and Taste not observed. Spore print white. 

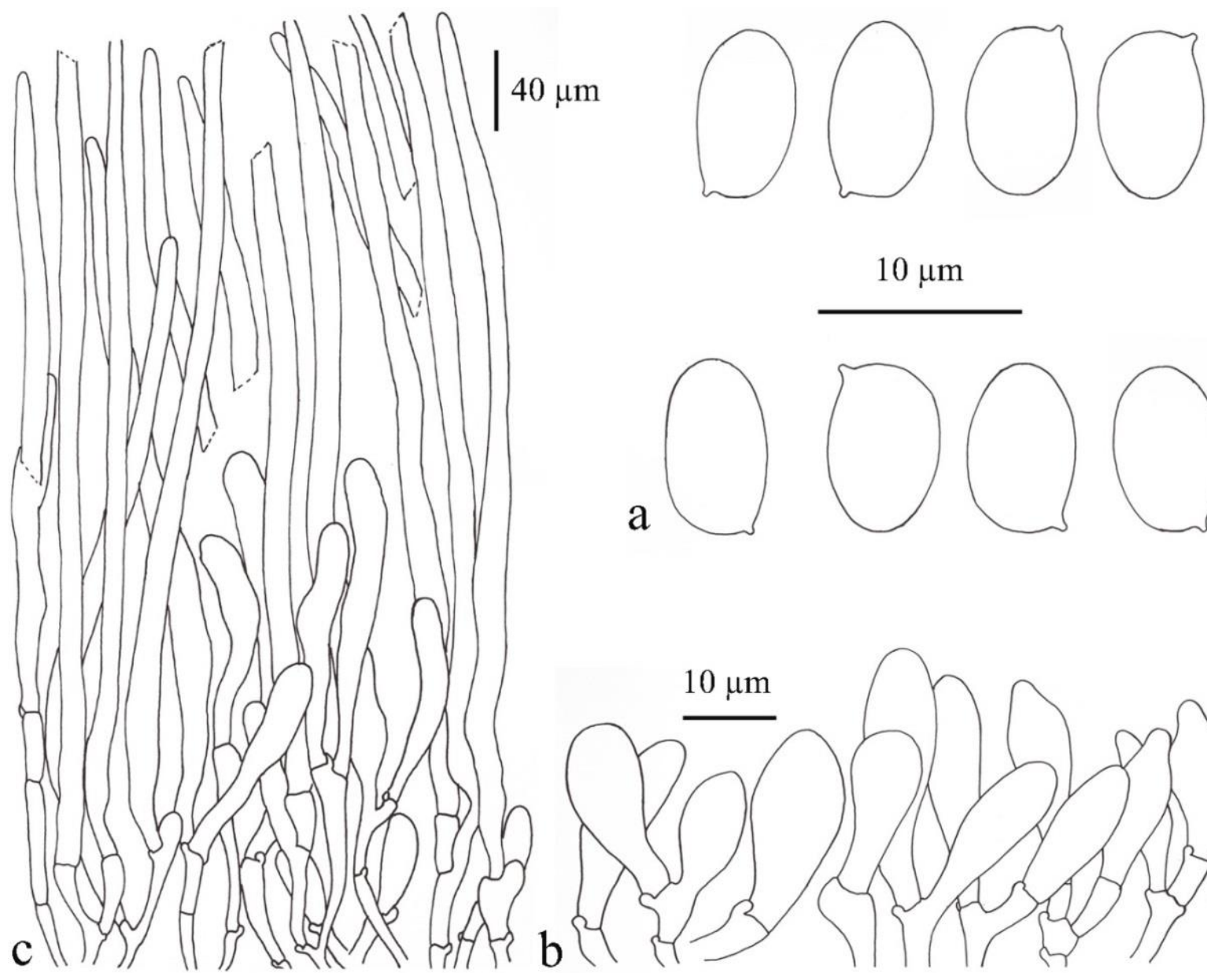

$10 \mu \mathrm{m}$
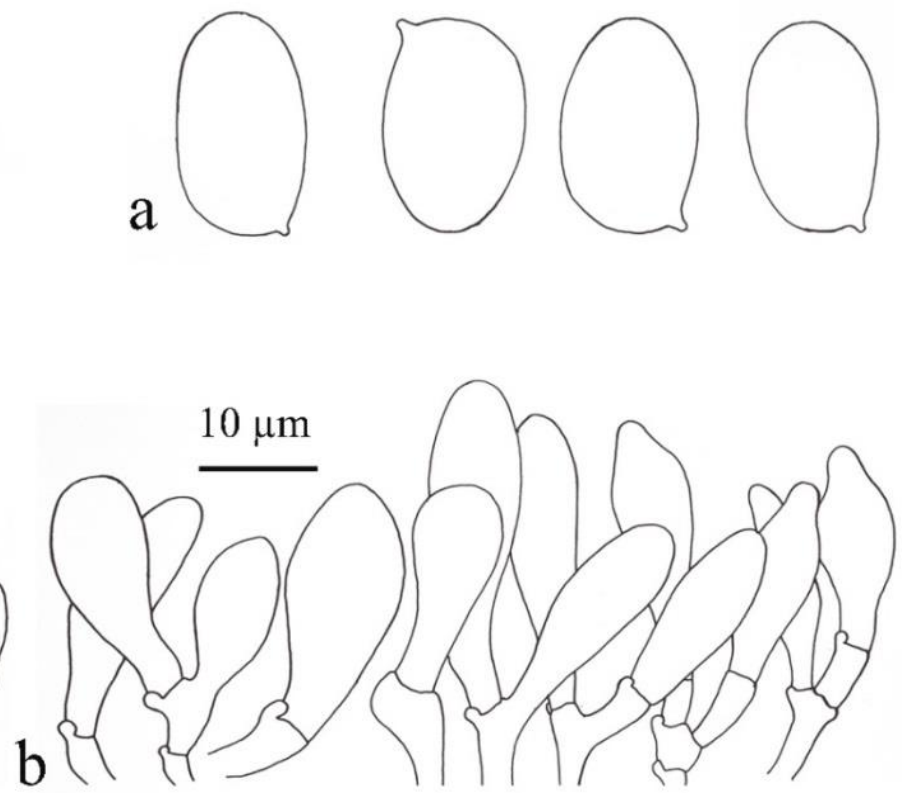

Fig. 176 - Microcharacters of Lepiota angusticystidiata (MFLU 09-02020). a Basidiospores. b Cheilocystidia. c A trichodermal pileus structure of pileus covering.

Basidiospores $16-19 \times 4.8-5.2 \mu \mathrm{m}$, avl $\times$ avw $=17.32 \times 5.02 \mu \mathrm{m}, \mathrm{Q}=3.2-3.6$, avQ $=3.45$, in side-view ellipsoid penguin-shaped with straight adaxial side and a suprahilar depression, with attenuate to apex, in frontal view fusiform, hyaline, slightly thick-walled, dextrinoid, congophilous, cyanophilous, not metachromatic. Basidia 28-32 × 7-10 $\mu \mathrm{m}$, clavate, 4-spored, rarely 2-spored, hyaline, thick walled. Cheilocystidia 25-28 $\times 6-10 \mu \mathrm{m}$, clavate to utriform or fusiform, rarely with apical excrescence, often with short clavate and septate, 6-10 $\times 4-6 \mu \mathrm{m}$. Pileus covering a trichoderm made up of narrowly cylindrical elements with tapering to apex, $120-300 \times 7.5-11.5$ $\mu \mathrm{m}$, sometimes septate at base, thick-walled, with pale brown parietal pigment; with concolorous short clavate elements with septate under these long elements. Stipe covering not observed. Clampconnections present in all tissues.

Known distribution (based on molecular data) - China (Liang et al. 2011), Northern Thailand (this study).

Known hosts (based on molecular data) - terrestrial on soil or soil mixed with decayed leaves and wood (Liang et al. 2011, this study).

Material examined - Thailand, Chiang Rai Province, Muang District, Mae Fah Luang University, N $18^{\circ} 05^{\prime}$ 59.1", E 102 40' 22,9", alt. 488 m., 6 November 2008. P. Sysouphanthong, PS123 (MFLU 090-196); ibidem, 6 Nov. 2008. P. Sysouphanthong, PS126 (MFLU 09-0199).

GenBank numbers - ITS (MFLU 09-0196 = MN240413, MFLU 09-0199 = MN240412).

Notes - Thai specimens of Lepiota attenuata are characterized by greyish brown to brownish orange squamulose to fibrillose squamules on the pileus, a striate or sulcate pileus margin, with a white to yellowish white fibrillose stipe, with an annulus, penguin-shaped basidiospores, clavate to utriform cheilocystidia, a trichodermal pileus cover, and clamp-connections. 
Lepiota attenuata was described from southern China by Liang et al. (2011). The species is placed in section Lepiota which comprises species with long basidiospores and a trichodermal pileus covering, and taxa similar to this species are Lepiota metulispora, Lepiota thrombophora, Lepiota cortinarius, Lepiota magnispora and Lepiota spheniscispora. Lepiota attenuata however, has basidiospores which are distinctly narrowed at the apex (Vellinga 2001, Wang 2004, Liang et al. 2011).

The nrITS sequences analysis showed that two Thai specimens clustered with type specimens from south China (see Fig. 174). The species is a new record for Chiang Rai of northern Thailand.
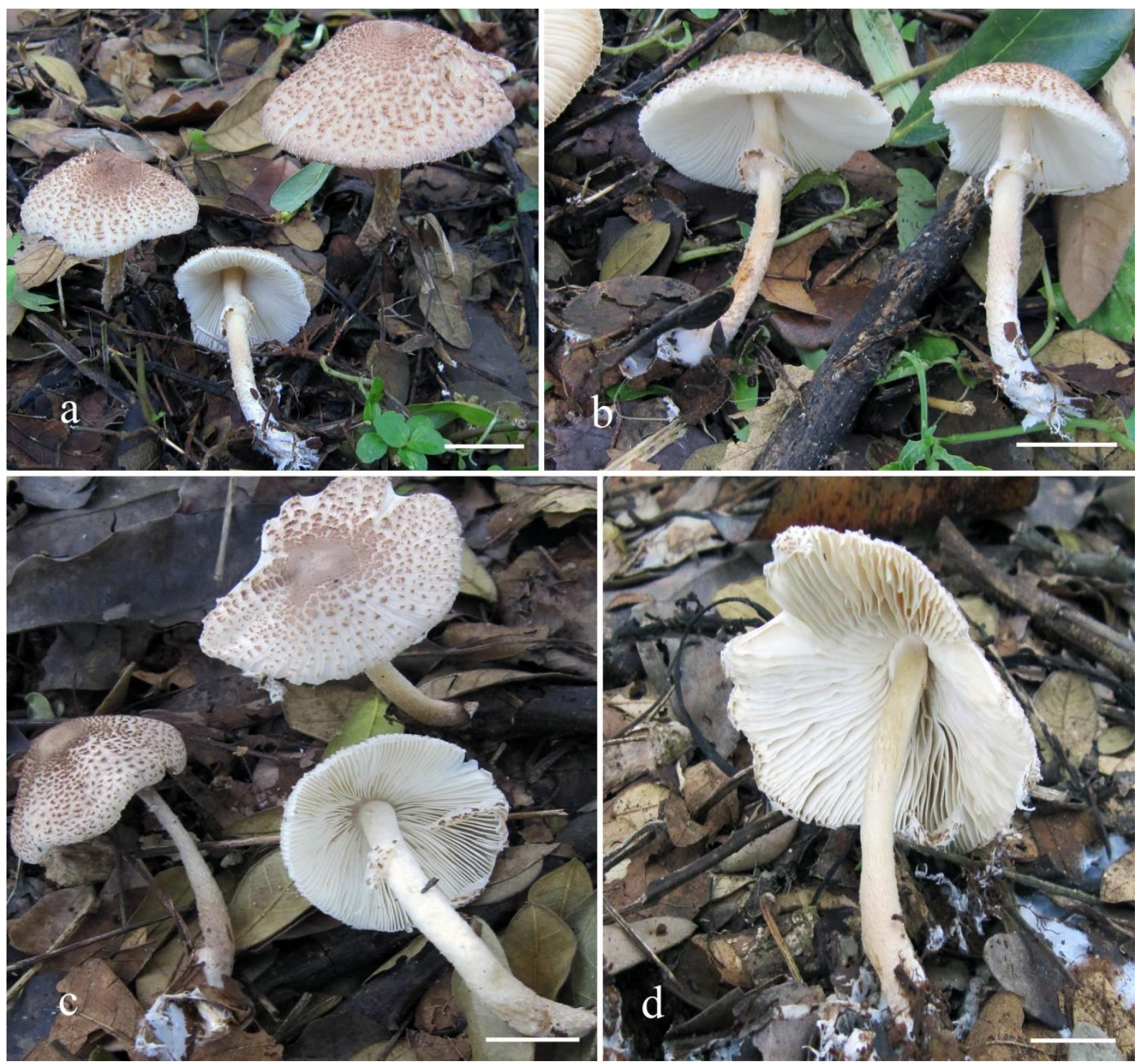

Fig. 177 - Fresh basidiomata of Lepiota attenuata on field. a, b MFLU 09-0196. c, d MFLU 090199. Scale bars: $a-d=20 \mathrm{~mm}$.

Lepiota magnispora Murrill, Mycologia 4: 237. 1912.

Figs 179, 180

Facesoffungi number: FoF06195

Pileus $65 \mathrm{~mm}$ diameter, umbonate, with straight or slightly inflexed margin; covered with light brown to golden brown (5D5-7) granulose at center, with orange white to light orang (6A2-4) granulose, with crowded squamules towards the margin on white background; margin with white to light orange (6A4) floccules. Lamellae free, crowded, ventricose, up to $6 \mathrm{~mm}$ wide, white, with colored eroded edge. Stipe $85 \times 5-10 \mathrm{~mm}$, tapering to apex, covered with white to light orang (6A4) granulose, very crowded from base to annular zone, with light brown to golden brown (5D57) squamules at base zone on orange white (6A2) background. Annulus an annular zone with white 
to light orang (6A4) granulose. Context white in pileus, $5 \mathrm{~mm}$ wide; white to orange white (6A2) in stipe, hollow, with white fibrils in central cavity. Smell and Taste not observed. Spore print white. Basidiospores 14-18 $\times 4.5-5.5 \mu \mathrm{m}$, avl $\times$ avw $=15.22 \times 5.02 \mu \mathrm{m}, \mathrm{Q}=2.8-3.4$, avQ $=3.05$, in sideview ellipsoid penguin-shaped with straight adaxial side and a suprahilar depression, with slightly attenuate to apex, in frontal view fusiform, hyaline, slightly thick-walled, dextrinoid, congophilous, cyanophilous, not metachromatic. Basidia 25-31 × 9-11 $\mu \mathrm{m}$, clavate, 4-spored, rarely 2-spored, hyaline, thick walled. Cheilocystidia 23-38 × 6-12 $\mu \mathrm{m}$, normally utriform or fusiform, rarely with clavate, often with 2-3 septate at base. Pileus covering a trichoderm made up of narrowly cylindrical elements with rounded apex, 175-310 × 11-15 $\mu \mathrm{m}$, sometimes septate, with encrusted at base layer, thick-walled, with pale brown parietal pigment; with concolorous short clavate elements with septate under these long elements. Clamp-connections present in all tissues.

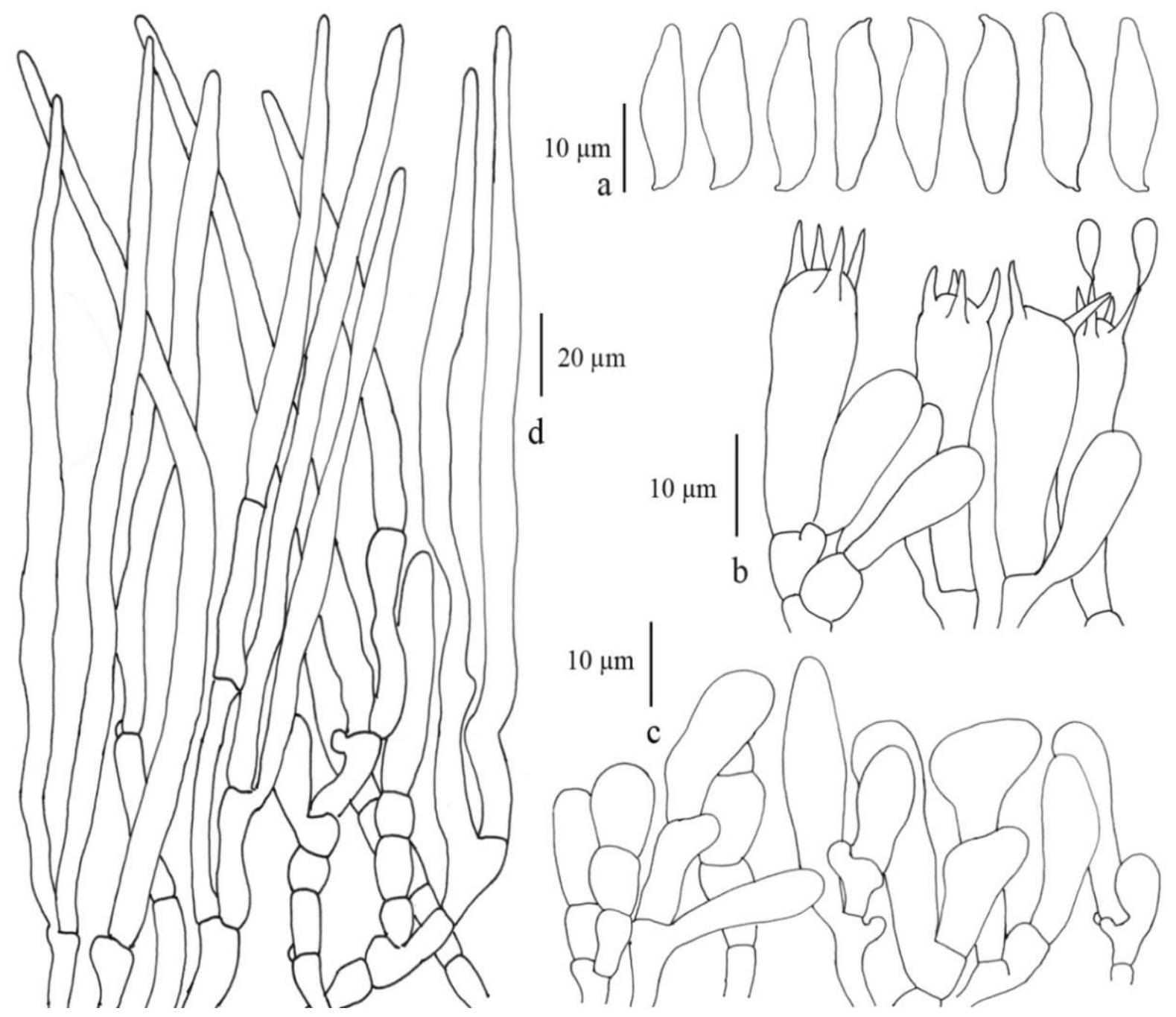

Fig. 178 - Microcharacteristics of Lepiota attenuata (MFLU 09-0196). a Basidiospores. b Basidia and basidiomes. c Cheilocystidia. $\mathrm{d}$ A trichodermal pileus structure of pileus covering.

Known distribution (based on molecular data) - China, Germany, Netherlands, USA (Liang et al. 2011, Vellinga 2004), Thailand (this study).

Known hosts (based on molecular data) - Terrestrial on soil or soil mixed with decayed leaves and wood (this study).

Material examined - Thailand; Chiang Mai Province, Mae Taeng District, Phadeng Village, N 19 07' 13.7", E $98^{\circ} 43^{\prime}$ 52,9", alt. 905 m., 9 July 2008. P. Sysouphanthong, PS51 (MFLU 090124).

GenBank numbers - ITS (MFLU 09-0124 = MN240411). 
Notes - The description of Lepiota magnispora is based on a single collection. The species is characterized by a light brown to golden brown pileus with white to light orange floccose margins, white and free lamellae, a white to light orange floccose stipe, ellipsoid penguin-shaped, utriform or fusiform cheilocystidia, a trichodermal pileus cover made up of narrowly cylindrical elements with rounded apices and clamp-connections.

Lepiota magnispora is mostly found in temperate countries, and taxonomical history of the species was discussed in Vellinga (2000). With regards to distribution in Asia, Lepiota magnispora is distributed in China and Japan (Sysouphanthong et al. 2011a). In this study, Lepiota magnispora is also found in quite high elevation $(905 \mathrm{~m}$.) in north Thailand. Phylogenetic analysis based on nrITS sequences indicate that our specimen of Lepiota magnispora from Thailand is identical and clustered with those specimens from temperate countries such as Belgium, Canada, China and USA.
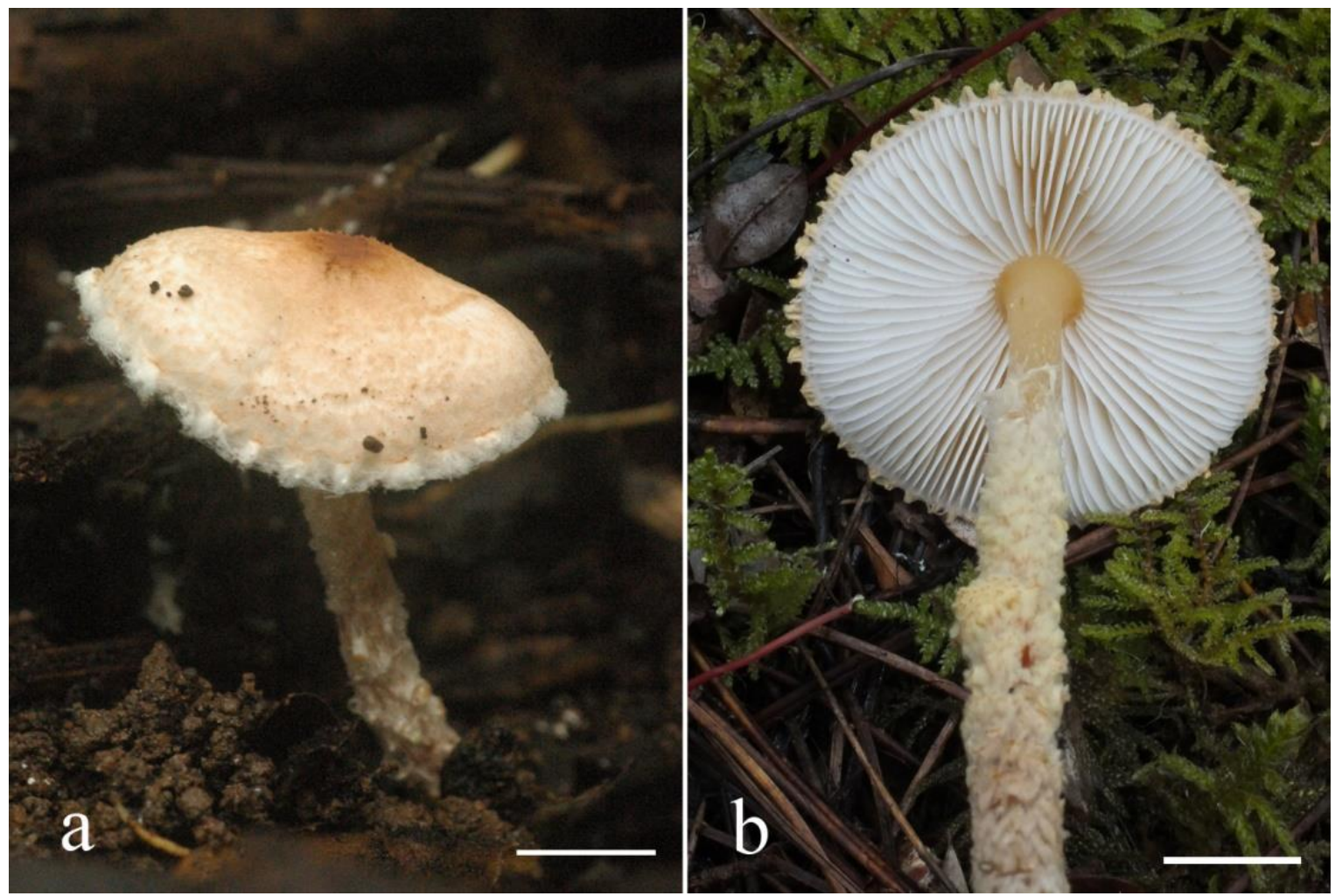

Fig. 179 - Fresh basidiomata of Lepiota magnispora on field. a, b MFLU 09-0124. Scale bars: a-b $=20 \mathrm{~mm}$.

Lepiota thrombophora (Berk. \& Broome) Sacc., Syll. fung. (Abellini) 5: 53 (1887)

Figs 181, 182

Facesoffungi number: FoF06196

Pileus $25 \mathrm{~mm}$ diameter, parabolic to hemispherical, with straight margin; covered with light brown to golden brown (5D5-7) granulose or crowded squamules at center, with pale orange to light orang (5A3-4) granulose to squamules towards the margin on white background; margin slightly sulcate, with white floccules or cortinate. Lamellae free, crowded, ventricose, up to $4 \mathrm{~mm}$ wide, white, with colored eroded edge. Stipe $45 \times 4-6 \mathrm{~mm}$, cylindrical or slightly tapering to apex; covered with white granulose or fibrillose, very crowded from base to annular zone, with pale orange to light orang (5A3-4) granulose to squamules at base zone on orange white (6A2) background. Annulus an annular zone with white fibrillose or cortinate. Context white in pileus, 2 mm wide; white in stipe, hollow, with white fibrils in central cavity. Smell and Taste not observed. Spore print white. 


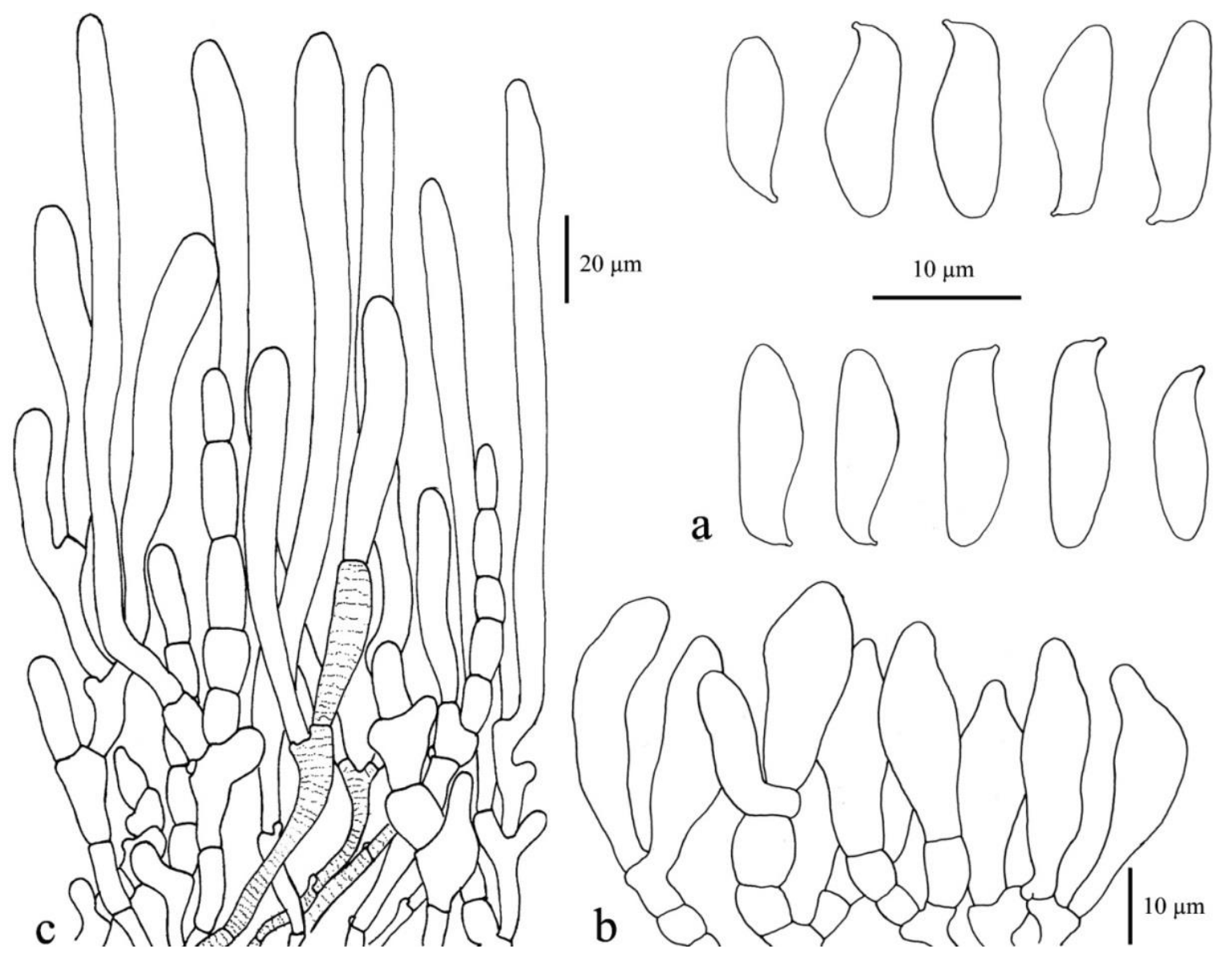

Fig. 180 - Microcharacteristics of Lepiota magnispora (MFLU 09-0124). a Basidiospores. b Cheilocystidia. c A trichodermal pileus structure of pileus covering.

Basidiospores $10-14 \times 3-5 \mu \mathrm{m}$, avl $\times$ avw $=11.8 \times 4.1 \mu \mathrm{m}, \mathrm{Q}=2.4-4.3$, avQ $=2.89$, in sideview ellipsoid penguin-shaped with straight adaxial side and a suprahilar depression, with slightly attenuate or rounded apex, in frontal view fusiform, hyaline, slightly thick-walled, not dextrinoid, congophilous, cyanophilous, not metachromatic. Basidia 18-22 × 6-8 $\mu \mathrm{m}$, clavate, 4-spored, sometime 2-spored, rarely 1-spored, hyaline, thick-walled. Cheilocystidia 18-30 × 10-12 $\mu \mathrm{m}$, short clavate to clavate, often utriform or fusiform. Pileus covering a trichoderm made up of narrowly cylindrical elements with rounded apex, 110-190 × 12-15 $\mu \mathrm{m}$, thick-walled, with pale brown parietal pigment; with concolorous short clavate elements under these long elements, 30-40 × 10$16 \mu \mathrm{m}$. Stipe covering in squamules a trichoderm similar to pileus covering. Clamp-connections present in all tissues.

Known distribution (based on molecular data) - China (Liang et al. 2011), Thailand (this study).

Known hosts (based on molecular data) - terrestrial on humus soil mixed with decayed leaves (Liang et al. 2011, this study).

Material examined - Thailand, Chiang Mai Province, Mae Rim District, Mae Sae Village, 18 July 2008. P. Sysouphanthong, PS89 (MFLU 09-0162).

GenBank numbers: ITS (MFLU 09-0162 = MN240410).

Notes - A specimen of a Thai Lepiota thrombophora was observed in a single young collection. It is characterized by pale orange to light orange granulose to squamules on the pileus, white and free lamellae, penguin-shaped basidiospores, clavate cheilocystidia, a trichoderm made up of narrowly cylindrical elements with rounded apex and clamp-connections (Figs 181, 182).

The microcharacters of the Thai specimen closely matches the holotype from Sri Lanka in shapes and size, including elements of the pileus covering being cylindrical with a rounded apex. However, specimens of Lepiota thrombophora from China has cylindrical elements with an 
attenuated apex (Liang et al. 2011). In analysis of nrITS sequence data, the Thai specimen clustered with the two specimens from China (see Fig. 174).

Lepiota thrombophora is distributed in tropical Asia in China, India and Sri Lanka (Liang et al. 2011, Pegler \& Young 1986, Natarajan \& Manjula 1983, Sysouphanthong et al. 2011a). Lepiota thrombophora is a new record to Thailand.

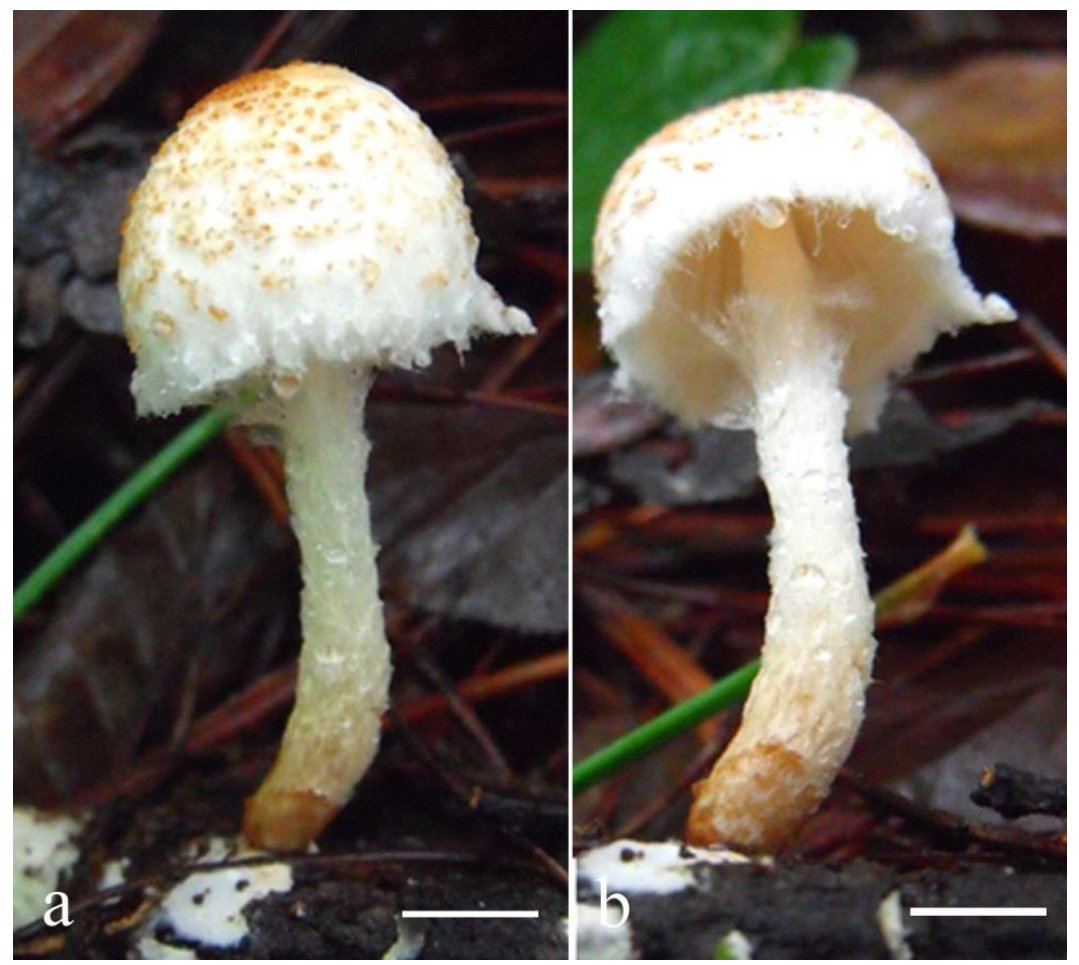

Fig. 181 - Fresh basidiomata of Lepiota thrombophora on field. a, b MFLU 09-0162. Scale bars: $\mathrm{a}-\mathrm{b}=10 \mathrm{~mm}$.

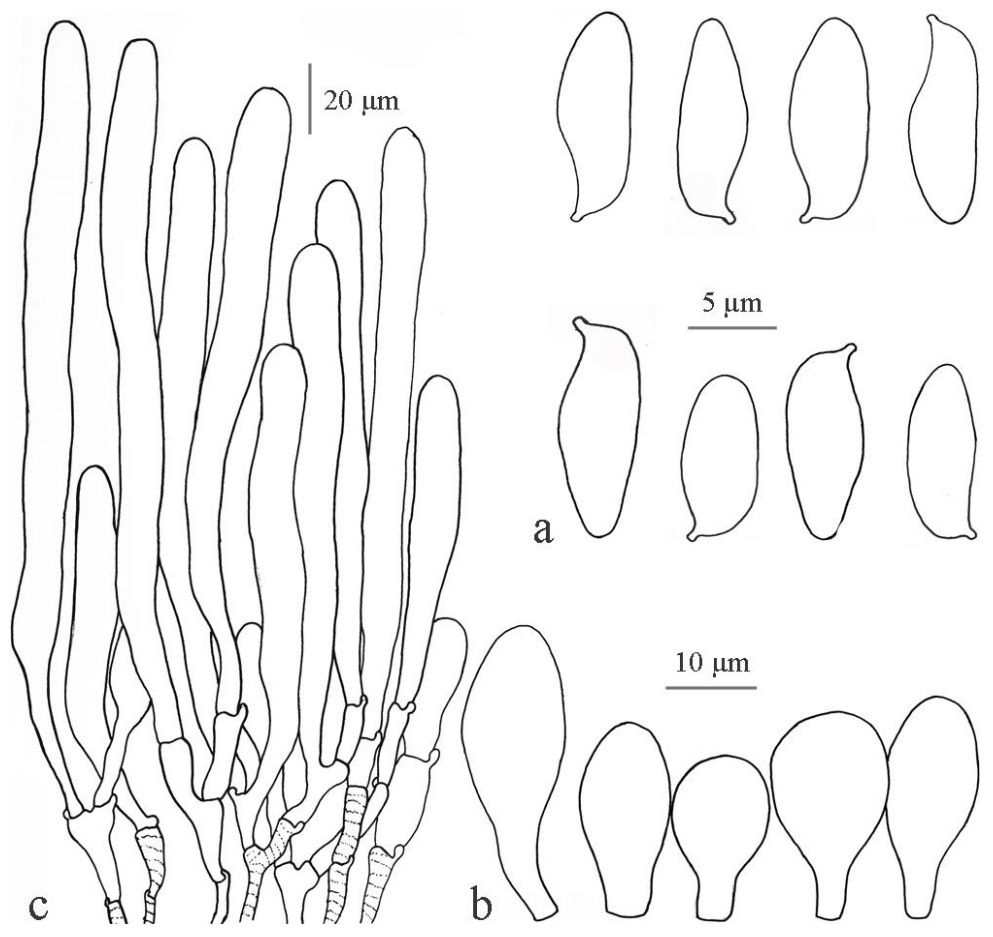

Fig. 182 - Microcharacteristics of Lepiota thrombophora (MFLU 09-0162). a Basidiospores. b Cheilocystidia. c A trichodermal pileus structure of pileus covering. 


\section{Acknowledgements}

Kevin D. Hyde would like to thank 'Impact of climate change on fungal diversity and biogeography in the Greater Mekong Subregion' (grant no: RDG6130001) and 'Macrofungi diversity research from the Lancang-Mekong Watershed and surrounding areas' (grant no. DBG6280009). Saranyaphat Boonmee would like to thank the Thailand Research Fund (No. TRG6180001), Plant Genetic Conservation Project under the Royal Initiation of Her Royal Highness Princess Maha Chakri Sirindhorn-Mae Fah Luang University and the National Research Council of Thailand (projects No. 61215320013 and No. 61215320023). Rungtiwa Phookamsak thanks CAS President's International Fellowship Initiative (PIFI) for young staff (grant no. Y9215811Q1), and National Science Foundation of China (NSFC) project code 31850410489 (grant no. Y81I982211) for financial research support. Napalai Chaiwan would like to thank the Royal Golden Jubilee PhD Program under Thailand Research Fund (RGJ) The scholarship no. PHD60K0147. V.V. Sarma would like to thank Ministry of Earth Sciences (MOES), Govt. of India for funding a project (Sanction order: MOES/36/OO1S/Extra/40/ 2014/PC-IV dt. 14.01.2015); the Department of Biotechnology, Pondicherry University for facilities. B. Devadatha would like to thank MOES for a fellowship. Mingkwan Doilom thanks the 5th batch of Postdoctoral Orientation Training Personnel in Yunnan Province (Number Y934283261) and the 64th batch of China Postdoctoral Science Foundation (Number Y913082271) for funding her postdoctoral research. Lakmali Dissanayake would like to thank the National Natural Science Foundation of China (NSFC Grants nos. 31670027, 31460011, 30870009). Rajesh Jeewon thanks the University of Mauritius and Mae Fah Luang University for research support. Monika C. Dayarathne would like to thank the grants National Natural Science Foundation of China (No. 31972222, 31560489), and Talent project of Guizhou Science and Technology Cooperation Platform ([2017]5788-5 and [2019]5641) and Guizhou Science, Technology Department International Cooperation Basic project ([2018]5806). Samantha C. Karunarathna thanks CAS President's International Fellowship Initiative (PIFI) for funding his postdoctoral research (No. 2018PC0006) and the National Science Foundation of China (NSFC) for partially funding this work under the project code 31851110759. J.K. Liu would like to thank National Natural Science Foundation of China (Project No.31600032).

\section{References}

Abbott EV. 1927 - Scolecobasidium, a new genus of soil fungi. Mycologia 19:29-31

Abdollahzadeh J, Goltapeh EM, Javadi A, Shams-bakhsh M et al. 2009 - Barriopsis iraniana and Phaeobotryon cupressi: two new species of the Botryosphaeriaceae from trees in Iran. Persoonia 23, 1-8.

Adams GC, Roux J, Wingfield MJ. 2006 - Cytospora species (Ascomycota, Diaporthales, Valsaceae): introduced and native pathogens of trees in South Africa. Australasian Plant Pathology, 35, 521-548.

Adams GC, Wingfield MJ, Common R, Roux J. 2005 - Phylogenetic relationships and morphology of Cytospora species and related teleomorphs (Ascomycota, Diaporthales, Valsaceae) from Eucalyptus. Studies in Mycology 52, 1-144.

Ahmed SI, Cain RF. 1972 - Revision of the genera Sporormia and Sporormiella. Canadian Journal of Botany 50(3), 419-477.

Alam MW, Rehman A, Gleason ML, Riaz K et al. 2017a - First report of Nigrospora sphaerica causing leaf spot of kinnow mandarin in Pakistan. Journal of Plant Pathology 99, 295.

Alam MW, Rehman A, Saira M, Kan NA et al. 2017b - First report of leaf spots in Aloe vera caused by Nigrospora oryzae in Pakistan. Plant Disease 101(5), 841.

Al-Sadi AM, Al-Wehaibi AN, Al-Shariqi RM, Al-Hammadi MS et al. 2013 - Population Genetic Analysis Reveals Diversity in Lasiodiplodia Species Infecting Date Palm, Citrus, and Mango in Oman and the UAE. Plant Disease 97, 1363-1369.

Alvarez LV, Groenewald JZ, Crous PW. 2016 - Revising the Schizoparmaceae: Coniella and its synonyms Pilidiella and Schizoparme. Studies in mycology 85, 1-34. 
Alves A, Barradas C, Phillips AJ, Correia A. 2013 - Diversity of Botryosphaeriaceae species associated with conifers in Portugal. European journal of plant pathology 135, 791-804.

Alves A, Phillips AJL, Henriques I, Correia A. 2005 - Evaluation of amplified ribosomal DNA restriction analysis as a method for the identification of Botryosphaeria species. FEMS Microbiology Letters 245, 221-229.

Anonymous 1960 - Index of Plant Diseases in the United States. U.S.D.A. Agric Handb 165, 1531.

Ariyawansa HA, Camporesi E, Thambugala KM, Mapook A et al. 2014a - Confusion surrounding Didymosphaeria phylogenetic and morphological evidence suggest Didymosphaeriaceae is not a distinct family. Phytotaxa 176, 102-119.

Ariyawansa HA, Hyde KD, Jayasiri SC, Buyck B et al. 2015 - Fungal diversity notes 111-252 taxonomic and phylogenetic contributions to fungal taxa. Fungal Diversity 75, 27-274.

Ariyawansa HA, Kang JC, Alias SA, Chukeatirote E, Hyde KD. 2014b - Pyrenophora. Mycosphere 5, 351-362.

Ariyawansa HA, Tanaka K, Thambugala KM, Phookamsak R et al. 2014c - A molecular phylogenetic reappraisal of the Didymosphaeriaceae (= Montagnulaceae). Fungal Diversity 68, 69-104.

Ariyawansa HA, Thambugala KM, Manamgoda DS, Jayawardena R et al. 2015 - Towards a natural classification and backbone tree for Pleosporaceae. Fungal Diversity 71, $85-139$.

Arjona-Girona I, Lopez-Herrera CJ. 2016 - First report of branch dieback in mango trees caused by Neofusicoccum parvum in Spain. Plant Disease 100, 2529-2530.

Bae JS, Jin BR, Lee HO, Park EJ et al. 2002 - Determination of the Ribosomal DNA Internal Transcribed Spacers and 5.85 rDNA Sequences of Cordyceps Species. International Journal of Industrial Entomology 5 (1), 85-91.

Bakhshi M, Arzanlou M, Groenewald JZ, Quaedvlieg W, Crous PW. 2019 - Parastagonosporella fallopiae gen. et sp. nov. (Phaeosphaeriaceae) on Fallopia convolvulus from Iran. Mycological Progress 18, 203-214.

Barkat EH, St J, Hardy GE, Ren Y et al. 2016 - Fungal contaminants of stored wheat vary between Australian states. Australas. Australasian Plant Pathology 45(6), 621-628.

Barnett HL, Hunter BB. 1998 - Illustrated genera of imperfect fungi. APS Press, Minnesota.

Barr ME. 1978 - The Diaporthales in North America: with emphasis on Gnomonia and its segregates. Mycologia Memoirs 7, 1-232.

Barr ME. 1987 - New taxa and combinations in the Loculoascomycetes. Mycotaxon. 29, 501-505.

Barr ME. 1990a - Prodromus to nonlichenized, pyrenomycetous members of class Hymenoascomycetes. Mycotaxon 39: 98-100

Barr ME. 1990b - Melanommatales (Loculoascomycetes). American Flora Ser. II, vol 13. New York Botanical Garden, 129 pp.

Barron GI, Busch LV. 1962 - Studies on the soil hyphomycete Scolecobasidium. Can J Bot 40:7784.

Begoude ADB, Gryzenhout M, Wingfield MJ, Roux J. 2010 - Aurifilum, a new fungal genus in the Cryphonectriaceae from Terminalia species in Cameroon. Antonie van Leeuwenhoek 98, 263-278.

Benny GL, Kimbrough JW. 1980 - A synopsis of the orders and families of Plectomycetes with keys to genera. Mycotaxon 12(1), 1-91.

Berbee M, Pirseyedi M, Hubbard S. 1999 - Cochliobolus phylogenetics and the origin of known, highly virulent pathogens, inferred from ITS and glyceraldehyde-3-phosphate dehydrogenase gene sequences. Mycologia 91, 964-977.

Berkeley MJ, Broome CE. 1850 - Notices of British fungi (438-501). Annals and Magazine of Natural History 5, 455-466.

Bhatt GC, KendrickWB. 1968 - The generic concepts of Diplorhinotrichum and Dactylaria, and a new species of Dactylaria from soil. Can J Bot 46:1253-1257. 
Bhunjun CS, Jayawardena RS, Wei D-P, Huanraluek N et al. 2019 - Multigene phylogenetic characterissation of Colletotrichum artocarpicola sp. nov. from Artocarpus heterophyllus in northern Thailand. Phytotaxa 418, 273-286.

Boedijn KB. 1933 - Über einige phragmosporen Dematiazen. Bulletin du Jardin botanique de Buitenzorg 13, 120-134.

Boehm EW, Mugambi GK, Miller AN, Huhndorf SM et al. 2009a - A molecular phylogenetic reappraisal of the Hysteriaceae, Mytilinidiaceae and Gloniaceae (Pleosporomycetidae, Dothideomycetes) with keys to world species. Studies in Mycology 64, 49-83.

Boehm EW, Schoch, CL, Spatafora JW. 2009b - On the evolution of the Hysteriaceae and Mytilinidiaceae (Pleosporomycetidae, Dothideomycetes, Ascomycota) using four nuclear genes. Mycological Research 113, 461-479.

Boerema GH, de Gruyter J, Noordeloos ME, Hamers MEC. 2004 - Phoma identification manual. Differentiation of specific and infra-specific taxa in culture. CABI Publishing, Wallingford, UK.

Boerema GH, de Gruyter J, Noordeloos ME. 1997 - Contributions towards a monograph of Phoma (Coelomycetes) - IV. Section Heterospora: Taxa with large sized conidial dimorphs, in vivo sometimes as Stagonosporopsis synanamorphs. Persoonia - Molecular Phylogeny and Evolution of Fungi 16, 335-371.

Boerema GH, Kesteren HAV. 1964 - The nomenclature of two fungi parasitizing brassica. Persoonia 3, 17-28.

Boonmee S, D'souza MJ, Luo ZL, Pinruan U et al. 2016 - Dictyosporiaceae fam. nov. Fungal Diversity 80, 457-482.

Boonmee S, Phookamsak R, Hongsanan S, Doilom M et al. 2017 - Mycosphere notes 51-101. Revision of genera in Perisporiopsidaceae and Pseudoperisporiaceae and other Ascomycota genera incertae sedis. Mycosphere 8(10), 1695-1801.

Burgess TI, Tan YP, Garnas J, Edwards J et al. 2019 - Current status of the Botryosphaeriaceae in Australia. Australasian Plant Pathology 48: 35-44.

Cao X, Xu X, Che H, West JS, Luo D. 2019 - Three Colletotrichum species, including a new species, are associated to leaf anthracnose of rubber tree in Hainan China. Plant Disease 103, 117-124.

Castlebury LA, Rossman AY, Jaklitsch WJ, Vasilyeva LN. 2002 - A preliminary overview of the Diaporthales based on large subunit nuclear ribosomal DNA sequences. Mycologia 94, 10171031.

Chander J, Singla N, Kundu R, Handa U, Spoorthy RCY. 2017 - Phaeohyphomycosis caused by Rhytidhysteron rufulum and review of literature. Mycopathologia 182, 403-407.

Chen KL, Kirschner R. 2018 - Fungi from leaves of lotus (Nelumbo nucifera). Mycological Progress 17(1-2), 275-293.

Chen Q, Hou LW, Duan WJ, Crous PW, Cai L. 2017 - Didymellaceae revisited. Studies in Mycology 87, 105-159.

Chen Q, Jiang JR, Zhang GZ, Cai L et al. 2015 - Resolving the Phoma enigma. Studies in Mycology 82, 137-217.

Chen SF, Morgan DP, Michailides TJ. 2013 - First report of Phoma fungicola associated with stem canker and fruit blight of pistachio in Arizona. Journal of Plant Pathology 95, 450.

Chen Y, Yang X, Zhang AF, Zang HY et al. 2016 - First report of leaf spot caused by Nigrospora sphaerica on kiwifruit in China. Plant Disease 100, 2326.

Chesters CGC, Bell A. 1970 - Studies in the Lophiostomataceae Sacc. Mycological Papers 120, 155.

Chethana KWT, Jayawardene RS, Zhang W, Zhou YY et al. 2019 - Molecular characterization and pathogenicity of fungal taxa associated with cherry leaf spot disease. Mycosphere 10, 490530.

Chethana KWT, Zhou Y, Zhang W, Liu M et al. 2017 - Coniella vitis sp. nov. is the common pathogen of white rot in Chinese vineyards. Plant disease 101, 2123-2136. 
Chethana T, Liu M, Ariyawansa HA, Konta S et al. 2015 - Splanchnonema-like species in Pleosporales: introducing Pseudosplanchnonema gen. nov. in Massarinaceae. Phytotaxa 231, 133-144.

Chevallier FF. 1826 - Flore Ge'ne'rale des Environs de Paris [General Flora of the Area Around Paris]. Ferra Jeune, France, 1-674.

Chomnunti P, Bhat DJ, Jones EG, Chukeatirote E et al. 2012a - Trichomeriaceae, a new sooty mould family of Chaetothyriales. Fungal Diversity 56, 63-76.

Chomnunti P, Hongsanan S, Aguirre-Hudson B, Tian Q et al. 2014 - The sooty moulds. Fungal Diversity 66(1), 1-36.

Chomnunti P, Ko TWK, Chukeatirote E, Hyde KD et al. 2012b - Phylogeny of Chaetothyriaceae in northern Thailand including three new species. Mycologia 104, 382-395.

Ciuffetti LM, Tuori RP. 1999 - Advances in the characterization of the Pyrenophora triticirepentis - wheat interaction. Phytopathology 89(6), 444-449.

Colombier MA, Alanio A, Denis B, Melica G et al. 2015 - Dual invasive infection with Phaeoacremonium parasiticum and Paraconiothyrium cyclothyrioides in a renal transplant recipient: case report and comprehensive review of the literature of Phaeoacremonium phaeohyphomycosis. Journal of clinical microbiology 53, 2084-2094.

Cooke MC, Plowright CB. 1879 - British Sphaeriacei. Grevillea 7, 77-89.

Corda AKJ. 1831 - Die Pilze Deutschlands. In: Sturm J., ed., Deutschlands Flora 3. Abtheilung 3(12), 33-64.

Corredor AH. 2011 - Ecological and molecular studies of fungal communities associated with roots of Salix spp. grown under high density, short rotation intensive culture. Thesis. University of Saskatchewan.

Costa JFO, Kamei SH, Silva JRA, Miranda ARGS et al. 2019 - Species diversity of Colletotrichum infecting Annona spp. in Brazil. European journal of plant pathology 153, 1119-1130.

Crous PC, Giraldo A, Hawksworth DL et al. 2014a - The genera of fungi: fixing the application of type species of generic names. IMA Fungus 5, 141-160.

Crous PW, Carris LM, Giraldo A, Groenewald JZ et al. 2015a - The genera of fungi-fixing the application of the type species of generic names-G 2: Allantophomopsis, Latorua, Macrodiplodiopsis, Macrohilum, Milospium, Protostegia, Pyricularia, Robillarda, Rotula, Septoriella, Torula, and Wojnowicia. IMA fungus 6, 163-198.

Crous PW, Carnegie AJ, Wingfield MJ, Sharma R et al. 2019a - Fungal Planet description sheets: 868-950. Persoonia - Molecular Phylogeny and Evolution of Fungi 42(1), 291-473.

Crous PW, Schumacher RK, Akulov A, Thangavel R et al. 2019b - New and Interesting Fungi.2. Fungal Systematics and Evolution 3, 57-134.

Crous PW, Schumacher RK, Wingfield MJ, Lombard L et al. 2015b - Fungal systematics and evolution: FUSE 1. Sydowia 67, 81-118.

Crous PW, Giraldo A, Hawksworth DL, Robert V et al. 2014b - The Genera of Fungi: fixing the application of type species of generic names. IMA fungus 5, 141-160.

Crous PW, Shivas RG, Quaedvlieg W, van der Bank M et al. 2014b - Fungal Planet description sheets: 214-280. Persoonia 32, 184-306.

Crous PW, Slippers B, Wingfield MJ, Rheeder J et al. 2006a - Phylogenetic lineages in the Botryosphaeriaceae. Studies in Mycology 55, 235-253.

Crous PW, Verkley GJM, Groenewald JZ. 2006b - Eucalyptus microfungi known from culture. 1. cladoriella and fulvoflamma genera nova, with notes on some other poorly known taxa. Studies in Mycology 55, 53- 63.

Crous PW, Wingfield MJ, Burgess TI, Hardy GESJ et al. 2017 - Fungal Planet description sheets: 558-624. Persoonia 38, 240-384.

Crous PW, Wingfield MJ, Burgess TI, Hardy GESTJ et al. 2018 - Fungal Planet description sheets: 716-784. Persoonia 40, 240-393.

Crous PW, Wingfield MJ, Richardson DM, Le Roux JJ et al. 2016a - Fungal Planet description sheets: 400-468. Persoonia 36, 316-458. 
Crous PW, Wingfield MJ, Burgess TI, Hardy GESJ et al. 2016b - Fungal Planet description sheets: 469-557. Persoonia - Molecular Phylogeny and Evolution of Fungi 37(1), 218-403.

Cui YP, Wu B, Peng AT, Li ZL et al. 2018 - First report of Nigrospora leaf blight on sugarcane caused by Nigrospora sphaerica in China. Plant Disease 102, 824.

Dai DQ, Bahkali AH, Ariyawansa HA, Li WJ et al. 2015 - Neokalmusia didymospora (Didymosphaeriaceae), a new species from bamboo. Sydowia 68, 17-25.

Dai DQ, Jiang HB, Tang LZ, Bhat DJ. 2016 - Two new species of Arthrinium (Apiosporaceae, Xylariales) associated with bamboo from Yunnan, China. Mycosphere 7(9), 1332-1345.

Damm U, Fourie PH, Crous PW. 2007 - Aplosporella prunicola, a novel species of anamorphic Botryosphaeriaceae. Fungal Diversity 27, 35-43.

Dar MA, Rai MK. 2014 - Cytospora castanae sp. nov., associated with perennial cankers of Castanea sativa. Mycosphere 5(6), 747-757.

Daranagama DA, Hyde KD, Thambugala KM, Tian Q et al. 2018 - Towards a natural classification and backbone tree for Graphostromataceae, Hypoxylaceae, Lopadostomataceae and Xylariaceae. Fungal Diversity 88, 11-65.

Dayarathne MC, Jones EBG, Maharachchikumbura SSN, Devadatha B et al. 2020 - Morphomolecular characterization of microfungi associated with marine based habitats. Mycosphere 11(1), 1-188.

De Almeida DAC, Gusmao LFP, Miller AN. 2014 - A new genus and three new species of hysteriaceous ascomycetes from the semiarid region of Brazil. Phytotaxa 176, 298-308.

De Beer ZW, Seifert KA, Wingfield MJ. 2013 - A nomenclator for ophiostomatoid genera and species in the Ophiostomatales and Microascales. Ophiostomatoid fungi: Expanding frontiers. Utrecht, CBS-KNAW Fungal Biodiversity Centre. CBS Biodiversity Series 12, 261-268.

De Gruyter J, Aveskamp MM, Woudenberg JHC, Verkley GJ et al. 2009 - Molecular phylogeny of Phoma and allied anamorph genera: towards a reclassification of the Phoma complex. Mycological Research 113, 508-519.

De Gruyter J, Woudenberg JHC, Aveskamp MM, Verkley GJM et al. 2013 - Redisposition of Phoma-like anamorphs in Pleosporales. Studies in Mycology 75, 1-36.

de Hoog GS, Guarro J, Gené J, Figueras MJ. 2000 - Atlas of clinical fungi, 2nd edn. Centraalbureau voor Schimmelcultures, Universitat Rovira i Virgili, Utrecht.

de Hoog GS, von Arx JA. 1973 - Revision of Scolecobasidium and Pleurophragmium. Kavaka 1, $55-60$.

De Notaris G. 1844 - Cenni sulla tribù dei Pirenomiceti sferiacei e descrizione di alcuni generi spettanti alla medesima. Giornale Botanico Italiano 1, 322-355.

De Oliveira Costa VS, Michereff SJ, Martins RB, et al. 2010 - Species of Botryosphaeriaceae associated on mango in Brazil. European Journal of Plant Pathology 127, 509-519.

de Silva NI, Phillips AJ, Liu JK, Lumyong S, Hyde KD. 2019 - Phylogeny and morphology of Lasiodiplodia species associated with Magnolia forest plants. Scientific reports 9(1), 1-11.

de Silva NI, Thambugala KM, Jeewon R, Phookamsak R et al. 2018 - Morphology and phylogeny of Atrocalyx acervatus sp. nov. (Lophiotremataceae) from Acer species. Phytotaxa 333, 199208.

Dennis RWG. 1952 - Lepiota and allied genera in Trinidad, British West Indies. Kew Bull. 7, 459499.

Devadatha B, Sarma VV, Jeewon R, Jones EBG. 2018 - Morosphaeria muthupetensis sp. nov. (Morosphaeriaceae) from India: morphological characterization and multigene phylogenetic inference. Botanica Marina 61, 395-405.

Dissanayake AJ, Phillips AJ, Li XH, Hyde KD. 2016 - Botryosphaeriaceae: Current status of genera and species. Mycosphere 7, 1001-1073.

Dissanayake AJ, Phillips AJL, Hyde KD, Yan JY et al. 2017 - The current status of species in Diaporthe. Mycosphere 8, 1106-115.

Dissanayake AJ, Zhang W, Liu M, Hyde KD et al. 2017 - Diaporthe species associated with peach tree dieback in Hubei, China. Mycosphere 8(5), 533-549. 
Dissing H. 1992 - Notes on the coprophilous pyrenomycete Sporormia fimetaria. PersooniaMolecular Phylogeny and Evolution of Fungi 14(4), 389-394.

Dixon LJ, Schlub RL, Pernezny K, Datnoff LE. 2009 - Host specialization and phylogenetic diversity of Corynespora cassiicola. Phytopathology 99, 1015-1027.

Doilom M, Manawasinghe IS, Jeewon R, Jayawardena RS et al. 2017ba- Can ITS sequence data identify fungal endophytes from cultures? A case study from Rhizophora apiculata. Mycosphere 8, 1869-1892.

Doilom M, Dissanayake AJ, Wanasinghe DN, Boonmee S et al. 2017b - Microfungi on Tectona grandis (teak) in Northern Thailand. Fungal Diversity 82, 107-182.

Doilom M, Shuttleworth L, Roux J, Chukeatirote E, Hyde KD. 2015 - Botryosphaeriaceae associated with Tectona grandis (teak) in Northern Thailand. Phytotaxa 233, 1-26.

Doilom M, Shuttleworth LA, Roux J, Chukeatirote E, Kevin HD. 2014 - Barriopsis tectonae sp. nov. a new species of Botryosphaeriaceae from Tectona grandis (teak) in Thailand. Phytotaxa 176, 81-91.

Doilom M, Taylor JE, Bhat DJ, Chukeatirote E et al. 2016 - Checklist of fungi on teak. Mycosphere 7, 656-678.

Dou ZP, Lu M, Wu JR, He W, Zhang Y. 2017a - A new species and interesting records of Aplosporella from China. Sydowia 69, 1-7.

Dou ZP, He W, Zhang Y. 2017b - Does morphology matter in taxonomy of Lasiodiplodia? An answer from Lasiodiplodia hyalina sp. nov. Mycosphere 8(8), 1014-1027.

Du Z, Fan XL, Yang Q, Hyde KD, Tian CM. 2017 - Aplosporella ginkgonis (Aplosporellaceae, Botryosphaeriales), a new species isolated from Ginkgo biloba in China. Mycosphere 8, 1246-1252.

Dwivedi RS. 1959 - Occurrence of the genus Scolecobasidium Abbott in India. Curr Sci Bangalore 28:374-375.

Ehrenberg CG. 1818 - Sylvae Mycologicae Berolinenses. Formis Theophili Bruschcke, Berlin, Germany. [In Latin]

Ekanayaka AH, Dissanayake AJ, Jayasiri SC, To-anun C et al. 2016 - Aplosporella thailandica; a novel species revealing the sexual-asexual connection in Aplosporellaceae (Botryosphaeriales). Mycosphere 7, 440-447.

Ekanayaka AH, Hyde KD, Gentekaki E, McKenzie EHC et al. 2019 - Preliminary classification of Leotiomycetes. Mycosphere 10, 310-489.

Eken C, Spanbayev A, Tulegenova Z, Yechshzhanov T. 2016 - First report of Nigrospora oryzae on wheat in Kazakhstan. Plant Disease 100(4), 861.

El-Deeb HM, Lashin SM, Arab YA. 2007 - Distribution and pathogenesis of date palm fungi in Egypt. Acta Horticulturae 736, 421-429.

Ellis MB. 1971 - Dematiaceous hyphomycetes. Commonwealth Mycological Institute, Kew, Surrey, England.

Ellis MB. 1976 - More Dematiaceous Hyphomycetes. Commonwealth Mycological Institute, Kew, Surrey, England.

Eo JK, Choi MS, Eom AH. 2014 - Diversity of endophytic fungi isolated from korean ginseng leaves. Mycobiology 42, 147-151.

Evans HC. 2013 - Fungal pathogens of spiders. In: Nentwig W (ed) Ecophysiology of spiders. Springer, Berlin-Heidelberg. 107-121.

Fan XL, Du Z, Liang YM, Tian CM. 2016 - Melanconis (Melanconidaceae) associated with Betula spp. in China. Mycological Progress 15, 1-9.

Fan XL, Hyde KD, Yang Q, Liang YM, Ma R, Tian CM. 2015a - Cytospora species associated with canker disease of three anti-desertification plants in northwestern China. Phytotaxa 197, 227-244.

Fan XL, Qin Y, Cao B, Liang YM, Tian CM. 2015b - New record of Aplosporella javeedii on five hosts in China based on multi-gene analysis and morphology. Mycotaxon 130, 749-756.

Farr DF, O'Neill NR, van Berkum PB. 2000 - Morphological and molecular studies on 
Dendryphion penicillatum and Pleospora papaveracea, pathogens of Papaver somniferum. Mycologia 92, 145-153.

Farr DF, Rossman AY. 2020 - Fungal Databases, U.S. National Fungus Collections, ARS, USDA. Retrieved November $20^{\text {th }}, 2019$, from https://nt.ars-grin.gov/fungaldatabases/

Fuckel L. 1870 - Symbolae mycologicae. Beiträge zur Kenntniss der Rheinischen Pilze. Jahrbücher des Nassauischen Vereins für Naturkunde. 23-24, 1-459.

Gadallah MF, White R, El-Shahawy MA, Abreo F, Oberle A, Work J. 1995 - Peritoneal dialysis complicated by Bipolaris hawaiiensis peritonitis: successful therapy with catheter removal and oral itraconazol without the use of amphotericin-B. American Journal of Nephrology 15, 348-352.

Gangadevi V, Muthumary J. 2008 - Taxol, an anticancer drug produced by an endophytic fungus Bartalinia robillardoides Tassi, isolated from a medicinal plant, Aegle marmelos Correa ex Roxb. World Journal of Microbiology and Biotechnology 24, 717-724.

Garcia-Hermoso D,Valenzuela-Lopez N, Rivero-Menendez O, AlastrueyIzquierdo A et al. 2019 Diversity of coelomycetous fungi in human infections: a 10-year experience of two European reference centres, Fungal Biology 123, 341-349.

Glawe DA, Rogers JD. 1986 - Conidial states of some species of Diatrypaceae and Xylariaceae. Canadian journal of botany 64, 1493-1498.

Ge YP, Lv GX, Shen YN, Li M et al. 2012 - First report of subcutaneous phaeohyphomycosis caused by Ochroconis tshawytschae in an immunocompetent patient. Med Mycol 50, 637640 .

Georg LK, Bierer BW, Cooke WB. 1964 - Encephalitis in turkey poults due to a new fungus species. Sabouraudia 3, 239-244.

Giambra S, Piazza G, Alves A, Mondello V, et al. 2016 - Botryosphaeriaceae species associated with diseased loquat trees in IItaly and Description of Diplodia rosacearum sp.nov. Mycosphere 7, 978-989.

Gilgado F, Gene J, Cano J, Guarro J. 2007 - Reclassification of Graphium tectonae as Parascedosporium tectonae gen. nov., comb. nov., Pseudallescheria africana as Petriellopsis africana gen. nov., comb. nov. and Pseudallescheria fimeti as Lophotrichus fimeti comb. nov. International journal of systematic and evolutionary microbiology 57(9), 2171-2178.

Golzar H, Burgess TI. 2011 - Neofusicoccum parvum, a causal agent associated with cankers and decline of Norfolk Island pine in Australia. Australasian Plant Pathology 40(5), 484.

Gordon RA, Sutton DA, Thompson EH, Shrikanth V et al. 2012 - Cutaneous phaeohyphomycosis caused by Paraconiothyrium cyclothyrioides. Journal of clinical microbiology 50, 37953798.

Grudzinska-Sterno M, Yuen J, Stenlid J, Djurle A. 2016 - Fungal communities in organically grown winter wheat affected by plant organ and development stage. European Journal of Plant Pathology 146, 401-417.

Grünig CR, Queloz V, Duò A, Sieber TN. 2009 - Phylogeny of Phaeomollisia piceae gen. sp. nov.: a dark, septate, conifer-needle endophyte and its relationships to Phialocephala and Acephala. Mycological Research 113(2), 207-221.

Gryzenhout M, Myburg H, Wingfield BD, Wingfield MJ. 2006 - Cryphonectriaceae (Diaporthales), a new family including Cryphonectria, Chrysoporthe, Endothia and allied genera. Mycologia 98, 239-249.

Guégan S, Garcia-Hermoso D, Sitbon K, Ahmed S et al. 2016 - Ten-year experience of cutaneous and/or subcutaneous infections due to coelomycetes in France. In Open forum infectious diseases Oxford University Press 3, 2.

Gupta S, Loughman R. 2001 - Current virulence of Pyrenophora teres on barley in Western Australia. Plant Disease 85, 960-966.

Güssow HT. 1906 - Über eine neue Krankheit an Gurken in England (Corynespora mazei, Güssow gen. et sp. nov.). Zeitschrift für Pflanzenkrankheiten 16, 10-13. 
Hao L, Chen C, Zhang R, Zhu M et al. 2013 - A new species of Scolecobasidium associated with the sooty blotch and flyspeck complex on banana from China. Mycological progress 12(3), 489-495.

Hashemian KT, Jahani M, Zare R, Mirzaee MR. 2014 - First report of Nigrospora leaf spot on Pennisetum americanum in Iran. Journal of Plant Pathology 96(3), 606.

Hashimoto A, Matsumura M, Hirayama K, Tanaka K. 2017 - Revision of Lophiotremataceae (Pleosporales, Dothideomycetes): Aquasubmersaceae, Cryptocoryneaceae, and Hermatomycetaceae fam. nov. Persoonia 39, 51-73.

Hauptman T, Piskur B, De Groot M, Ogris N et al. 2013 - Temperature effect on Chalara fraxinea: heat treatment of saplings as a possible disease control method. Forest Pathology 43, 360370.

Hawksworth D. 1981 - Astrosphaeriella Sydow, a misunderstood genus of melanommataceous pyrenomycetes. Botanical Journal of the Linnean Society 82, 35-59.

Hawksworth DL, Lücking R. 2017 - Fungal diversity revisited: 2.2 to 3.8 million species. The fungal kingdom, 79-95.

Hayova VP, Minter DW.iiuiiu 1998 - Valsa malicola. In 'IMI Descriptions of Fungi and Bacteria', No. 1368. (CAB International: Surrey).

He BY, Cernava T, He HD, Li HX et al. 2019a - First report of leaf spots on Photinia serrulata caused by Nigrospora oryzae in China. Plant Disease 103(9), 2480-2480.

He MQ, Zhao RL, Hyde KD, Begerow D et al. 2019b - Notes, outline and divergence times of Basidiomycota. Fungal Diversity 99, 105-367.

Heald FD. 1909 - A Species of Discosia on Living Bull Pine Seedlings. Mycologia 1, 215-217.

Hedjaroude GA. 1968 - Études taxonomiques sur les Phaeosphaeria Miyake et leurs formes voisines (Ascomycètes). Sydowia 22, 57-107.

Heidari K, Mehrabi-Koushki M, Farokhinejad R. 2018 - Curvularia mosaddeghii sp nov., a novel species from the family Pleosporaceae. Mycosphere 9, 635-646.

Hirayama K, Tanaka K. 2011 - Taxonomic revision of Lophiostoma and Lophiotrema based on reevaluation of morphological characters and molecular analyses. Mycoscience 52, 401-412.

Hirayama Y, Asano S, Okayama K, Ohki ST, Tojo M. 2018 - Weeds as the potential inoculum source of Colletotrichum fructicola responsible for strawberry anthracnose in Nara, Japan. Journal of general plant pathology 84, 12-19.

Höhnel F von. 1918 - Dritte vorläufige Mitteilung mycologischer Ergebnisse (nr. 201-304). Berichte der Deutschen Botanischen Gesellschaft. 36, 309-317.

Hongsanan S, Li YM, Liu JK, Hofmann T et al. 2014 - Revision of genera in Asterinales. Fungal diversity $68,1-68$.

Hongsanan S, Zhao RL, Hyde KD. 2017a - A new species of Chaetothyrina on branches of mango, and introducing Phaeothecoidiellaceae fam. nov. Mycosphere 8, 137-146.

Hongsanan S, Maharachchikumbura SS, Hyde KD, Samarakoon MC et al. 2017b - An updated phylogeny of Sordariomycetes based on phylogenetic and molecular clock evidence. Fungal Diversity 84, 25-41.

Hongsanan S, Tian Q, Hyde KD, Hu DM. 2016 - The asexual morph of Trichomerium gloeosporum. Mycosphere 7, 1473-1479.

Hongsanan S, Hyde KD, Phookamsak R, Wanasinghe DN et al. 2020 - Refined families of Dothideomycetes. Fungal divers (submitted).

Horré R, de Hoog GS, Klucsny C, Marklein G et al. 1999 - rDNA diversity and physiology of Ochroconis and Scolecobasidium species reported from humans and other vertebrates. Stud Mycol 43, 194-205.

Hosford RM. 1970 - A Form of Pyrenophora trichostoma Pathogenic to Wheat and Other Grasses. Department of Plant Pathology, North Dakota State University, Fargo 58102; Phytopathology $61,28-32$.

Hu HL. 2010 - Morphological and molecular studies of selected Dothideomycetes. PhD diss. The University of Hong Kong, Pokfulam. 
Huang SK, Maharachchikumbura SSN, Jeewon R, Bhat DJ et al. 2018 - Lecanicillium subprimulinum (Cordycipitaceae, Hypocreales), a novel species from Baoshan, Yunnan. Phytotaxa 348(2), 99-108.

Huang F, Udayanga D, Wang X, Hou et al. 2015 - Endophytic Diaporthe associated with Citrus: A phylogenetic reassessment with seven new species from China. Fungal Biology 119(5), 331347.

Huanraluek N, Thambugala KM, Wang Y, Hyde KD. 2018 - Introducing Massarioramusculicola, a novel genus in Massariaceae. Phytotaxa 371, 17-24.

Hughes SJ. 1958 - Revisiones hyphomycetum aliquot cum appendice de nominibus rejiciendis. Canadian Journal of Botany 36, 727-836.

Huhndorf SM, Miller AN, Fernández FA. 2004 - Molecular systematics of the Sordariales: the order and the family Lasiosphaeriaceae redefine. Mycologia 96, 368-387

Hyde KD, Cai L, McKenzie EHC, Yang YL et al. 2009 - Colletotrichum: a catalogue of confusion. Fungal Diversity 39(1), 1-17.

Hyde KD, Chaiwan N, Norphanphoun C, Boonmee S et al. 2018 - Mycosphere notes 169-224. Mycosphere 9(2), 271-430.

Hyde KD, Dong Y, Phookamsak R, Jeewon R et al. 2020 - Fungal diversity notes 1151-1275: taxonomic and phylogenetic contributions on genera and species of fungal taxa. Fungul Diversity (submitted: under review).

Hyde KD, Fröhlich J, Taylor JE. 1998 - Fungi from palms. XXXVI. Reflections on unitunicate ascomycetes with apiospores. Sydowia 50, 21-80.

Hyde KD, Hongsanan S, Jeewon R et al. 2016 - Fungal diversity notes 367-490: taxonomic and phylogenetic contributions to fungal taxa. Fungal Diversity 80, 1-270.

Hyde KD, Jones EBG, Liu JK, Ariyawansa H et al. 2013 - Families of Dothideomycetes. Fungal Diversity 63, 1-313.

Hyde KD, Nilsson RH, Alias SA, Ariyawansa HA et al. 2014 - One stop shop: backbones trees for important phytopathogenic genera: I. Fungal Diversity, 67(1), 21-125.

Hyde KD, Norphanphoun C, Abreu VP, Bazzicalupo A et al. 2017 - Fungal diversity notes 603-708: taxonomic and phylogenetic notes on genera and species. Fungal Diversity 87, 1-235.

Hyde KD, Soytong K. 2008 - The fungal endophyte dilemma. Fungal Diversity 33(163), 173.

Hyde KD, Taylor JE, Frohlich J. 2000 - Genera of ascomycetes from palms. Fungal Diversity Press, Hong Kong

Hyde KD, Tennakoon DS, Jeewon R, Bhat DJ et al. 2019 - Fungal diversity notes 1036-1150: taxonomic and phylogenetic contributions on genera and species of fungal taxa. Fungal Diversity $96,1-242$.

Inderbitzin P, Bostock RM, Trouillas FP, Michailides TJ. 2010 - A six locus phylogeny reveals high species diversity in Botryosphaeriaceae from California almond. Mycologia 102(6), 1350-1368.

Index Fungorum. 2020 - Available from: http://www.indexfungorum.org/names/Names.asp.

Ismail AM, Cirvilleri G, Lombard L, Crous PW et al. 2013 - Characterisation of Neofusicoccum species causing mango dieback in Italy. Journal of Plant Pathology 95, 549-557.

Isola D, Zucconi L, Onofri S, Santamaria U et al. 2016 - Extremotolerant rock-inhabiting black fungi from Italian monumental sites. Fungal Diversity 76, 75-96.

Iturrieta-González I, Gené J, Guarro J, Castañeda-Ruiz RF, García D. 2018 - Neodendryphiella, a novel genus of the Dictyosporiaceae (Pleosporales). MycoKeys 37, 19-38.

Jaklitsch W, Gardiennet A, Voglmayr H. 2016a - Resolution of morphology-based taxonomic delusions: Acrocordiella, Basiseptospora, Blogiascospora, Clypeosphaeria, Hymenopleella, Lepteutypa, Pseudapiospora, Requienella, Seiridium and Strickeria. Persoonia: Molecular Phylogeny and Evolution of Fungi 37, 82-105.

Jaklitsch WM, Fournier F, Voglmayr H. 2018 - Two unusual new species of Pleosporales: Anteaglonium rubescens and Atrocalyx asturiensis. Sydowia 70, 129-140. 
Jaklitsch WM, Voglmayr H. 2012 - Phylogenetic relationships of five genera of Xylariales and Rosasphaeria gen. nov. (Hypocreales). Fungal Diversity 52, 75-98.

Jaklitsch WM, Voglmayr H. 2016 - Hidden diversity in Thyridaria and a new circumscription of the Thyridariaceae. Stud Mycol 85, 35-64.

Jami F, Migliorini D, Burgess TI, Slippers B et al. 2018 - Diversity of Botryosphaeriaceae on Proteaceae in South Africa, Australia and Portugal. Pytopathology 108(10), 92-93.

Jami F, Slippers B, Wingfiled MJ, Gryzenhout M. 2013 - Patterns of host infection in Botryosphaeriaceae: A case study on three unrelated native hosts in South Africa. Fungal Biology 118, 168-179.

Jami F, Slippers B, Wingfield MJ, Loots MT et al. 2015 - Temporal and spatial variation of Botryosphaeriaceae associated with Acacia karroo in South Africa. Fungal ecology 15, 5162.

Javier-Alva J, Gramaje D, Alvarez LA, Armengol J. 2009 - First report of Neofusicoccum parvum associated with dieback of mango trees in Peru. Plant Disease 93, 426.

Jayasiri SC, Hyde KD, Jones EBG, Jeewon R. 2017 - Taxonomy and multigene phylogenetic evaluation of novel species in Boeremia and Epicoccum with new records of Ascochyta and Didymella (Didymellaceae). Mycosphere 8, 1080-1101.

Jayasiri SC, Hyde KD, Jones EBG, McKenzie EHC et al. 2019 - Diversity, morphology and molecular phylogeny of Dothideomycetes on decaying wild seed pods and fruits. Mycosphere $10,1-186$.

Jayasiri SC, Hyde KD, Jones EBG, Peršoh D et al. 2018 - Taxonomic novelties of hysteriform Dothideomycetes. Mycosphere 9, 803-837.

Jayasiri SC, Hyde KD, Ariyawansa HA, Bhat J et al. 2015 - The Faces of Fungi database: fungal names linked with morphology, phylogeny and human impacts. Fungal Diversity 74, 3-18.

Jayasiri SC, Jones EBG, Kang JC, Promputtha I et al. 2016 - A new species of genus Anteaglonium (Anteagloniaceae, Pleosporales) with its asexual morph. Phytotaxa 263, 233-244.

Jayawardena RS, Hyde KD, Damm U, Cai L et al. 2016 - Notes on currently accepted species of Colletotrichum. Mycosphere 7(8), 1192-1260.

Jayawardena RS, Hyde KD, Jeewon R, Ghobad-Nejhad M et al. 2019a - One stop shop II: taxonomic update with molecular phylogeny for important phytopathogenic genera: $26-50$. Fungal Diversity 94, 41-129.

Jayawardena RS, Hyde KD, McKenzie EH, Jeewon R et al. 2019b - One stop shop III: taxonomic update with molecular phylogeny for important phytopathogenic genera: 51-75. Fungal Diversity 98, 77-160.

Jeewon R, Hyde KD. 2016 - Establishing species boundaries and new taxa among fungi: recommendation to resolve taxonomic ambiguities. Mycosphere 7, 1669-1677.

Jeewon R, Yeung QS, Wannasinghe DN, Rampadarath S et al. 2018 - Hidden mycota of pine needles: Molecular signatures from PCR-DGGE and Ribosomal DNA phylogenetic characterization of novel phylotypes. Scientific reports, 8: 1-12.

Jeewon R, Liew ECY, Hyde KD. 2002 - Phylogenetic relationships of Pestalotiopsis and allied genera inferred from ribosomal DNA sequences and morphological characters. Molecular Phylogenetics and Evolution 25, 378-392.

Jeewon R, Liew ECY, Hyde KD. 2003 - Molecular systematics of the Amphisphaeriaceae based on cladistic analyses of partial LSU rDNA sequences. Fungal Biology 107, 1392-1402.

Jeewon R, Liew, ECY, Hyde KD. 2004 - Phylogenetic evaluation of species nomenclature of Pestalotiopsis in relation to host association. Fungal Diversity 17, 39-55.

Jeewon R, Wanasinghe DN, Rampadaruth S, Puchooa D et al. 2017 - Nomenclatural and identification pitfalls of endophytic mycota based on DNA sequence analyses of ribosomal and protein genes phylogenetic markers: A taxonomic dead end?. Mycosphere 8(10), 1802 1817. 
Jia H, Liu Z, Sungbom O, Yao C et al. 2019 - First report of Aplosporella javeedii causing branch blight disease of Mulberry (Morus alba) in China. Journal of Plant Diseases and Protection $126,475-477$.

Jiang HB, Hyde KD, Jayawardena RS, Doilom M et al. 2019 - Taxonomic and phylogenetic characterizations reveal two new species and two new records of Roussoella (Roussoellaceae, Pleosporales) from Yunnan. China. Mycol Prog 18, 577-591.

Jiang N, Yang Q, Fan XL, Tian CM. 2020 - Identification of six Cytospora species on Chinese chestnut in China. MycoKeys, 62, 1-25.

Jianyu B, Xiaoming W, Yanjiang S, Canxing D. 2016 - Occurrence and identification of Nothophoma quercina causing brown spot of jujube in China. Canadian Journal of Plant Pathology 38(4), 527-532.

Jie CY, Wang Y, Wu SP, Jinag YL. 2013 - Morphological characteristics and molecular phylogeny analysis of newly recorded fungal species Phialocephala humicla in China. Guizhou Agricultural Sciences 9, 86-89.

Jones EG, Pang KL, Abdel-Wahab MA, Scholz B et al. 2019 - An online resource for marine fungi. Fungal Diversity 96(1), 347-433.

Jong SC, Davis EE. 1972 - Phialocephala humicola, a new hyphomycete. Mycologia 64, 13511356.

Joshi S, Upreti, DK, Divakar PK. 2012 - Stictis palniense (Stictidaceae), a new species from India. Bryologist 115, 402-405.

Karunarathna A, Phookmasak R, Wanasinghe DN, Wijayawardene NN et al. 2017 - Taxonomy and phylogeny of Sparticola muriformis sp. nov. on decaying grass. Mycosphere 8, 603-614.

Kendrick WB. 1961 - The Leptographium complex. Phialocephala gen. nov. Canadian Journal of Botany 39(5), 1079-1085.

Kepler RM, Luangsa-Ard JJ, Hywel-Jones NL, Quandt CA et al. 2017 - A phylogenetically-based nomenclature for Cordycipitaceae (Hypocreales). IMA fungus 8(2), 335-353.

Kepler R, Sung GH, Ban S, Nakagiri A, et al. 2012 - New teleomorph combinations in the entomopathogenic genus Metacordyceps. Mycologia 104 (1), 182-197.

Kingsland GC. 1991 - Spot lesion of barley net blotch disease caused by Drechslera teres f. sp. maculata observed in South Carolina. Plant Disease 75, 537.

Kirk PM, Cannon PF, David JC, Stalpers JA. 2001 - Dictionary of the Fungi $9^{\text {th }}$ ed. London. CAB International.

Kirk P, Cannon PF, Minter DW, Stalpers JA. 2008a - Ainsworth \& Bisby's Dictionary of the Fungi, 10th edn. CAB International, Wallingford

Kirk PM, Cannon PF, Minter DW, Stalpers J. 2008b - Dictionary of the Fungi $10^{\text {th }}$ edn. CABI Bioscience, UK.

Kiyuna T, An KD, Kigawa R, Sano C et al. 2012 - Bristle-like fungal colonizers on the stone walls of the Kitoraand Takamatsuzuka Tumuli are identified as Kendrickiella phycomyces. Mycoscience 53, 446-459.

Kobayasi Y, Shimizu D. 1982 - Cordyceps species from Japan. 5. Bulletin of the National Science Museum Tokyo 8, 111-123.

Kodsueb R, Dhanasekaran V, Aptroot A, Lumyong S et al. 2006 - The family Pleosporaceae: intergeneric relationships and phylogenetic perspectives based on sequence analyses of partial 28S rDNA. Mycologia 98, 571-83.

Kodsueb R, Jeewon R, Lumyong P, McKenzie EHC et al. 2004 - Intergeneric relationships of Tubeufiaceae \& Pleosporaceae based on molecular data (LSU rDNA). Mycologia 98, 571583.

Kohlmeyer J. 1969 - Marine fungi of Hawaii including the new genus Helicascus. Canadian Journal of Botany 47, 1469-1487.

Kohlmeyer J, Kohlmeyer E. 1979 - Marine Mycology: The Higher Fungi. London: Academic Press. 
Konta S, Phillips A, Bahkali A, Jones E et al. 2016 - Botryosphaeriaceae from palms in ThailandBarriopsis archontophoenicis sp nov, from Archontophoenix alexandrae. Mycosphere 7, 921-932.

Korf RP. 1990 - Discomycete systematics today: a look at some unanswered questions in a group of unitunicate ascomycetes. Mycosystema 3, 19-27.

Kruys A, Eriksson OE, Wedin M. 2006 - Phylogenetic relationships of coprophilous Pleosporales (Dothideomycetes, Ascomycota), and the classification of some bitunicate taxa of unknown position. Mycological Research 110(5), 527-536.

Kumar S, Singh R, Saini DC, Kamal. 2012 - A new species of Corynespora from terai forest of Northeastern Uttar Pradesh, India. Mycosphere 3(4), 410-412.

Kumar S, Singh R. 2016 - Biodiversity, distribution and taxonomy of conidial fungus Corynespora (Corynesporascaceae) associated with Malvaceae. Journal of Biodiversity \& Endangered Species 2016, 1-3.

Kumar V, Cheewangkoon R, Thambugala KM, Jones GE et al. 2019 - Rhytidhysteron mangrovei (Hysteriaceae), a new species from mangroves in Phetchaburi Province, Thailand. Phytotaxa 401(3), 166-178.

Kushwaha P, Singh R, Kumar S. 2017 - Corynespora titarpaniensis sp. nov., on Lepidagathis from Central India. Mycotaxon. 132(2), 271-279.

Lackner M, de Hoog GS. 2011 - Parascedosporium and its relatives: phylogeny and ecological trends. IMA fungus, 2(1), 39-48.

Lamari L, Bernier CC. 1989 - Virulence of isolates of Pyrenophora tritici-repentis on 11 wheat cultivars and cytology of the differential host reactions. Canadian Journal of Plant Pathology 11, 284-90.

Lateef AA, Sepiah M, Bolhassan MH. 2015 - Description of Pseudopestalotiopsis kubahensis sp. nov., a new species of microfungi from Kubah National Park, Sarawak, Malaysia. Curr Res Environ Appl Mycol 5, 376-381.

Li CQ, Liu FF, Li JQ, Liu QL, Chen SF. 2018 - Botryosphaeriaceae from Eucalyptus plantations and adjacent plants in China. Persoonia 40, 63-95.

Li GJ, Hyde KD, Zhao RN, Hongsanan S et al. 2016a - Fungal divers notes 253-366: taxonomic and phylogenetic contributions to fungal taxa. Fungal Diversity 78, 1-237.

Li H, Zhou G-Y, Liu J-A, Xu J. 2016b - Populaiton genetic analyses of the fungal pathogen Colletotrichum fructicola on tea-oil trees in China. PLoS ONE 11, e0156841.

Li JF, Bhat DJ, Phookamsak R, Mapook A et al. 2016c - Sporidesmioides thailandica gen. et sp. nov. (Dothideomycetes) from northern Thailand. Mycological Progress 15, 1169-1178.

Li JF, Jeewon R, Luo Z, Phookamsak R et al. 2017a - Morphological characterization and DNA based taxonomy of Fusiconidium gen. nov with two novel taxa within Melanommataceae (Pleosporales). Phytotaxa 308, 206-218.

Li J, Jeewon R, Mortimer PE, Doilom M et al. 2020 - Multigene phylogeny and taxonomy of Dendryphion hydei and Torula hydei spp. nov. from herbaceous litter in northern Thailand. PloS one 15(2), e0228067.

Li JF, Phookamsak R, Jeewon R, Bhat DJ et al. 2017b - Molecular taxonomy and morphological characterization reveal new species and new host records of Torula species (Torulaceae, Pleosporales). Mycological Progress 16, 447-461.

Li JF, Phookamsak R, Mapook A, Boonmee S et al. 2016d - Seifertia shangrilaensis sp. nov. (Melanommataceae), a new species from Southwest China. Phytotaxa 273, 34-42.

Liang ZQ, Liu AY, Liu JL. 1991 - A new species of the genus Cordyceps and its Metarhizium anamorph. Acta Mycologica Sinica 10, 257-262.

Liang JF, Yang ZL, Xu DP. 2011 - A New Species in Lepiota from China. Mycologia 103, 820830.

Liang JL, Yu F, Lu JK, Wang SK, Song J. 2018 - Morphological and molecular evidence for two new species in Lepiota from China. Mycologia 110, 494- 501.

Libert MA. 1837 - Plantae Cryptogamae, quas in Arduenna collegit. Fasc. 4, 301-400. 
Lichtemberg PSF, Moral J, Sherman J, Nouri MT et al. 2019 - Characterizing Beltrania pseudorhombica the causal agent of pistachio leaf and fruit spot in Arizona. European Journal of Plant Pathology 154(3), 849-854.

Liew ECY, Aptroot A, Hyde KD. 2002 - An evaluation of the monophyly of Massarina based on ribosomal DNA sequences. Mycologia 94, 803-813.

Lin CG, Dai DQ, Bhat DJ, Hyde KD et al. 2017a - Subsessila turbinata gen. et. sp. nov. (Beltraniaceae), a Beltrania-like fungus from Thailand. Mycological progress 16(4), 393401.

Lin C-G, Hyde KD, Lumyong S, McKenzie EHC. 2017b - Beltrania-like taxa from Thailand. Cryptogamie, Mycologie 38(3), 301-319.

Linaldeddu BT, Maddau L, Franceschini A, Alves A et al. 2016 - Botryosphaeriaceae species associated with lentisk dieback in Italy and description of Diplodia insularis sp.nov. Mycosphere 7, 962-977.

Link HF. 1833 - Handbuch zur Erkennung der nutzbarsten und am häufigsten vorkommenden Gewächse. In der Haude und Spenerschen Buchhandlung. 2, 1-536.

Liu F, Bonthond G, Groenewald J, Cai L, Crous PW. 2019 - Sporocadaceae, a family of coelomycetous fungi with appendage-bearing conidia. Studies in mycology 92, 287-415.

Liu AR, Chen SC, Wu SY, Xu T et al. 2010 - Cultural studies coupled with DNA based sequence analyses and its implication on pigmentation as a phylogenetic marker in Pestalotiopsis taxonomy. Molecular Phylogenetics and Evolution 57, 528-535.

Liu F, Hou L, Raza M, Cai L. 2017a - Pestalotiopsis and allied genera from Camellia, with description of 11 new species from China. Scientific Reports 7, 1-19.

Liu F, Hu DM, Cai L. 2012a - Conlarium duplumascospora gen. et. sp. nov. and Jobellisia guangdongensis sp. nov. from freshwater habitats in China. Mycologia 104, 1178-1186.

Liu JK, Hyde KD, Jeewon R, Phillips AJL et al. 2017b - Ranking higher taxa using divergence times: a case study in Dothideomycetes. Fungal Diversity 84, 75-99.

Liu JK, Hyde KD, Jones EBG, Ariyawansa HA et al. 2015 - Fungal diversity notes 1-110: taxonomic and phylogenetic contributions to fungal species. Fungal Diversity 72, 1-197.

Liu JK, Luo ZL, Liu NG, Cheewangkoon R, To-Anun C. 2018a - Two novel species of Paradictyoarthrinium from decaying wood. Phytotaxa 338, 285-293.

Liu JK, Phookamsak R, Dai DQ, Tanaka K et al. 2014 - Roussoellaceae, a new pleosporalean family to accommodate the genera Neoroussoella gen. nov., Roussoella and Roussoellopsis. Phytotaxa 181, 1-33.

Liu JK, Phookamsak R, Doilom M, Wiki S et al. 2012b - Towards a natural classification of Botryosphaeriales. Fungal Diversity 57, 149-210.

Liu JK, Phookamsak R, Jones EBG, Zhang Y et al. 2011 - Astrosphaeriella is polyphyletic, with species in Fissuroma gen. nov., and Neoastrosphaeriella gen. nov. Fungal Diversity 51, 135154.

Liu M, Zhang W, Manawasighe IS, Zhou Y et al. 2018b - First report of Nothophoma quercina causing trunk canker on crabapple (Malus micromalus) in China. Plant Disease 102(7), 1462.

Liu NG, Hongsanan S, Yang J, Lin CG et al. 2017c - Dendryphiella fasciculata sp. nov. and notes on other Dendryphiella species. Mycosphere 8(9), 1575-1586.

Lopes A, Barradas C, Phillips AJL, Alves A. 2016 - Diversity and phylogeny of Neofusicoccum species occurring in forest and urban environments in Portugal. Mycosphere 7, 906-920.

Lorenzini M, Cappello MS, Logrieco A, Zapparoli G. 2016 - Polymorphism and phylogenetic species delimination in filamentous fungi from predominant mycobiota in withered grapes. International Journal of Food Microbiology 238, 56-62.

Lu B, Hyde KD, Ho WH, Tsui KM et al. 2000 - Checklist of Hong Kong Fungi. Fungal Diversity Press, Hong Kong, 207

Lumbsch HT, Huhndorf SM. 2010 - Myconet Volume 14. Part One. Outline of Ascomycota-2009. Part Two. Notes on Ascomycete Systematics. Nos. 4751-5113. Fieldiana Life and Earth Sciences, 2010(1), 1-64. 
Luo ZL, Hyde KD, Liu JK, Bhat DJ et al. 2018 - Lignicolous freshwater fungi from China II: Novel Distoseptispora (Distoseptisporaceae) species from northwestern Yunnan Province and a suggested unified method for studying lignicolous freshwater fungi. Mycosphere, 9(3), 444-461.

Luo ZL, Yang J, Liu JK, Su HY et al. 2016 - Two new species of Helicascus (Morosphaeriaceae) from submerged wood in northern Thailand. Phytotaxa 270, 182-190.

Luttrell ES. 1951 - The morphology of Dothidea collecta. American Journal of Botany 460-471.

Madrid H, da Cunha KC, Gené J, Dijksterhuis J et al. 2014 - Novel Curvularia species from clinical specimens. Persoonia 33, 48-60.

Maharachchikumbura SSN, Ariyawansa HA, Wanasinghe DN, Dayarathne MC et al. 2019 Phylogenetic classification and generic delineation of Hydeomyces desertipleosporoides gen. et sp. nov. (Phaeosphaeriaceae) from Jebel Akhdar Mountain in Oman. Phytotaxa. 391, 28 38 .

Maharachchikumbura SSN, Guo LD, Liu ZY, Hyde KD. 2016b - Pseudopestalotiopsis ignota and Ps. camelliae spp. nov. associated with grey blight disease of tea in China. Mycological Progress 15, 22.

Maharachchikumbura SSN, Haituk S, Pakdeeniti P, Al-Sadi AM et al. 2018 - Phaeosaccardinula coffeicola and Trichomerium chiangmaiensis, two new species of Chaetothyriales (Eurotiomycetes) from Thailand. Mycosphere 9(4), 769-778.

Maharachchikumbura SSN, Hyde KD, Groenewald JZ, Xu J, Crous PW. 2014 - Pestalotiopsis revisited. Studies Mycology 79, 121-186.

Maharachchikumbura SN, Hyde KD, Jones EBG, McKenzie EHC et al. 2016a - Families of Sordariomycetes. Fungal Diversity 79, 1-317.

Maharachchikumbura SS, Hyde KD, Jones EBG, McKenzie EH et al. 2015 - Towards a natural classification and backbone tree for Sordariomycetes. Fungal Diversity. 72, 199-301.

Malloch D. 1970 - New concepts in the Microascaceae illustrated by two new species. Mycologia 62(4), 727-740.

Manamgoda DS, Rossman AY, Castlebury LA, Chukeatirote E et al. 2015 - A taxonomic and phylogenetic re-appraisal of the genus Curvularia (Pleosporaceae): human and plant pathogens. Phytotaxa 212, 175-198.

Manamgoda DS, Rossman AY, Castlebury LA, Crous PW et al. 2014 - The genus Bipolaris. Studies of Mycology 79, 221-288.

Manawasinghe IS, Dissanayake A, Xinghong Li, Liu M et al. 2019 - High Genetic Diversity and Species Complexity of Diaporthe Associated with Grapevine Dieback in China. Frontiers in Microbiology.

Manawasinghe IS, Zhang W, Li X, Zhao W et al. 2018 - Novel microsatellite markers reveal multiple origins of Botryosphaeria dothidea causing the Chinese grapevine trunk disease. Fungal Ecology 33, 134-142.

Marincowitz S, Groenewald JZ, Wingfield MJ, Crous PW. 2008 - Species of Botryosphaeriaceae occurring on Proteaceae. Persoonia: Molecular Phylogeny and Evolution of Fungi 21, 111.

Marin-Felix Y, Senwanna C, Cheewangkoon R, Crous PW. 2017a - New species and records of Bipolaris and Curvularia from Thailand. Mycosphere 8(9), 1555-1573.

Marin-Felix Y, Groenewald JZ, Cai L, Chen Q, et al. 2017b - Genera of phytopathogenic fungi: GOPHY 1. Studies in mycology. 86,99-216.

Marin-Felix Y, Hernández-Restrepo M, Iturrieta-González I, García D et al. 2019 - Genera of phytopathogenic fungi: GOPHY 3. Studies in Mycology 94, 1-124.

Mason EW. 1927 - On species of the genus Nigrospora Zimmermann recorded on monocotyledons. Transactions of the British Mycological Society 12, 152-165.

Massonnet M, Figueroa-Balderas R, Galarneau ER, Miki S et al. 2017 - Neofusicoccum parvum colonization of the grapevine woody stem triggers asynchronous host responses at the site of infection and in the leaves. Frontiers in Plant Science 8, 1117. 
Matheny PB, Wang Z, Binder M, Curtis JM et al. 2007 - Contributions of rpb2 and tef1 to the phylogeny of mushrooms and allies (Basidiomycota, Fungi). Molecular phylogenetics and evolution 43, 430-451.

Mathur RS. 1979 - The Coelomycetes of India. Bishen Singh Mahendra Pal Singh, Delhi, India., 460.

Matsushima T. 1975 - Icones Microfungorum a Matsushima Lectorum Kobe.

Matsushima T. 1980 - Matsushima Mycological Memoirs No. 1. Published by the author.

Matsushima T. 1989 - Matsushima Mycological Memoirs No. 6. Published by the author.

Matsushima T. 1996 - MatsushimaMycological Memoirs 9. Matsushima Mycological Memoirs 9, $1-30$.

Mehl JW, Slippers B, Roux J, Wingfield MJ. 2017 - Overlap of latent pathogens in the Botryosphaeriaceae on a native and agricultural host. Fungal biology 121(4), 405-419.

Mehrabi M, Goltapeh ME, Fotouhifar KB. 2011 - Studies on Cytospora canker disease of apple trees in Semirom region of Iran. Journal of Agricultural Technology 7(4), 967-982.

Meng Z, Wen T, Kang J, Lei B et al. 2014 - Cordyceps pruinosa produces cordycepin and N6-(2hydroxyethyl)-adenosine in culture. Archives of Biological Sciences 66 (4), 1411-1421.

Mijeong P, Hong SB, Hyeondong S. 2015 - Lecanicillium uredinophilum sp. nov. associated with rust fungi from Korea. Mycotaxon 130(4), 997-1005.

Miller AN, Huhndorf SM. 2005 - Multi-gene phylogenies indicate ascomal wall morphology is a better predictor of phylogenetic relationships than ascospore morphology in the Sordariales (Ascomycota, Fungi). Molecular phylogenetics and evolution. 35, 60-75.

Mongkolsamrit S, Noisripoom W, Thanakitpipattana D, Wutikhun T et al. 2018 - Disentangling cryptic species with isaria-like morphs in Cordycipitaceae. Mycologia 110(1), 230-257.

Moon BC, Kim WJ, Park I, Sung G-H, et al. 2018 - Establishment of a PCR assay for the detection and discrimination of authentic Cordyceps and adulterant species in food and herbal medicines. Molecules 23 (8),1932.

Moral J, Agusti-Brisach C, Perez-Rodriguez M, Xavier C et al. 2017 - Identification of fungal species associated with branch dieback of olive and resistance of table cultivars to Neofusicoccum mediterraneum and Botryosphaeria dothidea. Plant Disease 101, 306-316.

Morelet M. 2001 - Beltrania Penzig: B. magnoliae sp. nov., with a taxonomic key to species. Cryptogamie Mycologie 22, 29-33.

Moreno MV, Arambarri AM, Perelló A. 2011 - Diversity of Pyrenophora tritici-repentis isolates from the Argentinian wheat growing area: morphocultural and pathogenic analysis. International Research Journal of Agricultural Science and Soil Science, 1.

Moricca S, Uccello A, Ginetti B, Ragazzi A. 2012 - Isolation and growth temperature requirements of oomycetes and Botryosphaeriaceae from the same oak hosts: evidence for a vicariant pathogenic action?.76, 79-84.

Mugambi GK, Huhndorf SM. 2009 - Parallel evolution of hysterothecial ascomata in ascolocularous fungi (Ascomycota, Fungi). Systematics and Biodiversity 7, 453-464.

Munk A. 1956 - On Metasphaeria coccodes (Karst.) Sacc. and other fungi probably related to Massarina Sacc. (Massarinaceae n. fam.) Friesia 5, 303-308.

Munk A. 1957 - Danish Pyrenomycetes. A preliminary flora. Dansk Botanisk Arkives 17(1): 1491.

Nag Raj TR. 1993 - Coelomycetous anamorphs with appendage-bearing con- idia. Mycologue publications, Canada.

Natarajan K, Manjula B. 1983 - South Indian Agaricales XII-Lepiota. Bibliotheca Mycologica, 91, 563-581.

Ni HF, Yang HR, Chen RS, Liou RF, Hung TH. 2012 - New Botryosphaeriaceae fruit rot of mango in Taiwan: identification and pathogenicity. Bot Stud 53, 467-478.

Nikoh N, Fukatsu T. 2001 - Evolutionary dynamics of multiple group I introns in nuclear ribosomal RNA genes of endoparasitic fungi of the genus Cordyceps. Molecular Biology and Evolution 18 (9),1631-1642. 
Nitschke TR. 1869 - Pleosporaceae. Verh. Naturhist. Ver Preuss Rheinl 26, 74.

Nonaka K, Kaifuchi S, Ōmura S, Masuma R. 2013 - Five new Simplicillium species (Cordycipitaceae) from soils in Tokyo, Japan. Mycoscience 54(1), 42-53.

Norphanphoun C, Doilom M, Daranagama DA, Phookamsak R et al. 2017 - Revisiting the genus Cytospora and allied species. Mycosphere 8, 51-97.

Norphanphoun C, Jayawardena RS, Chen Y, Wen TC et al. 2019 - Morphological and phylogenetic characterization of novel pestalotioid species associated with mangroves in Thailand. Mycosphere, 10, 531-578.

Norphanphoun C, Raspé O, Jeewon R, Wen TC et al. 2018 - Morphological and phylogenetic characterisation of novel Cytospora species associated with mangroves. MycoKeys (38), 93120.

Oo MM, Oh SK. 2017 - Identification and characterization of new record of grape ripe rot disease caused by Colletotrichum viniferum in Korea. Mycobiology 45, 421-425.

Osorio JA, Crous CJ, De Beer ZW, Wingfield MJ et al. 2017 - Endophytic Botryosphaeriaceae, including five new species, associated with mangrove trees in South Africa. Fungal biology, 121(4) 361-393.

Padhye AA, Amster RL, Browning M, Ewing EP. 1994 - Fatal encephalitis caused by Ochroconis gallopava in a domestic cat. J Med Vet Mycol 32:141-145.

Pang K, Jheng J, Jones EBG. 2011 - Marine mangrove fungi of Taiwan. National Taiwan Ocean University Keelung, Taiwan

Pem D, Hongsanan S, Doilom M, Tibpromma S et al. 2019 - https://www. dothideomycetes. org: An online taxonomic resource for the classification, identification, and nomenclature of Dothideomycetes. Asian Journal of Mycology 2(1), 287-297.

Peng LJ, Sun T, Yang YL, Cai L et al. 2013 - Colletotrichum species on grape in Guizhou and Yunnan provinces, China. Mycoscience 54, 29-41.

Pegler DN, Young TWK. 1986 - Classification of Oudemansiella (Basidiomycota: Tricholomataceae), with special reference to spore structure. Transactions of the British Mycological Society 87, 583-602.

Pennycook SR, Samuels GJ. 1985 - Botryosphaeria and Fusicoccum species associated with ripe fruit rot of Actinidia deliciosa (kiwifruit) in New Zealand. Mycotaxon 24, 445-458.

Perera RH, Hyde KD, Jones EBG, Liu JK et al. 2018 - Additions to wild seed and fruit fungi 2: Parascedosporium putredinis: a new Thailand record from Delonix regia seed pods. Studies in Fungi 3, 192-201.

Petch T. 1924 - Studies in entomogenous fungi. IV. Some Ceylon Cordyceps. Transactions of the British Mycological Society10, 28-45.

Petrak F. 1952 - Beiträge zur Pilzflora von Sierra Leone.

Petrak VF. 1983 - Beiträge zur Kenntnis einiger Asterineen der Philippinen. Sydowia 12, 472-486.

Petrini LE, Petrini O. 2012 - Rosellinia species (Xylariaceae) from South and Central America. An annotated list. Kurtziana 37, 127-139.

Petrini LE. 1992 - Rosellinia species of the temperate zones. Sydowia 44, 169-281.

Petrini LE. 2003 - Rosellinia and related genera in New Zealand. New Zealand Journal of Botany 41, 71-138.

Petrini LE. 2013 - Rosellinia: a world monograph. Gebruder Borntraeger Verlagsbuchhandlung, Science Publishers. 13

Phillips A, Alves A, Correia A, Luque J. 2005 - Two new species of Botryosphaeria with brown, 1-septate ascospores and Dothiorella anamorphs. Mycologia 97, 513-529

Phillips AJ, Hyde KD, Alves A, Liu JK. 2019 - Families in Botryosphaeriales: a phylogenetic, morphological and evolutionary perspective. Fungal diversity 94, 1-22.

Phillips AJL, Alves A, Abdollahzadeh J, Slippers B et al. 2013 - The Botryosphaeriaceae: genera and species known from culture. Study in Mycology 76, 51-167. 
Phillips AJL, Alves A, Pennycook SR, Johnston PR et al. 2008 - Resolving the phylogenetic and taxonomic status of dark-spored teleomorph genera in the Botryosphaeriaceae. Persoonia 21, $29-55$.

Phookamsak R, Hyde KD, Jeewon R, Bhat DJ et al. 2019 - Fungal diversity notes 929-1035: taxonomic and phylogenetic contributions on genera and species of fungi. Fungal Diversity 95, 1-273.

Phookamsak R, Norphanphoun C, Tanaka K, Dai DQ et al. 2015 - Towards a natural classification of Astrosphaeriella-like species; introducing Astrosphaeriellaceae and Pseudoastrosphaeriellaceae fam. nov. and Astrosphaeriellopsis, gen. nov. Fungal Diversity 74, 143-197.

Phookamsak R, Wanasinghe DN, Hongsanan S, Phukhamsakda C et al. 2017 - Towards a natural classification of Ophiobolus and ophiobolus-like taxa; introducing three novel genera Ophiobolopsis, Paraophiobolus and Pseudoophiobolus in Phaeosphaeriaceae (Pleosporales). Fungal diversity 87(1), 299-339.

Phukhamsakda C, Ariyawansa HA, Phillips AJL, Wanasinghe DN et al. 2016 - Additions to Sporormiaceae: Introducing Two Novel Genera, Sparticola and Forliomyces, from Spartium. Cryptogamie Mycologie 37(1), 75-97.

Phukhamsakda C, Jeewon R, McKenzie EHC, Xu JC. 2019 - Morphology and phylogeny of Phaeoseptum mali sp. nov. (Phaeoseptaceae, Pleosporales) on bark of Malus halliana. Asian Journal of Mycology 2(1), 118-128

Pinruan U, Hyde KD, Lumyong S, McKenzie EHC, Jones EBG. 2007 - Occurrence of fungi on tissues of the peat swamp palm Licuala longicalycata. Fungal Divers 25, 157-173

Pirozynski KA. 1963 - Beltrania and related genera. Mycological Papers 90, 1-37.

Prabhugaonkar A, Bhat DJ. 2011 - New record of Megacapitula villosa and Paradictyoarthrinium diffractum from India. Mycosphere 2, 463-467.

Preuss GT. 1851 - Ubersicht untersuchter pilze, besonders aus der umgegend Hoyerswerda. In Linnaea 24, 99-153.

Promputtha I, Jeewon R, Lumyong S, McKenzie EHC et al. 2005 - Ribosomal DNA fingerprinting in the identification of non sporulating endophytes from Magnolia liliifera (Magnoliaceae). Fungal Diversity 20, 167-186.

Promputtha I, Lumyong S, Dhanasekaran V, McKenzie EHC et al. 2007 - A phylogenetic evaluation of whether endophytes become saprotrophs at host senescence. Microbial ecology 53(4), 579-590.

Rabenhorst GL. 1857 - Klotzschii herbarium vivum mycologicum sistens fungorum per totam Germaniam crescentium collectionem perfectam. Editio nova. Centuria VI. Klotzschii Herbarium Vivum Mycologicum 6, 501-600.

Rao VG, Reddy KA. 1981 - Two new Hyphomycetes. Indian Journal of Botany 4, 108-114.

Rashmi M, Kushveer JS, Sarma VV. 2019 - A worldwide list of endophytic fungi with notes on ecology and diversity. Mycosphere 10(1), 798-1079.

Raza M, Zhang ZF, Hyde KD, Diao YZ et al. 2019 - Culturable plant pathogenic fungi associated with sugarcane in southern China. Fungal Diversity, 99(1), 1-104.

Rebentisch JF 1804 - Prodromus Flora Neomarchicae, Schüppel, Berlin.

Réblová M, Gams W, Seifert KA. 2011 - Monilochaetes and allied genera of the Glomerellales, and a reconsideration of families in the Microascales. Studies in Mycology 68(1), 163-191.

Reynolds DR, Gilbert GS. 2005 - Epifoliar fungi from Queensland, Australia Australian. Systematic Botany 18, 265-289.

Rogers JD. 2000 - Thoughts and musings about tropical Xylariaceae. Mycological Research 104, 1412-1420.

Rogers JD, Ju YM, San Martín González F. 1997 - Jumillera and Whalleya, new genera segregated from Biscogniauxia. Mycotaxon 64, 39-50. 
Rossman AY, Seifert KA, Samuels GJ, Minnis AM et al. 2013 - Genera in Bionectriaceae, Hypocreaceae, and Nectriaceae (Hypocreales) proposed for acceptance or rejection. IMA fungus 4, 41-51.

Rossman AY, Crous PW, Hyde KD, Hawksworth DL et al. 2015 - Recommended names for pleomorphic genera in Dothideomycetes. IMA Fungus 6, 507-523.

Rossman AY, Farr DF, Castlebury LA. 2007 - A review of the phylogeny and biology of the Diaporthales. Mycoscience 48, 135-144.

Saccardo PA. 1883 - Flora briologica della Venezia. Stabilimento di G. Antonelli.

Salkin IF, Dixon DM, Kemna ME, Danneman PJ et al. 1990 - Fatal encephalitis caused by Dactylaria constricta var. gallopava in a snowy owl chick (Nectea scandiaca). J Clin Microbiol 28, 2845-2847.

Samarakoon MC, Persoh D, Hyde KD, Bulgakov TS et al. 2018 - Colletotrichum acidae sp nov from northern Thailand and a new record of $C$. dematium on Iris sp. Mycosphere 9(3): 583597.

Samerpitak K, Gerrits van den Ende AHG, Menken SBJ, de Hoog GS. 2015 - Three New Species of the Genus Ochroconis. Mycopathologia 180, 7-17.

Sandoval-Sanchez M, Nieto-Angel D, Sandoval-Islas JS, Teliz-Ortiz D et al. 2013 - Fungi associated to stem-end rot and dieback of mango (Mangifera indica L.). Agrociencia 47, 6173.

Santos L, Alves A, Alves R. 2017 - Evaluating multi-locus phylogenies for species boundaries determination in the genus Diaporthe. PeerJ 5, 3120.

Seaver FJ, Waterston JM. 1946 - Contributions to the Mycoflora of Bermuda-IV. Mycologia 38, 180-201.

Seephueak P, Phongpaichit S, Hyde KD, Petcharat V. 2011 - Diversity of saprobic fungi on decaying rubber logs (Hevea brasiliensis). Sydowia 63, 249-282.

Seifert K, Jones MG, Gams W, Kendrick B. 2011 - The genera of hyphomycetes. CBS biodiversity series no. 9, 1-997.

Senanayake IC, Crous PW, Groenewald JC, Maharachchikumbura SSN et al. 2017 - Families of Diaporthales based on morphological and phylogenetic evidence. Studies in Mycology 86, 217-296.

Senanayake IC, Jeewon R, Chomnunti P, Wanasinghe DN et al. 2018 - Taxonomic circumscription of Diaporthales based on multigene phylogeny and morphology. Fungal Diversity 93, 241443.

Senanayake IC, Maharachchikumbura SSN, Hyde KD, Bhat DJ et al. 2015 - Towards unraveling relationships in Xylariomycetidae (Sordariomycetes). Fungal Diversity 73, 73-144.

Serrato-Diaz LM, Perez-Cuevas M, Rivera-Vargas LI, French-Monar RD. 2013 - First Report of Neofusicoccum parvum Causing Rachis Necrosis of Mango (Mangifera indica) in Puerto Rico. Pl Dis 97, 1381

Shang QJ, Hyde KD, Camporesi E, Maharachchikumbura SSN et al. 2020 - Additions to the genus Cytospora with sexual morph in Cytosporaceae. Mycosphere 11(1), 189-224.

Sharma R, Kulkarni G, Sinawane MS. 2017 - Alanomyces, a new genus of Aplosporellaceae based on four loci phylogeny. Phytotaxa 297, 168-175

Sharma R, Kulkarni G, Sonawane MS, Shouche YS. 2014 - A new endophytic species of Arthrinium (Apiosporaceae) from Jatropha podagrica. Mycoscience 55, 118-123.

Shear CL. 1902 - Mycological notes and new species. Bulletin of the Torrey Botanical Club 29, 449-457.

Shen YM, Liu HL, Huang TC, Pai KF. 2016 - First report of Aurifilum marmelostoma causing cankers on Terminalia mantaly in Taiwan. Journal of General Plant Pathology 82, 216-219.

Sherwood MA. 1977 - The Ostropalean fungi. Mycotaxon 5, 1-277.

Shirouzu T, Hirose D, Tokumasu S, To-Anun C et al. 2010 - Host affinity and phylogenetic position of a new anamorphic fungus Beltraniella botryospora from living and fallen leaves of evergreen oaks. Fungal Diversity 43, 85-92. 
Shivas RG, Tan YP, Edwards J, Dinh Q et al. 2016 - Colletotrichum species in Australia. Australasian Plant Pathology 45, 447-464.

Shoemaker RA. 1959 - Nomenclature of Drechslera and Bipolaris, grass parasites segregated from 'Helminosporium'. Canadian Journal of Botany. 37, 879-887.

Shoemaker RA. 1976 - Canadian and some extralimital Ophiobolus species. Canadian Journal of Botany 54, 2365-2404.

Singh K, Flood J, Welsh RD, Wyckoff JH et al. 2006 - Fatal systemic phaeohyphomycosis caused by Ochroconis gallopavum in a dog (Canis familaris). Vet Pathol 43:988-992.

Singh A, Kumar S, Singh R, Dubey NK. 2012 - A new species of Corynespora causing foliar disease on Ficus religiosa from forest of Sonebhadra, Uttar Pradesh, India. Mycosphere 3(5), $890-892$.

Singtripop C, Hongsanan S, Li JF, de Silva NI et al. 2016 - Chaetothyrina mangiferae sp. nov., a new species of Chaetothyrina. Phytotaxa 255, 21-33

Sivanesan A. 1996 - Corynesporasca caryotae gen. et sp. nov. with a Corynespora anamorph, and the family Corynesporascaceae. Mycol Res 100, 783-788.

Sivanesan A. 1984 - The bitunicate ascomycetes and their anamorphs, J. Cramer, Vaduz.

Slippers B, Boissin E, Phillips AJL, Groenewald JZ et al. 2013 - Phylogenetic lineages in the Botryosphaeriales: A systematic and evolutionary framework. Studies in Mycology 76, 31-49.

Slippers B, Fourie G, Crous PW, Coutinho TA et al. 2004 - Speciation and distribution of Botryosphaeria spp. on native and introduced Eucalyptus trees in Australia and South Africa. Studies in Mycology 50, 343-358

Slippers B, Wingfield MJ. 2007 - Botryosphaeriaceae as endophytes and latent pathogens of woody plants: diversity, ecology and impact. Fungal Biology Reviews 21, 90-106.

Species Fungorum. 2020 - Available from: http://www.speciesfungorum.org/Names/Names.asp

Spegazzini C. 1880 - Fungi argentini. Pugillus tertius (Continuacion). Anales de la Sociedad Científica Argentina 10, 145-168.

Spegazzini C. 1881 - Fungi Argentini. An dela Soc Cient Argentinaica Argentina 179-189:

Spegazzini CL. 1918 - Notas mycological. Physics 4, 281-295.

Stadler M, Kuhnert E, Peršoh D, Fournier J. 2013 - Te Xylariaceae as model example for a unifed nomenclature following the "One fungus-one name" (1F1N) concept. Mycology 4, 5-21.

Stamatakis A, Hoover P, Rougemont J. 2008 - A rapid bootstrap algorithm for the RAxML webservers. Systematic Biology 75, 758-771.

Steyaert RL. 1949 - Contribution à l'étude monographique de Pestalotia de Not. et Monochaetia Sacc.(Truncatella gen. nov. et Pestalotiopsis gen. nov.). Bulletin du Jardin botanique de l'Etat, Bruxelles/Bulletin van den Rijksplantentuin, Brussel, 285-347.

Stevens NE. 1926 - Two species of Physalospora on citrus and other hosts. Mycologia 18, 206-217. Sturm J. 1829 - Deutschlands Flora, Abt. III. Die Pilze Deutschlands 2, 1-136.

$\mathrm{Su}$ H, Li QR, Kang JC, Wen TC et al. 2016a - Rosellinia convexa, sp. nov. (Xylariales, Pezizomycotina) from China. Mycoscience 57, 164-170.

Su HY, Hyde KD, Maharachchikumbura SSN, Ariyawansa HA et al. 2016b - The families Distoseptisporaceae fam. nov., Kirschsteiniotheliaceae, Sporormiaceae and Torulaceae, with new species from freshwater in Yunnan Province, China. Fungal Diversity 80, 375-409.

Su HY, Udayanga D, Luo ZL, Manamgoda DS et al. 2015 - Hyphomycetes from aquatic habitats in Southern China: species of Curvularia (Pleosporaceae) and Phragmocephala (Melannomataceae). Phytotaxa 226, 201-216.

Su XJ, Luo ZL, Jeewon R, Bhat DJ et al. 2018 - Morphology and multigene phylogeny reveal new genus and species of Torulaceae from freshwater habitats in northwestern Yunnan China. Mycological Progress 16(4), 1-15.

Sue PK, Gurda GT, Lee R, Watkins T et al. 2014 - First report of Westerdykella dispersa as a cause of an angioinvasive fungal infection in a neutropenic host. Journal of clinical microbiology, 52(12), 4407-4411.

Subramanian CV. 1994 - Hyphomycetes from South East Asia - novelties from Singapore and 
Malaysia. Kavaka 22/23, 52-76.

Suetrong S, Schoch CL, Spatafora JW, Kohlmeyer J et al. 2009 - Molecular systematics of the marine Dothideomycetes. Studies in Mycology 64, 155-173.

Sun X, Guo L-D, Hyde KD. 2011 - Community composition of endophytic fungi in Acer truncatum and their role in decomposition. Fungal Diversity 47, 85-95.

Sung GH, Hyweljones NL, Sung JM, Luangsaard JJ et al. 2007 - Phylogenetic classification of Cordyceps and the clavicipitaceous fungi. Studies in Mycology 57 (57), 5-59.

Sydow H, Sydow P, Butler EJ. 1916 - Fungi Indiae orientalis pars V. Annales Mycologici. 14(3-4), 177-220.

Sydow H, Sydow P. 1913 - Novae fungorum species - X. Annales Mycologici. 11, 254-271

Sysouphanthong P, Guo J, Hyde KD, Xu J, Mortimer PE. 2016 - Lepiota thailandica (Agaricaceae), a new species from Thailand. Phytotaxa 245, 262-270.

Sysouphanthong P, Hyde KD, Vellinga EC, Chukeatirote E. 2013 - Diversity of Lepiota (Agaricales) in northern Thailand. Mycology 4, 22-28.

Sysouphanthong P, Hyde KD, Chukeatirote E, Bahkali AH et al. 2011b - Lepiota (Agaricales) in northern Thailand-1 Lepiota section Stenosporae. Mycotaxon 117, 53-85.

Sysouphanthong P, Hyde KD, Chukeatirote E, Vellinga EC. 2011a - A review of genus Lepiota and its distribution in Asia. Current Research in Environmental \& Applied Mycology 1, 161-176.

Sysouphanthong P. Hyde KD, Chukeatirote E, Bahkali AH, Vellinga EC. 2012 - Lepiota Agaricales in northern Thailand-2 Lepiota section Lepiota. Cryptogamie Mycologie 33, 25-42.

Tanaka K, Endo M, Hirayama K, Okane I et al. 2011 - Phylogeny of Discosia and Seimatosporium, and introduction of Adisciso and Immersidiscosia genera nova. Persoonia: Molecular Phylogeny and Evolution of Fungi 26, 85-98.

Tanaka K, Harada Y. 2003 - Pleosporales in Japan (2): the genus Lophiotrema. Mycoscience 44, $115-121$.

Tanaka K, Harada Y. 2005 - Bambusicolous fungi in Japan (4): a new combination, Astrosphaeriella aggregata. Mycoscience 46, 114-118.

Tanaka K, Hirayama K, Yonezawa H, Hatakeyama S et al. 2009 - Molecular taxonomy of bambusicolous fungi: Tetraplosphaeriaceae, a new pleosporalean family with Tetraploa-like anamorphs. Studies in Mycology 64, 175-209.

Tanaka K, Hirayama K, Yonezawa H, Sato G. 2015 - Revision of the Massarineae (Pleosporales, Dothideomycetes). Studies in Mycology 82, 75-36.

Tang AM, Jeewon R, Hyde KD. 2005 - Succession of microfungal communities on decaying leaves of Castanopsis fissa. Canadian journal of microbiology 51, 967-974.

Tang AMC, Jeewon R, Hyde KD. 2007 - Phylogenetic utility of protein (RPB2, B-tubulin) and ribosomal $(18 \mathrm{~S}, 28 \mathrm{~S})$ gene sequences in the systematics of Sordariomycetes (Ascomycota, Fungi). Antonie van Leeuwenhoek 91, 327-349.

Tanney JB, Douglas B, Seifert KA. 2016 - Sexual and asexual states of some endophytic Phialocephala species of Picea. Mycologia 108(2), 255-280.

Tekauz A. 1983 - Reaction of Canadian barley cultivars to Pyrenophora graminea, in the incitant of leaf stripe. Canadian Journal of Physiology and Pharmacology 5, 294-301.

Tennakoon DS, Jeewon R, Kuo CH, Hyde KD. 2018 - Phylogenetic and morphology characterization of Byssosphaeria macarangae sp. and B. musae sp. from Macaranga tanarius. Phytotaxa 364, 211-226.

Tennakoon DS, Phillips AJL, Phookamsak R, Ariyawansa HA et al. 2016 - Sexual morph of Lasiodiplodia pseudotheobromae (Botryosphaeriaceae, Botryosphaeriales, Dothideomycetes) from China. Mycosphere 7(7), 990-1000.

Tennakoon DS, Phookamsak R, Wanasinghe DN, Yang JB et al. 2017 - Morphological and phylogenetic insights resolve Plenodomus sinensis (Leptosphaeriaceae) as a new species. Phytotaxa 324 (1), 073-082. 
Thambugala KM, Bulgakov TS, Eungwanichayapant PD, Liu ZY, Hyde KD. 2016a Camarosporium uniseriatum nom. nov., from Celtis occidentalis in European Russia. Studies in Fungi 1, 90-98.

Thambugala KM, Hyde KD, Eungwanichayapant PD, Romero AI et al. 2016b - Additions to the Genus Rhytidhysteron in Hysteriaceae. Cryptogamie Mycologie 37, 99-116.

Theissen F, Sydow H. 1915 - Die Dothideales. Kritisch-systematische Originaluntersuchungen. Annales mycologici 13, 147-746.

Tian Q, Liu JK, Hyde KD, Wanasinghe DN et al. 2015 - Phylogenetic relationships and morphological reappraisal of Melanommataceae (Pleosporales). Fungal Diversity 74, 267324.

Tibpromma S, Hyde KD, Jeewon R, Maharachchikumbura SSN et al. 2017 - Fungal diversity notes 491-602: taxonomic and phylogenetic contributions to fungal taxa. Fungal Diversity 83, 1261.

Tibpromma S, Hyde K, Bhat J, Mortimer P et al. 2018a - Identification of endophytic fungi from leaves of Pandanaceae based on their morphotypes and DNA sequence data from southern Thailand. MycoKeys 33, 25-67.

Tibpromma S, Hyde KD, McKenzie EH, Bhat DJ et al. 2018b - Fungal diversity notes 840-928: micro-fungi associated with Pandanaceae. Fungal Diversity 93, 1-60.

Trakunyingcharoen T, Lombard L, Groenewald JZ, Cheewangkoon R et al. 2015 - Caulicolous Botryosphaeriales from Thailand. Persoonia 34, 87-99.

Tode HJ. 1791 - Fungi Mecklenburgenses Selecti. 2, 1-64.

Torres MS, Bergen M, Singh S, Bischoff J et al. 2005 - Plenodomus morganjonesii sp. nov. and a discussion of the genus Plenodomus. Mycotaxon 93, 333-344.

Tuckerman E. 1847 - A synopsis of the lichenes of the northern United States and British America. Proceedings of the American Academy of Arts and Sciences 1, 195-285.

Tulasne LR, Tulasne C. 1863 - Selecta Fungorum Carpologia, Tomus Secundus. Xylariei - ValseiSphaeriei 2, 1-319.

U'Ren JM, Miadlikowska J, Zimmerman NB, Lutzoni F et al. 2016 - Contributions of North American endophytes to the phylogeny, ecology, and taxonomy of Xylariaceae (Sordariomycetes, Ascomycota). Molecular Phylogenetics \& Evolution 98, 210-232.

Udayanga D, Castlebury LA, Rossman AY, Chukeatirote E, et al. 2015 -The Diaporthe sojae species complex: Phylogenetic re-assessment of pathogens associated with soybean, cucurbits and other field crops. Fungal Biology, 119(5), 383-407.

Udayanga D, Castlebury LA, Rossman AY, Hyde KD. 2014 - Species limits in Diaporthe: molecular re-assessment of $D$. citri, D. cytosporella, D. foeniculina and D. rudis. Persoonia 32, 83-101.

Úrbez-Torres JR, Gubler WD, Luque J. 2007 - First report of Botryosphaeria iberica and $B$. viticola associated with grapevine decline in California. Plant Disease, 91(6), 772-772.

Valenzuela-Lopez N, Cano-Lira JF, Guarro J, Sutton DA et al. 2018 - Coelomycetous Dothideomycetes with emphasis on the families Cucurbitariaceae and Didymellaceae. Studies in Mycology 90, 1-69.

Van Niekerk JM, Crous PW, Groenewald JZ, Fourie PH et al. 2004 - DNA phylogeny, morphology and pathogenicity of Botryosphaeria species on grapevines. Mycologia 96(4), 781-798.

Vanev SG. 1991 - Species conception and sections delimitation of genus Discosia. Mycotaxon 41, 387-396.

Vellinga EC, Sysouphanthong S, Hyde KD. 2011 - The family Agaricaceae: phylogenies and two new white-spored genera. Mycologia 103, 494-509.

Vellinga EC. 2000 - Notes on Lepiota and Leucoagaricus: Type studies on Lepiota magnispora, Lepiota barssii, and Agaricus americanus. Mycotaxon 76, 429-438.

Vellinga EC. 2001 - Agaricaceae. In: Noordeloos ME, Kuyper TW, Vellinga EC, eds. Flora Agaricina Neerlandica 5. Rotterdam: A.A. Balkema Publishers 76-151. 
Vellinga EC. 2003 - Phylogeny of Lepiota (Agaricaceae) - evidence from nrITS and nrLSU sequences. Mycological Progress 2, 305-322.

Vellinga EC. 2004 - Genera in the family Agaricaceae - evidence from nrITS and nrLSU sequences. Mycological Research 108, 354-377.

Veloso JS, Camara MPS, Lima WG, Michereff SJ, Doyle VP. 2018 - Why species delimitation matters for fungal ecology: Colletotrichum diversity on wild and cultivated cashew in Brazil. Fungal Biology 122, 677-691.

Valenzuela-Lopez N, Sutton DA, Cano-Lira JF, Paredes K et al. 2017 - Coelomycetous fungi in the clinical setting: morphological convergence and cryptic diversity. Journal of clinical microbiology 55, 552-567.

Valenzuela-Lopez N, Cano-Lira JF, Guarro J, Sutton DA et al. 2018 - Coelomycetous Dothideomycetes with emphasis on the families Cucurbitariaceae and Didymellaceae. Studies in mycology 90, 1-69.

Verkley GJM, da Silva M, Wicklow DT, Crous PW. 2004 - Paraconiothyrium, a new genus to accommodate the mycoparasite Coniothyrium minitans, anamorphs of Paraphaeosphaeria, and four new species. Studies in Mycology 50, 323-335.

Verkley GJM, Dukik K, Renfurm R, Göker M, Stielow JB. 2014 - Novel genera and species of coniothyrium-like fungi in Montagnulaceae (Ascomycota). Persoonia: Molecular Phylogeny and Evolution of Fungi 32, 25-51.

Vermeulen M, Gryzenhout M, Wingfield MJ, Roux J. 2011 - New records of the Cryphonectriaceae from southern Africa including Latruncellus aurorae gen. sp. nov. Mycologia 103, 554-569.

Voglmayr H, Jaklitsch WM. 2017 - Corynespora, Exosporium and Helminthosporium revisited New species and generic reclassification. Stud Mycol 87, 43-76.

Voglmayr H, Jaklitsch WM. 2011 - Molecular data reveal high host specificity in the phylogenetically isolated genus Massaria (Ascomycota, Massariaceae). Fungal Diversity 46, 133-170.

von Ranojevic N. 1914 - Dritter beitrag zur pilzflora serbiens. Annales Mycologici 12, 393-421.

Vu D, Groenewald M, De Vries M, Gehrmann T et al. 2019 - Large-sale generation and analysis of filamentous fungal DNA barcodes boosts coverage for kingdom fungi and reveals thresholds for fungal species and higher taxon delimitation. Studies in Mycology 92, 135-154.

Wallroth CFW. 1833 - Flora Cryptogamica Germaniae 2, 1-923.

Wanasinghe DN, Camporesi E, Hu DM. 2016 - Neoleptosphaeria jonesii sp nov., a novel saprobic sexual species. Leptosphaeriaceae. Mycosphere 7, 1368-1377.

Wanasinghe DN, Hyde KD, Jeewon R, Crous PW et al. 2017 - Phylogenetic revision of Camarosporium (Pleosporineae, Dothideomycetes) and allied genera. Studies in Mycology 87, 207-256.

Wanasinghe DN, Jeewon R, Jones EBG, Boonmee S et al. 2018a - Novel palmicolous taxa within Pleosporales: multigene phylogeny and taxonomic circumscription. Mycological Progress 17, 571-590.

Wanasinghe DN, Phukhamsakda C, Hyde KD, Jeewon R et al. 2018b - Fungal diversity notes 709839: taxonomic and phylogenetic contributions to fungal taxa with an emphasis on fungi on Rosaceae. Fungal Diversity 89, 1-238.

Wang HC. 2004 - Lepiota cortinarius, a species new to China. Mycosystema 23, 439-440.

Wang M, Liu F, Crous PW, Cai L. 2017 - Phylogenetic reassessment of Nigrospora: ubiquitous endophytes, plant and human pathogens. Persoonia: Molecular Phylogeny and Evolution of Fungi 39, 118.

Wang L, Zhang W, Hu B, Chen Y et al. 2008 - Genetic variation of Cordyceps militaris and its allies based on phylogenetic analysis of rDNA ITS sequence data. Fungal Diversity 31, 147155. 
Wedin M, Döring H, Könberg K, Gilenstam G. 2005 - Generic delimitations in the amily Stictidaceae (Ostropales, Ascomycota): The Stictis-Conotrema problem. Lichenologist 37, $67-75$.

Wei DP, Wanasinghe DN, Chaiwat TA, Hyde KD. 2018 - Lecanicillium uredinophilium known from rusts, also occurs animal hosts with chitinous bodies. Asian Journal Mocology 1, 63-73.

Weir BS, Johnston PR, Damm U. 2012 - The Colletotrichum gloeosporioides species complex. Studies in Mycology 73, 115-180.

Wendt L, Sir EB, Kuhnert E, Heitkämper S et al. 2017- Resurrection and emendation of the Hypoxylaceae, recognised from a multigene phylogeny of the Xylariales. Mycological Progress, 1-40.

Whalley AJS. 1996 - The Xylariaceous way of life. Mycological Research 100, 897-922.

Wijayawardene NN, Crous PW, Kirk PM, Hawksworth DL et al. 2014 - Naming and outline of Dothideomycetes-2014 including proposals for the protection or suppression of generic names. Fungal Diversity 69, 1-55.

Wijayawardene NN, Hyde KD, Lumbsch HT, Liu JK et al. 2018 - Outline of Ascomycota: 2017. Fungal Diversity 88, 167-263.

Wijayawardene NN, Hyde KD, Rajeshkumar KC, Hawksworth DL et al. 2017 - Notes for genera: Ascomycota. Fungal Diversity 86, 1-594.

Wijayawardene NN, Hyde KD, Wanasinghe DN, Papizadeh M et al. 2016 - Taxonomy and phylogeny of dematiaceous coelomycetes. Fungal Diversity 77, 1-316.

Wijayawardene NN, Hyde KD, Al-Ani LKT, Tedersoo L et al. 2020 - Outline of Fungi and fungilike taxa. Mycosphere 11, 1060-1456.

Winter G. 1886 - Fungi Australienses. Revue Mycologique Toulouse 8, $207-213$.

Wong PTW, Dong C, Martin PM, Sharp PJ. 2015 - Fairway patch - a serious emerging disease of couch (syn. bermudagrass) [Cynodon dactylon] and kikuyu (Pennisetum clandestinum) turf in Australia caused by Phialocephala bamuru P.T.W. Wong \& C. Dong sp. nov. Australasian Plant Pathology 44(5), 545-555.

Woudenberg JHC, Aveskamp MM, de Gruyter J, Spiers AG, Crous PW. 2009 - Multiple Didymella teleomorphs are linked to the Phoma clematidina morphotype. Persoonia 22, 5662.

Xie L, Chen YL, Long YY, Zhang Y et al. 2019 - Three new species of Conlarium from sugarcane rhizosphere in southern China. Mycokeys 56, 1-11.

Yacharoen S, Tian Q, Chomnunti P, Boonmee S et al. 2015 - Patellariaceae revisited. Mycosphere 6, 290-326.

Yan JY, Jayawardena MMRS, Goonasekara ID, Wang Y et al. 2015 - Diverse species of Colletotrichum associated with grapevine anthracnose in China. Fungal diversity 71(1), 233246.

Yang C, Xu XL, Wanasinghe DN, Jeewon R et al. 2019 - Neostagonosporella sichuanensis gen. et sp. nov. (Phaeosphaeriaceae, Pleosporales) on Phyllostachys heteroclada (Poaceae) from Sichuan Province, China. MycoKeys 46, 119-150.

Yang H, Chomnunti P, Ariyawansa H, Wu HX et al. 2014 - The genus Phaeosaccardinula (Chaetothyriales) from Yunnan, China, introducing two new species. Chiang Mai Journal of Science 41, 873-884.

Yang J, Liu JK, Hyde KD, Jones EBG, Liu ZY. 2018 - New species in Dictyosporium, new combinations in Dictyocheirospora and an updated backbone tree for Dictyosporiaceae. MycoKeys 36, 83-105.

Yang T, Groenewald JZ, Cheewangkoon R, Jami F et al. 2017 - Families, genera, and species of Botryosphaeriales. Fungal Biology 121, 322-246.

Yang YL, Liu ZY, Cai L, Hyde KD et al. 2009 - Colletotrichum anthracnose of Amaryllidaceae. Fungal Diversity 39, 123-146.

Yarita K, Sano A, Murata Y, Takayama A et al. 2007 - Pathogenicity of Ochroconis gallopava isolated from hot spring in Japan and a review of published reports. Mycopathologia 164, 
$135-147$.

You CJ, Liu X, Li LX, Tsui CK, Tian CM. 2017 - Dothiorella magnoliae, a new species associated with dieback of Magnolia grandiflora from China. Mycosphere 8, 1031-1041.

Zare R, Gams W. 2016 - More white verticillium-like anamorphs with erect conidiophores. Mycological Progress 15, 993-1030.

Zeng M, Huang SK, Hyde KD, Zhao Q. 2018 - Helicascus alatus (Morosphaeriaceae), a new freshwater species from south-western China. Phytotaxa 351, 210-218.

Zeng XY, Wu HX, Hongsanan S, Jeewon R et al. 2019 - Taxonomy and the evolutionary history of Micropeltidaceae. Fungal Diversity 97, 393-436.

Zha LS, Wen TC, Huang SK, Boonmee S et al. 2019 - Taxonomy and biology of Cordyceps qingchengensis sp. nov. and its allies. Phytotaxa 416 (1), 14-24.

Zhang G, Berbee ML. 2001 - Pyrenophora phylogenetics inferred from ITS and glyceradehyde-3phosphate dehydrogenase gene sequences. Mycologia 93, 1048-1063.

Zhang H, Dong W, Hyde KD, Maharachchikumbura SSN et al. 2017 - Towards a natural classification of Annulatascaceae-like taxa: introducing Atractosporales ord. Nov. and six new families. Fungal Diversity 85, 75-110.

Zhang Y, Jeewon R, Fournier J, Hyde KD. 2008 - Multi-gene phylogeny and morphotaxonomy of Amniculicola lignicola: novel freshwater fungus from France and its relationships to the Pleosporales. Fungal Biology 112, 1186-1194.

Zhang JF, Liu JK, Jeewon R, Wanasinghe DN, Liu ZY. 2019a - Fungi from Asian Karst formations III. Molecular and morphological characterization reveal new taxa in Phaeosphaeriaceae. Mycosphere 10, 202-220.

Zhang JF, Liu JK, Ran HY, Khongphinitbunjong K, Liu ZY. 2018 - A new species and new record of Lophiotrema (Lophiotremataceae, Dothideomycetes) from karst landforms in southwest China. Phytotaxa 379, 169-179.

Zhang Z, Liu F, Zhou X, Liu X, Liu S, Cai L. 2017 - Culturable mycobiota from Karst caves in China, with descriptions of 20 new species. Persoonia-Molecular Phylogeny an Evolution of Fungi 39, 1-31.

Zhang LQ, Jiang S, Meng JJ, An HS et al. 2019b - First report of leaf spot caused by Nigrospora oryzae on blueberry in Shanghai, China. Plant Diseases 103(9), 2473-2474.

Zhang M, He W, Wu JR, Zhang Y. 2016 - Two new species of Spencermartinsia (Botryosphaeriaceae, Botryosphaeriales) from China. Mycosphere 7, 942-949.

Zhang N, Castlebury LA, Miller AN, Huhndorf SM et al. 2006 - An overview of the systematics of the Sordariomycetes based on a four gene phylogeny. Mycologia 98, 1076-1087.

Zhang Y, Crous PW, Schoch CL, Hyde KD. 2012 - Pleosporales. Fungal Diversity 53, 1-221.

Zhang Y, Schoch CL, Fournier J, Crous PW et al. 2009 - Multi-locus phylogeny of Pleosporales: a taxonomic, ecological and evolutionary re-evaluation. Studies in Mycology 64, 85-102.

Zhao H, Liu HY, Yang XS, Liu YX et al. 2014 - First report of Nigrospora leaf blight on sesame caused by Nigrospora sphaerica in China. Plant Disease 98, 842.

Zheng HD, Zhuang WY. 2017 - Chlorovibrissea korfii sp. nov. from northern hemisphere and Vibrissea flavovirens new to China. MycoKeys 26, 1-11.

Zhou DQ, Cai L, Hyde KD. 2003 - Astrosphaeriella and Roussoella species on bamboo from Hong Kong and Yunnan, China, including a new species of Roussoella. Cryptogamie Mycologie 24, 191-197.

Zhuang WY. 2001 - Higher Fungi of Tropical China. Mycotaxon, Ltd., Ithaca, NY 485.

Zimmerman A. 1902 - Ueber einige an tropischen Kulturpflanzen beobachtete Pilze III. Zentralblatt für Bakteriologie, Parasitenkunde 8, 216-221. 